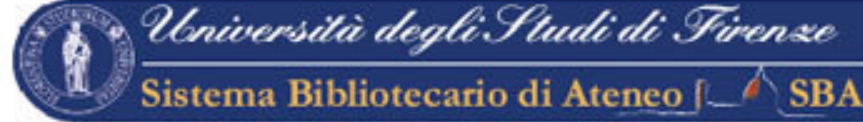

\section{Dalla misurazione dei servizi alla customer satisfaction}

La valutazione della qualità nel Sistema Bibliotecario di Ateneo dell'Università di Firenze

a cura di Roberto Ventura

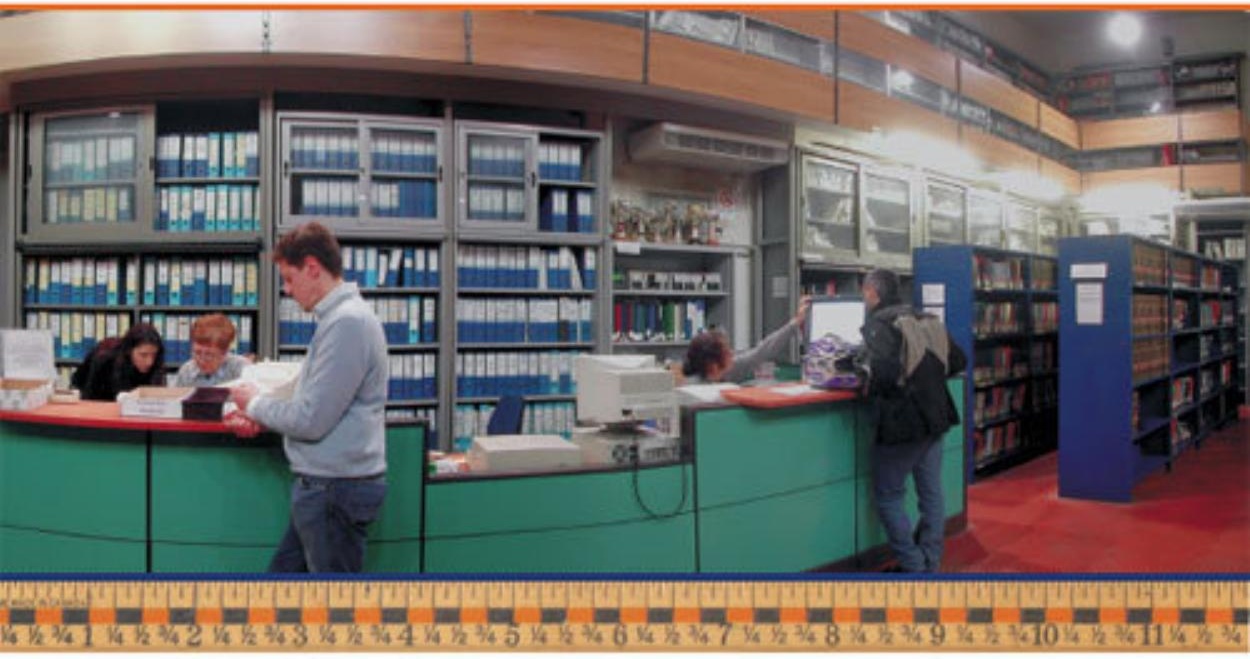




\title{
Dalla misurazione dei servizi alla customer satisfaction
}

\section{La valutazione della qualità nel Sistema Bibliotecario di Ateneo dell'Università di Firenze}

\author{
A cura di \\ ROBERTO VENTURA
}

Testi di

Silvana Benedetti, Eleonora Giusti, Francesca Landi, M. Giulia Maraviglia, Chiara Melani, Roberto Ventura

Firenze University Press

2004 
Dalla misurazione dei servizi alla customer satisfaction : la valutazione della qualità nel Sistema Bibliotecario di Ateneo dell'Università di Firenze/ a cura di Roberto Ventura. - Firenze : Firenze University Press, 2004.

http://digital.casalini.it/fulltext/is.asp?isbn=8884531489

Stampa a richiesta disponibile su http://epress.unifi.it

ISBN 88-8453-148-9 (online)

ISBN 88-8453-147-0 (print)

025.5 (ed. 20)

Biblioteche-Firenze-Università

Progetto grafico di copertina: Gabriella Migliore

In copertina: Biblioteca di Scienze tecnologiche, sede di Ingegneria

(C) 2004 Firenze University Press

Università degli Studi di Firenze

Firenze University Press

Borgo Albizi, 28, 50122 Firenze, Italy 


\section{SOMMARIO}

M. Giulia Maraviglia

Misurare e valutare: uno strumento per la gestione $\ldots \ldots \ldots \ldots \ldots$

Chiara Melani

L'esperienza del Sistema Bibliotecario dell'Ateneo fiorentino e

l'indagine sulla soddisfazione dell'utenza . . . . . . . . . . . . . 11

Roberto Ventura

Un'indagine sull'uso della statistica bibliotecaria nel Sistema

Bibliotecario di Ateneo . ......................... 27

Silvana Benedetti

Aspetti tecnici e metodologici di un'indagine di qualità . . . . . . . . 55

Francesca Landi

L'indagine sulla soddisfazione degli utenti del Sistema bibliotecario

dell'Università degli studi di Firenze: analisi dei risultati e studio

delle frequenze ........................... 73

Roberto Ventura

L'indagine sulla soddisfazione degli utenti del Sistema Bibliotecario di Ateneo dell'Università degli Studi di Firenze: metodologia e valutazione

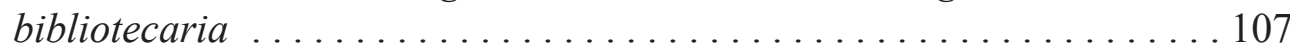

Eleonora Giusti

Analisi di un case study: la Biblioteca di Scienze Sociali . . . . . . . . . 165

Appendice A

Dati e indicatori in uso presso il Sistema Bibliotecario di Ateneo

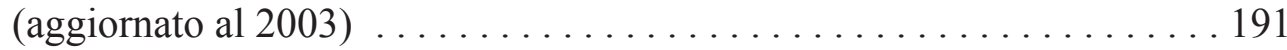

Appendice B

Questionari dell'indagine sulla soddisfazione degli utenti $\ldots \ldots \ldots 197$ 
Appendice C

Spoglio statistico dei dati del Sistema Bibliotecario di Ateneo . . . . . . 215

Appendice D

Stima degli errori di campionamento dell'indagine sulla soddisfazione degli utenti . . . . . . . . . . . . . . . . . . . . . . . . 249

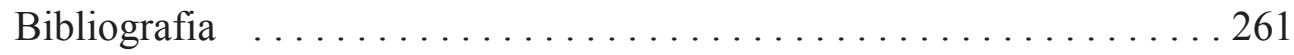




\title{
Misurare e valutare: uno strumento per la gestione
}

\author{
di M. Giulia Maraviglia
}

\begin{abstract}
Ma una biblioteca per quanto si voglia copiosa, se si vuole istruttiva, non conterrà molti libri. I libri son come gli uomini: non la moltiplicità, ma la scelta fa il loro pregio.

F. Milizia. Principi di architettura civile
\end{abstract}

Siamo bravi bibliotecari? La biblioteca in cui lavoriamo parla per noi. La qualità del lavoro restituisce qualità al servizio. La produttività fine a se stessa produce efficienza ma non efficacia, il "fare" da solo non determina la qualità del servizio e della struttura che lo eroga, è il "saper fare" che introduce l'elemento qualità. E il "saper fare" non può prescindere dalla conoscenza di ciò che l'utenza si aspetta dal servizio, di ciò che l'utenza ritiene essere di primaria importanza, di quanto sia ritenuto utile e apprezzato il servizio reso. La biblioteca non è un organismo autoreferenziale: la sua funzionalità si basa sulla interazione con l'utenza.

È questa la scommessa più difficile da vincere: dialogare con la propria utenza per raggiungere un buon livello qualitativo nei servizi sapendo di non poter disporre di risorse aggiuntive. La limitatezza delle risorse sia umane sia finanziarie è una amara certezza del nostro tempo.

In ambito accademico da tempo siamo passati dalle cosiddette "risorse vincolate" al "vincolo delle risorse". Ottenere buoni risultati è oggi molto difficile se non si applicano metodi gestionali innovativi che fondano gran parte della loro credibilità nello strumento della misurazione e della valutazione che come tale è, in molte strutture, divenuto prassi di lavoro.

Il Sistema Bibliotecario dell'Università di Firenze utilizza questo strumento dalla sua prima applicazione, nel $1996^{1}$.

In quegli anni ha iniziato a diffondersi anche in Italia la "cultura della misurazione" intesa come uno strumento di sostegno delle scelte politico-

1 Tammaro \& Landi 1997. 
strategiche, di supporto e di indirizzo per le scelte gestionali più che uno strumento di controllo dell'operato del gestore e del suo staff.

Alla esperienza dell'Università di Firenze, negli stessi anni si aggiungeva quella dell'ateneo di Sassari ${ }^{2}$ mentre la letteratura nazionale e internazionale affrontava con sempre maggiore dettaglio e analisi, metodi, strumenti e standard già sperimentati e adottati da altri paesi per rendere la prassi della rilevazione un percorso guidato e gettare le basi per poi poter avviare dialoghi e confronti tra strutture analoghe.

Oggi possiamo affermare che il processo legato alla misurazione e alla valutazione, seppur spesso condotto in maniera scoordinata tra le istituzioni che lo promuovono, ha acquisito un'importanza strategica soprattutto quando l'analisi dei risultati si ripercuote seriamente sull'attività di programmazione influendo nella definizione degli obiettivi, nella assegnazione delle risorse umane e finanziarie, nella organizzazione del lavoro e dei servizi.

Ma, ad oggi, nel Sistema Bibliotecario fiorentino, tali rilevazioni hanno affrontato l'aspetto quantitativo del problema, hanno isolato il dato rapportato al contesto. Per questo è stato necessario individuare una serie di indicatori che di anno in anno ci hanno consentito confronti credibili e comprensibili con il rendimento degli anni precedenti permettendoci di tracciare un percorso di sviluppo dei servizi nel corso del tempo. La rilevazione della qualità è stata affidata all'analisi dello sviluppo quantitativo del servizio associando alla crescita delle transazioni, relative soprattutto ai servizi di front office, l'ipotesi del gradimento da parte dell'utenza.

Il processo è ormai divenuto prassi di lavoro. La raccolta e l'elaborazione annuale dei dati sono divenute un supporto vitale per meglio operare le scelte gestionali che investono il Sistema bibliotecario e le sue biblioteche, dalla organizzazione dei servizi allo sviluppo delle tecnologie e della formazione del personale.

Anche le rilevazioni più semplici come quelle che derivano dalla quantificazione del numero dei prestiti possono essere fonte di preziose notizie per indirizzare le scelte dei responsabili. Il numero dei prestiti indica in generale il livello di circolazione della collezione, la percentuale del patrimonio documentale che viene consultato dall'utenza. A sua volta, se legato al numero di utenti che hanno usufruito del servizio ci dice anche la percentuale di utilizzo delle biblioteche rispetto al numero complessivo degli utenti, i così detti utenti potenziali.

\footnotetext{
2 Pilia 1997.
} 
Per esempio una rilevazione dei prestiti effettuati sulla documentazione acquisita negli ultimi tre anni ci darà la possibilità di valutare quanto lo sviluppo della collezione è venuto incontro alle esigenze dell'utenza e quanto eventualmente esso può essere modificato.

All'interno di ciascuna biblioteca la scomposizione del dato può a sua volta introdurre preziosi elementi per definire le "regole" dei servizi.

Ancora, per rimanere nell'esempio del prestito, questo servizio si scompone a sua volta in prestito esterno, prestito interno (per la lettura in sede) a volte in prestito notturno, ovvero la procedura che consente all'utente di portarsi a casa il libro, altrimenti escluso dal servizio, in concomitanza della chiusura della biblioteca.

L'analisi dei dati derivanti dalla scomposizione del prestito nelle varie tipologie associata a sua volta al numero di utenti che ne hanno usufruito nonché alla rilevazione della percentuale rispetto al dato complessivo (volumi acquisiti nell'anno/volumi prestati, prestiti per la lettura/patrimonio complessivo della biblioteca escluso dal prestito) possono darci spunti chiari per la gestione e l'incremento della collezione.

In tutto ciò giocano un ruolo molto importante sia l'utilizzo della componente informatica (il dato è tanto più affidabile quanto prodotto meccanicamente), sia la valorizzazione delle competenze del bibliotecario in ambito socio-statistico.

Dalla rilevazione applicata ai servizi di front office, e quindi associata al contesto dei propri utenti, dunque, si possono ricavare dati per determinare gli indici di utilizzo e quindi entrare, se pur in maniera parziale, in quel mondo ben più complesso che determina la qualità dei servizi.

Entrare nel terreno della misurazione della qualità significa tuttavia entrare nel mondo delle indagini sull'utenza, della interazione con quelle che sono le aspettative sui servizi e la loro effettiva corrispondenza. Significa estendere la competenza bibliometrica a fattori più strettamente sociologici o comunque significa poter disporre della consulenza statistica e sociologica, significa avviare un processo di raccolta dei dati e di analisi degli stessi fortemente condizionato dal contesto in cui si opera. Da qui la maggiore complessità e onerosità del processo.

L'acquisizione completa della conoscenza dell'ambiente in cui si opera, la formula quantità/qualità è in ogni caso l'obiettivo cui tendere nella definizione del processo di misurazione e valutazione. Fatto questo sarà più facile anche definire la carta dei servizi per i propri utenti. 
Per quanto l'analisi delle "performances di rendimento" produca dunque un risultato utile per chi utilizza i dati a supporto delle scelte decisionali e per chi deve politicamente dare un valore all'operato del Sistema nel suo complesso, rimane evidente la assoluta limitatezza di tale procedimento rispetto a due fondamentali considerazioni: la limitazione delle rilevazioni e dell'analisi al contesto locale; l'assenza di un processo valutativo indirizzato alla rilevazione del gradimento da parte dell'utente coinvolgendo quest'ultimo in maniera diretta in modo da determinare il livello di efficacia nei servizi resi associato al livello di efficienza.

Ovvero rimane valida l'analisi del confronto del rendimento del Sistema anno per anno ma pesa l'assenza del confronto con altri Sistemi bibliotecari, rimane aperto il problema di individuare gli esempi cui tendere nel definire gli obiettivi da raggiungere nella redazione del piano di sviluppo del Sistema stesso. E per poter avviare un processo più ampio che introduca il tema del dialogo e del confronto è fondamentale fondare il processo sugli stessi procedimenti attuativi che vanno dalla definizione dei dati e degli indicatori al metodo della rilevazione nonché alla procedura dell'analisi per una valutazione omogenea tra strutture diverse.

La prima indagine condotta su molti atenei italiani dall'allora Osservatorio per la valutazione del sistema universitario del $\mathrm{MURST}^{3}$ ha prodotto un risultato incoraggiante coinvolgendo biblioteche e sistemi bibliotecari italiani nel periodo compreso tra il 1995 e il 1997. È stato il punto di partenza non solo per definire un quadro dei Sistemi Bibliotecari universitari nazionali, ma anche per dare a ciascuno di essi la traccia su cui impostare la propria attività di misurazione e valutazione del rendimento.

L'assenza da allora di una prosecuzione dell'indagine, di una sua affermazione costante negli anni ha finito per rendere quei dati obsoleti e tradito le aspettative degli attori interessati al processo che avrebbero auspicato una sede permanente a livello nazionale, un ufficio aperto per la valorizzazione del sistema-biblioteca nazionale nella consapevolezza che si rende noto e si valuta ciò che realmente interessa. L'abbandono della rilevazione dei dati e dell'analisi finisce per far cadere la tensione costruttiva intorno a un ambito di investimento e di sviluppo riaprendo quell'antipatica forbice che ormai contraddistingue la politica culturale del nostro paese rispetto a quella della maggior parte degli altri paesi europei, per non citare l'esempio americano.

3 Ministero dell'Università e della Ricerca Scientifica e Tecnologica 1999. 
E nell'attesa di istituzionalizzare il processo a livello nazionale per i sistemi e le biblioteche delle università, proseguono le iniziative. Il Comitato nazionale per la valutazione del sistema universitario istituito all'interno del MIUR nel 1999 ha sostituito l'Osservatorio (Legge 19 ottobre 1999 n. 370, "Disposizioni in materia di Università e Ricerca scientifica tecnologica", art. 2). La CRUI ha dato vita ad un gruppo di lavoro per la valutazione e la misurazione delle biblioteche universitarie, mentre, a livello interuniversitario, per iniziativa dell'Università di Padova, si è creato il gruppo GIM (Gruppo Interuniversitario per il Monitoraggio dei sistemi bibliotecari) ${ }^{4}$ cui il Comitato Nazionale per la Valutazione del sistema universitario ha affidato la ricerca "Misurazione e valutazione dell'offerta bibliotecaria degli Atenei italiani".

Incoraggia il fatto che la ricerca si ponga nella stessa linea di sviluppo dell'attività condotta dall'Osservatorio in modo da venire incontro alla pesante assenza di una continuazione di quella indagine e alla definizione quindi di un processo permanente di rilevazione, una delle necessità più sentite dalle strutture bibliotecarie universitarie e ancora di più dai sistemi bibliotecari che nella loro logica sistemica individuano nella rilevazione, nell'analisi e nella valutazione dei dati una delle attività permanenti più importanti.

L'indagine sulla qualità dei servizi condotta all'interno del Sistema Bibliotecario dell'ateneo fiorentino ha sicuramente evidenziato l'aspetto sperimentale della procedura. Ma, nella ormai consolidata prassi operativa che rileva di anno in anno i dati quantitativi legati alle performances di rendimento dei servizi, ha significato ribaltare la concezione della efficacia e della efficienza del servizio ponendosi totalmente dalla parte dell'utente.

Il risultato che viene analiticamente presentato nei saggi che seguono, offre spunti ancora più interessanti per i gestori che adesso possono contare non solo sul dato quantitativo legato al servizio ma anche sul dato legato alle aspettative dell'utenza, a come quel servizio funziona, a quanto quel determinato servizio è gradito, a come si possa estendere e/o ridurre rispetto alle esigenze emerse.

Certo i dati invecchiano precocemente e sarebbe quanto meno opportuno ripetere l'indagine almeno a cadenza triennale nonostante i costi ancora effettivamente sostenuti che l'attuazione di tale processo impone alle strutture.

Il prepotente ingresso delle risorsa elettronica, il suo veloce sviluppo, la necessità di scegliere cosa acquisire rispetto a un'offerta sempre più vasta $\mathrm{e}$

4 Fanno parte del Gruppo GIM, costituitosi nel 2000, le Università di Bologna, Firenze, Milano Bicocca, Padova, Parma, il Politecnico di Torino e l'Università di Trento. 
costosa attribuisce allo strumento della misurazione un'importanza ancora più strategica. Ma anche in questo settore della documentazione, che già di per sé offre molte potenziali di sviluppo per la creazione di un servizio di qualità, la valutazione delle aspettative e della soddisfazione dell'utenza rispetto ai contenuti e alle tecnologie disponibili per la gestione della risorsa e per l'organizzazione dell'accesso torna a imporsi come un elemento fondamentale. Rimane sempre assolutamente imprescindibile l'interazione tra utente e bibliotecario, tra utente e servizio.

E resta infine indispensabile estendere il processo di misurazione della quantità/qualità a più Sistemi bibliotecari, utilizzando gli stessi metodi di rilevazione nonché gli stessi metodi di analisi ed elaborazione per dare avvio a un dialogo che perfezioni le scelte gestionali dei singoli sistemi alla luce del confronto con l'analisi dei risultati ottenuti da altre analoghe istituzioni. Il confronto fa risparmiare tempo e porta alla migliore soluzione dei problemi. 


\title{
L'esperienza del Sistema Bibliotecario dell'Ateneo fiorentino e l'indagine sulla soddisfazione dell'utenza*
}

\author{
di Chiara Melani
}

La cultura e la pratica della misurazione e valutazione dei servizi sembrano essersi ormai ampiamente diffuse presso le biblioteche universitarie italiane. Il nuovo quadro normativo su temi quali l'autonomia e la valutazione nella Pubblica Amministrazione, la necessità di gestire budget limitati a fronte della pressione di un'utenza sempre più esigente e di risorse documentarie sempre più costose, lo stimolo rappresentato da alcune iniziative promosse a livello nazionale, quali le rilevazioni condotte dalla Conferenza dei Rettori delle Università Italiane (CRUI) e dall'Osservatorio per la valutazione del sistema universitario, hanno prodotto un crescente interesse su questo tema e contribuito alla realizzazione di numerose esperienze. Negli ultimi anni l'attenzione sembra essersi decisamente spostata verso la misurazione della customer satisfaction ${ }^{1}$. Anche l'indagine sulla soddisfazione dell'utenza per i servizi offerti dal Sistema Bibliotecario d'Ateneo (SBA) dell'Università di Firenze, condotta ad opera del Gruppo di lavoro Monitoraggio e Valutazione a cavallo tra i mesi finali del 2001 e quelli iniziali del 2002, non costituisce un episodio valutativo isolato. Essa si colloca all'interno di un'esperienza ormai consolidata di misurazione e valutazione, concretizzatasi tuttavia fino ad oggi unicamente nella rilevazione periodica di dati e nell'elaborazione di relativi indicatori.

Le biblioteche dell'Università di Firenze hanno iniziato a raccogliere e ad elaborare dati statistici sulle attività svolte e sui servizi resi fin dal 1986 ad opera dell'ufficio Servizio Biblioteche. In base al Regolamento del Sistema dei servizi bibliotecari d'Ateneo allora in vigore spettava infatti al Servizio

* Questo testo è ampiamente debitore nei confronti dei documenti di lavoro del SBA - Piani di lavoro, Relazioni, Rapporti redatti dal Coordinatore generale, disponibili in linea ad accesso riservato all'indirizzo <http://www.unifi.it/universita/biblioteche/progetto/prog_ind.htm>-e nei confronti dei Piani di lavoro e delle Relazioni annuali del gruppo di lavoro Misurazione e Valutazione - MIS.

1 Alcune biblioteche universitarie hanno già effettuato indagini sulla soddisfazione dell'utenza, altre ne annunciano la futura attuazione. Nelli \& Sada 1998, Mamoli 2001, Corbolante 2000, Bellini \& Rizzi 2001, Catinella Corbolante \& Romeo 2001, Sacchetti \& Gava 2003. 
Biblioteche il coordinamento dell'attività bibliotecaria e lo svolgimento a livello centrale di alcune attività, tra le quali appunto l'elaborazione e l'aggiornamento dei dati. È soltanto a partire dal 1995, tuttavia, con l'avvio del Progetto di riorganizzazione del SBA che l'attività di misurazione e valutazione diventa momento essenziale e consapevole del processo di gestione, azione svolta ordinariamente secondo un'ottica manageriale, per mezzo della creazione e del progressivo aggiornamento di un sistema informativo.

Il Progetto di riorganizzazione del SBA ha avuto come fine ultimo il miglioramento della qualità dei servizi, in nome della centralità dell'utente $\mathrm{e}$ dei suoi bisogni, con un forte accento posto sull'importanza dei servizi al pubblico, "prodotto finale di ogni processo svolto nelle biblioteche e [loro] funzione prioritaria"2. Strumenti di realizzazione degli obiettivi specifici del Progetto, individuati di anno in anno in un Piano di lavoro redatto dal Coordinatore, sono stati essenzialmente l'innovazione strutturale, nel senso di un progressivo accentramento amministrativo, da una parte, l'introduzione a tutti i livelli di una nuova organizzazione del lavoro basata su di un modello partecipato, a scapito del precedente modello gerarchico, dall'altra. Lo scenario che ha fatto da sfondo al cambiamento è la riforma legislativa che, introducendo il principio di separazione tra funzioni di indirizzo politico, affidati agli organi accademici, e responsabilità di gestione, affidata ai bibliotecari, ha promosso nelle biblioteche universitarie la gestione per budget e per obiettivi, richiedendo altresì l'attivazione di strumenti di valutazione finale, sia interna che esterna, dei risultati raggiunti. "L'operazione qualità è [stata] intesa come un percorso graduale attuato attraverso la progettazione e la valutazione periodica del grado di raggiungimento degli obiettivi"3. Il Progetto quindi ha avuto "una natura dinamica, con un approccio metodologico che tende al raggiungimento del miglioramento dei servizi in un contesto organizzativo flessibile, in continua sperimentazione"4.

La situazione di partenza era costituita da un discreto numero di Biblioteche di Facoltà e Fondi di Istituto o di Dipartimento, sparsi sul territorio fiorentino, di varie dimensioni ed estremamente eterogenei quanto a livello di automazione, servizi offerti, risorse assegnate, organizzazione interna. Solo poche sedi avevano completato l'inserimento in SBN ed i prestiti continuavano ad essere gestiti prevalentemente con procedura non automatizzata.

\footnotetext{
Tammaro 1995.

Tammaro 1997b.

Tammaro 1998.
} 
L'organizzazione del materiale librario era per lo più a deposito chiuso e ovunque gli spazi risultavano carenti sia in vista di un ampliamento dei depositi stessi, sia della disponibilità di sale di lettura o dell'eventuale allestimento di sale a scaffale aperto. La maggior parte dei Fondi presentava restrizioni di accesso o limitazioni di servizio. L'informazione bibliografica tramite banche dati in linea e su cd-rom, ma anche il servizio di prestito interbibliotecario e fornitura di documenti non erano uniformemente diffusi. Le politiche di acquisizione e le procedure interne inerenti la gestione delle collezioni erano autonomamente gestite dalle diverse strutture, senza un coordinamento. Il personale rivestiva ruoli rigidi e soprattutto era nettamente distinto tra addetti al pubblico e addetti alle procedure interne. ${ }^{5}$

L'Ordinanza 96 del 14 dicembre 1994 comprendeva tutte le Biblioteche e Fondi in un costituendo Sistema bibliotecario articolato in sette Poli gestionali, individuati in base a un criterio di vicinanza geografica e guidati da un coordinatore (vedi Tab. 1). Il personale, precedentemente attribuito alle ventuno biblioteche, veniva da ora in avanti assegnato ai Poli, un primo passo nella direzione di una maggiore razionalità ed efficienza nella distribuzione $\mathrm{e}$ nell'utilizzo delle risorse, nonché della semplificazione e centralizzazione delle procedure; questo primo cambiamento organizzativo permetteva per esempio l'immediato avvio di un processo di centralizzazione a livello di Polo delle attività di acquisizione e catalogazione.

L'architettura attuale del Sistema, articolata non più in sette Poli, ma in sole cinque Biblioteche di Area, viene tuttavia raggiunta solo ad opera del nuovo Regolamento del SBA approvato nel $1998^{6}$ e dei cinque decreti rettorali 666-670 del 12 maggio 1999 che lo attuano, spingendo ancora oltre il processo di ristrutturazione ${ }^{7}$. Le Biblioteche di Area aggregano i punti di servizio che le compongono (cioè le ventuno ex Biblioteche e gli ex Fondi librari) non più in base a un criterio geografico, ma alle aree disciplinari individuate dallo Statuto (Titolo I, art. 8) ${ }^{8}$ : biomedica, di scienze, di scienze sociali, tecnologica, umanistica (vedi Tab. 2). Esse sono unità amministrativamente autonome e gestiscono non soltanto il personale, ma anche le risor-

5 Per questa analisi della situazione di partenza vedi le sintesi delle relazioni dei coordinatori di Polo inserite all'interno di: Tammaro 1995.

6 Università degli Studi di Firenze 2000c.

7 I decreti di costituzione delle cinque Biblioteche di Area sono consultabili a partire da: Università degli Studi di Firenze 2001a. La definizione delle Biblioteche di Area è contenuta nell'art. 2.

8 Università degli Studi di Firenze 2002b. 
Tab. 1 - I poli

\begin{tabular}{|c|c|c|}
\hline \multirow{6}{*}{$\begin{array}{l}\text { Coordinamento } \\
\text { centrale } \\
\text { biblioteche }\end{array}$} & Polo 1 & $\begin{array}{l}\text { Biblioteca di Agraria } \\
\text { Biblioteca di Economia } \\
\text { Biblioteca di Statistica }\end{array}$ \\
\hline & Polo 2 & $\begin{array}{l}\text { Biblioteca di Architettura } \\
\text { Biblioteca di Ingegneria }\end{array}$ \\
\hline & Polo 3 & $\begin{array}{l}\text { Biblioteca di Giurisprudenza } \\
\text { Biblioteca di Scienze politiche } \\
\text { Emeroteca }\end{array}$ \\
\hline & Polo 4/5 & $\begin{array}{l}\text { Biblioteca di Geografia } \\
\text { Biblioteca di Lettere e Filosofia } \\
\text { Biblioteca di Nordamericana } \\
\text { Biblioteca di Scienze della Formazione }\end{array}$ \\
\hline & Polo 6 & $\begin{array}{l}\text { Biblioteca di Farmacia } \\
\text { Biblioteca di Matematica } \\
\text { Biblioteca di Medicina }\end{array}$ \\
\hline & Polo 7 & $\begin{array}{l}\text { Biblioteca di Antropologia } \\
\text { Biblioteca di Biologia animale } \\
\text { Biblioteca di Botanica } \\
\text { Biblioteca di Chimica } \\
\text { Biblioteca di Fisica } \\
\text { Biblioteca di Geomineralogia }\end{array}$ \\
\hline
\end{tabular}

se finanziarie in un regime di autonomia contabile; hanno un unico bilancio e un unico Comitato di gestione.

Di pari passo alla ristrutturazione amministrativa, veniva lentamente a modificarsi anche l'organizzazione interna del lavoro. L'Ordinanza 97 del 15 gennaio 1995 istituiva il ruolo del Coordinatore generale del SBA, mentre 1'Ordinanza 98 del 10 luglio dello stesso anno gli attribuiva il compito della programmazione, pianificazione e valutazione dei servizi. Il Coordinamento centrale di Ateneo iniziava quindi a centralizzare alcune funzioni di interesse generale per tutte le strutture del Sistema, che nel corso degli anni si sono tradotte in alcuni degli obiettivi specifici individuati nel Piano di lavoro annuale, tra i quali anche la realizzazione e il mantenimento di un sistema informativo per il monitoraggio e la valutazione. Per lo svolgimento di queste funzioni il Coordinatore centrale si avvaleva di gruppi di lavoro, cosiddetti "di Ateneo", composti da personale appartenente ai singoli Poli e, in un secondo momento, alle singole Biblioteche. La formazione di gruppi di lavo- 
Tab. 2 - Le Biblioteche di Area

\begin{tabular}{|c|c|}
\hline \multicolumn{2}{|r|}{ Coordinamento centrale biblioteche } \\
\hline Biblioteche di Area & $\begin{array}{l}\text { Punti di servizio correlati a facoltà, a corsi di laurea, a } \\
\text { dipartimenti }\end{array}$ \\
\hline Biblioteca Biomedica & $\begin{array}{l}\text { Farmacia } \\
\text { Medicina } \\
\text { Dipartimento di Farmacologia preclinica e clinica } \\
\text { Dipartimento di Pediatria } \\
\text { Dipartimento di Sanità pubblica, Epidemiologia e Clinica } \\
\text { analitica } \\
\text { Dipartimento di Scienze neurologiche e psichiatriche }\end{array}$ \\
\hline Biblioteca di Scienze & $\begin{array}{l}\text { Antropologia } \\
\text { Biologia animale } \\
\text { Botanica } \\
\text { Polo scientifico di Sesto F.no (Chimica e Fisica) } \\
\text { Geomineralogia } \\
\text { Matematica }\end{array}$ \\
\hline $\begin{array}{l}\text { Biblioteca di } \\
\text { Scienze sociali }\end{array}$ & $\begin{array}{l}\text { Economia } \\
\text { Dipartimento di Diritto dell'Economia } \\
\text { Giurisprudenza } \\
\text { Scienze politiche } \\
\text { Dipartimento di Diritto comparato e penale e Diritto privato } \\
\text { e processuale } \\
\text { Dipartimento di Diritto pubblico } \\
\text { Dipartimento di Teoria e Storia del diritto } \\
\text { Statistica } \\
\text { Polo universitario di Prato - Centro servizi bibliotecari di Ateneo }\end{array}$ \\
\hline $\begin{array}{l}\text { Biblioteca di } \\
\text { Scienze tecnologiche }\end{array}$ & $\begin{array}{l}\text { Agraria } \\
\text { Dipartimento economico estimativo agrario e forestale } \\
\text { Architettura } \\
\text { Dipartimento di Costruzioni } \\
\text { Dipartimento di Tecnologie dell'Architettura e Design } \\
\text { Dipartimento di Progettazione dell'Architettura } \\
\text { Dipartimento di Storia e Restauro delle Strutture } \\
\text { architettoniche } \\
\text { Dipartimento di Urbanistica e Pianificazione del Territorio } \\
\text { Ingegneria }\end{array}$ \\
\hline Biblioteca Umanistica & $\begin{array}{l}\text { Filosofia } \\
\text { Geografia } \\
\text { Lettere e Filosofia } \\
\text { Nordamericana } \\
\text { Psicologia } \\
\text { Scienze della Formazione } \\
\text { Storia dell'Arte }\end{array}$ \\
\hline
\end{tabular}


ro veniva inoltre promossa anche all'interno dei Poli stessi, in un primo momento solo in via sperimentale e semplicemente affiancati alla struttura precedente.

Lavorare per gruppi è una prassi ormai consolidata all'interno del Sistema bibliotecario dell'Ateneo fiorentino. Oltre ai gruppi di Ateneo, dipendenti direttamente dal Coordinamento centrale, anche le cinque Biblioteche sono articolate in gruppi di lavoro. L'organigramma attuale del Sistema è visibile nella Fig. 1.

L'evoluzione da un'organizzazione di tipo gerarchico, in cui prevalgono qualifiche, mansioni, la correttezza delle procedure, ad una basata sulla condivisione delle responsabilità, la partecipazione di tutti, l'orientamento al risultato, è stata tuttavia lenta e estremamente faticosa. Una tappa importante del processo è stato un accordo siglato nel febbraio 1996 tra le Organizzazioni Sindacali e l'Amministrazione che ha introdotto nella gestione del personale i concetti di flessibilità nello svolgimento delle mansioni stabilite dal mansionario e mobilità tra più sedi di servizio all'interno del Polo di afferenza; in cambio, il personale che dichiarava di aderire al Progetto e di collaborare al raggiungimento dei suoi obiettivi, accettando di svolgere mansioni anche sensibilmente superiori o inferiori rispetto a quelle previste per la propria qualifica, riceveva in busta paga un piccolo incentivo. L'accordo ha realizzato le condizioni essenziali per il miglioramento dei servizi in generale e l'avvio di nuovi: la turnazione al pubblico di tutto il personale nei diversi punti di servizio ha permesso l'ampliamento dell'orario di apertura delle strutture; è stato possibile intervenire nel senso di un generale snellimento delle procedure, precedentemente ingolfate da un'eccessiva parcellizzazione dipendente dalle mansioni; le attività sono state nel complesso ridistribuite non più in base alle qualifiche, ma in base alle competenze e conoscenze possedute. L'intero processo è stato sostenuto e incoraggiato dal costante impegno del Coordinamento nell'aggiornamento e nella formazione permanente. La generale crescita professionale che ne è derivata ha condotto nel 1997 al reinquadramento, tramite corso-concorso, nella VI qualifica funzionale della quasi totalità di quanti erano precedentemente inquadrati nelle ex III, IV e V qualifica.

In una prima fase del Progetto, negli anni 1995-1997, "la qualità del servizio è stata identificata in un livello minimo e basilare di servizi bibliotecari"9. Gli obiettivi specifici individuati sono stati l'ampliamento dell'orario di apertura, il miglioramento del servizio di prestito, soprattutto tramite la generaliz-

9 Tamaro 1998. 
L'ESPERIENZA DEL SisTeMA BiblioteCARIO DELL'ATENEO FIORENTINO

Fig. 1 - L'organigramma attuale del SBA

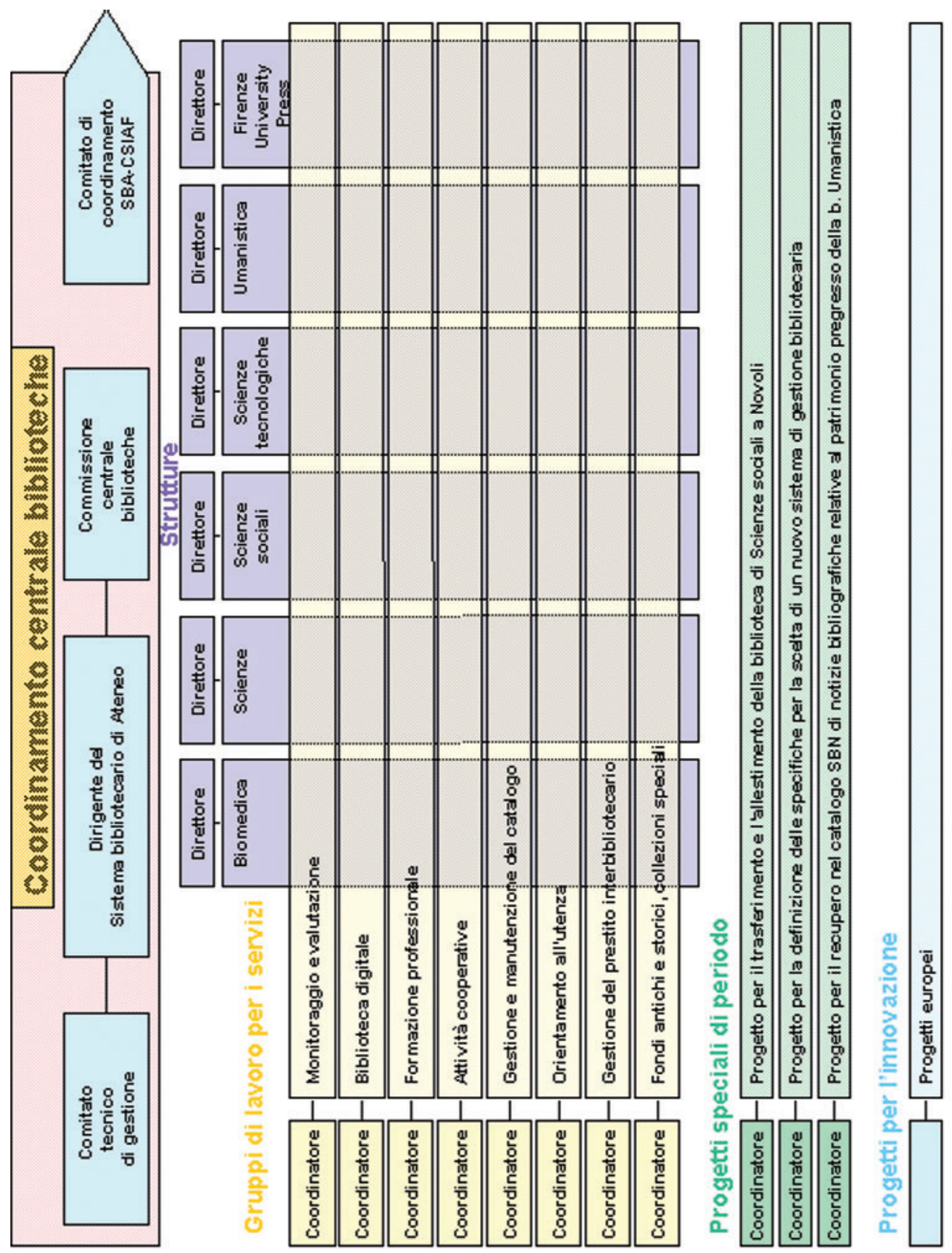


zazione della procedura automatizzata, la semplificazione dell'iter del libro, in modo da ridurre i tempi di attesa per la disponibilità a scaffale delle nuove acquisizioni, lo sviluppo dei servizi di informazione bibliografica e prestito interbibliotecario/fornitura di documenti. Il piano di lavoro per il 1998 segna quindi una svolta, individuando per il futuro due distinte linee di sviluppo. La prima continua ad identificarsi nel raggiungimento e progressivo consolidamento di un livello standard accettabile di servizi, la cui realizzazione è affidata ai Poli/Biblioteche e ai gruppi di lavoro interni ad essi. La seconda linea di sviluppo prevede invece l'attivazione di un processo continuo di miglioramento della qualità del servizio, al fine di ottenere una sempre maggiore soddisfazione dell'utenza. Tra i diversi sotto-obiettivi individuati nel corso degli anni, affidati ai gruppi di lavoro di Ateneo, possiamo ricordare un insieme di iniziative per l'assistenza e l'orientamento all'utenza (corsi per studenti, depliant e altro materiale informativo, pagine web), l'organizzazione nei Poli di un servizio audiovisivi e multimediali di carattere tecnico scientifico, il trattamento automatizzato delle tesi, la gestione e lo sviluppo del sistema automatizzato, l'acquisto cooperativo e l'incremento delle risorse elettroniche (banche dati e periodici elettronici), la semplificazione delle procedure amministrative dei Poli, il potenziamento dei servizi di documentazione sulla letteratura periodica, il monitoraggio e la valutazione del SBA, la formazione del personale. È degli ultimi tempi una forte tendenza verso l'innovazione tecnologica e la biblioteca digitale: "il progetto di riorganizzazione persegue lo sviluppo continuo dei servizi al pubblico organizzando o migliorando i servizi di accesso diretto da parte dell'utente (disintermediazione) favorendo l'aumento delle risorse elettroniche disponibili"'10.

L'avvio del Progetto ha determinato la creazione di un sistema informativo gestionale, strumento a supporto delle attività di monitoraggio e valutazione, articolato in una banca dati, aggiornata con continuità, e in un set di indicatori. La sua realizzazione e mantenimento sono compito del Coordinamento centrale; a partire dal 1999 è stato creato un apposito gruppo di lavoro di Ateneo, il Gruppo per il Monitoraggio e la Valutazione ${ }^{11}$. Annualmente vengono effettuate due rilevazioni quadrimestrali e un'ulteriore rilevazione alla fine dell'anno, finalizzata alla redazione della relazione e del rapporto annuali del SBA ad opera del Coordinatore. I risultati vengono resi noti ai

10 Università degli Studi di Firenze 2000a.

11 Il gruppo di lavoro comprende allo stato attuale gli autori dei testi di questa monografia. 
direttori di biblioteca e ai coordinatori dei gruppi d'Ateneo in modo tale da permettere il confronto con gli obiettivi prefissati.

Negli anni antecedenti al Progetto i rilevamenti venivano effettuati mensilmente mediante un questionario cartaceo che i responsabili delle singole biblioteche erano invitati a compilare; i dati raccolti venivano trasferiti su fogli elettronici. Si trattava di un'attività estremamente laboriosa in termini di tempo e soggetta ai rischi dell'approssimazione e dell'errore. A partire dal 1995 la selezione dei dati da raccogliere è stata fatta invece in base a criteri di semplicità, velocità e oggettività di rilevazione, criteri seguiti anche in occasione delle modifiche operate successivamente. È sempre stata dunque data preferenza a quei dati che fosse possibile estrarre automaticamente dai programmi di automazione in uso. Pochi dati vengono forniti dai direttori di biblioteca e dai coordinatori dei gruppi di Ateneo in base a moduli standardizzati; altri provengono da fonte esterna, cioè da alcuni uffici amministrativi dell'Ateneo. Ulteriore innovazione introdotta dal Progetto è la batteria di indicatori. "Gli indicatori devono rappresentare le dinamiche dei processi di cambiamento in atto, cioè sono misure sistematiche di quanto il sistema bibliotecario d'Ateneo ha raggiunto i suoi obiettivi in un periodo determinato di tempo e, se non li ha raggiunti, indicano perché ed aiutano a rimuovere gli ostacoli. In altre parole, gli indicatori di rendimento devono migliorare il rendimento" 12 .

Una prima fotografia del sistema informativo gestionale del SBA di Firenze è contenuta in un articolo pubblicato nel 1997 su Biblioteche Oggi da Anna Maria Tammaro con la collaborazione di Francesca Landi. L'Appendice 3 riporta l'elenco dei dati raccolti, suddivisi in Dati di interesse generale (che corrispondono a quelli selezionati dalla CRUI), Dati relativi alle risorse patrimoniali e alle strutture in dotazione al SBA, Input, Output, Personale, Uten$\mathrm{za}^{13}$. L'Appendice 5 riporta la batteria degli indicatori di rendimento $\operatorname{adottati~}^{14}$. Negli anni successivi il sistema informativo è stato più volte modi-

12 Tammaro \& Landi 1997, p. 69.

13 Tammaro \& Landi 1997, p. 76.

14 Tammaro \& Landi 1997, p. 77. Gli indicatori generali equivalgono a quelli definiti dalla CRUI per i servizi bibliotecari delle università: posti di lettura/iscritti, metri quadrati delle biblioteche/iscritti, personale/numero delle biblioteche. A questi si aggiungono indicatori di economicità e di efficienza, funzionali a quantificare le risorse impiegate e la modalità del loro impiego: costi totali/iscritti, costi totali in percentuale del budget Università (economicità), costo percentuale per singola attività, costo per ora di apertura (efficienza). Seguono alcuni indicatori di efficacia, atti a valutare l'utilizzo dei servizi erogati: prestiti/iscritti, ricerche in OPAC/iscritti, soddisfazione utenza (indagine da realizzare). Gli indicatori di produttività correlano la quantità dei prodotti al tempo impiegato dallo staff espresso in FTE: 
ficato e ampliato in funzione di nuove esigenze. Nel 1997, per esempio, è stato individuato in uno standard prodotto dall'americana Association of College and Research Libraries, a preferenza di altri e con i dovuti adattamenti alla realtà fiorentina, lo strumento idoneo per la periodica quantificazione del fabbisogno di personale delle singole biblioteche ${ }^{15}$. L'esigenza di fondo era ottimizzare l'utilizzo delle risorse umane; "lo standard dei college universitari americani è stato ritenuto quello che più si avvicinava alle priorità di servizio del Progetto, perché prende le mosse dal numero di studenti effettivi e dalle loro esigenze di servizio" ${ }^{16}$. Ulteriori dati e indicatori sono stati quindi introdotti per monitorare l'offerta e l'utilizzo di nuovi servizi progressivamente attivati. È il caso per esempio dei corsi rivolti agli studenti sull'utilizzo delle risorse informative offerte dalle singole biblioteche ${ }^{17}$, o del servizio riservato ad utenti interni di fornitura di copia elettronica di articoli contenuti in riviste possedute in formato cartaceo dal $\mathrm{SBA}^{18}$. A differenza di altre esperienze $^{19}$, il set di indicatori attualmente in uso è stato creato confrontando pragmaticamente vari standard e strumenti di lavoro e attingendone di volta in volta le indicazioni che sembravano più conformi al caso specifico. Il sistema informativo gestionale attualmente in uso è riportato nell'Appendice $A$.

Negli ultimi tempi l'attenzione del gruppo di lavoro Monitoraggio e Valutazione si è concentrata per lo più su due fronti: garantire un controllo costan-

FTE/volumi catalogati, FTE/numero inventari, FTE/ore apertura, FTE/prestito interbibliotecario. Infine, alcuni indicatori specifici permettono il confronto tra gli obiettivi del Progetto e i risultati raggiunti: orario apertura, tempestività processi, volumi ad accesso libero/volumi totali, $\mathrm{PC}$ destinati al servizio/iscritti, velocità del prestito interbibliotecario.

15 ACRL \& ALA 1995.

16 Tammaro 1997a. All'interno del modello americano, “per le esigenze di personale sono considerati fattori determinanti gli studenti iscritti a tempo pieno (FTE) ed il numero dei volumi. Possono essere aggiunti bibliotecari supplementari in considerazione di fattori organizzativi ed istituzionali: servizi avanzati, dislocazione dei punti di servizio, ore di apertura dei servizi, corsi di laurea, numero dei docenti, corsi di supporto. Sono stati inoltre considerati i seguenti fattori di aggravio organizzativi ed istituzionali: nuovi servizi avanzati da attivare nelle biblioteche, media ponderata dell'apertura dei punti di servizio aperti nel Polo" (ibidem).

17 Quadrimestralmente i responsabili del servizio per le singole biblioteche forniscono al gruppo di lavoro il numero dei corsi effettuati e il numero dei partecipanti (vedi Appendice A, Dato D4.12).

18 Il servizio di fornitura di copia elettronica di articoli, effettuato tramite il software Ariel, è riservato a quegli utenti che possiedono una casella di posta elettronica su un server dell'Università di Firenze. Per il contesto teorico all'interno del quale il servizio è stato creato: Tammaro 1999. Quadrimestralmente i responsabili del servizio per le singole biblioteche forniscono il numero dei documenti inviati, come risulta dal sistema elettronico di gestione del servizio stesso (vedi Appendice A, Dato D4.08).

19 Bellini \& Rizzi 2001. 
te sui tempi necessari per la disponibilità a scaffale delle nuove acquisizioni, in modo tale da impedire l'accumulo di arretrati difficili da smaltire, già noti all'esperienza del SBA fiorentino; studiare nuovi strumenti di valutazione per la documentazione elettronica, cioè per banche dati e soprattutto periodici elettronici, a fronte di un forte investimento di risorse in questi settori ad opera del Coordinamento.

Il monitoraggio dei tempi dell'iter del libro veniva effettuato fin dai tempi della migrazione nell'Indice SBN tramite un software fornito dall'Istituto Centrale per il Catalogo Unico, che non è stato più disponibile a partire dall'anno 2001. Il nuovo sistema di rilevazione è stato messo a punto con la collaborazione dei Servizi Informatici per l'Amministrazione e le Biblioteche, sperimentato nel 2001 e messo a regime nel 2002. Mensilmente esso permette di calcolare la percentuale di documenti resi disponibili nell'arco dei quindici giorni successivi ad una settimana presa come campione, tra tutti i nuovi inventari creati nell'arco della settimana campione stessa ${ }^{20}$.

Per quanto concerne i periodici elettronici, dal 2001 vengono regolarmente effettuate rilevazioni semestrali relativamente agli accessi a partire dalle statistiche fornite da alcuni editori e distributori; l'irregolarità e la disomogeneità delle informazioni fornite dagli editori stessi fino ad oggi non hanno permesso in questo caso rilevazioni quadrimestrali. La raccolta dei dati riguarda il numero totale dei titoli messi a disposizione e il numero totale degli accessi distinto per TOC, abstract e full-text (il dato sul full text è a sua volta distinto, dove possibile, in PDF e HTML). Le statistiche di accesso ci vengono fornite o come totale degli accessi all'intero pacchetto di titoli attivato presso un determinato editore, oppure, in alcuni casi, per singolo titolo. Benché l'esame della recente letteratura professionale nel campo della misurazione delle risorse elettroniche abbia rivelato come a tutt'oggi manchi ancora un insieme standard di misure e di indicatori consolidato, il gruppo di lavoro si sta orientando verso il tentativo di sperimentare alcuni strumenti che rispondano al bisogno di avere indicazioni circa il grado di utilizzo dei periodici acquistati, soprattutto in rapporto al costo. Si sente infatti piuttosto pressante l'esigenza di valutare quanto l'investimento effettuato valga il $\operatorname{costo}^{21}$.

Il sistema informativo gestionale è stato utilizzato fin dalle origini per scopi di valutazione sia interna che esterna. Da una parte esso ha soddisfatto

20 Vedi Appendice A, Dato D4.05.

21 Vedi Appendice A, Indicatori I2.11, I2.12. Sulle problematiche inerenti la valutazione della biblioteca ibrida e digitale: Tammaro 2000, Galluzzi 2001a, Galluzzi 2001b. 
l'esigenza si supportare i processi decisionali del Coordinatore generale, dei direttori di biblioteca, dei coordinatori dei gruppi di lavoro d'Ateneo permettendo la quantificazione delle risorse e il monitoraggio della loro distribuzione e utilizzazione, la verifica periodica sullo stato di avanzamento delle attività previste dal Progetto, la misurazione del rendimento del SBA, la comparazione dei risultati raggiunti in tempi diversi. Dall'altra, tuttavia, lo stesso sistema informativo ha permesso di far fronte con precisione e prontezza alle richieste di informazioni provenienti dai vari uffici dell'Amministrazione dell'Ateneo, e ha facilitato la raccolta dei dati per le rilevazioni nazionali promosse dalla CRUI, nonché dal Gruppo di lavoro sui servizi bibliotecari degli Atenei dell'Osservatorio per la valutazione del sistema universitario ${ }^{22}$.

Nel 2000 il sistema informativo è stato presentato, insieme a numerose altre esperienze di valutazione effettuate localmente presso altri Atenei italiani, al Convegno Le biblioteche accademiche del futuro. Idee, progetti e risorse organizzato a Roma dalla $\mathrm{CRUI}^{23}$. In questa occasione è stata avviata una collaborazione con le università di Bologna, Parma, Torino Politecnico, Milano Bicocca, Trento, Padova finalizzata a costituire un gruppo di lavoro interuniversitario con lo scopo di mettere a punto criteri condivisi di rilevazione che consentano il confronto tra le performance dei Sistemi bibliotecari degli Atenei italiani. Il GIM (Gruppo Interuniversitario sul Monitoraggio dei Sistemi Bibliotecari) lavora per costruire un sistema permanente di monitoraggio sulle biblioteche universitarie, attraverso la definizione di una metodologia unitaria di raccolta ed elaborazione dei dati e l'effettuazione di una rilevazione a livello nazionale. Il fine è consentire al Comitato Nazionale per la Valutazione del Sistema Universitario del Ministero dell'Istruzione, dell'Università e della Ricerca di valutare l'offerta dei servizi bibliotecari degli atenei, in linea con le finalità delle rilevazioni dei Nuclei interni alle università, attraverso strumenti metodologici e dati aggiornati rispetto a quelli prodotti dall'Osservatorio. La definizione delle misure da rilevare, obiettivo intermedio al cui raggiungimento nello specifico ha contribuito in una prima fase del lavoro l'Università di Firenze, è partita proprio dall'analisi e dal confronto dei sistemi di monitoraggio in uso presso tutti e otto i sistemi bibliotecari delle Università partecipanti al GIM, selezionando i dati maggiormente utilizzati e ritenuti condivisibili e confrontandoli con gli standard nazionali e internazionali e la recente letteratura sull'argomento.

22 Ministero dell'Università e della Ricerca Scientifica e Tecnologica 1999.

23 Papa \& Scimone 2000. 
La consapevolezza della necessità di integrare il sistema informativo del SBA con indicatori di qualità sulla soddisfazione dell'utenza è stata forte fin dalle origini del Progetto. L'ingente investimento nel miglioramento e nell'incremento dei servizi andava valutato non solo in base alla misura del loro utilizzo, ma anche in base alla qualità realmente percepita dagli utenti: "non c'è qualità se il singolo utente non ne percepisce la presenza" 24 . Già il piano di lavoro per il 1995 prevedeva l'avvio di un'indagine, in relazione alla prospettata riorganizzazione dei servizi al pubblico. Il sopra citato articolo di Anna Maria Tammaro con la collaborazione di Francesca Landi inserisce la soddisfazione dell'utenza, da misurarsi tramite un'apposita indagine, tra gli indicatori di efficacia ${ }^{25}$. In un primo momento tuttavia il previsto progetto di trasmissione di un questionario è stato inglobato all'interno di un progetto più ampio di misurazione della soddisfazione dell'utenza per tutti i servizi dell'Ateneo, realizzato ad opera del Nucleo di Valutazione dell'Università di Firenze.

Il Nucleo di Valutazione, istituito presso l'Ateneo fiorentino con delibera del Consiglio di Amministrazione n. 181 del 5 maggio 1995, ha dimostrato fin da subito un vivo interesse nei confronti del SBA. Tra le prime attività svolte, infatti, ha iniziato alla fine del 1996 alcune verifiche in settori di particolare rilievo per il funzionamento dell'Ateneo o che erano stati oggetto di recenti riforme, e ha quindi effettuato anche uno studio sul funzionamento del SBA a seguito delle riforme subite appunto negli anni precedenti ${ }^{26}$. Il secondo rapporto redatto dal Nucleo nel 1999 presenta per la prima volta, oltre a dati inerenti la didattica, anche dati riguardanti le risorse logistiche di supporto alla didattica, tra le quali appunto le biblioteche ${ }^{27}$. Lo stesso Rapporto 1999 contiene inoltre i risultati di un'indagine sulla soddisfazione degli studenti riguardo a molteplici aspetti della funzionalità dell'Ateneo, indagine ripetuta anche l'anno successivo e realizzata in ottemperanza alla legge 370 del 19 ottobre 1999; in base a tale legge i Nuclei devono acquisire periodicamente le opinioni degli studenti sulle attività didattiche e devono trasmetterne annuale relazione al Ministero dell'Università e della Ricerca scientifica e tecnologica e al Comitato Nazionale per la Valutazione del Sistema universitario. Altra esigenza alla base delle indagini era saggiare lo studente in quanto "cliente che più misura sulla base della propria diretta esperienza la qualità della prestazione e dell'organizzazione didattica e formativa di un'università

\footnotetext{
24 Di Domenico 1996, p. 56.

25 Tammaro \& Landi. 1997, p. 77.

26 Università degli Studi di Firenze 1998, p. 9-15.

27 Università degli Studi di Firenze 2000b, p. 71-72.
} 
e influisce di conseguenza sugli orientamenti contingenti del mercato e del dislocarsi della domanda nella gamma sempre più ampia di offerte formative che il mercato nazionale ed europeo allo studente può offrire"28.

Le rilevazioni sono state effettuate tramite un questionario appositamente formulato e distribuito ad un campione di popolazione studentesca costruito sulla base del numero totale di studenti iscritti almeno al secondo anno di uno qualunque dei corsi di laurea e di diploma gestiti da ognuna delle Facoltà dell'Università di Firenze. I questionari sono stati distribuiti tramite interviste individuali dirette presso molteplici spazi pubblici delle differenti Facoltà universitarie, al fine di raccogliere la tipologia di studenti più ampia e il più possibile casuale. Nel questionario del 1999 le domande relative alle biblioteche erano quattro su un totale di cinquantaquattro e concernevano la disponibilità di libri e riviste in rapporto alle necessità di studio locale e al panorama nazionale e internazionale sulla materia di interesse, la disponibilità di banche dati, l'orario d'apertura ${ }^{29}$. Nel 2000 alle biblioteche venivano dedicate solo due domande su sessantanove: disponibilità e competenza del personale, tempestività del servizio nel reperimento dei volumi, facilità di consultazione venivano indagati con la prima domanda; la seconda riproponeva il tema dell'orario di apertura chiedendo all'intervistato di esprimersi propositivamente su quello che ritenesse come il più idoneo. Sia nel 1999 che nel 2000 il servizio bibliotecario è stato giudicato nel complesso abbastanza soddisfacente. Tra le strutture e i servizi offerti dall'Ateneo agli studenti, quale supporto all'attività didattica, le biblioteche sembrano riscuotere il maggior successo.

Benché avessero fornito alcuni primi utilissimi strumenti di riscontro, nel complesso le indagini condotte dal Nucleo non erano sufficienti a soddisfare a pieno le esigenze valutative del SBA. Gli aspetti del servizio presi in considerazione sono limitati e la popolazione di riferimento è stata individuata nei soli studenti, tagliando fuori quella rilevante fetta di utenza bibliotecaria costituita dal corpo docente. Nel 1999 il Gruppo di lavoro d'Ateneo per l'Orientamento all'utenza ha quindi iniziato a lavorare fattivamente all'elaborazione di un questionario specifico, nell'ambito di una serie di interventi volti

28 Università degli Studi di Firenze 2000b, p. 89.

29 "Generalmente, quanto spesso si trovano in biblioteca i libri e le riviste accademiche necessarie per i suoi studi?"; "Quante riviste accademiche nazionali e internazionali sulla sua materia sono disponibili in biblioteca, fra quelle più importanti?"; "Come giudica le possibilità offerte dalla biblioteca universitaria di ricercare libri o riviste attraverso banche dati informatizzate?"; “Come giudica l'orario di apertura della biblioteca universitaria?". Università degli Studi di Firenze 2000b, p. 93. 
a garantire la qualità dei servizi su di un livello standard, interventi che hanno condotto tra le altre cose anche alla redazione della Carta dei servizi ${ }^{30}$. Fondamentale punto di partenza del lavoro di elaborazione di questa versione preliminare di questionario, così come del resto di quella definitiva raggiunta successivamente, è stata la ricerca svolta da Roberto Ventura per il conseguimento della tesi di diploma in Biblioteconomia presso la Scuola Speciale per Archivisti e Bibliotecari ${ }^{31}$. Allo stesso tempo si iniziava a prendere coscienza delle problematiche insite nell'effettuazione di un'indagine e a porsi di conseguenza una serie di domande: se e in che misura rivolgersi a una ditta esterna per lo svolgimento delle interviste, come individuare la popolazione di riferimento e come raggiungerla, se integrare il questionario con altri metodi di ricerca qualitativa, come intervenire sui servizi una volta valutati i risultati dell'indagine.

Nel 2000 un'indagine interna volta alla verifica del grado di soddisfazione degli utenti del sistema informativo gestionale, cioè del coordinatore generale, dei direttori delle biblioteche e dei responsabili dei gruppi di lavoro di Ateneo, tra le altre cose, ha ulteriormente confermato il bisogno di rilevare la soddisfazione degli utenti per la qualità dei servizi, in modo tale da integrare le misure quantitative in uso ${ }^{32}$. Nel 2001 l'indagine sulla soddisfazione dell'utenza è stata quindi recepita all'interno del piano di lavoro annuale del Gruppo Monitoraggio e Valutazione, e nello stesso anno finalmente si sono realizzate alcune condizioni favorevoli per la sua effettiva realizzazione. Se da una parte infatti è stato possibile stanziare le risorse finanziare necessarie, dall'altra si è resa disponibile la collaborazione dello staff del Dipartimento di Statistica che ha messo a disposizione la propria competenza, ma anche le attrezzature e tecnologie del proprio laboratorio.

Obiettivo fondamentale dell'indagine è stata una verifica complessiva, in termini di percezione e grado di apprezzamento da parte dell'utenza, degli sforzi messi in atto con il Progetto per migliorare il livello qualitativo dei servizi. In particolare, le domande del questionario rispecchiano molte delle problematiche affrontate dal 1995 ad oggi: dalla comunicazione con il personale della front-line, alla cui preparazione professionale sono state dedicate numerose energie tramite programmi di formazione permanente, a servizi quali il prestito interbibliotecario e l'informazione bibliografica, che si è cercato di diffondere uniformemente presso tutte le sedi; dal comfort degli spazi,

30 Università degli Studi di Firenze 2002a.

31 Ventura 1998.

32 Si veda: Roberto VenturA. Un'indagine sull'uso della statistica bibliotecaria nel Sistema Bibliotecario di Ateneo, in questo volume. 
che nonostante i ripetuti tentativi di ampliamento rimangono a tutt'oggi una nota dolente per il SBA a causa della loro esiguità, alla disponibilità di libri a scaffale aperto, che si è cercato parimenti di ampliare; dai servizi di base, standardizzati su livelli accettabili, ad alcuni servizi avanzati, come l'accesso a banche dati su web o su cd-rom, o l'abbonamento a periodici elettronici, al cui incremento soprattutto nel corso degli ultimi anni è stata dedicata particolare attenzione, promuovendo anche interventi specifici a livello informatico, un adeguamento qualitativo e quantitativo del parco macchine, un'intensa attività cooperativa con altri atenei italiani per stipulare licenze di accesso vantaggiose $^{33}$.

${ }^{33}$ Per un bilancio complessivo dei risultati raggiunti dal Progetto: Maraviglia 2001. 


\title{
Un'indagine sull'uso della statistica bibliotecaria nel Sistema Bibliotecario di Ateneo
}

\author{
di ROBERTO VENTURA
}

Qual è l'impatto della misurazione dei servizi sul lavoro dei bibliotecari e, più in particolare, sulla gestione della biblioteca? Rispetto a quali decisioni e con quale frequenza i dati statistici riguardanti il funzionamento di un sistema bibliotecario sono usati da coloro che ricoprono responsabilità organizzative? In altri termini, è possibile misurare e valutare l'efficacia di un sistema di misurazione (nello specifico, la selezione dei dati periodicamente raccolti, gli indicatori che si costruiscono a partire dai dati) nel contesto lavorativo ordinario?

La misurazione e la valutazione dei servizi bibliotecari hanno una duplice natura: da un lato costituiscono uno strumento teso a motivare la spesa per le biblioteche e la richiesta di nuovi stanziamenti all'ente di cui la biblioteca fa parte, dall'altro sono strumenti tecnici, a valenza prettamente biblioteconomica e bibliotecaria, finalizzati a rendere più consapevoli le decisioni gestionali. Non è pacifico stabilire i confini che separano queste due dimensioni: esse si integrano, dal momento che la prima può influenzare la scelta degli indicatori mentre la costruzione degli indicatori, nonché la valutazione del loro andamento, può aprire nuove opportunità di promozione "interna" di determinati servizi.

A partire da un'ottica fornitore-cliente interno, i bibliotecari che si occupano di misurare e di valutare i servizi e che intendono migliorare l'erogazione di tale attività, hanno bisogno di indagare l'impatto effettivo che il loro lavoro ha sulle attività dei colleghi, anche per meglio comprendere il valore aggiunto di cui la misurazione si connota. Tale esigenza è avvertita particolarmente nelle strutture bibliotecarie di grandi dimensioni, dove le attività bibliotecarie risultano suddivise tanto dal punto della localizzazione quanto dal punto di vista della loro natura informativa (si pensi ad un sistema bibliotecario di ateneo), con evidenti implicazioni sul piano delle pratiche gestionali.

Chi si occupa di misurazione in un contesto operativo eroga un servizio a terzi ed ha la necessità di strutturare il processo di raccolta ed elaborazione dei dati in modo da fornire un prodotto che si integri con le attività gestiona- 
li affidate alla responsabilità di coloro che ricoprono incarichi di carattere direttivo a livello di sistema bibliotecario. Ciò significa offrire informazioni e valutazioni di effettiva utilità nei processi decisionali a tali responsabilità correlati. Se questo non accade, la misurazione e la valutazione dei servizi bibliotecari non oltrepassa il confine dello studio o dell'esercitazione.

Come qualsivoglia attività di servizio, la statistica bibliotecaria per essere efficace ha bisogno di informazioni di feedback sugli effetti da essa prodotti e, particolarmente, sulle aspettative e l'uso degli indicatori. Tale genere di informazione è necessaria a maggior ragione qualora si manifesti l'esigenza di revisionare un sistema di misurazione: ciò accade, nel contesto bibliotecario, ad intervalli di tempo più o meno regolari, perché la biblioteca, essendo un servizio "sociale" deve adeguarsi ai mutamenti ambientali e culturali che, nei tempi correnti, sono sempre più rapidi, improvvisi ed imprevedibili. Con tutta probabilità, un sistema di misurazione obsoleto è indice della obsolescenza di una biblioteca.

La ricerca qui descritta riguarda la percezione del sistema di misurazione in uso nel Sistema Bibliotecario di Ateneo (da qui: SBA) dell'Università degli Studi di Firenze ed è stata condotta nel corso del $2000^{1}$.

Sinteticamente, l'organizzazione amministrativa e funzionale del SBA si struttura su due livelli: uno relativo all'articolazione delle biblioteche, l'altro relativo ai gruppi di lavoro su attività che riguardano, in modo trasversale alle singole biblioteche, il sistema bibliotecario nel suo complesso. Entrambi i livelli fanno capo al Coordinamento centrale biblioteche, l'ufficio dirigenziale del SBA.

Nel 1999 le biblioteche appartenenti al SBA sono state riorganizzate in cinque unità amministrative, per settori disciplinari omogenei e trasversali all'organizzazione didattica dell'ateneo fiorentino, e sono state affidate alla responsabilità di altrettanti direttori ${ }^{2}$. I gruppi di lavoro di ateneo sono formati dai bibliotecari in servizio presso le biblioteche del SBA. Il coordinamento dei gruppi è affidato alla responsabilità di un bibliotecario.

Per un quadro aggiornato al 2002 del sistema di misurazione si veda l'Allegato A.

2 L'attuale organizzazione del SBA è illustrata nella Fig. 1, p. 17. 
Tab. 1 - I gruppi di lavoro nel 2000

\begin{tabular}{|c|c|}
\hline Gruppi di lavoro & Obiettivi \\
\hline $\begin{array}{l}\text { Attività cooperativa } \\
\text { nazionali e internazionali }\end{array}$ & Gestione dei progetti cooperativi con altri enti \\
\hline Formazione professionale & $\begin{array}{l}\text { Formazione e implementazione professionale dei } \\
\text { bibliotecari attraverso la progettazione e l'esecuzione dei } \\
\text { corsi di formazione }\end{array}$ \\
\hline $\begin{array}{l}\text { Gestione amministrativo } \\
\text { contabile }\end{array}$ & $\begin{array}{l}\text { Gestione centralizzata e standardizzata a livello di SBA dei } \\
\text { procedimenti amministrativi-contabili delle biblioteche }\end{array}$ \\
\hline Gestione biblioteca digitale & $\begin{array}{l}\text { Sviluppo della collezione digitale (periodici elettronici, } \\
\text { basi dati bibliografiche e risorse di rete) con la creazione } \\
\text { di un ambiente informativo integrato con la collezione } \\
\text { cartacea. Organizzazione dell'accesso e promozione dei } \\
\text { servizi digitali }\end{array}$ \\
\hline Gestione dell'automazione & $\begin{array}{l}\text { Analisi, implementazione, manutenzione, sviluppo e } \\
\text { integrazione, in collaborazione con il Settore informatico, } \\
\text { di sistemi gestionali e informativi automatizzati del SBA; } \\
\text { applicazione della tecnologia ai servizi bibliotecari }\end{array}$ \\
\hline $\begin{array}{l}\text { Gestione del prestito } \\
\text { interbibliotecario e del } \\
\text { document delivery }\end{array}$ & $\begin{array}{l}\text { Progettazione standardizzata a livello di SBA dei processi } \\
\text { di lavoro, della modulistica e delle griglie di rilevazione } \\
\text { dei dati. Partecipazione del SBA a sperimentazioni ed a } \\
\text { progetti cooperativi o consortili in materia di prestito } \\
\text { interbibliotecario o document delivery }\end{array}$ \\
\hline $\begin{array}{l}\text { Gestione e manutenzione } \\
\text { del catalogo }\end{array}$ & $\begin{array}{l}\text { Manutenzione del catalogo SBN del polo Università e } \\
\text { predisposizione di corsi di aggiornamento per il personale. } \\
\text { Definizione di progetti mirati al recupero e alla } \\
\text { valorizzazione del patrimonio storico delle biblioteche } \\
\text { dell'Università }\end{array}$ \\
\hline $\begin{array}{l}\text { Orientamento all'utenza e } \\
\text { servizi agli studenti }\end{array}$ & $\begin{array}{l}\text { Attività di assistenza e orientamento sui servizi delle } \\
\text { biblioteche, in particolare sulle risorse elettroniche, e di } \\
\text { promozione dei servizi }\end{array}$ \\
\hline $\begin{array}{l}\text { Progetto Firenze } \\
\text { University Press }\end{array}$ & Editore cartaceo e digitale dell'ateneo \\
\hline Sistema informativo & $\begin{array}{l}\text { Produce e mantiene aggiornato un sistema di misurazione } \\
\text { e valutazione dei servizi del SBA }\end{array}$ \\
\hline $\begin{array}{l}\text { Web del sistema } \\
\text { bibliotecario }\end{array}$ & $\begin{array}{l}\text { Creazione e manutenzione del sito internet del SBA; } \\
\text { coordinamento del lavoro dei gruppi che si occupano della } \\
\text { creazione e manutenzione delle pagine web delle singole } \\
\text { biblioteche di area }\end{array}$ \\
\hline
\end{tabular}


L'indagine sulla percezione del sistema di misurazione delle biblioteche universitarie di Firenze ha riguardato il coordinatore generale, i direttori di biblioteca ed i coordinatori dei gruppi di lavoro, con l'eccezione del gruppo che ha promosso l'indagine. I dati sono stati raccolti durante il periodo estivo. I questionari sono stati compilati dal $75 \%$ degli intervistati (12 persone su 16).

Nel testo del questionario, riportato di seguito, è descritta la struttura del sistema di misurazione. Esso si compone di una batteria di dati e di una batteria di indicatori. Per ogni elemento è riportata la denominazione, l'oggetto della misurazione e, nel caso degli indicatori, la formula di calcolo.

\section{Il testo del questionario}

Gentile collega,

il gruppo di lavoro che cura il sistema informativo del SBA vorrebbe conoscere le tue opinioni sulle statistiche bibliotecarie in uso nel nostro ateneo.

Se, da un lato, è necessario misurare la performance delle biblioteche, dall'altro è utile valutare l'effettivo impatto che le misurazioni hanno sul lavoro bibliotecario di tutti i giorni.

Il questionario si articola in varie sezioni.

La prima e la seconda riguardano, rispettivamente, i dati e gli indicatori in uso. Di ogni misura è riportata la definizione e la formula di calcolo. Per rispondere non è necessaria la diretta conoscenza tecnica delle misure in questione: lo scopo principale di questa intervista è di valutare "che cosa", al di là del metodo di calcolo, può essere utile misurare e quali "settori" della misurazione sviluppare o ridimensionare.

Le altre sezioni definiscono un quadro di riferimento per meglio interpretare le risposte precedenti. Non ci sono risposte giuste o sbagliate, è per noi importante conoscere le opinioni reali.

Il sistema statistico è in fase di revisione: osservazioni e critiche, sia generali sia sui singoli indicatori, costituiscono un importante contributo al miglioramento del nostro servizio.

Grazie per la collaborazione

Il gruppo di lavoro Sistema informativo 


\begin{tabular}{|c|c|c|c|c|c|c|c|c|c|c|}
\hline Istruzioni per la compilazione & \multicolumn{5}{|c|}{$a$} & \multicolumn{5}{|c|}{$b$} \\
\hline \multirow{2}{*}{$\begin{array}{l}\text { Considera le attività delle quali sei responsabile a } \\
\text { livello di SBA (direttore di biblioteca o coordina- } \\
\text { tore di gruppo di lavoro). Nella colonna a chie- } \\
\text { diamo il tuo parere sulle informazioni che un } \\
\text { sistema gestionale eccellente, in relazione alle } \\
\text { attività da te coordinate, dovrebbe presentare: se } \\
\text { ritieni che, sotto il profilo teorico, un dato sia del } \\
\text { tutto inutile, barra il valore } 1 \text {. Se ritieni che il } \\
\text { dato sia importante alla gestione di un servizio } \\
\text { bibliotecario, barra il numero } 5 \text {. Nella colonna b } \\
\text { ti chiediamo di indicare l'effettivo uso che fai dei } \\
\text { dati e degli indicatori del nostro sistema. }\end{array}$} & \multicolumn{5}{|c|}{$\begin{array}{l}\text { Da un punto di vista } \\
\text { biblioteconomico, } \\
\text { quanto ritieni che } \\
\text { siano importanti i } \\
\text { seguenti dati per la } \\
\text { buona gestione del } \\
\text { servizio affidato alla } \\
\text { tua responsabilità? }\end{array}$} & \multicolumn{5}{|c|}{$\begin{array}{l}\text { Quale uso fai dei } \\
\text { seguenti dati nella } \\
\text { gestione dei servizi } \\
\text { affidati alla tua } \\
\text { responsabilità? }\end{array}$} \\
\hline & 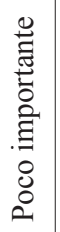 & $\vdots$ & $\vdots$ & & 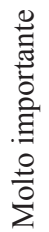 & 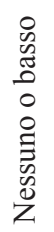 & $\vdots$ & $\vdots$ & & $\stackrel{8}{\frac{8}{4}}$ \\
\hline
\end{tabular}

\begin{tabular}{|l|l|l|l|l|l|l|l|l|l|l|l|}
\hline DATI & 1 & 2 & 3 & 4 & 5 & 1 & 2 & 3 & 4 & 5 \\
\hline $\begin{array}{l}\text { N1.01 - Docenti e ricercatori } \\
\text { Numero dei docenti delle tre fasce che } \\
\text { lavorano presso il nostro ateneo }\end{array}$ & 1 & 2 & 3 & 4 & 5 & 1 & 2 & 3 & 4 & 5 \\
\hline $\begin{array}{l}\text { D1.02 - Studenti } \\
\text { Numero degli iscritti ai corsi di laurea, } \\
\text { di diploma universitario ed alle scuole } \\
\text { di specializzazione per l'anno accademico } \\
\text { di riferimento }\end{array}$ & & & & & & & & & & & \\
\hline $\begin{array}{l}\text { D2.01 - Patrimonio bibliografico } \\
\text { Il dato risulta dalla somma di: a) numero di } \\
\text { volumi dichiarati dai Direttori delle Biblioteche } \\
\text { prima dell'automazione; b) il numero di } \\
\text { inventari inseriti annualmente in SBN }\end{array}$ & 1 & 2 & 3 & 4 & 5 & 1 & 2 & 3 & 4 & 5 \\
\hline $\begin{array}{l}\text { D2.02 - Periodici correnti } \\
\text { Numero degli abbonamenti correnti nell'anno } \\
\text { di riferimento }\end{array}$ & 1 & 2 & 3 & 4 & 5 & 1 & 2 & 3 & 4 & 5 \\
\hline $\begin{array}{l}\text { D2.03 - Periodici disdetti } \\
\text { Numero degli abbonamenti non rinnovati } \\
\text { nell'anno di riferimento }\end{array}$ & 1 & 2 & 3 & 4 & 5 & 1 & 2 & 3 & 4 & 5 \\
\hline $\begin{array}{l}\text { D2.04 - Volumi e periodici ad accesso libero } \\
\text { Stima del numero di volumi collocati a scaffale } \\
\text { aperto (a partire dalla lunghezza in metri lineari } \\
\text { degli scaffali) }\end{array}$ & 1 & 2 & 3 & 4 & 5 & & 1 & 2 & 3 & 4 & 5 \\
\hline $\begin{array}{l}\text { D2.05 - Posti di lettura per Biblioteca } \\
\text { Numero dei posti a sedere nelle sale di lettura } \\
\text { delle biblioteche }\end{array}$ & 1 & 2 & 3 & 4 & 5 & 1 & 2 & 3 & 4 & 5 \\
\hline $\begin{array}{l}\text { D2.06 - PC destinati al pubblico } \\
\text { Numero dei personal e dei network computer } \\
\text { destinati al pubblico per biblioteca }\end{array}$ & 1 & 2 & 3 & 4 & 5 & 1 & 2 & 3 & 4 & 5 \\
\hline
\end{tabular}




\begin{tabular}{|c|c|c|c|c|c|c|c|c|c|c|}
\hline $\begin{array}{l}\text { D2.07- } m^{2} \text { al pubblico } \\
\text { Dimensione in metri quadrati degli spazi delle } \\
\text { biblioteche destinati al pubblico (sale di lettura, } \\
\text { sale cataloghi, distribuzione, eccetera) }\end{array}$ & 1 & 2 & 3 & 4 & 5 & 1 & 2 & 3 & 4 & 5 \\
\hline $\begin{array}{l}\text { D2.08- } m^{2} \text { totali } \\
\text { Dimensione in metri quadrati degli spazi delle } \\
\text { biblioteche }\end{array}$ & 1 & 2 & 3 & 4 & 5 & 1 & 2 & 3 & 4 & 5 \\
\hline $\begin{array}{l}\text { D2.09 - Punti di servizio } \\
\text { Numero dei punti di servizio del Sistema } \\
\text { bibliotecario di ateneo (biblioteche di facoltà, } \\
\text { di dipartimento, di istituto) aperti al pubblico } \\
\text { stabiliti dall'Ordinanza n. } 102 \text { del } 29 \text { marzo } 1996\end{array}$ & 1 & 2 & 3 & 4 & 5 & 1 & 2 & 3 & 4 & 5 \\
\hline $\begin{array}{l}\text { D2.10 - Ore settimanali di apertura } \\
\text { Orari ordinari dei punti di servizio del SBA } \\
\text { aperti al pubblico ad opera dei bibliotecari }\end{array}$ & 1 & 2 & 3 & 4 & 5 & 1 & 2 & 3 & 4 & 5 \\
\hline $\begin{array}{l}\text { D2.11 - Personale per qualifica funzionale } \\
\text { Personale attribuito alle unità amministrative del } \\
\text { SBA per qualifiche funzionali }\end{array}$ & 1 & 2 & 3 & 4 & 5 & 1 & 2 & 3 & 4 & 5 \\
\hline $\begin{array}{l}\text { D2.12 - Personale suddiviso per attività } \\
\text { Stima del tempo di lavoro dedicato alle varie } \\
\text { attività da parte dei bibliotecari }\end{array}$ & 1 & 2 & 3 & 4 & 5 & 1 & 2 & 3 & 4 & 5 \\
\hline $\begin{array}{l}\text { D2.13 - Formule di ripartizione } \\
\text { delle risorse umane } \\
\text { Unità di personale necessarie per lo svolgimento } \\
\text { delle attività secondo uno standard della } \\
\text { Association of College \& Research Libraries }\end{array}$ & 1 & 2 & 3 & 4 & 5 & 1 & 2 & 3 & 4 & 5 \\
\hline $\begin{array}{l}\text { D3.01 - Budget Università } \\
\text { Indica la dotazione finanziaria annuale dell'ateneo }\end{array}$ & 1 & 2 & 3 & 4 & 5 & 1 & 2 & 3 & 4 & 5 \\
\hline $\begin{array}{l}\text { D3.02 - Budget Sistema bibliotecario d'Ateneo } \\
\text { Spese sostenute dalle biblioteche }\end{array}$ & 1 & 2 & 3 & 4 & 5 & 1 & 2 & 3 & 4 & 5 \\
\hline $\begin{array}{l}\text { D3.03-04 Spese libri, periodici, } \\
\text { rilegature, risorse elettroniche } \\
\text { Spese sostenute nelle voci sopra indicate } \\
\text { aggregate per biblioteche di area e SBA }\end{array}$ & 1 & 2 & 3 & 4 & 5 & 1 & 2 & 3 & 4 & 5 \\
\hline $\begin{array}{l}\text { D4.01 - Inventari } \\
\text { Numero dei nuovi inventari in un anno, dunque } \\
\text { il numero dei singoli volumi acquisiti dalla } \\
\text { biblioteca }\end{array}$ & 1 & 2 & 3 & 4 & 5 & 1 & 2 & 3 & 4 & 5 \\
\hline $\begin{array}{l}\text { D4.02 - Catalogazioni } \\
\text { Numero di inventari catalogati e collocati in } \\
\text { un anno }\end{array}$ & 1 & 2 & 3 & 4 & 5 & 1 & 2 & 3 & 4 & 5 \\
\hline $\begin{array}{l}\text { D4.03 - Catture } \\
\text { Numero delle catture di notizie bibliografiche }\end{array}$ & 1 & 2 & 3 & 4 & 5 & 1 & 2 & 3 & 4 & 5 \\
\hline $\begin{array}{l}\text { D4.04 - Recupero del pregresso } \\
\text { Numero dei recuperi del pregresso }\end{array}$ & 1 & 2 & 3 & 4 & 5 & 1 & 2 & 3 & 4 & 5 \\
\hline $\begin{array}{l}\text { D4.05 - Tempi dell'iter del libro } \\
\text { Tempi delle fasi di catalogazione, dalla ricezione } \\
\text { del libro alla sua collocazione }\end{array}$ & 1 & 2 & 3 & 4 & 5 & 1 & 2 & 3 & 4 & 5 \\
\hline
\end{tabular}




\begin{tabular}{|l|l|l|l|l|l|l|l|l|l|l|l|}
\hline $\begin{array}{l}\text { D4.06 - Prestiti } \\
\text { Numero dei prestiti in un anno e le statistiche } \\
\text { relative ai 50 inventari più prestati }\end{array}$ & 1 & 2 & 3 & 4 & 5 & 1 & 2 & 3 & 4 & 5 \\
\hline $\begin{array}{l}\text { D4.07 - Prestiti interbibliotecari } \\
\text { Attività ILL e DD secondo gli schemi } \\
\text { attualmente in uso }\end{array}$ & 1 & 2 & 3 & 4 & 5 & 1 & 2 & 3 & 4 & 5 \\
\hline $\begin{array}{l}\text { D4.09-Ricerche in OPAC } \\
\text { Si riporta il numero degli accessi all’OPAC } \\
\text { in un anno }\end{array}$ & 1 & 2 & 3 & 4 & 5 & 1 & 2 & 3 & 4 & 5 \\
\hline $\begin{array}{l}\text { D4.10 - Ricerche in Ultranet } \\
\text { Numero totale degli accessi alle banche dati } \\
\text { di Ultranet in un anno }\end{array}$ & 1 & 2 & 3 & 4 & 5 & 1 & 2 & 3 & 4 & 5 \\
\hline $\begin{array}{l}\text { D4.12 - Corsi per studenti } \\
\text { Numero dei corsi organizzati dalle biblioteche } \\
\text { e dal coordinamento e numero dei partecipanti }\end{array}$ & 1 & 2 & 3 & 4 & 5 & 1 & 2 & 3 & 4 & 5 \\
\hline
\end{tabular}

\begin{tabular}{|c|c|c|c|c|c|c|c|c|c|c|}
\hline Istruzioni per la compilazione & \multicolumn{5}{|c|}{$a$} & \multicolumn{5}{|c|}{$b$} \\
\hline \multirow{2}{*}{$\begin{array}{l}\text { Considera le attività delle quali sei responsabile a } \\
\text { livello di SBA (direttore di biblioteca o coordina- } \\
\text { tore di gruppo di lavoro). Nella colonna a chie- } \\
\text { diamo il tuo parere sulle informazioni che un } \\
\text { sistema gestionale eccellente, in relazione alle } \\
\text { attività da te coordinate, dovrebbe presentare: se } \\
\text { ritieni che, sotto il profilo teorico, un indicatore } \\
\text { sia del tutto inutile, barra il valore } 1 \text {. Se ritieni } \\
\text { che l'indicatore sia importante alla gestione di un } \\
\text { servizio bibliotecario, barra il numero } 5 \text {. Nella } \\
\text { colonna b ti chiediamo di indicare l'effettivo uso } \\
\text { che fai dei dati e degli indicatori del nostro siste- } \\
\text { ma. }\end{array}$} & \multicolumn{5}{|c|}{$\begin{array}{l}\text { Da un punto di vista } \\
\text { biblioteconomico, } \\
\text { quanto ritieni che } \\
\text { siano importanti i } \\
\text { seguenti indicatori } \\
\text { per la buona gestione } \\
\text { di un servizio biblio- } \\
\text { tecario? }\end{array}$} & \multicolumn{5}{|c|}{$\begin{array}{l}\text { Quale uso fai dei } \\
\text { seguenti indicatori } \\
\text { nella gestione dei } \\
\text { servizi affidati alla } \\
\text { tua responsabilità? }\end{array}$} \\
\hline & 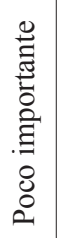 & $\vdots$ & $\vdots$ & $\vdots$ & 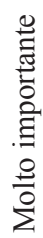 & $\begin{array}{l}0 \\
0 \\
0 \\
0 \\
0 \\
0 \\
0 \\
\vdots \\
\vdots \\
0 \\
0 \\
0\end{array}$ & $\vdots$ & $\vdots$ & $\vdots$ & $\frac{E}{2}$ \\
\hline
\end{tabular}

\begin{tabular}{|l|l|l|l|l|l|l|l|l|l|l|l|}
\hline INDICATORI & & & & & & & & & & & \\
\hline $\begin{array}{l}\text { Il.01 - Iscritti / posti di lettura } \\
\text { Indica il numero di studenti che potenzialmente } \\
\text { gravitano su ogni posto a sedere delle sale } \\
\text { di lettura. }\end{array}$ & 1 & 2 & 3 & 4 & 5 & 1 & 2 & 3 & 4 & 5 \\
\hline $\begin{array}{l}\text { I1.01 = D1.02 / D2.05 } \\
\text { Il.02 - Iscritti / mq Biblioteche } \\
\begin{array}{l}\text { Indica il numero di studenti che potenzialmente } \\
\text { gravitano su ogni metro quadrato della biblioteca. } \\
\text { I1.02 = D1.02 / D2.07 }\end{array}\end{array}$ & 1 & 2 & 3 & 4 & 5 & 1 & 2 & 3 & 4 & 5 \\
\hline $\begin{array}{l}\text { I1.03 - Volumi / utenza } \\
\text { Indica per ogni biblioteca il numero di volumi } \\
\text { disponibili per ogni studente. } \\
\text { I1.03 = D2.01 / D1.02 }\end{array}$ & 1 & 2 & 3 & 4 & 5 & & 1 & 2 & 3 & 4 & 5 \\
\hline
\end{tabular}


I1.04 - Spesa del Sistema bibliotecario /

Spesa dell'Ateneo

Indica la percentuale del budget di ateneo destinata al SBA

$\mathrm{I} 1.04=(\mathrm{D} 3.02 / \mathrm{D} 3.01) \times 100$

I1.05 - Iscritti / Corsi per gli studenti

Indica il numero di studenti che potenzialmente potrebbero frequentare un singolo corso organizzato dalle biblioteche o dal coordinamento.

$\mathrm{I} 1.05=\mathrm{D} 1.02 / \mathrm{D} 4.12$

I1.06 - Volumi ad accesso libero / volumi totali Indica la percentuale del patrimonio bibliografico collocata a scaffale aperto rispetto al posseduto. $\mathrm{I} 1.06=(\mathrm{D} 2.04 / \mathrm{D} 2.01) \times 100$

I2.01 - Indice di circolazione Indica la percentuale del patrimonio documentario dato in prestito (esterno, interno, notturno) con gestione in SBN.

$\mathrm{I} 2.01=(\mathrm{D} 4.06 / \mathrm{D} 2.01) \times 100$

I2.02-Prestiti / BID

Indica quante volte ogni edizione posseduta $\mathrm{e}$ registrata in SBN è andata potenzialmente in prestito, indipendentemente dal numero di esemplari posseduti.

$\mathrm{I} 2.02=\mathrm{D} 4.06 /$ totale dei BID

I2.03 - Indice di penetrazione ambientale

Stima della percentuale degli studenti che usano le biblioteche (il servizio di prestito) rispetto al totale degli studenti universitari.

I2.03 = totale delle iscrizioni al prestito / D1.02

I2.04-Prestiti / iscritti

Stima del numero di volumi mediamente presi in prestito (esterno, interno, notturno) da ogni studente.

$\mathrm{I} 2.04=\mathrm{D} 4.06 / \mathrm{D} 1.02$

\section{I2.05 - Prestiti interbibliotecari / iscritti}

Stima del numero medio annuale di prestiti interbibliotecari (borrowing + lending) fruiti da ogni studente.

$\mathrm{I} 2.05=\mathrm{D} 4.07 / \mathrm{D} 1.02$

I2.08 - Ricerche in OPAC / iscritti

Stima del numero medio annuale degli accessi al server WWW-OPAC da parte di ogni studente. $\mathrm{I} 2.08=\mathrm{D} 4.09 / \mathrm{D} 1.02$

I2.09 - Ricerche in Ultranet / iscritti

Stima del numero medio annuale delle ricerche condotte in Ultranet da ogni studente.

$\mathrm{I} 2.09=\mathrm{D} 4.10 / \mathrm{D} 1.02$ 


\begin{tabular}{|c|c|c|c|c|c|c|c|c|c|c|}
\hline $\begin{array}{l}\text { I2.10 - Percentuale degli iscritti partecipanti } \\
\text { ai corsi } \\
\text { Indica la percentuale degli studenti che ha } \\
\text { partecipato ai corsi di formazione per gli utenti. } \\
\text { I1.10 = (D4.12 / D1.02) x } 100\end{array}$ & 1 & 2 & 3 & 4 & 5 & 1 & 2 & 3 & 4 & 5 \\
\hline $\begin{array}{l}\text { I2.11 - Percentuale di recupero } \\
\text { Indica la percentuale dei recuperi sul totale delle } \\
\text { catalogazioni. } \\
\text { I2.11 = }\end{array}$ & 1 & 2 & 3 & 4 & 5 & 1 & 2 & 3 & 4 & 5 \\
\hline $\begin{array}{l}\text { I2.12 - Percentuale di catalogazione derivata } \\
\text { Indica la percentuale delle notizie bibliografiche } \\
\text { catturate da SBN sul totale delle catalogazioni. } \\
\text { I2.12=(D4.03 / D4.02) } \times 100\end{array}$ & 1 & 2 & 3 & 4 & 5 & 1 & 2 & 3 & 4 & 5 \\
\hline $\begin{array}{l}\text { I2.13 - Tempo medio dell'iter del libro } \\
\text { Indica i tempi di catalogazione, dalla fase della } \\
\text { ricezione in biblioteca del libro alla sua } \\
\text { collocazione sugli scaffali. } \\
\text { METODO DI CALCOLO ABROGATO E } \\
\text { DA SOSTITUIRE }\end{array}$ & 1 & 2 & 3 & 4 & 5 & 1 & 2 & 3 & 4 & 5 \\
\hline $\begin{array}{l}\text { I4.01 - Numero delle catalogazioni in un'ora } \\
\text { Indica il numero medio di volumi che la } \\
\text { biblioteca è riuscita a catalogare in un'ora di } \\
\text { lavoro dedicata alla catalogazione. } \\
\text { I4.01 = (D4.02 + D4.04) / "Carichi" } 203\end{array}$ & 1 & 2 & 3 & 4 & 5 & 1 & 2 & 3 & 4 & 5 \\
\hline $\begin{array}{l}\text { I4.02 - Numero delle acquisizioni in un'ora } \\
\text { Indica il numero medio di acquisizioni che la } \\
\text { biblioteca è riuscita ad effettuare in un'ora di } \\
\text { lavoro dedicata alle acquisizioni. } \\
\text { I4.02 = D4.01 / "Carichi" } 201\end{array}$ & 1 & 2 & 3 & 4 & 5 & 1 & 2 & 3 & 4 & 5 \\
\hline $\begin{array}{l}\text { I4.03 - Numero di operazioni ILL + Prestito } \\
\text { in un'ora } \\
\text { Indica il numero medio di operazioni di prestito } \\
\text { interbibliotecario, document delivery e prestito } \\
\text { locale che la biblioteca è riuscita ad effettuare } \\
\text { in un'ora di lavoro dedicata a queste attività. } \\
\text { I4.03 = (D4.06 + D4.07) / "Carichi" } 211\end{array}$ & 1 & 2 & 3 & 4 & 5 & 1 & 2 & 3 & 4 & 5 \\
\hline
\end{tabular}




\begin{tabular}{|c|c|c|c|c|c|c|c|c|c|c|}
\hline \multirow{3}{*}{$\begin{array}{l}\text { Le prossime aree della misurazione non fanno } \\
\text { ancora parte del sistema informativo. Indica } \\
\text { quanto le ritieni importanti e che uso ne faresti } \\
\text { per la gestione dei servizi affidati alla tua respon- } \\
\text { sabilità. }\end{array}$} & \multicolumn{5}{|c|}{$a$} & \multicolumn{5}{|c|}{$b$} \\
\hline & \multicolumn{5}{|c|}{$\begin{array}{l}\text { Quanto ritieni che } \\
\text { siano importanti gli } \\
\text { indicatori sulle } \\
\text { seguenti dimensioni? }\end{array}$} & \multicolumn{5}{|c|}{$\begin{array}{l}\text { Quale uso ne faresti } \\
\text { per la gestione dei } \\
\text { servizi affidati alla } \\
\text { tua responsabilità? }\end{array}$} \\
\hline & 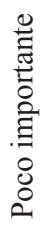 & $\vdots$ & $\vdots$ & $\vdots$ & 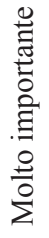 & 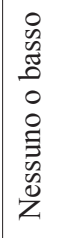 & $\vdots$ & $\vdots$ & $\vdots$ & $\stackrel{\circ}{\frac{O}{4}}$ \\
\hline $\begin{array}{l}\text { I3.01 - Soddisfazione per la qualità delle } \\
\text { raccolte } \\
\text { Indicatori sulla percezione della qualità delle } \\
\text { raccolte rispetto alle necessità documentarie degli } \\
\text { utenti (la qualità della gestione di acquisizioni, } \\
\text { patrimonio corrente e retrospettivo dal punto di } \\
\text { vista delle sue potenzialità informative) }\end{array}$ & 1 & 2 & 3 & 4 & 5 & 1 & 2 & 3 & 4 & 5 \\
\hline $\begin{array}{l}\text { I3.02 - Soddisfazione per la circolazione } \\
\text { Indicatori sulla percezione della qualità del } \\
\text { sistema di circolazione documentaria (locale, } \\
\text { interbibliotecaria, cartacea, elettronica) }\end{array}$ & 1 & 2 & 3 & 4 & 5 & 1 & 2 & 3 & 4 & 5 \\
\hline $\begin{array}{l}\text { I3.03 - Soddisfazione per il reference } \\
\text { Indicatori sulla percezione qualitativa del servizio } \\
\text { di orientamento e assistenza bibliografica }\end{array}$ & 1 & 2 & 3 & 4 & 5 & 1 & 2 & 3 & 4 & 5 \\
\hline $\begin{array}{l}\text { I3.04 - Soddisfazione per le risorse tecniche } \\
\text { e umane } \\
\text { Indicatori sulla percezione qualitativa della } \\
\text { disponibilità di risorse tecniche e umane presso } \\
\text { i punti di servizio }\end{array}$ & 1 & 2 & 3 & 4 & 5 & 1 & 2 & 3 & 4 & 5 \\
\hline
\end{tabular}


Quali aree della misurazione sono a tuo parere importanti per le attività bibliotecarie affidate alla tua responsabilità? Distribuisci un punteggio totale di 100 punti tra le sei aree sotto indicate relativamente all'importanza attribuita da te ad ognuna di esse. Controlla che la somma totale sia di 100 punti.

\begin{tabular}{|c|c|c|}
\hline 1 & Misure sull'ambiente ${ }^{3}$ da servire & Punti \\
\hline 2 & Misure sulle risorse ${ }^{4}$ documentarie, materiali e umane della biblioteche & Punti \\
\hline 3 & Misure sui costi ${ }^{5}$ delle risorse & Punti \\
\hline 4 & Misure sull'accessibilità ${ }^{6}$ delle biblioteche & Punti \\
\hline 5 & Misure sugli usi ${ }^{7}$ delle risorse da parte del pubblico & Punti \\
\hline 6 & Misure sulla produttività ${ }^{8}$ del personale & Punti \\
\hline \multirow[t]{2}{*}{7} & Misure sulla soddisfazione ${ }^{9}$ degli utenti & Punti \\
\hline & Totale dei punti assegnati & 100 punti \\
\hline
\end{tabular}

Con quale frequenza consulti il sistema informativo per prendere decisioni?

\begin{tabular}{|l|l|}
\hline Mai & \\
\hline una volta ogni__ giorni & \\
\hline
\end{tabular}

Come vorresti comunicare i dati che il Coordinamento centrale biblioteche periodicamente ti chiede?

(una sola risposta)

\begin{tabular}{|l|l|}
\hline Tramite form (sul web del SBA) da aggiornare quando necessario & \\
\hline Con questionari da compilare e inviare al coordinamento & \\
\hline Comunicando i dati volta per volta che il coordinamento li chiede & \\
\hline
\end{tabular}

3 L'ambiente è costituito dall'utenza istituzionale da servire, nelle sue caratteristiche culturali, sociali ed economiche.

4 Le risorse sono costituite da tutti gli "input" o beni (documentari, materiali, tecnici e umani) che, attraverso i processi lavorativi, sono trasformati in "output" o prestazioni di servizio.

5 I costi si riferiscono agli investimenti monetari necessari a finanziare l'acquisizione delle risorse.

6 L'accessibilità si riferisce alla capacità della biblioteca di rendere fruibili i suoi servizi nel tempo (es.: orario di apertura) e nello spazio (es: n. dei posti di lettura, disponibilità di servizi a distanza).

7 Gli usi si riferiscono alla fruizione delle risorse da parte dell'utenza.

8 La produttività indica la quantità di lavoro bibliotecario prodotta dal personale.

9 La soddisfazione è la percezione della qualità dei servizi bibliotecari da parte degli utenti rispetto alle loro aspettative. 
Come vorresti consultare il sistema informativo?

(una sola risposta)

\begin{tabular}{|l|l|}
\hline Sul web del SBA & \\
\hline Su report annuale cartaceo & \\
\hline Chiedendo i dati al coordinamento ogni volta che servono & \\
\hline
\end{tabular}

Qual è il tuo ruolo nel SBA tra quelli sotto presentati?

\begin{tabular}{|l|l|}
\hline Coordinatore generale & \\
\hline Direttore della Biblioteca ... & \\
\hline Coordinatore di Gruppo del lavoro di ateneo ... & \\
\hline
\end{tabular}

Quali misure non incluse nei primi due elenchi vorresti che fossero inserite nel sistema informativo?

Altre tue osservazioni sul sistema informativo:

Fine del questionario

Grazie per la collaborazione 


\section{I risultati}

I dati sono stati trattati sulla falsariga del metodo SERVQUAL ${ }^{10}$. L'indagine si proponeva di rilevare l'eventuale scostamento esistente tra l'aspettativa e l'uso riguardanti i dati e gli indicatori del sistema di misurazione. Si è chiesto inoltre di indicare la frequenza di consultazione del sistema statistico e il metodo preferito per comunicare e consultare i dati, nonché di effettuare osservazioni non strutturate inerenti l'attuale sistema.

Dati e indicatori sono stati attribuiti a sette dimensioni della misurazione, come sotto indicato:

1. Ambiente: D1.01, D1.02, I2.03, I2.10.

2. Risorse: D2.01, D2.02, D2.03, D2.06, D2.11, D2.13, D4.01, D4.12, I1.03, I1.05.

3. Costi: D3.01, D3.02, D3.03-4, I1.04.

4. Accessibilità: D2.04, D2.05, D2.07, D2.08, D2.09, D2.10, I1.01, I1.02, I1.06.

5. Usi: D4.06, D4.07, D4.09, D4.10, I2.01, I2.02, I2.04, I2.05, I2.08, I2.09.

6. Produttività: D2.12, D4.02, D4.03, D4.04, D4.05, I2.11, I2.12, I2.13, I4.01, I4.02, I4.03.

7. Soddisfazione: I3.01, I3.02, I3.03, I3.04.

Per ogni dato e indicatore è stata proposta una coppia di enunciati. Il primo enunciato della coppia evidenzia l'aspettativa del bibliotecario sotto il profilo biblioteconomico rispetto al dato o indicatore in questione, il secondo enunciato chiede di dichiarare con che frequenza il bibliotecario usa quel dato o indicatore nelle sue attività. L'intervistato ha a disposizione una scala di cinque risposte predefinite, delle quali la prima indica una bassa aspettativa o un basso uso, la quinta indica alta aspettativa o alto uso, le altre risposte denotano valori intermedi. Sottraendo al punteggio dell'uso il punteggio dell'aspettativa, otteniamo lo scostamento tra uso e aspettativa. Un valore positivo denota un ricorso al dato o all'indicatore superiore all'importanza ad esso attribuita (la qual cosa viene assunta come indice di qualità del dato o indicatore), un valore negativo denota al contrario un sotto-utilizzo.

Ad ogni intervistato è richiesto di dichiarare l'importanza relativa assunta dalle sette dimensioni della qualità (delle quali una, quella sulla soddisfazione degli utenti, non è ancora in uso). Nel questionario non appare quali dati e

10 Zeithaml, Parasuraman \& Berry 1991. 
indicatori facciano parte di ogni dimensione, ciò per ottenere risposte non condizionate dall'appartenenza dell'elemento.

I risultati sono stati sintetizzati in quattro serie di grafici. La prima serie illustra le risposte degli intervistati, prescindendo dall'importanza del ruolo ricoperto ed attribuendo ad ognuno di essi il medesimo peso. La seconda serie prende in considerazione le risposte date dai direttori di biblioteca. La terza serie considera i risultati dei coordinatori dei gruppi di lavoro. L'ultima serie presenta i dati con riferimento al ruolo ricoperto dagli intervistati. I ruoli di coordinatore generale, direttore di biblioteca e coordinatore di gruppo di lavoro, pur operando in modo integrato, pesano in modo diverso sulla politica gestionale del SBA. In relazione all'ultima serie, si è dunque deciso di ponderare i dati per ruoli, col seguente criterio: il $35 \%$ del peso è attribuito alle risposte date dal coordinatore generale, il 30\% è stato attribuito ai direttori di biblioteca $(7,5 \%$ per direttore, poiché hanno risposto quattro direttori su cinque), il 35\% ai coordinatori di gruppi di lavoro (5\% per coordinatore, poiché hanno risposto sette coordinatori su undici).

\begin{tabular}{|lcccc|}
\hline Sintesi dei risultati & Dati & Indicatori & $\begin{array}{c}\text { Importanza } \\
\text { dimensioni }\end{array}$ & $\begin{array}{c}\text { Performance } \\
\text { dimensioni }\end{array}$ \\
\hline Non ponderati per ruoli & Tab. 1 & Tab. 5 & Tab. 9 & Tab. 13 \\
\hline Direttori di biblioteca & Tab. 2 & Tab. 6 & Tab. 10 & Tab. 14 \\
\hline Coordinatori di gruppo di lavoro & Tab. 3 & Tab. 7 & Tab. 11 & Tab. 15 \\
\hline Ponderati per ruoli & Tab. 4 & Tab. 8 & Tab. 12 & Tab. 16 \\
\hline
\end{tabular}

Il calcolo dei valori relativi alle tabelle di sintesi è stato effettuato come segue:

\begin{tabular}{|ll|}
\hline Tab. 1 & $\begin{array}{l}\text { Lo scostamento è calcolato sottraendo, per ogni dato, la media aritmetica dell'aspettativa } \\
\text { alla media aritmetica dell'uso, a partire dalle risposte date da tutti gli intervistati. }\end{array}$ \\
\hline Tab. 2 & $\begin{array}{l}\text { Lo scostamento è calcolato sottraendo, per ogni dato, la media aritmetica dell'aspettativa } \\
\text { alla media aritmetica dell'uso, a partire dalle risposte date dai direttori di biblioteca. }\end{array}$ \\
\hline Tab. 3 & $\begin{array}{l}\text { Lo scostamento è calcolato sottraendo, per ogni dato, la media aritmetica dell'aspettativa } \\
\text { alla media aritmetica dell'uso, a partire dalle risposte date dai coordinatori dei gruppi di } \\
\text { lavoro. }\end{array}$ \\
\hline Tab. 4 & $\begin{array}{l}\text { Lo scostamento è calcolato sottraendo, per ogni dato, la somma delle aspettative } \\
\text { ponderate per ruoli alla somma degli usi ponderati per ruoli, a partire dalle risposte date } \\
\text { da tutti gli intervistati. }\end{array}$ \\
\hline Tab. 5 & $\begin{array}{l}\text { Lo scostamento è calcolato sottraendo, per ogni indicatore, la media aritmetica dell'aspetta- } \\
\text { tiva alla media aritmetica dell'uso, a partire dalle risposte date da tutti gli intervistati. }\end{array}$ \\
\hline Tab. 6 & $\begin{array}{l}\text { Lo scostamento è calcolato sottraendo, per ogni indicatore, la media aritmetica } \\
\text { dell'aspettativa alla media aritmetica dell'uso, a partire dalle risposte date dai direttori di } \\
\text { biblioteca. }\end{array}$ \\
\hline
\end{tabular}




\begin{tabular}{|c|c|}
\hline Tab. 7 & $\begin{array}{l}\text { Lo scostamento è calcolato sottraendo, per ogni indicatore, la media aritmetica } \\
\text { dell'aspettativa alla media aritmetica dell'uso, a partire dalle risposte date dai coordinatori } \\
\text { dei gruppi di lavoro. }\end{array}$ \\
\hline Tab. 8 & $\begin{array}{l}\text { Lo scostamento è calcolato sottraendo, per ogni indicatore, la somma delle aspettative } \\
\text { ponderate per ruoli alla somma degli usi ponderati per ruoli, a partire dalle risposte date } \\
\text { da tutti gli intervistati. }\end{array}$ \\
\hline Tab. 9 & $\begin{array}{l}\text { L'importanza è calcolata con la media aritmetica dei valori dichiarati dagli intervistati } \\
\text { relativamente a ciascuna dimensione. }\end{array}$ \\
\hline Tab. 10 & $\begin{array}{l}\text { L'importanza è calcolata con la media aritmetica dei valori dichiarati dai direttori di } \\
\text { biblioteca relativamente a ciascuna dimensione. }\end{array}$ \\
\hline Tab. 11 & $\begin{array}{l}\text { L'importanza è calcolata con la media aritmetica dei valori dichiarati dai coordinatori dei } \\
\text { gruppi di lavoro relativamente a ciascuna dimensione. }\end{array}$ \\
\hline Tab. 12 & $\begin{array}{l}\text { L'importanza per ogni dimensione è calcolata con la somma ponderata per ruoli dei valori } \\
\text { dell'importanza, a partire dalle risposte date da tutti gli intervistati. }\end{array}$ \\
\hline Tab. 13 & $\begin{array}{l}\text { La performance è calcolata con la media aritmetica degli scostamenti in ciascuna } \\
\text { dimensione, ponderati sul valore dell'importanza attribuita da ogni intervistato. }\end{array}$ \\
\hline Tab. 14 & $\begin{array}{l}\text { La performance è calcolata con la media aritmetica degli scostamenti in ciascuna } \\
\text { dimensione, ponderati sul valore dell'importanza attribuita dai direttori di biblioteca. }\end{array}$ \\
\hline Tab. 15 & $\begin{array}{l}\text { La performance è calcolata con la media aritmetica degli scostamenti in ciascuna dimen- } \\
\text { sione, ponderati sul valore dell'importanza attribuita dai coordinatori di gruppo di ateneo. }\end{array}$ \\
\hline Tab. 16 & $\begin{array}{l}\text { La performance è calcolata con la somma ponderata per ruoli degli scostamenti in } \\
\text { ciascuna dimensione, ponderati sul valore dell'importanza attribuita da ogni intervistato. }\end{array}$ \\
\hline
\end{tabular}

\section{I grafici sono riportati nelle pagine $48-53$}

Sul piano generale si nota una buona percezione del sistema statistico da parte degli intervistati. Considerando le tabelle 1, 4, 5 e 8, notiamo che l'aspettativa si attesta su valori elevati per la quasi totalità dei dati e degli indicatori. L'uso, tuttavia, appare leggermente sottodimensionato rispetto alle aspettative, sia nelle tabelle che presentano i risultati non ponderati per ruolo, sia nelle tabelle che presentano i risultati ponderati per ruolo.

L'uso dei dati è appena superiore a quello che si fa degli indicatori, come dimostrano gli scostamenti complessivi tratti dalle tabelle sopra indicate: per le tabelle 1 e 4 rispettivamente di $-0,80$ e $-0,50$; per le tabelle 5 e 8 rispettivamente di $-1,00$ e $-0,71$.

Gli scostamenti delle tabelle 1 e 5 (risultati non ponderati per ruoli) si presentano in modo speculare e in misura ridotta nelle tabelle 4 e 8 (risultati ponderati per ruoli) per l'effetto delle ponderazioni alle quali i risultati sono stati sottoposti.

Gli scostamenti negativi più evidenti si notano in D4.05 e in I2.13, misure riguardanti i tempi dell'iter del libro, a causa della riconosciuta inaffidabilità 
dei metodi di calcolo dei valori connessi: si tratta difatti di misure ottenute interrogando il gestionale con un programma importato da altro ente ed i cui criteri di elaborazione sono oscuri, anche per il fatto che SBN non mantiene in memoria le date relative al completamento di alcune fasi del trattamento del libro. L'uso in sede gestionale di queste due misure, così come attualmente si calcolano, è percepito negativamente.

Per i dati, seguono D2.10 (Ore settimanali di apertura) e D4.06 (Numero dei prestiti) col medesimo scostamento. La performance di questi dati è legata alla recente riorganizzazione del SBA ed alla circostanza che ulteriori miglioramenti sono legati ad una maggiore disponibilità di personale (da destinare prevalentemente ai servizi al pubblico), materia che, da un lato, non rientra nelle esclusive competenze del settore amministrativo bibliotecario e dall'altro, difficilmente può essere oggetto di interventi immediati e pacifici. Anche D4.09 (Ricerche in OPAC) e D4.12 (Numero dei corsi organizzati dalle biblioteche e dal coordinamento e numero dei partecipanti) presentano una performance negativa accentuata. Il primo risultato è collegato alla performance negativa di D4.06, per il secondo si vedano le successive considerazioni svolte sull'indicatore collegato. Si noti comunque che apertura, ricerche, prestito e formazione costituiscono funzioni di base della biblioteca caratterizzate da una diretta esposizione all'utenza.

Per gli indicatori, la tabella dei valori non ponderati - dove ha maggior peso il punto di vista dei coordinatori dei gruppi di lavoro - presenta la seguente classifica: I2.13 (tempo medio dell'iter del libro), I2.01 (indice di circolazione) e, con uno scostamento minore, I1.01 (iscritti / posti di lettura), I2.04 (prestiti / iscritti), I2.05 (prestiti interbibliotecari / iscritti), I2.10 (percentuale degli iscritti partecipanti ai corsi) e I2.11 (percentuale di recupero). La tabella dei valori ponderati per ruoli presenta: I2.13 (tempo medio dell'iter del libro) I4.01 (numero delle catalogazioni in un'ora), I4.02 (numero delle acquisizioni in un'ora), I2.05 (prestiti interbibliotecari / iscritti), I2.01 (indice di circolazione) e I2.04 (prestiti / iscritti).

Tentiamo di tracciare alcune linee interpretative sugli indicatori. Sul sottoutilizzo di I2.13 abbiamo già detto.

Per quanto riguarda gli indicatori legati alla circolazione documentaria (I2.01, I2.04, I2.05) la performance negativa può dipendere dal fatto che il congestionamento degli spazi, l'entità dei fondi finanziari e le consuetudini amministrative legate alle acquisizioni cartacee non consentono ampie possibilità per l'innovazione delle usuali politiche. Inoltre, con la recente introduzione in catalogo dei prodotti dell'editoria elettronica, tra cui i numerose edizioni elettroniche di periodici scientifici, i dati relativi alla circolazione 
cartacea vanno perdendo di significato, soprattutto in relazione all'utenza più qualificata ed operante nella ricerca (laureandi, borsisti di varia natura, ricercatori e docenti) piuttosto che nella mera didattica (studenti non ancora giunti alla laurea). Produttività e area della catalogazione (I2,11, I4.01, I4.02) risultano legate in modo consequenziale alle vicissitudini appena accennate.

L'indicatore I1.01 presenta un fenomeno in relazione al quale il miglioramento può dipendere o da una diminuzione degli iscritti o dalla crescita dello spazio fisico a disposizione della biblioteca, per cui le relative politiche gestionali risultano vincolate da un altro numero di fattori e attori.

Lo scostamento di I2.10 denota probabilmente una difficoltà a rendere la formazione dell'utenza una prassi diffusa "a livello di massa": è evidente che la mancata valutazione (al momento dell'indagine) di tali corsi in sede di curriculum didattico degli studenti costituisce un forte disincentivo alla frequenza e solo una ristrettissima minoranza di studenti, forse i più sensibili alle potenzialità dei servizi bibliotecari, decide di frequentarli. Ne deriva che l'uso del dato e dell'indicatore risulta meno collegato di altri alle pratiche di gestione, non perché non si avverta l'importanza della formazione, ma per il fatto che la formazione coinvolge una quota residuale della popolazione universitaria e richiede un uso intenso di risorse umane usualmente impegnate nello svolgimento dei servizi di base.

Per quanto riguarda la performance delle dimensioni della misurazione (tabelle 13,14, 15 e 16) ci si attesta su scostamenti non inferiori a -0,30. Notiamo che l'uso della dimensione qualitativa, pur essendo riconosciuta come la più importante (tabelle 9, 10, 11 e 12), presenterebbe una performance negativa qualora fosse integrata nel sistema statistico (si sono confrontati l'aspettativa e l'uso che di queste misurazioni si sarebbe fatto), con divario maggiore per i coordinatori dei gruppi di lavoro, forse a causa della specializzazione e della minore esposizione diretta all'utenza di alcuni processi da costoro gestiti. Anche in questo caso, con tutta probabilità, giocano a sfavore della dimensione i succitati vincoli alla gestione bibliotecaria. Le altre dimensioni si attestano su una posizione di sostanziale equilibrio sebbene con un leggero scarto in negativo tra uso e aspettativa particolarmente per quanto concerne gli usi della biblioteca e la produttività del personale e comunque con valori non inferiori a $-0,22$.

I risultati relativi ai direttori delle biblioteche (tabelle 2, 6, 10 e 14) rivelano una situazione equilibrata sia nell'uso dei dati sia nell'uso degli indicatori. Tre sono i dati con performance positiva, e legati a due funzioni di base della biblioteca, l'aggiornamento scientifico e la catalogazione: D2.02 
(periodici correnti), D4.03 (catture) e D4.04 (recupero del pregresso), per i quali si registra un uso superiore all'importanza attribuita a tali funzioni. Il primo dato riguarda la principale fonte di aggiornamento scientifico della biblioteca, sulla quale l'inflazione costringe a tagli o spese in crescita, gli altri due dati riguardano la produttività ed interessano la velocità della catalogazione.

I2.03 (indice di penetrazione ambientale) e I2.05 (prestiti interbibliotecari fruiti da ogni studente) sono gli indicatori che rivelano il sottoutilizzo più marcato.

La dimensione di cui si fa un uso di pochissimo inferiore alla sua importanza, subito dopo quella della soddisfazione, è quella degli usi della biblioteca: probabilmente il congestionamento di molte strutture non consente politiche di incentivazione della frequenza delle sedi.

La performance complessiva del sistema statistico è, relativamente ai direttori di biblioteca, soddisfacente (tabella 14), senza sostanziali squilibri tra le dimensioni contemplate.

I coordinatori dei gruppi di lavoro di ateneo presentano un maggiore stacco tra l'aspettativa e l'uso dei dati e degli indicatori (tabelle 3 e 7), confermato dalla performance nelle dimensioni della misurazione (tabella 15). Tale situazione si giustifica con il fatto che il questionario chiedeva di indicare aspettative ed uso di dati e indicatori in stretta connessione con le attività svolte dal gruppo di lavoro, le quali riguardano processi trasversali sul piano della diffusione in ateneo ma settoriali su quello delle competenze e dei risultati. In altri termini, l'ambito degli interessi del coordinatore di un gruppo di lavoro non può riguardare la totalità dei processi bibliotecari né, pur riconoscendo l'importanza teorica degli indicatori, estendersi all'intera gamma delle misurazioni previste dal sistema statistico, per quanto i gruppi di lavoro operino in modo integrato con il SBA.

Il dato meno usato dai coordinatori è quello sull'orario di apertura dei punti di servizio (D2.10), riconducibile all'accessibilità. Prestiti (D4.06) e corsi per studenti (D4.12), periodici correnti (D2.02), personale suddiviso per attività (D2.12) e tempi dell'iter del libro (D4.05) riscuotono anch'essi performance negative.

L'indicatore meno usato ha ad oggetto il patrimonio bibliografico posseduto dell'università (I2.01).

Lo scostamento complessivo per quanto riguarda i dati è di $-1,30$ punti, per gli indicatori è -1,56. La dimensione della soddisfazione riscuote la performance peggiore, seguita da quelle degli usi e della produttività, sebbene con scostamenti non elevati (tabella 15). 
In conclusione, l'impatto del sistema statistico sui coordinatori può essere migliorato.

Esaminiamo da ultimo l'importanza attribuita alle dimensioni della misurazione, dal momento che i grafici rivelano una tendenza consolidata quale che sia la modalità di aggregazione dei risultati: la soddisfazione dell'utente è il tipo di misurazione che riscuote il maggior successo (tabelle 9, $10,11,12$ ) e, al contempo, nel complesso, la peggiore performance (si tratta, come è stato detto, di una performance virtuale, in quanto è stato chiesto quale uso l'intervistato avrebbe fatto della suddetta). Ciò evidenzia come vi sia una diffusa sensibilità alle questioni della qualità in biblioteca e un corrispondente desiderio di includere misurazioni di tal sorta nel sistema statistico, nonostante una qualche incertezza - o scarsi margini d'azione? - sull'uso di tali valori. La conoscenza dell'effettivo impatto che i servizi bibliotecari hanno sul pubblico è considerata importante e, probabilmente, tale da conferire significato alle altre dimensioni quantitative. I valori parano da soli: siamo generalmente attorno al $24 \%$ con una punta del $27 \%$ per quanto riguarda i direttori di biblioteche, su un totale di sette dimensioni.

Il sistema statistico è consultato almeno una volta ogni 70,89 giorni (secondo la media d'uso non ponderata per ruoli), con una maggiore frequenza per i direttori di biblioteca (che lo consultano almeno una volta ogni 43,33 giorni) ed una minore frequenza per i coordinatori di gruppo di lavoro (che lo consultano una volta ogni 101 giorni). La media ponderata per ruoli presenta un valore di 49,40 giorni. Per quanto riguarda la comunicazione dei dati e la consultazione del sistema statistico abbiamo una netta prevalenza del medium web.

Nel questionario si chiedeva agli intervistati di esprimere osservazioni non strutturate sia sui singoli dati e indicatori sia sul sistema statistico nel suo complesso.

Sui singoli dati e indicatori appaiono più rilevanti le seguenti osservazioni:

- D1.01 - D1.02. Rivedere i criteri di individuazione per una più esaustiva scansione del mercato potenziale.

- D2.02. Includere i periodici elettronici.

- D2.10. Il valore dell'orario di apertura va messo in relazione all'uso dei servizi.

- D2.12. Rivedere lo schema delle attività, perché alcune di esse di fatto si sovrappongono.

- D4.06. Il dato andrebbe posto in relazione con le consultazioni dello scaffale aperto. 
- I1.03. Eliminare dal calcolo dell'indicatore i volumi obsoleti nelle biblioteche scientifiche, data la loro utilità prettamente storiografica.

Sono state inoltre manifestate le seguenti esigenze:

- Monitorare la movimentazione dello scaffale aperto, anche con indagini a campione.

- Indagare l'efficacia della segnaletica e delle attività di promozione dei servizi.

- Segmentare l'utenza in modo più dettagliato al fine di una più accurata individuazione delle aspettative.

- Collaborare con il Settore budgeting, il Nucleo di valutazione e il Dipartimento di Statistica.

- Introdurre nel sistema statistico misurazioni sulla biblioteca digitale.

- Effettuare attività di Benchmarking con altri sistemi bibliotecari universitari.

I risultati dell'indagine sono da considerarsi complessivamente buoni: emerge difatti che la rilevazione statistica nelle biblioteche dell'ateneo è un'esperienza condivisa dai responsabili di biblioteche e dei gruppi di lavoro sia sul piano teorico sia su quello operativo, qualora dati e indicatori interessino processi o funzioni suscettibili di concrete opportunità di sviluppo e siano in grado di cogliere gli aspetti qualificanti delle specifiche attività svolte.

Per concludere, possono essere individuate le seguenti priorità di implementazione:

1. Effettuare un'indagine sulla qualità dei servizi, orientata alla rilevazione della soddisfazione dei clienti ed i cui risultati possano integrarsi con le attuali misure quantitative per completare il quadro interpretativo dell'andamento dei servizi bibliotecari dell'ateneo. L'indagine dovrà avere una promozione interna particolarmente incentrata sui risvolti applicativi dei risultati e sull'uso dei metodi di management dedicati al miglioramento della qualità, focalizzando su quei processi bibliotecari in relazione ai quali è possibile operare dei cambiamenti.

2. Implementare gli indicatori relativi alla dimensione dell'uso delle risorse documentarie con particolare attenzione alla biblioteca digitale e allo scaffale aperto.

3. Rivedere i dati e gli indicatori relativi alla produttività del personale, a partire dal processo di trattamento del materiale documentario. 
4. Predisporre un'interfaccia di comunicazione via web, sia per l'inserimento dei dati da parte degli interessati sia per la consultazione del sistema statistico.

5. Promuovere l'integrazione tra le attività gestionali e il sistema statistico (gli indicatori dovrebbero essere legati in modo più chiaro alla verifica del grado di raggiungimento degli obiettivi), anche attraverso la comunicazione degli eventuali cambiamenti a cui il sistema statistico sarà sottoposto. 
RoBerto VENTURA
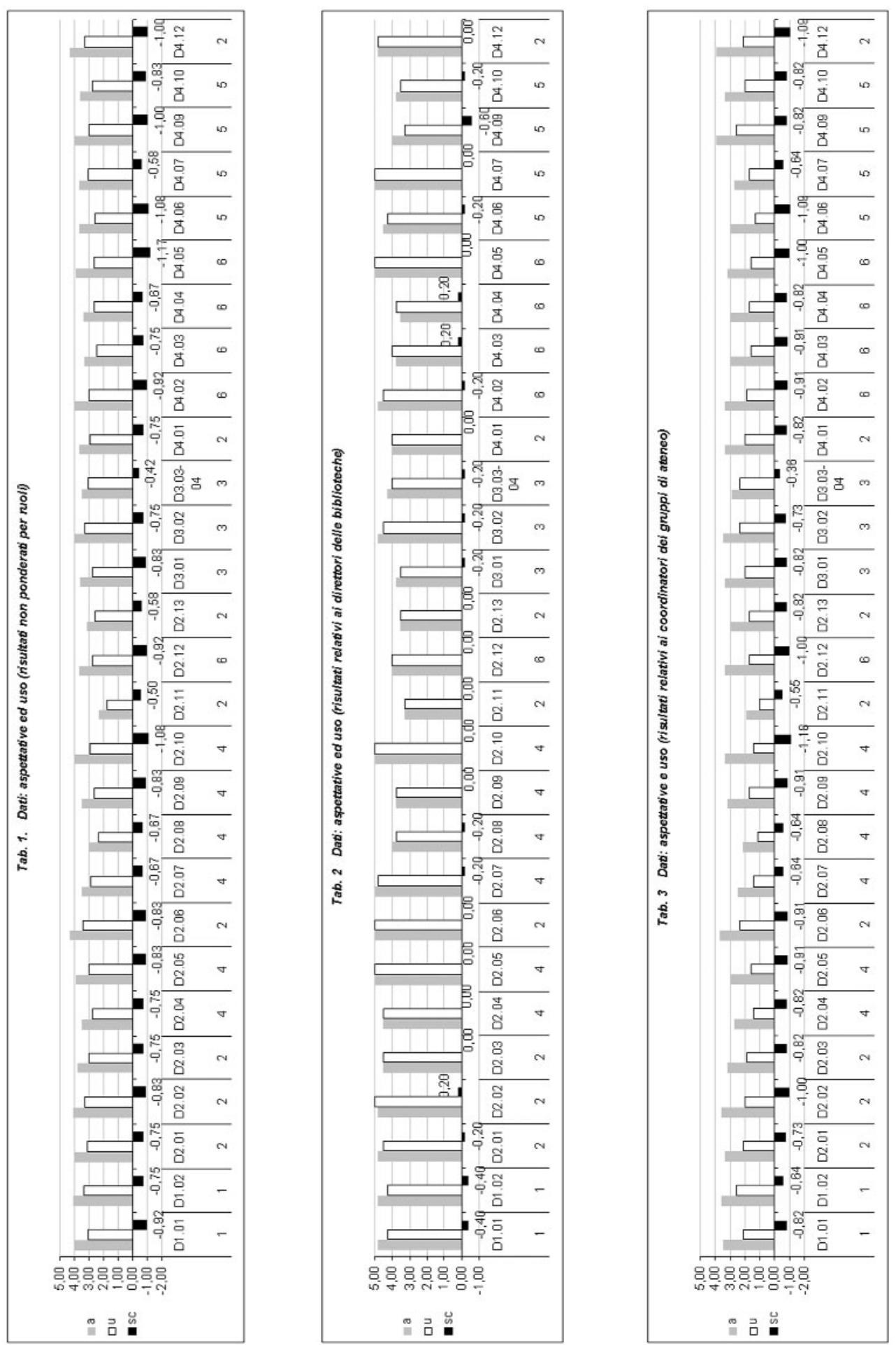

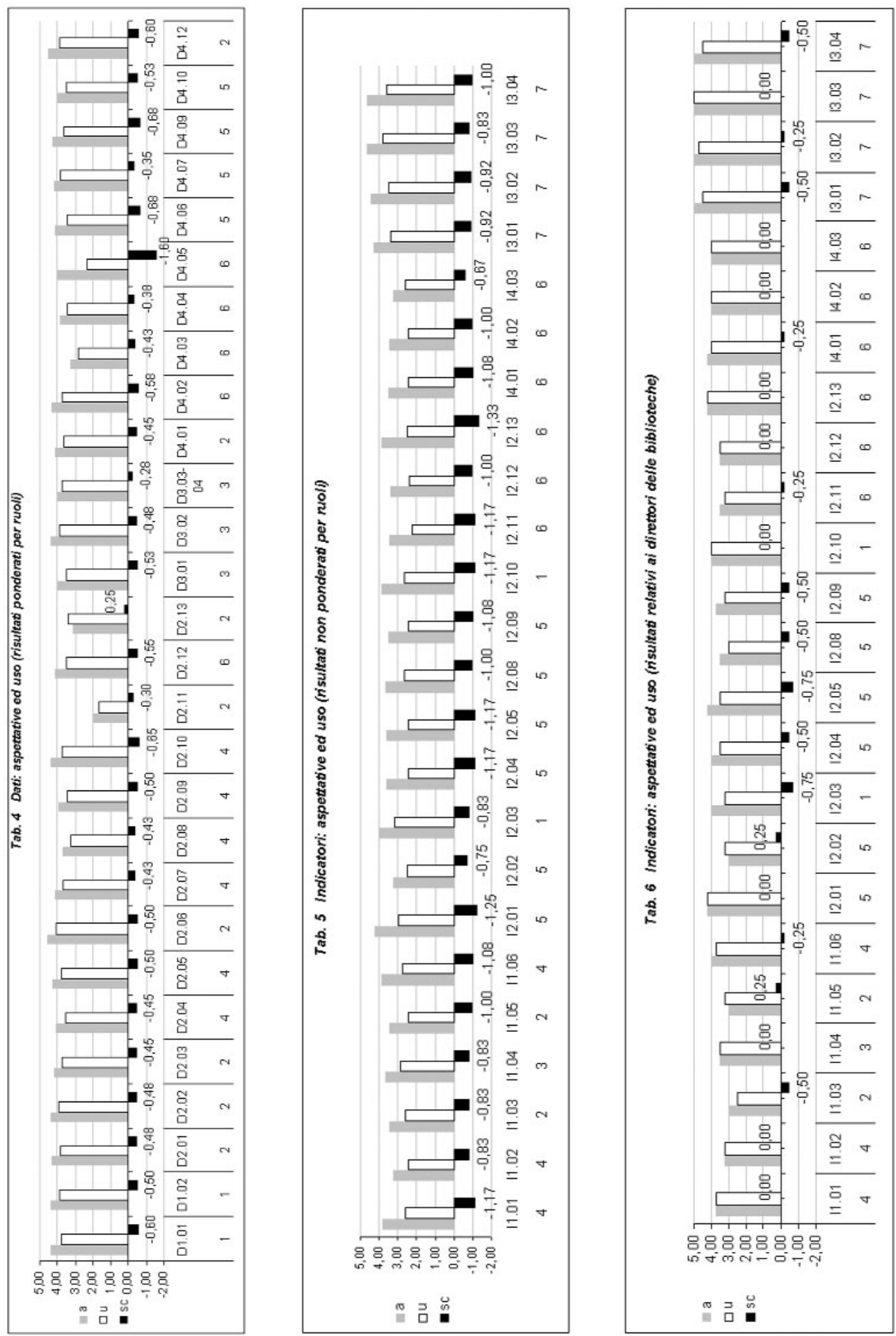
RoBerto VENTURA
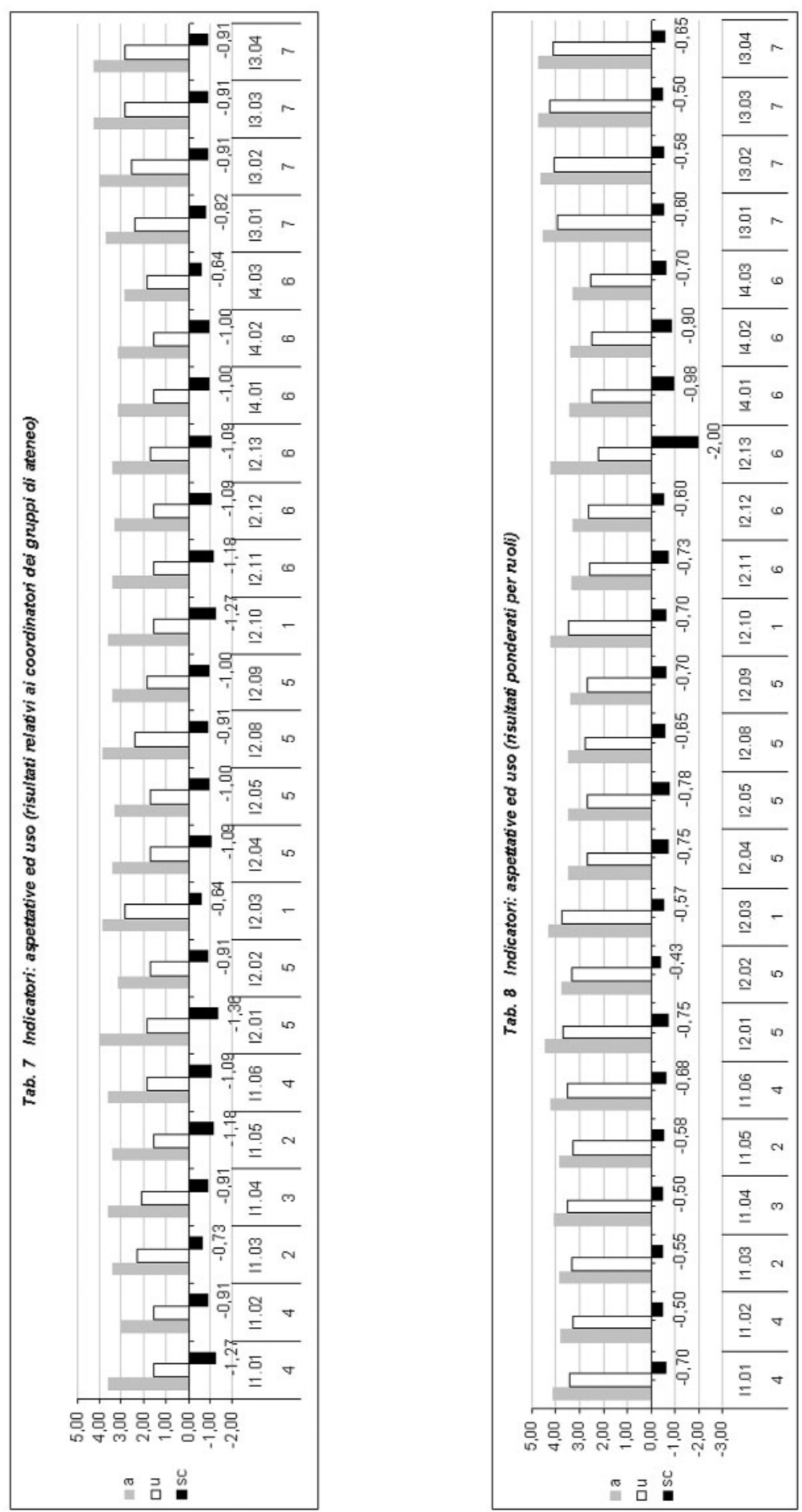


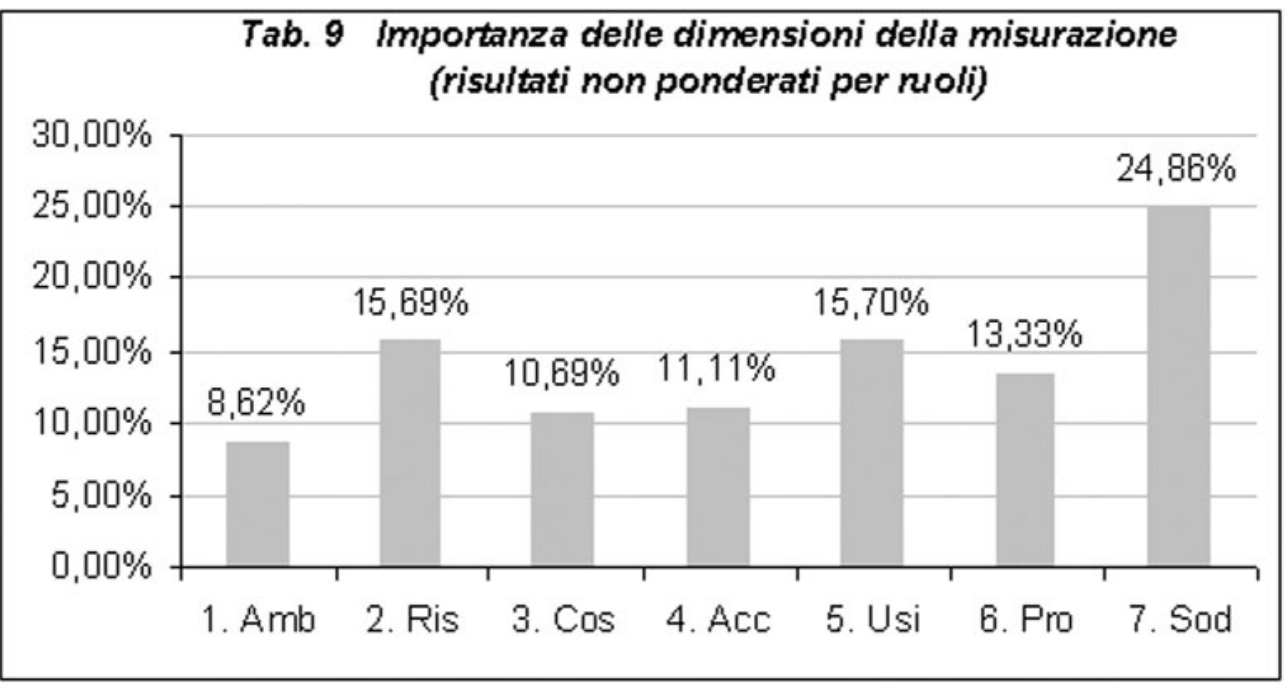

Tab. 10 Importanza delle dimensioni della misurazione (direttori delle biblioteche)

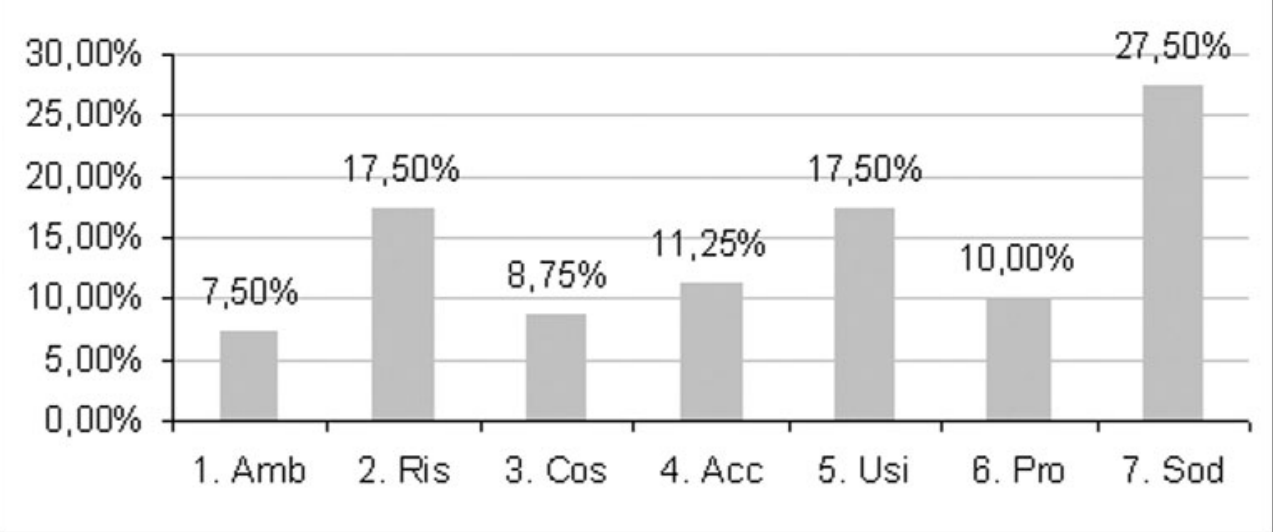



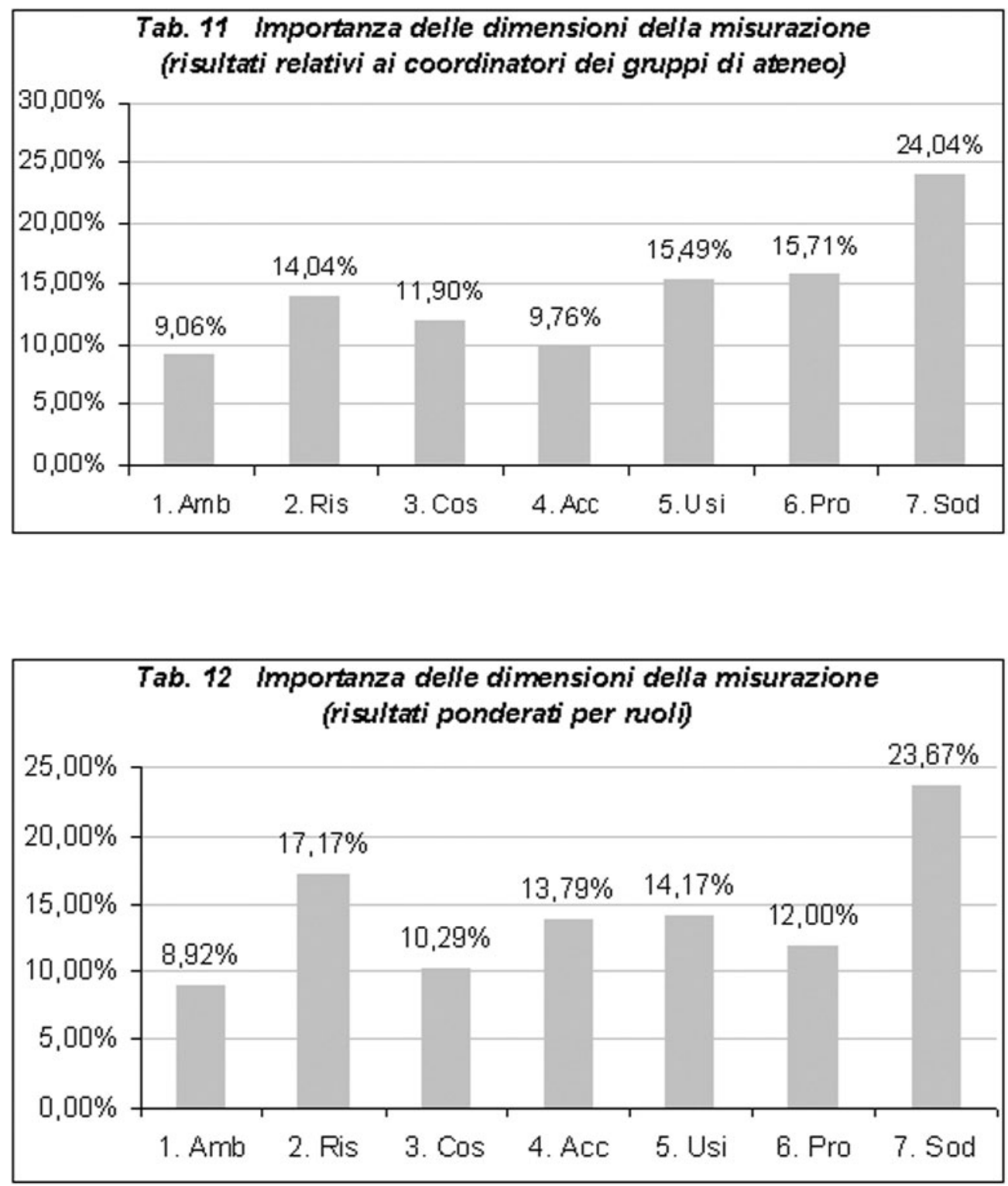
Tab. 13 Perfomance nelle dimensioni della misurazione (risultati non ponderati per ruoli)

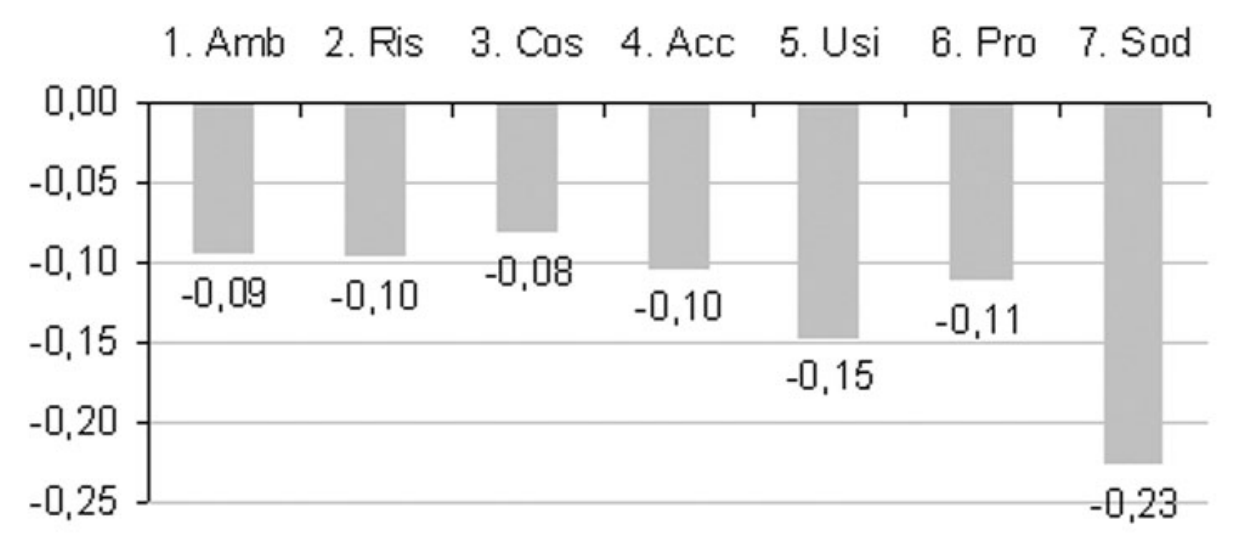

\section{Tab. 14 Perfomance nelle dimensioni della misurazione (risultati relativi ai diretton delle biblioteche)}

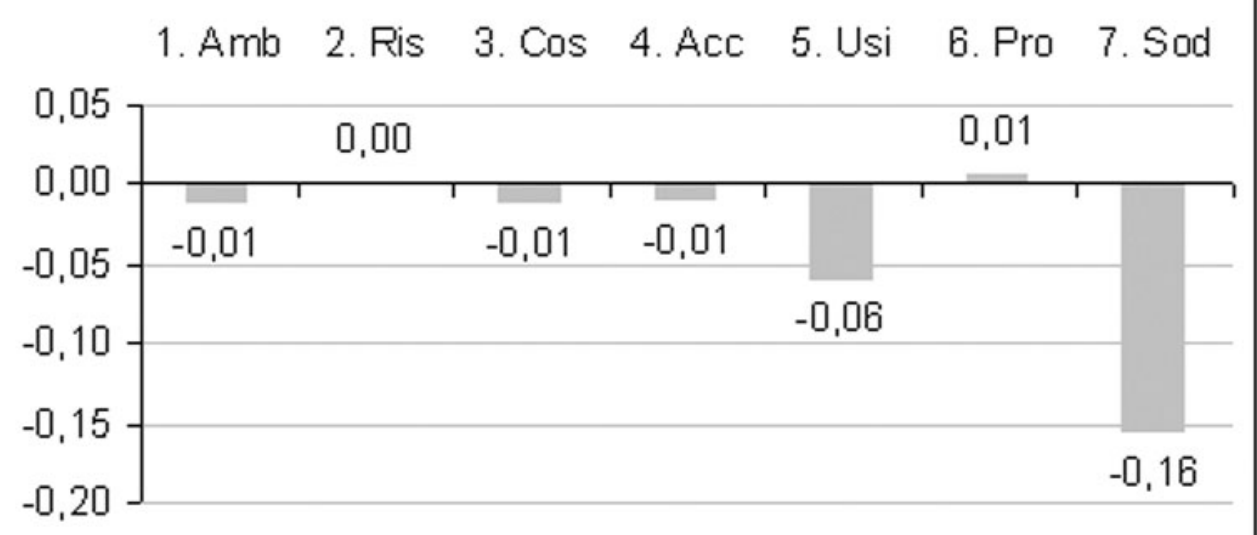


Tab. 15 Penformance nelle dimensioni della misurazione (nisultati relativi ai coordinatori dei gruppi di ateneo)

1. Amb 2. Ris 3. Cos 4. Acc 5. Usi 6. Pro 7. Sod

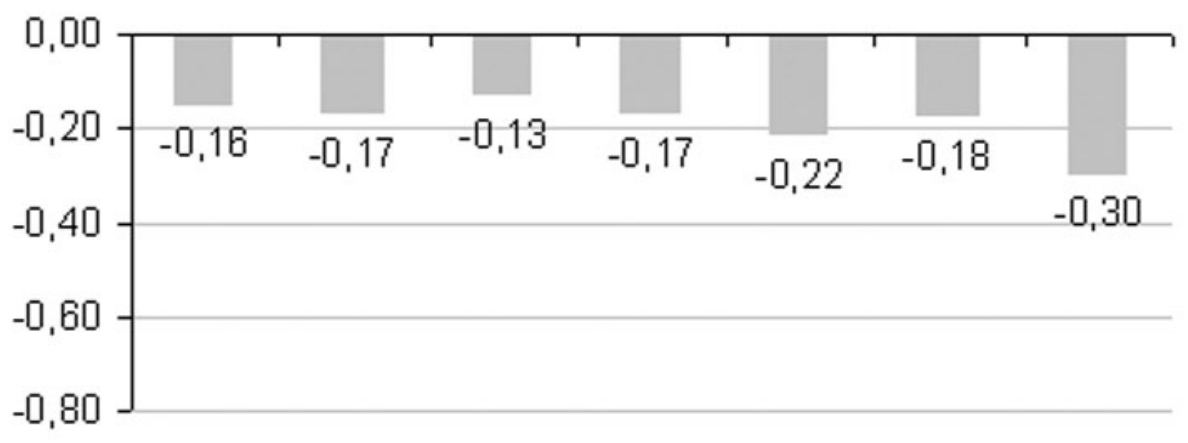

Tab. 16 Performance nelle dimensioni della misurazione (risultati ponderati per ruoli)

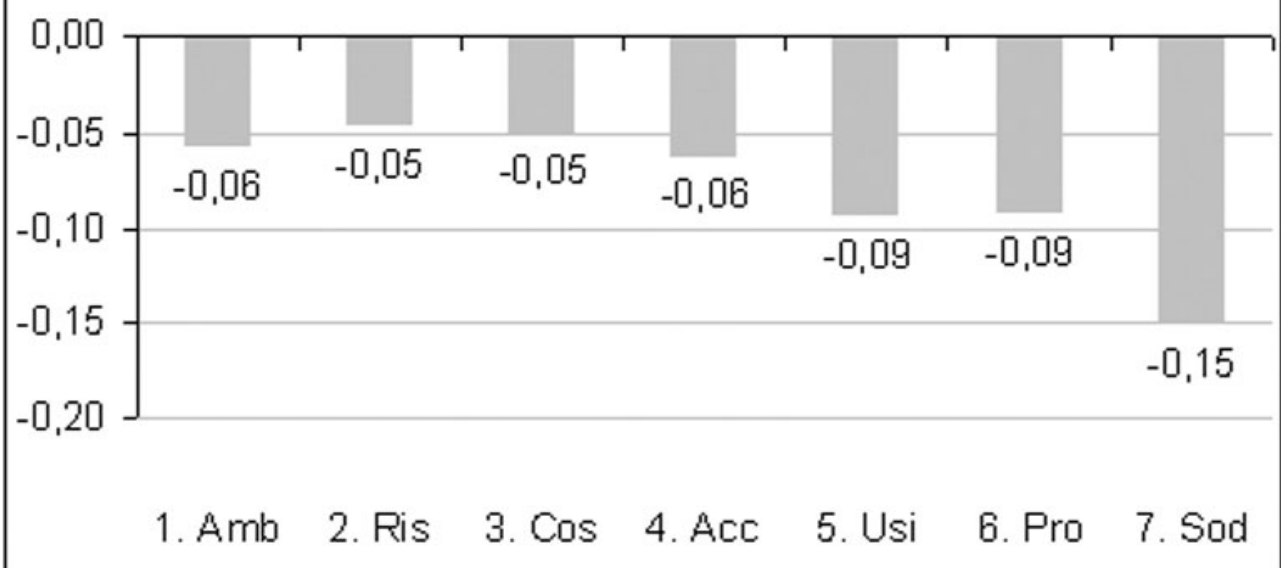




\title{
Aspetti tecnici e metodologici di un'indagine di qualità
}

\author{
di Silvana Benedetti
}

L'indagine sulla qualità dei servizi del Sistema Bibliotecario di Ateneo è stata rivolta a due particolari categorie di utenti:

- gli studenti che nel corso dell'anno 2000 hanno usufruito del servizio di prestito in uno dei punti di servizio del Sistema bibliotecario di Ateneo;

- il personale docente dell'ateneo fiorentino.

Per le due categorie suddette la somministrazione del questionario è stata condotta separatamente: dal 27 novembre al 11 dicembre 2001 per la prima, nei mesi di febbraio-marzo 2002 per la seconda. Sono stati inoltre utilizzati due diversi metodi di indagine: intervista telefonica per gli studenti e questionario online per i docenti; indagine campionaria per gli studenti, completa per i docenti e ricercatori.

Partendo dal presupposto iniziale di compiere un'indagine per tutto il Sistema bibliotecario di Ateneo e di utilizzare per la rilevazione dei dati un questionario da sottoporre agli utenti, il gruppo di lavoro sulla misurazione dei servizi si è dovuto confrontare su una serie di problematiche relative a:

- definizione della popolazione target e del campione;

- scelta delle tecniche di rilevazione da utilizzare;

- costruzione e verifiche del questionario, somministrazione di un pre-test.

La customer satisfaction in ambito biblioteconomico è un argomento ampiamente trattato nella letteratura professionale degli ultimi anni ma, ad oggi, è assente un modello unanimemente condiviso a cui attenersi per la sua rilevazione. Pertanto nella fase progettuale dell'indagine si è reso necessario, oltre all'esame dei contributi e delle esperienze di settore, un breve studio delle problematiche inerenti le tecniche ed i metodi di somministrazione di questionari utilizzati in ambito della ricerca sociale ${ }^{1}$.

1 Di particolare interesse, tra le innumerevoli pubblicazioni sull'argomento, sono state: Corbetta 2000 e Zammuner 1998. 
Oltre alla letteratura sull'argomento abbiamo ritenuto utile di avvalerci della consulenza, durata per tutta l'indagine, del Dipartimento di Statistica ${ }^{2}$ al quale sono stati affidati il disegno di campionamento, la somministrazione del questionario e l'elaborazione statistica di una parte dei dati.

Volendo misurare il grado di soddisfazione per i servizi forniti piuttosto che le aspettative dell'utenza potenziale, abbiamo ritenuto opportuno rivolgere la nostra indagine ai soli utenti reali, a coloro che avevano usato almeno uno dei servizi di biblioteca nell'anno considerato. La scelta successiva è stata quella di somministrare il questionario non a tutta la popolazione target ma ad un campione significativo.

La letteratura di settore ci insegna che uno degli elementi fondamentali di un'indagine campionaria è la rappresentatività. Il campione cui sottoporre l'indagine deve essere estratto da una lista che contenga tutti gli individui a cui si vuol generalizzare i dati raccolti, nello specifico tra tutti gli utenti potenziali solo quelli che si recano veramente in biblioteca per usufruire dei servizi offerti. Ma come individuare i nostri utenti reali? Come nella maggior parte delle biblioteche universitarie non è previsto alcun tipo di registrazione degli utenti che entrano in biblioteca. L'unico servizio per il quale gli utenti vengono registrati al momento in cui ne usufruiscono in modo automatizzato e in tutti i punti di servizio del Sistema Bibliotecario è il prestito (esterno, interno o notturno).

Alla luce di questa realtà ci restava da scegliere fra due possibilità: limitare la popolazione d'interesse ai soli utenti del servizio di prestito oppure, in alternativa, somministrare il questionario durante settimane-campione presso le varie sedi delle biblioteche.

Nel primo caso sarebbe stata rappresentata solo una parte degli utenti dei servizi bibliotecari; sarebbero stati esclusi, infatti, tutti gli utenti che pur non avendo usufruito del prestito, hanno utilizzato altri servizi forniti dal Sistema Bibliotecario. Con il vantaggio però di poter disporre di liste, le quali potevano essere agevolmente arricchite da una serie di informazioni utili successivamente per l'interpretazione dei dati. Avere tali informazioni evitava una serie di domande all'interno del questionario con un notevole snellimento di questo ultimo. L'estrazione del campione avrebbe rispettato il principio della casualità.

L'eventuale scelta di effettuare l'indagine durante settimane-campione non avrebbe assicurato una maggiore attendibilità della rilevazione. Avrebbe

2 Nello specifico sono stati nostri referenti il Prof. Bruno Chiandotto, la Prof.ssa Monica Pratesi e la Dott.ssa Emilia Rocco. 
invece comportato costi maggiori soprattutto in caso di intervista diretta, mentre con l'autocompilazione si sarebbe sicuramente registrato un tasso di non-risposta più elevato. Tenendo conto anche del fatto che questa possibilità è prevista dagli stessi standard ${ }^{3}$ la decisione finale del gruppo è stata quella di individuare la popolazione target negli iscritti al servizio di prestito.

Contemporaneamente, al fine di sperimentare una modalità ancora abbastanza nuova come il questionario web, è nata l'idea di scindere in due l'indagine, adottando questa ultima tecnica per il personale docente. L'aspettativa è che, dato la diffusione dell'utilizzo del computer, di internet e della posta elettronica tra la comunità accademica questa tecnica possa diventare uno strumento agile e a basso costo per poter ripetere questo tipo di indagine anche a cadenza regolare.

Infine, considerato il numero (2.293 unità), relativamente piccolo dal punto di vista statistico, è stato deciso di individuare la popolazione target con il totale dei docenti e ricercatori dell'ateneo fiorentino e di somministrare il questionario alla totalità dei componenti la categoria.

\section{Popolazione studentesca oggetto di indagine}

Come abbiamo già detto, la popolazione studentesca oggetto dell'indagine, a seguito delle scelte operate, è costituita da tutti gli studenti che nel corso dell'anno 2000 hanno preso in prestito almeno un libro in una delle biblioteche del Sistema Bibliotecario. In termini numerici è risultata pari a 27.746 individui ${ }^{4}$.

Operativamente ci siamo dotati di un file, estratto dal sistema gestionale SBN, ed elaborato dal settore biblioteche dello CSIAF (Centro Servizi Informatici dell'Ateneo Fiorentino), contenente gli studenti iscritti al prestito ${ }^{5}$ assegnati alle ex-biblioteche di facoltà e ad altri punti di servizio dipartimentali, poi raggruppati nelle 5 biblioteche di area del Sistema bibliotecario: Biblioteca Biomedica, Biblioteca di Scienze, Biblioteca di Scienze sociali, Biblioteca di Scienze tecnologiche, Biblioteca Umanistica. Con una ulteriore

3 Sia IFLA Poll \& te Boekhorst 1996 che ISO 1998 nella definizione di utente attivo danno la possibilità di farlo coincidere con gli utenti del servizio di prestito.

4 Nell'anno accademico 1999/2000, gli iscritti all'Università sono stati 58.848.

5 L'archivio del prestito SBN viene creato attraverso la cattura dei dati dall'archivio della segreteria studenti quindi il file conteneva per ogni utente oltre ai dati anagrafici e al numero di prestiti, anche il codice di biblioteca di ex-facoltà e di area, il codice di facoltà di iscrizione, il numero di matricola e l'anno di iscrizione. 
elaborazione sono stati successivamente individuati gli utenti unici eliminando le ricorrenze di studenti che risultavano iscritti al prestito in più biblioteche considerandoli utenti di quella da cui avevano preso in prestito il maggior numero di libri. In questo modo siamo arrivati alle 27.746 unità così ripartite nelle 5 biblioteche di area:

Utenti-studenti iscritti al prestito nell'anno 2000 suddivisi nelle ex-biblioteche di facoltà e raggruppati per le attuali biblioteche di area ${ }^{6}$

\begin{tabular}{|l|l|l|c|c|c|}
\hline \multicolumn{1}{|c|}{ Punti di servizio } & \multicolumn{5}{c|}{ Biblioteche di area } \\
\hline & Biomedica & Scienze & $\begin{array}{c}\text { Scienze } \\
\text { sociali }\end{array}$ & $\begin{array}{c}\text { Scienze } \\
\text { tecnolog. }\end{array}$ & Umanistica \\
\hline Agraria & & & & 1.365 & \\
\hline Architettura & & & & 5.027 & \\
\hline Economia & & & 2.497 & & \\
\hline Farmacia & 746 & & & & \\
\hline Ingegneria & & & & 2.797 & \\
\hline Lettere & & & & & 4.323 \\
\hline Medicina & 1.644 & & & & \\
\hline Scienze della Formazione & & & & & 2.439 \\
\hline Scienze & & 2.345 & & & \\
\hline Scienze Politiche e Giurisprudenza & & & $1.593+2.970$ & & \\
\hline Totali & 2.390 & 2.345 & 7.060 & 9.189 & 6.762 \\
\hline
\end{tabular}

\section{Il disegno di campionamento}

Sulla base della popolazione d'interesse così definita è stato selezionato un campione di 1.063 unità mediante un disegno di campionamento probabilistico $^{7}$. Nello specifico il disegno di campionamento è stato di tipo stratificato utilizzando come variabili di stratificazione la ex-biblioteca di facoltà e l'area di appartenenza della stessa. Ma essendo le aree raggruppamenti di biblioteche, gli strati, di fatto, coincidono con queste ultime.

La numerosità del campione è stata calcolata tenendo conto delle variabili: costo dell'indagine, variabilità dei fenomeni da analizzare, attendibilità dei

6 Come si vede nella tabella le biblioteche Scienze politiche e Giurisprudenza sono state accorpate in un'unica biblioteca fittizia perché le due biblioteche sono situate in un'unica sede.

7 Perché un campione sia probabilistico tutti i componenti della popolazione target devono avere la stessa probabilità di entrare a far parte del campione. 
risultati delle analisi successive. Dai calcoli effettuati su queste variabili si è ottenuta una numerosità totale di 1.000 interviste anche se di fatto sono stati estratti 1.063 individui. Tale risultato dipende dalle regole seguite in ambito CASES (Computer Assisted Survey Method Program sviluppato dalla University of California di Berkley): per raggiungere le numerosità previste per strato si continua a contattare membri di uno stesso strato finché non viene raggiunta la numerosità prevista ma il controllo viene fatto solo al termine di ogni seduta di lavoro per cui, nell'ambito di una stessa seduta, tale numerosità può essere anche leggermente superata. Allo scopo di fare inferenza si è cercato di rendere la stratificazione più omogenea possibile individuando approssimativamente la stessa numerosità dei campioni per le cinque aree, circa 200 individui. Quindi per ciascuno strato è stato selezionato un campione di dimensione proporzionale a quella dello strato medesimo rispetto alla sua area di afferenza.

In ciascuno strato gli studenti da intervistare sono stati selezionati con un'estrazione casuale semplice senza ripetizione.

Il risultato di questi calcoli ha dato una numerosità campionaria per biblioteca così ripartita:

\begin{tabular}{|l|c|}
\hline \multicolumn{1}{|c|}{ Biblioteche } & Numerosità campionaria \\
\hline Farmacia & 71 \\
\hline Medicina & 139 \\
\hline Economia & 75 \\
\hline Giurisprudenza e Scienze Politiche & 131 \\
\hline Scienze & 206 \\
\hline Agraria & 32 \\
\hline Architettura & 128 \\
\hline Ingegneria & 83 \\
\hline Lettere & 126 \\
\hline Scienze della Formazione & 72 \\
\hline Totale & 1.063 \\
\hline
\end{tabular}

\section{Caratteristiche del campione}

Sulla base delle informazioni contenute nella lista degli utenti attivi del servizio di prestito e di alcune domande poste agli utenti stessi nel corso dell'intervista, è stato possibile dedurre alcune caratteristiche del campione: il $60 \%$ è costituito da donne; il $40 \%$ da maschi. 
Composizione del campione per sesso

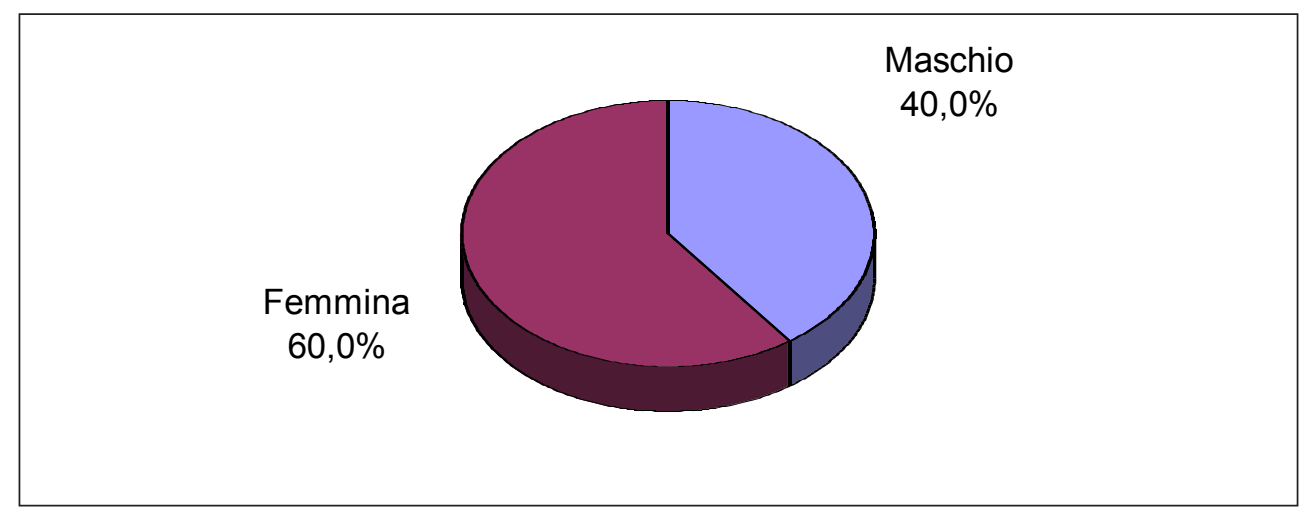

Il 42,5\% degli intervistati ha dichiarato di essere residente nel comune di Firenze, e solo il 9,6\% di non essere residente in Toscana.

Residenza degli intervistati

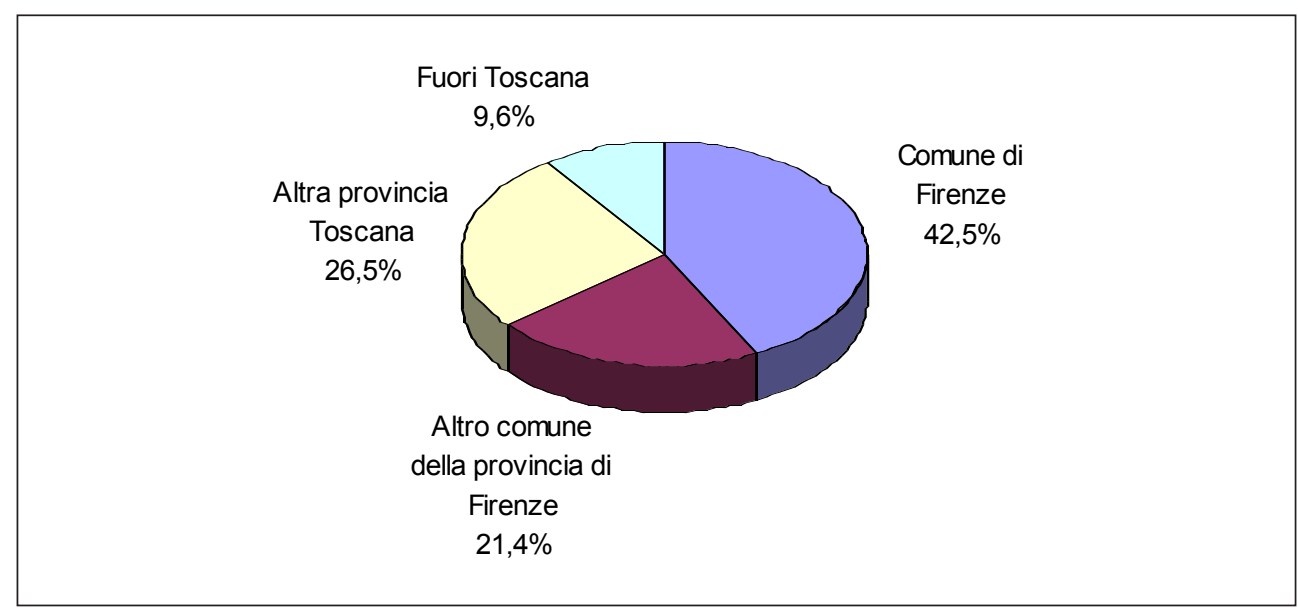

Relativamente al loro percorso di studio risulta che il 44,5\% in corrispondenza dell'intervista si era già laureato o aveva già chiesto la tesi. 
Prossimità alla laurea

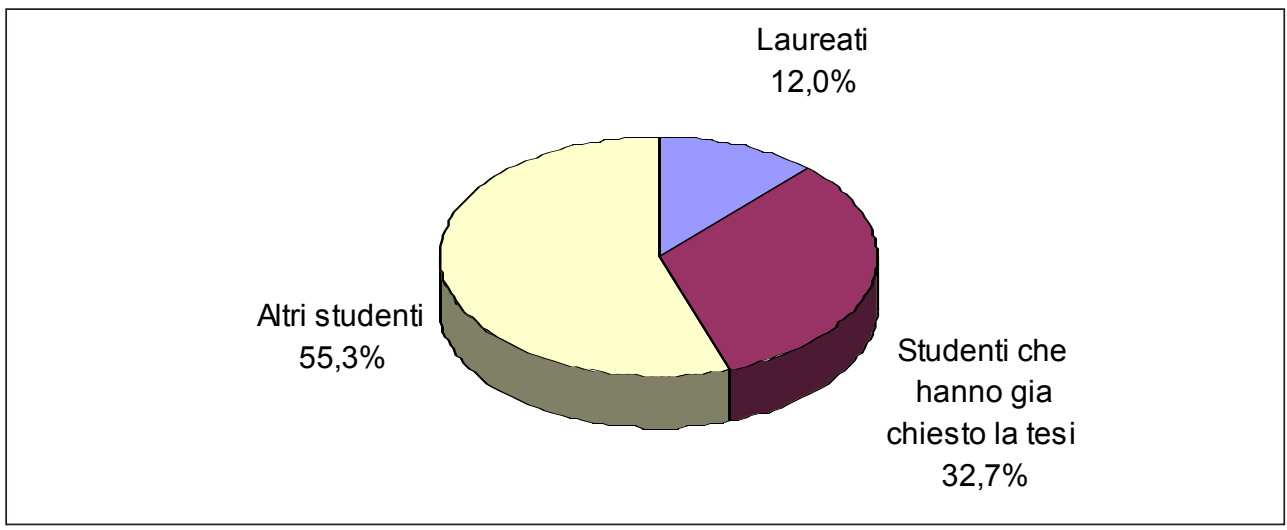

In merito alla condizione lavorativa ben il $32,5 \%$ degli intervistati è uno studente lavoratore.

Condizione lavorativa dei rispondenti

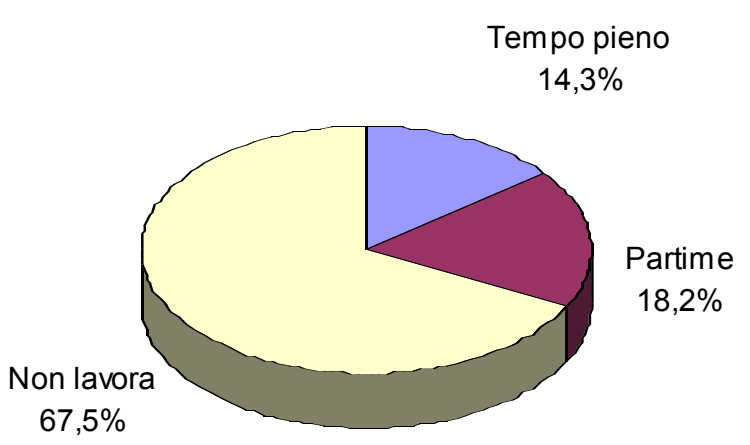

Le ultime osservazioni riguardano la mobilità degli studenti all'interno delle varie biblioteche in esame. Dall'indagine risulta che la maggior parte degli studenti, il 52,3\%, frequenta abitualmente solo la biblioteca di cui risulta utente, il restante $47,7 \%$ ha dichiarato di frequentare altre biblioteche in numero variabile da 1 a 7 , scelte quasi sempre nella stessa area disciplinare della biblioteca di utenza. 
Mobilità degli studenti tra biblioteche appartenenti ad aree diverse

da cinque a sette

Se poi confrontiamo la facoltà di iscrizione e la biblioteca di utenza risulta che la maggior parte degli studenti sono iscritti alla stessa facoltà di appartenenza della biblioteca.

Utenti del prestito per facoltà

\begin{tabular}{|l|c|c|c|}
\hline \multicolumn{1}{|c|}{ Facoltà } & $\begin{array}{c}\text { Iscritti che hanno } \\
\text { usufruito del } \\
\text { prestito }(a)\end{array}$ & $\begin{array}{c}\text { Iscritti che hanno } \\
\text { usufruito del prestito } \\
\text { nella stessa facoltà }(b)\end{array}$ & $(a / b) * 100$ \\
\hline Agraria & 1.334 & 1.289 & $96.63 \%$ \\
\hline Architettura & 5.157 & 4.890 & $94.82 \%$ \\
\hline Economia & 2.373 & 2.164 & $91.19 \%$ \\
\hline Farmacia & 661 & 602 & $91.07 \%$ \\
\hline Giurisprudenza & 2.624 & 2.403 & $91.58 \%$ \\
\hline Ingegneria & 2.995 & 2.754 & $91.95 \%$ \\
\hline Lettere e Filosofia & 4.331 & 3.704 & $85.52 \%$ \\
\hline Medicina e Chirurgia & 1.669 & 1.545 & $92.57 \%$ \\
\hline Scienze della Formazione & 2.426 & 1.909 & $78.69 \%$ \\
\hline Scienze MFN & 2.175 & 2.011 & $92.46 \%$ \\
\hline Scienze Politiche & 2.001 & 1.392 & $69.57 \%$ \\
\hline Totale & 27.746 & 24.663 & $88.89 \%$ \\
\hline
\end{tabular}




\section{Popolazione docente oggetto di indagine}

La popolazione relativa alla categoria personale docente nell'anno d'interesse era costituita da 2.293 individui così distribuiti tra le diverse categorie:

Docenti e ricercatori interni per categoria

\begin{tabular}{|l|r|}
\hline \multicolumn{1}{|c|}{ Categoria } & Numero \\
\hline Assistente di ruolo & 28 \\
\hline Professore associato confermato & 437 \\
\hline Professore associato confermato fuori ruolo & 11 \\
\hline Professore associato soggetto a conferma & 266 \\
\hline Professore ordinario & 547 \\
\hline Professore straordinario & 238 \\
\hline Ricercatore confermato & 574 \\
\hline Ricercatore non confermato & 192 \\
\hline Totale & 2.293 \\
\hline
\end{tabular}

Relativamente a questa categoria di utenti, avendo deciso di prendere in considerazione la totalità della popolazione, si devono sottolineare due aspetti: per problemi legati alla difficoltà di ottenere indirizzi e-mail o postali, come si può vedere dalla tabella, sono esclusi dall'indagine non solo i docenti esterni o a contratto ma anche gli assegnisti e i dottorandi, quindi una parte, se pur piccola, di coloro che si occupano di attività di ricerca e docenza nell'ambito della stessa Università. Inoltre, la scelta di somministrare il questionario tramite autocompilazione, tra l'altro associata all'utilizzo della posta elettronica e la compilazione su web, ha dato un basso tasso di risposta $(30,09 \%)$. La percentuale di risposta riscontrata rientra nei tassi mediamente ottenuti in indagini di questo tipo, ma si deve tenere presente che i risultati dell'indagine, pur fornendoci utili indicazioni da prendere senz'altro in considerazione, non possono essere generalizzati all'intera popolazione di questa categoria di utenti.

Delle 690 valutazioni ottenute 674 sono di docenti e ricercatori che hanno dichiarato di frequentare almeno una biblioteca del Sistema bibliotecario (nella tabella si può vedere la loro distribuzione tra le diverse biblioteche), 16 di docenti e ricercatori che hanno dichiarato di non frequentare nessuna biblioteca. Le motivazioni addotte per quest'ultima dichiarazione sono: la frequenza di altre biblioteche non appartenenti all'Università ( 8 casi), la indi- 
sponibilità degli strumenti/servizi di interesse (2 casi), la preferenza di strumenti disponibili su internet ( 2 casi), la dispersione delle varie sedi sul territorio (1 caso), un generico "altro" (3 casi).

Frequenza di almeno una biblioteca del Sistema bibliotecario

\begin{tabular}{|l|c|}
\hline \multicolumn{1}{|c|}{ Frequenza biblioteche del SBA } & Numero rispondenti \\
\hline Almeno una biblioteca del SBA & 674 \\
\hline Nessuna biblioteca del SBA & 16 \\
\hline Totale & 690 \\
\hline
\end{tabular}

Frequenza delle biblioteche del $\mathrm{SBA}^{8}$

\begin{tabular}{|l|c|c|}
\hline \multicolumn{1}{|c|}{ Nome biblioteca } & $\begin{array}{c}\text { Numero docenti e } \\
\text { ricercatori che frequentano } \\
\text { abitualmente la biblioteca }\end{array}$ & $\begin{array}{c}\text { Numero di volte in cui } \\
\text { la biblioteca è stata indicata } \\
\text { come quella principale } \\
\text { tra quelle frequentate }\end{array}$ \\
\hline Agraria & 69 & 42 \\
\hline Antropologia & 10 & 3 \\
\hline Architettura & 45 & 16 \\
\hline Biologia Animale & 42 & 16 \\
\hline Botanica & 33 & 12 \\
\hline Chimica & 92 & 48 \\
\hline Dip. Costruzioni & 16 & 4 \\
\hline Dip. di Diritto comparato e penale e & & 7 \\
\hline Dip. di Diritto privato e processuale & 21 & 3 \\
\hline Dip. di Diritto dell'economia & 7 & 10 \\
\hline Dip. di Diritto Pubblico & 31 & 4 \\
\hline Dip. Economico estimativo & 8 & 15 \\
\hline Dip. Farmacologia & 48 & 1 \\
\hline Dip. Filosofia & 15 & 1 \\
\hline Dip. Ing. Civile & 15 & 5 \\
\hline Dip. Linguistica & 13 & \\
\hline
\end{tabular}

(continua)

831 tra gli intervistati hanno dichiarato di frequentare più biblioteche ma nessuna più delle altre, 13 intervistati hanno indicato più scelte come biblioteca maggiormente frequentata per un totale complessivo di 30 anziché 13 scelte. Questo spiega perché la somma è diverso da 690 (totale delle interviste), tale valore è infatti dato da 690-31+30-13. 
(continua)

\begin{tabular}{|c|c|c|}
\hline $\begin{array}{l}\text { Dip. Matematica applicata } \\
\text { "G. Sansone" }\end{array}$ & 21 & 3 \\
\hline Dip. Meccanica & 12 & 4 \\
\hline Dip. Pediatria & 8 & 5 \\
\hline Dip. Processi e metodi & 9 & 8 \\
\hline Dip. Progettazione & 13 & 5 \\
\hline Dip. Psicologia & 10 & 5 \\
\hline Dip. Sanità pubblica & 11 & 7 \\
\hline Dip. Scienze dell'antichità & 21 & 11 \\
\hline $\begin{array}{l}\text { Dip. Scienze neurologiche } \\
\text { e psichiatriche }\end{array}$ & 16 & 4 \\
\hline Dip. Sistemi & 9 & 3 \\
\hline Dip. Storia dell'Architettura & 10 & 1 \\
\hline $\begin{array}{l}\text { Dip. Storia delle arti e dello } \\
\text { spettacolo }\end{array}$ & 14 & 3 \\
\hline Dip. Studi sul Medioevo & 20 & 0 \\
\hline Dip. Teoria e storia del diritto & 23 & 1 \\
\hline Dip. Urbanistica & 8 & 5 \\
\hline Economia & 100 & 38 \\
\hline Emeroteca & 10 & 2 \\
\hline Farmacia & 53 & 12 \\
\hline Fisica & 69 & 37 \\
\hline Geografia & 20 & 4 \\
\hline Geomineralogia & 27 & 16 \\
\hline Giurisprudenza & 47 & 4 \\
\hline Ingegneria & 87 & 42 \\
\hline Lettere & 103 & 37 \\
\hline Matematica & 98 & 44 \\
\hline Medicina & 191 & 110 \\
\hline Scienze della formazione & 45 & 11 \\
\hline Scienze politiche & 73 & 24 \\
\hline Statistica & 58 & 25 \\
\hline Storia e lett. Nordamericana & 10 & 2 \\
\hline \multirow[t]{2}{*}{ Nessuna } & 16 & 16 \\
\hline & & 676 \\
\hline
\end{tabular}




\section{Tecniche di rilevazione utilizzate}

Per la somministrazione del questionario alla categoria "studenti" è stata scelta l'intervista telefonica in quanto particolarmente vantaggiosa rispetto ad altre tecniche di rilevazione per il basso costo delle interviste, la tempestività dei risultati, una generale maggiore attendibilità e la possibilità di contenere il tasso di non-risposta. Quest'ultimo aspetto è particolarmente importante, perché, come si è già detto, un alto tasso di non-risposta impedirebbe l'estensione dei risultati all'intera popolazione d'interesse. Nel nostro caso solo lo $0,43 \%$ degli individui contattati ha rifiutato di partecipare all'indagine.

Andamento delle interviste per strato

\begin{tabular}{|l|c|c|c|c|c|}
\hline \multicolumn{1}{|c|}{ Strato } & $\begin{array}{c}\text { Numerosità } \\
\text { prevista }\end{array}$ & $\begin{array}{c}\text { Interviste } \\
\text { completate }\end{array}$ & $\begin{array}{c}\text { Copertura } \\
\text { campionaria }\end{array}$ & $\begin{array}{c}\text { Numero } \\
\text { di rifiuti }\end{array}$ & $\begin{array}{c}\% \\
\text { di rifiuti }\end{array}$ \\
\hline Agraria & 30 & 32 & 106,7 & 0 & 0,00 \\
\hline Architettura & 109 & 128 & 117,4 & 1 & 0,24 \\
\hline Economia & 71 & 75 & 105,6 & 0 & 0,00 \\
\hline Farmacia & 62 & 71 & 114,5 & 3 & 0,77 \\
\hline Lettere & 128 & 126 & 98,4 & 3 & 0,50 \\
\hline Ingegneria & 61 & 83 & 136,1 & 0 & 0,00 \\
\hline Medicina & 138 & 139 & 100,7 & 1 & 0,30 \\
\hline Psicologia e formazione & 72 & 72 & 100,0 & 1 & 0,55 \\
\hline $\begin{array}{l}\text { Scienze Politiche e } \\
\text { Giurisprudenza }\end{array}$ & 129 & 131 & 101,6 & 4 & 1,04 \\
\hline Scienze & 200 & 206 & 103,0 & 1 & 0,46 \\
\hline Totale & 1.000 & 1.063 & 106,3 & 13 & 0,43 \\
\hline
\end{tabular}

In particolare le interviste sono state condotte utilizzando un sistema CATI (Computer Assisted Telephone Interviewing), per il quale il Dipartimento di Statistica ha messo a punto un insieme integrato di programmi specifico per questa indagine, nell'ambiente di lavoro CASES.

Questo metodo si avvale dell'uso del computer già in fase di rilevazione per cui il questionario da noi formulato è stato tradotto, sempre a cura del Dipartimento di Statistica, in termini informatici per renderlo idoneo alla somministrazione ed inserito nel programma di rilevazione. L'intervistatore memorizza direttamente su supporto magnetico i dati raccolti durante l'intervista. Il programma gestisce lo svolgimento stesso dell'intervista passando automaticamente alla domanda giusta in caso di domande condizionanti 
garantendo una maggiore velocità dell'intervista e riducendo al minimo la possibilità di errori da parte dell'intervistatore.

Il sistema CATI nella gestione dei contatti e degli appuntamenti telefonici è stato impostato in modo da seguire alcune regole:

- il rispondente, se impossibilitato ad effettuare l'intervista al momento della chiamata, poteva fissare fino a due appuntamenti telefonici in due diverse date e momenti della giornata;

- il contatto con la persona selezionata poteva essere effettuato entro 6 telefonate (non considerando la condizione di numero occupato) effettuate dal sistema CATI in diversi momenti della giornata tramite un sistema automatico di scheduling delle chiamate;

- i numeri occupati venivano riproposti agli intervistatori dopo 15 minuti.

Prima della rilevazione gli intervistatori sono stati istruiti circa le finalità dell'indagine, la struttura del questionario e sono stati addestrati all'uso della modalità CATI di intervista con sessioni di lavoro di prova.

Per quanto concerne l'indagine web, l'invito a partecipare è stato effettuato tramite un messaggio di posta elettronica in cui si presentavano gli obiettivi e si assicurava il rispetto della normativa sulla privacy.

I destinatari erano invitati a compilare il questionario direttamente in rete: nella e-mail veniva fornito l'indirizzo a cui connettersi, l'identificativo e la password di accesso.

Questa procedura ha interessato 1' $85 \%$ dei casi. Per il rimanente $15 \%$, per i quali non disponevamo dell'indirizzo e-mail, il questionario è stato spedito per posta. Solo successivamente i dati ottenuti sono stati immessi nell'archivio.

Rispetto ai 1.953 messaggi e-mail inviati solo 715 hanno suscitato una reazione nel destinatario i restanti 1.238 non hanno dato alcuna reazione (i diversi esiti delle e-mail sono riportati nella tabella).

Risposte dell'indagine Web

\begin{tabular}{|l|c|r|}
\hline \multicolumn{1}{|c|}{ Reazione } & Numero destinatari & \multicolumn{1}{c|}{$\%$} \\
\hline Mail delivery & 20 & 1,0 \\
\hline Rifiuti espliciti & 24 & 1,2 \\
\hline $\begin{array}{l}\text { Hanno comunicato via e-mail di avere problemi di } \\
\text { incompatibilità con il browser o nella compilazione o altri }\end{array}$ & 36 & 1,8 \\
\hline Hanno avuto accesso al questionario ma non lo hanno compilato & 5 & 0,3 \\
\hline Hanno compilato totalmente o parzialmente il questionario & 630 & 32,3 \\
\hline Nessuna reazione & 1.238 & 63,4 \\
\hline Totale & 1.953 & 100,0 \\
\hline
\end{tabular}


In definitiva dei 2.293 docenti e ricercatori invitati a partecipare all'indagine solo 690 , cioè il $30,09 \%$, hanno collaborato: 635 sono quelli che hanno compilato il questionario tramite il Web e 60 quelli che hanno compilato e rispedito il questionario cartaceo.

Il tempo utile per la compilazione del questionario è stato di 4 settimane. In questo periodo sono stati inviati tre solleciti. Il piano dei solleciti, a cadenza quasi settimanale, ha avuto un ruolo importante nell'indagine: il $25.2 \%$ dei rispondenti ha aderito al progetto in base al messaggio iniziale mentre il $74.8 \%$ ha ricevuto almeno un sollecito.

Autointerviste per numero di solleciti

\begin{tabular}{|l|c|c|c|}
\hline \multicolumn{1}{|c|}{ Numero di solleciti } & Frequenza & $\%$ & Frequenza cumulata \\
\hline 0 & 160 & 25,2 & 25,2 \\
\hline Solo 1 & 168 & 26,5 & 51,7 \\
\hline Solo 2 & 173 & 27,2 & 78,9 \\
\hline 3 & 134 & 21,1 & 100,0 \\
\hline Totale & 635 & & \\
\hline
\end{tabular}

\section{Il questionario}

Relativamente alla costruzione del questionario abbiamo fatto riferimento al modello elaborato da Roberto Ventura ${ }^{9}$. Secondo il modello adottato per ciascuno dei servizi o delle caratteristiche indagate l'utente è stato chiamato ad esprimersi non solo circa il proprio grado di soddisfazione ma relativamente alle seguenti tre dimensioni:

- l'importanza attribuita al servizio indipendentemente dal modo in cui è gestito in biblioteca;

- una stima del reale uso del servizio espresso in percentuale;

- il grado di soddisfazione rispetto a quel servizio.

In ambito bibliotecario ad oggi questo tipo di rilevazione non è stato trattato in maniera esauriente dagli standard in vigore. L'IFLA ${ }^{10}$, pur prendendo in considerazione questi aspetti suggerendo la possibilità di inserire una domanda a risposta libera in cui l'utente possa spiegare le ragioni della propria

\footnotetext{
9 Ventura 1998.

10 IFLA Poll \& te Boekhorst 1996.
} 
insoddisfazione, non li traduce in un campo strutturato e quindi difficilmente quantificabile. ISO $11620^{11}$ non dà alcuna indicazione in merito. Rilevare queste tre dimensioni risulta particolarmente interessante in quanto permette, come vedremo dettagliatamente nell'esame dei risultati, di calcolare la soddisfazione complessiva dell'utente. Ogni aspetto sottoposto ad indagine, letto mettendo in relazione le tre dimensioni rilevate, può essere analizzato con maggiore precisione, i bisogni degli utenti sono meglio definiti.

Il questionario è costruito a risposte chiuse, vale a dire viene posta una domanda a cui l'intervistato non risponde liberamente ma deve scegliere fra un certo numero di risposte predefinite assegnando un punteggio. Per tale punteggio abbiamo adottato la scala di Likert ${ }^{12}$ con la possibilità di esprimere l'importanza attribuita al servizio e la propria soddisfazione per quel servizio con una scala di valori da 1 a 5 . Per rilevare la dimensione di contatto è stato chiesto di indicare la percentuale d'uso scegliendo tra cinque valori a scalare da 0 a 100. Nel dettaglio, le risposte si articolano come segue:

- importanza attribuita al servizio indipendentemente dal modo in cui è gestito in biblioteca: 1 = per nulla importante, $2=$ poco importante, $3=$ indifferente, 4 = importante, $5=$ molto importante;

- percentuale dell'utilizzo del servizio sul totale delle visite in biblioteca: $0 \%, 25 \%, 50 \%, 75 \%, 100 \%$;

- grado di soddisfazione per il servizio: 1 = insoddisfatta/o, 2 = poco insoddisfatta/o, 3 = indifferente, 4 = abbastanza soddisfatta/o, 5 = soddisfatta/o.

Per distinguere chi non ha utilizzato il servizio, rilevato nella dimensione di contatto, da chi si è rifiutato di rispondere il rilevatore ha registrato $i$ seguenti casi: $96=$ Non sa, $97=$ Non vuole rispondere, $98=$ Interrompe l'intervista, $99=$ Cade la linea.

Queste casistiche naturalmente nel questionario web si trovavano fra le opzioni da poter barrare.

Nella fase progettuale dell'indagine, dopo avere individuato i bisogni informativi utili alla gestione del Sistema bibliotecario, si è proceduto alla stesura del questionario. Il questionario si articola in tre parti, la prima contiene domande sui servizi offerti o caratteristiche della biblioteca di riferimento, la seconda contiene quesiti riferibili all'intero sistema bibliotecario e l'ultima rileva alcuni dati anagrafici.

11 ISO 1998.

12 I criteri di misurazione delle risposte sono chiamate in gergo "tecniche di scaling", fra le più conosciute quelle di Likert, di Thurstone e di Guttman. 
A livello di singola biblioteca abbiamo sottoposto ad indagine la qualità delle risorse documentarie (libri e periodici posseduti dalla biblioteca; disponibilità in biblioteca delle novità librarie; disponibilità in biblioteca degli ultimi numeri dei periodici), il prestito interbibliotecario (recupero di libri e articoli da altre biblioteche), l'accessibilità del materiale, la logistica, l'adeguatezza o comfort degli spazi (facilità di recupero di libri e periodici a scaffale aperto; disponibilità in sala lettura di posti a sedere; silenzio, comfort e luminosità della sala lettura; utilità di depliant e segnaletica per facilitare l'accesso ai servizi), i rapporti tra gli utenti e il personale delle biblioteche (precisione e rapidità nella distribuzione di libri e periodici dai magazzini; la comunicazione con il personale delle biblioteche; l'importanza dei servizi di assistenza personalizzata da parte del bibliotecario).

A livello di Sistema bibliotecario abbiamo chiesto di valutare i servizi avanzati: l'uso dell'OPAC (ricerca per titolo e/o autore, ricerca per argomento, ricerca per parola chiave; uso dell'OPAC per prenotazione e proroga del prestito di libri); le banche dati messe a disposizione (accesso remoto o locale); l'uso dei periodici elettronici; il sito web del Sistema bibliotecario.

Tra la prima e la seconda parte, al fine di evidenziare eventuali spostamenti tra biblioteche appartenenti ad aree diverse, è stato chiesto allo studente se frequentasse altre biblioteche del Sistema Bibliotecario e quali. Per il personale docente, non essendo stata identificata la biblioteca di utilizzo anteriormente all'indagine, prima di iniziare il questionario si è chiesto quali biblioteche l'intervistato frequentasse e quale, tra quelle utilizzate, frequentasse in maggior misura, identificando quest'ultima come biblioteca di riferimento per le domande della prima parte del questionario ${ }^{13}$.

Per ottenere una compilazione veloce del questionario sono state inserite alcune domande "filtro" che hanno guidato l'intervistatore ed i docenti, nello svolgimento dell'indagine. Se, ad esempio, alla domanda 1.7 (Disponibilità in sala di lettura di posti a sedere per l'uso di materiale della biblioteca) l'utente dichiarava di non utilizzare la sala di lettura (percentuale di uso uguale a $0 \%$ ) nel questionario di trovava l'indicazione di passare alla domanda 1.10 saltando in questo modo tutte le domande relative alle caratteristiche della sala di lettura.

13 Nel caso in cui un intervistato dichiari di non frequentare alcuna biblioteca del Sistema bibliotecario ne viene chiesto il motivo e poi si spassa alle domande relative al sito WEB del Sistema Bibliotecario di Ateneo. 


\section{Verifiche e PRE-TEST}

La versione definitiva del questionario è stata sottoposta al Comitato di gestione del $\mathrm{SBA}^{14}$, dal quale, in quanto fruitore principale dei risultati dell'indagine, si poteva avere un importante apporto circa la corretta individuazione delle aree tematiche da sottoporre a indagine viste anche in relazione alle possibili azioni da intraprendere nel caso i risultati dell'indagine lo richiedessero.

Il Dipartimento di Statistica ha curato in particolare l'organizzazione del questionario nei livelli previsti, l'ordine e la forma in cui presentare le domande.

Pur avendo prestato attenzione alla formulazione delle domande cercando di usare un linguaggio semplice, evitando sigle e termini troppo tecnici o in inglese, come verifica finale, allo scopo di testare la facilità di comprensione e la non ambiguità delle domande, è stato effettuato anche un pre-test somministrato ad un gruppo di studenti e docenti. In particolare, a un gruppo di docenti del Dipartimento di Statistica, agli studenti che lavoravano part-time presso l'Ufficio di Coordinamento centrale delle biblioteche, agli studenti che nell'arco di tre giorni hanno frequentato le biblioteche di Economia, Lettere e Architettura.

14 Il Comitato di Gestione del Sistema Bibliotecario è composto dal coordinatore generale, dai direttori delle 5 biblioteche di area e dai coordinatori dei gruppi di lavoro di Ateneo. 



\title{
L'indagine sulla soddisfazione degli utenti del Sistema bibliotecario dell'Università degli Studi di Firenze: analisi dei risultati e studio delle frequenze
}

\author{
di FRANCESCA LANDI
}

Obiettivo di questo contributo è presentare un'analisi delle risposte fornite dagli studenti-utenti del Sistema bibliotecario sulla base degli spogli effettuati dallo staff del Dipartimento di Statistica.

Benché il Dipartimento di Statistica abbia fornito gli spogli sia per l'intero Sistema bibliotecario d'Ateno ${ }^{1}$ sia per le singole biblioteche di area $^{2}$, si intende qui analizzare unicamente i risultati relativi al Sistema Bibliotecario nel suo complesso. Vengono prese in considerazione inoltre unicamente le dichiarazioni degli studenti che, in fase di spoglio dei dati, sono state riportate all'universo di riferimento costituito dai 27.746 utenti del prestito nell'anno 2000. Non vengono analizzate invece le risposte dei docenti a cui il questionario è stato somministrato in via sperimentale tramite internet ${ }^{3 ;}$ data la bassa percentuale di coloro che hanno risposto, infatti, non è stato possibile estendere i risultati dell'indagine a tutto l'universo di riferimento ${ }^{4}$.

L'analisi delle risposte è stata effettuata individuando le seguenti dimensioni della qualità dei servizi erogati dalle biblioteche, corrispondenti agli obiettivi su cui maggiormente ha investito il Sistema bibliotecario in tutte le fasi della sua riorganizzazione a partire dal 1995:

- qualità dell'offerta documentaria;

- qualità dei servizi avanzati;

- accessibilità dell'offerta documentaria;

- qualità dell'OPAC;

- qualità degli ambienti: la sala di lettura;

- personale: empatia e competenza.

\footnotetext{
1 Vedi l'Appendice $C$.

2 Vedi il case study affrontato da Eleonora Giusti.

3 Vedi il contributo di Silvana Benedetti.

4 L'analisi delle risposte fornite dai docenti si può leggere nel successivo contributo di Roberto Ventura.
} 
Le singole domande del questionario costituiscono le voci che caratterizzano le suddette dimensioni. Per ciascuna voce sono stati "misurati" tre aspetti: importanza, percentuale d'uso e livello di soddisfazione. In fase di elaborazione del questionario è stata scelta la modalità di risposta chiusa, utilizzando il formato tipo Likert, un metodo di misurazione nel quale la scala rappresenta un continuo bipolare che consente all'intervistato di rispondere con diverse gradazioni di intensità ${ }^{5}$; nella rappresentazione grafica, tuttavia, relativa all'importanza e alla soddisfazione, le risposte negative sono state sommate, così come le positive, in modo da avere una lettura più sintetica dei risultati.

Il giudizio espresso sui singoli servizi in merito all'importanza e alla soddisfazione è stato inoltre soppesato mettendolo in relazione, oltre che con il numero di utenti che lo hanno espresso, anche con la frequenza d'uso. Per rappresentare questa relazione è stato scelto il grafico a bolle, nel quale sull'asse delle ordinate sono rappresentati gli studenti-utenti, su quella delle ascisse il grado di risposta dato, mentre l'ampiezza dei cerchi indica la percentuale media di uso del servizio.

\section{Valutazioni degli studenti-utenti sulla qualità dell'offerta documentaria della biblioteca di cui sono utenti}

Relativamente a questa dimensione, gli intervistati sono stati invitati a rispondere, riferendosi alla biblioteca di cui risultavano utenti del prestito (nel caso di utenti che avevano effettuato prestiti in più biblioteche risultano utenti di quella dove hanno preso più libri).

L'offerta documentaria di ciascuna biblioteca è stata identificata da 4 voci:

1. libri, periodici e cd-rom posseduti dalla biblioteca;

2. disponibilità in biblioteca delle novità librarie nelle discipline di interesse dell'intervistato;

3. disponibilità in biblioteca degli ultimi numeri dei periodici nelle discipline di interesse dell'intervistato;

4. prestito interbibliotecario: recupero di libri e articoli da altre biblioteche universitarie.

Il Sistema bibliotecario d'Ateneo, nel 2000, offriva alla sua utenza un patrimonio documentario di 3.400 .000 volumi (monografie e annate di perio-

5 Vedi il contributo di Silvana Benedetti. 
dici) distribuiti in 38 punti di servizio gestiti dalle biblioteche di area; i periodici attivi erano circa 8.300. L'incremento inventariale complessivo è di 30.000 unità. L'offerta documentaria comprende anche i documenti resi accessibili tramite il prestito interbibliotecario, un servizio sul quale si è investito molto nell'ottica di un Sistema bibliotecario basato sull'accesso oltre che sul possesso; dal 1995 al 2000 il servizio di prestito interbibliotecario ha avuto infatti un incremento del $46 \%$.

Fig. 1 - Importanza attribuita alle risorse documentarie

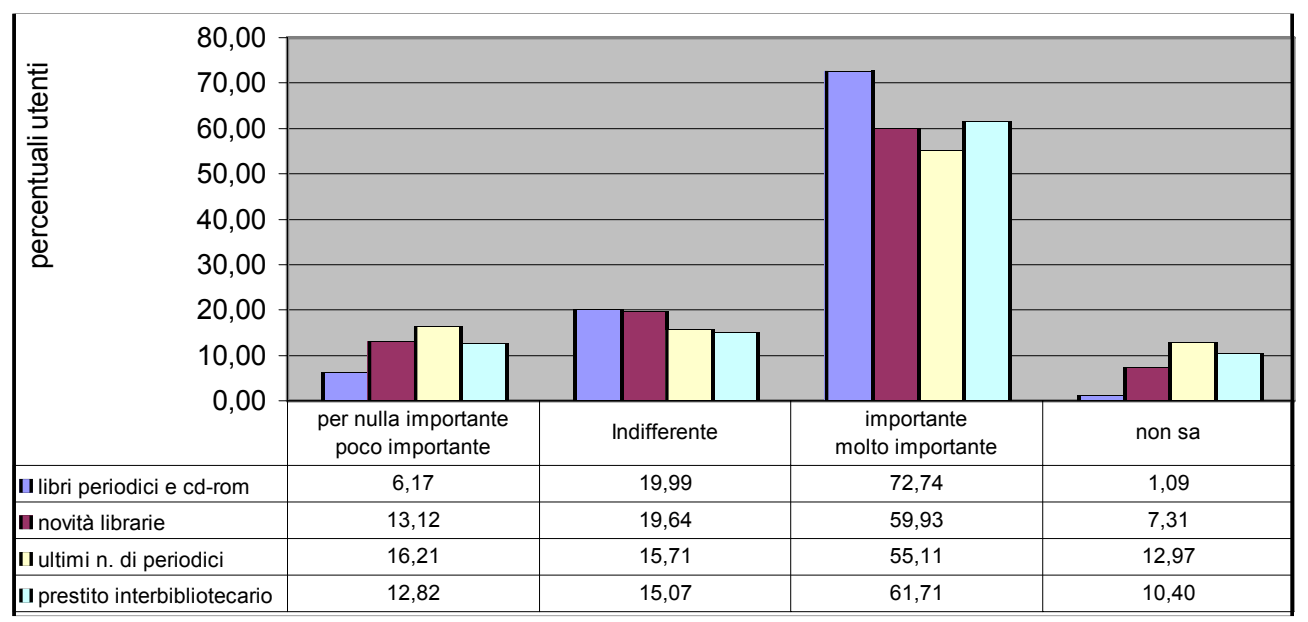

La dotazione documentaria della biblioteca di riferimento è una dimensione della qualità del servizio per la quale c'è un'alta aspettativa; oltre la metà della popolazione di riferimento considera tutte e quattro le voci importanti o molto importanti. Mediamente solo il 12\% degli studenti-utenti si esprime con un giudizio di poco o per nulla importante, il 18\% è indifferente, ma ben il $62 \%$ considera importante o molto importante la dotazione documentaria della biblioteca. Questa dimensione della qualità inoltre è ben conosciuta dagli studenti-utenti; infatti solo 1'8\% non sa esprimere un giudizio. Delle quattro voci che definiscono questa dimensione, quella relativa ai libri, periodici e cd-rom è quella per cui si evidenzia la maggior aspettativa; il 73\% della popolazione di riferimento infatti, la considera importante o molto importante, mentre la percentuale dei non sa scende all'1\% (Fig. 1).

I libri, periodici e cd-rom della biblioteca sono anche la risorsa documentaria maggiormente utilizzata; oltre il 65\% degli studenti li utilizzano almeno 
Fig. 2 - Utilizzo delle risorse documentarie

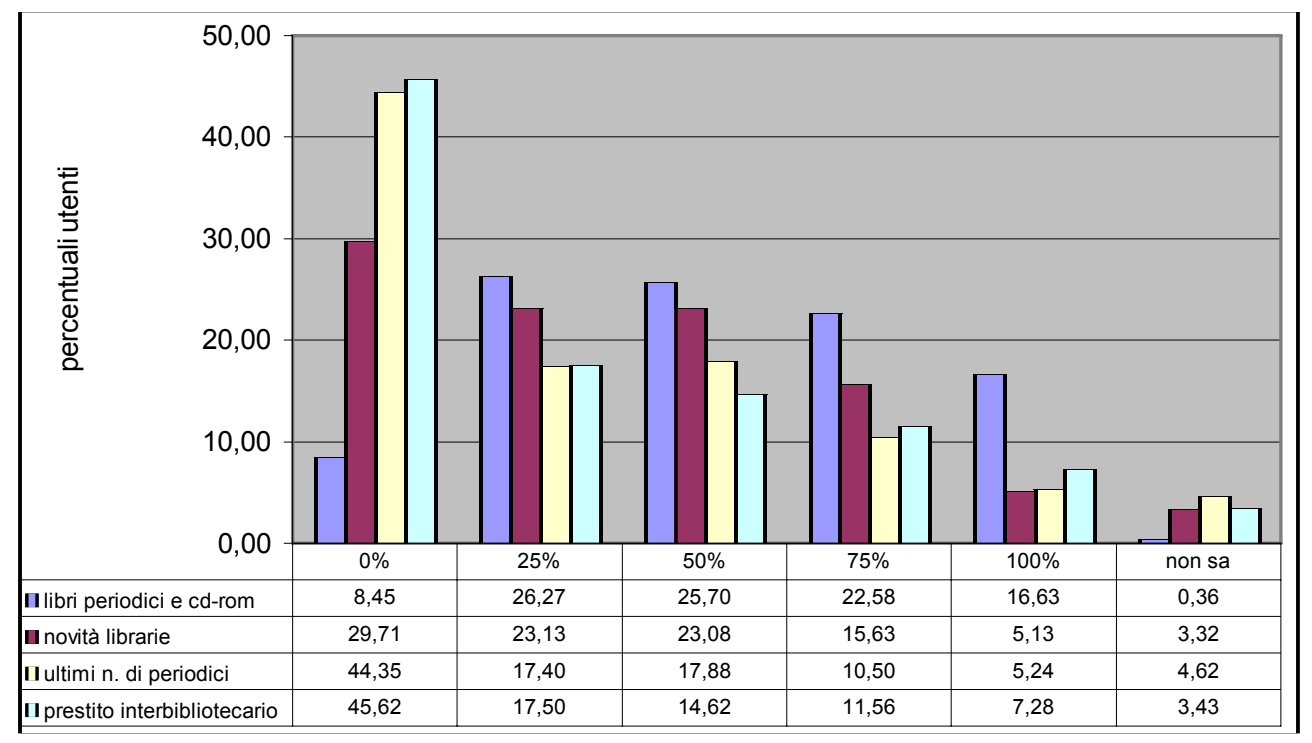

una volta ogni 2 visite in biblioteca. Sono tutt'altro che trascurabili le percentuali di studenti-utenti che dichiarano di non utilizzare (ovvero una percentuale d'uso pari a 0) le altre tre risorse elencate: oltre il $40 \%$ degli studenti-utenti dichiara di non utilizzare gli ultimi numeri dei periodici e il prestito interbibliotecario. È da rilevare come la presenza del servizio di prestito interbibliotecario sia avvertito dal $62 \%$ degli utenti come importante o molto importante, ma per nulla utilizzata nel $46 \%$ dei casi (Fig. 2).

Il possesso di libri, periodici e cd-rom è anche, tra i quattro elementi assunti a rappresentare le risorse documentarie di ciascuna biblioteca, quello che ha dato i risultati migliori in termini di grado di soddisfazione (Fig. 3). Tale elemento, infine, si conferma come quello più conosciuto da parte degli studenti-utenti o comunque quello rispetto al quale si sentono più sicuri di rispondere: la percentuale dei non so o dei rifiuti è la più bassa e decisamente trascurabile rispetto ai tre aspetti valutati.

Per quanto riguarda le risposte relative al grado di soddisfazione occorre ulteriormente sottolineare che per tutte le voci scelte per rappresentare questa dimensione i giudizi positivi prevalgono su quelli negativi. Mediamente solo il $17 \%$ si dichiara insoddisfatto o poco insoddisfatto mentre il $49 \%$ esprime un giudizio di soddisfazione, anche se coloro che si dichiarano 
Fig. 3 - Soddisfazione per le risorse della biblioteca di utenza

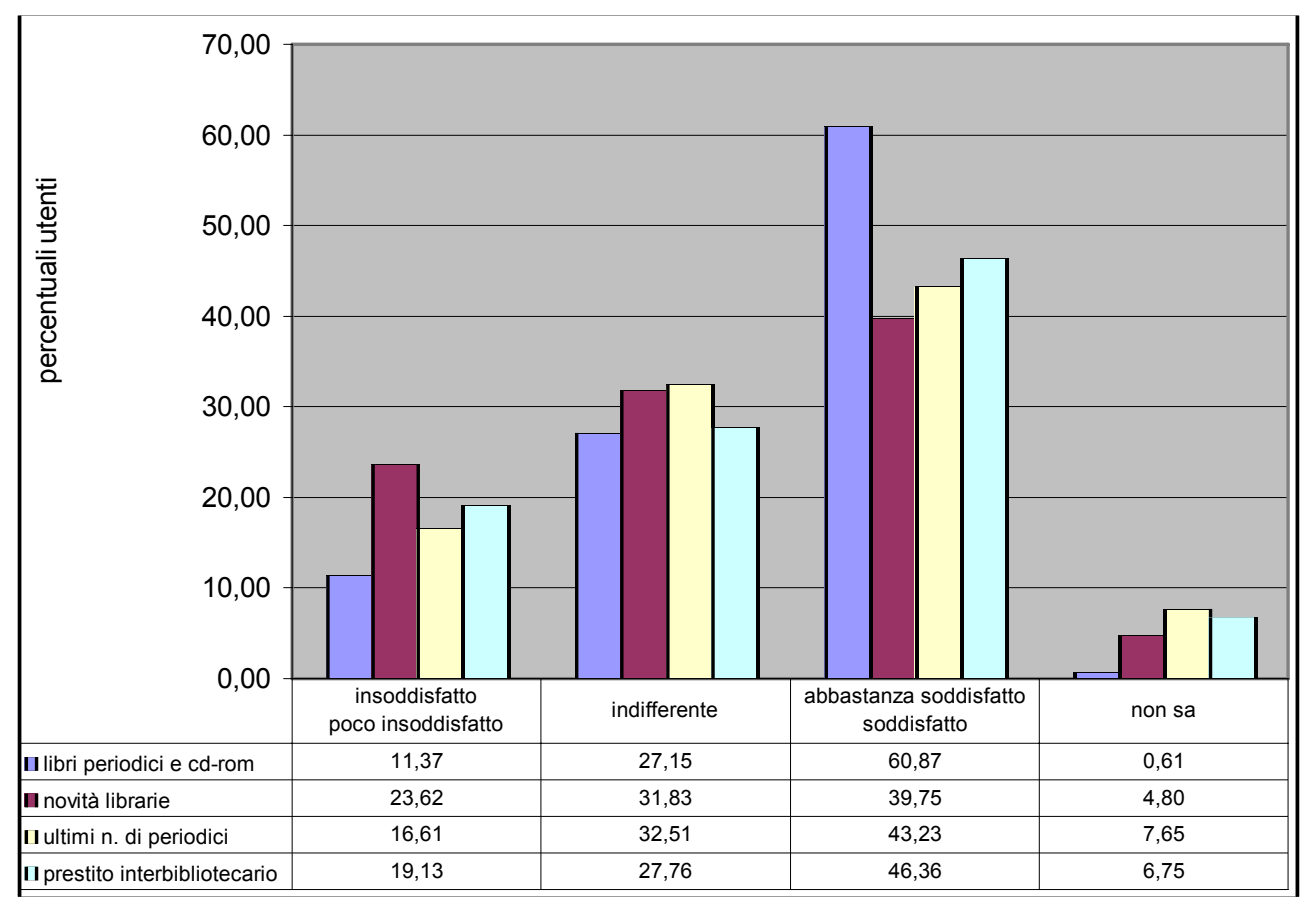

abbastanza soddisfatti sono molti di più di coloro che esprimono un giudizio di piena soddisfazione. Un risultato, invece, sicuramente meno confortante è rappresentato da quella parte di studenti che si dichiarano indifferenti, in media circa il 30\% di utenti. Quest'ultimo risultato è comunque conforme a quello che accade per tutte le voci esaminate nel questionario per le quali la percentuale di coloro che si dichiarano indifferenti, relativamente alla soddisfazione, non scende quasi mai al di sotto del $20 \%$ e in pochi casi supera anche il $35 \%{ }^{6}$.

Passando ora ad esaminare la relazione tra la percentuale d'uso di un servizio e il livello di importanza attribuito allo stesso (Fig. 4) e quella tra la percentuale d'uso di un servizio e il livello di soddisfazione dichiarato per esso (Fig. 5), si rileva che per tutti e quattro gli elementi assunti a rappresentare l'offerta documentaria della biblioteca di utenza, la percentuale media d'uso

6 Vedi l'Appendice $C$. 
Fig. 4 - Relazione fra uso, importanza attribuita alle risorse e numero di utenti

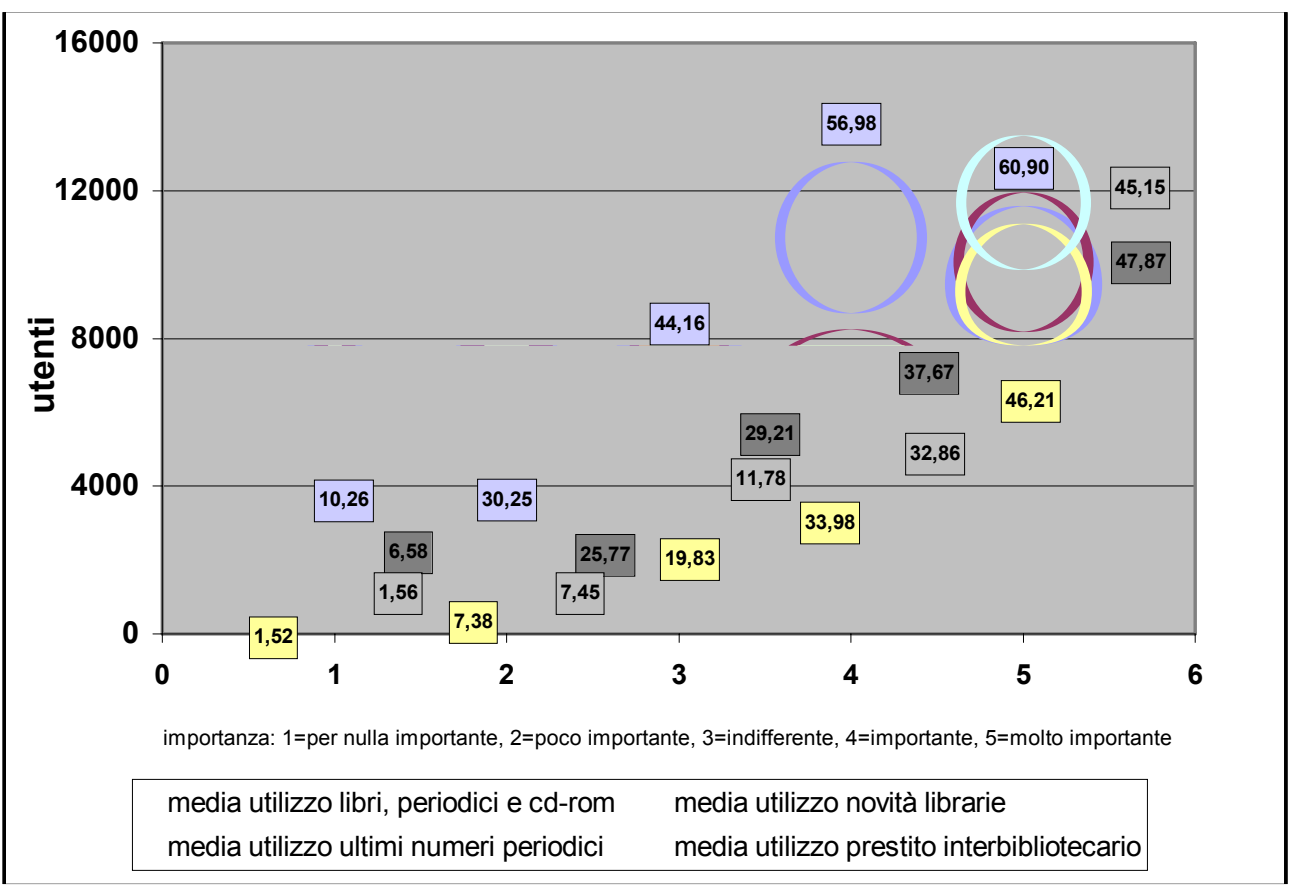

cresce al crescere dell'importanza assegnata all'elemento in questione (nel grafico infatti, al crescere dell'ascissa cresce il diametro dei cerchi). Si rileva inoltre, che, non solo coloro che esprimono un giudizio di soddisfazione positivo (abbastanza soddisfatto o soddisfatto) sono la maggioranza (come si vede dal numero di utenti riportati in ordinata nel grafico) ma sono anche quelli a cui corrisponde una maggiore percentuale media di utilizzo (un cerchio di maggior diametro), e quindi si esprimono sulla base di una buona conoscenza del servizio. Il grafico evidenzia anche, la tendenza, da parte degli studenti-utenti ad esprimersi con un giudizio di abbastanza soddisfatto, piuttosto che dichiarare la piena soddisfazione. 
Fig. 5 - Relazione fra uso, soddisfazione percepita per le risorse e numero di utenti

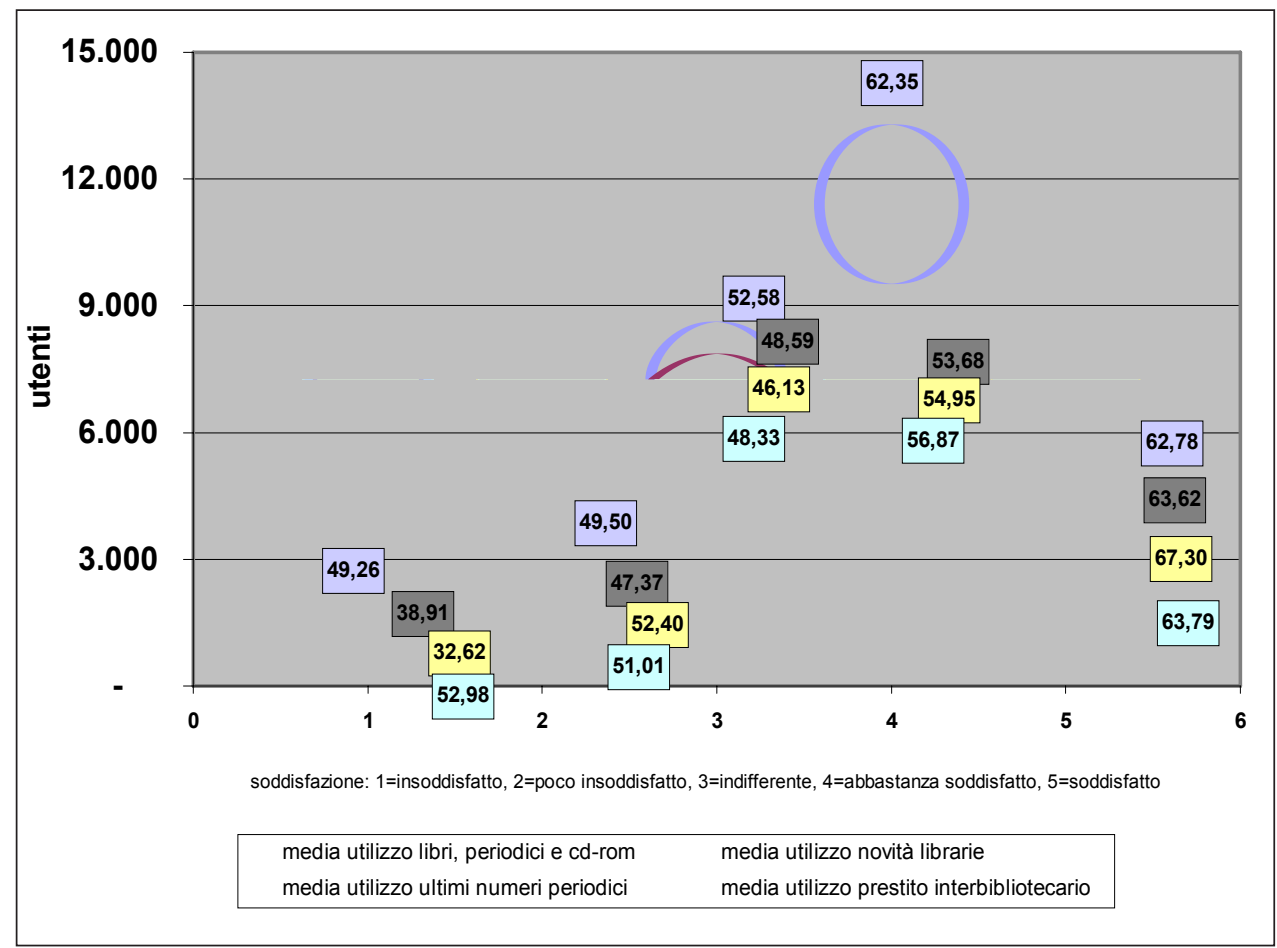

\section{Valutazioni degli studenti-utenti per la qualità dei servizi avanzati offerti dal Sistema Bibliotecario d'Ateneo}

Le risposte relative alla qualità di questi servizi erano molto attese poiché è su questi che si è concentrata l'attività di gestione del Sistema bibliotecario negli ultimi anni. L'incremento esponenziale della documentazione disponibile in rete o comunque su supporto elettronico ha reso necessario dedicare tempo e risorse, finanziarie e umane, all'integrazione di questi nuovi servizi con quelli tradizionali già erogati dalle biblioteche, alla loro promozione, e alla formazione sia dei bibliotecari che degli utenti. Le pagine web del Sistema bibliotecario, all'interno del sito dell'Università di Firenze, danno accesso sia a pagine informative sulle biblioteche di area e i relativi punti di servizio (orari, servizi offerti, modalità di utilizzo, ecc.), sia a risorse bibliografiche e documentali; in particolare, già nel 2000, gli utenti potevano acce- 
dere dalle pagine web del Sistema bibliotecario a 46 banche dati in rete, alle informazioni relative ad altre 39 in locale e a 2160 periodici elettronici.

La dimensione della qualità dei "servizi avanzati" offerti dal Sistema bibliotecario nel suo complesso comprende le seguenti voci:

1. il sito web del Sistema Bibliotecario di Ateneo;

2. le banche dati disponibili nelle biblioteche (via web, su cd-rom);

3. risultati ottenuti dall'interrogazione delle banche dati;

4. uso dei periodici elettronici nelle discipline di interesse dell'intervistato.

Gli utenti sono stati invitati a rispondere per l'intero Sistema bibliotecario; per ciascuna voce sono stati misurati tre aspetti: importanza, percentuale d'uso (esclusa la voce n. 3), livello di soddisfazione. Mentre tutti gli intervistati hanno espresso le loro valutazioni sul sito web, i quesiti sugli altri tre servizi sono stati posti solo a coloro che hanno dichiarato una percentuale di utilizzo positiva per quest'ultimo. In una prima fase di sperimentazione del questionario, infatti, si è rilevato che coloro che non utilizzano il sito web generalmente non utilizzano alcun servizio informatico; le domande relative ai servizi informatici sono state, quindi, poste solo a coloro che dichiaravano per il sito web una percentuale di utilizzo diversa da 0 .

In merito all'importanza attribuita ai servizi avanzati, l'elemento per il quale abbiamo la più alta aspettativa da parte degli studenti-utenti è il sito

Fig. 6 - Importanza attribuita ai servizi avanzati offerti dal Sistema bibliotecario d'Ateneo

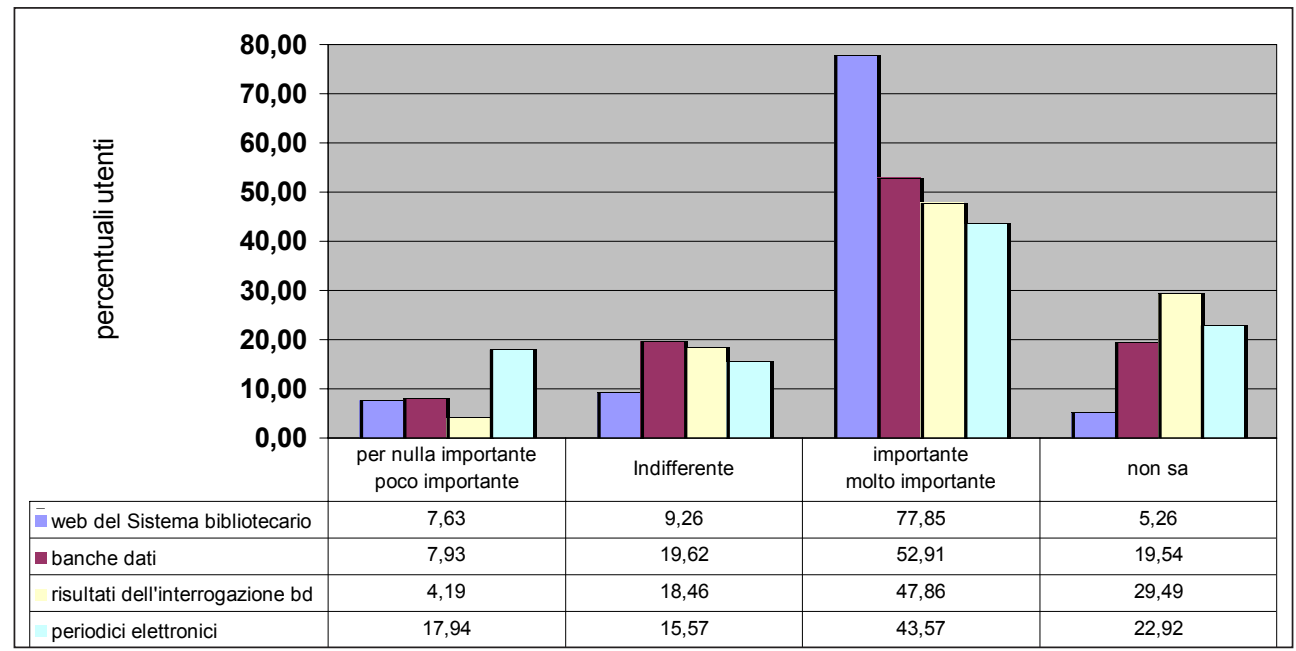


web del Sistema bibliotecario, che il $78 \%$ della popolazione di riferimento considera importante o molto importante. Il sito web, tra i servizi avanzati, è anche quello più conosciuto dagli studenti; infatti ottiene la minor percentuale di non sa fra le voci qui esaminate.

Anche per questa dimensione della qualità possiamo valutare mediamente l'aspettativa degli utenti. I servizi avanzati sono considerati per nulla o poco importanti dal 9\% degli studenti-utenti, gli indifferenti sono il 15\% della popolazione di riferimento, mentre il $57 \%$ li ritiene importanti o molto importanti. C'è inoltre un $18 \%$ di utenti che non sa esprimere un giudizio, ad evidente dimostrazione della necessità di promuovere ulteriormente l'uso di queste risorse fra gli utenti (Fig. 6).

Fig. 7 - Utilizzo dei servizi avanzati offerti dal Sistema bibliotecario d'Ateneo

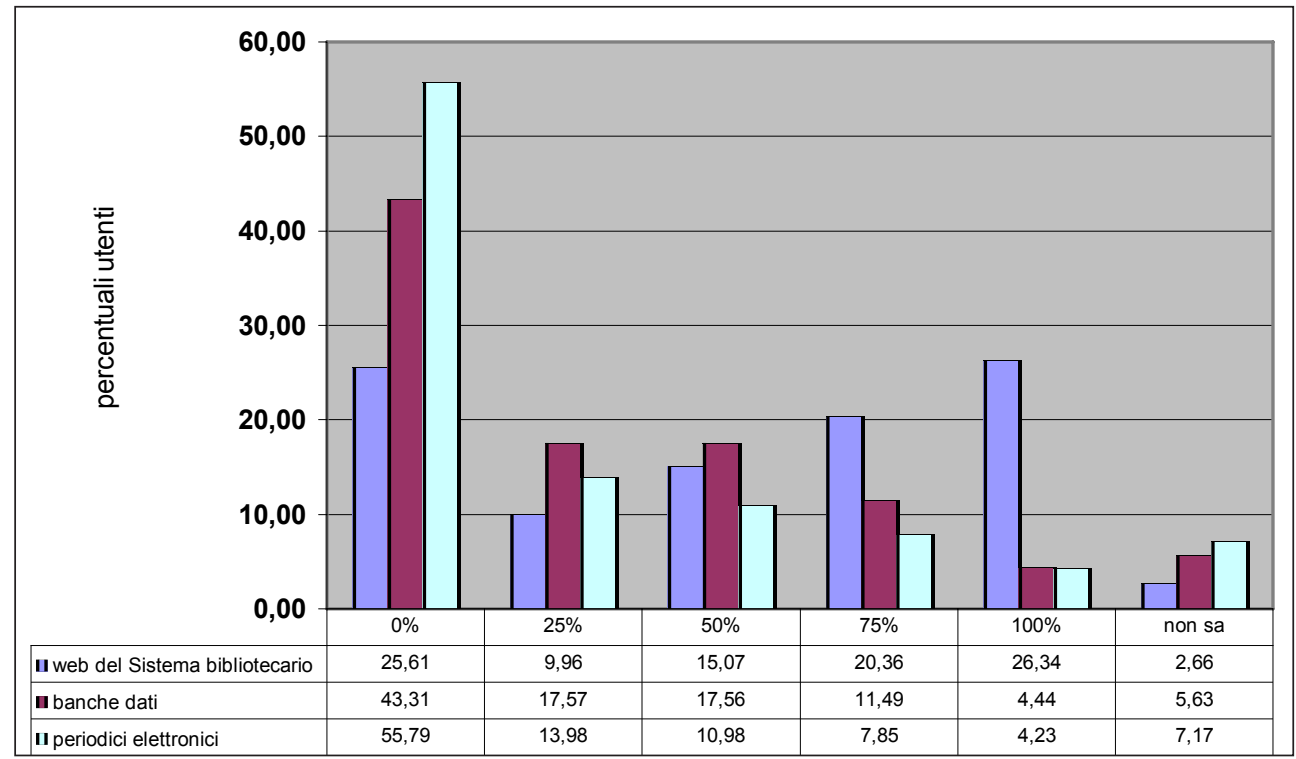

L'analisi dei risultati relativi all'utilizzo dei servizi avanzati rileva l'alto numero di studenti-utenti che non li usano mai. In particolare, i periodici elettronici e le banche dati non sono mai stati interrogati rispettivamente dal $56 \%$ e $43 \%$ della popolazione di riferimento; un risultato particolarmente grave per il Sistema bibliotecario è tuttavia rappresentato da quel $26 \%$ di coloro che non usano mai il sito web (Fig. 7). Potrebbe essere interessate analizzare l'evoluzione nel tempo di questo risultato, dato il rapido diffondersi di internet $\mathrm{e}$ il forte aumento dei servizi disponibili via web. 
Fig. 8 - Soddisfazione per i servizi avanzati offerti dal Sistema bibliotecario d'Ateneo

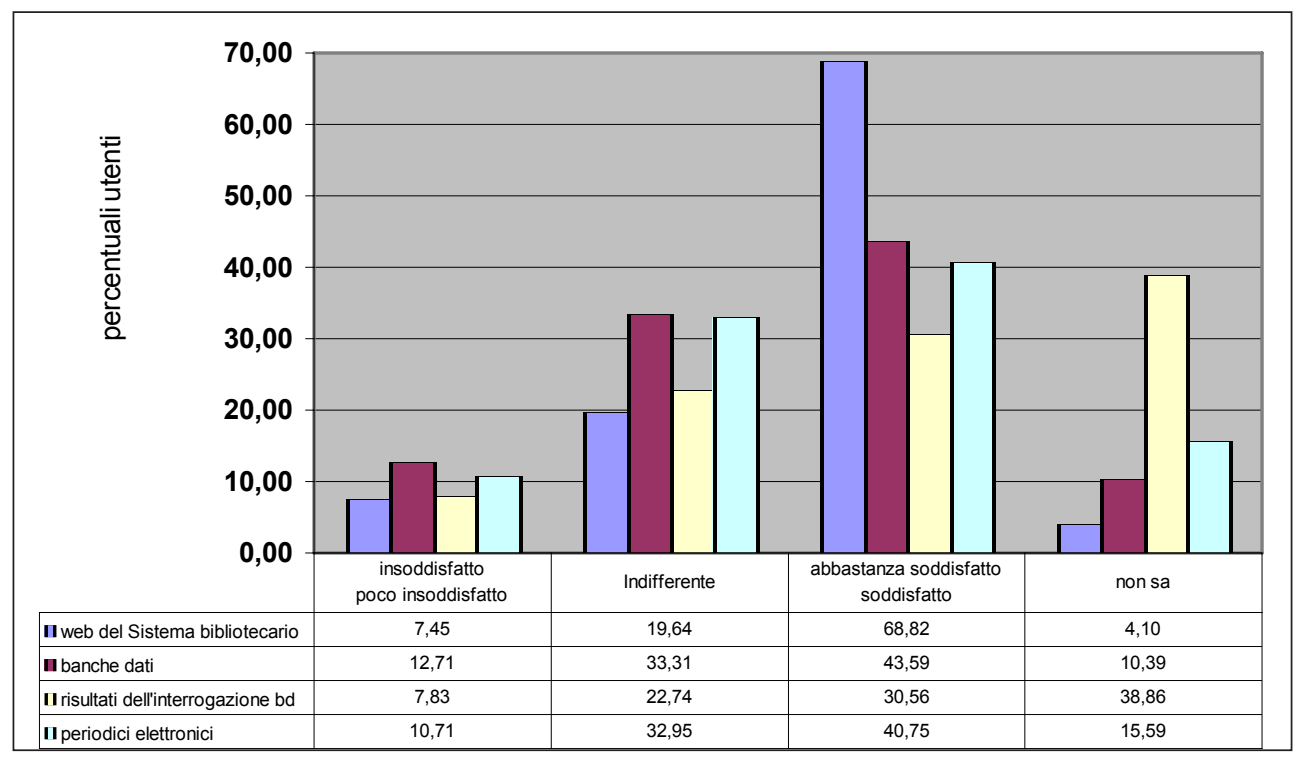

In termini di soddisfazione per tutti i servizi avanzati i giudizi positivi prevalgono su quelli negativi. Mediamente questo aspetto della dimensione della qualità è valutato abbastanza soddisfacente o soddisfacente dal $47 \%$ degli studenti; il 9\% si dichiara invece insoddisfatto o poco insoddisfatto. $\mathrm{Si}$ conferma piuttosto alta la percentuale degli indifferenti, pari al 25\%; alta anche, il 19\%, la percentuale di coloro che pur avendo usato questi servizi non sono in grado di esprimersi. In quest'ultimo caso contribuisce in maniera determinante la difficoltà di esprimere un giudizio sui risultati ottenuti dall'interrogazione delle banche dati, pari al 39\%.

Il sito web si conferma essere il servizio per il quale si registra la percentuale più alta di risposte positive (69\%) (Fig. 8).

Rispetto alla relazione tra la percentuale d'uso di un servizio e il livello di importanza attribuito allo stesso (Fig. 9) occorre notare che, per tutti i servizi avanzati la percentuale media d'uso cresce considerevolmente nel passare da coloro che giudicano il servizio poco o per nulla importante a coloro che lo valutano abbastanza importante o molto importante. 
Fig. 9 - Relazione fra uso, importanza attribuita ai servizi avanzati e numero utenti

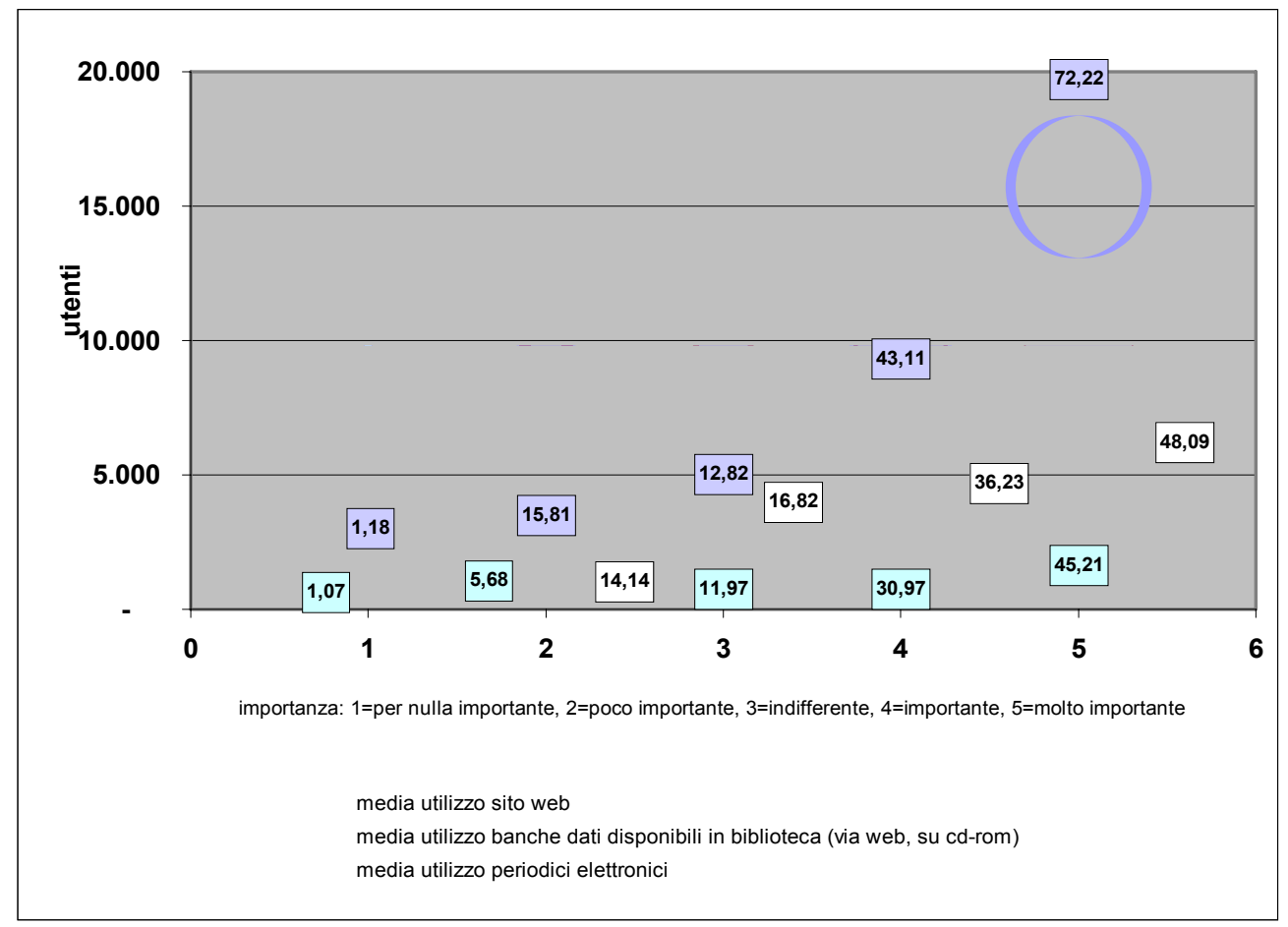

Fig. 10 - Relazione fra uso, soddisfazione percepita per i servizi avanzati e numero di utenti

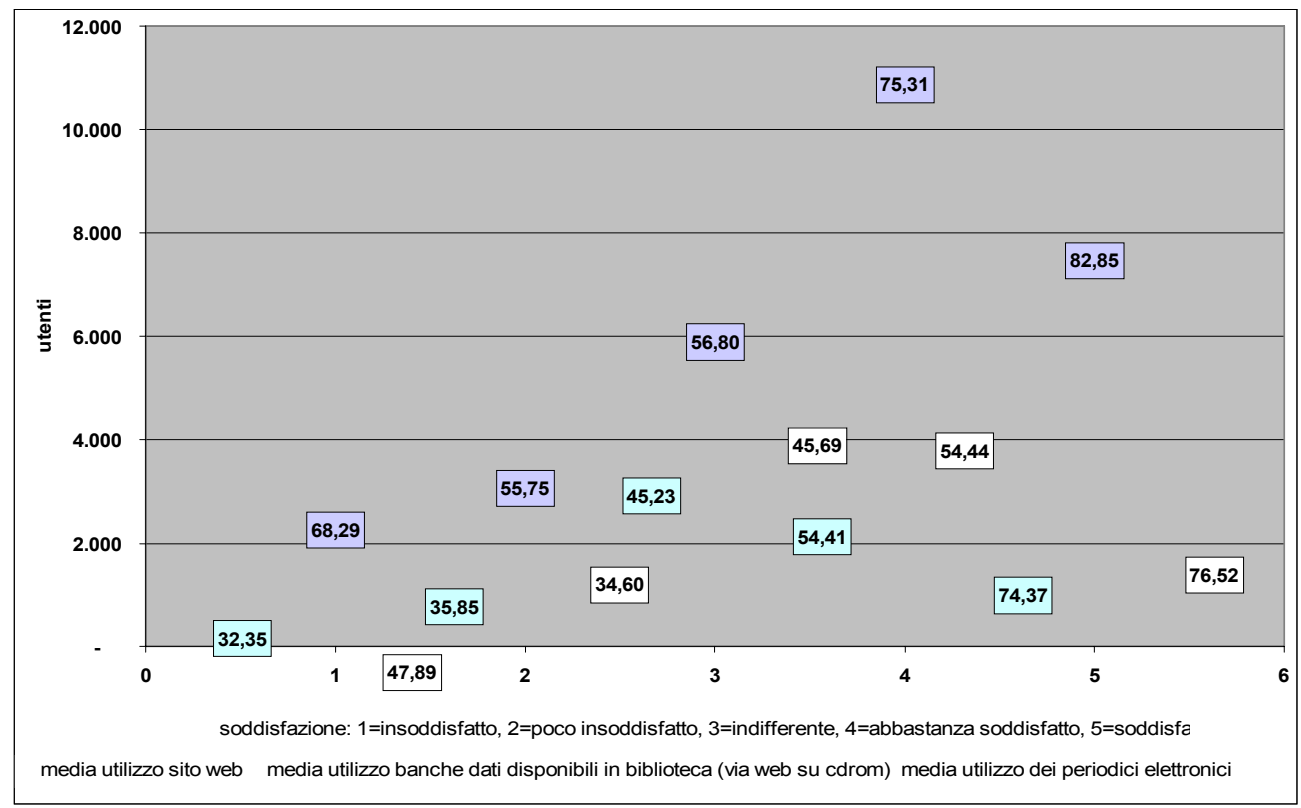


Infine, rispetto alla relazione tra la percentuale d'uso di un servizio e il livello di soddisfazione dichiarato per esso (Fig. 10), si nota che, mentre per le banche dati e i periodici elettronici il grado di soddisfazione aumenta all'aumentare della percentuale media di utilizzo (quindi chi li utilizza intensamente ne è anche soddisfatto), per il sito web la percentuale media di utilizzo è alta per tutti, anche per coloro che si dichiarano insoddisfatti o poco insoddisfatti. Occorre comunque aggiungere che coloro che utilizzano tale servizio e esprimono un giudizio negativo su di esso in termini di soddisfazione sono pochi rispetto a coloro che utilizzano il servizio e manifestano un giudizio positivo su di esso in termini di soddisfazione.

\section{Valutazioni degli studenti-utenti sulla accessibilità dell'offerta documen- taria della biblioteca di cui sono utenti}

Uno degli obiettivi del progetto di riorganizzazione del Sistema bibliotecario d'Ateneo è stato offrire il maggior numero possibile di documentazione cartacea a scaffale aperto. L'attivazione dei servizi on line ha inoltre richiesto un ulteriore sforzo da parte delle biblioteche per recuperare spazi attrezzati per la ricerca in internet e per la consultazione di banche dati sia su cd-rom che in rete. Nel 2000 i volumi a scaffale aperto nelle sale di lettura e consultazione delle biblioteche di area ammontavano a 395.000, circa il 12\% del patrimonio totale. I pc a disposizione del pubblico per la navigazione in rete e la consultazione del catalogo OPAC erano circa 200. Gli utenti sono stati invitati a rispondere, in riferimento a questa dimensione, relativamente alla biblioteca di cui risultavano utenti del prestito; gli studenti che avevano effettuato prestiti in più biblioteche risultano utenti di quella dove hanno preso più libri.

L'accessibilità della documentazione per ciascuna biblioteca è stata delineata da due voci per il materiale cartaceo e due per quello elettronico:

1. facilità di recupero di libri e periodici dagli scaffali liberamente accessibili dal pubblico;

2. facilità del recupero e consultazione di bibliografie, repertori, enciclopedie;

3. accesso ad internet in biblioteca;

4. banche dati: modo in cui sono messe a disposizione del pubblico. 
Fig. 11 - Importanza attribuita all'accessibilità dell'offerta documentaria

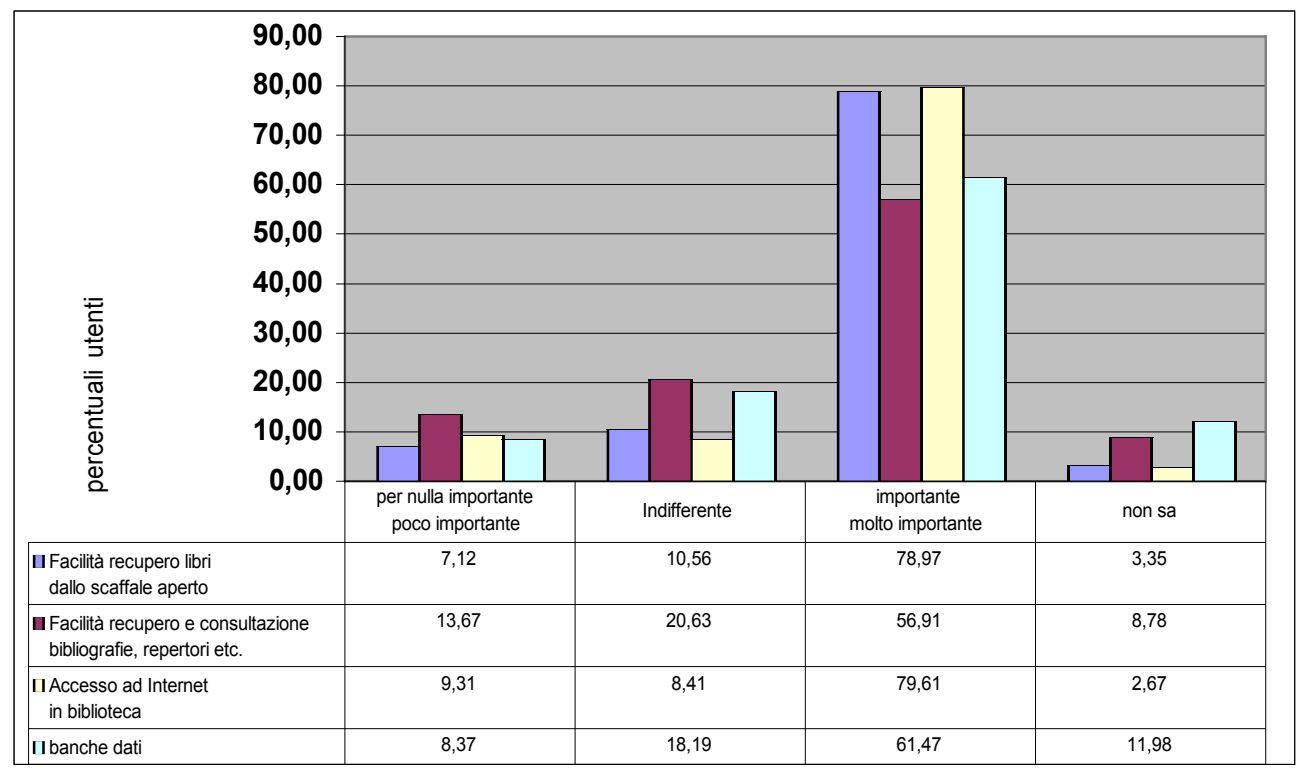

L'analisi dei risultati relativi all'aspettativa per questa dimensione della qualità mette in rilievo quanto tutte le voci considerate siano ritenute importanti o molto importanti dalla popolazione di riferimento; la percentuale media di coloro che hanno espresso un giudizio positivo per questo aspetto dell'accessibilità dell'offerta documentaria corrisponde infatti al 69\% degli studenti-utenti, mentre un $10 \%$ ha espresso un giudizio negativo. Anche in questo caso gli indifferenti sono in media il 14\%; coloro che non sono in grado di dare una valutazione il 7\% (Fig. 11).

Le voci che ottengono il miglior risultato sono lo scaffale aperto (79\%) per la documentazione cartacea e l'accesso ad internet (80\%) per quella elettronica; l'aspettativa per l'accesso alle bibliografie, repertori, ecc., da una parte, e alle banche dati, dall'altra, ottiene un risultato più basso, conformemente a quanto emerso dall'analisi delle risposte relative alla qualità dei servizi avanzati.

I libri a scaffale aperto sono anche la risorsa documentaria che è maggiormente utilizzata dalla popolazione di riferimento (Fig. 12); il 70\% degli studenti-utenti se ne serve almeno una volta su due visite in biblioteca. Il materiale a scaffale aperto è anche la voce che ha la percentuale più bassa di non utilizzo, a differenza di bibliografie e repertori che non risultano mai utilizzati durante le visite in biblioteca da ben il $40 \%$ della popolazione di riferimento. 


\section{FRANCESCA LANDI}

Fig. 12 - Utilizzo delle risorse documentarie accessibili direttamente in biblioteca

\begin{tabular}{|cc|c|c|c|c|c|c|c|}
\hline & $\mathbf{5 0 , 0 0}$ \\
\hline & & & \\
\hline
\end{tabular}

Fig. 13 - Soddisfazione percepita per l'accessibilità delle risorse documentaria della biblioteca di utenza

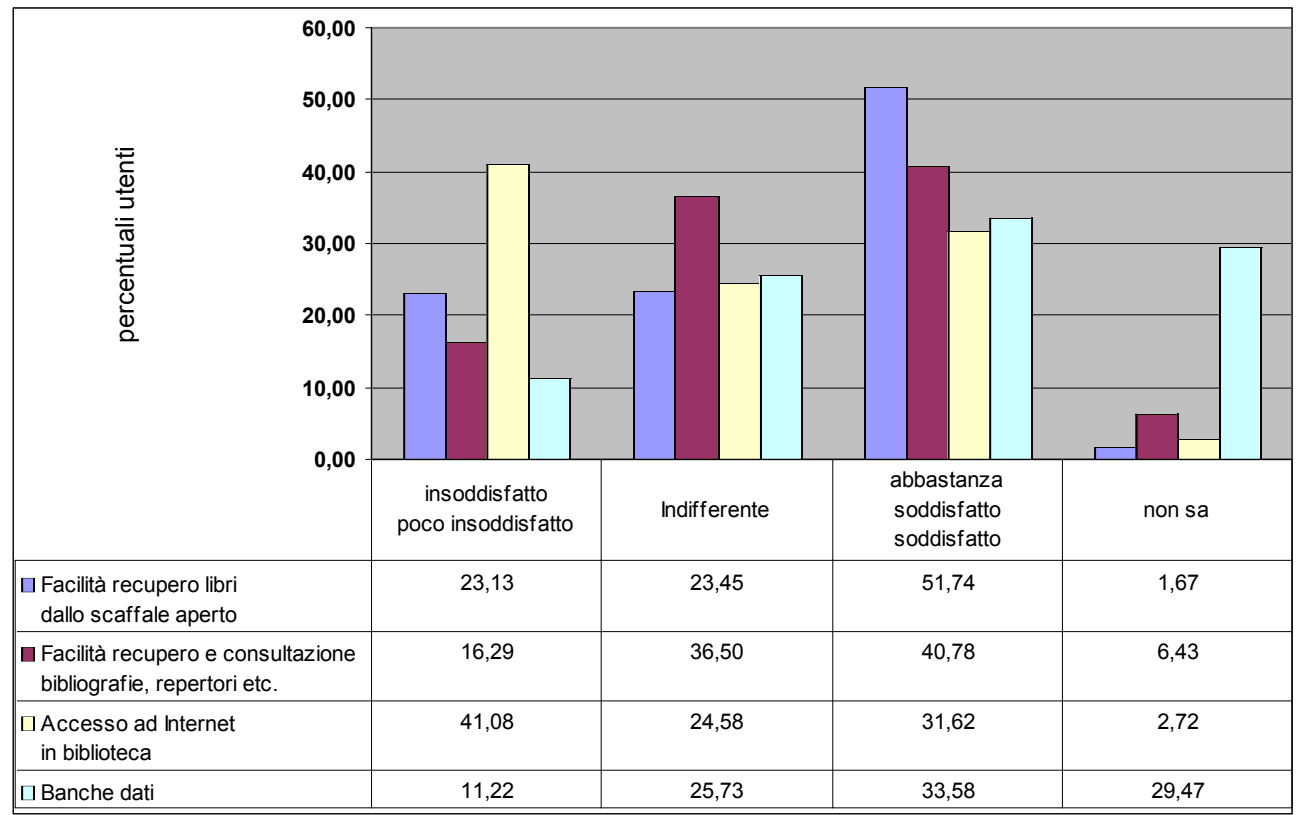


Ciò che maggiormente emerge dall'esame dei risultati sulla soddisfazione per questa dimensione è l'alta percentuale di insoddisfatti per l'accessibilità ad internet (Fig. 13). Il 40\% degli studenti-utenti dichiara la propria insoddisfazione per la facilità di accesso ad internet in biblioteca. Questo giudizio, che concorda con quanto emerso da un'indagine fatta dal Nucleo di valutazione $^{7}$ nel 2000, probabilmente è legato in parte ad un numero insufficiente di postazioni collegate alla rete, in parte, alle limitazioni poste in molte biblioteche alla navigazione degli utenti; presso alcuni punti di servizio si è tentato infatti di regolamentare l'utilizzo di internet poiché gli studenti spesso non si limitano alle sole ricerche di natura bibliografica o comunque legate a motivi di studio.

Fig. 14 - Relazione fra uso, importanza attribuita all'accessibilità dell'offerta documentaria e numero di utenti

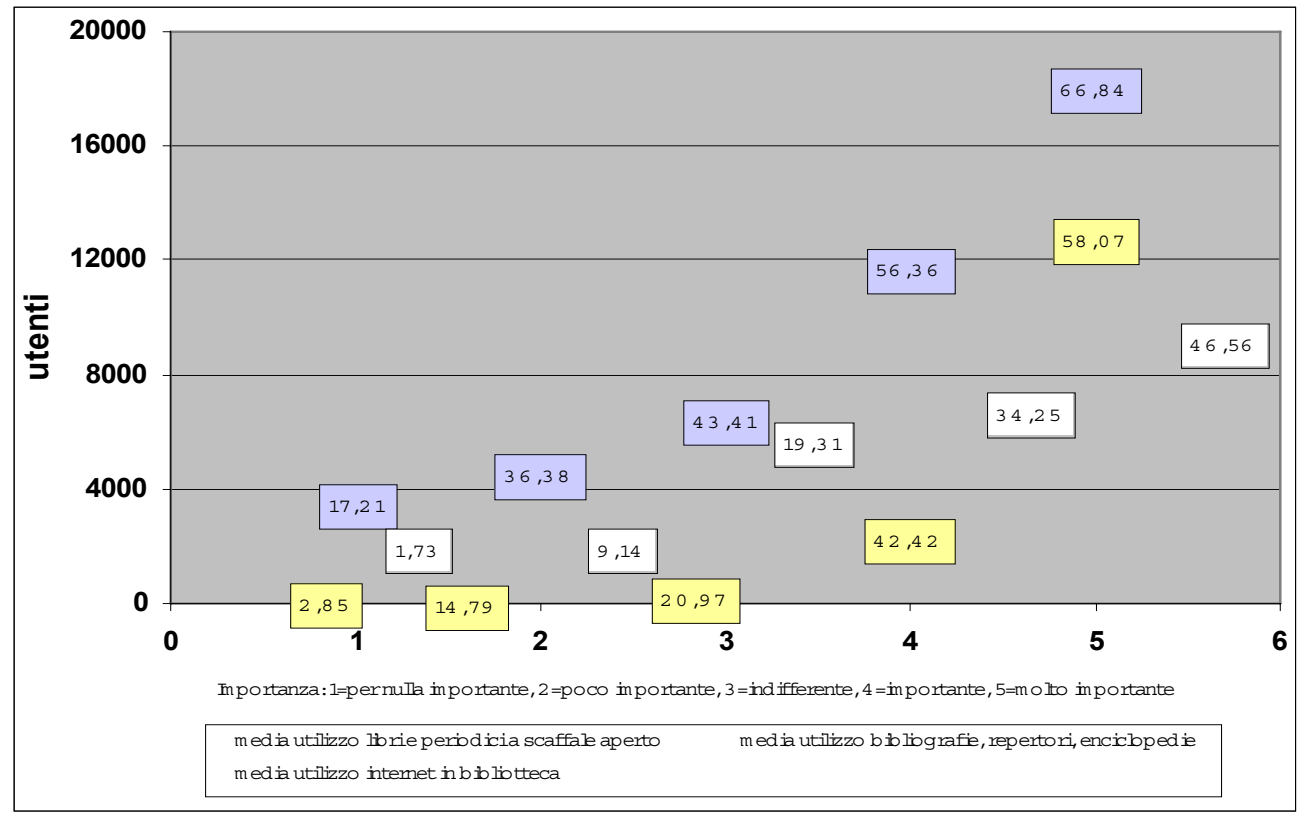

L'analisi della relazione fra uso e importanza (Fig. 14) e quella fra uso e soddisfazione percepita per questa dimensione della qualità è stata fatta solo per tre voci poiché la formulazione della domanda sulle banche dati escludeva la possibilità di raccogliere un dato di utilizzo. La relazione fra uso e importanza attribuita al servizio conferma la tendenza generale in base alla quale la percentuale d'uso dei servizi cresce al crescere dell'importanza attribuita agli stessi (il diametro dei cerchi cresce lungo l'ascissa); inoltre, coloro 
che usano i servizi e che al tempo stesso li ritengono importanti o molto importanti, sono anche la maggioranza degli studenti (cerchi posizionati più in alto sull'asse delle ordinate).

Fig. 15 - Relazione fra uso, soddisfazione percepita per l'accessibilità dell'offerta documentaria e numero di utenti

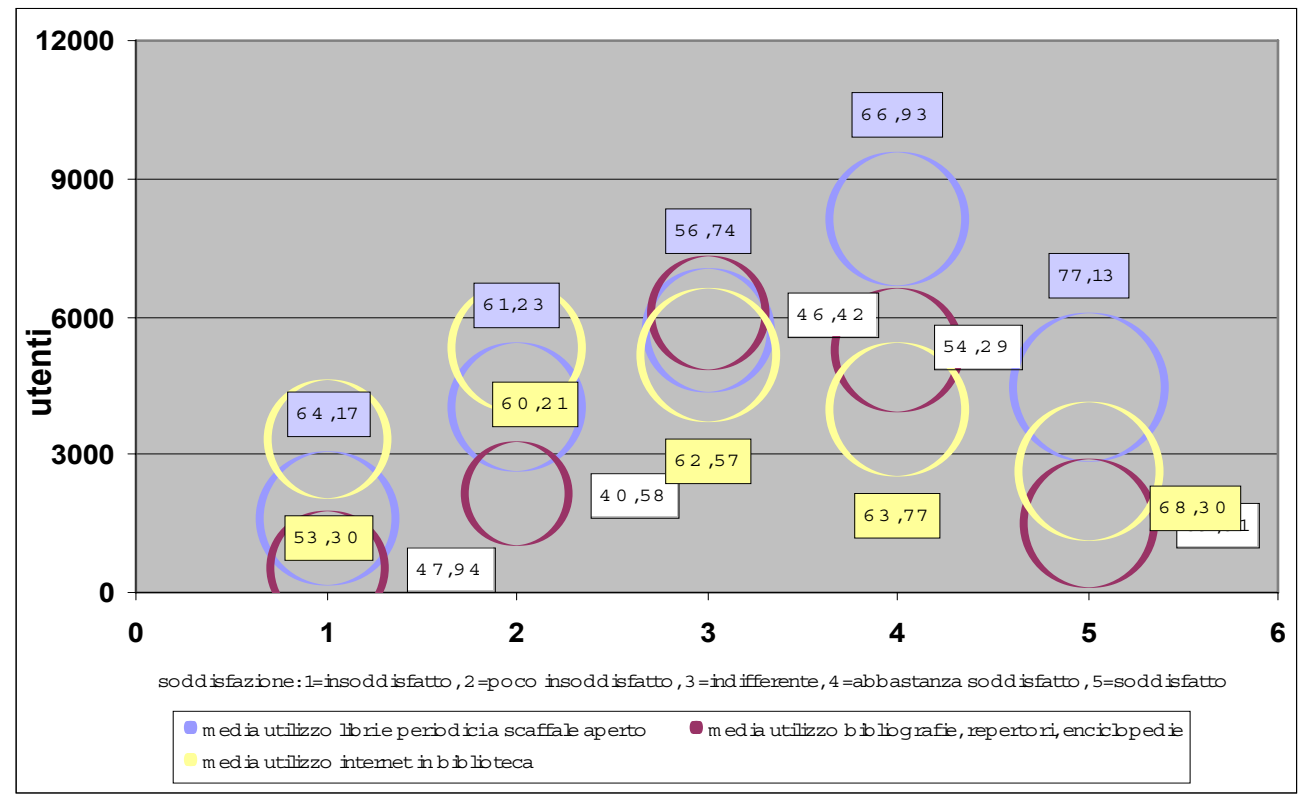

Passando ad analizzare la relazione fra uso e soddisfazione percepita, emerge chiaramente come questi servizi siano molto utilizzati anche da chi esprime un giudizio negativo su di essi (Fig. 15). La percentuale media di utilizzo, per tutte e tre le voci esaminate, è superiore al $40 \%$ sia per chi esprime il giudizio poco insoddisfatto che per chi si dichiara totalmente insoddisfatto. Conforta comunque rilevare che coloro che esprimono un giudizio negativo sono molti meno di coloro che si dichiarano abbastanza soddisfatti o soddisfatti.

7 Università degli Studi di Firenze 2001b, p. 135-224. In questo caso la domanda chiedeva la facilità o meno di accedere ad internet da postazioni rese disponibili per gli studenti anche in facoltà. 


\section{Valutazioni degli studenti-utenti sulla qualità dell'OPAC}

Benché riconducibile anch'esso alla dimensione dell'accessibilità dell'offerta documentaria, l'OPAC merita una riflessione a parte, date le risorse investite nella sua elaborazione e mantenimento dal Sistema bibliotecario in quanto principale strumento di mediazione fra il patrimonio e l'utenza. Il catalogo in linea, unico per tutte le biblioteche dell'Ateneo, è stato sviluppato a partire dal 1995 e si è alimentato inizialmente con le notizie provenienti dal gestionale SBN in uso; successivamente è stato incrementato con record, non gestibili in SBN, relativi ai periodici elettronici. Nel 2000 era possibile interrogare una base dati di circa 420.000 notizie bibliografiche; le pagine web relative al catalogo sono state poi arricchite con ulteriori servizi quali la ricerca per classe Dewey ${ }^{8}$, la visualizzazione dei prestiti in corso, la possibilità di effettuare prenotazioni ${ }^{9}$.

Sono state scelte quattro voci per valutare la qualità del catalogo in linea:

1. uso del catalogo della biblioteca su internet: ricerca di libri o periodici per titolo e ricerca di libri per autore;

2. uso del catalogo della biblioteca su internet: ricerca di libri per argomento (campo soggetto) o materia (campo classificazione);

3. uso del catalogo della biblioteca su internet: ricerca di libri o periodici nel campo parole chiave;

4. uso del catalogo della biblioteca su internet: prenotazione o proroga del prestito di un libro.

L'aspettativa per questo strumento di ricerca (Fig. 16) è veramente alta. L'OPAC risulta infatti la dimensione che ottiene mediamente la più alta percentuale (78\%) di utenti che lo giudicano importante o molto importante ${ }^{10} \mathrm{e}$ una fra le più basse $(7 \%)$ per quelli che lo considerano per nulla o poco importante; inoltre solo il 5\% di utenti non sa rispondere in merito.

Fra le voci considerate, la ricerca per autore e titolo risulta essere quella per la quale l'aspettativa è più alta; oltre il $94 \%$ degli studenti-utenti si esprimono con un giudizio di importante o molto importante.

8 È possibile effettuare la ricerca sia sui numeri di classificazione che sui descrittori associati, ed anche effettuare il browse sui descrittori.

9 È possibile visualizzare lo stato dei propri prestiti, ottenere una proroga, prenotare un libro in scadenza da un altro utente.

10 Vedi Tab. 1. 
Fig. 16 - Importanza attribuita all'OPAC del Sistema bibliotecario d'Ateneo

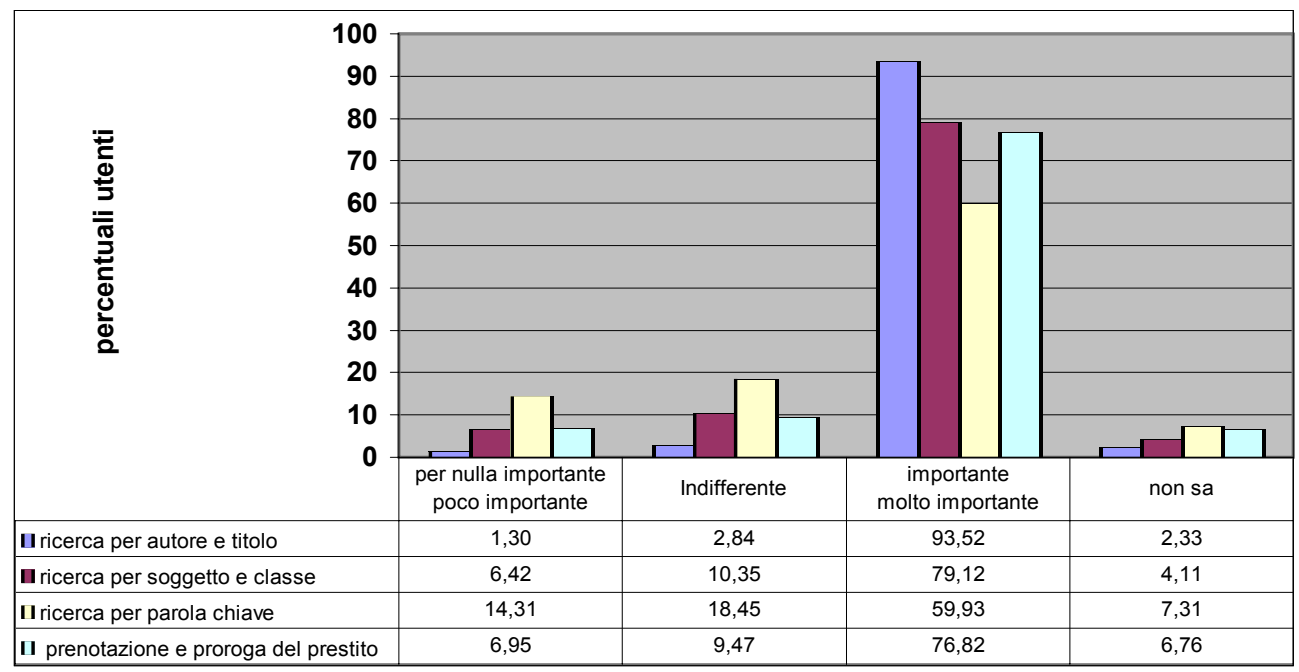

Fig. 17 - Utilizzo dell'OPAC del Sistema bibliotecario d'Ateneo

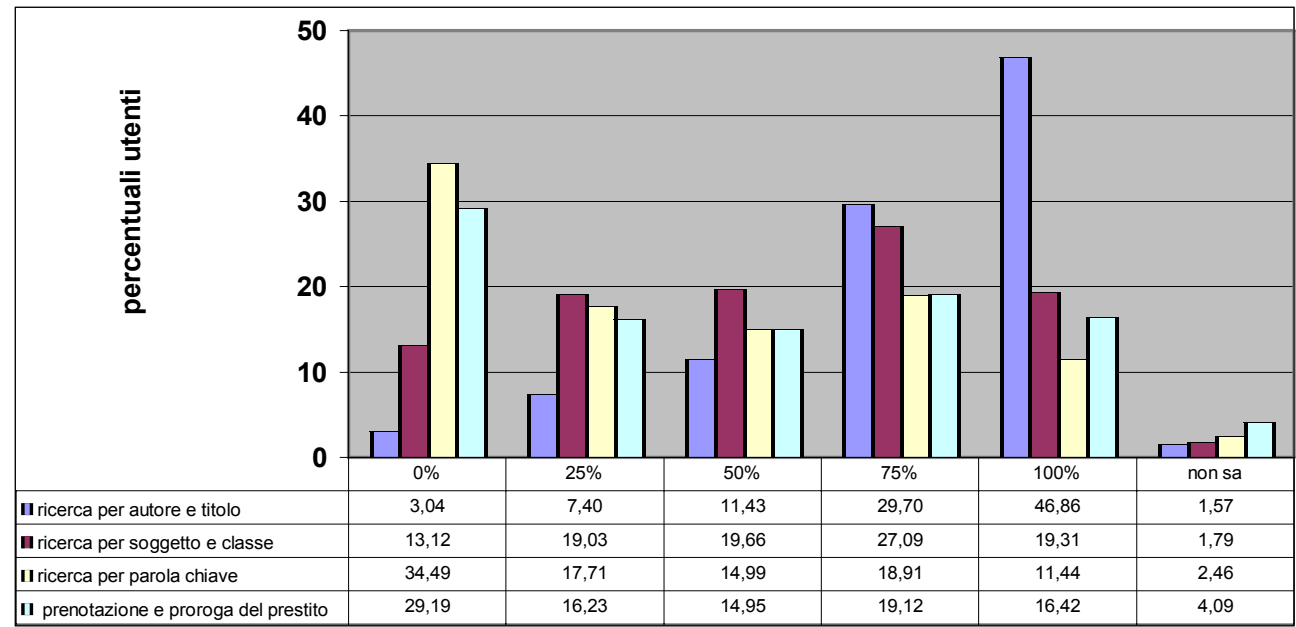

La ricerca per autore e titolo risulta anche essere quella maggiormente utilizzata; solo il 3\% degli studenti-utenti non la usa mai, mentre 1' $88 \%$ interroga l'OPAC tramite i campi di ricerca autore o titolo almeno una volta ogni due visite in biblioteca (Fig. 17). La ricerca per parole chiave risulta essere invece la meno utilizzata; ben il 34\% degli studenti-utenti non ha mai utilizzato questo tipo di accesso, a causa probabilmente dell'eccessivo rumore che genera questo tipo di ricerca. 
Fig. 18 - Soddisfazione percepita per l'OPAC del Sistema bibliotecario d'Ateneo

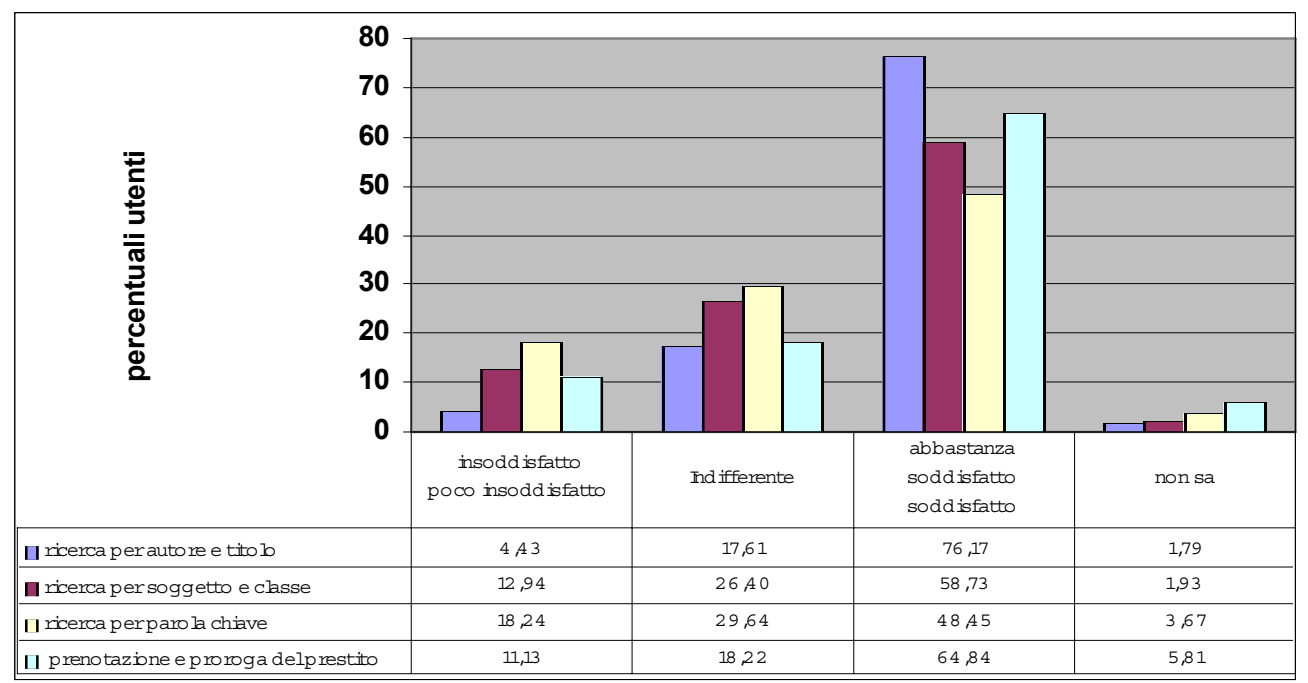

La ricerca nel campo parole chiave registra anche la percentuale più alta (18\%) della popolazione di riferimento che esprime un giudizio di insoddisfazione. La ricerca per autore o titolo è invece la voce per la quale abbiamo la più alta percentuale $(76 \%)$ di utenti abbastanza soddisfatti o soddisfatti (Fig. 18).

L'OPAC, fra tutte le dimensioni considerate, riporta il miglior risultato in termini di soddisfazione, con, mediamente, il $63 \%$ di studenti-utenti che si esprimono con un giudizio positivo; è inoltre la dimensione che presenta la più bassa percentuale di insoddisfatti, in media l'11\% della popolazione di riferimento ${ }^{11}$.

L'analisi della relazione fra uso e importanza (Fig. 19) evidenzia, come per le dimensioni precedentemente affrontate, un forte incremento della percentuale media di utilizzo (ampiezza dei cerchi) di pari passo con l'aumentare dell'importanza attribuita. Si può inoltre aggiungere che coloro che esprimono un giudizio positivo per il servizio e lo usano frequentemente sono molti di più di coloro che lo giudicano negativamente e lo usano poco (posizione dei cerchi sull'asse delle ordinate).

11 Vedi Tab. 2. 


\section{FRANCESCA LANDI}

Fig. 19 - Relazione fra uso, importanza attribuita all'OPAC e numero di utenti

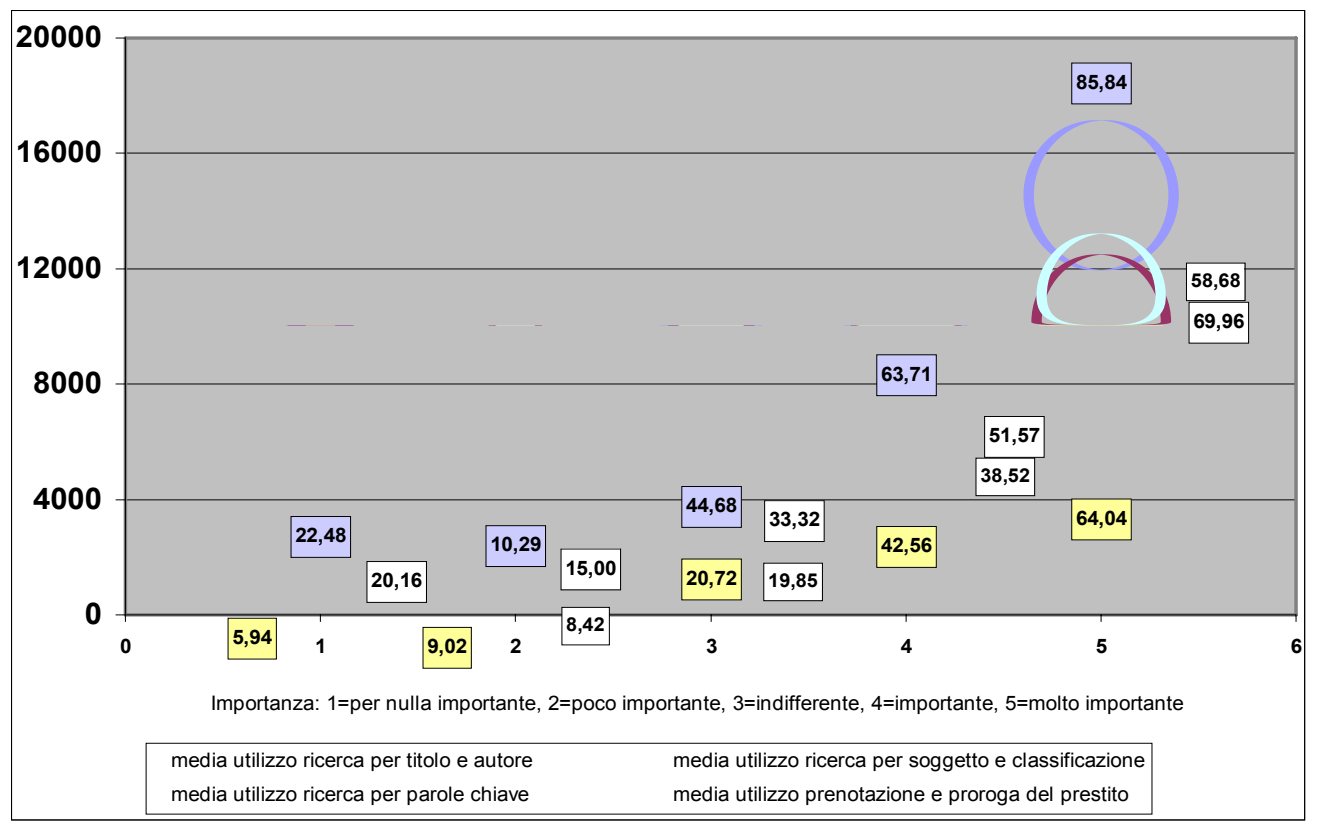

Fig. 20 - Relazione fra uso, soddisfazione per l'OPAC e numero di utenti

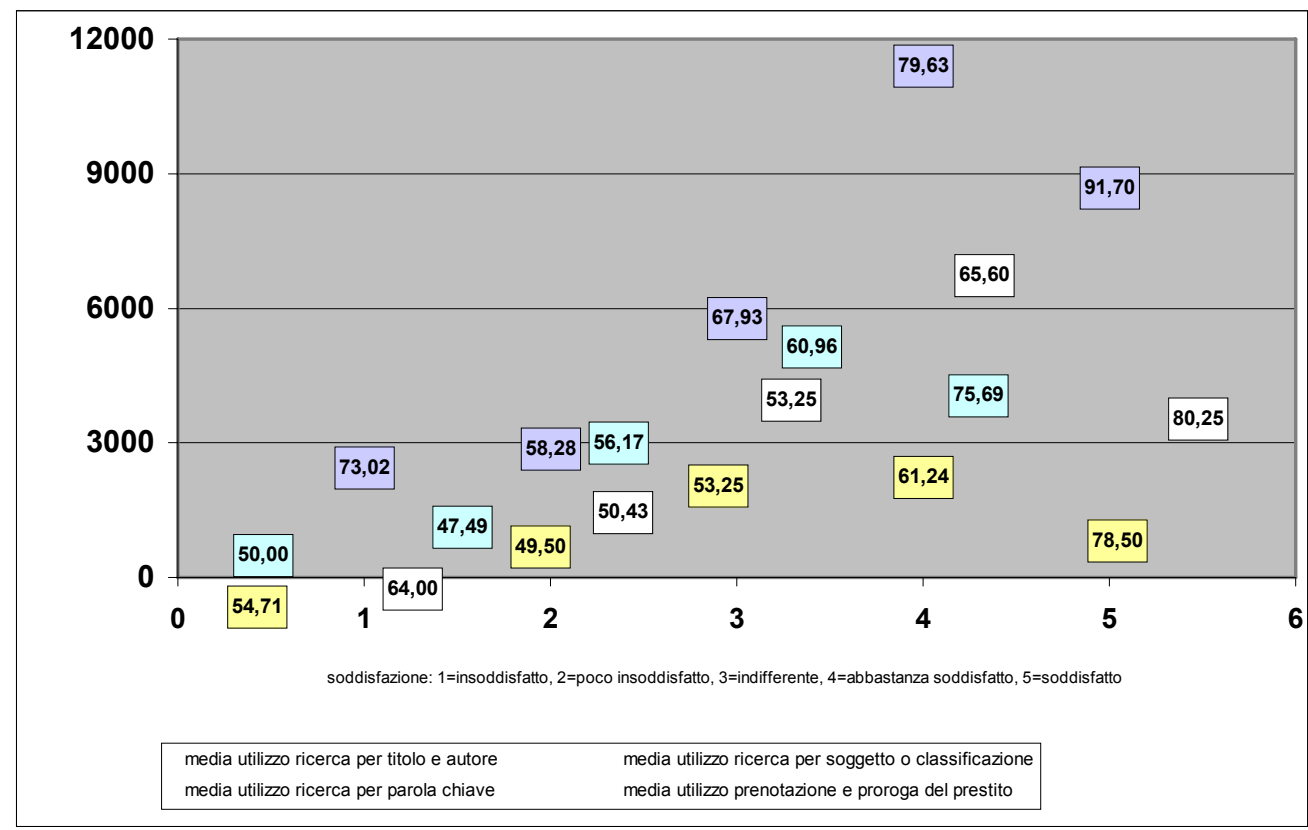


Relativamente alla relazione fra uso e soddisfazione percepita (Fig. 20) dobbiamo rilevare che, benché coloro che si dichiarano soddisfatti e usano il servizio in più del $60 \%$ delle loro visite in biblioteca siano la maggioranza, l'OPAC risulta comunque essere uno strumento indispensabile per la ricerca dei documenti e viene utilizzato anche da coloro che lo considerano insoddisfacente in almeno il $48 \%$ delle loro visite in biblioteca.

\section{Valutazioni degli studenti-utenti sulla qualità degli ambienti: la sala di lettura e la segnaletica della biblioteca}

Nel 2000 le 5 biblioteche di area hanno messo a disposizione degli utenti circa 65 sale di lettura, distribuite in 38 punti di servizio, per un totale di 3000 posti a sedere. Agli studenti-utenti è stato chiesto di esprimere un giudizio sulla biblioteca di cui risultavano utenti.

La dimensione della qualità degli ambienti a stata individuata in 3 voci che sono di pertinenza della sala di lettura e in una più generale relativa alla segnaletica presente in tutti gli ambienti della biblioteca e ai depliant predisposti per offrire informazioni su ogni biblioteca di area e sui servizi offerti, sia a livello di Biblioteca che di Sistema. Le voci scelte per rappresentare questa dimensione sono:

1. disponibilità in sala di lettura di posti a sedere per l'uso di materiale della biblioteca;

2. silenzio in sala di lettura;

3. comfort e luminosità della sala di lettura;

4. depliant sulle biblioteche e segnaletica in biblioteca per facilitare l'uso dei servizi.

Per ciascuna di tali voci sono stati "misurati" $i$ tre aspetti sopra richiamati: importanza, percentuale d'uso e livello di soddisfazione. Tutti gli intervistati hanno espresso la loro valutazione sulla disponibilità in sala lettura di posti a sedere, mentre il quesito sul silenzio e quello sul comfort e luminosità sono stati posti solo a chi ha dichiarato di utilizzare i posti a sedere.

In base ai risultati ottenuti, nonostante la popolazione oggetto di indagine, costituita solo dagli utenti del prestito, non comprenda tutti gli utenti delle sale di lettura, tutte e tre le voci ad essa relative sono considerate importanti o molto importanti da oltre i due terzi degli studenti appartenenti alla popolazione d'indagine (Fig. 21). Scende invece al 50\% la percentuale di coloro che ritengono importante o molto importante la segnaletica e i 
Fig. 21 - Importanza attribuita all'ambiente in biblioteca

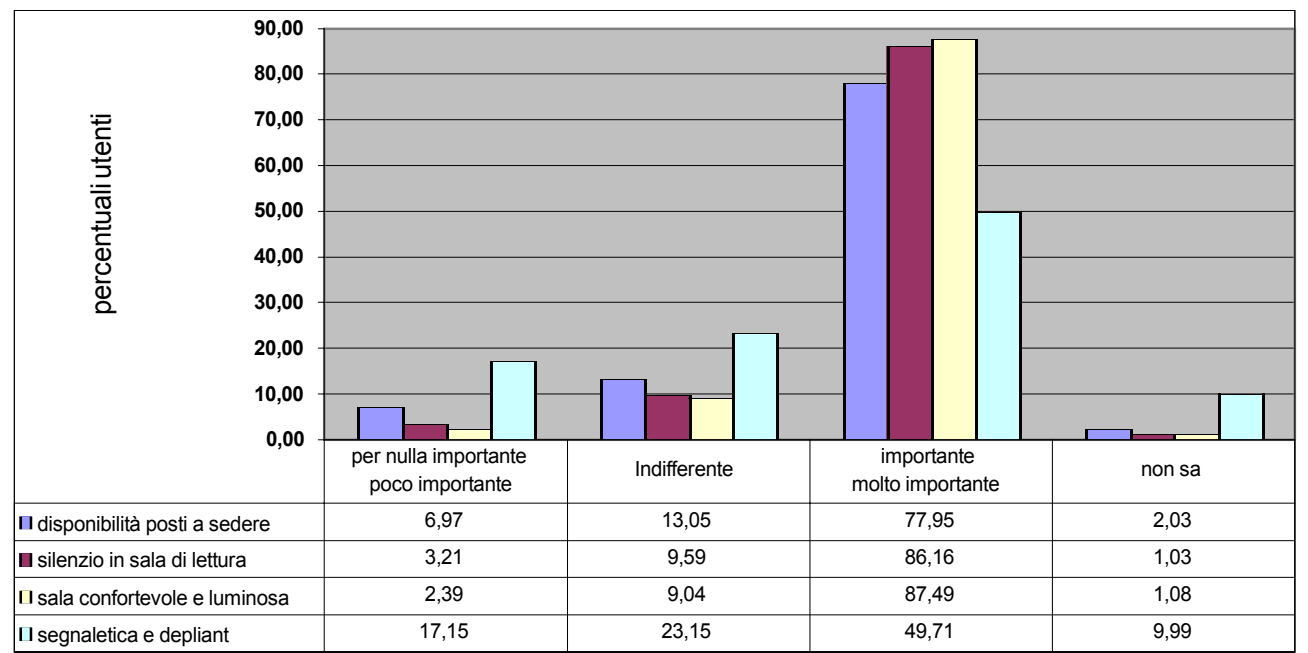

depliant. Mediamente il 74\% degli utenti considera importante o molto importante questa dimensione della qualità e solo il $4 \%$ non è in grado di esprimere un giudizio al riguardo.

Fig. 22 - Utilizzo della sala di lettura

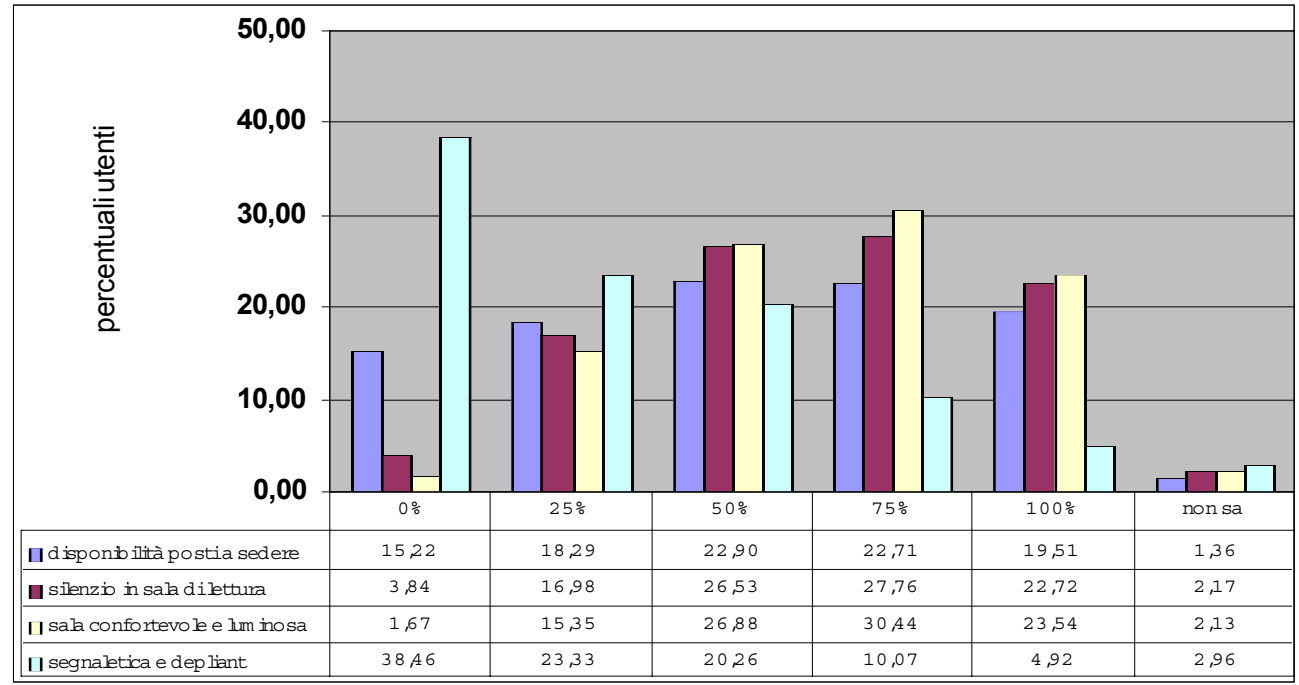


Oltre il 65\% degli studenti usa la sala di lettura almeno una volta ogni due visite in biblioteca e, fra coloro che hanno dichiarato una percentuale positiva di utilizzo, più del 50\% la trova silenziosa, nonché confortevole e luminosa in occasione di quasi tutte le sue visite (Fig. 22). La segnaletica e i depliant invece non risultano mai utilizzati da ben il $38 \%$ della popolazione di riferimento; anche chi li usa, lo fa saltuariamente.

Fig. 23 - Soddisfazione percepita per la sala di lettura

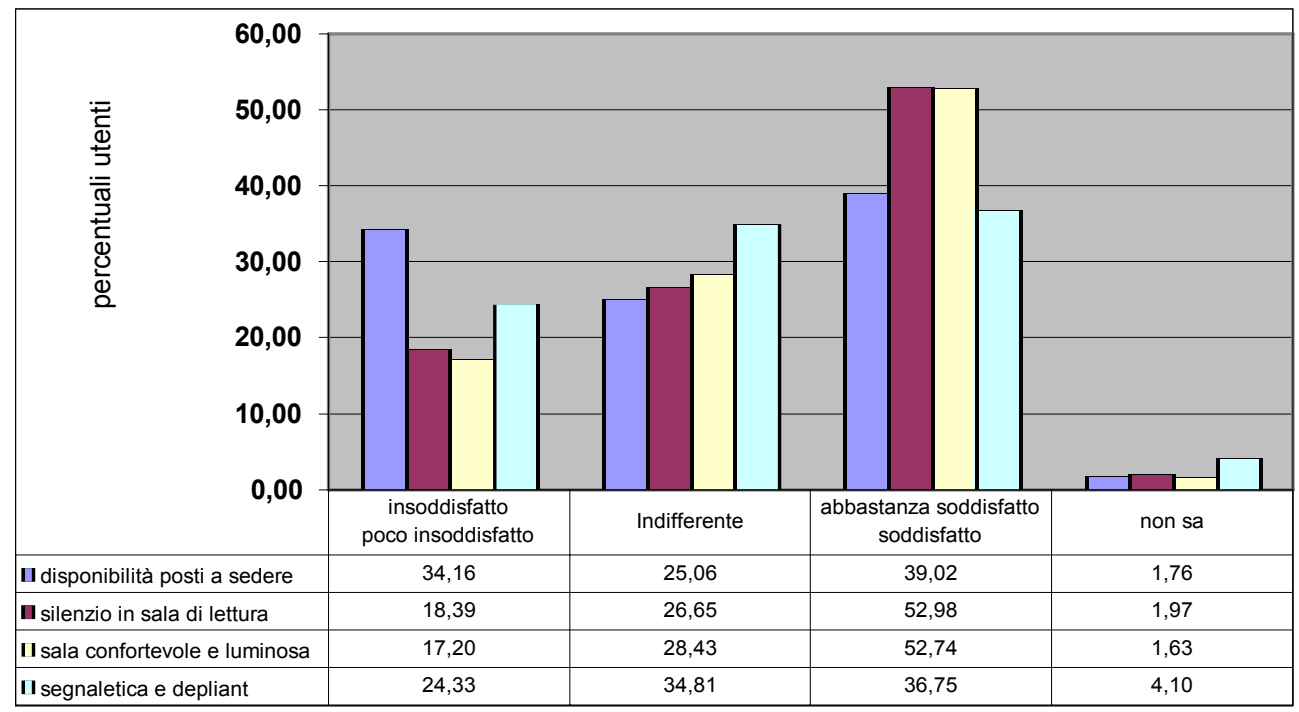

Dall'analisi dei risultati relativi alla soddisfazione emerge chiaramente come, a fronte di un'aspettativa molto alta (il 74\% della popolazione di riferimento giudica importante o molto importante la qualità degli ambienti), la percentuale di insoddisfatti o poco insoddisfatti sia la più elevata fra tutte le dimensioni considerate $^{12}$ (Fig. 23). Il 24\% degli studenti infatti si esprime con un giudizio negativo, in linea con quanto emerso dalla precedente indagine del Nucleo di Valutazione ${ }^{13}$ dove ben il 50\% degli studenti esprimeva la propria insoddisfazione per gli ambienti; solo il $46 \%$ della popolazione oggetto di indagine si dichiara abbastanza soddisfatto o soddisfatto. Fra le voci considerate, quella per la quale si riscontra la più alta percentuale di insoddisfatti $(34 \%)$ è la disponibilità di posti a sedere. Sotto questo aspetto

12 Vedi Tab. 2.

13 Università degli Studi di Firenze 2001b. 
l'indagine conferma un problema già più volte emerso nella gestione quotidiana dei servizi, per il quale è allo stato attuale estremamente arduo proporre soluzioni, data la carenza generale di spazi nell'Ateneo e la conseguente difficoltà di ampliare quelli assegnati alle biblioteche.

Fig. 24 - Relazione fra uso, importanza attribuita all'ambiente e numero di utenti

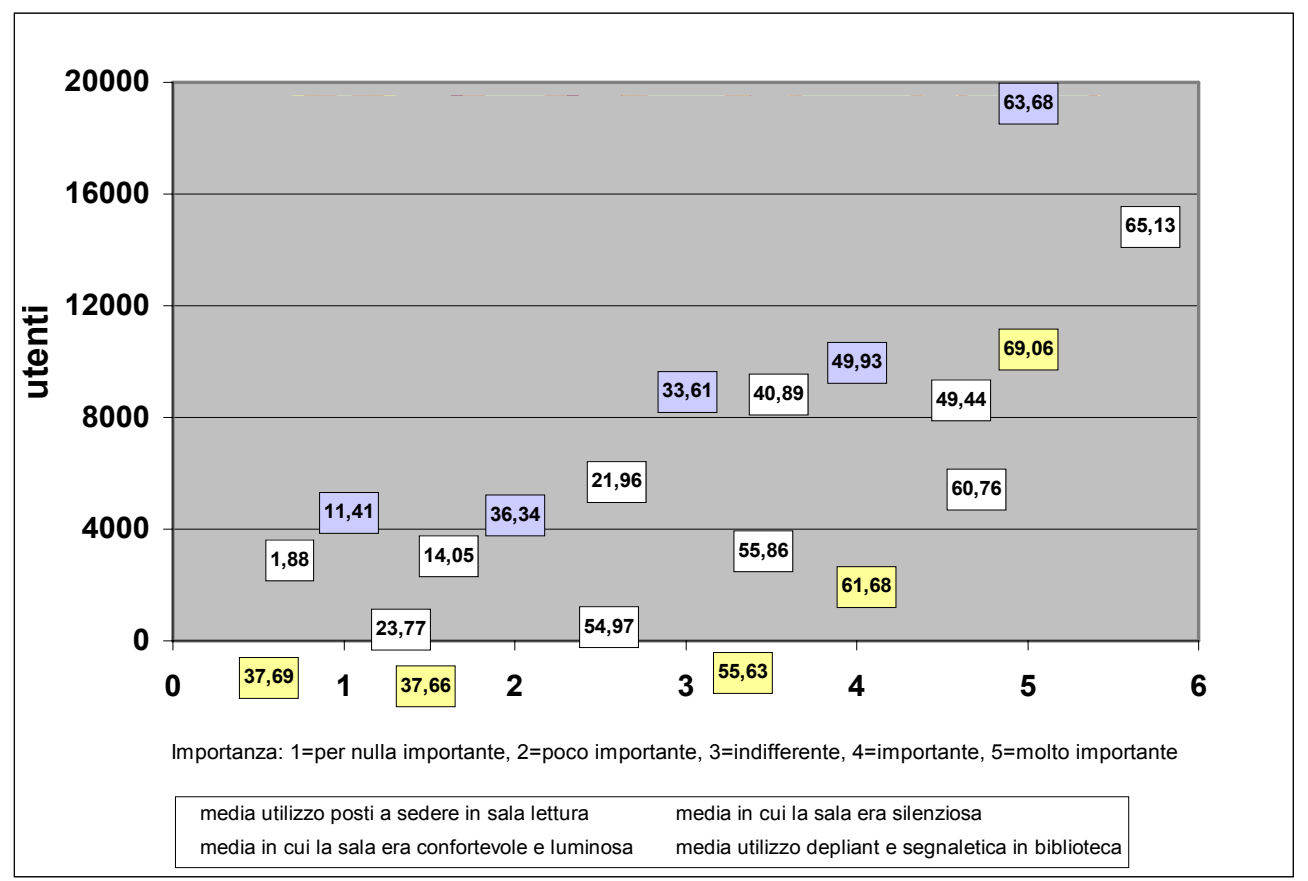

Per quanto riguarda la relazione tra la percentuale di visite in biblioteca, in cui sono stati utilizzati o rilevati gli aspetti della sala lettura oggetto di indagine, e l'importanza ad essi attribuita, si rileva che la maggioranza degli utenti ha fornito valutazioni positive in termini di importanza per tutti e tre gli aspetti e contemporaneamente ha dichiarato di averli rilevati in quasi o più del $50 \%$ delle proprie visite in biblioteca (Fig. 24). 
Per quanto riguarda invece la relazione tra la percentuale di visite in biblioteca, in cui sono stati utilizzati o rilevati gli aspetti della sala lettura oggetto di indagine, e il grado di soddisfazione per essi espresso, occorre rilevare un elemento alquanto negativo: oltre 2500 utenti dichiarano una percentuale d'uso superiore al $50 \%$ e si dicono insoddisfatti per quanto riguarda la disponibilità di posti a sedere; oltre 5000 utenti inoltre dichiarano una percentuale d'uso superiore al $50 \%$ e si dicono poco insoddisfatti (Fig. 25). Relativamente agli altri due aspetti invece il numero di utenti cresce al crescere del grado di soddisfazione e aumenta anche la percentuale d'uso; coloro che si dichiarano molto soddisfatti, pur avendo una percentuale d'uso più elevata, sono tuttavia meno di coloro che si dichiarano abbastanza soddisfatti. Una ulteriore conferma, quest'ultima, di quanto accade in generale per le dimensioni analizzate nel questionario: coloro che in termini di soddisfazione esprimono un giudizio positivo preferiscono comunque non dare un giudizio di piena soddisfazione.

Fig. 25 - Relazione fra uso, soddisfazione percepita per l'ambiente e numero di utenti

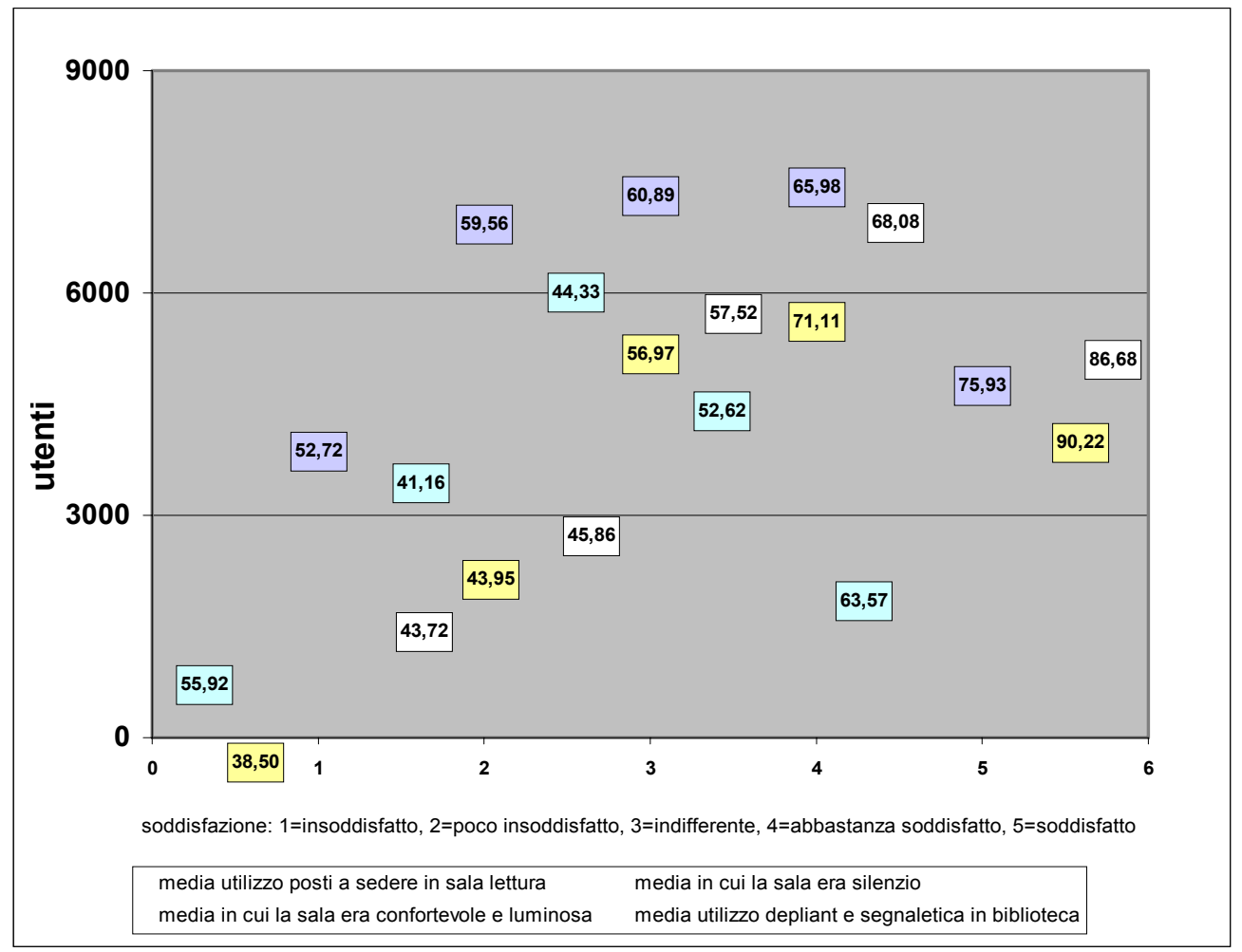




\section{Valutazioni degli studenti-utenti sull'empatia e competenza del persona- le della biblioteca di cui sono utenti}

Condizione necessaria e punto di forza del Progetto di riorganizzazione del Sistema bibliotecario per lo sviluppo dei servizi e il miglioramento della qualità è stata la formazione del personale bibliotecario. Il Coordinamento centrale biblioteche ha inserito annualmente, fra gli obiettivi del progetto, corsi di formazione realizzati ad hoc per sviluppare le conoscenze e le capacità del personale, nonché orientare il servizio sui bisogni e sulla soddisfazione dell'utente.

Nel 2000 i bibliotecari in servizio nelle strutture del Sistema bibliotecario d'Ateneo erano 202; tutti avevano partecipato ad uno o più corsi di formazione.

Per valutare la qualità di questa dimensione gli studenti sono stati invitati a rispondere, relativamente alla biblioteca di cui risultavano utenti, su i seguenti elementi:

1. precisione e rapidità nella distribuzione di libri e periodici dai magazzini;

2. comunicazione con il personale delle biblioteche (disponibilità e cortesia dei bibliotecari nei confronti del pubblico);

3. servizi e assistenza personalizzata al lettore da parte del bibliotecario.

Fig. 26 - Importanza attribuita all'empatia e competenza dei bibliotecari

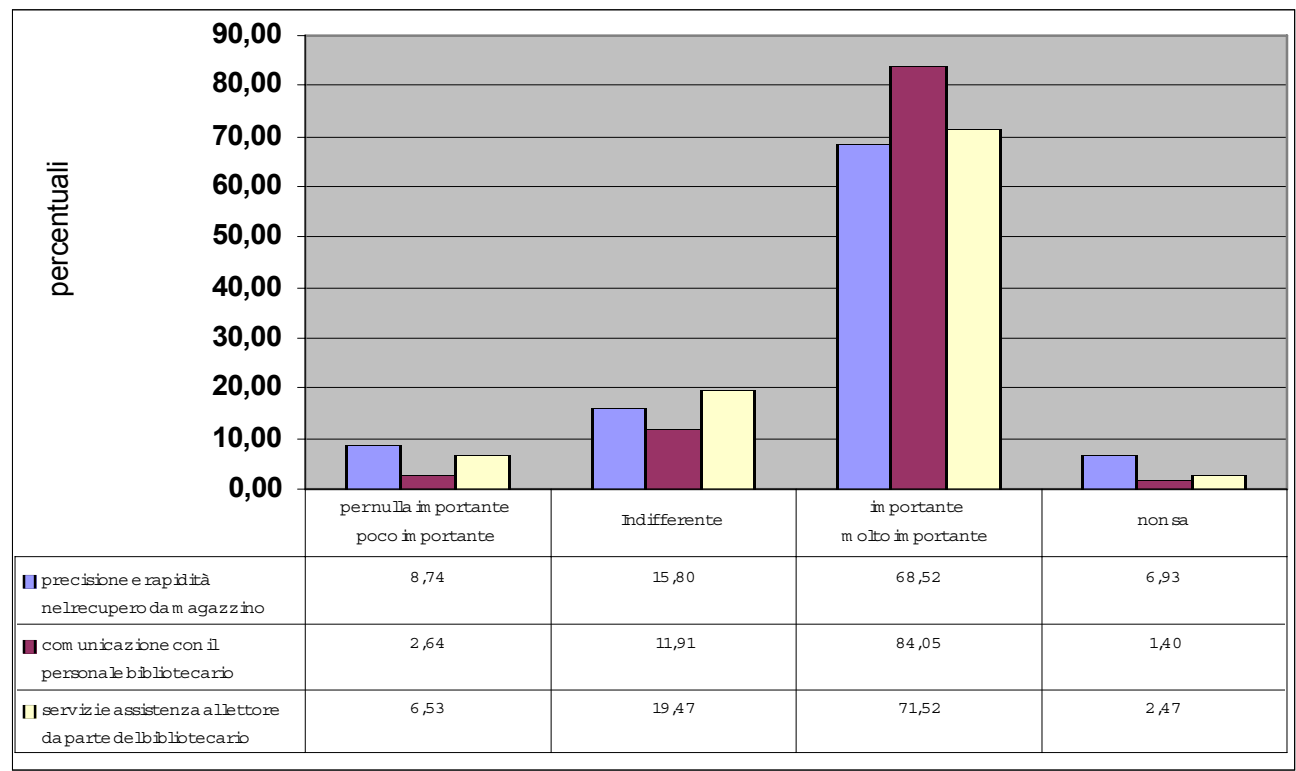


L'aspettativa per l'interazione tra studenti e personale bibliotecario risulta molto alta (Fig. 26). Mediamente il $75 \%$ della popolazione di riferimento fornisce un giudizio di importante o molto importante, contro il $6 \%$ di studenti che giudicano per nulla o poco importante questa dimensione; solo il $4 \%$ non sa esprimersi al riguardo. Delle tre voci considerate, la comunicazione con il personale bibliotecario è quella per la quale abbiamo la percentuale più alta di risposte positive $(84 \%)$ e la percentuale più bassa di risposte negative (solo $3 \%$ di per nulla o poco importante); la comunicazione con il personale bibliotecario è anche la voce per cui si riscontra la maggior consapevolezza (solo 1'1\% di non sa).

Fig. 27 - Contatti con i bibliotecari nelle visite in biblioteca

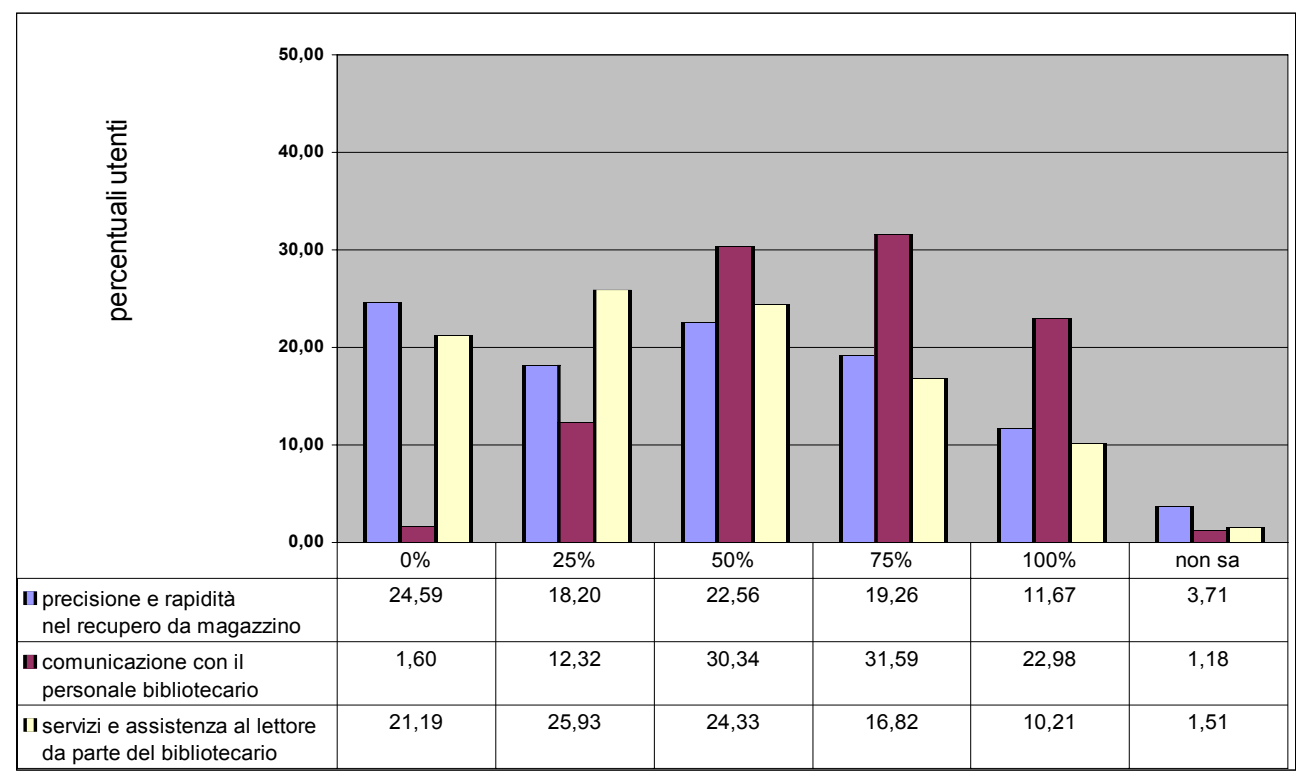

La comunicazione con il personale delle biblioteche è ovviamente molto frequente: solo il 2\% degli studenti ha dichiarato che nelle sue visite in biblioteca non ha contatti diretti con i bibliotecari. Per le altre due voci si registra invece una percentuale superiore al 20\% di utenti che non hanno mai usato i relativi servizi (Fig. 27).

Oltre il 50\% degli utenti si sono dichiarati soddisfatti o abbastanza soddisfatti di tutti e tre gli elementi connessi ai rapporti con il personale (Fig. 28); gli insoddisfatti o poco insoddisfatti sono in tutti e tre i casi meno del $18 \%$. Volendo comunque fare un confronto tra i tre elementi, i risultati migliori riguardano la comunicazione con il personale delle biblioteche e quindi la dis- 


\section{FRANCESCA LANDI}

Fig. 28 - Soddisfazione percepita per l'empatia e competenza dei bibliotecari

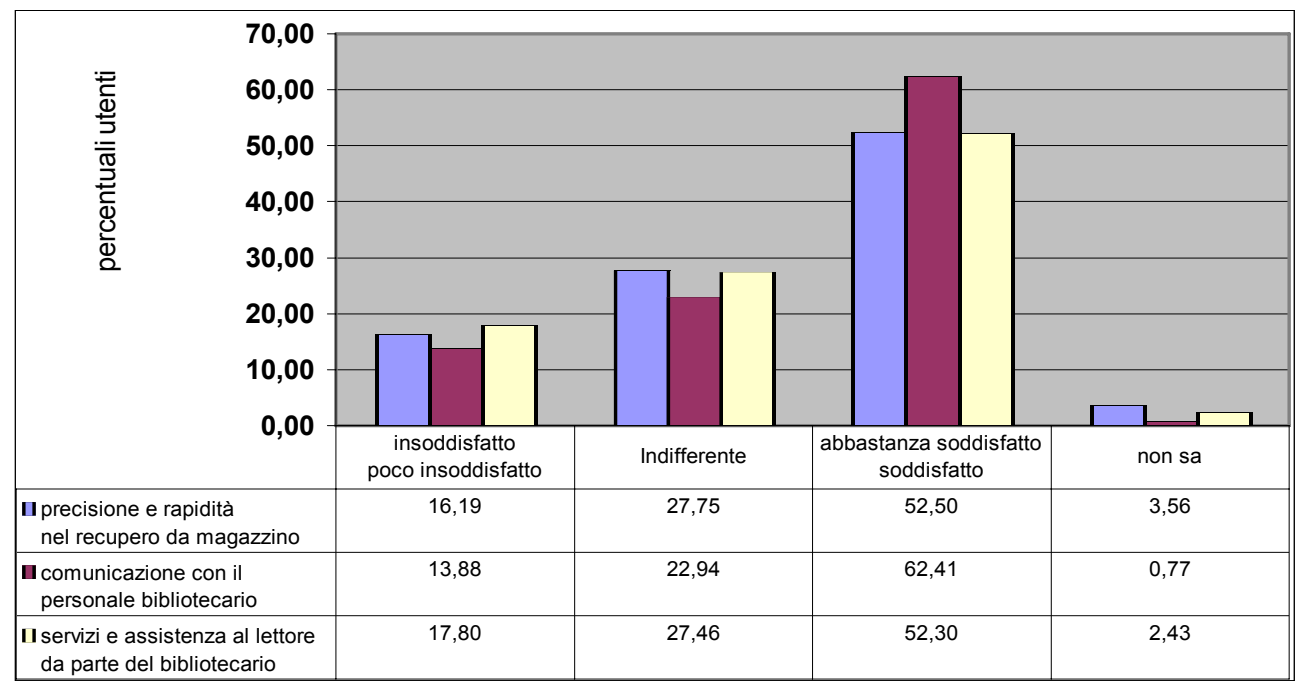

Fig. 29 - Relazione fra uso, importanza attribuita all'empatia e alla competenza dei bibliotecari e numero di utenti

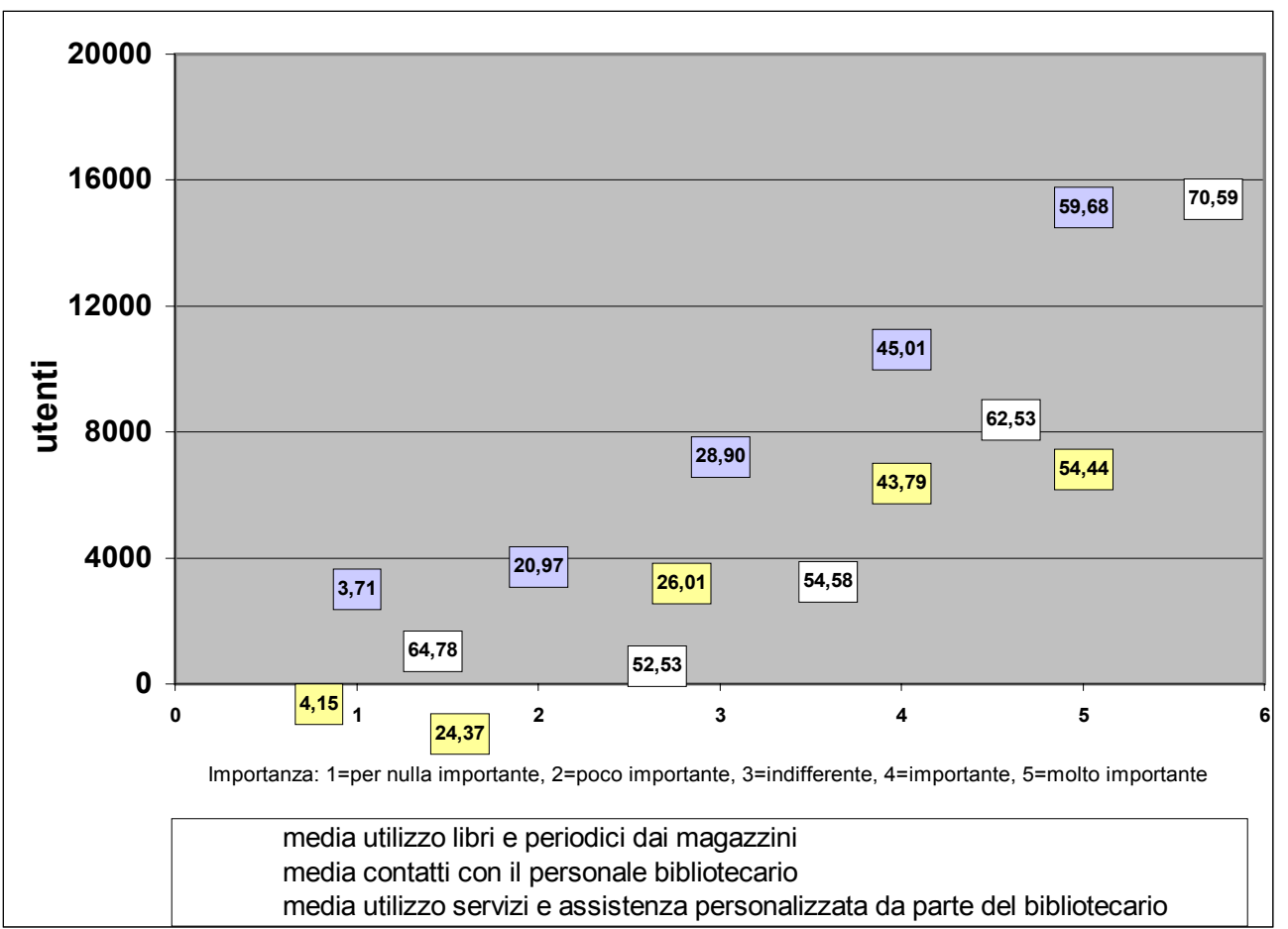


ponibilità e cortesia dei bibliotecari nei confronti del pubblico che è giudicata positivamente dal $62 \%$ degli studenti-utenti. Anche questo dato è in linea con i risultati emersi dall'indagine del Nucleo di valutazione ${ }^{14}$ relativamente a questo argomento. Questa dimensione è, dopo l'OPAC, quella che ottiene il miglior risultato in termini di soddisfazione con, in media, il $56 \%$ di popolazione di riferimento che si esprime positivamente e solo il $26 \%$ di indifferenti.

Relativamente alla distribuzione di materiale dai magazzini e al ricorso a servizi personalizzati da parte del bibliotecario, la percentuale d'uso cresce al crescere dell'importanza attribuita al servizio (Fig. 29). Al contrario, relativamente ai contatti con il personale delle biblioteche, si registra una percentuale d'uso più alta per coloro (pochi) che giudicano il servizio per nulla importante rispetto a coloro che lo giudicano poco importante o indifferente. Qualunque sia la valutazione in termini di importanza, si registrano comun-

Fig. 30 - Relazione fra uso, soddisfazione percepita nei contatti con i bibliotecari e numero di utenti

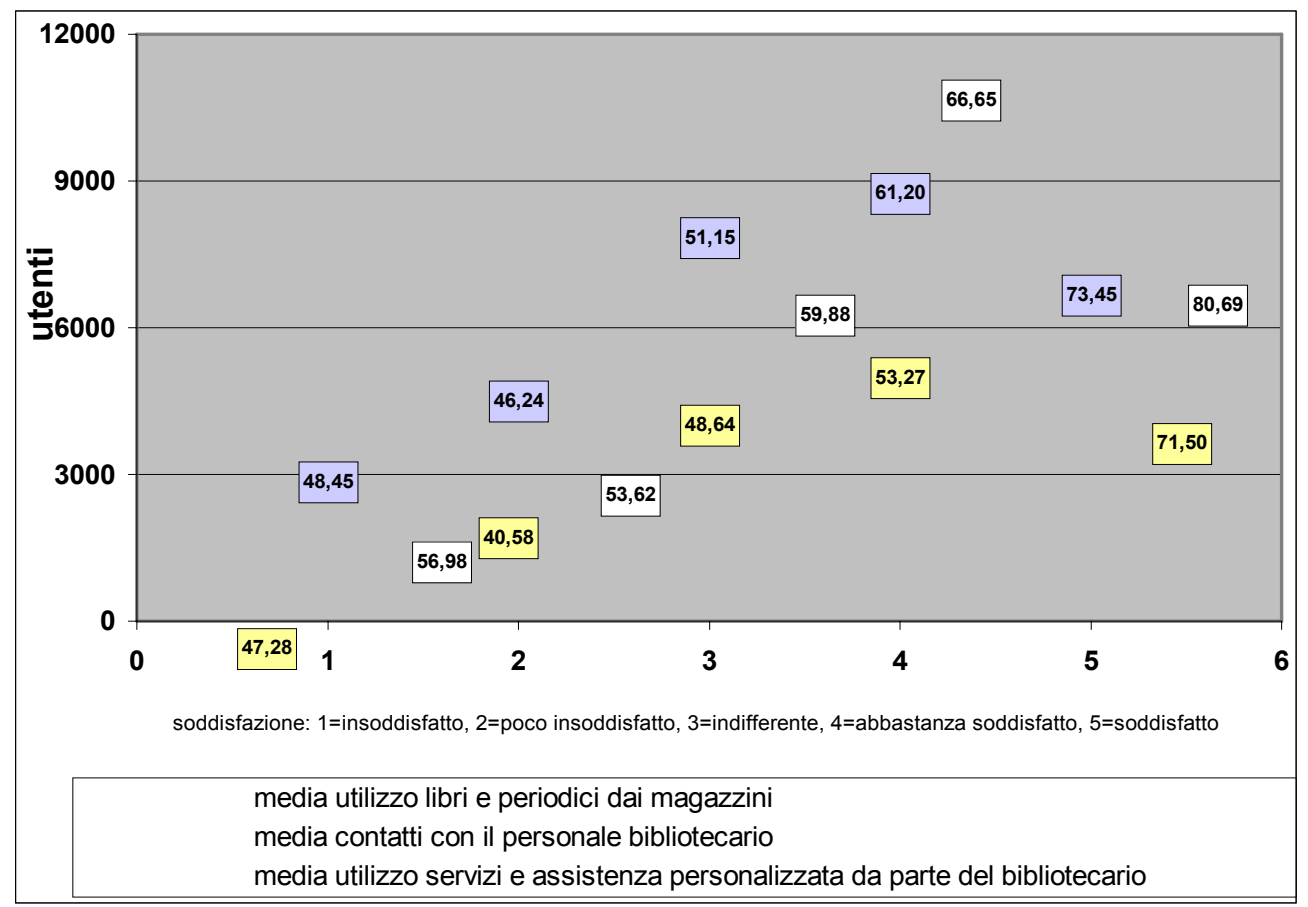

14 Università degli studi di Firenze 2001b. 
que percentuali di utilizzo superiori al 50\%: indipendentemente da quello che pensano in termini di importanza, tutti gli studenti-utenti interagiscono con $\mathrm{i}$ bibliotecari in oltre metà delle loro visite in biblioteca.

Rileviamo inoltre percentuali di utilizzo comunque alte (sempre superiori al $40 \%$ ) qualunque sia il giudizio in termini di soddisfazione (Fig. 30); un po' più alte sono comunque le percentuali associate ai giudizi di soddisfazione positivi, e questi sono espressi da un maggior numero di utenti.

\section{Conclusioni}

In ultimo, si è provveduto a inserire il valore medio ${ }^{15}$ assunto da ciascuna dimensione in una tabella per effettuare un'analisi complessiva della qualità attesa e della qualità percepita dagli utenti per le dimensioni analizzate e formulare alcune ipotesi d'insieme, sia relativamente all'importanza che alla soddisfazione cercando di rilevare i punti di forza e le criticità.

Nella tabella 1 i dati sono ordinati in quattro serie, ciascuna in ordine decrescente rispetto ad una delle possibili risposte; la prima relativa al valore "importante /molto importante", poi quella relativa a "per nulla importante/ poco importante", successivamente la risposta "indifferente", in ultimo quella "non $s a$ ".

Tab. 1 - Importanza

\begin{tabular}{|l|c|c|c|c|}
\cline { 2 - 5 } \multicolumn{1}{c|}{} & $\begin{array}{c}\text { per nulla } \\
\text { importante / } \\
\text { poco importante }\end{array}$ & indifferente & $\begin{array}{c}\text { importante / } \\
\text { molto } \\
\text { importante }\end{array}$ & non sa \\
\hline OPAC & 7 & 10 & $\mathbf{7 8}$ & 5 \\
\hline Personale & 6 & 16 & $\mathbf{7 5}$ & 3 \\
\hline Sala lettura & 8 & 14 & $\mathbf{7 4}$ & 4 \\
\hline Accessibilità risorse & 10 & 14 & $\mathbf{6 9}$ & 7 \\
\hline Offerta documentaria & 12 & 18 & $\mathbf{6 2}$ & 8 \\
\hline Servizi avanzati & 9 & 15 & $\mathbf{5 8}$ & 18 \\
\hline
\end{tabular}

15 I valori medi assunti da ciascuna dimensione per tutte le variabili di risposta sono ottenuti dalla ponderazione delle medie di ogni voce che concorre a quella dimensione sul totale delle frequenze 
L'aspettativa che gli utenti hanno dichiarato per le varie dimensioni analizzate ci fa ipotizzare che la dimensione OPAC, sia quella a cui attribuiscono maggior importanza, seguita a breve distanza dall'aspettativa per le interazioni che si instaurano fra l'utente e il personale della biblioteca; entrambe queste dimensioni possono essere considerate quali strumenti per accedere all'informazione bibliografica ed ottenere risultati più qualificati; dunque l'aspettativa dello studente-utente sembra coincidere con uno degli obiettivi fondamentali della biblioteca. Per queste dimensioni inoltre, è molto bassa la percentuale di coloro che non sono in grado di valutare la qualità attesa.

\begin{tabular}{|l|c|c|c|c|}
\cline { 2 - 5 } \multicolumn{1}{c|}{} & $\begin{array}{c}\text { per nulla } \\
\text { importante / } \\
\text { poco importante }\end{array}$ & indifferente & $\begin{array}{c}\text { importante / } \\
\text { molto } \\
\text { importante }\end{array}$ & non sa \\
\hline Offerta documentaria & $\mathbf{1 2}$ & 18 & 62 & 8 \\
\hline $\begin{array}{l}\text { Accessibilità dell'of- } \\
\text { ferta documentaria }\end{array}$ & $\mathbf{1 0}$ & 14 & 69 & 7 \\
\hline Servizi avanzati & $\mathbf{9}$ & 15 & 58 & 18 \\
\hline Sala lettura & $\mathbf{8}$ & 14 & 74 & 4 \\
\hline OPAC & $\mathbf{7}$ & 10 & 78 & 5 \\
\hline Personale & $\mathbf{6}$ & 16 & 75 & 3 \\
\hline
\end{tabular}

\begin{tabular}{|l|c|c|c|c|}
\cline { 2 - 5 } \multicolumn{1}{c|}{} & $\begin{array}{c}\text { per nulla } \\
\text { importante/ } \\
\text { poco importante }\end{array}$ & indifferente & $\begin{array}{c}\text { importante/ } \\
\text { molto } \\
\text { importante }\end{array}$ & non sa \\
\hline Offerta documentaria & 12 & $\mathbf{1 8}$ & 62 & 8 \\
\hline Personale & 6 & $\mathbf{1 6}$ & 75 & 3 \\
\hline Servizi avanzati & 9 & $\mathbf{1 5}$ & 58 & 18 \\
\hline Sala lettura & 8 & $\mathbf{1 4}$ & 74 & 4 \\
\hline $\begin{array}{l}\text { Accessibilità dell'of- } \\
\text { ferta documentaria }\end{array}$ & 10 & $\mathbf{1 4}$ & 69 & 7 \\
\hline OPAC & 7 & $\mathbf{1 0}$ & 78 & 5 \\
\hline
\end{tabular}

L'offerta documentaria invece sembra essere la dimensione verso cui c'è una minore aspettativa, poiché raccoglie la più alta percentuale di indifferenti e di coloro che la definiscono poco o per nulla importante. Un giudizio che lascia abbastanza perplessi, anche se è stato rilevato in altre indagini sulla customer satisfaction una certa tendenza ad esprimersi così nei confronti di 
servizi che sono percepiti già in linea con le proprie esigenze; in particolare, questa dimensione risulta al terzo posto in termini di soddisfazione ${ }^{16}$.

\begin{tabular}{|l|c|c|c|c|}
\cline { 2 - 5 } \multicolumn{1}{c|}{} & $\begin{array}{c}\text { per nulla } \\
\text { importante } \\
\text { poco importante }\end{array}$ & indifferente & $\begin{array}{c}\text { importante / } \\
\text { molto } \\
\text { importante }\end{array}$ & non sa \\
\hline Servizi avanzati & 9 & 15 & 58 & $\mathbf{1 8}$ \\
\hline Offerta documentaria & 12 & 18 & 62 & $\mathbf{8}$ \\
\hline $\begin{array}{l}\text { Accessibilità dell'of- } \\
\text { ferta documentaria }\end{array}$ & 10 & 14 & 69 & 7 \\
\hline OPAC & 7 & 10 & 78 & $\mathbf{5}$ \\
\hline Sala lettura & 8 & 14 & 74 & $\mathbf{4}$ \\
\hline Personale & 6 & 16 & 75 & $\mathbf{3}$ \\
\hline
\end{tabular}

I servizi avanzati infine si collocano al primo posto se ordiniamo i dati secondo la risposta "non sa" e all'ultimo posto quando si analizza la risposta "importante/molto importante"; questo ci lascia ipotizzare che la conoscenza degli strumenti disponibili in rete fosse ancora poco diffusa fra studenti-utenti delle biblioteche al momento dell'intervista. Ipotesi che pare confermata anche analizzando la fig.9 dove si rileva che comunque coloro che usano questi servizi ne sono molto soddisfatti.

Anche nella tabella 2 i dati sono ordinati in quattro serie, ciascuna in ordine decrescente rispetto ad una delle possibili risposte; la prima relativa al valore "abbastanza soddisfatto/ soddisfatto", poi quella relativa a "insoddisfatto / poco insoddisfatto", successivamente la risposta "indifferente", in ultimo quella "non sa".

Analizzando i dati relativi a questo aspetto, rileviamo che è ancora la dimensione OPAC quella che ottiene la più alta percentuale di soddisfatti o abbastanza soddisfatti seguita dalla dimensione Personale. Da ciò sembra emergere che siano queste le dimensioni da considerarsi come punti di forza del Sistema bibliotecario; anche in questo caso si registra, per entrambe le dimensioni, basse percentuali di risposte "non sa", ovvero una buona conoscenza del servizio su cui sono chiamati a dare un giudizio.

La lettura della tabella ordinata per la risposta "insoddisfatto / poco insoddisfatto" sembra confermare che il sovraffollamento delle sale di lettu-

16 Vedi tab. 2 
Tab. 2 - Soddisfazione

\begin{tabular}{|l|c|c|c|c|}
\cline { 2 - 5 } \multicolumn{1}{c|}{} & $\begin{array}{c}\text { insoddisfatto / } \\
\text { poco } \\
\text { insoddisfatto }\end{array}$ & indifferente & $\begin{array}{c}\text { abbastanza } \\
\text { soddisfatto / } \\
\text { soddisfatto }\end{array}$ & non sa \\
\hline OPAC & 11 & 23 & $\mathbf{6 3}$ & 3 \\
\hline Personale & 16 & 26 & $\mathbf{5 6}$ & 2 \\
\hline Offerta documentaria & 17 & 30 & $\mathbf{4 9}$ & 4 \\
\hline Servizi avanzati & 9 & 25 & $\mathbf{4 7}$ & 19 \\
\hline Sala lettura & 24 & 28 & $\mathbf{4 6}$ & 2 \\
\hline $\begin{array}{l}\text { Accessibilità dell'of- } \\
\text { ferta documentaria }\end{array}$ & 22 & 27 & $\mathbf{4 0}$ & 11 \\
\hline
\end{tabular}

ra, la perdurante mancanza di posti a sedere è l'elemento che più di ogni altro viene percepito negativamente dall'utente; è infatti la voce disponibilità di posti a sedere in sala di lettura quella che pesa di più nel giudizio negativo per questa dimensione. Di poco più bassa la percentuale relativa all'insoddisfazione per l'accessibilità delle risorse, per la quale contribuisce prevalentemente la voce accesso ad internet in biblioteca ad alzare la percentuale di insoddisfatti.

\begin{tabular}{|l|c|c|c|c|}
\cline { 2 - 5 } \multicolumn{1}{c|}{} & $\begin{array}{c}\text { insoddisfatto / } \\
\text { poco } \\
\text { insoddisfatto }\end{array}$ & indifferente & $\begin{array}{c}\text { abbastanza } \\
\text { soddisfatto / } \\
\text { soddisfatto }\end{array}$ & non sa \\
\hline Sala lettura & $\mathbf{2 4}$ & 28 & 46 & 2 \\
\hline $\begin{array}{l}\text { Accessibilità dell'of- } \\
\text { ferta documentaria }\end{array}$ & $\mathbf{2 2}$ & 27 & 40 & 11 \\
\hline Offerta documentaria & $\mathbf{1 7}$ & 30 & 49 & 4 \\
\hline Personale & $\mathbf{1 6}$ & 26 & 56 & 2 \\
\hline OPAC & $\mathbf{1 1}$ & 23 & 63 & 3 \\
\hline Servizi avanzati & $\mathbf{9}$ & 25 & 47 & 19 \\
\hline
\end{tabular}

L'offerta documentaria conferma, per questo aspetto, quanto già espresso relativamente all' aspettativa raccogliendo ben il $30 \%$ di utenti che si dichiarano indifferenti. 


\begin{tabular}{|l|c|c|c|c|}
\cline { 2 - 5 } \multicolumn{1}{c|}{} & $\begin{array}{c}\text { insoddisfatto / } \\
\text { poco } \\
\text { insoddisfatto }\end{array}$ & indifferente & $\begin{array}{c}\text { abbastanza } \\
\text { soddisfatto / } \\
\text { soddisfatto }\end{array}$ & non sa \\
\hline Offerta documentaria & 17 & $\mathbf{3 0}$ & 49 & 4 \\
\hline Sala lettura & 24 & $\mathbf{2 8}$ & 46 & 2 \\
\hline $\begin{array}{l}\text { Accessibilità dell'of- } \\
\text { ferta documentaria }\end{array}$ & 22 & $\mathbf{2 7}$ & 40 & 11 \\
\hline Personale & 16 & $\mathbf{2 6}$ & 56 & 2 \\
\hline Servizi avanzati & 9 & $\mathbf{2 5}$ & 47 & 19 \\
\hline OPAC & 11 & $\mathbf{2 3}$ & 63 & 3 \\
\hline
\end{tabular}

\begin{tabular}{|l|c|c|c|c|}
\cline { 2 - 5 } & $\begin{array}{c}\text { insoddisfatto / } \\
\text { poco } \\
\text { insoddisfatto }\end{array}$ & indifferente & $\begin{array}{c}\text { abbastanza } \\
\text { soddisfatto / } \\
\text { soddisfatto }\end{array}$ & non sa \\
\hline Servizi avanzati & 9 & 25 & 47 & $\mathbf{1 9}$ \\
\hline $\begin{array}{l}\text { Accessibilità dell'of- } \\
\text { ferta documentaria }\end{array}$ & 22 & 27 & 40 & $\mathbf{1 1}$ \\
\hline Offerta documentaria & 17 & 30 & 49 & $\mathbf{4}$ \\
\hline OPAC & 11 & 23 & 63 & $\mathbf{3}$ \\
\hline Sala lettura & 24 & 28 & 46 & $\mathbf{2}$ \\
\hline Personale & 16 & 26 & 56 & $\mathbf{2}$ \\
\hline
\end{tabular}

Anche per la soddisfazione, così come per l'importanza, la più alta percentuale di utenti che non sanno esprimere un giudizio si riferisce alla dimensione Servizi avanzati.

Per un ulteriore approfondimento dell'analisi delle tabelle e per uno specifico confronto fra le dimensioni, che concorrono alla percezione della qualità dei servizi in biblioteca da parte degli utenti, si rimanda comunque all'appendice $\mathrm{D}$, "Stima degli errori di campionamento dell'Indagine sulla soddisfazione degli utenti", dove si riporta, per ciascuna variabile assunta da ogni voce del questionario, la stima dell'errore campionario che consente di calcolare l'intervallo di confidenza. 


\title{
L'indagine sulla soddisfazione degli utenti del Sistema Bibliotecario di Ateneo all'Università degli Studi di Firenze: metodologia e valutazione bibliotecaria
}

\author{
di ROBERTO VENTURA
}

\section{Introduzione}

La misurazione dei servizi bibliotecari, intesa come strumento gestionale atto a prendere decisioni commisurate, da un lato, alle finalità istituzionali della biblioteca e, dall'altro, alle esigenze del pubblico che la frequenta, mostra negli ultimi anni un deciso orientamento verso la quantificazione e la valutazione della customer satisfaction, vale a dire dei giudizi qualitativi che l'utente forma nell'ambito della esperienza di fruizione e del grado di adeguatezza del servizio alle esigenze di cui egli è portatore. Il concetto di base è che, da sole, le tradizionali misurazioni quantitative sulla performance delle attività bibliotecarie non sembrano in grado di estrinsecare adeguatamente le modalità di integrazione della biblioteca con l'ambiente di riferimento, dal momento che un determinato volume di transazioni con il cliente, posto in essere dalla biblioteca, non costituirebbe di per sé un indice di qualità quanto piuttosto si limiterebbe ad esprimere il volume di lavoro e di movimentazione di mezzi tecnici e finanziari posto in essere a fronte di un dato volume di domanda.

La decisione di procedere ad un'indagine qualitativa dedicata al Sistema Bibliotecario di Ateneo (SBA) promana dall'esigenza di conoscere il giudizio del pubblico sui servizi offerti al di là, per così dire, della estensione física che essi assumono, anche al fine di meglio interpretare le misure tradizionalmente raccolte ed elaborate all'interno delle batterie di dati ed indicatori di performance bibliotecaria localmente in uso da qualche anno a questa parte ${ }^{1}$.

L'indagine è stata effettuata, a due riprese, sull'utenza reale studentesca del SBA e sul personale docente e di ricerca dell'Ateneo ${ }^{2}$. In sede di selezione degli attributi qualitativi da sottoporre ad indagine, è stato deciso di valu-

\footnotetext{
1 La batteria di dati ed indicatori, aggiornata al 2002, è riportata nell'Appendice A.

2 Sul metodo statistico applicato in fase di definizione della popolazione e del campione nonché di realizzazione dell'indagine, si rimanda al contributo di Silvana Benedetti in
} 
tare tre aree fondamentali di servizio, tre "sub-sistemi" che generalmente costituiscono l'articolazione organizzativa della biblioteca universitaria ${ }^{3}$ :

1. Qualità delle acquisizioni cartacee ed elettroniche, al fine di esplorare la gestione delle raccolte dal punto di vista della loro configurazione bibliografica, semantica e nominale, e della politica degli acquisti;

2. Qualità della circolazione documentaria, al fine di valutare l'efficacia dei processi tesi a favorire l'uso delle raccolte, dalla ricerca dei documenti tramite 1'OPAC di Ateneo al recupero ed alla fruizione delle notizie bibliografiche individuate e dei documenti identificati;

3. Qualità del servizio di reference nella sua più ampia accezione, dall'orientamento bibliografico alla comunicazione all'utenza, al fine di valutare l'efficacia dell'apparato di consultazione cartaceo ed elettronico, dei servizi di consulenza bibliografica e bibliotecaria "avanzata" sull'uso delle risorse possedute o accessibili nonché sulla generalità dei servizi che il SBA mette a disposizione del pubblico.

Ponendosi l'indagine come momento di valutazione gestionale del settore bibliotecario di ateneo nel suo complesso, all'interno di ciascuna area sono stati individuati singoli attributi qualitativi riscontrabili in ogni componente del SBA, prescindendo dalle particolarità che una biblioteca o un punto di servizio presenta in virtù dello specifico ambito bibliografico e disciplinare di competenza.

I singoli attributi qualitativi sono stati "tradotti" in espressioni del linguaggio naturale, scevre del gergo teorico e tecnico, affinché le domande ad essi collegate risultassero immediatamente comprensibili a chi non dispone delle conoscenze e della terminologia delle discipline biblioteconomiche e bibliografiche.

Le domande effettuate riguardano la biblioteca che l'intervistato dichiara di frequentare in via principale. È evidente che una serie di attributi, ad esem-

questo volume. Riassumiamo qui i principali dati riguardanti la popolazione oggetto dell'indagine. Iscritti all'Università nell'anno accademico 1999/2000: 58.848 persone; utenza reale del SBA: 27.746 utenti; campione d'indagine: 1.063 individui; personale docente e di ricerca in servizio al dicembre 2001: 2.293 persone, di cui 630 hanno accettato di collaborare all'indagine. I risultati relativi al personale docente e di ricerca di seguito riportati sono da interpretare senza pretesa di rappresentatività statistica ma possono tuttavia fornire utili informazioni riguardo all'impatto che i servizi del SBA hanno su questa parte qualificata dell'utenza bibliotecaria. Al Dipartimento di Statistica dell'Università degli Studi di Firenze è stato affidato il compito di definire il campione, di somministrare il questionario e di raccogliere i dati. Sulla struttura e le attività del SBA si rimanda al sito internet dello stesso: $<$ http://www.unifi.it/universita/biblioteche/>. SBA.

Sono state dedicate a tale scopo varie riunioni del Comitato tecnico di gestione del 
pio quelli relativi alle caratteristiche dell'OPAC di Ateneo, si riferiscono direttamente al SBA nel suo complesso e sono suscettibili, al limite, di rivelare eventuali diversità d'uso in relazione a diversi percorsi di studio.

La sezione conclusiva del questionario è dedicata alle caratteristiche anagrafiche ed alla carriera universitaria degli intervistati.

Nel dettaglio, la struttura dell'indagine e del questionario si articola come segue:

\begin{tabular}{|l|l|c|}
\hline \multicolumn{3}{|c|}{ 1. Qualità delle acquisizioni cartacee ed elettroniche } \\
\hline \multicolumn{1}{|c|}{ Attributo qualitativo } & \multicolumn{1}{|c|}{ Formulazione dell'attributo } & $\begin{array}{c}\text { Codice domanda } \\
\text { (Appendice B) }\end{array}$ \\
\hline $\begin{array}{l}\text { Configurazione } \\
\text { bibliografica delle raccolte }\end{array}$ & $\begin{array}{l}1.1 \text { - Libri, periodici e cd-rom } \\
\text { attualmente posseduti dalla biblioteca }\end{array}$ & $\begin{array}{l}\text { QU02A1 } \\
\text { QU02B1 } \\
\text { QU02C1 }\end{array}$ \\
\hline $\begin{array}{l}\text { Grado di aggiornamento } \\
\text { delle raccolte librarie }\end{array}$ & $\begin{array}{l}1.2 \text { - Disponibilità in biblioteca delle } \\
\text { novità librarie nelle discipline di } \\
\text { interesse }\end{array}$ & $\begin{array}{l}\text { QU02A2 } \\
\text { QU02B2 } \\
\text { QU02C2 }\end{array}$ \\
\hline $\begin{array}{l}\text { Grado di aggiornamento } \\
\text { delle raccolte seriali }\end{array}$ & $\begin{array}{l}1.3 \text { - Disponibilità in biblioteca degli } \\
\text { ultimi numeri dei periodici nelle } \\
\text { discipline di interesse }\end{array}$ & $\begin{array}{l}\text { QU02A3 } \\
\text { QU02B3 } \\
\text { QU02C3 }\end{array}$ \\
\hline $\begin{array}{l}\text { Impatto dei periodici } \\
\text { elettronici }\end{array}$ & $\begin{array}{l}1.4 \text { - Uso dei periodici elettronici } \\
\text { nelle discipline di interesse }\end{array}$ & $\begin{array}{l}\text { QU07A24 } \\
\text { QU07B24 } \\
\text { QU07C24 }\end{array}$ \\
\hline
\end{tabular}

\begin{tabular}{|l|l|c|}
\hline \multicolumn{3}{|c|}{ 2. Qualità della circolazione documentaria } \\
\hline \multicolumn{1}{|c|}{ Attributo qualitativo } & \multicolumn{1}{|c|}{ Formulazione dell'attributo } & $\begin{array}{c}\text { Codice domanda } \\
\text { (Appendice B) }\end{array}$ \\
\hline $\begin{array}{l}\text { Uso dell'OPAC attraverso } \\
\text { chiavi di ricerca nominali }\end{array}$ & $\begin{array}{l}\text { 2.1 - Uso del catalogo della biblioteca } \\
\text { su internet: ricerca di libri o periodici } \\
\text { per titolo e ricerca di libri per autore }\end{array}$ & $\begin{array}{l}\text { QU06aA18 } \\
\text { QU06aB18 } \\
\text { QU06aC18 }\end{array}$ \\
\hline $\begin{array}{l}\text { Uso dell'OPAC attraverso } \\
\text { chiavi di ricerca semantiche }\end{array}$ & $\begin{array}{l}\text { 2.2 - Uso del catalogo su internet: } \\
\text { ricerca di libri per argomento (nel } \\
\text { campo: soggetto) o materia (nel } \\
\text { campo: classificazione) }\end{array}$ & $\begin{array}{l}\text { QU06aA19 } \\
\text { QU06aB19 } \\
\text { QU06aC19 }\end{array}$ \\
\hline $\begin{array}{l}\text { Uso dell'OPAC attraverso } \\
\text { chiavi di ricerca di } \\
\text { ricorrenza }\end{array}$ & $\begin{array}{l}2.3 \text { - Uso del catalogo della biblioteca } \\
\text { su internet: ricerca di libri o periodici } \\
\text { nel campo parole chiave }\end{array}$ & $\begin{array}{l}\text { QU06aA20 } \\
\text { QU06aB20 } \\
\text { QU06aC20 }\end{array}$ \\
\hline $\begin{array}{l}\text { Interfaccia dell'OPAC: } \\
\text { facilità delle operazioni di } \\
\text { prenotazione di documenti } \\
\text { o proroga del prestito. }\end{array}$ & $\begin{array}{l}\text { 2.4 - Uso del catalogo della biblioteca } \\
\text { su internet: prenotazione o proroga del } \\
\text { prestito di un libro }\end{array}$ & $\begin{array}{l}\text { QU07A21 } \\
\text { QU07B21 } \\
\text { QU07C21 }\end{array}$ \\
\hline
\end{tabular}




\begin{tabular}{|c|c|c|}
\hline $\begin{array}{l}\text { Recupero locale di } \\
\text { monografie e periodici dalla } \\
\text { collocazione in magazzino }\end{array}$ & $\begin{array}{l}2.5 \text { - Precisione e rapidità nella } \\
\text { distribuzione di libri e periodici dai } \\
\text { magazzini }\end{array}$ & $\begin{array}{l}\text { QU02A4 } \\
\text { QU02B4 } \\
\text { QU02C4 }\end{array}$ \\
\hline $\begin{array}{l}\text { Recupero locale di } \\
\text { monografie e periodici dalla } \\
\text { collocazione a scaffali } \\
\text { aperti }\end{array}$ & $\begin{array}{l}2.6 \text { - Facilità del recupero di libri e } \\
\text { periodici dagli scaffali liberamente } \\
\text { accessibili ai lettori }\end{array}$ & $\begin{array}{l}\text { QU02A5 } \\
\text { QU02B5 } \\
\text { QU02C5 }\end{array}$ \\
\hline \multirow{3}{*}{$\begin{array}{l}\text { Condizioni ambientali } \\
\text { (qualità delle sale di lettura) } \\
\text { della fruizione dei } \\
\text { documenti locali }\end{array}$} & $\begin{array}{l}2.7 \text { - Disponibilità in sala di lettura di } \\
\text { posti a sedere per l'uso di materiale } \\
\text { della biblioteca }\end{array}$ & $\begin{array}{l}\text { QU03A6 } \\
\text { QU03B6 } \\
\text { QU03C6 }\end{array}$ \\
\hline & 2.8 - Silenzio in sala di lettura & $\begin{array}{l}\text { QU03A7 } \\
\text { QU03B7 } \\
\text { QU03C7 }\end{array}$ \\
\hline & $\begin{array}{l}2.9 \text { - Comfort e luminosità della sala } \\
\text { di lettura }\end{array}$ & $\begin{array}{l}\text { QU03A8 } \\
\text { QU03B8 } \\
\text { QU03C8 } \\
\end{array}$ \\
\hline $\begin{array}{l}\text { Uso di internet in } \\
\text { biblioteca: disponibilità e } \\
\text { collegamento per motivi di } \\
\text { studio }\end{array}$ & 2.10 - Accesso a internet in biblioteca & $\begin{array}{l}\text { QU03A9 } \\
\text { QU03B9 } \\
\text { QU03C9 }\end{array}$ \\
\hline $\begin{array}{l}\text { Uso e percezione del } \\
\text { prestito interbibliotecario e } \\
\text { del document delivery }\end{array}$ & $\begin{array}{l}2.11 \text { - Prestito interbibliotecario: } \\
\text { recupero di libri e articoli da altre } \\
\text { biblioteche universitarie }\end{array}$ & $\begin{array}{l}\text { QU03A10 } \\
\text { QU03B10 } \\
\text { QU03C10 }\end{array}$ \\
\hline
\end{tabular}

\begin{tabular}{|l|l|l|}
\hline \multicolumn{2}{|c|}{ 3. Qualità del reference, dell'orientamento e della comunicazione all'utenza } \\
\hline \multicolumn{1}{|c|}{ Attributo qualitativo } & \multicolumn{1}{|c|}{ Formulazione dell'attributo } & $\begin{array}{l}\text { Codice domanda } \\
\text { (Appendice B) }\end{array}$ \\
\hline $\begin{array}{l}\text { Fruibilità dei repertori } \\
\text { cartacei (collocazione e } \\
\text { facilità del recupero della } \\
\text { risorsa) }\end{array}$ & $\begin{array}{l}\text { 3.1 - Facilità del recupero e } \\
\text { consultazione di bibliografie, } \\
\text { repertori, enciclopedie }\end{array}$ & $\begin{array}{l}\text { QU03A11 } \\
\text { QU03B11 } \\
\text { QU03C11 }\end{array}$ \\
\hline $\begin{array}{l}\text { Politica della selezione } \\
\text { degli accessi alle banche- } \\
\text { dati }\end{array}$ & $\begin{array}{l}\text { 3.2 - Banche dati attualmente } \\
\text { disponibili nelle biblioteche (via } \\
\text { web, su cd-rom) }\end{array}$ & $\begin{array}{l}\text { QU07A22 } \\
\text { QU07B22 } \\
\text { QU07C22 }\end{array}$ \\
\hline $\begin{array}{l}\text { Qualità dei risultati } \\
\text { (quantità di errori e rumore } \\
\text { nei record recuperati) }\end{array}$ & $\begin{array}{l}3.3 \text { - Risultati ottenuti } \\
\text { dall'interrogazione delle banche } \\
\text { dati }\end{array}$ & $\begin{array}{l}\text { QU07A23 } \\
\text { QU07B22 } \\
\text { QU07C23 }\end{array}$ \\
\hline $\begin{array}{l}\text { Fruibilità delle banche dati } \\
\text { collocazione e facilità del } \\
\text { recupero della risorsa) }\end{array}$ & $\begin{array}{l}3.4 \text { - Banche dati: modo in cui sono } \\
\text { messe a disposizione del pubblico }\end{array}$ & $\begin{array}{l}\text { QU03A12 } \\
\text { QU07B22 } \\
\text { QU03C12 }\end{array}$ \\
\hline $\begin{array}{l}\text { Interazione bibliotecario- } \\
\text { lettore: efficacia } \\
\text { comunicativa del front- } \\
\text { office (orientamento dei } \\
\text { lettori) }\end{array}$ & $\begin{array}{l}\text { 3.5 - Comunicazione con il } \\
\text { personale delle biblioteche } \\
\text { (disponibilità e cortesia dei } \\
\text { bibliotecari nei confronti del } \\
\text { pubblico) }\end{array}$ & $\begin{array}{l}\text { QU03A13 } \\
\text { QU03B13 } \\
\text { QU03C13 }\end{array}$ \\
\hline
\end{tabular}




\begin{tabular}{|l|l|l|}
\hline $\begin{array}{l}\text { Servizio di orientamento } \\
\text { "avanzato" }\end{array}$ & $\begin{array}{l}\text { 3.6 - Servizi e assistenza } \\
\text { personalizzata al lettore da parte del } \\
\text { bibliotecario }\end{array}$ & $\begin{array}{l}\text { QU04A14 } \\
\text { QU04B14 } \\
\text { QU04C14 }\end{array}$ \\
\hline $\begin{array}{l}\text { Depliant e segnaletica sui } \\
\text { servizi bibliotecari come } \\
\text { "utility" alla localizzazione } \\
\text { e all'uso di risorse e servizi }\end{array}$ & $\begin{array}{l}\text { 3.7 - Depliant sulle biblioteche e } \\
\text { segnaletica in biblioteca per } \\
\text { facilitare l'uso dei servizi }\end{array}$ & $\begin{array}{l}\text { QU04A15 } \\
\text { QU04B15 } \\
\text { QU04C15 }\end{array}$ \\
\hline $\begin{array}{l}\text { Sito web del SBA (escluso } \\
\text { OPAC): percezione } \\
\text { dell'informazione erogata } \\
\text { on-line. }\end{array}$ & $\begin{array}{l}\text { 3.8 - Sito web del Sistema } \\
\text { Bibliotecario di Ateneo (esclusi } \\
\text { catalogo, prenotazioni e rinnovi) }\end{array}$ & $\begin{array}{l}\text { QU06aA17 } \\
\text { QU06aB17 } \\
\text { QU06aC17 }\end{array}$ \\
\hline
\end{tabular}

\begin{tabular}{|c|c|c|}
\hline \multicolumn{3}{|c|}{ 4. Dati anagrafici dell'intervistato } \\
\hline Attributo qualitativo & Formulazione dell'attributo & $\begin{array}{c}\text { Codice domanda } \\
\text { (Appendice B) }\end{array}$ \\
\hline \multirow{8}{*}{$\begin{array}{l}\text { Dati anagrafici e } \\
\text { informazioni su domicilio e } \\
\text { carriera universitaria degli } \\
\text { intervistati. }\end{array}$} & $4.1-$ Sesso & QU08sex \\
\hline & 4.2 - Età & QU08anno \\
\hline & 4.3 - Domicilio & QU08dom \\
\hline & $\begin{array}{l}4.4 \text { - L'intervistata/o risiede a } \\
\text { Firenze }\end{array}$ & QU08resfi \\
\hline & $\begin{array}{l}4.5 \text { - Provincia di residenza } \\
\text { intervistato/a }\end{array}$ & Qu08respr \\
\hline & $\begin{array}{l}4.6 \text { - Se studente, l'intervistata/o } \\
\text { lavora? }\end{array}$ & QU08lav \\
\hline & $\begin{array}{l}4.7 \text { - Tipo di lavoro } \\
\text { dell'intervistata/o, se studente. }\end{array}$ & QU08tlav \\
\hline & $\begin{array}{l}4.8 \text { - Se studente, l'intervistata/o ha } \\
\text { già chiesto la tesi? }\end{array}$ & QU08tesi \\
\hline
\end{tabular}

In fase operativa, l'ordine degli attributi e delle relative domande è stato adattato dai nostri consulenti statistici ad una sequenza diversa e ad una struttura articolata su domande "filtro": ad esempio, a chi dichiarava di non frequentare le sale di lettura delle biblioteche universitarie (l'intensità del contatto per la domanda 2.7) non sono state somministrate le domande relative alle condizioni ambientali delle stesse (domande 2.7-2.9). Tale riorganizzazione, altresì scaturente dalla specificità del medium di somministrazione, non comporta effetti sulla struttura concettuale dell'indagine e sulla valutazione biblioteconomica dei risultati, che sono qui presentate secondo la specifica chiave interpretativa del bibliotecario ${ }^{4}$.

4 L'Appendice $B$ riporta il testo del questionario così come è stato riadattato e somministrato al campione d'indagine. 


\section{Il modello di valutazione dei dati}

Per ogni attributo qualitativo è stato richiesto all'intervistato di esprimere il livello dell'aspettativa, una stima dell'intensità del contatto con l'attributo in questione rispetto alla totalità delle visite in biblioteca, il grado di soddisfazione derivante dall'esperienza del servizio al quale l'attributo si riferisce.

L'indagine attribuisce un preciso significato ai termini "aspettativa", "intensità del contatto" e "soddisfazione", prevedendo per tali dimensioni una serie di risposte predefinite, riportate nelle tabelle sottostanti:

\begin{tabular}{|l|l|}
\hline \multirow{4}{*}{$\begin{array}{l}\text { Aspettativa } \\
\text { l'importanza che gli studenti frequentatori delle }\end{array}$} & 1 = per nulla importante \\
\cline { 2 - 2 } $\begin{array}{l}\text { biblioteche attribuiscono ai servizi del SBA sotto- } \\
\text { posti ad indagine, indipendentemente da come essi } \\
\text { sono gestiti }\end{array}$ & $3=$ poco importante \\
\cline { 2 - 2 } & $4=$ importante \\
\cline { 2 - 2 } & $5=$ molto importante \\
\hline
\end{tabular}

\begin{tabular}{|l|c|}
\hline & $0 \%$ \\
& $\begin{array}{c}\text { Intensità del contatto } \\
\text { la percentuale delle visite dedicate all'uso dei ser- } \\
\text { vizi sul totale delle visite che gli studenti effet- } \\
\text { tuano nelle biblioteche dell'Università di Firenze }\end{array}$ \\
\cline { 2 - 2 } & $25 \%$ \\
\cline { 2 - 2 } & $50 \%$ \\
\hline
\end{tabular}

\begin{tabular}{|c|c|}
\hline \multirow{5}{*}{$\begin{array}{l}\text { Soddisfazione } \\
\text { il grado di soddisfazione che gli studenti frequen- } \\
\text { tatori delle biblioteche attribuiscono ai servizi del } \\
\text { SBA }\end{array}$} & $1=$ insoddisfatta $/ o$ \\
\hline & $2=$ poco insoddisfatta $/ 0$ \\
\hline & $3=$ indifferente \\
\hline & $4=$ abbastanza soddisfatta/o \\
\hline & $5=$ soddisfatta $/ 0$ \\
\hline
\end{tabular}

L'integrazione dei valori dell'aspettativa, della frequenza o intensità dei contatti e della soddisfazione del cliente dà origine ad un indicatore della soddisfazione complessiva rispetto al quale le tre componenti enunciate svolgono, ognuna nei confronti delle altre, una funzione di ponderazione. I valori assunti dalle componenti della soddisfazione complessiva possono essere confrontati e ciò consente al bibliotecario di inoltrare le sue valutazioni gestionali verso determinati precorsi di interpretazione e di ricerca delle cause del livello effettivo di rendimento qualitativo della biblioteca. 
A fondamento della qualità sta l'adattamento del servizio bibliotecario alle esigenze ambientali, interpretate come opportunità: le opportunità devono essere indagate, l'adattamento deve essere gestito attraverso il miglioramento continuo o l'innovazione dei fattori critici del processo di produzione del servizio. La qualità è il luogo della congiunzione tra tecnica e "mercato" (o sistema) dell'informazione. In esso, le 'specifiche' tecniche di un servizio assumono un significato più ampio di quello conferito dalla disciplina specialistica di pertinenza e divengono fattori governabili della gestione bibliotecaria e dell'efficacia dei servizi bibliotecari. In un'ottica ispirata alla qualità, è più rilevante il valore assunto dalla soddisfazione piuttosto che il numero dei libri acquistati, il numero dei libri consultati o prestati nelle diverse aree disciplinari: le connotazioni tecniche della selezione e acquisizione del materiale documentario sono subordinate dalla biblioteca eccellente al paradigma dell'efficacia, quand'anche ciò dovesse comportare una strategica e momentanea riduzione quantitativa della performance.

La soddisfazione complessiva è qui graficamente rappresentata dal cubo della soddisfazione. Esso può essere applicato: alle finalità istituzionali; alle aree principali di servizio; alle singole attività esercitate nell'ambito di ciascun sub-sistema; per sintesi, alla biblioteca o al sistema bibliotecario nel suo complesso, attraverso il confronto delle medie aritmetiche ottenute a partire da gruppi omogenei di dati.

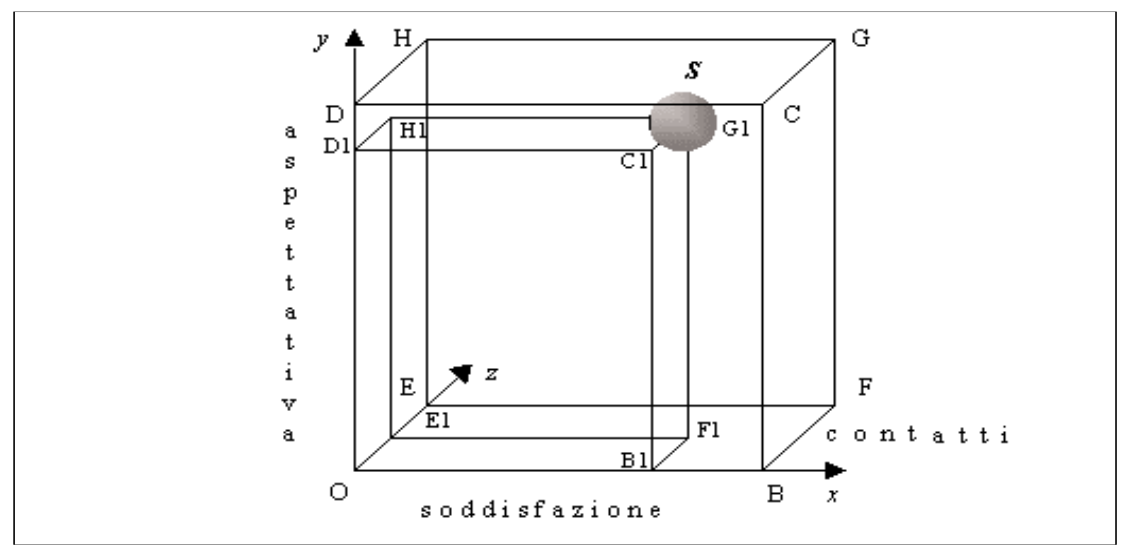

Il cubo della soddisfazione è una gabbia virtuale delimitata da valori massimi e minimi dell'aspettativa (sull'asse y), della soddisfazione (sull'asse x) e dell'intensità del contatto (sull'asse z) all'interno della quale si muove la sfera della soddisfazione complessiva $(S)$. La posizione di $S$ è determinata dall'interazione di $\mathrm{x}, \mathrm{y}, \mathrm{z}$, secondo una formula del tipo $[S=(x ; y ; z)]$ 
Con il passare del tempo, $S$ si muoverà tracciando una curva nello spazio. Per esempio, dal tempo $t_{0}$ (momento al quale è riferito il primo rilevamento della customer satisfaction) al tempo $\mathrm{t}_{1}$, la sfera della soddisfazione traccerà uno spezzone della curva, da $t_{1}$ a $t_{2}$ la curva si prolungherà, e così via, assumendo un andamento che potrà essere più o meno regolare ma visibile e riducibile ad una formula matematica, e quindi governabile.

Interpretando in via generale il significato assunto dal movimento di $S$ verso ognuno dei vertici del cubo, si possono formulare i seguenti enunciati:

Tendenza verso il punto $O$ : aspettativa, intensità del contatto e soddisfazione hanno valori pari a zero. La biblioteca abbandonerà l'attività misurata o intraprenderà una ricerca delle cause di tale situazione.

Tendenza verso il punto $B$ : aspettativa e intensità del contatto sono uguali a zero mentre la soddisfazione è massima; potrebbe costituire una prospettiva non certo auspicabile per una biblioteca oppure essere il segnale che la mera presenza del servizio misurato soddisfa un bisogno insorto casualmente e non gestibile dal bibliotecario.

Tendenza verso il punto $C$ : aspettativa e soddisfazione sono massimi, ma l'intensità del contatto è nulla. Il lettore nutre evidentemente aspettative simboliche le quali risultano soddisfatte stante la tendenza ad un contatto inesistente.

Tendenza verso il punto $D$ : massima aspettativa, nessuna intensità del contatto e nessuna soddisfazione. $\mathrm{O}$ il servizio desiderato dal pubblico non è attivo oppure la biblioteca deve risolvere un problema di comunicazione con i suoi clienti.

Tendenza verso il punto E: il lettore ricorre intensamente ad un dato servizio per il quale non nutre alcuna aspettativa e per il quale prova grande insoddisfazione. A questo proposito, si possono formulare due ipotesi. La biblioteca offre un servizio che il lettore è costretto ad usare suo malgrado: probabilmente esso si presenta come alternativa immediata ad un servizio atteso ed assente in biblioteca. Il servizio in questione dovrà essere sostituito con un servizio più idoneo e soddisfacente. Come seconda ipotesi, un servizio è usato in sostituzione di un servizio effettivamente presente in biblioteca: la biblioteca dovrà svolgere un lavoro di comunicazione per dirottare i lettori verso il servizio pertinente e atteso.

Tendenza verso il punto $F$ : Il lettore non nutre nessuna aspettativa, ma il contatto si rivela assai intenso e soddisfacente; si tratta, per il cliente, della scoperta di un servizio che si è rivelato utile e verso il quale è stato condotto da una buona organizzazione dei processi bibliotecari. Non resta che operare per la consapevolezza delle aspettative da parte del pubblico.

Tendenza verso il punto $G$ : per il servizio sussiste un'alta aspettativa, un uso intenso ed una soddisfazione elevata. Siamo nella situazione in cui la biblioteca esprime, per quell'attributo, un ideale grado di eccellenza. 
Tendenza verso il punto $H$ : il servizio suscita alte aspettative e viene intensamente usato, tuttavia la soddisfazione è pari a zero. La biblioteca dovrà ricercare le cause dell'insoddisfazione e rimuoverle per rendere il servizio più efficace oppure guiderà i lettori verso un servizio alternativo.

Per esemplificare, simuliamo un'applicazione del cubo della customer satisfaction alla valutazione del servizio di prestito interbibliotecario e document delivery in una biblioteca universitaria. È opportuno premettere che i numeri, anche se riferiti alla qualità, vanno sempre interpretati e che le valutazioni che scaturiscono dall'esame delle posizioni di $S$ possono variare a seconda del servizio, delle attività e del contesto a cui questo strumento è applicato.

Tendenza verso il punto $O$. Aspettativa, intensità del contatto e soddisfazione hanno valori pari a zero. I lettori non sanno che la biblioteca svolge un servizio di prestito interbibliotecario, favorendo l'accessibilità ed il recupero di documentazione presente in altre biblioteche, né conoscono le opportunità che tale servizio può costituire per soddisfare determinati bisogni informativi. Probabilmente vi è un vuoto di comunicazione tra la biblioteca ed i potenziali utenti del servizio, che non sono consapevoli di tale opportunità.

Tendenza verso il punto $B$. Il pubblico è informato della presenza del servizio ma non della capacità di recupero interbibliotecario o extrabibliotecario che la biblioteca può offrire: pur non avendo bisogno del servizio né ricorrendo ad esso, i lettori esprimono soddisfazione per la mera presenza, tangibile e rassicurante, del servizio stesso.

Tendenza verso il punto $C$. I contatti, come per il punto precedente, rimangono bassi: il servizio soddisfa un bisogno di presenza slegato dall'uso. È probabile che, in questo caso, la biblioteca sia generale e che per richieste mirate di prestito o recupero documentario il pubblico esperto si rivolga a biblioteche più specializzate. Il pubblico attribuisce al servizio un valore elevato, più simbolico che pratico, e desidera che esso sia mantenuto indipendentemente dall'utilizzo.

Tendenza verso il punto $D$. Sussiste un'aspettativa elevata per il prestito interbibliotecario, ma sia l'uso sia la soddisfazione tendono a valori negativi. Questo perché: il servizio non è presente e va introdotto; il servizio è presente ma non è visibile, promosso, agevolmente fruibile. Una migliore comunicazione tra biblioteca e lettore sulle aspettative di quest'ultimo potrebbe essere la leva su cui fare affidamento per il miglioramento della percezione qualitativa.

Tendenza verso il punto $E$. Il pubblico entra in contatto con il servizio di prestito interbibliotecario e recupero documentario senza conoscerne l'esistenza (da qui l'assenza di aspettativa), dirottato ad esso dal front-office della biblioteca nel caso in cui non riesca trovare in sede i documenti che servono, ma, nonostante la domanda, il servizio non funziona. La chiave per migliorare la performance è una riorganizzazione del servizio accompagnata da un rapporto comunicativo più amichevole con il pubblico. 
Tendenza verso il punto $F$. Il lettore usa il servizio pur in assenza di una consapevole aspettativa e, contrariamente al punto precedente, ne rimane soddisfatto: si tratta, per il cliente, della scoperta di un servizio non atteso che si è rivelato soddisfacente; il bibliotecario lavorerà per far crescere l'aspettativa attraverso una adeguata campagna di promozione.

Tendenza verso il punto $G$. Il pubblico nutre alte aspettative e la biblioteca è in grado di soddisfarle appieno, sapendo gestire efficacemente l'intenso uso del servizio. Il bibliotecario ha saputo interpretare e prevedere con successo i gusti del pubblico, ha saputo comunicare efficacemente al pubblico la concezione della biblioteca come punto di accesso all'universo documentario ovunque collocato.

Tendenza verso il punto $H$. Questo caso è inequivocabile: i lettori ricorrono intensamente al servizio di prestito interbibliotecario, sono consapevoli della sua utilità e ne rimangono costantemente insoddisfatti. La biblioteca offre prestazioni inadeguate e questo può dipendere da molteplici cause: (a) il servizio è impostato su giusti parametri tecnici, ma i bibliotecari non riescono a tradurre le istruzioni di lavoro in prestazioni adeguate; (b) le attività previste per questo servizio sono poco pertinenti ed i lettori vi ricorrono perché non sono disponibili risorse alternative; (c) parametri tecnici e metodi di lavoro sono applicati correttamente, tuttavia qualcosa non funziona: potrebbe essere opportuno ripensare la "filosofia" del servizio.

Una volta accertata la tendenza di $S$, il bibliotecario deciderà verso quale altro vertice del cubo indurre il movimento della sfera. Questa considerazione, è ovvio, non vale nel caso in cui $S$ coincida o sia molto vicina a coincidere con il punto $\mathrm{G}$, a meno di uno spostamento di risorse verso servizi ritenuti più strategici.

\section{I risultati dell'indagine: i dati del SBA}

Si presentano qui i risultati dell'indagine elaborati a livello di SBA, quest'ultimo inteso come sistema di servizi bibliotecari omogenei rivolti al pubblico dell'Ateneo nel suo complesso. Si prescinderà pertanto da considerazioni inerenti singole biblioteche o punti di servizio.

Ogni attributo qualitativo è illustrato in quattro tabelle: le prime tre riguardano i dati relativi all'utenza studentesca, la quatra riporta i dati relativi al personale docente e di ricerca e mostra la sola elaborazione quantitativa dei risultati dato che tali numeri non sono dotati di rappresentatività statistica e possono fornire informazioni relative esclusivamente alle persone che hanno accettato di rispondere al questionario. La prima tabella è corredata da un 
commento sulla performance ottenuta dall'attributo e da una figura che mostra la posizione assunta dalla soddisfazione complessiva $(S)$ in virtù delle tre variabili della percezione qualitativa (aspettativa, intensità del contatto, soddisfazione).

Nell'elaborazione dei dati qui effettuata si è tenuto conto delle sole risposte valide - recanti cioè un valore quantitativo tra quelli predefiniti - espresse dagli intervistati: quelle previste dal questionario per ogni domanda con l'esclusione della risposta "non so / non rispondo" nonché dell'eventuale abbandono dell'intervista o delle eventuali omissioni di risposta provocate dalle domande filtro. Nelle prime due tabelle le risposte valide sono state "proiettate" sull'universo indagato, stimando il numero di risposte che si sarebbero ottenute se ad essere intervistati fossero stati i 27.746 utenti reali del SBA nonché ponderando la diversa dimensione assunta dai dieci strati che compongono il campione. Successivamente, per ogni domanda, si è calcolato la somma complessiva di punteggio risultante dalle citate stime e si è proceduto ad assegnare alle tre variabili di ciascun attributo la media aritmetica dei relativi punteggi validi. La terza tabella riporta l'elaborazione delle sole risposte del campione, senza ponderazione degli strati. È dunque possibile che nelle tabelle il numero di risposte valide risulti talvolta inferiore alla dimensione dell'universo indagato o del campione d'indagine e che le percentuali indicate per ogni risposta non raggiungano la somma del 100\%: ciò significa che una parte del campione ha interrotto l'intervista, oppure non ha risposto alla domanda, oppure non è stato sottoposto a talune domande per l'effetto di precedenti domande filtro.

Le medie aritmetiche così ottenute sono state confrontate senza procedere ad ulteriori ponderazioni. La chiave interpretativa dei dati mira ad offrire la valutazione non già del valore 'assoluto' assunto dalle tre variabili e dalla soddisfazione complessiva (S), ma la tendenza di quest'ultima verso i punti A, B, C, D, E, F, G. Data la natura anche psicologica, oltre che sociale, di queste indagini, è opportuno considerare i numeri con una qualche elasticità rispetto ai valori assoluti che rispettivamente rappresentano, tentando di superare la soglia interpretativa del determinismo quantitativo per raggiungere una visuale "sistemica" in grado di cogliere le interazioni tra fattori processuali di diversa natura e le implicazioni di politica gestionale bibliotecaria che coinvolgono un insieme di servizi erogati in un contesto assai ampio e composito.

Che il metodo adottato possa risultare perfettibile è coerente con la circostanza che nell'ambito della ricerca teorica ed applicata sussiste disaccordo sull'esatto significato da attribuire al termine «qualità»: c'è chi lo identifica 
con lo scostamento esistente tra aspettativa e soddisfazione del cliente; chi ritiene invece che solo quest'ultima vada considerata portatrice di indicazioni qualitative; chi, ancora, pensa che la percezione di un servizio sia condizionata anche da fattori probabilistici che sfuggono al controllo umano sia dal lato della domanda sia da quello dell'offerta.

In definitiva, non esistono ricette sicure ma metodi che, seppure non sempre coincidenti, possono suggerire strade da percorrere alla luce del tipo di conoscenza che si desidera acquisire sull'ambiente in cui si è chiamati ad operare. L'importante è che un metodo sia scelto o costruito, che esso sia esposto nella maniera più chiara possibile, che siano dati gli strumenti per perfezionare ed affinare la capacità, da parte degli operatori dei servizi pubblici statali o privati, di offrire una prestazione sempre più competitiva e vicina alle esigenze ed alle aspirazioni dei propri "clienti".

Nelle pagine che seguono sono riportate le considerazioni bibliotecarie e biblioteconomiche scaturenti dall'elaborazione e dall'esame dei risultati grezzi dell'indagine.

\section{Qualità delle acquisizioni cartacee ed elettroniche}

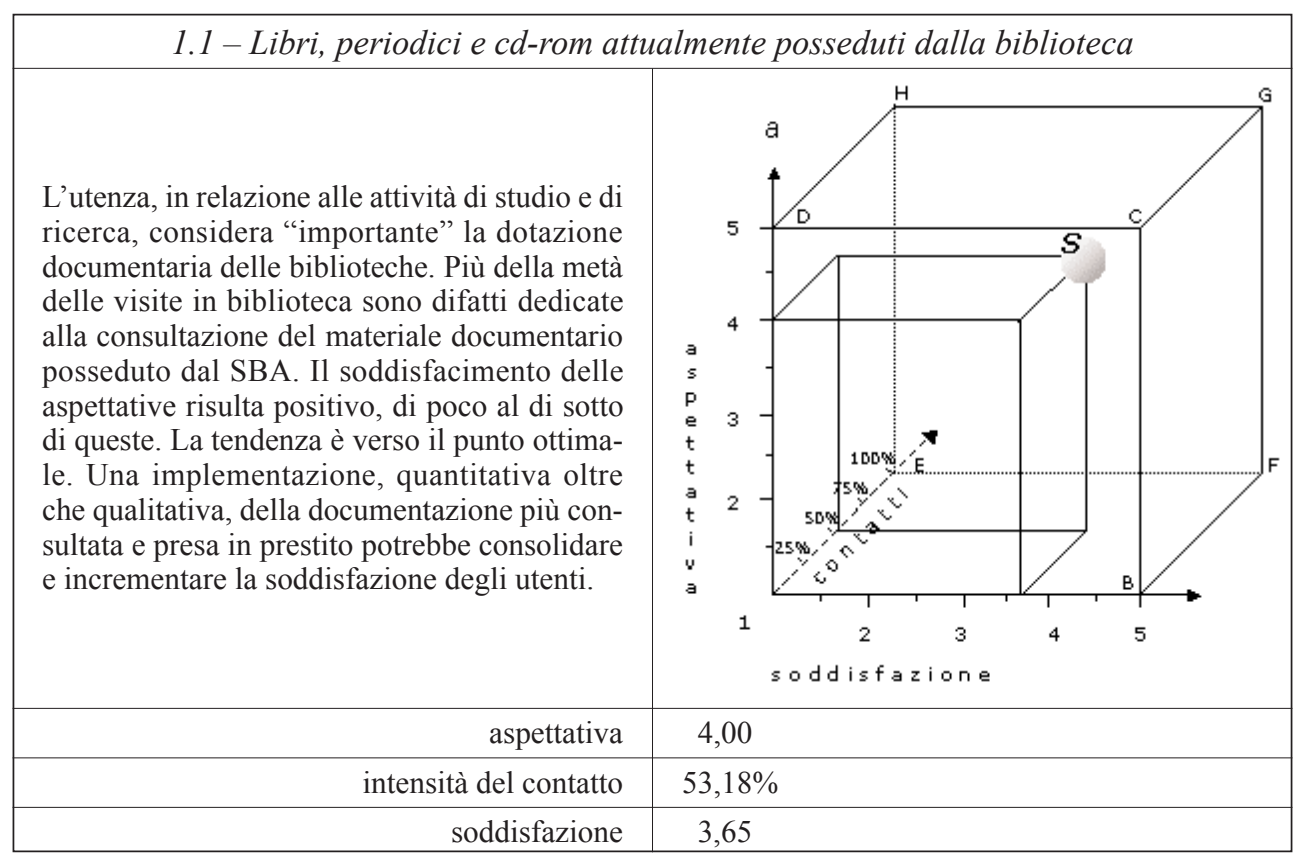


L'INDAGINE SULLA SODDISFAZIONE DEGLI UTENTI: METODOLOGIA E VALUTAZIONE BIBLIOTECARIA

\begin{tabular}{|c|c|c|c|c|c|c|c|}
\hline \multirow[b]{3}{*}{1} & \multicolumn{6}{|c|}{ distribuzione delle risposte (stime su 27746 utenti) } & \multirow[b]{3}{*}{$0 \%$} \\
\hline & \multicolumn{2}{|c|}{ aspettativa } & \multicolumn{2}{|c|}{ soddisfazione } & \multicolumn{2}{|c|}{ intensità } & \\
\hline & $2,18 \%$ & 605 & $1,24 \%$ & 315 & $8,45 \%$ & 2345 & \\
\hline 2 & $3,99 \%$ & 1108 & $10,13 \%$ & 2574 & $26,27 \%$ & 7290 & $25 \%$ \\
\hline 3 & $19,99 \%$ & 5548 & $27,14 \%$ & 6895 & $25,70 \%$ & 7131 & $50 \%$ \\
\hline 4 & $38,64 \%$ & 10721 & $44,96 \%$ & 11421 & $22,58 \%$ & 6265 & $75 \%$ \\
\hline 5 & $34,10 \%$ & 9462 & $15,90 \%$ & 4040 & $16,63 \%$ & 4614 & $100 \%$ \\
\hline
\end{tabular}

\begin{tabular}{|c|r|r|r|r|r|r|r|}
\cline { 2 - 7 } \multicolumn{1}{c|}{} & \multicolumn{6}{c|}{ distribuzione delle risposte (su 1063 interviste) } & \multirow{2}{*}{} \\
\cline { 2 - 7 } \multicolumn{1}{c|}{} & \multicolumn{2}{c|}{ aspettativa } & \multicolumn{2}{c|}{ soddisfazione } & \multicolumn{2}{c|}{ intensità } & \multicolumn{1}{c|}{} \\
\hline 1 & $2,07 \%$ & 22 & $1,13 \%$ & 12 & $8,56 \%$ & 91 & $0 \%$ \\
\hline 2 & $3,95 \%$ & 42 & $9,13 \%$ & 97 & $26,62 \%$ & 283 & $25 \%$ \\
\hline 3 & $20,51 \%$ & 218 & $24,84 \%$ & 264 & $25,12 \%$ & 267 & $50 \%$ \\
\hline 4 & $38,01 \%$ & 404 & $41,02 \%$ & 436 & $23,14 \%$ & 246 & $75 \%$ \\
\hline 5 & $35,37 \%$ & 376 & $15,33 \%$ & 163 & $16,27 \%$ & 173 & $100 \%$ \\
\hline
\end{tabular}

\begin{tabular}{|c|c|c|c|c|c|c|c|}
\hline & \multicolumn{6}{|c|}{ risposte del personale docente e di ricerca (su 690 interviste) } & \\
\hline & \multirow{2}{*}{\multicolumn{2}{|c|}{ aspettativa }} & \multirow{2}{*}{\multicolumn{2}{|c|}{$\begin{array}{c}\text { soddisfazione } \\
3,71 \\
\end{array}$}} & \multirow{2}{*}{\multicolumn{2}{|c|}{$\begin{array}{c}\text { intensità } \\
76,93 \% \\
\end{array}$}} & \\
\hline & & & & & & & \\
\hline 1 & $1,88 \%$ & 13 & $3,48 \%$ & 24 & $2,32 \%$ & 16 & $0 \%$ \\
\hline 2 & $4,49 \%$ & 31 & $10,43 \%$ & 72 & $8,99 \%$ & 62 & $25 \%$ \\
\hline 3 & $3,77 \%$ & 26 & $11,74 \%$ & 81 & $10,87 \%$ & 75 & $50 \%$ \\
\hline 4 & $31,88 \%$ & 220 & $48,55 \%$ & 335 & $25,22 \%$ & 174 & $75 \%$ \\
\hline 5 & $49,42 \%$ & 341 & $16,38 \%$ & 113 & $42,75 \%$ & 295 & $100 \%$ \\
\hline
\end{tabular}




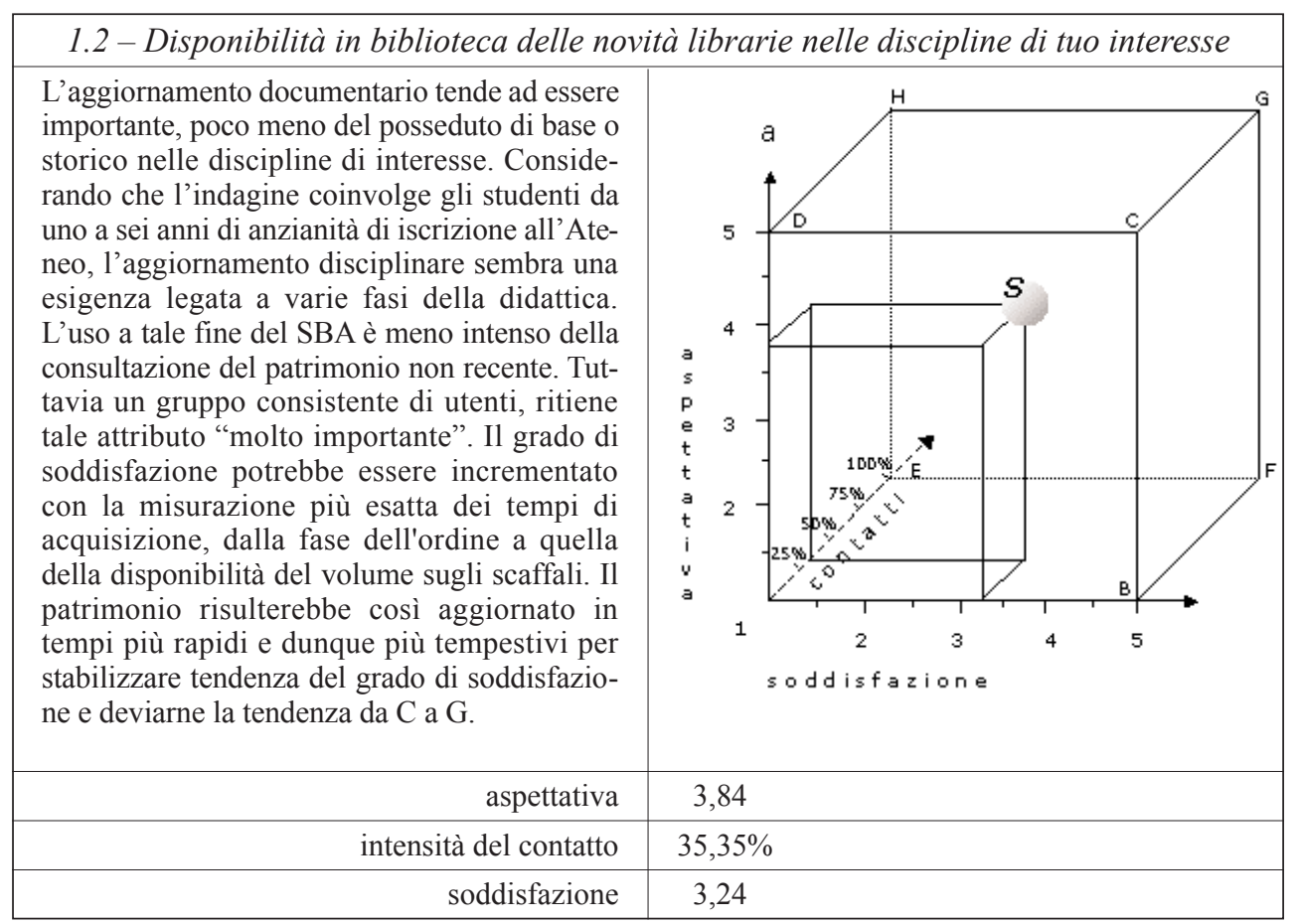

\begin{tabular}{|c|c|c|c|c|c|c|c|}
\hline \multirow[b]{3}{*}{1} & \multicolumn{6}{|c|}{ distribuzione delle risposte (stime su 27746 utenti) } & \multirow[b]{3}{*}{$0 \%$} \\
\hline & \multicolumn{2}{|c|}{ aspettativa } & \multicolumn{2}{|c|}{ soddisfazione } & \multicolumn{2}{|c|}{ intensità } & \\
\hline & $5,40 \%$ & 1497 & $5,20 \%$ & 1014 & $29,71 \%$ & 8243 & \\
\hline 2 & $7,73 \%$ & 2144 & $18,42 \%$ & 3593 & $23,13 \%$ & 6419 & $25 \%$ \\
\hline 3 & $19,64 \%$ & 5449 & $31,83 \%$ & 6207 & $23,08 \%$ & 6403 & $50 \%$ \\
\hline 4 & $23,67 \%$ & 6567 & $28,24 \%$ & 5508 & $15,63 \%$ & 4337 & $75 \%$ \\
\hline 5 & $36,26 \%$ & 10062 & $11,51 \%$ & 2244 & $5,13 \%$ & 1423 & $100 \%$ \\
\hline
\end{tabular}

\begin{tabular}{|c|c|c|c|c|c|c|c|}
\hline \multirow[b]{3}{*}{1} & \multicolumn{6}{|c|}{ distribuzione delle risposte (su 1063 interviste) } & \multirow[b]{3}{*}{$0 \%$} \\
\hline & \multicolumn{2}{|c|}{ aspettativa } & \multicolumn{2}{|c|}{ soddisfazione } & \multicolumn{2}{|c|}{ intensità } & \\
\hline & $4,70 \%$ & 50 & $3,57 \%$ & 38 & $29,35 \%$ & 312 & \\
\hline 2 & $7,71 \%$ & 82 & $12,89 \%$ & 137 & $24,18 \%$ & 257 & $25 \%$ \\
\hline 3 & $27,75 \%$ & 295 & $25,21 \%$ & 268 & $22,48 \%$ & 239 & $50 \%$ \\
\hline 4 & $22,58 \%$ & 240 & $20,51 \%$ & 218 & $14,86 \%$ & 158 & $75 \%$ \\
\hline 5 & $37,25 \%$ & 396 & $8,47 \%$ & 90 & $5,55 \%$ & 59 & $100 \%$ \\
\hline
\end{tabular}


L'INDAGINE SULLA SODDISFAZIONE DEGLI UTENTI: METODOLOGIA E VALUTAZIONE BIBLIOTECARIA

\begin{tabular}{|c|c|c|c|c|c|c|c|}
\hline & \multicolumn{6}{|c|}{ risposte del personale docente e di ricerca (su 690 interviste) } & \\
\hline & \multirow{2}{*}{\multicolumn{2}{|c|}{$\begin{array}{c}\text { aspettativa } \\
4,13\end{array}$}} & \multirow{2}{*}{\multicolumn{2}{|c|}{$\begin{array}{c}\text { soddisfazione } \\
2,95 \\
\end{array}$}} & \multirow{2}{*}{\multicolumn{2}{|c|}{$\begin{array}{c}\text { intensità } \\
44,70 \% \\
\end{array}$}} & \\
\hline & & & & & & & \\
\hline 1 & $2,46 \%$ & 17 & $9,86 \%$ & 68 & $13,62 \%$ & 94 & $0 \%$ \\
\hline 2 & $8,26 \%$ & 57 & $21,74 \%$ & 150 & $26,09 \%$ & 180 & $25 \%$ \\
\hline 3 & $6,81 \%$ & 47 & $19,28 \%$ & 133 & $20,87 \%$ & 144 & $50 \%$ \\
\hline 4 & $26,81 \%$ & 185 & $26,38 \%$ & 182 & $14,64 \%$ & 101 & $75 \%$ \\
\hline 5 & $42,32 \%$ & 292 & $5,36 \%$ & 37 & $10,29 \%$ & 71 & $100 \%$ \\
\hline
\end{tabular}

\begin{tabular}{|l|l|l|l|}
\hline \multicolumn{3}{|c|}{ 1.3-Disponibilità degli ultimi numeri dei periodici nelle discipline di tuo interesse } \\
\hline L'aggiornamento delle raccolte seriali è solita- \\
mente considerato una componente importante \\
per valutare l'efficacia di una biblioteca uni- \\
versitaria. Esso indica che l'utenza segue il \\
dibattito disciplinare per ragioni di studio e di \\
ricerca ed ha bisogno di servizi tempestivi di \\
informazione scientifica. Questo attributo è \\
avvertito e usato con minore intensità rispetto \\
al materiale monografico di recente accessio- \\
ne, rispondendo probabilmente ad interessi \\
maggiormente specializzati. Per interpretare \\
tali misure, può essere utile un confronto con \\
l'attributo successivo. \\
antensità del contatto
\end{tabular}

\begin{tabular}{|c|c|c|c|c|c|c|c|}
\hline \multirow[b]{3}{*}{1} & \multicolumn{6}{|c|}{ distribuzione delle risposte (stime su 27746 utenti) } & \multirow[b]{3}{*}{$0 \%$} \\
\hline & \multicolumn{2}{|c|}{ aspettativa } & \multicolumn{2}{|c|}{ soddisfazione } & \multicolumn{2}{|c|}{ intensità } & \\
\hline & $9,39 \%$ & 2604 & $5,08 \%$ & 784 & $44,35 \%$ & 12305 & \\
\hline 2 & $6,83 \%$ & 1894 & $11,53 \%$ & 1781 & $17,40 \%$ & 4827 & $25 \%$ \\
\hline 3 & $15,71 \%$ & 4359 & $32,51 \%$ & 5020 & $17,88 \%$ & 4962 & $50 \%$ \\
\hline 4 & $21,72 \%$ & 6027 & $32,32 \%$ & 4991 & $10,50 \%$ & 2913 & $75 \%$ \\
\hline 5 & $33,39 \%$ & 9263 & $10,91 \%$ & 1685 & $5,24 \%$ & 1455 & $100 \%$ \\
\hline
\end{tabular}




\section{ROBERTO VENTURA}

\begin{tabular}{|c|c|c|c|c|c|c|c|}
\hline \multirow[b]{3}{*}{1} & \multicolumn{6}{|c|}{ distribuzione delle risposte (su 1063 interviste) } & \multirow[b]{3}{*}{$0 \%$} \\
\hline & \multicolumn{2}{|c|}{ aspettativa } & \multicolumn{2}{|c|}{ soddisfazione } & \multicolumn{2}{|c|}{ intensità } & \\
\hline & $9,03 \%$ & 96 & $2,63 \%$ & 28 & $46,10 \%$ & 490 & \\
\hline 2 & $6,11 \%$ & 65 & $5,74 \%$ & 61 & $17,03 \%$ & 181 & $25 \%$ \\
\hline 3 & $31,61 \%$ & 336 & $21,92 \%$ & 233 & $16,93 \%$ & 180 & $50 \%$ \\
\hline 4 & $21,73 \%$ & 231 & $17,69 \%$ & 188 & $10,16 \%$ & 108 & $75 \%$ \\
\hline 5 & $31,51 \%$ & 335 & $5,93 \%$ & 63 & $4,80 \%$ & 51 & $100 \%$ \\
\hline
\end{tabular}

\begin{tabular}{|c|c|c|c|c|c|c|c|}
\hline & \multicolumn{6}{|c|}{ risposte del personale docente e di ricerca (su 690 interviste) } & \\
\hline & \multicolumn{2}{|c|}{ aspettativa } & \multicolumn{2}{|c|}{ soddisfazione } & \multicolumn{2}{|c|}{ intensità } & \\
\hline & \multicolumn{2}{|c|}{4,29} & \multicolumn{2}{|c|}{3,24} & \multicolumn{2}{|c|}{$55,86 \%$} & \\
\hline 1 & $2,46 \%$ & 17 & $6,52 \%$ & 45 & $7,39 \%$ & 51 & $0 \%$ \\
\hline 2 & $4,64 \%$ & 32 & $16,23 \%$ & 112 & $17,68 \%$ & 122 & $25 \%$ \\
\hline 3 & $6,81 \%$ & 47 & $17,68 \%$ & 122 & $21,88 \%$ & 151 & $50 \%$ \\
\hline 4 & $22,90 \%$ & 158 & $35,51 \%$ & 245 & $23,04 \%$ & 159 & $75 \%$ \\
\hline 5 & $48,41 \%$ & 334 & $6,67 \%$ & 46 & $14,64 \%$ & 101 & $100 \%$ \\
\hline
\end{tabular}

\begin{tabular}{|l|l|l|}
\hline \multicolumn{3}{|c|}{$1.4-$ Uso dei periodici elettronici nelle discipline di tuo interesse } \\
La soddisfazione per i periodici elettronici è \\
maggiore rispetto a quanto manifestato per \\
l'aggiornamento seriale in edizione cartacea. \\
Come è noto, l'aggiornamento scientifico in \\
tempo reale è il punto di forza di questa nuova \\
forma di distribuzione della stampa periodica, \\
non solo in ambito accademico. La frequenza \\
dell'uso potrebbe essere incrementata con una \\
maggiore promozione. Si potrà valutare se \\
investire maggiori risorse lavorative nella \\
velocizzazione del processo di trattamento dei \\
periodici cartacei ovvero se dirottare col \\
tempo gli sforzi e l'utenza verso un uso più \\
intenso ed assistito della raccolta seriale elet- \\
tronica. \\
intensità del contatto \\
soddisfazione
\end{tabular}




\begin{tabular}{|r|r|r|r|r|r|r|r|}
\cline { 2 - 7 } \multicolumn{1}{c|}{} & \multicolumn{5}{c|}{ distribuzione delle risposte (stime su 27746 utenti) } & \multirow{2}{*}{} \\
\cline { 2 - 7 } \multicolumn{1}{c|}{ aspettativa } & \multicolumn{2}{c|}{ soddisfazione } & \multicolumn{2}{c|}{ intensità } & \multicolumn{1}{c|}{} \\
\hline 1 & $9,20 \%$ & 1899 & $1,74 \%$ & 159 & $55,79 \%$ & 11514 & $0 \%$ \\
\hline 2 & $8,74 \%$ & 1804 & $8,97 \%$ & 818 & $13,98 \%$ & 2885 & $25 \%$ \\
\hline 3 & $15,57 \%$ & 3213 & $32,95 \%$ & 3006 & $10,98 \%$ & 2266 & $50 \%$ \\
\hline 4 & $18,75 \%$ & 3869 & $27,06 \%$ & 2469 & $7,85 \%$ & 1620 & $75 \%$ \\
\hline 5 & $24,82 \%$ & 5122 & $13,70 \%$ & 1250 & $4,23 \%$ & 873 & $100 \%$ \\
\hline
\end{tabular}

\begin{tabular}{|c|c|c|c|c|c|c|c|}
\hline \multirow[b]{3}{*}{1} & \multicolumn{6}{|c|}{ distribuzione delle risposte (su 1063 interviste) } & \multirow[b]{3}{*}{$0 \%$} \\
\hline & \multicolumn{2}{|c|}{ aspettativa } & \multicolumn{2}{|c|}{ soddisfazione } & \multicolumn{2}{|c|}{ intensità } & \\
\hline & $6,68 \%$ & 71 & $0,56 \%$ & 6 & $41,77 \%$ & 444 & \\
\hline 2 & $5,74 \%$ & 61 & $3,01 \%$ & 32 & $10,63 \%$ & 113 & $25 \%$ \\
\hline 3 & $29,63 \%$ & 315 & $16,09 \%$ & 171 & $8,18 \%$ & 87 & $50 \%$ \\
\hline 4 & $14,11 \%$ & 150 & $9,03 \%$ & 96 & $5,83 \%$ & 62 & $75 \%$ \\
\hline 5 & $18,44 \%$ & 196 & $4,14 \%$ & 44 & $3,01 \%$ & 32 & $100 \%$ \\
\hline
\end{tabular}

\begin{tabular}{|c|c|c|c|c|c|c|c|}
\hline & \multicolumn{6}{|c|}{ risposte del personale docente e di ricerca (su 690 interviste) } & \\
\hline & \multicolumn{2}{|c|}{ aspettativa } & \multicolumn{2}{|c|}{ soddisfazione } & \multicolumn{2}{|c|}{ intensità } & \\
\hline & \multicolumn{2}{|c|}{4,32} & \multicolumn{2}{|c|}{3,92} & \multicolumn{2}{|c|}{$64,10 \%$} & \\
\hline 1 & $3,19 \%$ & 22 & $2,03 \%$ & 14 & $7,54 \%$ & 52 & $0 \%$ \\
\hline 2 & $2,32 \%$ & 16 & $3,62 \%$ & 25 & $7,97 \%$ & 55 & $25 \%$ \\
\hline 3 & $3,91 \%$ & 27 & $7,97 \%$ & 55 & $7,97 \%$ & 55 & $50 \%$ \\
\hline 4 & $13,62 \%$ & 94 & $25,07 \%$ & 173 & $15,94 \%$ & 110 & $75 \%$ \\
\hline 5 & $37,83 \%$ & 261 & $16,67 \%$ & 115 & $20,43 \%$ & 141 & $100 \%$ \\
\hline
\end{tabular}




\section{Qualità della circolazione documentaria}

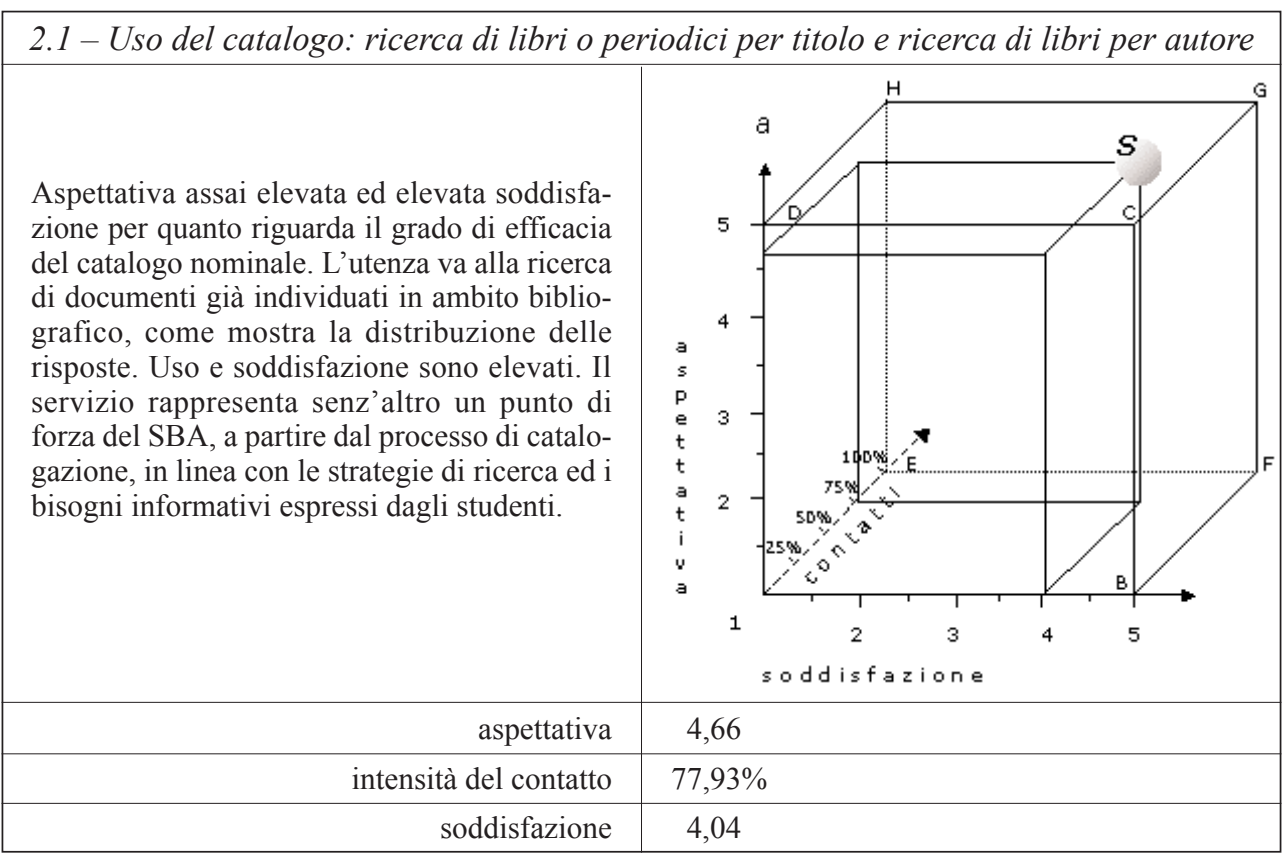

\begin{tabular}{|c|c|c|c|c|c|c|c|}
\hline \multirow[b]{3}{*}{1} & \multicolumn{6}{|c|}{ distribuzione delle risposte (stime su 27746 utenti) } & \multirow[b]{3}{*}{$0 \%$} \\
\hline & \multicolumn{2}{|c|}{ aspettativa } & \multicolumn{2}{|c|}{ soddisfazione } & \multicolumn{2}{|c|}{ intensità } & \\
\hline & $0,37 \%$ & 77 & $0,80 \%$ & 161 & $3,04 \%$ & 627 & \\
\hline 2 & $0,93 \%$ & 192 & $3,63 \%$ & 726 & $7,40 \%$ & 1527 & $25 \%$ \\
\hline 3 & $2,84 \%$ & 587 & $17,61 \%$ & 3524 & $11,43 \%$ & 2360 & $50 \%$ \\
\hline 4 & $23,10 \%$ & 4767 & $45,14 \%$ & 9034 & $29,69 \%$ & 6129 & $75 \%$ \\
\hline 5 & $70,42 \%$ & 14535 & $31,02 \%$ & 6209 & $46,86 \%$ & 9672 & $100 \%$ \\
\hline
\end{tabular}

\begin{tabular}{|c|c|c|c|c|c|c|c|}
\hline \multirow[b]{3}{*}{1} & \multicolumn{6}{|c|}{ distribuzione delle risposte (su 1063 interviste) } & \multirow[b]{3}{*}{$0 \%$} \\
\hline & \multicolumn{2}{|c|}{ aspettativa } & \multicolumn{2}{|c|}{ soddisfazione } & \multicolumn{2}{|c|}{ intensità } & \\
\hline & $0,19 \%$ & 2 & $0,75 \%$ & 8 & $2,07 \%$ & 22 & \\
\hline 2 & $0,56 \%$ & 6 & $2,63 \%$ & 28 & $5,74 \%$ & 61 & $25 \%$ \\
\hline 3 & $3,95 \%$ & 42 & $14,02 \%$ & 149 & $9,03 \%$ & 96 & $50 \%$ \\
\hline 4 & $17,87 \%$ & 190 & $31,61 \%$ & 336 & $22,58 \%$ & 240 & $75 \%$ \\
\hline 5 & $52,02 \%$ & 553 & $23,52 \%$ & 250 & $33,87 \%$ & 360 & $100 \%$ \\
\hline
\end{tabular}




\begin{tabular}{|c|c|c|c|c|c|c|c|}
\hline & \multicolumn{6}{|c|}{ risposte del personale docente e di ricerca (su 690 interviste) } & \\
\hline & \multirow{2}{*}{\multicolumn{2}{|c|}{$\begin{array}{c}\text { aspettativa } \\
4,62 \\
\end{array}$}} & \multirow{2}{*}{\multicolumn{2}{|c|}{$\begin{array}{c}\text { soddisfazione } \\
3,97 \\
\end{array}$}} & \multirow{2}{*}{\multicolumn{2}{|c|}{$\begin{array}{c}\text { intensità } \\
69,36 \% \\
\end{array}$}} & \\
\hline & & & & & & & \\
\hline 1 & $0,43 \%$ & 3 & $0,72 \%$ & 9 & $1,30 \%$ & 5 & $0 \%$ \\
\hline 2 & $1,16 \%$ & 8 & $3,91 \%$ & 61 & $8,84 \%$ & 27 & $25 \%$ \\
\hline 3 & $2,90 \%$ & 20 & $8,70 \%$ & 78 & $11,30 \%$ & 60 & $50 \%$ \\
\hline 4 & $13,91 \%$ & 96 & $33,77 \%$ & 163 & $23,62 \%$ & 233 & $75 \%$ \\
\hline 5 & $46,52 \%$ & 321 & $16,67 \%$ & 128 & $18,55 \%$ & 115 & $100 \%$ \\
\hline
\end{tabular}

\begin{tabular}{|l|l|}
\hline \multicolumn{3}{|c|}{$2.2-$ Uso del catalogo: ricerca di libri per argomento o materia } \\
L'efficacia del catalogo semantico tende verso \\
punto ottimale, sebbene con esigenze informa- \\
tive meno frequenti rispetto a 2.1. Si potrebbe \\
verificare quali categorie editoriali della colle- \\
zione sono più ricercate nelle varie discipline e \\
il grado di coincidenza della attuale configura- \\
zione bibliografica rispetto all'insieme dei \\
testi d'esame e per le tesi. La ricerca semantica \\
richiede consapevolezza della struttura dei \\
sistemi di classificazione e di soggettazione ed \\
è probabile che l'organizzazione didattica dei \\
corsi costituisca un riferimento più immediato \\
per quanto riguarda l'accesso alla documenta- \\
zione. Una maggiore enfasi sui sistemi di \\
comunicazione tra i due metodi di organizza- \\
zione della conoscenza potrebbe consolidare \\
un servizio già efficace.
\end{tabular}

\begin{tabular}{|c|c|c|c|c|c|c|c|}
\hline \multirow[b]{3}{*}{1} & \multicolumn{6}{|c|}{ distribuzione delle risposte (stime su 27746 utenti) } & \multirow[b]{3}{*}{$0 \%$} \\
\hline & \multicolumn{2}{|c|}{ aspettativa } & \multicolumn{2}{|c|}{ soddisfazione } & \multicolumn{2}{|c|}{ intensità } & \\
\hline & $1,65 \%$ & 340 & $2,47 \%$ & 442 & $13,12 \%$ & 2708 & \\
\hline 2 & $4,77 \%$ & 984 & $10,47 \%$ & 1878 & $19,03 \%$ & 3928 & $25 \%$ \\
\hline 3 & $10,35 \%$ & 2137 & $26,40 \%$ & 4733 & $19,66 \%$ & 4057 & $50 \%$ \\
\hline 4 & $30,02 \%$ & 6195 & $37,10 \%$ & 6653 & $27,09 \%$ & 5591 & $75 \%$ \\
\hline 5 & $49,10 \%$ & 10134 & $21,63 \%$ & 3878 & $19,31 \%$ & 3985 & $100 \%$ \\
\hline
\end{tabular}




\section{ROBERTO VENTURA}

\begin{tabular}{|c|c|c|c|c|c|c|c|}
\hline \multirow[b]{3}{*}{1} & \multicolumn{6}{|c|}{ distribuzione delle risposte (su 1063 interviste) } & \multirow[b]{3}{*}{$0 \%$} \\
\hline & \multicolumn{2}{|c|}{ aspettativa } & \multicolumn{2}{|c|}{ soddisfazione } & \multicolumn{2}{|c|}{ intensità } & \\
\hline & $1,03 \%$ & 11 & $1,32 \%$ & 14 & $9,88 \%$ & 105 & \\
\hline 2 & $3,10 \%$ & 33 & $7,15 \%$ & 76 & $14,21 \%$ & 151 & $25 \%$ \\
\hline 3 & $10,82 \%$ & 115 & $18,53 \%$ & 197 & $15,15 \%$ & 161 & $50 \%$ \\
\hline 4 & $22,58 \%$ & 240 & $23,71 \%$ & 252 & $20,32 \%$ & 216 & $75 \%$ \\
\hline 5 & $37,06 \%$ & 394 & $14,02 \%$ & 149 & $13,55 \%$ & 144 & $100 \%$ \\
\hline
\end{tabular}

\begin{tabular}{|c|c|c|c|c|c|c|c|}
\hline & \multicolumn{6}{|c|}{ risposte del personale docente e di ricerca (su 690 interviste) } & \\
\hline & \multirow{2}{*}{\multicolumn{2}{|c|}{$\begin{array}{c}\text { aspettativa } \\
3,80 \\
\end{array}$}} & \multirow{2}{*}{\multicolumn{2}{|c|}{$\begin{array}{c}\text { soddisfazione } \\
3,54 \\
\end{array}$}} & \multirow{2}{*}{\multicolumn{2}{|c|}{$\begin{array}{c}\text { intensità } \\
43,14 \% \\
\end{array}$}} & \\
\hline & & & & & & & \\
\hline 1 & $3,04 \%$ & 21 & $2,46 \%$ & 17 & $9,57 \%$ & 66 & $0 \%$ \\
\hline 2 & $7,68 \%$ & 53 & $5,65 \%$ & 39 & $19,86 \%$ & 137 & $25 \%$ \\
\hline 3 & $7,97 \%$ & 55 & $14,35 \%$ & 99 & $13,19 \%$ & 91 & $50 \%$ \\
\hline 4 & $21,59 \%$ & 149 & $22,90 \%$ & 158 & $10,29 \%$ & 71 & $75 \%$ \\
\hline 5 & $20,29 \%$ & 140 & $8,41 \%$ & 58 & $6,23 \%$ & 43 & $100 \%$ \\
\hline
\end{tabular}

\section{3 - Uso del catalogo della biblioteca su internet: ricerca di libri o periodici nel campo parole chiave}

Il campo "parole chiave" consente una ricerca ispirata alle tecniche dell'information retrieval. Trattandosi di una modalità di accesso all'informazione basata sulla mera rispondenza letterale delle chiavi alle parole contenute nei record bibliografici, essa richiede un maggiore impegno nella fase della valutazione dei risultati. Maggiore istruzione per un corretto uso di tale metodo potrebbero migliorare $\mathrm{i}$ risultati di questo servizio.

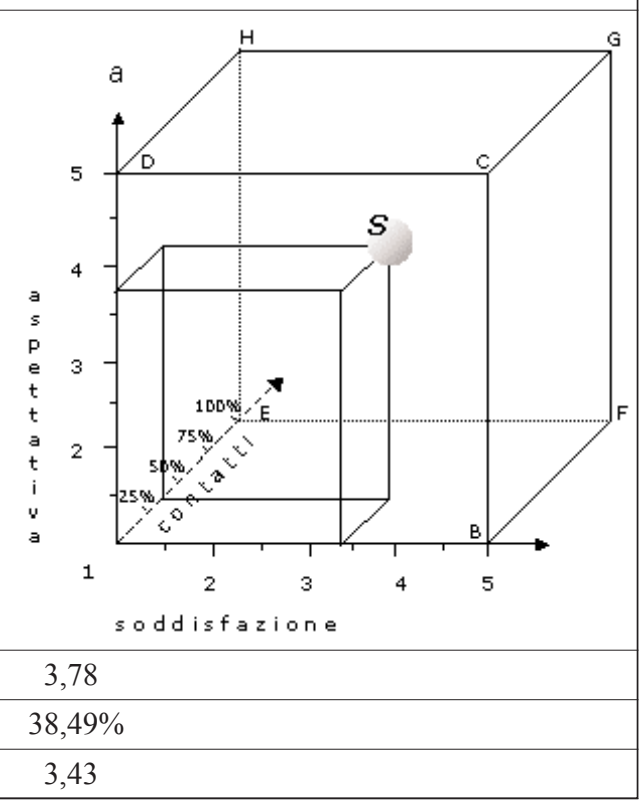




\begin{tabular}{|c|c|c|c|c|c|c|c|}
\hline \multirow[b]{3}{*}{1} & \multicolumn{6}{|c|}{ distribuzione delle risposte (stime su 27746 utenti) } & \multirow[b]{3}{*}{$0 \%$} \\
\hline & \multicolumn{2}{|c|}{ aspettativa } & \multicolumn{2}{|c|}{ soddisfazione } & \multicolumn{2}{|c|}{ intensità } & \\
\hline & $6,98 \%$ & 1440 & $4,57 \%$ & 618 & $34,49 \%$ & 7118 & \\
\hline 2 & $7,33 \%$ & 1513 & $13,67 \%$ & 1848 & $17,71 \%$ & 3656 & $25 \%$ \\
\hline 3 & $18,45 \%$ & 3807 & $29,64 \%$ & 4007 & $14,99 \%$ & 3093 & $50 \%$ \\
\hline 4 & $26,57 \%$ & 5484 & $32,77 \%$ & 4430 & $18,91 \%$ & 3903 & $75 \%$ \\
\hline 5 & $33,36 \%$ & 6886 & $15,69 \%$ & 2121 & $11,44 \%$ & 2362 & $100 \%$ \\
\hline
\end{tabular}

\begin{tabular}{|c|c|c|c|c|c|c|c|}
\hline \multirow[b]{3}{*}{1} & \multicolumn{6}{|c|}{ distribuzione delle risposte (su 1063 interviste) } & \multirow[b]{3}{*}{$0 \%$} \\
\hline & \multicolumn{2}{|c|}{ aspettativa } & \multicolumn{2}{|c|}{ soddisfazione } & \multicolumn{2}{|c|}{ intensità } & \\
\hline & $4,89 \%$ & 52 & $1,98 \%$ & 21 & $26,06 \%$ & 277 & \\
\hline 2 & $5,27 \%$ & 56 & $6,30 \%$ & 67 & $13,45 \%$ & 143 & $25 \%$ \\
\hline 3 & $19,38 \%$ & 206 & $17,12 \%$ & 182 & $11,57 \%$ & 123 & $50 \%$ \\
\hline 4 & $20,79 \%$ & 221 & $15,05 \%$ & 160 & $13,83 \%$ & 147 & $75 \%$ \\
\hline 5 & $24,27 \%$ & 258 & $8,09 \%$ & 86 & $7,71 \%$ & 82 & $100 \%$ \\
\hline
\end{tabular}

\begin{tabular}{|c|c|c|c|c|c|c|c|}
\hline & \multicolumn{6}{|c|}{ risposte del personale docente e di ricerca (su 690 interviste) } & \\
\hline & \multicolumn{2}{|c|}{ aspettativa } & \multicolumn{2}{|c|}{ soddisfazione } & \multicolumn{2}{|c|}{ intensità } & \\
\hline & \multicolumn{2}{|c|}{3,78} & \multicolumn{2}{|c|}{3,57} & \multicolumn{2}{|c|}{44,06} & \\
\hline 1 & $3,48 \%$ & 24 & $2,32 \%$ & 16 & $8,84 \%$ & 61 & $0 \%$ \\
\hline 2 & $7,25 \%$ & 50 & $4,93 \%$ & 34 & $18,12 \%$ & 125 & $25 \%$ \\
\hline 3 & $7,68 \%$ & 53 & $13,04 \%$ & 90 & $12,46 \%$ & 86 & $50 \%$ \\
\hline 4 & $19,28 \%$ & 133 & $21,74 \%$ & 150 & $10,87 \%$ & 75 & $75 \%$ \\
\hline 5 & $19,86 \%$ & 137 & $8,26 \%$ & 57 & $5,80 \%$ & 40 & $100 \%$ \\
\hline
\end{tabular}


2.4 - Uso del catalogo della biblioteca su internet: prenotazione o proroga del prestito di un libro

La possibilità di effettuare operazioni "a distanza" relativamente al prestito è apprezzata ed utilizzata con una elevata frequenza, probabilmente in seguito alla forte pressione sui servizi costruiti attorno alla circolazione documentaria e alla comodità di questa opzione. La tendenza della soddisfazione complessiva potrebbe essere spostata verso il punto ottimale e potrebbe essere consolidata con la promozione e la risoluzione dei problemi talvolta derivanti dalla gestione delle password.

4,27

\begin{tabular}{|c|c|c|c|c|c|c|c|}
\hline \multirow[b]{3}{*}{1} & \multicolumn{6}{|c|}{ distribuzione delle risposte (stime su 27746 utenti) } & \multirow[b]{3}{*}{$0 \%$} \\
\hline & \multicolumn{2}{|c|}{ aspettativa } & \multicolumn{2}{|c|}{ soddisfazione } & \multicolumn{2}{|c|}{ intensità } & \\
\hline & $4,73 \%$ & 977 & $3,39 \%$ & 496 & $29,19 \%$ & 6024 & \\
\hline 2 & $2,21 \%$ & 457 & $7,74 \%$ & 1131 & $16,23 \%$ & 3349 & $25 \%$ \\
\hline 3 & $9,47 \%$ & 1954 & $18,21 \%$ & 2662 & $14,95 \%$ & 3086 & $50 \%$ \\
\hline 4 & $23,28 \%$ & 4804 & $32,89 \%$ & 4807 & $19,12 \%$ & 3946 & $75 \%$ \\
\hline 5 & $53,54 \%$ & 11051 & $31,95 \%$ & 4669 & $16,42 \%$ & 3389 & $100 \%$ \\
\hline
\end{tabular}

\begin{tabular}{|c|c|c|c|c|c|c|c|}
\hline \multirow[b]{3}{*}{1} & \multicolumn{6}{|c|}{ distribuzione delle risposte (su 1063 interviste) } & \multirow[b]{3}{*}{$0 \%$} \\
\hline & \multicolumn{2}{|c|}{ aspettativa } & \multicolumn{2}{|c|}{ soddisfazione } & \multicolumn{2}{|c|}{ intensità } & \\
\hline & $3,39 \%$ & 36 & $1,79 \%$ & 19 & $20,60 \%$ & 219 & \\
\hline 2 & $1,32 \%$ & 14 & $3,67 \%$ & 39 & $11,38 \%$ & 121 & $25 \%$ \\
\hline 3 & $11,38 \%$ & 121 & $12,14 \%$ & 129 & $11,19 \%$ & 119 & $50 \%$ \\
\hline 4 & $17,22 \%$ & 183 & $18,53 \%$ & 197 & $14,86 \%$ & 158 & $75 \%$ \\
\hline 5 & $41,30 \%$ & 439 & $17,87 \%$ & 190 & $13,55 \%$ & 144 & $100 \%$ \\
\hline
\end{tabular}




\begin{tabular}{|c|c|c|c|c|c|c|c|}
\hline & \multicolumn{6}{|c|}{ risposte del personale docente e di ricerca (su 690 interviste) } & \\
\hline & \multirow{2}{*}{\multicolumn{2}{|c|}{ aspettativa }} & \multirow{2}{*}{\multicolumn{2}{|c|}{$\begin{array}{c}\text { soddisfazione } \\
3,35\end{array}$}} & \multirow{2}{*}{\multicolumn{2}{|c|}{$\begin{array}{c}\text { intensità } \\
20,61 \%\end{array}$}} & \\
\hline & & & & & & & \\
\hline 1 & $11,01 \%$ & 76 & $28,12 \%$ & 194 & $3,04 \%$ & 21 & $0 \%$ \\
\hline 2 & $8,55 \%$ & 59 & $8,84 \%$ & 61 & $1,88 \%$ & 13 & $25 \%$ \\
\hline 3 & $8,99 \%$ & 62 & $4,78 \%$ & 33 & $12,75 \%$ & 88 & $50 \%$ \\
\hline 4 & $12,46 \%$ & 86 & $3,33 \%$ & 23 & $8,70 \%$ & 60 & $75 \%$ \\
\hline 5 & $7,54 \%$ & 52 & $2,75 \%$ & 19 & $5,07 \%$ & 35 & $100 \%$ \\
\hline
\end{tabular}

\section{5 - Precisione e rapidità nella distribuzione di libri e periodici dai magazzini}

I valori assunti da questo attributo confermano l'importanza assunta dalla circolazione documentaria. La pressione esercitata su questo processo bibliotecario suggerisce una ottimizzazione dello stesso, spesso legato alle caratteristiche strutturali architettoniche delle biblioteche. Un esame delle procedure di recupero (ad esempio, sistema di raccolta delle richieste, orari o modalità di prelievo dai magazzini e di distribuzione dei libri, diversa organizzazione della risorsa umana) potrebbe migliorare la resa dell'attributo.

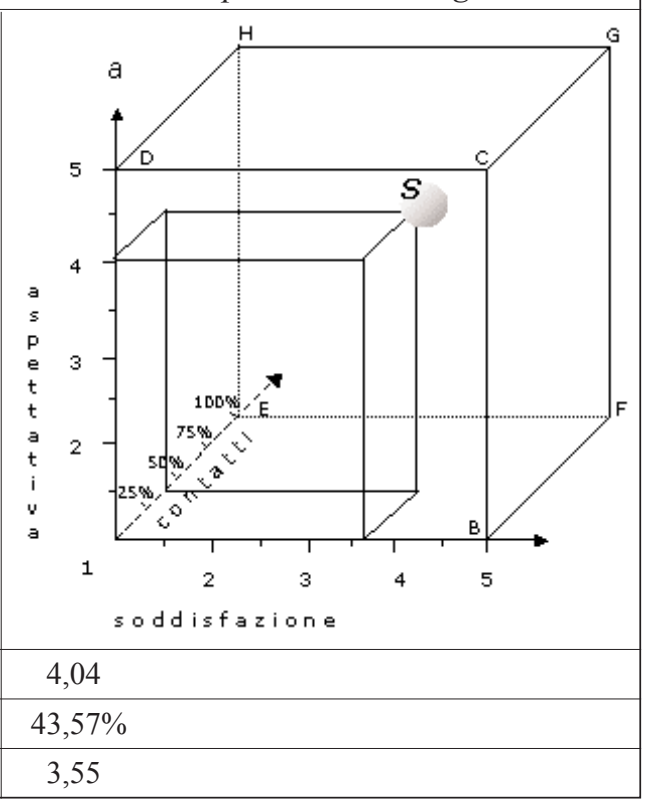

\begin{tabular}{|c|c|c|c|c|c|c|c|}
\hline \multirow[b]{3}{*}{1} & \multicolumn{6}{|c|}{ distribuzione delle risposte (stime su 27746 utenti) } & \multirow[b]{3}{*}{$0 \%$} \\
\hline & \multicolumn{2}{|c|}{ aspettativa } & \multicolumn{2}{|c|}{ soddisfazione } & \multicolumn{2}{|c|}{ intensità } & \\
\hline & $4,54 \%$ & 1259 & $4,07 \%$ & 852 & $24,59 \%$ & 6823 & \\
\hline 2 & $4,21 \%$ & 1167 & $12,12 \%$ & 2535 & $18,20 \%$ & 5051 & $25 \%$ \\
\hline 3 & $15,80 \%$ & 4384 & $27,75 \%$ & 5806 & $22,56 \%$ & 6259 & $50 \%$ \\
\hline 4 & $26,71 \%$ & 7411 & $31,53 \%$ & 6597 & $19,26 \%$ & 5344 & $75 \%$ \\
\hline 5 & $41,81 \%$ & 11601 & $20,97 \%$ & 4387 & $11,67 \%$ & 3239 & $100 \%$ \\
\hline
\end{tabular}




\section{ROBERTO VENTURA}

\begin{tabular}{|c|c|c|c|c|c|c|c|}
\hline \multirow[b]{3}{*}{1} & \multicolumn{6}{|c|}{ distribuzione delle risposte (su 1063 interviste) } & \multirow[b]{3}{*}{$0 \%$} \\
\hline & \multicolumn{2}{|c|}{ aspettativa } & \multicolumn{2}{|c|}{ soddisfazione } & \multicolumn{2}{|c|}{ intensità } & \\
\hline & $3,86 \%$ & 41 & $2,63 \%$ & 28 & $25,78 \%$ & 274 & \\
\hline 2 & $3,95 \%$ & 42 & $8,00 \%$ & 85 & $18,63 \%$ & 198 & $25 \%$ \\
\hline 3 & $20,13 \%$ & 214 & $20,60 \%$ & 219 & $21,83 \%$ & 232 & $50 \%$ \\
\hline 4 & $26,72 \%$ & 284 & $23,89 \%$ & 254 & $19,19 \%$ & 204 & $75 \%$ \\
\hline 5 & $41,49 \%$ & 441 & $16,27 \%$ & 173 & $10,82 \%$ & 115 & $100 \%$ \\
\hline
\end{tabular}

\begin{tabular}{|c|c|c|c|c|c|c|c|}
\hline & \multicolumn{6}{|c|}{ risposte del personale docente e di ricerca (su 690 interviste) } & \\
\hline & \multicolumn{2}{|c|}{ aspettativa } & \multicolumn{2}{|c|}{ soddisfazione } & \multicolumn{2}{|c|}{ intensità } & \\
\hline & \multicolumn{2}{|c|}{3,99} & \multicolumn{2}{|c|}{3,51} & \multicolumn{2}{|c|}{$43,47 \%$} & \\
\hline 1 & $2,90 \%$ & 20 & $4,49 \%$ & 89 & $12,90 \%$ & 31 & $0 \%$ \\
\hline 2 & $5,94 \%$ & 41 & $6,96 \%$ & 156 & $22,61 \%$ & 48 & $25 \%$ \\
\hline 3 & $10,87 \%$ & 75 & $18,12 \%$ & 115 & $16,67 \%$ & 125 & $50 \%$ \\
\hline 4 & $25,51 \%$ & 176 & $28,26 \%$ & 97 & $14,06 \%$ & 195 & $75 \%$ \\
\hline 5 & $31,01 \%$ & 214 & $11,74 \%$ & 52 & $7,54 \%$ & 81 & $100 \%$ \\
\hline
\end{tabular}

\section{6 - Facilità del recupero di libri e periodici dagli scaffali liberamente accessibili}

ai lettori

Aspettativa e uso per lo scaffale aperto sembrano evidenziare il favore degli studenti verso questo metodo di collocazione. La tendenza dell'attributo è buona e potrà essere migliorata con una manutenzione più frequente - ad esempio, durante le ore di chiusura della biblioteca - o una più efficace selezione dei libri da collocare.

4,24




\begin{tabular}{|c|c|c|c|c|c|c|c|}
\hline \multirow[b]{3}{*}{1} & \multicolumn{6}{|c|}{ distribuzione delle risposte (stime su 27746 utenti) } & \multirow[b]{3}{*}{$0 \%$} \\
\hline & \multicolumn{2}{|c|}{ aspettativa } & \multicolumn{2}{|c|}{ soddisfazione } & \multicolumn{2}{|c|}{ intensità } & \\
\hline & $2,83 \%$ & 784 & $6,62 \%$ & 1615 & $12,13 \%$ & 3365 & \\
\hline 2 & $4,29 \%$ & 1191 & $16,61 \%$ & 4025 & $15,03 \%$ & 4169 & $25 \%$ \\
\hline 3 & $10,56 \%$ & 2931 & $23,45 \%$ & 5717 & $23,38 \%$ & 6486 & $50 \%$ \\
\hline 4 & $28,28 \%$ & 7848 & $33,32 \%$ & 8123 & $28,30 \%$ & 7851 & $75 \%$ \\
\hline 5 & $50,69 \%$ & 14064 & $18,42 \%$ & 4492 & $18,90 \%$ & 5244 & $100 \%$ \\
\hline
\end{tabular}

\begin{tabular}{|c|c|c|c|c|c|c|c|}
\hline \multirow[b]{3}{*}{1} & \multicolumn{6}{|c|}{ distribuzione delle risposte (su 1063 interviste) } & \multirow[b]{3}{*}{$0 \%$} \\
\hline & \multicolumn{2}{|c|}{ aspettativa } & \multicolumn{2}{|c|}{ soddisfazione } & \multicolumn{2}{|c|}{ intensità } & \\
\hline & $2,54 \%$ & 27 & $4,70 \%$ & 50 & $12,32 \%$ & 131 & \\
\hline 2 & $4,80 \%$ & 51 & $12,51 \%$ & 133 & $15,80 \%$ & 168 & $25 \%$ \\
\hline 3 & $14,49 \%$ & 154 & $22,58 \%$ & 240 & $23,80 \%$ & 253 & $50 \%$ \\
\hline 4 & $28,50 \%$ & 303 & $30,39 \%$ & 323 & $28,22 \%$ & 300 & $75 \%$ \\
\hline 5 & $49,67 \%$ & 528 & $17,50 \%$ & 186 & $17,87 \%$ & 190 & $100 \%$ \\
\hline
\end{tabular}

\begin{tabular}{|c|c|c|c|c|c|c|c|}
\hline & \multicolumn{6}{|c|}{ risposte del personale docente e di ricerca (su 690 interviste) } & \\
\hline & \multicolumn{2}{|c|}{ aspettativa } & \multicolumn{2}{|c|}{ soddisfazione } & \multicolumn{2}{|c|}{ intensità } & \\
\hline & \multicolumn{2}{|c|}{4,08} & \multicolumn{2}{|c|}{3,40} & \multicolumn{2}{|c|}{$56,73 \%$} & \\
\hline 1 & $2,32 \%$ & 16 & $6,52 \%$ & 55 & $7,97 \%$ & 45 & $0 \%$ \\
\hline 2 & $6,23 \%$ & 43 & $15,22 \%$ & 70 & $10,14 \%$ & 105 & $25 \%$ \\
\hline 3 & $9,86 \%$ & 68 & $18,55 \%$ & 103 & $14,93 \%$ & 128 & $50 \%$ \\
\hline 4 & $24,64 \%$ & 170 & $24,35 \%$ & 197 & $28,55 \%$ & 168 & $75 \%$ \\
\hline 5 & $35,36 \%$ & 244 & $12,32 \%$ & 97 & $14,06 \%$ & 85 & $100 \%$ \\
\hline
\end{tabular}




\begin{tabular}{|l|l|l|}
\hline \multicolumn{2}{|c|}{2.7 - Disponibilità in sala di lettura di posti a sedere per l'uso di materiale } \\
della biblioteca \\
L'attributo in questione è legato alle caratteri- \\
stiche strutturali ed architettoniche della bi- \\
blioteca, sulle quali non è sempre possibile \\
intervenire. Lo stacco tra aspettativa e soddi- \\
sfazione è notevole, ed il grado di soddisfazio- \\
ne è quasi coincidente con l'indifferenza: la \\
performance è dunque suscettibile di scivolare \\
dell'area dell'insoddisfazione. L'utenza di- \\
chiara di consultare materiale della biblioteca \\
in occasione di più del 50\% delle visite. O si \\
aumenta la disponibilità di posti nelle sale di \\
lettura o si vincola, nei casi più critici, l'uso \\
della sala di lettura all'uso effettivo delle col- \\
lezioni.
\end{tabular}

\begin{tabular}{|c|c|c|c|c|c|c|c|}
\hline \multirow[b]{3}{*}{1} & \multicolumn{6}{|c|}{ distribuzione delle risposte (stime su 27746 utenti) } & \multirow[b]{3}{*}{$0 \%$} \\
\hline & \multicolumn{2}{|c|}{ aspettativa } & \multicolumn{2}{|c|}{ soddisfazione } & \multicolumn{2}{|c|}{ intensità } & \\
\hline & $2,63 \%$ & 731 & $10,68 \%$ & 2513 & $15,22 \%$ & 4224 & \\
\hline 2 & $4,34 \%$ & 1203 & $23,48 \%$ & 5523 & $18,29 \%$ & 5076 & $25 \%$ \\
\hline 3 & $13,05 \%$ & 3621 & $25,06 \%$ & 5895 & $22,90 \%$ & 6355 & $50 \%$ \\
\hline 4 & $22,81 \%$ & 6328 & $25,37 \%$ & 5967 & $22,71 \%$ & 6301 & $75 \%$ \\
\hline 5 & $55,14 \%$ & 15300 & $13,65 \%$ & 3211 & $19,51 \%$ & 5412 & $100 \%$ \\
\hline
\end{tabular}

\begin{tabular}{|c|c|c|c|c|c|c|c|}
\hline \multirow[b]{3}{*}{1} & \multicolumn{6}{|c|}{ distribuzione delle risposte (su 1063 interviste) } & \multirow[b]{3}{*}{$0 \%$} \\
\hline & \multicolumn{2}{|c|}{ aspettativa } & \multicolumn{2}{|c|}{ soddisfazione } & \multicolumn{2}{|c|}{ intensità } & \\
\hline & $2,63 \%$ & 28 & $9,13 \%$ & 97 & $15,33 \%$ & 163 & \\
\hline 2 & $4,14 \%$ & 44 & $19,29 \%$ & 205 & $18,06 \%$ & 192 & $25 \%$ \\
\hline 3 & $13,26 \%$ & 141 & $21,07 \%$ & 224 & $20,88 \%$ & 222 & $50 \%$ \\
\hline 4 & $22,48 \%$ & 239 & $21,83 \%$ & 232 & $23,89 \%$ & 254 & $75 \%$ \\
\hline 5 & $57,48 \%$ & 611 & $13,36 \%$ & 142 & $20,79 \%$ & 221 & $100 \%$ \\
\hline
\end{tabular}


L'INDAGINE SULLA SODDISFAZIONE DEGLI UTENTI: METODOLOGIA E VALUTAZIONE BIBLIOTECARIA

\begin{tabular}{|c|c|c|c|c|c|c|c|}
\hline & \multicolumn{6}{|c|}{ risposte del personale docente e di ricerca (su 690 interviste) } & \\
\hline & \multicolumn{2}{|c|}{ aspettativa } & \multicolumn{2}{|c|}{ soddisfazione } & \multicolumn{2}{|c|}{ intensità } & \\
\hline & \multicolumn{2}{|c|}{2,86} & \multicolumn{2}{|c|}{3,16} & \multicolumn{2}{|c|}{33,65} & \\
\hline 1 & 2,86 & 98 & $7,25 \%$ & 50 & $20,58 \%$ & 142 & $0 \%$ \\
\hline 2 & $14,20 \%$ & 143 & $9,57 \%$ & 66 & $27,54 \%$ & 190 & $25 \%$ \\
\hline 3 & $20,72 \%$ & 102 & $24,35 \%$ & 168 & $14,20 \%$ & 98 & $50 \%$ \\
\hline 4 & $14,78 \%$ & 100 & $16,81 \%$ & 116 & $8,99 \%$ & 62 & $75 \%$ \\
\hline 5 & $14,49 \%$ & 83 & $9,13 \%$ & 63 & $4,93 \%$ & 34 & $100 \%$ \\
\hline
\end{tabular}

\section{8 - Silenzio in sala di lettura}

Nonostante la pressione dell'utenza sulle sale di lettura, gli ambienti risultano in generale abbastanza silenziosi, sebbene l'attributo possa essere migliorato. Nelle biblioteche italiane non è infrequente il sovrapporsi di più funzioni nel medesimo spazio e ciò può creare una eccessiva animazione: maggiore attenzione da parte del personale nonché una maggiore sensibilizzazione dell'utenza per questo tratto qualitativo potrebbero produrre un miglioramento dei risultati.

4,47 soddisfazione

\begin{tabular}{|c|c|c|c|c|c|c|c|}
\hline \multirow[b]{3}{*}{1} & \multicolumn{6}{|c|}{ distribuzione delle risposte (stime su 27746 utenti) } & \multirow[b]{3}{*}{$0 \%$} \\
\hline & \multicolumn{2}{|c|}{ aspettativa } & \multicolumn{2}{|c|}{ soddisfazione } & \multicolumn{2}{|c|}{ intensità } & \\
\hline & $0,86 \%$ & 202 & $6,34 \%$ & 1435 & $3,82 \%$ & 898 & \\
\hline 2 & $2,63 \%$ & 554 & $12,05 \%$ & 2757 & $16,86 \%$ & 3967 & $25 \%$ \\
\hline 3 & $9,60 \%$ & 2257 & $26,65 \%$ & 6030 & $26,35 \%$ & 6199 & $50 \%$ \\
\hline 4 & $23,14 \%$ & 5444 & $30,45 \%$ & 6889 & $27,57 \%$ & 6486 & $75 \%$ \\
\hline 5 & $63,06 \%$ & 14823 & $22,54 \%$ & 5099 & $22,57 \%$ & 5309 & $100 \%$ \\
\hline
\end{tabular}




\section{ROBERTO VENTURA}

\begin{tabular}{|c|c|c|c|c|c|c|c|}
\hline \multirow[b]{3}{*}{1} & \multicolumn{6}{|c|}{ distribuzione delle risposte (su 1063 interviste) } & \multirow[b]{3}{*}{$0 \%$} \\
\hline & \multicolumn{2}{|c|}{ aspettativa } & \multicolumn{2}{|c|}{ soddisfazione } & \multicolumn{2}{|c|}{ intensità } & \\
\hline & $0,85 \%$ & 9 & $5,55 \%$ & 59 & $3,57 \%$ & 38 & \\
\hline 2 & $2,07 \%$ & 22 & $9,13 \%$ & 97 & $13,45 \%$ & 143 & $25 \%$ \\
\hline 3 & $8,18 \%$ & 87 & $22,58 \%$ & 240 & $21,92 \%$ & 233 & $50 \%$ \\
\hline 4 & $20,04 \%$ & 213 & $25,02 \%$ & 266 & $23,99 \%$ & 255 & $75 \%$ \\
\hline 5 & $53,53 \%$ & 569 & $18,81 \%$ & 200 & $19,47 \%$ & 207 & $100 \%$ \\
\hline
\end{tabular}

\begin{tabular}{|c|c|c|c|c|c|c|c|}
\hline & \multicolumn{6}{|c|}{ risposte del personale docente e di ricerca (su 690 interviste) } & \\
\hline & \multirow{2}{*}{\multicolumn{2}{|c|}{$\begin{array}{c}\text { aspettativa } \\
3,95\end{array}$}} & \multirow{2}{*}{\multicolumn{2}{|c|}{$\begin{array}{c}\text { soddisfazione } \\
3,52\end{array}$}} & \multirow{2}{*}{\multicolumn{2}{|c|}{$\begin{array}{c}\text { intensità } \\
63,04 \% \\
\end{array}$}} & \\
\hline & & & & & & & \\
\hline 1 & $1,59 \%$ & 11 & $3,48 \%$ & 24 & $2,32 \%$ & 16 & $0 \%$ \\
\hline 2 & $5,51 \%$ & 38 & $7,54 \%$ & 52 & $7,25 \%$ & 50 & $25 \%$ \\
\hline 3 & $8,12 \%$ & 56 & $11,16 \%$ & 77 & $13,77 \%$ & 95 & $50 \%$ \\
\hline 4 & $19,42 \%$ & 134 & $22,03 \%$ & 152 & $21,16 \%$ & 146 & $75 \%$ \\
\hline 5 & $21,16 \%$ & 146 & $10,43 \%$ & 72 & $9,42 \%$ & 65 & $100 \%$ \\
\hline
\end{tabular}

\section{9 - Comfort e luminosità della sala di lettura}

La performance di questo attributo risulta collegata con la performance dei precedenti due: pur tendendo verso il punto ottimale è possibile un miglioramento attraverso una maggiore attenzione alla qualità delle infrastrutture (arredamento, disponibilità di guardaroba o armadietti per gli utenti, introduzione di postazioni di lettura e di lavoro singole ed adeguatamente attrezzate).

4,48


L'INDAGINE SULLA SODDISFAZIONE DEGLI UTENTI: METODOLOGIA E VALUTAZIONE BIBLIOTECARIA

\begin{tabular}{|c|c|c|c|c|c|c|c|}
\hline \multirow[b]{3}{*}{1} & \multicolumn{6}{|c|}{ distribuzione delle risposte (stime su 27746 utenti) } & \multirow[b]{3}{*}{$0 \%$} \\
\hline & \multicolumn{2}{|c|}{ aspettativa } & \multicolumn{2}{|c|}{ soddisfazione } & \multicolumn{2}{|c|}{ intensità } & \\
\hline & $0,75 \%$ & 176 & $4,12 \%$ & 953 & $1,67 \%$ & 392 & \\
\hline 2 & $1,64 \%$ & 386 & $13,08 \%$ & 3025 & $15,28 \%$ & 3595 & $25 \%$ \\
\hline 3 & $9,04 \%$ & 2127 & $28,43 \%$ & 6576 & $26,76 \%$ & 6295 & $50 \%$ \\
\hline 4 & $25,82 \%$ & 6074 & $30,77 \%$ & 7116 & $30,30 \%$ & 7128 & $75 \%$ \\
\hline 5 & $61,67 \%$ & 14506 & $21,97 \%$ & 5082 & $23,43 \%$ & 5512 & $100 \%$ \\
\hline
\end{tabular}

\begin{tabular}{|c|c|c|c|c|c|c|c|}
\hline \multirow[b]{3}{*}{1} & \multicolumn{6}{|c|}{ distribuzione delle risposte (su 1063 interviste) } & \multirow[b]{3}{*}{$0 \%$} \\
\hline & \multicolumn{2}{|c|}{ aspettativa } & \multicolumn{2}{|c|}{ soddisfazione } & \multicolumn{2}{|c|}{ intensità } & \\
\hline & $0,66 \%$ & 7 & $3,20 \%$ & 34 & $1,22 \%$ & 13 & \\
\hline 2 & $1,32 \%$ & 14 & $9,97 \%$ & 106 & $11,57 \%$ & 123 & $25 \%$ \\
\hline 3 & $8,37 \%$ & 89 & $23,33 \%$ & 248 & $22,01 \%$ & 234 & $50 \%$ \\
\hline 4 & $22,11 \%$ & 235 & $27,56 \%$ & 293 & $26,90 \%$ & 286 & $75 \%$ \\
\hline 5 & $52,21 \%$ & 555 & $19,38 \%$ & 206 & $20,88 \%$ & 222 & $100 \%$ \\
\hline
\end{tabular}

\begin{tabular}{|c|c|c|c|c|c|c|c|}
\hline & \multicolumn{6}{|c|}{ risposte del personale docente e di ricerca (su 690 interviste) } & \\
\hline & \multicolumn{2}{|c|}{ aspettativa } & \multicolumn{2}{|c|}{ soddisfazione } & \multicolumn{2}{|c|}{ intensità } & \\
\hline & \multicolumn{2}{|c|}{3,85} & \multicolumn{2}{|c|}{3,46} & \multicolumn{2}{|c|}{$62,60 \%$} & \\
\hline 1 & $1,30 \%$ & 9 & $3,77 \%$ & 26 & $1,74 \%$ & 12 & $0 \%$ \\
\hline 2 & $5,36 \%$ & 37 & $8,12 \%$ & 56 & $8,84 \%$ & 61 & $25 \%$ \\
\hline 3 & $10,00 \%$ & 69 & $11,59 \%$ & 80 & $13,33 \%$ & 92 & $50 \%$ \\
\hline 4 & $22,32 \%$ & 154 & $20,87 \%$ & 144 & $19,86 \%$ & 137 & $75 \%$ \\
\hline 5 & $16,38 \%$ & 113 & $9,71 \%$ & 67 & $9,71 \%$ & 67 & $100 \%$ \\
\hline
\end{tabular}




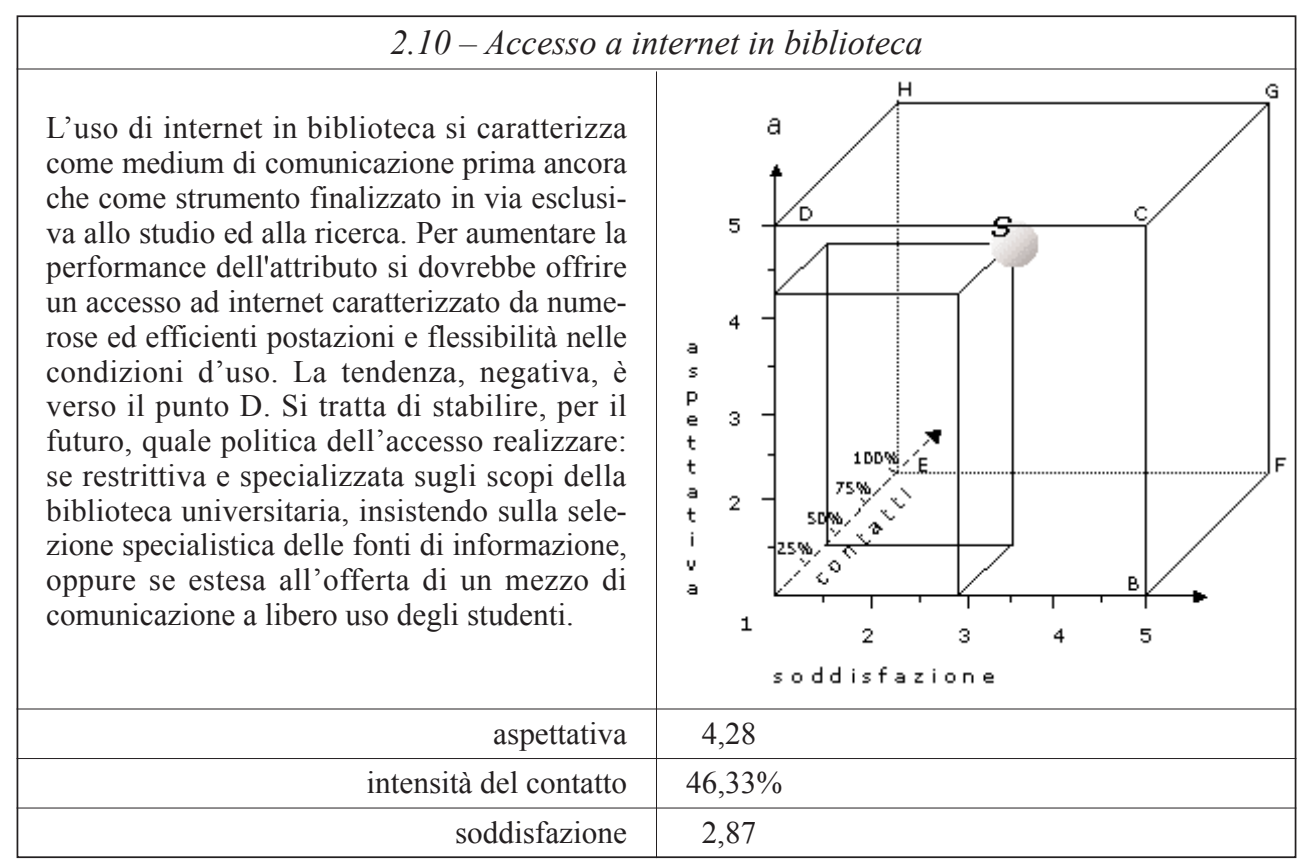

\begin{tabular}{|c|c|c|c|c|c|c|c|}
\hline \multirow[b]{3}{*}{1} & \multicolumn{6}{|c|}{ distribuzione delle risposte (stime su 27746 utenti) } & \multirow[b]{3}{*}{$0 \%$} \\
\hline & \multicolumn{2}{|c|}{ aspettativa } & \multicolumn{2}{|c|}{ soddisfazione } & \multicolumn{2}{|c|}{ intensità } & \\
\hline & $4,71 \%$ & 1307 & $15,70 \%$ & 3309 & $24,03 \%$ & 6666 & \\
\hline 2 & $4,60 \%$ & 1276 & $25,38 \%$ & 5350 & $19,29 \%$ & 5352 & $25 \%$ \\
\hline 3 & $8,41 \%$ & 2333 & $24,58 \%$ & 5182 & $18,09 \%$ & 5019 & $50 \%$ \\
\hline 4 & $20,19 \%$ & 5603 & $19,01 \%$ & 4007 & $20,44 \%$ & 5672 & $75 \%$ \\
\hline 5 & $59,42 \%$ & 16486 & $12,61 \%$ & 2659 & $16,25 \%$ & 4508 & $100 \%$ \\
\hline
\end{tabular}

\begin{tabular}{|c|c|c|c|c|c|c|c|}
\hline \multirow[b]{3}{*}{1} & \multicolumn{6}{|c|}{ distribuzione delle risposte (su 1063 interviste) } & \multirow[b]{3}{*}{$0 \%$} \\
\hline & \multicolumn{2}{|c|}{ aspettativa } & \multicolumn{2}{|c|}{ soddisfazione } & \multicolumn{2}{|c|}{ intensità } & \\
\hline & $4,42 \%$ & 47 & $11,38 \%$ & 121 & $23,89 \%$ & 254 & \\
\hline 2 & $4,70 \%$ & 50 & $18,16 \%$ & 193 & $20,04 \%$ & 213 & $25 \%$ \\
\hline 3 & $10,82 \%$ & 115 & $20,79 \%$ & 221 & $18,91 \%$ & 201 & $50 \%$ \\
\hline 4 & $21,54 \%$ & 229 & $15,43 \%$ & 164 & $19,76 \%$ & 210 & $75 \%$ \\
\hline 5 & $58,51 \%$ & 622 & $10,35 \%$ & 110 & $15,62 \%$ & 166 & $100 \%$ \\
\hline
\end{tabular}


L'INDAGINE SULLA SODDISFAZIONE DEGLI UTENTI: METODOLOGIA E VALUTAZIONE BIBLIOTECARIA

\begin{tabular}{|c|c|c|c|c|c|c|c|}
\hline & \multicolumn{6}{|c|}{ risposte del personale docente e di ricerca (su 690 interviste) } & \\
\hline & \multicolumn{2}{|c|}{ aspettativa } & \multicolumn{2}{|c|}{ soddisfazione } & \multicolumn{2}{|c|}{ intensità } & \\
\hline & \multicolumn{2}{|c|}{3,20} & \multicolumn{2}{|c|}{3,22} & \multicolumn{2}{|c|}{$29,81 \%$} & \\
\hline 1 & $17,68 \%$ & 122 & $5,51 \%$ & 38 & $32,32 \%$ & 223 & $0 \%$ \\
\hline 2 & $10,72 \%$ & 74 & $8,26 \%$ & 57 & $17,54 \%$ & 121 & $25 \%$ \\
\hline 3 & $9,42 \%$ & 65 & $19,86 \%$ & 137 & $9,13 \%$ & 63 & $50 \%$ \\
\hline 4 & $14,06 \%$ & 97 & $16,23 \%$ & 112 & $9,42 \%$ & 65 & $75 \%$ \\
\hline 5 & $23,77 \%$ & 164 & $7,97 \%$ & 55 & $6,23 \%$ & 43 & $100 \%$ \\
\hline
\end{tabular}

\subsection{1 - Prestito interbibliotecario: recupero di libri e articoli da altre biblioteche universitarie}

L'aspettativa per questo servizio è da ritenersi elevata, considerando che l'utenza ricorre ad esso, solitamente, per ragioni di ricerca piuttosto che di studio "manualistico". L'intensità del contatto è di conseguenza conforme al grado di specializzazione del servizio. La performance è suscettibile di un miglioramento, agendo sulla standardizzazione a livello di SBA o, nelle sedi locali. su una o più componenti del servizio (velocità di recupero, modalità di comunicazione con gli utenti).

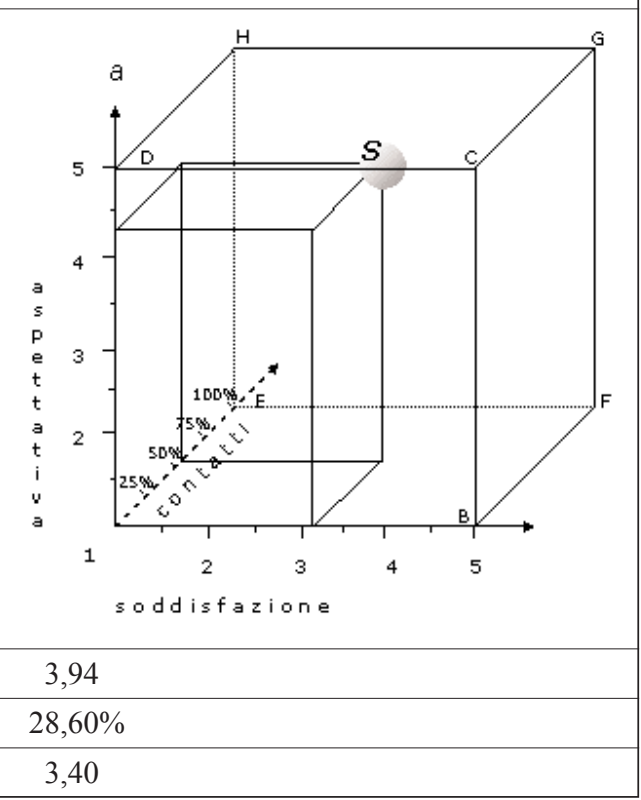

\begin{tabular}{|c|r|r|r|r|r|r|r|}
\cline { 2 - 7 } \multicolumn{1}{c|}{} & \multicolumn{6}{c|}{ distribuzione delle risposte (stime su 27746 utenti) } & \multirow{2}{*}{} \\
\cline { 2 - 7 } \multicolumn{1}{c|}{} & \multicolumn{2}{c|}{ aspettativa } & \multicolumn{2}{c|}{ soddisfazione } & \multicolumn{2}{c|}{ intensità } & \\
\hline 1 & $6,43 \%$ & 1783 & $5,35 \%$ & 808 & $45,60 \%$ & 12653 & $0 \%$ \\
\hline 2 & $6,40 \%$ & 1776 & $13,76 \%$ & 2077 & $17,49 \%$ & 4853 & $25 \%$ \\
\hline 3 & $15,07 \%$ & 4181 & $27,73 \%$ & 4186 & $14,61 \%$ & 4054 & $50 \%$ \\
\hline 4 & $19,61 \%$ & 5442 & $31,07 \%$ & 4690 & $11,55 \%$ & 3205 & $75 \%$ \\
\hline 5 & $42,09 \%$ & 11679 & $15,25 \%$ & 2302 & $7,28 \%$ & 2019 & $100 \%$ \\
\hline
\end{tabular}




\section{ROBERTO VENTURA}

\begin{tabular}{|c|r|r|r|r|r|r|r|}
\cline { 2 - 7 } \multicolumn{1}{c|}{} & \multicolumn{6}{c|}{ distribuzione delle risposte (su 1063 interviste) } & \multicolumn{1}{c}{} \\
\cline { 2 - 7 } \multicolumn{1}{c|}{ aspettativa } & \multicolumn{2}{c|}{ soddisfazione } & \multicolumn{2}{c|}{ intensità } & \multicolumn{1}{c|}{} \\
\hline 1 & $6,30 \%$ & 67 & $3,10 \%$ & 33 & $45,44 \%$ & 483 & $0 \%$ \\
\hline 2 & $6,11 \%$ & 65 & $6,87 \%$ & 73 & $18,06 \%$ & 192 & $25 \%$ \\
\hline 3 & $25,78 \%$ & 274 & $18,16 \%$ & 193 & $14,02 \%$ & 149 & $50 \%$ \\
\hline 4 & $20,04 \%$ & 213 & $17,40 \%$ & 185 & $11,57 \%$ & 123 & $75 \%$ \\
\hline 5 & $41,77 \%$ & 444 & $9,03 \%$ & 96 & $7,34 \%$ & 78 & $100 \%$ \\
\hline
\end{tabular}

\begin{tabular}{|c|c|c|c|c|c|c|c|}
\hline & \multicolumn{6}{|c|}{ risposte del personale docente e di ricerca (su 690 interviste) } & \\
\hline & \multicolumn{2}{|c|}{ aspettativa } & \multirow{2}{*}{\multicolumn{2}{|c|}{$\begin{array}{c}\text { soddisfazione } \\
3,35 \\
\end{array}$}} & \multirow{2}{*}{\multicolumn{2}{|c|}{$\begin{array}{c}\text { intensità } \\
25,71\end{array}$}} & \\
\hline & \multicolumn{2}{|c|}{3,83} & & & & & \\
\hline 1 & $4,49 \%$ & 31 & $7,10 \%$ & 49 & $22,90 \%$ & 158 & $0 \%$ \\
\hline 2 & $9,42 \%$ & 65 & $8,41 \%$ & 58 & $32,32 \%$ & 223 & $25 \%$ \\
\hline 3 & $7,25 \%$ & 50 & $13,91 \%$ & 96 & $9,42 \%$ & 65 & $50 \%$ \\
\hline 4 & $25,94 \%$ & 179 & $20,87 \%$ & 144 & $4,93 \%$ & 34 & $75 \%$ \\
\hline 5 & $26,67 \%$ & 184 & $11,59 \%$ & 80 & $1,88 \%$ & 13 & $100 \%$ \\
\hline
\end{tabular}




\section{Qualità del reference, dell'orientamento e della comunicazione}

\section{1 - Facilità del recupero e consultazione di bibliografie, repertori, enciclopedie}

L'utenza tende a giudicare nel complesso importante la fruibilità dell'apparato cartaceo di consultazione, e l'intensità d'uso di tale materiale mostra le caratteristiche della specializzazione. La soddisfazione per questo attributo è probabilmente legata alle condizioni ambientali generali della fruizione del materiale documentario posseduto dalla biblioteca. L'aggiornamento di tale apparato e la collocazione a scaffale aperto, dove non attuata, potrebbero incrementare il grado di soddisfazione.

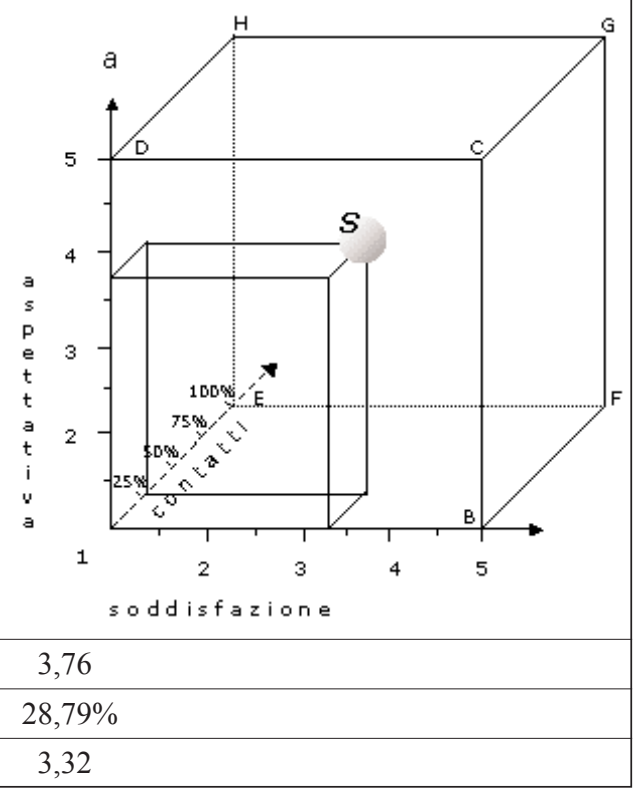

\begin{tabular}{|c|c|c|c|c|c|c|c|}
\hline \multirow[b]{3}{*}{1} & \multicolumn{6}{|c|}{ distribuzione delle risposte (stime su 27746 utenti) } & \multirow[b]{3}{*}{$0 \%$} \\
\hline & \multicolumn{2}{|c|}{ aspettativa } & \multicolumn{2}{|c|}{ soddisfazione } & \multicolumn{2}{|c|}{ intensità } & \\
\hline & $6,78 \%$ & 1882 & $3,28 \%$ & 546 & $40,08 \%$ & 11119 & \\
\hline 2 & $6,89 \%$ & 1912 & $13,00 \%$ & 2162 & $20,80 \%$ & 5771 & $25 \%$ \\
\hline 3 & $20,63 \%$ & 5725 & $36,50 \%$ & 6069 & $20,52 \%$ & 5692 & $50 \%$ \\
\hline 4 & $24,09 \%$ & 6683 & $31,83 \%$ & 5292 & $11,51 \%$ & 3193 & $75 \%$ \\
\hline 5 & $32,83 \%$ & 9109 & $8,96 \%$ & 1489 & $3,74 \%$ & 1039 & $100 \%$ \\
\hline
\end{tabular}

\begin{tabular}{|c|c|c|c|c|c|c|c|}
\hline \multirow[b]{3}{*}{1} & \multicolumn{6}{|c|}{ distribuzione delle risposte (su 1063 interviste) } & \multirow[b]{3}{*}{$0 \%$} \\
\hline & \multicolumn{2}{|c|}{ aspettativa } & \multicolumn{2}{|c|}{ soddisfazione } & \multicolumn{2}{|c|}{ intensità } & \\
\hline & $6,87 \%$ & 73 & $42,71 \%$ & 17 & $1,60 \%$ & 454 & \\
\hline 2 & $6,87 \%$ & 73 & $20,23 \%$ & 73 & $6,87 \%$ & 215 & $25 \%$ \\
\hline 3 & $30,10 \%$ & 320 & $19,57 \%$ & 224 & $21,07 \%$ & 208 & $50 \%$ \\
\hline 4 & $24,65 \%$ & 262 & $10,91 \%$ & 198 & $18,63 \%$ & 116 & $75 \%$ \\
\hline 5 & $31,42 \%$ & 334 & $3,01 \%$ & 54 & $5,08 \%$ & 32 & $100 \%$ \\
\hline
\end{tabular}




\section{ROBERTO VENTURA}

\begin{tabular}{|c|c|c|c|c|c|c|c|}
\hline & \multicolumn{6}{|c|}{ risposte del personale docente e di ricerca (su 690 interviste) } & \\
\hline & \multirow{2}{*}{\multicolumn{2}{|c|}{$\frac{\text { aspettativa }}{3,06}$}} & \multirow{2}{*}{\multicolumn{2}{|c|}{$\begin{array}{c}\text { soddisfazione } \\
3,20 \\
\end{array}$}} & \multirow{2}{*}{\multicolumn{2}{|c|}{$\begin{array}{c}\text { intensità } \\
27,24 \% \\
\end{array}$}} & \\
\hline & & & & & & & \\
\hline 1 & $11,16 \%$ & 77 & $3,33 \%$ & 23 & $22,17 \%$ & 153 & $0 \%$ \\
\hline 2 & $14,78 \%$ & 102 & $5,94 \%$ & 41 & $23,33 \%$ & 161 & $25 \%$ \\
\hline 3 & $10,43 \%$ & 72 & $23,48 \%$ & 162 & $9,42 \%$ & 65 & $50 \%$ \\
\hline 4 & $18,26 \%$ & 126 & $14,49 \%$ & 100 & $6,38 \%$ & 44 & $75 \%$ \\
\hline 5 & $11,30 \%$ & 78 & $4,20 \%$ & 29 & $1,88 \%$ & 13 & $100 \%$ \\
\hline
\end{tabular}

\section{2 - Banche dati attualmente disponibili nelle biblioteche (via web, su cd-rom)}

L'aspettativa è tendenzialmente elevata e l'intensità del contatto suggerisce un ricorso mirato a tali strumenti di ricerca. Considerando l'ampia offerta di banche dati che il SBA, anche in virtù delle politiche consortili in atto, mette a disposizione dell'utenza, il grado di soddisfazione appare sottodimensionato rispetto alle potenzialità: promozione del servizio e l'assistenza all'uso potrebbero essere le chiavi per incrementare la performance complessiva di questo attributo.

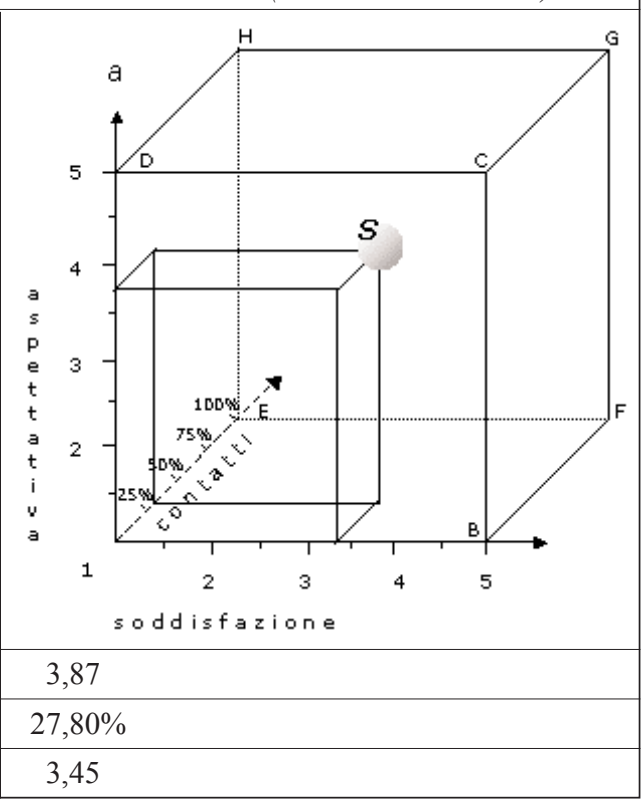

\begin{tabular}{|c|c|c|c|c|c|c|c|}
\hline \multirow[b]{3}{*}{1} & \multicolumn{6}{|c|}{ distribuzione delle risposte (stime su 27746 utenti) } & \multirow[b]{3}{*}{$0 \%$} \\
\hline & \multicolumn{2}{|c|}{ aspettativa } & \multicolumn{2}{|c|}{ soddisfazione } & \multicolumn{2}{|c|}{ intensità } & \\
\hline & $4,96 \%$ & 1024 & $2,43 \%$ & 284 & $43,31 \%$ & 8938 & \\
\hline 2 & $2,97 \%$ & 614 & $10,28 \%$ & 1203 & $17,57 \%$ & 3627 & $25 \%$ \\
\hline 3 & $19,62 \%$ & 4050 & $33,31 \%$ & 3897 & $17,56 \%$ & 3624 & $50 \%$ \\
\hline 4 & $22,77 \%$ & 4700 & $31,71 \%$ & 3710 & $17,49 \%$ & 2372 & $75 \%$ \\
\hline 5 & $30,54 \%$ & 6219 & $11,88 \%$ & 1390 & $4,44 \%$ & 917 & $100 \%$ \\
\hline
\end{tabular}




\begin{tabular}{|c|c|c|c|c|c|c|c|}
\hline \multirow[b]{3}{*}{1} & \multicolumn{6}{|c|}{ distribuzione delle risposte (su 1063 interviste) } & \multirow[b]{3}{*}{$0 \%$} \\
\hline & \multicolumn{2}{|c|}{ aspettativa } & \multicolumn{2}{|c|}{ soddisfazione } & \multicolumn{2}{|c|}{ intensità } & \\
\hline & $3,76 \%$ & 40 & $1,03 \%$ & 11 & $33,21 \%$ & 353 & \\
\hline 2 & $2,16 \%$ & 23 & $4,05 \%$ & 43 & $12,61 \%$ & 134 & $25 \%$ \\
\hline 3 & $28,97 \%$ & 308 & $17,87 \%$ & 190 & $13,08 \%$ & 139 & $50 \%$ \\
\hline 4 & $17,97 \%$ & 191 & $13,36 \%$ & 142 & $8,37 \%$ & 89 & $75 \%$ \\
\hline 5 & $21,73 \%$ & 231 & $5,08 \%$ & 54 & $3,29 \%$ & 35 & $100 \%$ \\
\hline
\end{tabular}

\begin{tabular}{|c|c|c|c|c|c|c|c|}
\hline & \multicolumn{6}{|c|}{ risposte del personale docente e di ricerca (su 690 interviste) } & \\
\hline & \multicolumn{2}{|c|}{ aspettativa } & \multicolumn{2}{|c|}{ soddisfazione } & \multicolumn{2}{|c|}{ intensità } & \\
\hline & \multicolumn{2}{|c|}{4,06} & \multicolumn{2}{|c|}{3,51} & \multicolumn{2}{|c|}{$45,69 \%$} & \\
\hline 1 & $3,77 \%$ & 26 & $2,61 \%$ & 18 & $10,72 \%$ & 74 & $0 \%$ \\
\hline 2 & $3,04 \%$ & 21 & $5,07 \%$ & 35 & $12,90 \%$ & 89 & $25 \%$ \\
\hline 3 & $5,65 \%$ & 39 & $12,90 \%$ & 89 & $11,16 \%$ & 77 & $50 \%$ \\
\hline 4 & $16,81 \%$ & 116 & $19,71 \%$ & 136 & $12,90 \%$ & 89 & $75 \%$ \\
\hline 5 & $26,23 \%$ & 181 & $7,54 \%$ & 52 & $6,09 \%$ & 42 & $100 \%$ \\
\hline
\end{tabular}

\section{3 - Risultati ottenuti dall'interrogazione delle banche dati}

L'assenza di "rumore" è tradizionalmente considerato uno dei parametri fondamentali per valutare la qualità di una banca dati. Tuttavia tale dato è direttamente connesso con le abilità di ricerca, che richiede la conoscenza degli operatori booleani e la redazione di una "stringa" efficace. Per il miglioramento della qualità di questo attributo, considerando che le banche dati possono presentare linguaggi di interrogazione ed interfacce dissimili, possono valere le medesime considerazioni formulate per l'attributo 3.2 .

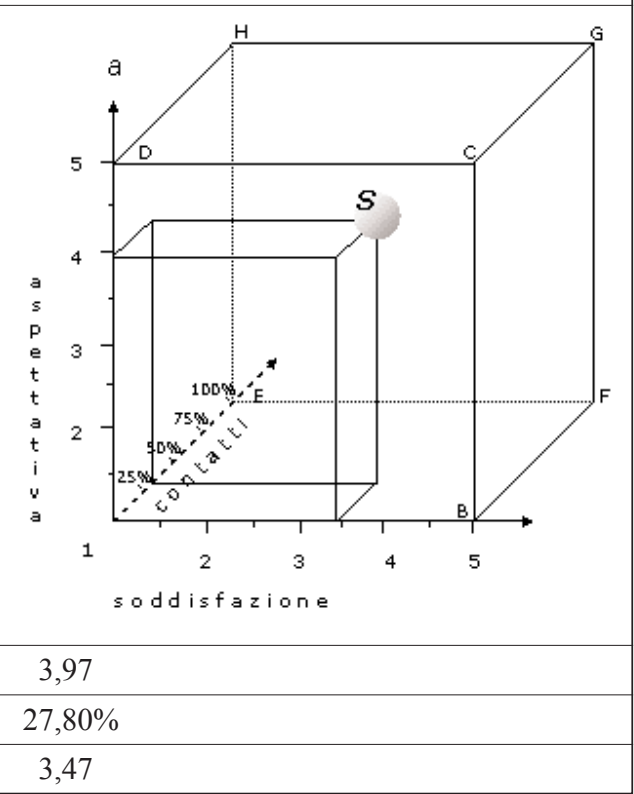




\section{ROBERTO VENTURA}

\begin{tabular}{|r|r|r|r|r|r|r|r|}
\cline { 2 - 7 } \multicolumn{1}{c|}{} & \multicolumn{5}{c|}{ distribuzione delle risposte (stime su 27746 utenti) } & \multirow{2}{*}{} \\
\cline { 2 - 7 } \multicolumn{1}{c|}{ aspettativa } & \multicolumn{2}{c|}{ soddisfazione } & \multicolumn{2}{c|}{ intensità } & \multicolumn{1}{c|}{} \\
\hline 1 & $2,03 \%$ & 419 & $2,91 \%$ & 601 & $43,31 \%$ & 8938 & $0 \%$ \\
\hline 2 & $2,16 \%$ & 446 & $4,92 \%$ & 1016 & $17,57 \%$ & 3627 & $25 \%$ \\
\hline 3 & $18,46 \%$ & 3810 & $22,74 \%$ & 4694 & $17,56 \%$ & 3624 & $50 \%$ \\
\hline 4 & $20,77 \%$ & 4286 & $21,79 \%$ & 4497 & $17,49 \%$ & 2372 & $75 \%$ \\
\hline 5 & $27,09 \%$ & 5591 & $8,77 \%$ & 1810 & $4,44 \%$ & 917 & $100 \%$ \\
\hline
\end{tabular}

\begin{tabular}{|c|c|c|c|c|c|c|c|}
\hline \multirow[b]{3}{*}{1} & \multicolumn{6}{|c|}{ distribuzione delle risposte (su 1063 interviste) } & \multirow[b]{3}{*}{$0 \%$} \\
\hline & \multicolumn{2}{|c|}{ aspettativa } & \multicolumn{2}{|c|}{ soddisfazione } & \multicolumn{2}{|c|}{ intensità } & \\
\hline & $1,51 \%$ & 16 & $2,26 \%$ & 24 & $33,21 \%$ & 353 & \\
\hline 2 & $1,60 \%$ & 17 & $3,67 \%$ & 39 & $12,61 \%$ & 134 & $25 \%$ \\
\hline 3 & $36,22 \%$ & 385 & $46,47 \%$ & 494 & $13,08 \%$ & 139 & $50 \%$ \\
\hline 4 & $15,33 \%$ & 163 & $15,80 \%$ & 168 & $8,37 \%$ & 89 & $75 \%$ \\
\hline 5 & $19,94 \%$ & 212 & $6,21 \%$ & 66 & $3,29 \%$ & 35 & $100 \%$ \\
\hline
\end{tabular}

\begin{tabular}{|c|c|c|c|c|c|c|c|}
\hline & \multicolumn{6}{|c|}{ risposte del personale docente e di ricerca (su 690 interviste) } & \\
\hline & \multirow{2}{*}{\multicolumn{2}{|c|}{$\begin{array}{c}\text { aspettativa } \\
4,01 \\
\end{array}$}} & \multirow{2}{*}{\multicolumn{2}{|c|}{$\begin{array}{c}\text { soddisfazione } \\
3,51 \\
\end{array}$}} & \multirow{2}{*}{\multicolumn{2}{|c|}{$\begin{array}{c}\text { intensità } \\
45,69 \\
\end{array}$}} & \\
\hline & & & & & & & \\
\hline 1 & $2,75 \%$ & 19 & $2,46 \%$ & 17 & $10,72 \%$ & 74 & $0 \%$ \\
\hline 2 & $3,33 \%$ & 23 & $5,07 \%$ & 35 & $12,90 \%$ & 89 & $25 \%$ \\
\hline 3 & $5,51 \%$ & 38 & $12,61 \%$ & 87 & $11,16 \%$ & 77 & $50 \%$ \\
\hline 4 & $19,13 \%$ & 132 & $21,01 \%$ & 145 & $12,90 \%$ & 89 & $75 \%$ \\
\hline 5 & $20,87 \%$ & 144 & $6,81 \%$ & 47 & $6,09 \%$ & 42 & $100 \%$ \\
\hline
\end{tabular}


L'INDAGINE SULLA SODDISFAZIONE DEGLI UTENTI: METODOLOGIA E VALUTAZIONE BIBLIOTECARIA

\begin{tabular}{|l|l|l|}
\hline 3.4 - Banche dati: modo in cui sono messe a disposizione del pubblico \\
La resa dell'attributo è legata alla qualità del- \\
l'accesso a internet in biblioteca, dal momento \\
che la consultazione delle banche dati avviene \\
attraverso i pc disponibili per il pubblico e per \\
molte di esse attraverso la rete stessa. Il mi- \\
glioramento potrebbe essere conseguito incen- \\
tivando un uso più consapevole di tali risorse $\mathrm{e}$ \\
migliorando la qualità dell'attrezzatura infor- \\
matica.
\end{tabular}

\begin{tabular}{|c|c|c|c|c|c|c|c|}
\hline \multirow[b]{3}{*}{1} & \multicolumn{6}{|c|}{ distribuzione delle risposte (stime su 27746 utenti) } & \multirow[b]{3}{*}{$0 \%$} \\
\hline & \multicolumn{2}{|c|}{ aspettativa } & \multicolumn{2}{|c|}{ soddisfazione } & \multicolumn{2}{|c|}{ intensità } & \\
\hline & $5,01 \%$ & 1389 & $3,74 \%$ & 1038 & $43,31 \%$ & 8938 & \\
\hline 2 & $3,36 \%$ & 932 & $7,48 \%$ & 2075 & $17,57 \%$ & 3627 & $25 \%$ \\
\hline 3 & $18,19 \%$ & 5046 & $25,73 \%$ & 7139 & $17,56 \%$ & 3624 & $50 \%$ \\
\hline 4 & $25,96 \%$ & 7202 & $24,03 \%$ & 6666 & $17,49 \%$ & 2372 & $75 \%$ \\
\hline 5 & $35,51 \%$ & 9853 & $9,56 \%$ & 2652 & $4,44 \%$ & 917 & $100 \%$ \\
\hline
\end{tabular}

\begin{tabular}{|c|c|c|c|c|c|c|c|}
\hline \multirow[b]{3}{*}{1} & \multicolumn{6}{|c|}{ distribuzione delle risposte (su 1063 interviste) } & \multirow[b]{3}{*}{$0 \%$} \\
\hline & \multicolumn{2}{|c|}{ aspettativa } & \multicolumn{2}{|c|}{ soddisfazione } & \multicolumn{2}{|c|}{ intensità } & \\
\hline & $4,80 \%$ & 51 & $3,48 \%$ & 37 & $33,21 \%$ & 353 & \\
\hline 2 & $3,57 \%$ & 38 & $7,24 \%$ & 77 & $12,61 \%$ & 134 & $25 \%$ \\
\hline 3 & $30,01 \%$ & 319 & $55,88 \%$ & 594 & $13,08 \%$ & 139 & $50 \%$ \\
\hline 4 & $26,62 \%$ & 283 & $23,42 \%$ & 249 & $8,37 \%$ & 89 & $75 \%$ \\
\hline 5 & $35,00 \%$ & 372 & $9,97 \%$ & 106 & $3,29 \%$ & 35 & $100 \%$ \\
\hline
\end{tabular}




\section{ROBERTO VENTURA}

\begin{tabular}{|c|c|c|c|c|c|c|c|}
\hline & \multicolumn{6}{|c|}{ risposte del personale docente e di ricerca (su 690 interviste) } & \\
\hline & \multirow{2}{*}{\multicolumn{2}{|c|}{$\begin{array}{c}\text { aspettativa } \\
4,01\end{array}$}} & \multirow{2}{*}{\multicolumn{2}{|c|}{$\begin{array}{c}\text { soddisfazione } \\
3,45 \\
\end{array}$}} & \multirow{2}{*}{\multicolumn{2}{|c|}{$\begin{array}{c}\text { intensità } \\
45,69 \% \\
\end{array}$}} & \\
\hline & & & & & & & \\
\hline 1 & $3,33 \%$ & 23 & $3,77 \%$ & 26 & $10,72 \%$ & 74 & $0 \%$ \\
\hline 2 & $7,10 \%$ & 49 & $8,12 \%$ & 56 & $12,90 \%$ & 89 & $25 \%$ \\
\hline 3 & $5,65 \%$ & 39 & $14,93 \%$ & 103 & $11,16 \%$ & 77 & $50 \%$ \\
\hline 4 & $23,04 \%$ & 159 & $26,81 \%$ & 185 & $12,90 \%$ & 89 & $75 \%$ \\
\hline 5 & $30,43 \%$ & 210 & $8,55 \%$ & 59 & $6,09 \%$ & 42 & $100 \%$ \\
\hline
\end{tabular}

\section{5 - Comunicazione con il personale delle biblioteche (disponibilità e cortesia dei bibliotecari nei confronti del pubblico)}

La tendenza è verso il punto ottimale: elevata aspettativa ed intensità di comunicazione col personale bibliotecario, grado di soddisfazione positivo. L'attributo coinvolge tutte le occasioni di contatto tra utenza e personale, dal frontoffice agli uffici. Il livello di soddisfazione può essere giudicato positivamente, anche alla luce del fatto che, in talune realtà, l'elevata pressione del pubblico comporta condizioni operative caratterizzate da criticità. Una maggiore standardizzazione delle modalità di contatto, coniugate con la frequenza di appositi corsi di formazione, potrebbe elevare la resa già buona di questo tratto fondamentale per l'immagine del SBA.

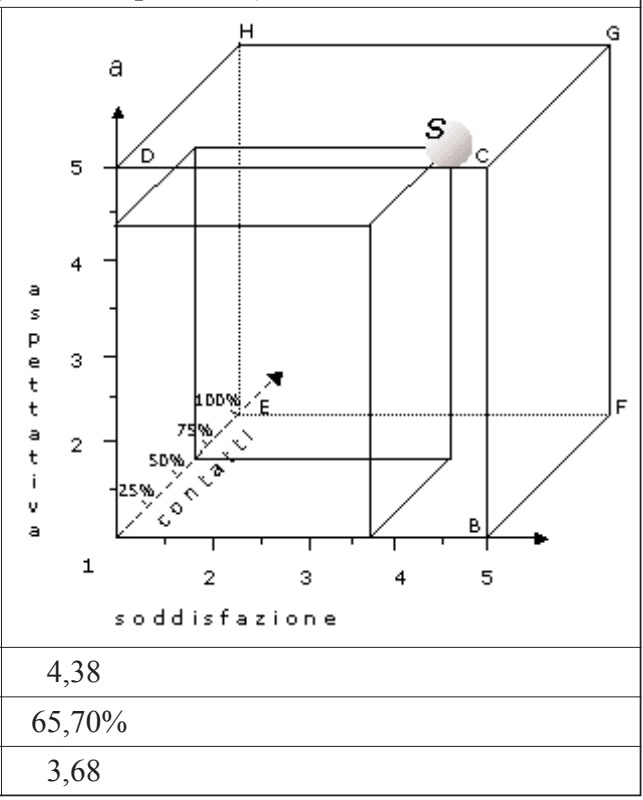

\begin{tabular}{|c|c|c|c|c|c|c|c|}
\hline \multirow[b]{3}{*}{1} & \multicolumn{6}{|c|}{ distribuzione delle risposte (stime su 27746 utenti) } & \multirow[b]{3}{*}{$0 \%$} \\
\hline & \multicolumn{2}{|c|}{ aspettativa } & \multicolumn{2}{|c|}{ soddisfazione } & \multicolumn{2}{|c|}{ intensità } & \\
\hline & $0,57 \%$ & 157 & $4,45 \%$ & 1216 & $1,60 \%$ & 443 & \\
\hline 2 & $2,07 \%$ & 575 & $9,43 \%$ & 2575 & $12,31 \%$ & 3417 & $25 \%$ \\
\hline 3 & $11,91 \%$ & 3304 & $22,94 \%$ & 6264 & $30,33 \%$ & 8417 & $50 \%$ \\
\hline 4 & $28,53 \%$ & 7915 & $38,73 \%$ & 10575 & $31,59 \%$ & 8766 & $75 \%$ \\
\hline 5 & $55,53 \%$ & 15406 & $23,68 \%$ & 6464 & $22,98 \%$ & 6376 & $100 \%$ \\
\hline
\end{tabular}




\begin{tabular}{|c|c|c|c|c|c|c|c|}
\hline \multirow[b]{3}{*}{1} & \multicolumn{6}{|c|}{ distribuzione delle risposte (su 1063 interviste) } & \multirow[b]{3}{*}{$0 \%$} \\
\hline & \multicolumn{2}{|c|}{ aspettativa } & \multicolumn{2}{|c|}{ soddisfazione } & \multicolumn{2}{|c|}{ intensità } & \\
\hline & $0,47 \%$ & 5 & $4,23 \%$ & 45 & $1,88 \%$ & 20 & \\
\hline 2 & $2,07 \%$ & 22 & $9,13 \%$ & 97 & $12,70 \%$ & 135 & $25 \%$ \\
\hline 3 & $12,98 \%$ & 138 & $24,65 \%$ & 262 & $29,63 \%$ & 315 & $50 \%$ \\
\hline 4 & $28,50 \%$ & 303 & $36,78 \%$ & 391 & $31,70 \%$ & 337 & $75 \%$ \\
\hline 5 & $55,97 \%$ & 595 & $23,33 \%$ & 248 & $22,95 \%$ & 244 & $100 \%$ \\
\hline
\end{tabular}

\begin{tabular}{|c|c|c|c|c|c|c|c|}
\hline & \multicolumn{6}{|c|}{ risposte del personale docente e di ricerca (su 690 interviste) } & \\
\hline & \multicolumn{2}{|c|}{ aspettativa } & \multicolumn{2}{|c|}{ soddisfazione } & \multicolumn{2}{|c|}{ intensità } & \\
\hline & \multicolumn{2}{|c|}{4,02} & \multicolumn{2}{|c|}{4,05} & \multicolumn{2}{|c|}{$67,00 \%$} & \\
\hline 1 & $0,87 \%$ & 6 & $2,32 \%$ & 16 & $2,03 \%$ & 14 & $0 \%$ \\
\hline 2 & $4,93 \%$ & 34 & $4,64 \%$ & 32 & $12,61 \%$ & 87 & $25 \%$ \\
\hline 3 & $10,14 \%$ & 70 & $9,13 \%$ & 63 & $15,22 \%$ & 105 & $50 \%$ \\
\hline 4 & $36,81 \%$ & 254 & $31,30 \%$ & 216 & $25,22 \%$ & 174 & $75 \%$ \\
\hline 5 & $23,77 \%$ & 164 & $29,28 \%$ & 202 & $21,88 \%$ & 151 & $100 \%$ \\
\hline
\end{tabular}

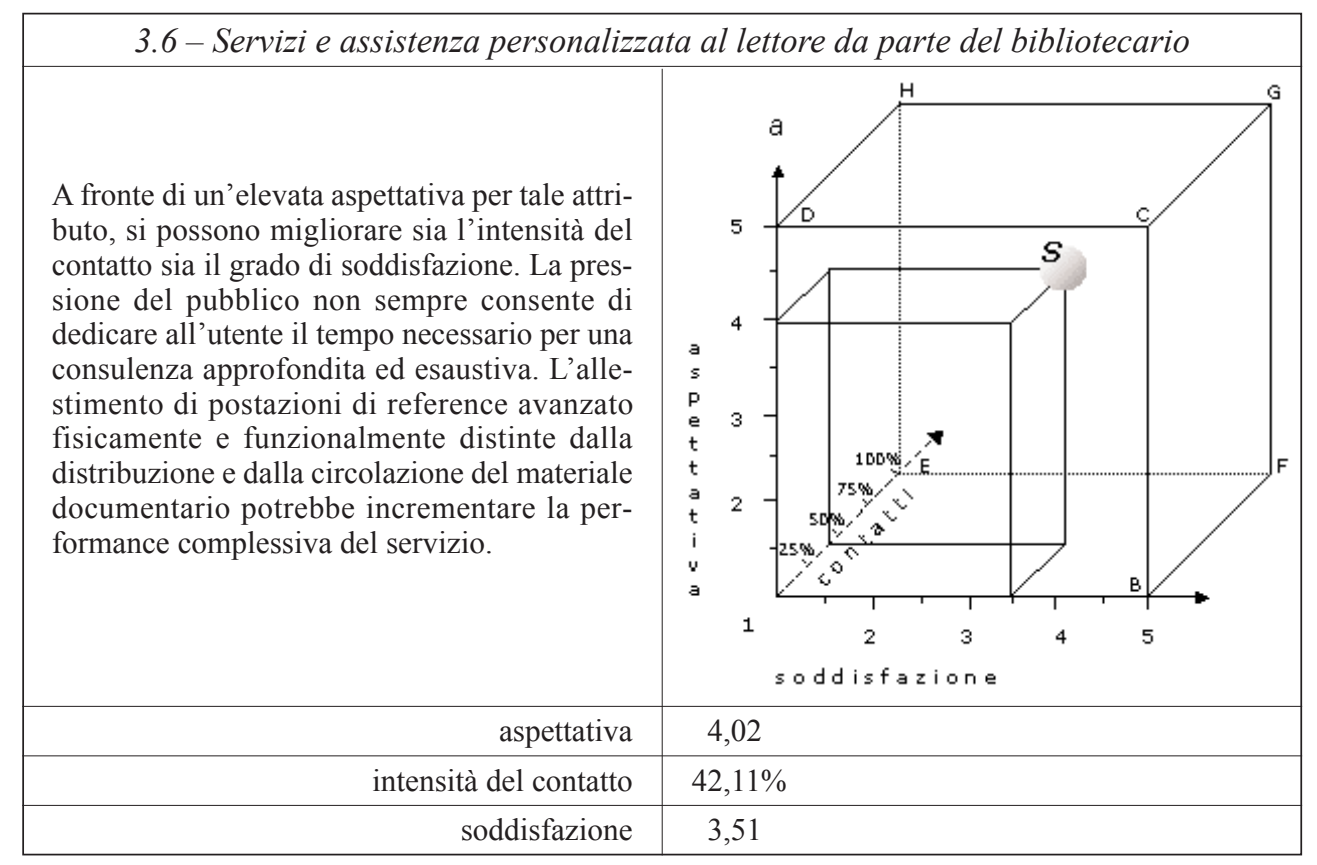




\section{ROBERTO VENTURA}

\begin{tabular}{|c|r|r|r|r|r|r|r|}
\cline { 2 - 7 } \multicolumn{1}{c|}{} & \multicolumn{5}{c|}{ distribuzione delle risposte (stime su 27746 utenti) } & \multirow{2}{*}{} \\
\cline { 2 - 7 } \multicolumn{1}{c|}{ aspettativa } & \multicolumn{2}{c|}{ soddisfazione } & \multicolumn{2}{c|}{ intensità } & \multirow{2}{*}{$0 \%$} \\
\hline 1 & $2,49 \%$ & 692 & $4,64 \%$ & 1014 & $21,19 \%$ & 5879 & $0 \%$ \\
\hline 2 & $4,04 \%$ & 1121 & $13,17 \%$ & 2879 & $25,93 \%$ & 7195 & $25 \%$ \\
\hline 3 & $19,47 \%$ & 5403 & $27,46 \%$ & 6005 & $24,33 \%$ & 6751 & $50 \%$ \\
\hline 4 & $34,49 \%$ & 9569 & $32,10 \%$ & 7019 & $16,82 \%$ & 4668 & $75 \%$ \\
\hline 5 & $37,03 \%$ & 10275 & $20,20 \%$ & 4418 & $10,21 \%$ & 2833 & $100 \%$ \\
\hline
\end{tabular}

\begin{tabular}{|c|c|c|c|c|c|c|c|}
\hline \multirow[b]{3}{*}{1} & \multicolumn{6}{|c|}{ distribuzione delle risposte (su 1063 interviste) } & \multirow[b]{3}{*}{$0 \%$} \\
\hline & \multicolumn{2}{|c|}{ aspettativa } & \multicolumn{2}{|c|}{ soddisfazione } & \multicolumn{2}{|c|}{ intensità } & \\
\hline & $2,73 \%$ & 29 & $3,86 \%$ & 41 & $21,83 \%$ & 232 & \\
\hline 2 & $4,52 \%$ & 48 & $9,88 \%$ & 105 & $26,15 \%$ & 278 & $25 \%$ \\
\hline 3 & $21,35 \%$ & 227 & $23,42 \%$ & 249 & $23,52 \%$ & 250 & $50 \%$ \\
\hline 4 & $34,05 \%$ & 362 & $25,12 \%$ & 267 & $17,40 \%$ & 185 & $75 \%$ \\
\hline 5 & $37,35 \%$ & 397 & $15,90 \%$ & 169 & $9,78 \%$ & 104 & $100 \%$ \\
\hline
\end{tabular}

\begin{tabular}{|c|c|c|c|c|c|c|c|}
\hline & \multicolumn{6}{|c|}{ risposte del personale docente e di ricerca (su 690 interviste) } & \\
\hline & \multirow{2}{*}{\multicolumn{2}{|c|}{$\begin{array}{c}\text { aspettativa } \\
3,21\end{array}$}} & \multicolumn{4}{|c|}{ soddisfazione intensità } & \\
\hline & & & \multicolumn{2}{|c|}{3,74} & \multicolumn{2}{|c|}{$36,92 \%$} & \\
\hline 1 & $8,70 \%$ & 60 & $2,75 \%$ & 19 & $17,97 \%$ & 124 & $0 \%$ \\
\hline 2 & $13,77 \%$ & 95 & $4,06 \%$ & 28 & $20,58 \%$ & 142 & $25 \%$ \\
\hline 3 & $12,03 \%$ & 83 & $14,64 \%$ & 101 & $15,22 \%$ & 105 & $50 \%$ \\
\hline 4 & $22,32 \%$ & 154 & $22,17 \%$ & 153 & $9,28 \%$ & 64 & $75 \%$ \\
\hline 5 & $11,74 \%$ & 81 & $15,65 \%$ & 108 & $5,65 \%$ & 39 & $100 \%$ \\
\hline
\end{tabular}




\section{7 - Depliant sulle biblioteche e segnaletica in biblioteca per facilitare l'uso dei servizi}

Questa interfaccia di comunicazione tra biblioteca e pubblico non è esclusiva e si sovrappone con la comunicazione diretta col personale e le informazioni erogate via internet. Il servizio può essere mantenuto e migliorato con una diversa modalità di distribuzione dei depliant, con una maggiore evidenziazione della segnaletica presente in biblioteca nonché abituando il lettore al suo corretto uso.

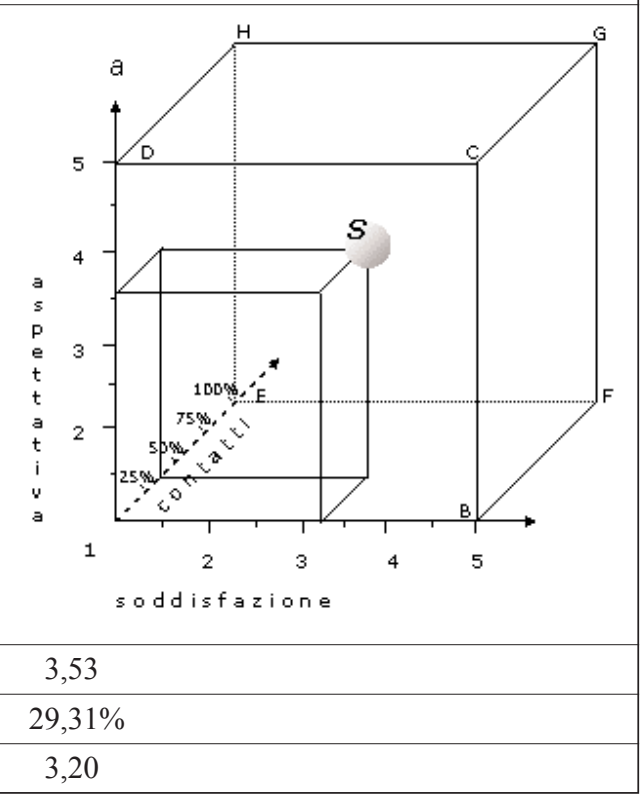

\begin{tabular}{|c|c|c|c|c|c|c|c|}
\hline \multirow[b]{3}{*}{1} & \multicolumn{6}{|c|}{ distribuzione delle risposte (stime su 27746 utenti) } & \multirow[b]{3}{*}{$0 \%$} \\
\hline & \multicolumn{2}{|c|}{ aspettativa } & \multicolumn{2}{|c|}{ soddisfazione } & \multicolumn{2}{|c|}{ intensità } & \\
\hline & $9,71 \%$ & 2693 & $4,17 \%$ & 712 & $38,46 \%$ & 10670 & \\
\hline 2 & $7,44 \%$ & 2065 & $20,16 \%$ & 3443 & $23,33 \%$ & 6473 & $25 \%$ \\
\hline 3 & $23,15 \%$ & 6424 & $34,81 \%$ & 5945 & $20,26 \%$ & 5621 & $50 \%$ \\
\hline 4 & $24,87 \%$ & 6901 & $25,94 \%$ & 4429 & $10,07 \%$ & 2795 & $75 \%$ \\
\hline 5 & $24,84 \%$ & 6892 & $10,82 \%$ & 1847 & $4,92 \%$ & 1366 & $100 \%$ \\
\hline
\end{tabular}

\begin{tabular}{|c|c|c|c|c|c|c|c|}
\hline \multirow[b]{3}{*}{1} & \multicolumn{6}{|c|}{ distribuzione delle risposte (su 1063 interviste) } & \multirow[b]{3}{*}{$0 \%$} \\
\hline & \multicolumn{2}{|c|}{ aspettativa } & \multicolumn{2}{|c|}{ soddisfazione } & \multicolumn{2}{|c|}{ intensità } & \\
\hline & $9,88 \%$ & 105 & $2,26 \%$ & 24 & $39,51 \%$ & 420 & \\
\hline 2 & $8,00 \%$ & 85 & $12,70 \%$ & 135 & $23,14 \%$ & 246 & $25 \%$ \\
\hline 3 & $32,83 \%$ & 349 & $22,67 \%$ & 241 & $19,85 \%$ & 211 & $50 \%$ \\
\hline 4 & $24,27 \%$ & 258 & $15,71 \%$ & 167 & $10,16 \%$ & 108 & $75 \%$ \\
\hline 5 & $25,02 \%$ & 266 & $7,15 \%$ & 76 & $4,42 \%$ & 47 & $100 \%$ \\
\hline
\end{tabular}




\section{ROBERTO VENTURA}

\begin{tabular}{|c|c|c|c|c|c|c|c|}
\hline & \multicolumn{6}{|c|}{ risposte del personale docente e di ricerca (su 690 interviste) } & \\
\hline & \multirow{2}{*}{\multicolumn{2}{|c|}{$\begin{array}{c}\text { aspettativa } \\
2,97\end{array}$}} & \multirow{2}{*}{\multicolumn{2}{|c|}{$\begin{array}{c}\text { soddisfazione } \\
3,14 \\
\end{array}$}} & \multirow{2}{*}{\multicolumn{2}{|c|}{$\begin{array}{c}\text { intensità } \\
28,97 \% \\
\end{array}$}} & \\
\hline & & & & & & & \\
\hline 1 & $10,72 \%$ & 74 & $2,75 \%$ & 19 & $20,58 \%$ & 142 & $0 \%$ \\
\hline 2 & $11,74 \%$ & 81 & $8,70 \%$ & 60 & $20,00 \%$ & 138 & $25 \%$ \\
\hline 3 & $16,09 \%$ & 111 & $21,01 \%$ & 145 & $12,61 \%$ & 87 & $50 \%$ \\
\hline 4 & $18,55 \%$ & 128 & $13,91 \%$ & 96 & $6,23 \%$ & 43 & $75 \%$ \\
\hline 5 & $6,38 \%$ & 44 & $3,62 \%$ & 25 & $1,74 \%$ & 12 & $100 \%$ \\
\hline
\end{tabular}

\section{8 - Sito web del Sistema Bibliotecario di Ateneo (esclusi catalogo, prenotazioni e rinnovi)}

La comunicazione via web è diventata un tratto saliente delle società avanzate ed il ricorso ad internet per acquisire informazioni sui servizi costituisce una strategia comunicativa più snella e rapida rispetto ai tradizionali mezzi di comunicazione. L'utenza mostra che il sito del SBA è usato più di una volta su due quando sorga la necessità di informarsi sull'uso dei servizi bibliotecari, sussistendo per tale opzione elevate aspettative. L'implementazione del sito web del SBA potrebbe rivelarsi un buon investimento, anche al fine di portare il pubblico ad un maggior grado di consapevolezza delle opportunità informative offerte dal SBA.

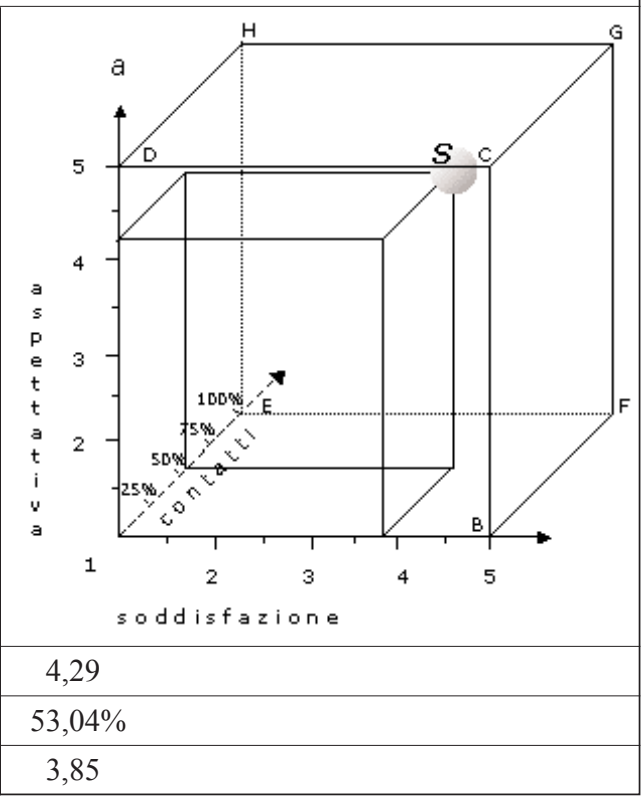

\begin{tabular}{|r|r|r|r|r|r|r|r|}
\cline { 2 - 7 } \multicolumn{1}{c|}{} & \multicolumn{5}{c|}{ distribuzione delle risposte (stime su 27746 utenti) } & \multirow{2}{*}{} \\
\cline { 2 - 7 } \multicolumn{1}{c|}{} & \multicolumn{2}{c|}{ aspettativa } & \multicolumn{2}{c|}{ soddisfazione } & \multicolumn{2}{c|}{ intensità } & \\
\hline 1 & $4,42 \%$ & 1227 & $1,41 \%$ & 292 & $25,61 \%$ & 7107 & $0 \%$ \\
\hline 2 & $3,21 \%$ & 891 & $6,03 \%$ & 1245 & $9,96 \%$ & 2764 & $25 \%$ \\
\hline 3 & $9,26 \%$ & 2570 & $19,64 \%$ & 4053 & $15,07 \%$ & 4180 & $50 \%$ \\
\hline 4 & $21,29 \%$ & 5908 & $42,79 \%$ & 8830 & $20,36 \%$ & 5650 & $75 \%$ \\
\hline 5 & $56,55 \%$ & 15692 & $26,03 \%$ & 5372 & $26,34 \%$ & 7307 & $100 \%$ \\
\hline
\end{tabular}


L'INDAGINE SULLA SODDISFAZIONE DEGLI UTENTI: METODOLOGIA E VALUTAZIONE BIBLIOTECARIA

\begin{tabular}{|c|c|c|c|c|c|c|c|}
\hline \multirow[b]{3}{*}{1} & \multicolumn{6}{|c|}{ distribuzione delle risposte (su 1063 interviste) } & \multirow[b]{3}{*}{$0 \%$} \\
\hline & \multicolumn{2}{|c|}{ aspettativa } & \multicolumn{2}{|c|}{ soddisfazione } & \multicolumn{2}{|c|}{ intensità } & \\
\hline & $4,61 \%$ & 49 & $1,41 \%$ & 15 & $25,40 \%$ & 270 & \\
\hline 2 & $2,73 \%$ & 29 & $3,76 \%$ & 40 & $10,25 \%$ & 109 & $25 \%$ \\
\hline 3 & $14,39 \%$ & 153 & $17,40 \%$ & 185 & $14,49 \%$ & 154 & $50 \%$ \\
\hline 4 & $21,73 \%$ & 231 & $32,55 \%$ & 346 & $21,17 \%$ & 225 & $75 \%$ \\
\hline 5 & $56,54 \%$ & 601 & $19,47 \%$ & 207 & $26,15 \%$ & 278 & $100 \%$ \\
\hline
\end{tabular}

\begin{tabular}{|c|c|c|c|c|c|c|c|}
\hline & \multicolumn{6}{|c|}{ risposte del personale docente e di ricerca (su 690 interviste) } & \\
\hline & \multicolumn{2}{|c|}{ aspettativa } & \multicolumn{2}{|c|}{ soddisfazione } & \multicolumn{2}{|c|}{ intensità } & \\
\hline & \multicolumn{2}{|c|}{4,18} & \multicolumn{2}{|c|}{3,92} & \multicolumn{2}{|c|}{66,19} & \\
\hline 1 & $1,16 \%$ & 8 & $0,58 \%$ & 4 & $2,61 \%$ & 18 & $0 \%$ \\
\hline 2 & $2,90 \%$ & 20 & $3,04 \%$ & 21 & $7,25 \%$ & 50 & $25 \%$ \\
\hline 3 & $6,38 \%$ & 44 & $9,86 \%$ & 68 & $13,19 \%$ & 91 & $50 \%$ \\
\hline 4 & $23,62 \%$ & 163 & $32,46 \%$ & 224 & $23,19 \%$ & 160 & $75 \%$ \\
\hline 5 & $26,81 \%$ & 185 & $12,90 \%$ & 89 & $14,20 \%$ & 98 & $100 \%$ \\
\hline
\end{tabular}




\section{Qualità complessiva del SBA}

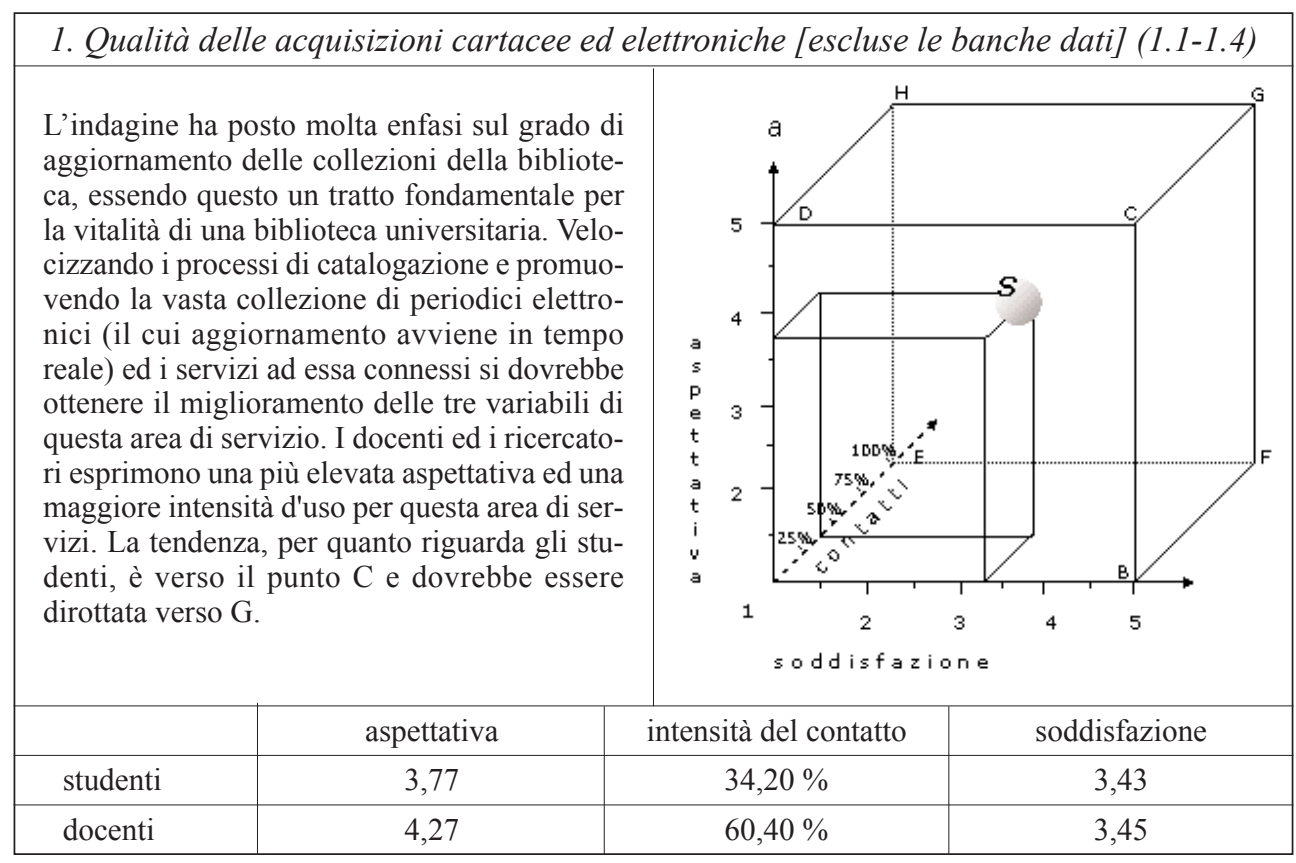

\section{Qualità della circolazione documentaria (2.1-2.11)}

Questa area di servizio costituisce senz'altro uno dei punti di forza del SBA, perché presenta un livello di aspettativa assai elevato e per il fatto che l'utenza dedica una visita su due in biblioteca per usare i servizi connessi alla circolazione documentaria. Docenti e ricercatori mostrano una minore aspettativa ed un uso meno intenso per questa area di servizi. La tendenza dell'indicatore è verso il punto ottimale, sebbene si riveli necessario l'incremento dell'intensità d'uso e del grado di soddisfazione.

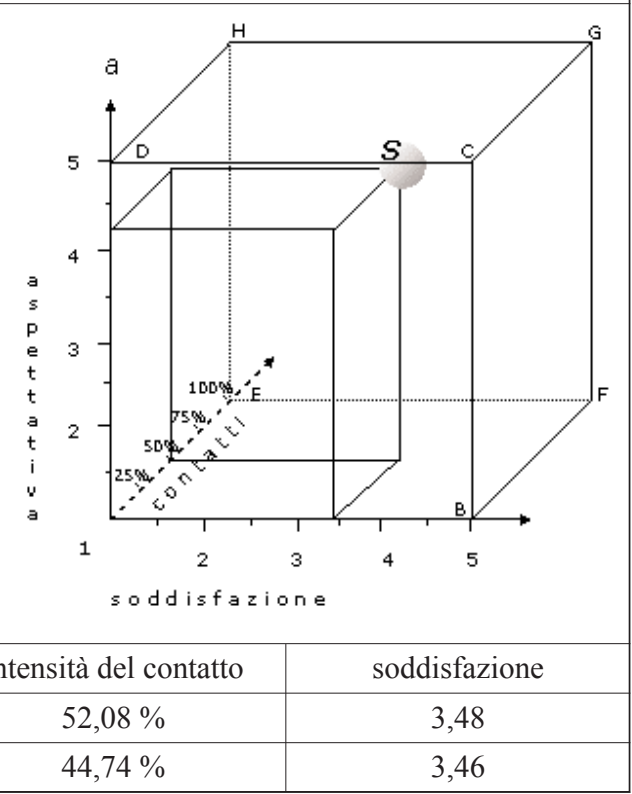




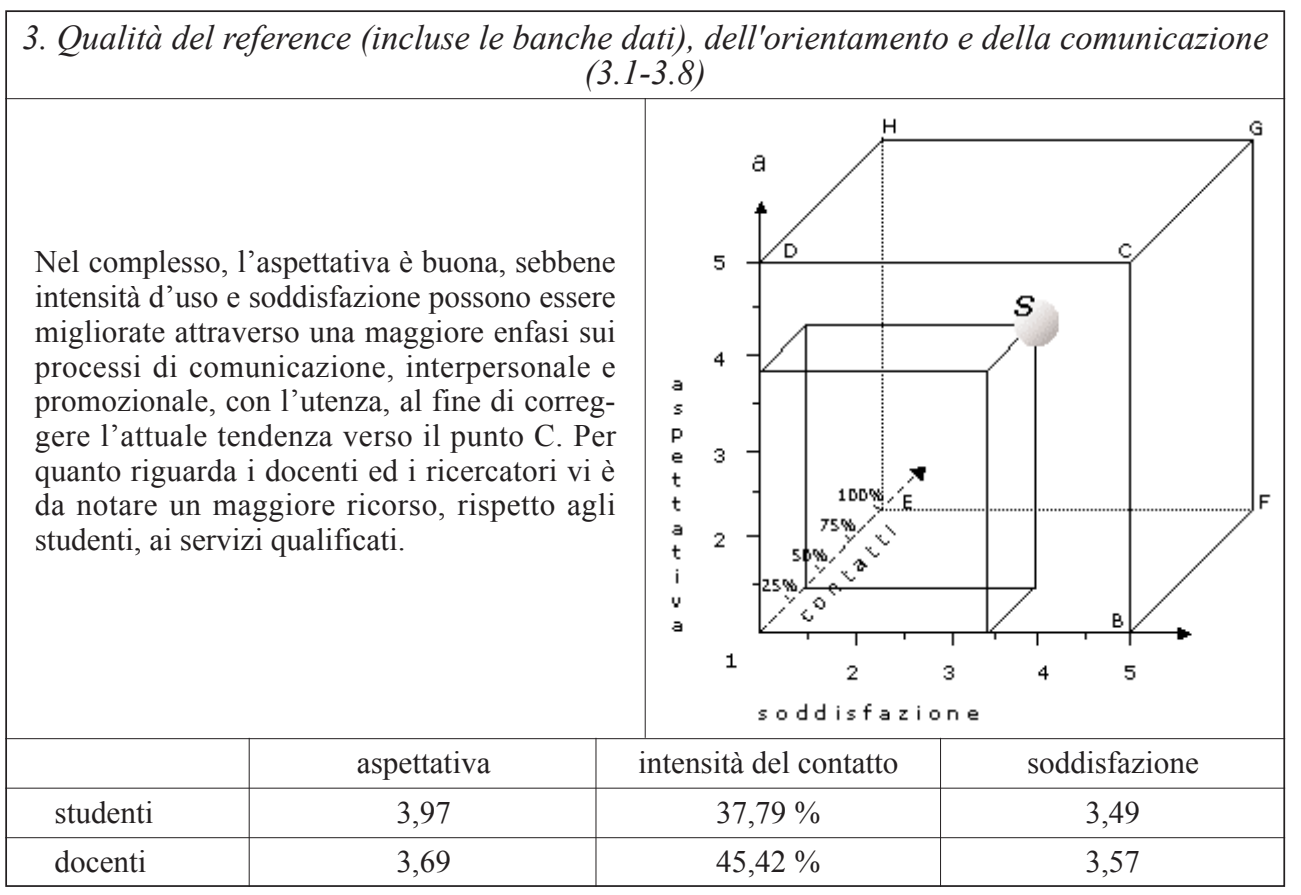

Qualità complessiva del SBA (1-3)

Questo indicatore unico della qualità del SBA sintetizza la performance dei tre indicatori precedenti. Il livello di aspettativa delle prestazioni bibliotecarie può ritenersi soddisfacente, sebbene emerga che più della metà dell'uso delle nostre strutture è dedicato a finalità diverse dalla fruizione dei servizi erogati. Oltre all'azione relativa ad ogni singolo attributo, potrebbe rivelarsi utile porre l'enfasi su ciò che caratterizza la biblioteca universitaria, con un maggiore coordinamento - e comunicazione interna - con la didattica. Difatti, su quest'ultimo fronte, si nota una maggiore intensità d'uso dei servizi bibliotecari: l'eventuale integrazione potrebbe produrre un virtuoso effetto di "trascinamento" dell'utenza studentesca verso usi più congrui dei servizi. La tendenza generale è tuttavia verso il punto $\mathrm{C}$ e ciò denota una performance "simbolica" dei servizi erogati alla quale, nonostante una buona aspettativa e una soddisfazione di valore positivo, non corrisponde una fruizione di pari intensità.

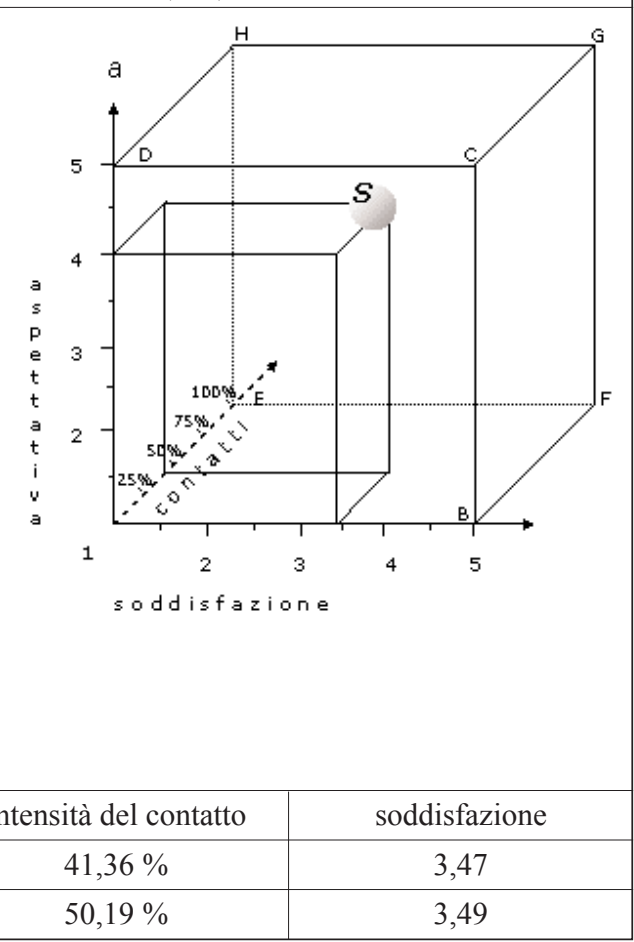




\section{I risultati dell'indagine: le biblioteche di area secondo gli studenti}

Il campionamento effettuato per l'indagine sull'utenza studentesca consente di procedere al trattamento dei dati relativi a ciascuna delle cinque biblioteche di area, senza perdita di rappresentatività dei risultati e delle valutazioni che da ciò possono scaturire. La valutazione ed il confronto della performance qualitativa tra le cinque unità amministrative bibliotecarie è utile al fine dell'individuazione delle aree di eccellenza o di criticità, secondo un approccio che aiuti, fatte salve le differenti proprietà strutturali che caratterizzano le biblioteche per storia, ambito disciplinare ed organizzazione, ad individuare i processi bibliotecari più efficaci, cosicché le esperienze organizzative e procedurali possano essere valorizzate, nell'ottica del benchmarking e del knowledge management, all'interno del SBA.

Da un altro punto di vista, forse il più immediato, le informazioni relative alla singola biblioteca di area costituiscono uno strumento di gestione che $\mathrm{i}$ responsabili dei servizi possono utilizzare per una riflessione relativa alle procedure interne ed all'organizzazione dell'ufficio affidato alla propria responsabilità. Se le valutazioni di customer satisfaction sono uno strumento finalizzato a gettare luce sulla capacità che la biblioteca ha di interagire con l'ambiente istituzionale di riferimento, una cultura della qualità diffusa, come si suol dire, a tutti i livelli dell'organizzazione è uno tra i fondamenti per la realizzazione della biblioteca eccellente. Il confronto e la valutazione tra le biblioteche prescinde dalla focalizzazione di responsabilità collettive o singole per gli eventuali malfunzionamenti - e, del resto, dalla enfatizzazione di meriti personalistici per eventuali successi - quanto piuttosto tende ad evidenziare eventuali errori nella organizzazione dei processi lavorativi i quali, in caso di performance critiche o posizionate attorno ai valori dell'indifferenza, dovrebbero essere rimossi per prevenire probabili sconfinamenti futuri degli indicatori nell'area dell'insoddisfazione.

All'esame ed alla valutazione dei risultati seguirà, ove lo si ritenga opportuno, la ricerca delle cause attraverso la tecnica del brainstorming ed il successivo intervento sull'organizzazione lavorativa tramite il diagramma di flusso, con conseguente riorganizzazione dei processi stessi.

Si tenga conto, inoltre, della circostanza che l'attuale organizzazione delle biblioteche e dei punti di servizio in un sistema bibliotecario dotato di un elevato grado di interazione tra le singole componenti - da un lato, alcuni processi come quelli catalografici sono dipendenti dall'adozione delle medesime procedure in qualunque punto del sistema e risultano in parte centralizzati; dall'altro l'organizzazione del SBA prevede gruppi di lavoro trasversali su 
processi avanzati e tipici della biblioteca universitaria - rende necessaria l'opportunità di una visione complessiva e comparativa tra le performance dei diversi uffici, sia per ragioni di gestione bibliotecaria del sistema, sia per l'allocazione delle risorse finanziarie.

La performance delle aree è legata, infine, alle diverse abitudini di comportamento dell'utenza, in virtù delle specifiche caratteristiche di studio, di didattica e di ricerca che la natura delle discipline praticate in Ateneo comporta.

Di seguito, si riportano i dati relativi alle tre aree di servizio e i dati relativi ad ognuna delle biblioteche di area, elaborati direttamente dalle risposte ottenute del campione d'indagine.

\begin{tabular}{|l|c|c|c|}
\hline \multicolumn{4}{|c|}{ 1. Qualità delle acquisizioni cartacee ed elettroniche } \\
\hline Biblioteca & aspettativa & intensità & soddisfazione $^{1}$ \\
\hline BIOMEDICA & 3,68 & $31,72 \%$ & 3,48 \\
\hline SCIENZE SOCIALI & 3,82 & $35,12 \%$ & 3,50 \\
\hline SCIENZE & 3,80 & $34,20 \%$ & 3,39 \\
\hline SC. TECNOLOGICHE & 3,71 & $36,65 \%$ & 3,70 \\
\hline UMANISTICA & 3,52 & $30,60 \%$ & 3,40 \\
\hline
\end{tabular}

\begin{tabular}{|l|c|c|c|}
\hline \multicolumn{4}{|c|}{ 2. Qualità della circolazione documentaria } \\
\hline Biblioteca & aspettativa & intensità & soddisfazione \\
\hline BIOMEDICA & 4,21 & $50,79 \%$ & 3,59 \\
\hline SCIENZE SOCIALI & 4,25 & $51,35 \%$ & 3,48 \\
\hline SCIENZE & 4,29 & $53,78 \%$ & 3,66 \\
\hline SC. TECNOLOGICHE & 4,20 & $52,73 \%$ & 3,65 \\
\hline UMANISTICA & 4,11 & $51,07 \%$ & 3,48 \\
\hline
\end{tabular}

\begin{tabular}{|l|c|c|c|}
\hline \multicolumn{4}{|c|}{ 3. Qualità del reference ${ }^{2}$, dell'orientamento e della comunicazione all'utenza } \\
\hline Biblioteca & aspettativa & intensità & soddisfazione \\
\hline BIOMEDICA & 3,84 & $35,72 \%$ & 3,45 \\
\hline SCIENZE SOCIALI & 3,97 & $39,56 \%$ & 3,46 \\
\hline SCIENZE & 3,87 & $36,32 \%$ & 3,51 \\
\hline SC. TECNOLOGICHE & 3,80 & $37,97 \%$ & 3,72 \\
\hline UMANISTICA & 3,83 & $36,94 \%$ & 3,41 \\
\hline
\end{tabular}

\footnotetext{
1 Escluse le banche dati.

2 Comprese le banche dati.
} 


\section{RoBERTO VENTURA}

\begin{tabular}{|l|c|c|c|}
\hline \multicolumn{4}{|c|}{ Qualità complessiva delle biblioteche } \\
\hline Biblioteca & aspettativa & intensità & soddisfazione \\
\hline BIOMEDICA & 3,91 & $39,41 \%$ & 3,51 \\
\hline SCIENZE SOCIALI & 4,02 & $42,01 \%$ & 3,48 \\
\hline SCIENZE & 3,99 & $41,43 \%$ & 3,52 \\
\hline SC. TECNOLOGICHE & 3,90 & $42,45 \%$ & 3,69 \\
\hline UMANISTICA & 3,82 & $39,54 \%$ & 3,43 \\
\hline
\end{tabular}




\section{Biblioteca biomedica}

\begin{tabular}{|l|c|c|c|}
\hline 1. Qualità delle acquisizioni cartacee ed elettroniche & $a$ & $i$ & $s$ \\
\hline $\begin{array}{l}\text { 1.1 - Libri, periodici e cd-rom attualmente posseduti dalla } \\
\text { biblioteca }\end{array}$ & 4,01 & $49,88 \%$ & 3,67 \\
\hline $\begin{array}{l}\text { 1.2 - Disponibilità in biblioteca delle novità librarie nelle } \\
\text { discipline di tuo interesse }\end{array}$ & 3,77 & $34,88 \%$ & 3,31 \\
\hline $\begin{array}{l}1.3 \text { - Disponibilità in biblioteca degli ultimi numeri dei } \\
\text { periodici nelle discipline di tuo interesse }\end{array}$ & 3,48 & $21,01 \%$ & 3,47 \\
\hline $\begin{array}{l}1.4-\text { Uso dei periodici elettronici nelle discipline di tuo } \\
\text { interesse }\end{array}$ & 3,46 & $21,11 \%$ & 3,46 \\
\hline
\end{tabular}

\begin{tabular}{|l|c|c|c|}
\hline 2. Qualità della circolazione documentaria & $a$ & $i$ & $s$ \\
\hline $\begin{array}{l}\text { 2.1 - Uso del catalogo della biblioteca su internet: ricerca } \\
\text { di libri o periodici per titolo e ricerca di libri per autore }\end{array}$ & 4,53 & $70,21 \%$ & 4,01 \\
\hline $\begin{array}{l}\text { 2.2 - Uso del catalogo su internet: ricerca di libri per } \\
\text { argomento (nel campo: soggetto) o materia (nel campo: } \\
\text { classificazione) }\end{array}$ & 4,26 & $50,53 \%$ & 3,62 \\
\hline $\begin{array}{l}\text { 2.3 - Uso del catalogo della biblioteca su internet: ricerca } \\
\text { di libri o periodici nel campo parole chiave }\end{array}$ & 3,64 & $32,37 \%$ & 3,37 \\
\hline $\begin{array}{l}2.4 \text { - Uso del catalogo della biblioteca su internet: } \\
\text { prenotazione o proroga del prestito di un libro }\end{array}$ & 4,42 & $55,00 \%$ & 3,92 \\
\hline $\begin{array}{l}2.5 \text { - Precisione e rapidità nella distribuzione di libri e } \\
\text { periodici dai magazzini }\end{array}$ & 4,08 & $37,75 \%$ & 3,76 \\
\hline $\begin{array}{l}2.6 \text { - Facilità del recupero di libri e periodici dagli scaffali } \\
\text { liberamente accessibili ai lettori }\end{array}$ & 4,10 & $52,52 \%$ & 3,70 \\
\hline $\begin{array}{l}2.7 \text { - Disponibilità in sala di lettura di posti a sedere per } \\
\text { l'uso di materiale della biblioteca }\end{array}$ & 4,41 & $57,38 \%$ & 3,31 \\
\hline 2.8 - Silenzio in sala di lettura & 4,47 & $63,57 \%$ & 3,44 \\
\hline $\begin{array}{l}2.9 \text { - Comfort e luminosità della sala di lettura } \\
\text { articoli da altre biblioteche universitarie }\end{array}$ & 4,47 & $72,41 \%$ & 3,83 \\
\hline 2.10 - Accesso a internet in biblioteca & 3,71 & $25,25 \%$ & 3,50 \\
\hline
\end{tabular}




\section{ROBERTO VENTURA}

\begin{tabular}{|l|c|c|c|}
\hline $\begin{array}{l}\text { 3. Qualità del reference, dell'orientamento e della } \\
\text { comunicazione all'utenza }\end{array}$ & $a$ & $i$ & $s$ \\
\hline $\begin{array}{l}3.1 \text { - Facilità del recupero e consultazione di bibliografie, } \\
\text { repertori, enciclopedie }\end{array}$ & 3,57 & $20,50 \%$ & 3,39 \\
\hline $\begin{array}{l}3.2-\text { Banche dati attualmente disponibili nelle } \\
\text { biblioteche (via web, su cd-rom) }\end{array}$ & 3,82 & $29,35 \%$ & 3,62 \\
\hline $\begin{array}{l}3.3-\text { Risultati ottenuti dall'interrogazione delle banche } \\
\text { dati }\end{array}$ & 3,65 & $29,35 \%$ & 3,26 \\
\hline $\begin{array}{l}3.4-\text { Banche dati: modo in cui sono messe a disposizione } \\
\text { del pubblico }\end{array}$ & 3,87 & $29,35 \%$ & 3,34 \\
\hline $\begin{array}{l}3.5-\text { Comunicazione con il personale delle biblioteche } \\
\text { (disponibilità e cortesia dei bibliotecari nei confronti del } \\
\text { pubblico) }\end{array}$ & 4,36 & $62,44 \%$ & 3,58 \\
\hline $\begin{array}{l}3.6-\text { Servizi e assistenza personalizzata al lettore da parte } \\
\text { del bibliotecario }\end{array}$ & 3,99 & $42,67 \%$ & 3,42 \\
\hline $\begin{array}{l}3.7-\text { Depliant sulle biblioteche e segnaletica in biblioteca } \\
\text { per facilitare l'uso dei servizi }\end{array}$ & 3,39 & $24,63 \%$ & 3,16 \\
\hline $\begin{array}{l}3.8-\text { Sito web del Sistema Bibliotecario di Ateneo } \\
\text { (esclusi catalogo, prenotazioni e rinnovi) }\end{array}$ & 4,10 & $47,44 \%$ & 3,83 \\
\hline
\end{tabular}




\section{Biblioteca di scienze}

\begin{tabular}{|l|c|c|c|}
\hline 1. Qualità delle acquisizioni cartacee ed elettroniche & $a$ & $i$ & $s$ \\
\hline $\begin{array}{l}\text { 1.1 - Libri, periodici e cd-rom attualmente posseduti } \\
\text { dalla biblioteca }\end{array}$ & 4,12 & $55,58 \%$ & 3,71 \\
\hline $\begin{array}{l}1.2 \text { - Disponibilità in biblioteca delle novità librarie nelle } \\
\text { discipline di tuo interesse }\end{array}$ & 3,97 & $36,11 \%$ & 3,29 \\
\hline $\begin{array}{l}\text { 1.3 - Disponibilità in biblioteca degli ultimi numeri dei } \\
\text { periodici nelle discipline di tuo interesse }\end{array}$ & 3,64 & $26,40 \%$ & 3,32 \\
\hline $\begin{array}{l}1.4-\text { Uso dei periodici elettronici nelle discipline di tuo } \\
\text { interesse }\end{array}$ & 3,49 & $18,71 \%$ & 3,25 \\
\hline
\end{tabular}

\begin{tabular}{|l|c|c|c|}
\hline 2. Qualità della circolazione documentaria & $a$ & $i$ & $s$ \\
\hline $\begin{array}{l}\text { 2.1 - Uso del catalogo della biblioteca su internet: ricerca } \\
\text { di libri o periodici per titolo e ricerca di libri per autore }\end{array}$ & 4,70 & $80,84 \%$ & 4,08 \\
\hline $\begin{array}{l}\text { 2.2 - Uso del catalogo su internet: ricerca di libri per } \\
\text { argomento (nel campo: soggetto) o materia (nel campo: } \\
\text { classificazione) }\end{array}$ & 4,34 & $55,42 \%$ & 3,69 \\
\hline $\begin{array}{l}\text { 2.3 - Uso del catalogo della biblioteca su internet: ricerca } \\
\text { di libri o periodici nel campo parole chiave }\end{array}$ & 3,85 & $36,60 \%$ & 3,60 \\
\hline $\begin{array}{l}2.4 \text { - Uso del catalogo della biblioteca su internet: } \\
\text { prenotazione o proroga del prestito di un libro }\end{array}$ & 4,27 & $50,15 \%$ & 4,07 \\
\hline $\begin{array}{l}2.5 \text { - Precisione e rapidità nella distribuzione di libri e } \\
\text { periodici dai magazzini }\end{array}$ & 4,07 & $39,09 \%$ & 3,73 \\
\hline $\begin{array}{l}2.6 \text { - Facilità del recupero di libri e periodici dagli } \\
\text { scaffali liberamente accessibili ai lettori }\end{array}$ & 4,22 & $57,64 \%$ & 3,70 \\
\hline $\begin{array}{l}2.7 \text { - Disponibilità in sala di lettura di posti a sedere per } \\
\text { 1'uso di materiale della biblioteca }\end{array}$ & 4,41 & $58,01 \%$ & 3,26 \\
\hline 2.8 - Silenzio in sala di lettura & 4,48 & $64,41 \%$ & 3,68 \\
\hline 2.9 - Comfort e luminosità della sala di lettura & 4,49 & $69,48 \%$ & 3,75 \\
\hline 2.10 - Accesso a internet in biblioteca & 4,33 & $47,52 \%$ & 3,14 \\
\hline $\begin{array}{l}2.11 \text { - Prestito interbibliotecario: recupero di libri e } \\
\text { articoli da altre biblioteche universitarie }\end{array}$ & 4,00 & $32,41 \%$ & 3,51 \\
\hline
\end{tabular}




\begin{tabular}{|l|c|c|c|}
\hline $\begin{array}{l}\text { 3. Qualità del reference, dell'orientamento e della } \\
\text { comunicazione all'utenza }\end{array}$ & $a$ & $i$ & $s$ \\
\hline $\begin{array}{l}3.1 \text { - Facilità del recupero e consultazione di } \\
\text { bibliografie, repertori, enciclopedie }\end{array}$ & 3,66 & $24,50 \%$ & 3,50 \\
\hline $\begin{array}{l}3.2-\text { Banche dati attualmente disponibili nelle } \\
\text { biblioteche (via web, su cd-rom) }\end{array}$ & 3,55 & $23,15 \%$ & 3,33 \\
\hline $\begin{array}{l}3.3-\text { Risultati ottenuti dall'interrogazione delle banche } \\
\text { dati }\end{array}$ & 3,64 & $23,15 \%$ & 3,22 \\
\hline $\begin{array}{l}3.4-\text { Banche dati: modo in cui sono messe a } \\
\text { disposizione del pubblico }\end{array}$ & 3,79 & $23,15 \%$ & 3,32 \\
\hline $\begin{array}{l}3.5-\text { Comunicazione con il personale delle biblioteche } \\
\text { (disponibilità e cortesia dei bibliotecari nei confronti del } \\
\text { pubblico) }\end{array}$ & 4,45 & $67,56 \%$ & 3,74 \\
\hline $\begin{array}{l}3.6 \text { - Servizi e assistenza personalizzata al lettore da } \\
\text { parte del bibliotecario }\end{array}$ & 3,98 & $38,78 \%$ & 3,61 \\
\hline $\begin{array}{l}3.7-\text { Depliant sulle biblioteche e segnaletica in } \\
\text { biblioteca per facilitare l'uso dei servizi }\end{array}$ & 3,51 & $30,28 \%$ & 3,42 \\
\hline $\begin{array}{l}3.8-\text { Sito web del Sistema Bibliotecario di Ateneo } \\
\text { (esclusi catalogo, prenotazioni e rinnovi) }\end{array}$ & 4,41 & $60,02 \%$ & 3,95 \\
\hline
\end{tabular}




\section{Biblioteca di scienze sociali}

\begin{tabular}{|l|c|c|c|}
\hline 1. Qualità delle acquisizioni cartacee ed elettroniche & $a$ & $i$ & $s$ \\
\hline $\begin{array}{l}\text { 1.1 - Libri, periodici e cd-rom attualmente posseduti } \\
\text { dalla biblioteca }\end{array}$ & 3,97 & $51,48 \%$ & 3,87 \\
\hline $\begin{array}{l}1.2-\text { Disponibilità in biblioteca delle novità librarie } \\
\text { nelle discipline di tuo interesse }\end{array}$ & 3,93 & $33,50 \%$ & 3,37 \\
\hline $\begin{array}{l}\text { 1.3 - Disponibilità in biblioteca degli ultimi numeri dei } \\
\text { periodici nelle discipline di tuo interesse }\end{array}$ & 3,83 & $30,51 \%$ & 3,42 \\
\hline $\begin{array}{l}1.4-\text { Uso dei periodici elettronici nelle discipline di tuo } \\
\text { interesse }\end{array}$ & 3,57 & $25,00 \%$ & 3,35 \\
\hline
\end{tabular}

\begin{tabular}{|l|c|c|c|}
\hline 2. Qualità della circolazione documentaria & $a$ & $i$ & $s$ \\
\hline $\begin{array}{l}\text { 2.1 - Uso del catalogo della biblioteca su internet: } \\
\text { ricerca di libri o periodici per titolo e ricerca di libri per } \\
\text { autore }\end{array}$ & 4,54 & $74,09 \%$ & 3,85 \\
\hline $\begin{array}{l}\text { 2.2 - Uso del catalogo su internet: ricerca di libri per } \\
\text { argomento (nel campo: soggetto) o materia (nel campo: } \\
\text { classificazione) }\end{array}$ & 4,20 & $56,57 \%$ & 3,60 \\
\hline $\begin{array}{l}\text { 2.3 - Uso del catalogo della biblioteca su internet: } \\
\text { ricerca di libri o periodici nel campo parole chiave }\end{array}$ & 3,90 & $46,53 \%$ & 3,39 \\
\hline $\begin{array}{l}2.4 \text { - Uso del catalogo della biblioteca su internet: } \\
\text { prenotazione o proroga del prestito di un libro }\end{array}$ & 4,13 & $40,77 \%$ & 3,78 \\
\hline $\begin{array}{l}2.5 \text { - Precisione e rapidità nella distribuzione di libri e } \\
\text { periodici dai magazzini }\end{array}$ & 4,19 & $48,61 \%$ & 3,57 \\
\hline $\begin{array}{l}2.6 \text { - Facilità del recupero di libri e periodici dagli } \\
\text { scaffali liberamente accessibili ai lettori }\end{array}$ & 4,24 & $52,88 \%$ & 3,73 \\
\hline $\begin{array}{l}2.7 \text { - Disponibilità in sala di lettura di posti a sedere per } \\
\text { 1'uso di materiale della biblioteca }\end{array}$ & 4,28 & $50,12 \%$ & 2,95 \\
\hline 2.8 - Silenzio in sala di lettura & 4,45 & $58,82 \%$ & 3,42 \\
\hline 2.9 - Comfort e luminosità della sala di lettura & 4,49 & $64,64 \%$ & 3,58 \\
\hline $\begin{array}{l}2.10 \text { - Accesso a internet in biblioteca } \\
\text { articoli da altre biblioteche universitarie }\end{array}$ & 4,42 & $45,50 \%$ & 2,98 \\
\hline
\end{tabular}




\begin{tabular}{|l|c|c|c|}
\hline $\begin{array}{l}\text { 3. Qualità del reference, dell'orientamento e della } \\
\text { comunicazione all'utenza }\end{array}$ & $a$ & $i$ & $s$ \\
\hline $\begin{array}{l}3.1 \text { - Facilità del recupero e consultazione di } \\
\text { bibliografie, repertori, enciclopedie }\end{array}$ & 3,79 & $28,44 \%$ & 3,43 \\
\hline $\begin{array}{l}3.2 \text { - Banche dati attualmente disponibili nelle } \\
\text { biblioteche (via web, su cd-rom) }\end{array}$ & 3,86 & $34,23 \%$ & 3,43 \\
\hline $\begin{array}{l}3.3 \text { - Risultati ottenuti dall'interrogazione delle banche } \\
\text { dati }\end{array}$ & 3,88 & $34,23 \%$ & 3,29 \\
\hline $\begin{array}{l}3.4-\text { Banche dati: modo in cui sono messe a } \\
\text { disposizione del pubblico }\end{array}$ & 3,86 & $34,23 \%$ & 3,29 \\
\hline $\begin{array}{l}3.5-\text { Comunicazione con il personale delle biblioteche } \\
\text { (disponibilità e cortesia dei bibliotecari nei confronti del } \\
\text { pubblico) }\end{array}$ & 4,50 & $66,67 \%$ & 3,79 \\
\hline $\begin{array}{l}3.6-\text { Servizi e assistenza personalizzata al lettore da } \\
\text { parte del bibliotecario }\end{array}$ & 4,03 & $42,49 \%$ & 3,47 \\
\hline $\begin{array}{l}3.7-\text { Depliant sulle biblioteche e segnaletica in } \\
\text { biblioteca per facilitare l'uso dei servizi }\end{array}$ & 3,64 & $27,90 \%$ & 3,13 \\
\hline $\begin{array}{l}3.8-\text { Sito web del Sistema Bibliotecario di Ateneo } \\
\text { (esclusi catalogo, prenotazioni e rinnovi) }\end{array}$ & 4,20 & $48,25 \%$ & 3,87 \\
\hline
\end{tabular}




\section{Biblioteca di scienze tecnologiche}

\begin{tabular}{|l|c|c|c|}
\hline 1. Qualità delle acquisizioni cartacee ed elettroniche & $a$ & $i$ & $s$ \\
\hline $\begin{array}{l}\text { 1.1 - Libri, periodici e cd-rom attualmente posseduti } \\
\text { dalla biblioteca }\end{array}$ & 4,05 & $54,63 \%$ & 3,86 \\
\hline $\begin{array}{l}1.2-\text { Disponibilità in biblioteca delle novità librarie } \\
\text { nelle discipline di tuo interesse }\end{array}$ & 3,70 & $37,39 \%$ & 3,64 \\
\hline $\begin{array}{l}1.3 \text { - Disponibilità in biblioteca degli ultimi numeri dei } \\
\text { periodici nelle discipline di tuo interesse }\end{array}$ & 3,63 & $32,42 \%$ & 3,58 \\
\hline $\begin{array}{l}1.4-\text { Uso dei periodici elettronici nelle discipline di tuo } \\
\text { interesse }\end{array}$ & 3,46 & $22,16 \%$ & 3,72 \\
\hline
\end{tabular}

\begin{tabular}{|l|c|c|c|}
\hline 2. Qualità della circolazione documentaria & $a$ & $i$ & $s$ \\
\hline $\begin{array}{l}\text { 2.1 - Uso del catalogo della biblioteca su internet: } \\
\text { ricerca di libri o periodici per titolo e ricerca di libri per } \\
\text { autore }\end{array}$ & 4,64 & $77,67 \%$ & 4,17 \\
\hline $\begin{array}{l}\text { 2.2 - Uso del catalogo su internet: ricerca di libri per } \\
\text { argomento (nel campo: soggetto) o materia (nel campo: } \\
\text { classificazione) }\end{array}$ & 4,35 & $56,42 \%$ & 3,80 \\
\hline $\begin{array}{l}\text { 2.3 - Uso del catalogo della biblioteca su internet: } \\
\text { ricerca di libri o periodici nel campo parole chiave }\end{array}$ & 3,83 & $39,32 \%$ & 3,77 \\
\hline $\begin{array}{l}2.4 \text { - Uso del catalogo della biblioteca su internet: } \\
\text { prenotazione o proroga del prestito di un libro }\end{array}$ & 4,17 & $45,68 \%$ & 3,90 \\
\hline $\begin{array}{l}2.5 \text { - Precisione e rapidità nella distribuzione di libri e } \\
\text { periodici dai magazzini }\end{array}$ & 3,69 & $35,85 \%$ & 3,46 \\
\hline $\begin{array}{l}2.6 \text { - Facilità del recupero di libri e periodici dagli } \\
\text { scaffali liberamente accessibili ai lettori }\end{array}$ & 4,46 & $70,06 \%$ & 3,44 \\
\hline $\begin{array}{l}2.7 \text { - Disponibilità in sala di lettura di posti a sedere per } \\
\text { 1'uso di materiale della biblioteca }\end{array}$ & 4,16 & $54,44 \%$ & 3,37 \\
\hline 2.8 - Silenzio in sala di lettura & 4,44 & $61,63 \%$ & 3,76 \\
\hline 2.9 - Comfort e luminosità della sala di lettura & 4,52 & $63,99 \%$ & 4,06 \\
\hline $\begin{array}{l}2.10 \text { - Accesso a internet in biblioteca } \\
\text { articoli da altre biblioteche universitarie }\end{array}$ & 3,77 & $29,10 \%$ & 3,59 \\
\hline
\end{tabular}




\begin{tabular}{|l|c|c|c|}
\hline $\begin{array}{l}\text { 3. Qualità del reference, dell'orientamento e della } \\
\text { comunicazione all'utenza }\end{array}$ & $a$ & $i$ & $s$ \\
\hline $\begin{array}{l}3.1 \text { - Facilità del recupero e consultazione di } \\
\text { bibliografie, repertori, enciclopedie }\end{array}$ & 3,64 & $30,44 \%$ & 3,70 \\
\hline $\begin{array}{l}3.2-\text { Banche dati attualmente disponibili nelle } \\
\text { biblioteche (via web, su cd-rom) }\end{array}$ & 3,52 & $26,51 \%$ & 3,78 \\
\hline $\begin{array}{l}3.3-\text { Risultati ottenuti dall'interrogazione delle banche } \\
\text { dati }\end{array}$ & 3,65 & $26,51 \%$ & 3,45 \\
\hline $\begin{array}{l}3.4-\text { Banche dati: modo in cui sono messe a } \\
\text { disposizione del pubblico }\end{array}$ & 3,68 & $26,51 \%$ & 3,43 \\
\hline $\begin{array}{l}3.5-\text { Comunicazione con il personale delle biblioteche } \\
\text { (disponibilità e cortesia dei bibliotecari nei confronti del } \\
\text { pubblico) }\end{array}$ & 4,33 & $65,13 \%$ & 3,82 \\
\hline $\begin{array}{l}3.6-\text { Servizi e assistenza personalizzata al lettore da } \\
\text { parte del bibliotecario }\end{array}$ & 3,98 & $41,11 \%$ & 3,84 \\
\hline $\begin{array}{l}3.7-\text { Depliant sulle biblioteche e segnaletica in } \\
\text { biblioteca per facilitare l'uso dei servizi }\end{array}$ & 3,34 & $33,09 \%$ & 3,71 \\
\hline $\begin{array}{l}3.8-\text { Sito web del Sistema Bibliotecario di Ateneo } \\
\text { (esclusi catalogo, prenotazioni e rinnovi) }\end{array}$ & 4,27 & $54,50 \%$ & 4,02 \\
\hline
\end{tabular}




\section{Biblioteca umanistica}

\begin{tabular}{|l|c|c|c|}
\hline 1. Qualità delle acquisizioni cartacee ed elettroniche & $a$ & $i$ & $s$ \\
\hline $\begin{array}{l}\text { 1.1 - Libri, periodici e cd-rom attualmente posseduti } \\
\text { dalla biblioteca }\end{array}$ & 3,97 & $53,16 \%$ & 3,67 \\
\hline $\begin{array}{l}1.2 \text { - Disponibilità in biblioteca delle novità librarie } \\
\text { nelle discipline di tuo interesse }\end{array}$ & 3,63 & $33,85 \%$ & 3,20 \\
\hline $\begin{array}{l}1.3 \text { - Disponibilità in biblioteca degli ultimi numeri dei } \\
\text { periodici nelle discipline di tuo interesse }\end{array}$ & 3,35 & $20,48 \%$ & 3,23 \\
\hline $\begin{array}{l}1.4 \text { - Uso dei periodici elettronici nelle discipline di tuo } \\
\text { interesse }\end{array}$ & 3,13 & $14,93 \%$ & 3,50 \\
\hline
\end{tabular}

\begin{tabular}{|l|c|c|c|}
\hline 2. Qualità della circolazione documentaria & $a$ & $i$ & $s$ \\
\hline $\begin{array}{l}\text { 2.1 - Uso del catalogo della biblioteca su internet: } \\
\text { ricerca di libri o periodici per titolo e ricerca di libri per } \\
\text { autore }\end{array}$ & 4,70 & $83,33 \%$ & 4,11 \\
\hline $\begin{array}{l}2.2 \text { - Uso del catalogo su internet: ricerca di libri per } \\
\text { argomento (nel campo: soggetto) o materia (nel campo: } \\
\text { classificazione) }\end{array}$ & 4,05 & $53,42 \%$ & 3,68 \\
\hline $\begin{array}{l}2.3 \text { - Uso del catalogo della biblioteca su internet: } \\
\text { ricerca di libri o periodici nel campo parole chiave }\end{array}$ & 3,44 & $32,59 \%$ & 3,31 \\
\hline $\begin{array}{l}2.4 \text { - Uso del catalogo della biblioteca su internet: } \\
\text { prenotazione o proroga del prestito di un libro }\end{array}$ & 4,11 & $39,16 \%$ & 3,75 \\
\hline $\begin{array}{l}2.5 \text { - Precisione e rapidità nella distribuzione di libri e } \\
\text { periodici dai magazzini }\end{array}$ & 4,07 & $52,23 \%$ & 3,66 \\
\hline $\begin{array}{l}2.6 \text { - Facilità del recupero di libri e periodici dagli } \\
\text { scaffali liberamente accessibili ai lettori }\end{array}$ & 3,91 & $43,91 \%$ & 3,22 \\
\hline $\begin{array}{l}2.7 \text { - Disponibilità in sala di lettura di posti a sedere per } \\
\text { l'uso di materiale della biblioteca }\end{array}$ & 4,13 & $50,78 \%$ & 3,28 \\
\hline 2.8 - Silenzio in sala di lettura & 4,46 & $66,15 \%$ & 3,66 \\
\hline $\begin{array}{l}2.11 \text { - Prestito interbibliotecario: recupero di libri e } \\
\text { articoli da altre biblioteche universitarie }\end{array}$ & 4,80 & $29,74 \%$ & 3,32 \\
\hline \begin{tabular}{l}
2.10 - Acomfort e luminosità della sala di lettura \\
\hline
\end{tabular} & 4,13 & $48,20 \%$ & 3,02 \\
\hline
\end{tabular}




\begin{tabular}{|l|c|c|c|}
\hline $\begin{array}{l}\text { 3. Qualità del reference, dell'orientamento e della } \\
\text { comunicazione all'utenza }\end{array}$ & $a$ & $i$ & $s$ \\
\hline $\begin{array}{l}3.1 \text { - Facilità del recupero e consultazione di } \\
\text { bibliografie, repertori, enciclopedie }\end{array}$ & 3,65 & $30,63 \%$ & 3,23 \\
\hline $\begin{array}{l}3.2-\text { Banche dati attualmente disponibili nelle } \\
\text { biblioteche (via web, su cd-rom) }\end{array}$ & 3,63 & $24,64 \%$ & 3,38 \\
\hline $\begin{array}{l}3.3 \text { - Risultati ottenuti dall'interrogazione delle banche } \\
\text { dati }\end{array}$ & 3,61 & $24,64 \%$ & 3,26 \\
\hline $\begin{array}{l}3.4-\text { Banche dati: modo in cui sono messe a } \\
\text { disposizione del pubblico }\end{array}$ & 3,87 & $24,64 \%$ & 3,25 \\
\hline $\begin{array}{l}3.5-\text { Comunicazione con il personale delle biblioteche } \\
\text { (disponibilità e cortesia dei bibliotecari nei confronti } \\
\text { del pubblico) }\end{array}$ & 4,32 & $65,61 \%$ & 3,62 \\
\hline $\begin{array}{l}3.6-\text { Servizi e assistenza personalizzata al lettore da } \\
\text { parte del bibliotecario }\end{array}$ & 3,98 & $43,56 \%$ & 3,54 \\
\hline $\begin{array}{l}3.7-\text { Depliant sulle biblioteche e segnaletica in } \\
\text { biblioteca per facilitare l'uso dei servizi }\end{array}$ & 3,38 & $26,17 \%$ & 3,09 \\
\hline $\begin{array}{l}3.8-\text { Sito web del Sistema Bibliotecario di Ateneo } \\
\text { (esclusi catalogo, prenotazioni e rinnovi) }\end{array}$ & 4,21 & $55,66 \%$ & 3,93 \\
\hline
\end{tabular}




\title{
Analisi di un case study: la Biblioteca di Scienze Sociali
}

\author{
di ELEONORA GiUsti
}

\section{Introduzione}

Nell'ambito dell'Indagine sulla soddisfazione degli utenti delle biblioteche dell'Ateneo fiorentino, questo contributo analizza il giudizio espresso dagli utenti/studenti della Biblioteca di Scienze sociali ${ }^{1}$ su alcune caratteristiche rilevanti della loro biblioteca di riferimento.

La Bss è il risultato dell'accorpamento di sette ex-biblioteche dislocate in varie zone della città 2 : Economia, Giurisprudenza (con la collezione distribuita nella sede centrale e in tre dipartimenti della facoltà), Scienze politiche, Statistica e l'Emeroteca di Scienze politiche. La biblioteca dalla sua costituzione ad oggi ha vissuto le non poche difficoltà dell'integrazione di realtà fra loro distanti fisicamente e molto diverse per storia, struttura, dimensioni, organizzazione e gestione dei servizi. Oggi siamo alla vigilia del trasloco, che farà confluire tutti i punti di servizio in un'unica nuova sede; è auspicio di questo lavoro che il parere degli utenti possa contribuire alla giusta valorizzazione del patrimonio e più in generale dei servizi della nuova biblioteca, ampliando così le potenzialità e i contributi che possono essere offerti allo sviluppo della didattica e della ricerca scientifica.

\section{La popolazione di interesse}

Oggetto dell'indagine ${ }^{3}$ sono state le sole ex-biblioteche di facoltà confluite nella Bss: Economia, Giurisprudenza e Scienze politiche. Dalle liste degli utenti del prestito dei suddetti punti di servizio sono stati individuati 7060 studenti, utenti unici del prestito ${ }^{4}$ nell'anno 2000, di cui 2497 di Economia,

1 D'ora in avanti Bss.

2 La costituzione della Biblioteca di area è avvenuta il 12 maggio 1999, col decreto rettorale n. 668 (Università degli Studi di Firenze 2001a).

3 Per tutte le problematiche relative alla metodologia applicata nell'indagine si veda il contributo di Silvana Benedetti.

4 Si parla di utenti unici, perché nel caso in cui lo studente è risultato iscritto al prestito in più punti di servizio, lo si è considerato utente di quello in cui ha preso in prestito il maggior numero di documenti. 
1593 di Scienze politiche e 2970 di Giurisprudenza. Dalla popolazione così individuata, mediante un disegno di campionamento probabilistico, è stato selezionato un campione rappresentativo a cui sottoporre il questionario. Complessivamente sono stati intervistati 75 utenti di Economia e 131 di Giurisprudenza e Scienze politiche; gli utenti di Giurisprudenza e di Scienze politiche sono stati infatti raggruppati dato che le due ex-biblioteche di facoltà, pur essendo dotate di collezioni distinte, sono situate in un'unica sede.

Le informazioni di carattere anagrafico, ricavate dallo spoglio delle interviste, hanno permesso di ricostruire alcune caratteristiche del profilo degli utenti/studenti del prestito della biblioteca. Qui di seguito vengono infatti rappresentate alcune distribuzioni semplici di frequenza delle variabili ritenute più significative: il sesso, la residenza, la prossimità alla laurea e la condizione lavorativa.

Il 66\% degli intervistati sono donne, confermando la tendenza riscontrata anche nella composizione del campione dell'intera indagine; altra caratteristica rilevata è che il $48 \%$ degli intervistati ha dichiarato di risiedere nel comune di Firenze e solo il 7\% di non essere residente in Toscana.

\section{Composizione del campione per sesso}

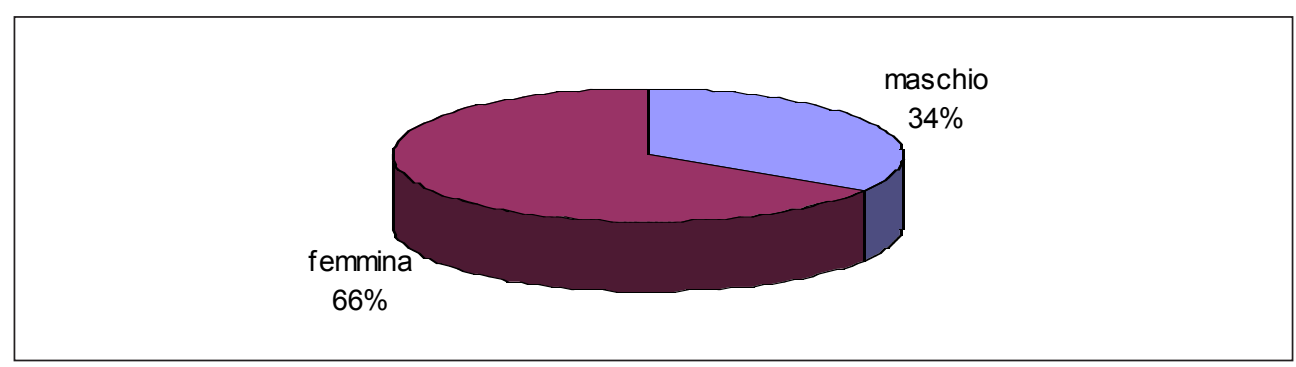

Residenza

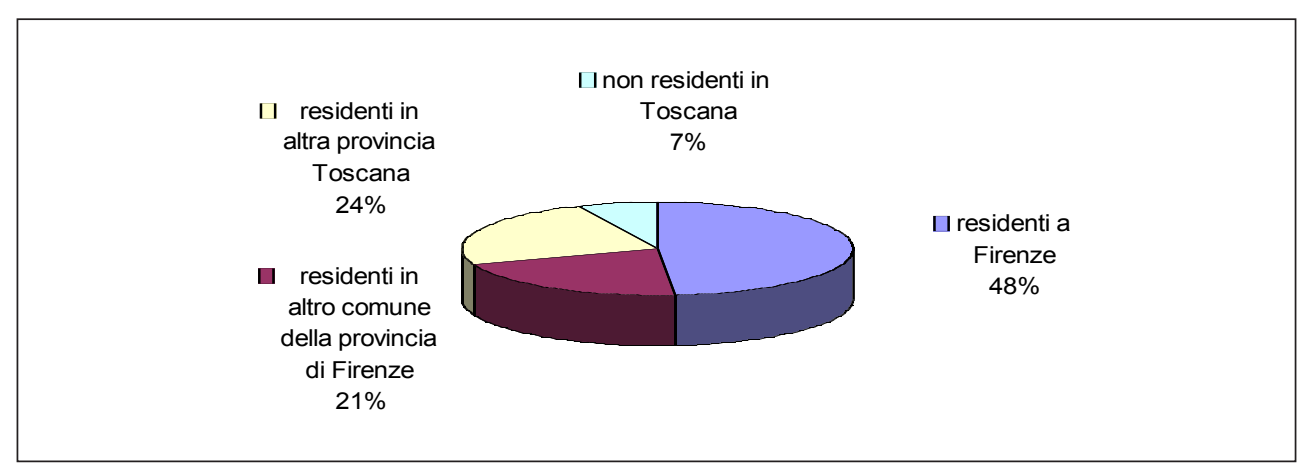


I componenti del campione sono fortemente caratterizzati dalla prossimità alla laurea, infatti il 61\% degli intervistati è o già laureato al momento dell'intervista o ha già chiesto il titolo della tesi. Il giudizio degli intervistati, almeno per oltre la metà di loro, dovrebbe quindi esprimere la valutazione di utenti "esperti" cioè di coloro che da più tempo usano la biblioteca e che molto probabilmente hanno avuto l'opportunità di conoscerne tutti i servizi. Ultima caratteristica rilevata è la condizione lavorativa: il 46\% degli intervistati ha un'occupazione a tempo pieno o part-time.

\section{Prossimità alla laurea}

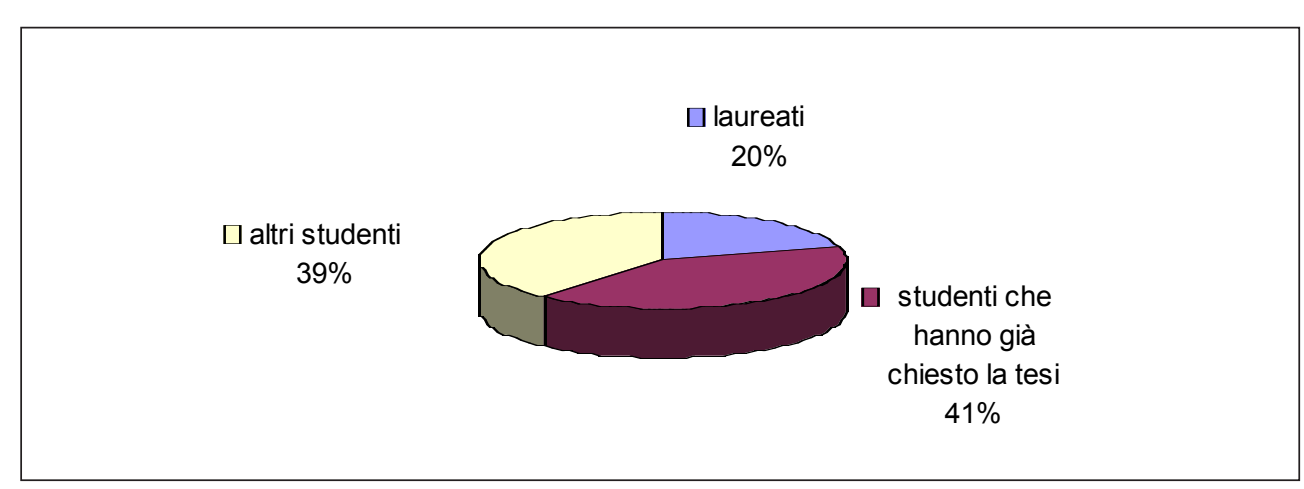

Condizione lavorativa

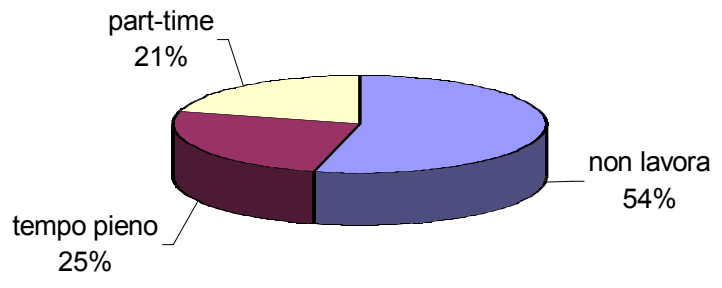




\section{L'analisi dei risultati}

In questo contributo vengono analizzate le valutazioni espresse dai 206 intervistati che, data la rappresentatività del campione, possono essere estese all'intera popolazione di utenti/studenti del prestito della Bss.

In particolare qui vengono analizzati i giudizi espressi su quattro delle sei dimensioni indagate complessivamente dall'indagine ${ }^{5}$ :

- Offerta documentaria;

- Accessibilità delle risorse;

- Ambienti;

- Personale.

Queste dimensioni sono infatti facilmente riconducibili ad una specifica biblioteca e, in fase di stesura del questionario, sono state individuate fra le caratteristiche più rilevanti dell'attività dell'organizzazione/biblioteca. L'analisi riguarda pertanto le risposte fornite dagli utenti/studenti alle 15 voci, cioè i quesiti del questionario, inerenti le suddette dimensioni; e per ogni voce ne vengono indagati i seguenti aspetti:

- livello di importanza attribuita (qualità attesa);

- percentuale d'uso (grado di utilizzo);

- soddisfazione (qualità percepita).

Per ciascuna dimensione si rappresentano graficamente il livello di importanza attribuito, le abitudini espresse con la percentuale d'uso, il giudizio sul grado di soddisfazione. La scelta di impiegare per le risposte relative all'importanza e alla soddisfazione la scala a 5 valori di Likert ci permette di stabilire la percentuale di risposte positive e negative e quindi di rappresentare graficamente in modo evidente i giudizi degli utenti ${ }^{6}$. Inoltre vengono messi in relazione e rappresentati con un grafico a bolle tutti i dati raccolti: il grado di giudizio espresso sull'importanza o sulla soddisfazione (sull'asse delle X), il numero di utenti che hanno espresso quel giudizio (asse delle Y), la frequenza di uso della risorsa rappresentata da cerchi (bolle), che nel punto d'incontro fra ascisse e ordinate indicano con la loro ampiezza la percentuale media di uso.

5 Sono quindi escluse le risposte degli utenti relative ai servizi avanzati e alla qualità dell'OPAC; per queste si rimanda al contributo di Francesca Landi.

6 Si ottiene questo sommando le due voci poste all'estremità della scala (ad esempio, combinando per nulla importante + poco importante da una parte; importante + molto importante dall'altra). Hayes 2000, p. 87. 
Infine vengono confrontate le valutazione raccolte per le quattro dimensioni e i risultati relativi alle voci più significative.

\section{Valutazione dell'Offerta documentaria}

\begin{tabular}{|l|l|}
\hline \multicolumn{2}{|l|}{ Offerta documentaria } \\
\hline Voci & $\begin{array}{l}\text { Libri, periodici e cd-rom posseduti dalla biblioteca; } \\
\text { Disponibilità in biblioteca delle novità librarie nelle discipli- } \\
\text { ne di interesse dell'intervistato; } \\
\text { Disponibilità in biblioteca degli ultimi numeri dei periodici } \\
\text { nelle discipline di interesse dell'intervistato; } \\
\text { Prestito interbibliotecario: recupero di libri e articoli da altre } \\
\text { biblioteche universitarie; }\end{array}$ \\
\hline Aspetti & $\begin{array}{l}\text { Importanza } \\
\text { Percentuale d'uso } \\
\text { Grado di soddisfazione }\end{array}$ \\
\hline
\end{tabular}

Con questa dimensione si vuole conoscere il parere sulle tradizionali risorse che gli utenti si aspettano di trovare in biblioteca; infatti si chiede di esprimere giudizi sulla qualità e sull'aggiornamento della documentazione disponibile, soprattutto in relazione alle discipline d'interesse. Inoltre si indaga anche sul servizio di prestito interbibliotecario, che ha proprio lo scopo di ampliare temporaneamente la disponibilità di documenti, in seguito a specifiche esigenze degli utenti.

Prima di esaminare i risultati dello spoglio, è opportuno offrire alcuni elementi quantitativi per descrivere l'offerta documentaria dei punti di servizio in oggetto nell'anno $2000^{7}$, periodo di riferimento dell'indagine. Complessivamente il patrimonio documentario, costituito da monografie e periodici, era pari a 709.568 volumi e rappresentava quasi il $22 \%$ dell'intero patrimonio del Sistema bibliotecario d'Ateneo. La collezione, caratterizzata anche da importanti e prestigiosi fondi storici, ha avuto, nel periodo di riferimento, un accrescimento pari a oltre 8000 documenti, incremento che rappresenta il $25 \%$ della crescita dell'intero patrimonio documentario del SBA. Nell'anno 2000 sono giunti in abbonamento 2131 periodici. Non è disponibile il dato relativo ai cd-rom. Il ser-

7 I dati sono stati forniti dal sistema informativo gestionale del Sistema bibliotecario di Ateneo. 
vizio di prestito interbibliotecario ha avuto una forte crescita proprio a partire dal 2000, anno in cui è stato riorganizzato il servizio, potenziando anche la fornitura dei documenti in versione elettronica; in particolare dal 1999 al 2000 il volume delle transazioni ha avuto un incremento complessivo del $10 \%{ }^{8}$

Dallo spoglio si rileva che mediamente il $65,5 \%$ degli utenti/studenti ritiene importante o molto importante questa dimensione, il 9,2\% esprime invece un giudizio negativo, in termini di importanza; e ben il 16,7\% si definisce indifferente (Fig. 1). Da una disamina delle singole voci emerge che la voce libri, periodici e cd-rom si differenzia dalle altre tre voci, che invece si attestano su valori molto ravvicinati fra loro; inoltre questa voce ottiene la maggior percentuale di giudizi positivi e la minore di giudizi negativi e di risposte non sa.

Fig. 1 - Importanza attribuita all'Offerta documentaria

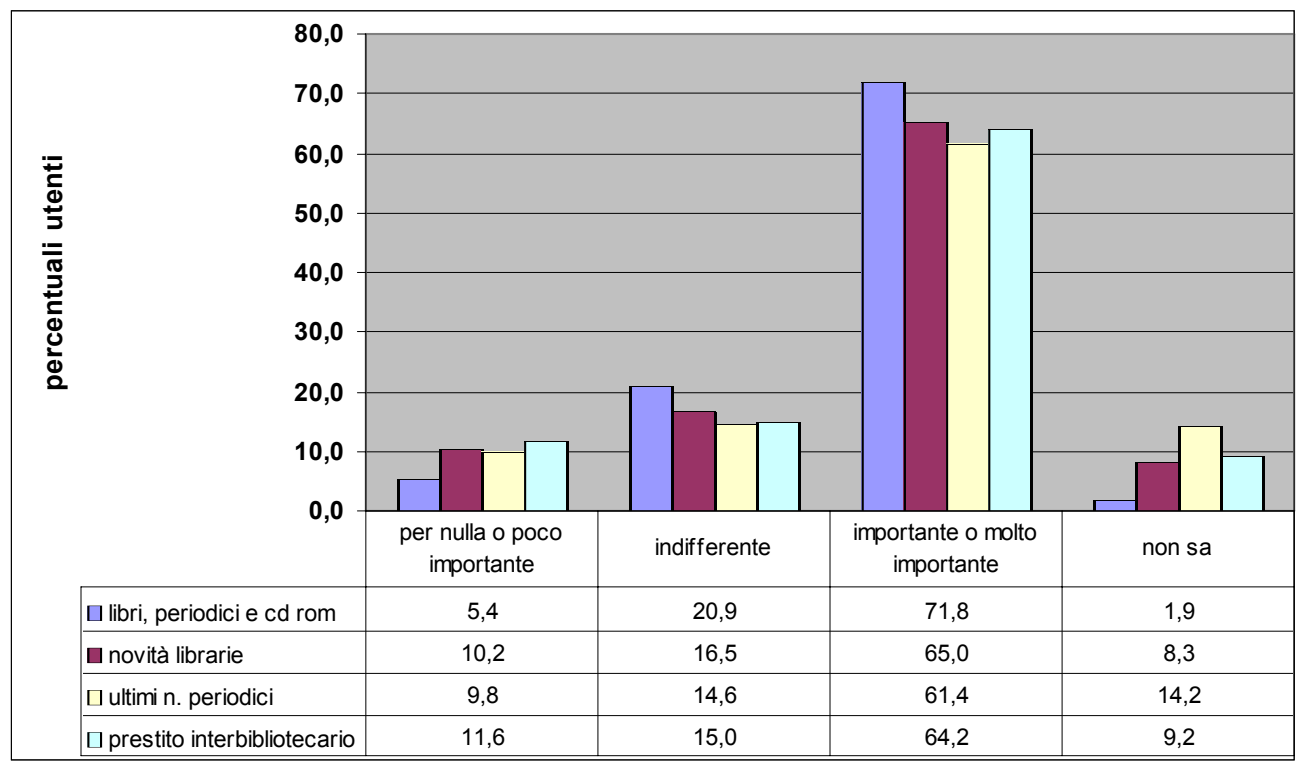

Riguardo alla frequenza d'uso, le risorse più utilizzate sono proprio i libri, i periodici e i cd-rom: oltre il 58\% degli utenti li usa almeno una volta su due che visita la biblioteca e solo il 9,25\% degli utenti non li utilizza mai (Fig. 2). Pochi invece utilizzano le novità librarie e gli ultimi numeri dei periodici, rispettivamente il $30 \%$ non usa mai le prime e oltre il $42 \%$ i secondi. Superio-

8 Il servizio ha continuato a crescere, in particolare dal 2000 al 2001 il borrowing ha avuto un incremento del 73\%; sarebbe quindi interessante conoscere la valutazione degli utenti anche per gli anni successivi. 
re il dato relativo al servizio di prestito interbibliotecario: oltre il $45 \%$ degli utenti dichiara di utilizzarlo almeno una volta su due, che si reca in biblioteca.

Fig. 2 - Utilizzo dell'Offerta documentaria

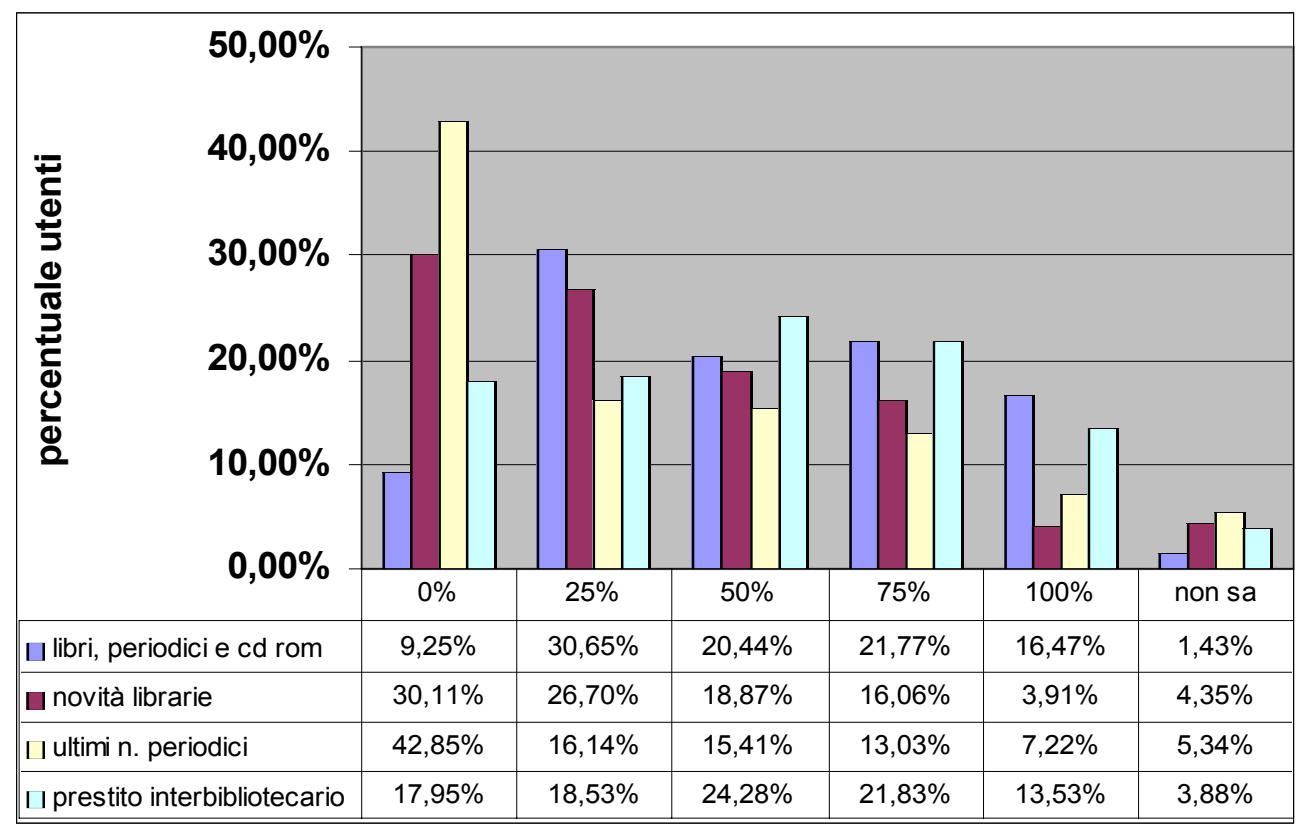

Passando all'aspetto relativo alla soddisfazione (Fig. 3), mediamente il $37,1 \%$ si ritiene abbastanza soddisfatto o soddisfatto, con una netta prevalenza dei soddisfatti; i giudizi positivi superano ampiamente quelli negativi, che sono espressi solo dall' $8,12 \%$. Anche in termini di soddisfazione emerge la voce relativa ai libri, periodici e cd-rom, per la quale il 63\% dichiara un giudizio positivo e il 3,9\% un giudizio negativo. Complessivamente la dimensione Offerta documentaria è ben conosciuta dagli utenti, che sono in grado di valutarne il grado di soddisfazione, infatti in media, solo il 3,7\% dichiara di non saper rispondere.

Rappresentando graficamente la relazione esistente fra importanza attribuita e frequenza d'uso (Fig. 4), è evidente che per tutte e quattro le voci indagate cresce la percentuale media di uso (rappresentata dall'ampiezza dei cerchi) di pari passo all'importanza attribuita; è pertanto la maggioranza della popolazione quella che usa, e quindi conosce la dimensione e le attribuisce importanza. Stessa relazione si evidenzia nel grafico (Fig. 5), che rappresenta invece la relazione fra l'uso e la soddisfazione percepita: gli 
abbastanza soddisfatti prevalgono sui soddisfatti e, anche in questo caso, coloro che esprimono un giudizio positivo sono proprio gli utenti/studenti, che abitualmente usano tali risorse.

Fig. 3 - Soddisfazione percepita per l'Offerta documentaria

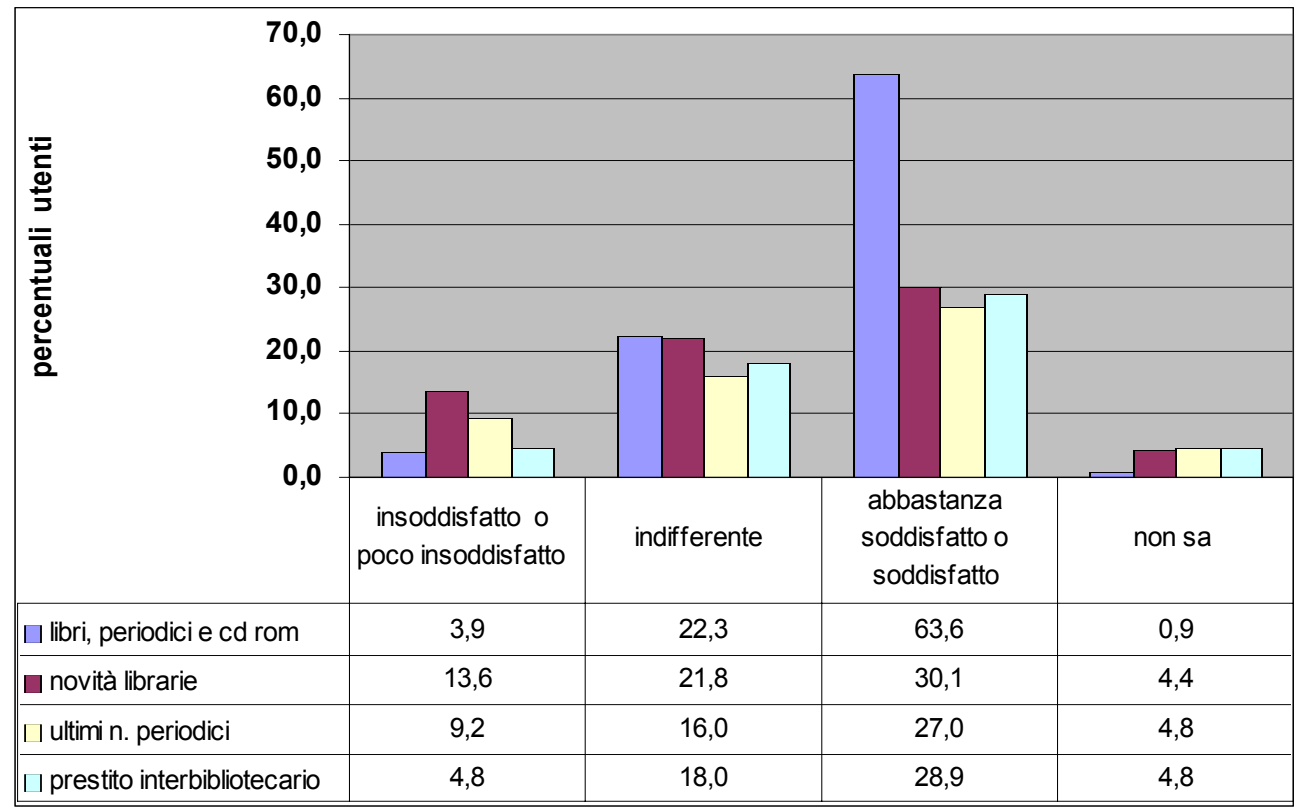

Fig. 4 - Relazione fra uso, importanza attribuita all'Offerta documentaria e numero di utenti

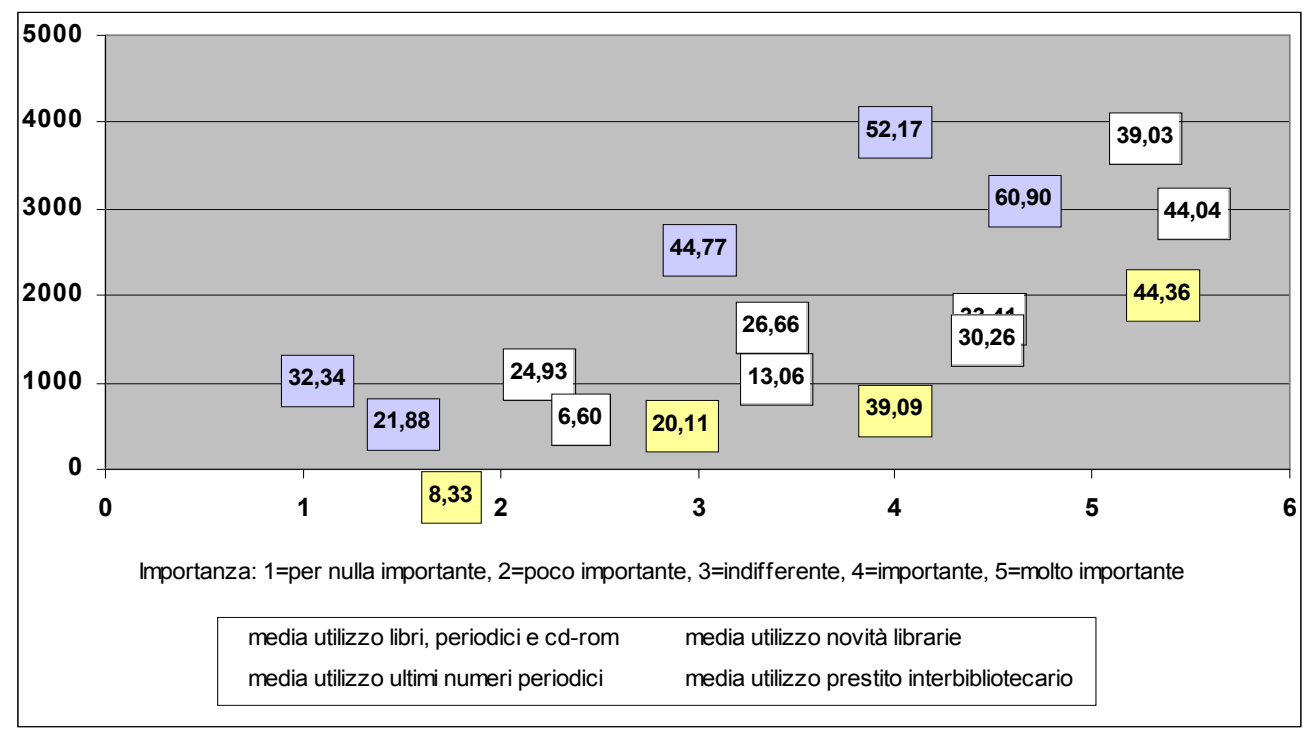


Fig. 5 - Relazione fra uso, soddisfazione percepita per l'Offerta documentaria e numero di utenti

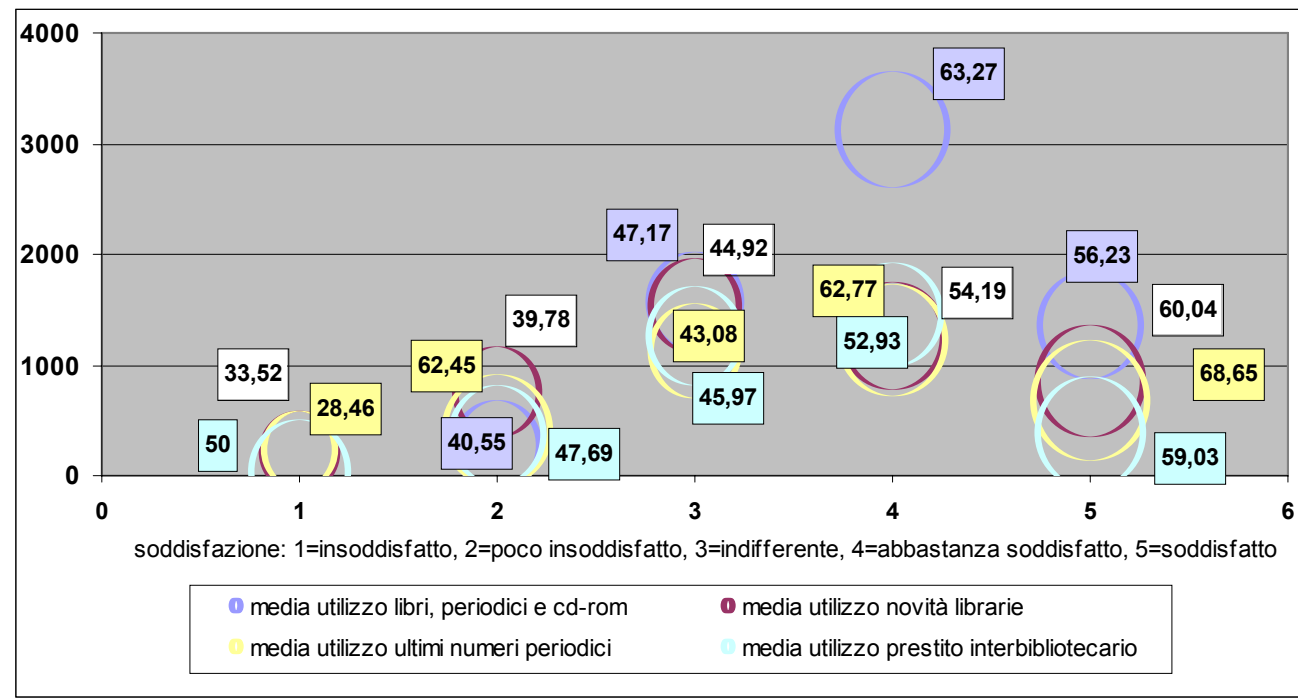

Valutazione dell'Accessibilità delle risorse

\begin{tabular}{|l|l|}
\hline \multicolumn{2}{|l|}{ Accessibilità delle risorse } \\
\hline Voci & $\begin{array}{l}\text { Facilità di recupero di libri e periodici dagli scaffali libera- } \\
\text { mente accessibili ai lettori; } \\
\text { Facilità di recupero e consultazione di bibliografie, repertori, } \\
\text { enciclopedie; } \\
\text { Accesso a Internet in biblioteca; } \\
\text { Banche dati: modo in cui sono messe a disposizione del pub- } \\
\text { blico; }\end{array}$ \\
\hline Aspetti & $\begin{array}{l}\text { Importanza } \\
\text { Percentuale d'uso (non rilevato per la voce banche dati) } \\
\text { Grado di soddisfazione }\end{array}$ \\
\hline
\end{tabular}

In questa dimensione sono raccolte voci fra loro "eterogenee": si indaga infatti sull'accessibilità di risorse sia cartacee che elettroniche. Lo scopo è quello di cogliere il giudizio degli utenti su alcune soluzioni che, in questi anni di riorganizzazione dei servizi, hanno impegnato molto le biblioteche: da un lato l'allestimento dello scaffale aperto, dall'altro il potenziamento 
delle postazioni dedicate alla ricerca in rete e alla consultazione delle banche dati.

In particolare per la Bss, nel punto di servizio di economia, nella sala del prestito e nella sala di lettura, sono state collocate a scaffale aperto le ultime annate (mediamente 10-15 anni per titolo) di circa 70 periodici fra quelli più utilizzati, che precedentemente erano collocati nel pozzo librario. Nel punto di servizio di giurisprudenza/scienze politiche sono stati collocati a scaffale aperto tutti i manuali per la didattica. Complessivamente i punti di servizio in oggetto, nell'anno 2000, offrivano oltre 5800 volumi a scaffale aperto ed erano dotati di 21 postazioni dedicate alla navigazione in rete e alla consultazione delle banche dati.

Passando alla valutazione si nota che per questa dimensione, più che per l'Offerta documentaria, vi è una forte aspettativa: infatti ben il $72 \%$ della popolazione attribuisce mediamente un giudizio positivo e solo il 7,6\% esprime un giudizio negativo, in termini di importanza (Fig. 6). Pesano sul risultato i giudizi espressi per la voce accesso a Internet con 1' $84,4 \%$ e per la voce relativa lo scaffale aperto $1^{\prime} 81,2 \%$, in ambedue i casi con una forte prevalenza dei molto importante su importante; conferma l'interesse per queste risorse la bassa percentuale di chi non sa rispondere: solo il 2,9\% per ambedue le voci. Su percentuali inferiori (intorno al 60\%), in termini di importanza, si attestano invece le voci relative alle banche dati e alle opere di reference.

Fig. 6 - Importanza attribuita all'Accessibilità delle risorse

\begin{tabular}{|c|c|c|c|c|}
\hline \multirow{11}{*}{$\begin{array}{r}90 \\
80 \\
70 \\
60 \\
50 \\
40 \\
30 \\
20 \\
10 \\
0\end{array}$} & \multicolumn{4}{|c|}{90,0} \\
\hline & \multicolumn{4}{|c|}{80,0} \\
\hline & \multicolumn{4}{|c|}{70,0} \\
\hline & \multicolumn{4}{|c|}{60,0} \\
\hline & \multicolumn{4}{|c|}{50,0} \\
\hline & \multicolumn{4}{|c|}{40,0} \\
\hline & \multicolumn{4}{|c|}{30,0} \\
\hline & \multicolumn{4}{|c|}{20,0} \\
\hline & \multicolumn{4}{|c|}{10,0} \\
\hline & & & \multirow{2}{*}{\multicolumn{2}{|c|}{$\begin{array}{c}\text { importante } \\
\text { molto importante }\end{array}$}} \\
\hline & poco importante & indifferente & & \\
\hline$\square$ scaffale aperto & 5,8 & 10,1 & 81,2 & 2,9 \\
\hline — opere di consultazione & 10,7 & 19,7 & 59,8 & 9,7 \\
\hline$\square$ accesso a Internet & 6,4 & 6,3 & 84,4 & 2,9 \\
\hline$\square$ banche dati & 7,8 & 16,4 & 62,7 & 13,1 \\
\hline
\end{tabular}


Significative le abitudini espresse con la percentuale d'uso: il $40 \%$ dei rispondenti non usa mai le opere di reference nelle sue visite; mentre oltre il $67,3 \%$ fa il browsing fra gli scaffali e il 54\% usa le postazioni per navigare in rete, almeno una volta su due che si reca in biblioteca (Fig. 7).

Fig. 7 - Utilizzo dell'Accessibilità delle risorse

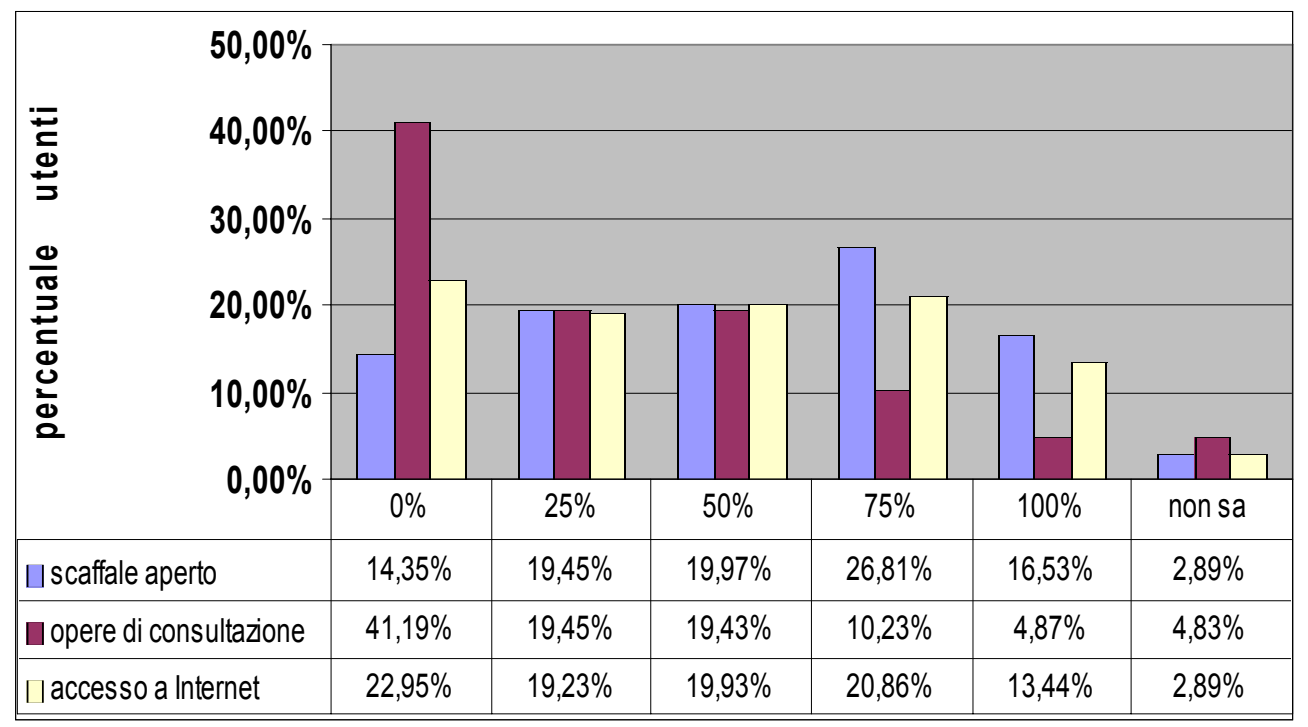

Mediamente il 35,4\% degli utenti/studenti attribuisce un giudizio positivo, in termini di soddisfazione, a questa dimensione (Fig. 8); e, come precedentemente osservato, anche in questo caso gli abbastanza soddisfatti superano massicciamente i soddisfatti. Esprime invece mediamente un giudizio negativo il $13,27 \%$ ed è la voce relativa alle postazioni dedicate alla navigazione in rete, che pesa sul giudizio, con una percentuale pari al $27,6 \%$ di insoddisfatti o poco insoddisfatti. Rispetto alle attese espresse, in termini di importanza, conforta invece il dato relativo allo scaffale aperto, per il quale il 54\% esprime un giudizio positivo. L'alta percentuale dei non sa, pari al 28,12\%, per la voce sulle banche dati conferma la scarsa familiarità con queste risorse.

Non resta che combinare l'aspetto importanza e uso con la percentuale delle risposte degli utenti; ed anche in questo caso viene confermata la tendenza, che vede crescere la percentuale d'uso (rappresentata dall'ampiezza dei cerchi) espressa da un maggior numero di utenti, in concomitanza con l'importanza attribuita alle risorse (Fig. 9). 
Fig. 8 - Soddisfazione percepita per l'Accessibilità delle risorse

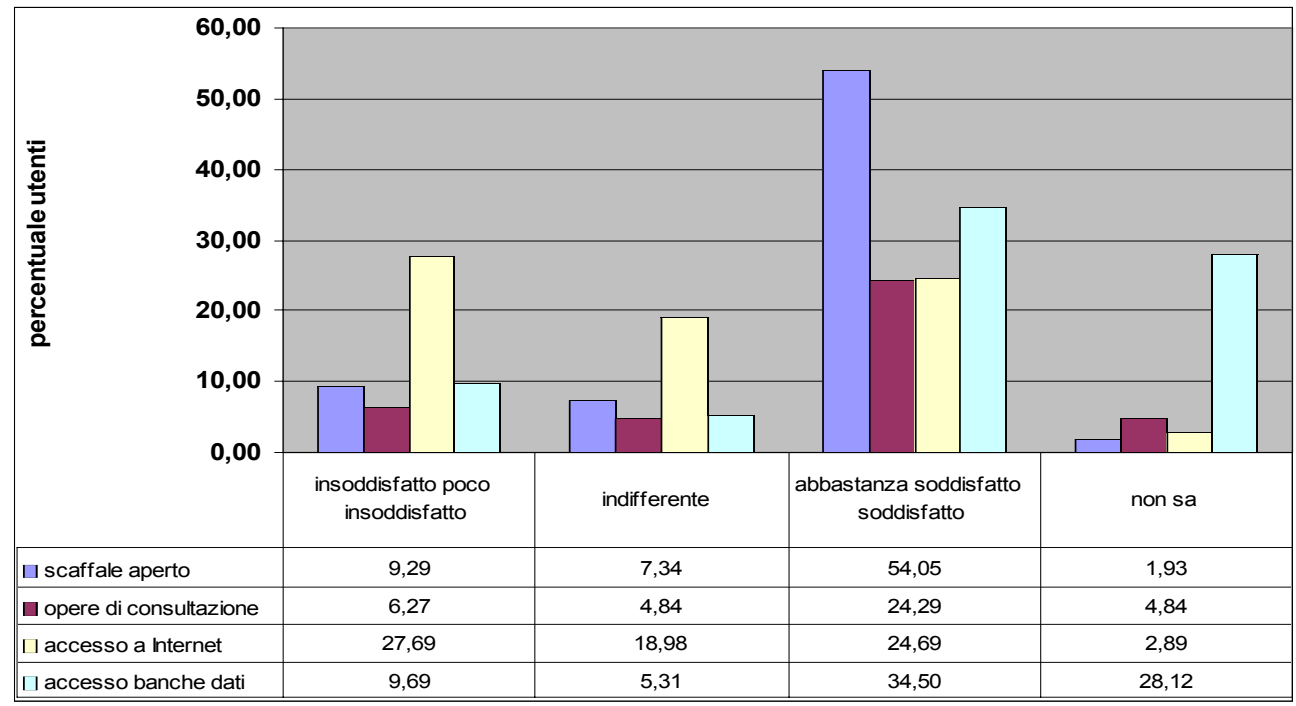

Fig. 9 - Relazione fra uso, importanza attribuita all'Accessibilità delle risorse e numero di utenti

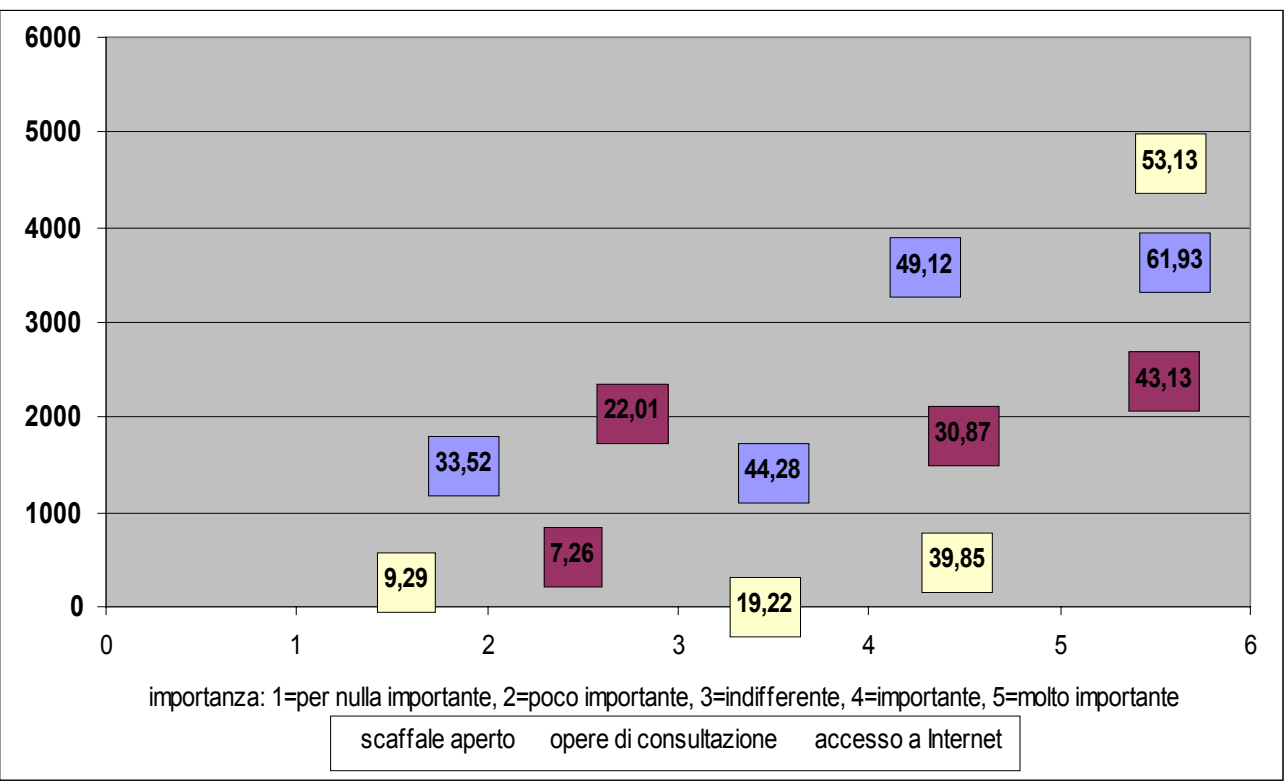

Se si vanno invece ad incrociare i dati relativi all'uso, alla soddisfazione e il numero di utenti si ha un'inversione di tendenza: le risorse risultano molto utilizzate anche da coloro che esprimono un giudizio negativo; in particolare 
si veda l'ampiezza dei cerchi relativi alla voci accesso a Internet e opere di consultazione laddove si incrociano con i giudizi insoddisfatto, poco insoddisfatto. Ed infine nel caso dell'accesso a Internet i giudizi negativi prevalgono su quelli positivi; unico elemento di comforto all'interno dei giudizi negativi i poco insoddisfatti sono oltre il doppio degli insoddisfatti (Fig. 10).

Fig. 10 - Relazione fra uso, soddisfazione percepita per l'Accessibilità delle risorse $\mathrm{e}$ numero di utenti

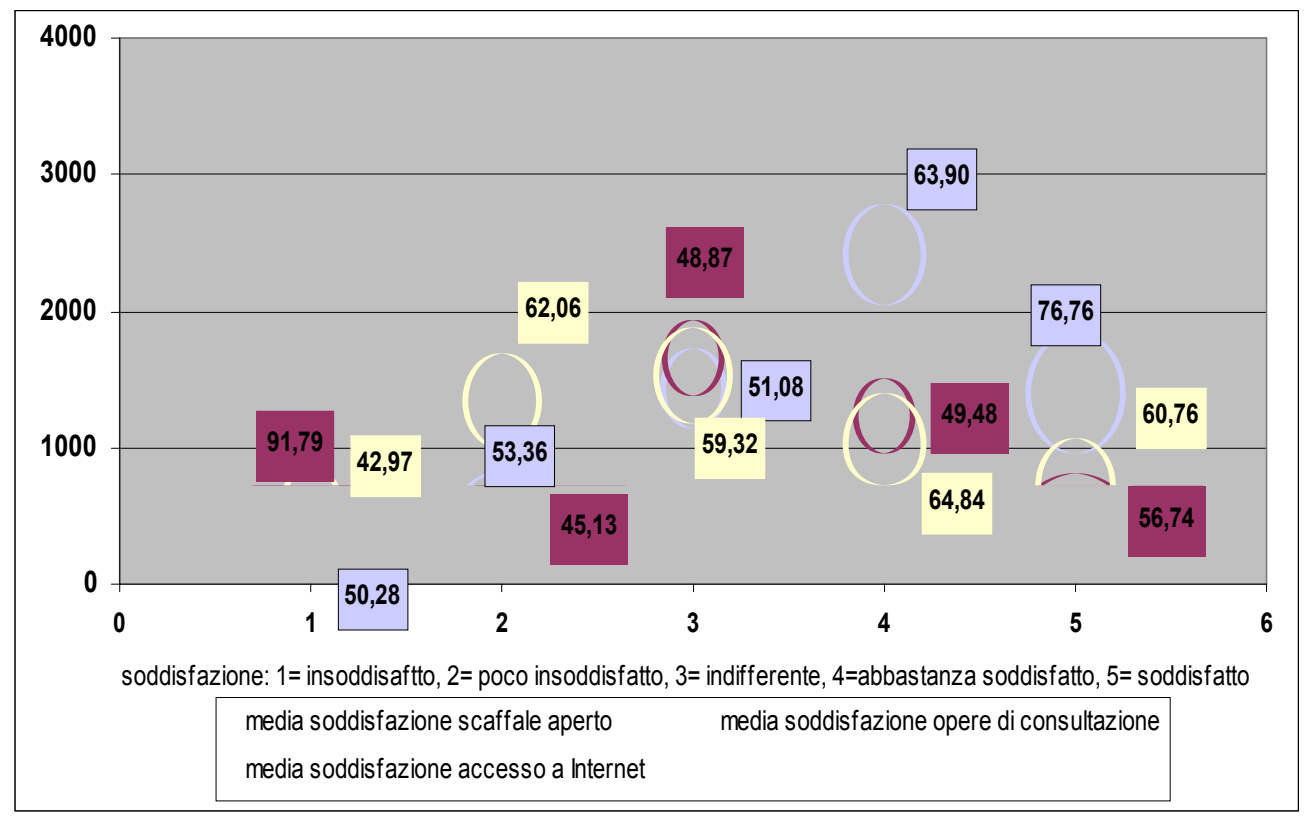

\section{Valutazione sugli Ambienti}

\begin{tabular}{|l|l|}
\hline Ambienti & \\
\hline Voci & $\begin{array}{l}\text { Disponibilità in sala di lettura di posti a sedere; } \\
\text { Silenzio in sala di lettura; } \\
\text { Comfort e luminosità della sala di lettura; } \\
\text { Depliant sulle biblioteche e segnaletica in biblioteca per faci- } \\
\text { litare l'uso dei servizi; }\end{array}$ \\
\hline Aspetti & $\begin{array}{l}\text { Grado di importanza } \\
\text { Percentuale d'uso } \\
\text { Grado di soddisfazione }\end{array}$ \\
\hline
\end{tabular}


Questa dimensione indaga su elementi che, per motivi oggettivi, non possono rappresentare un punto di forza della biblioteca; infatti la Bss nell'anno 2000 offriva ai suoi utenti circa 600 posti a sedere, con un'utenza potenziale studentesca di riferimento (gli iscritti alle facoltà di giurisprudenza, economia e scienze politiche) pari a 16.253 studenti.

Ovviamente la dimensione raccoglie mediamente il giudizio positivo in termini di importanza dal 70,8\% degli utenti, e solo il 4,3\% non sa esprimere un giudizio (Fig. 11). Mediamente è molto bassa la percentuale dei non sa (dall' $1 \%$ al 2\%), fatta eccezione per la voce relativa alla segnaletica, per la quale dichiara di non saper rispondere 1' $11,2 \%$ degli intervistati; e sempre per quest'ultima voce si registra anche un alto tasso di indifferenza, oltre il $20 \%$. Nonostante che la popolazione di riferimento dell'indagine sia costituita da utenti del prestito e non da frequentatori della biblioteca, oltre il 70\% attribuisce un giudizio positivo, in termini di importanza, alle voci relative alla sala di lettura.

Fig. 11 - Importanza attribuita agli Ambienti

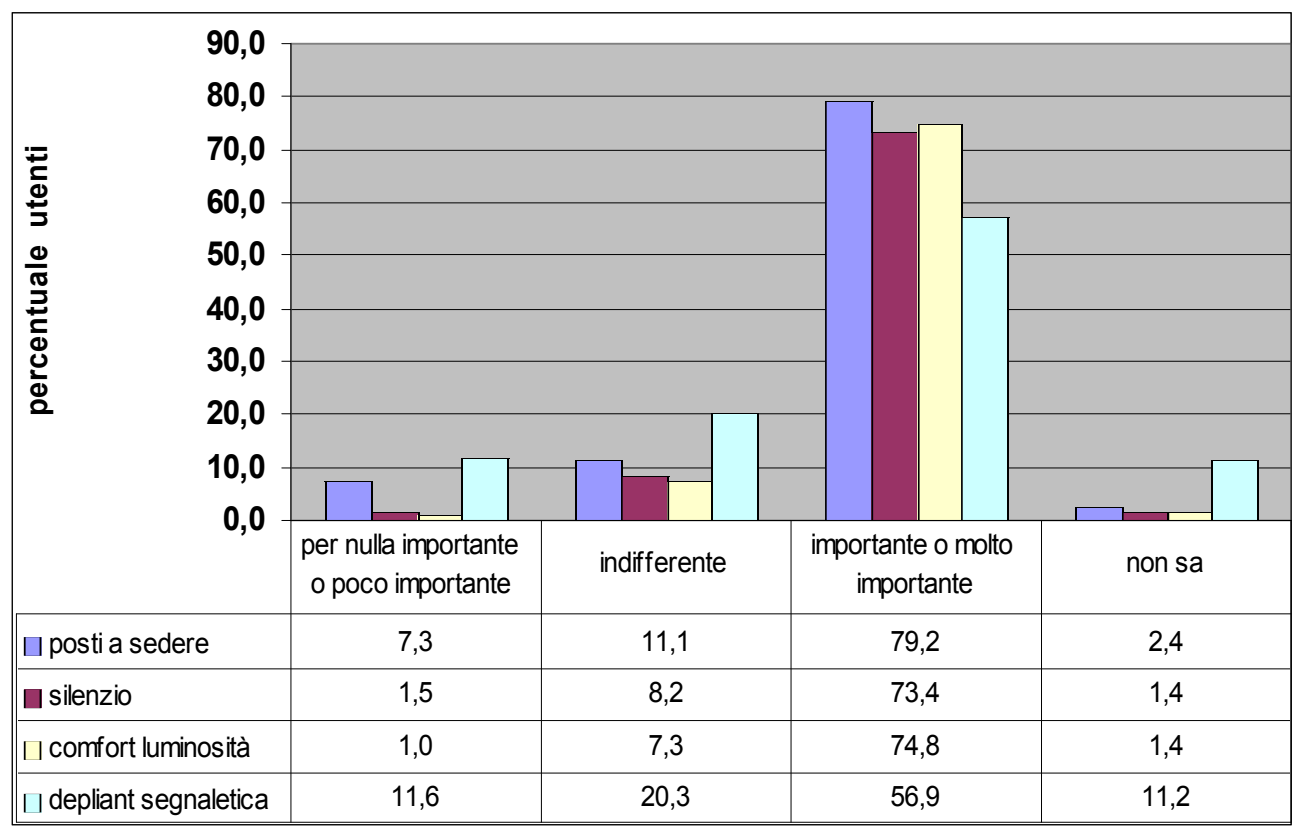

Oltre il $60 \%$ usa la sala di lettura almeno una volta su due, che si reca in biblioteca; il $74,4 \%$ la trova silenziosa e quasi 1' $80 \%$ ritiene che l'ambiente sia confortevole e luminoso per la metà delle volte che la frequenta. Si disco- 
sta anche per questo aspetto il dato per la voce depliant e segnaletica: ben il $38,4 \%$ dichiara di non usufruirne mai (Fig. 12).

Per quanto riguarda la soddisfazione questa dimensione raccoglie mediamente il giudizio positivo solo dal $27,2 \%$ degli utenti; sul risultato incide pesantemente la voce posti a sedere per la quale esprime un giudizio negativo oltre il $32 \%$ degli utenti/studenti. Dato che si sta valutando una dimensione tangibile, è bassa la percentuale dei non sa, mediamente il 2,2\% (Fig. 13).

Fig. 12 - Utilizzo degli Ambienti

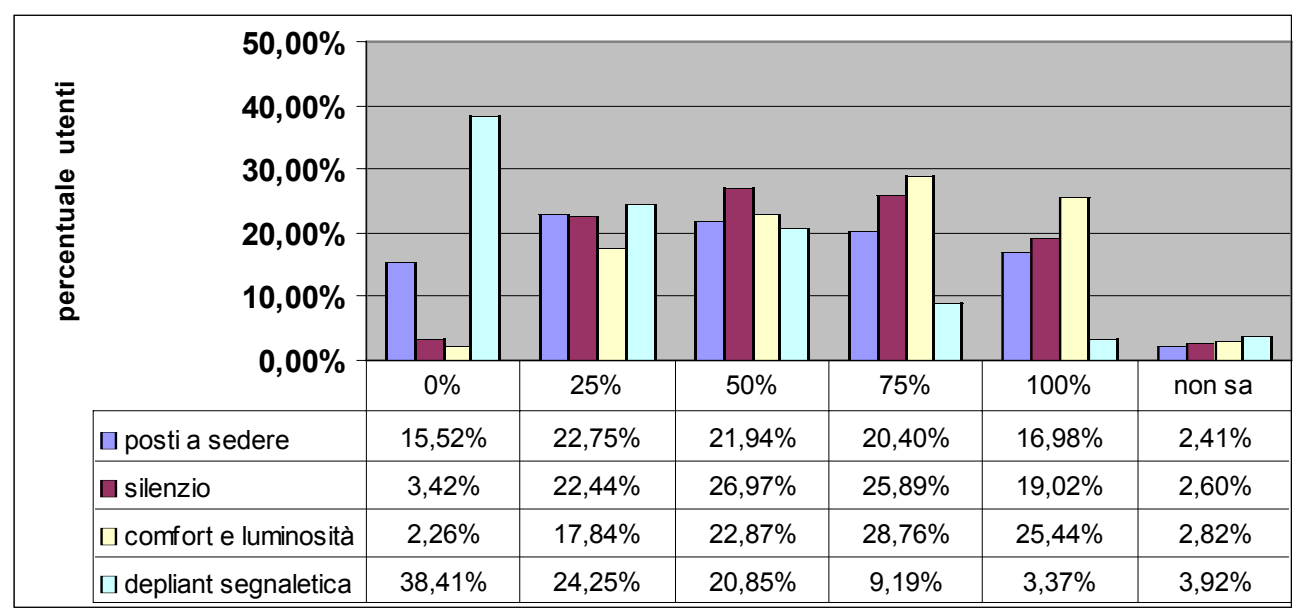

Fig. 13 - Soddisfazione percepita per gli Ambienti

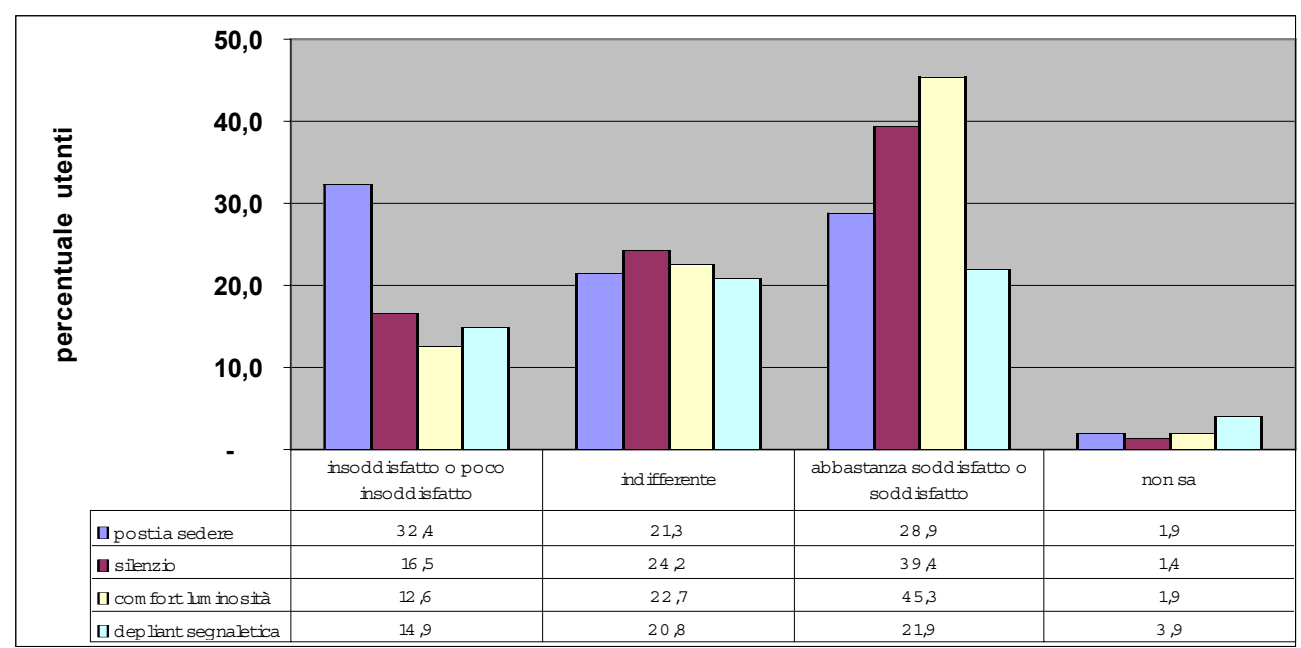


In questo caso la relazione fra l'importanza, la frequenza d'uso e il numero di utenti, non evidenzia una crescita con il maggior utilizzo: anche chi non attribuisce importanza alla voce dichiara di usarla abitualmente. Si differenzia invece la voce depliant e segnaletica dove ad un maggior uso, corrisponde anche un maggior grado di importanza attribuita (Fig. 14).

Fig. 14 - Relazione fra uso, importanza attribuita agli Ambienti e numero di utenti

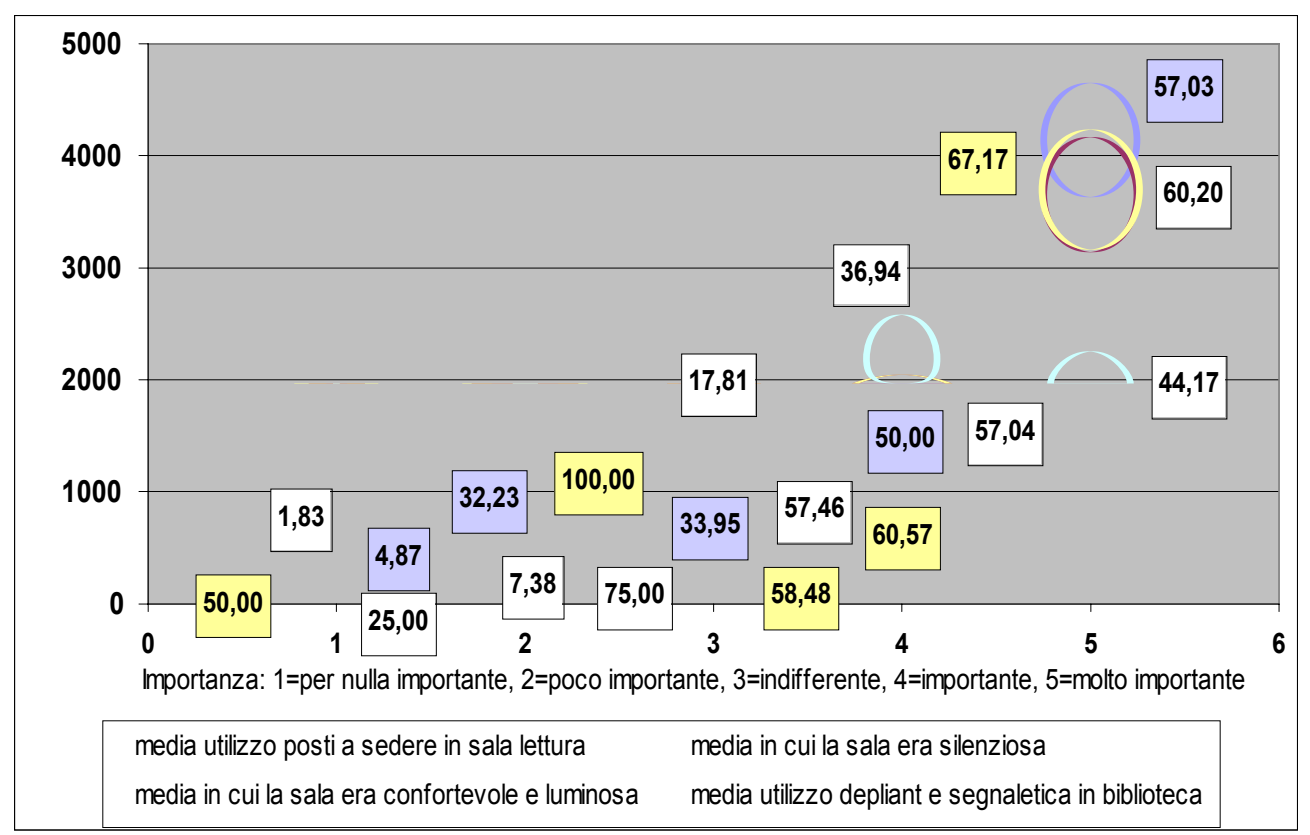

Come precedentemente segnalato, il giudizio negativo per la dimensione Ambienti è dovuto soprattutto alla voce riguardante i posti a sedere, in sala di lettura; infatti se si mettono in relazione il dato sulla soddisfazione e l'uso, emerge che in questo caso anche coloro che sono abitudinari frequentatori della sala di lettura (vedi l'ampiezza dei cerchi in prossimità dei giudizi negativi di soddisfazione) esprimono un giudizio negativo. Sono invece soddisfatti oltre il $50 \%$ degli utenti/studenti per quanto riguarda le voci che indagano sul comfort e il silenzio in sala (Fig. 15). 
Fig. 15 - Relazione fra uso, soddisfazione per gli Ambienti e numero di utenti

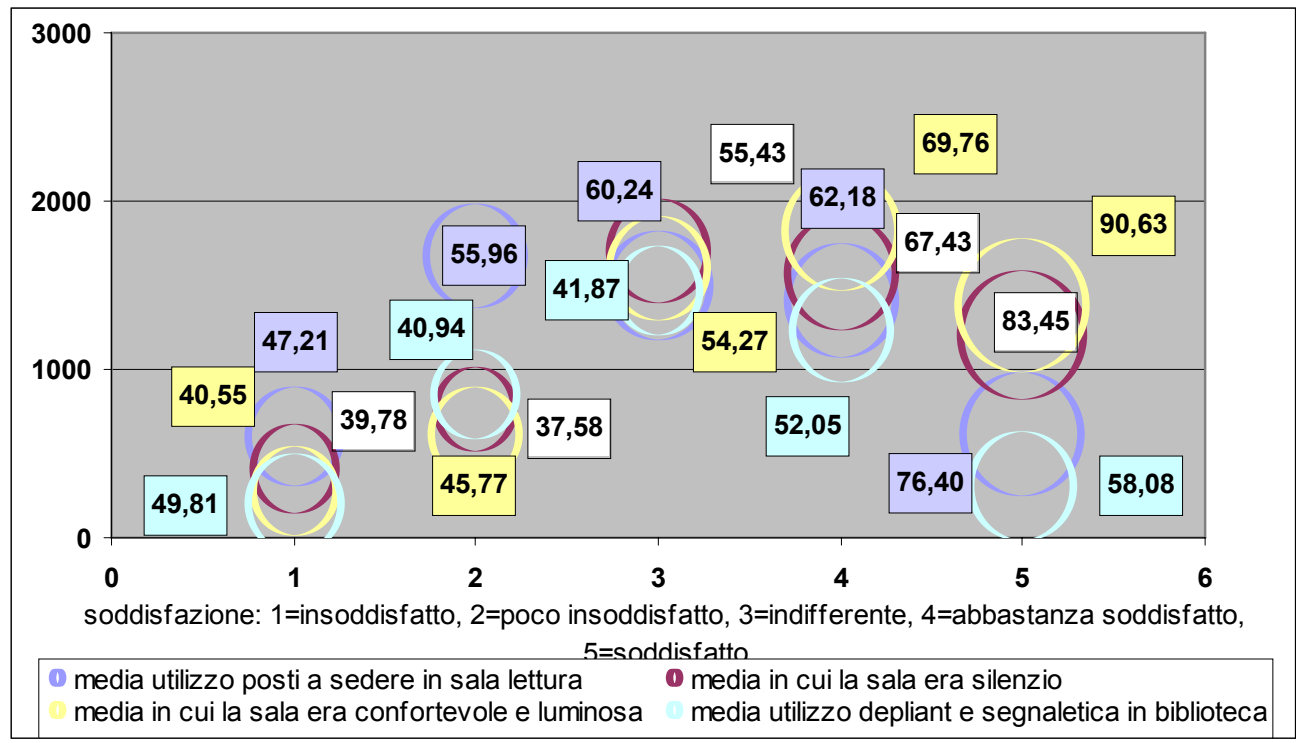

Valutazione sul Personale

\begin{tabular}{|l|l|}
\hline Personale & \\
\hline Voci & $\begin{array}{l}\text { Precisione e rapidità nella distribuzione del materiale; } \\
\text { Comunicazione con il personale delle biblioteche (disponibi- } \\
\text { lità e cortesia dei bibliotecari nei confronti del pubblico); } \\
\text { Servizi e assistenza personalizzata al lettore da parte del } \\
\text { bibliotecario; }\end{array}$ \\
\hline Aspetti & $\begin{array}{l}\text { Grado di importanza } \\
\text { Frequenza d'uso } \\
\text { Grado di soddisfazione }\end{array}$ \\
\hline
\end{tabular}

Il personale della Bss, che nel 2000 era costituito complessivamente da 54 unità ${ }^{9}$, ha partecipato ai corsi di riqualificazione e aggiornamento che si sono svolti, nell'ambito del Progetto di riorganizzazione del Sistema bibliotecario d'Ateneo $^{10}$.

9 Data l'organizzazione del lavoro, improntata alla mobilità e all'espletamento di attività diverse in più sedi, non è possibile indicare il numero di unità di personale assegnate ai punti di servizio di Giurisprudenza, Economia e Scienze politiche.

10 Per questo aspetto si veda il contributo di Francesca Landi. 
Mediamente 1' $80 \%$ degli utenti/studenti attribuisce un alto grado di importanza alla dimensione Personale alla quale corrisponde anche una bassa percentuale di giudizi negativi. Dal confronto delle voci emerge che per la voce comunicazione con il personale ben il 91,32\% esprime un giudizio positivo, mentre le altre due voci si attestano intorno al 70\% (Fig. 16). La capacità comunicativa del personale è la voce in assoluto alla quale il maggior numero di utenti/studenti attribuisce un alto grado d'importanza; la cortesia e la disponibilità sono più importanti anche delle competenze professionali, rappresentate in questo caso dalle altre due voci della dimensione.

Fig. 16 - Importanza attribuita al Personale

\begin{tabular}{|c|c|c|c|c|c|}
\hline \multirow{13}{*}{ 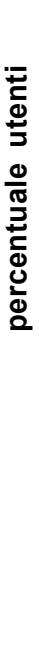 } & 100,0 & & & & \\
\hline & 90,0 & & & & \\
\hline & 80,0 & & & & \\
\hline & 70,0 & & & & \\
\hline & 60,0 & & & 1 & \\
\hline & $\begin{array}{l}50,0 \\
40,0\end{array}$ & & & & \\
\hline & 30,0 & & & 1 & \\
\hline & 20,0 & & & 1 & \\
\hline & 10,0 & ○—— & & & $\square$ \\
\hline & & $\begin{array}{c}\text { per nulla } \\
\text { importante o poco } \\
\text { importante }\end{array}$ & indifferente & $\begin{array}{c}\text { importante o molto } \\
\text { importante }\end{array}$ & non sa \\
\hline & $\begin{array}{l}\text { q precisione e rapidità nella } \\
\text { distribuzione del materiale }\end{array}$ & 6,37 & 13,57 & 74,72 & 5,34 \\
\hline & comunicazione con il personale & 2,47 & 5,75 & 91,32 & 0,47 \\
\hline & $\square$ servizi e assistenza personalizzata & 4,91 & 21,32 & 72,34 & 1,44 \\
\hline
\end{tabular}

La comunicazione col personale è ovviamente anche la voce che registra la maggiore percentuale d'uso, infatti oltre il 59\% degli utenti/studenti ha modo di entrare in contatto col personale, almeno una volta su due che frequenta la biblioteca; solo l'0,96\% non è in grado di quantificare la frequenza d'uso. Alta è invece la percentuale di coloro, che dichiarano di non avvalersi mai nelle loro visite in biblioteca del servizio di assistenza personalizzata, circa il 38\% (Fig. 17).

Per quanto riguarda la soddisfazione mediamente il 52,2\% esprime un giudizio positivo, e gli insoddisfatti o poco insoddisfatti non superano il $12,3 \%$; mentre alta è la percentuale degli indifferenti: $21,7 \%$. Fra le tre voci emerge il dato relativo alla comunicazione con il personale, per la quale oltre il $60 \%$ esprime un giudizio positivo (Fig. 18). 
Fig. 17 - Utilizzo del Personale

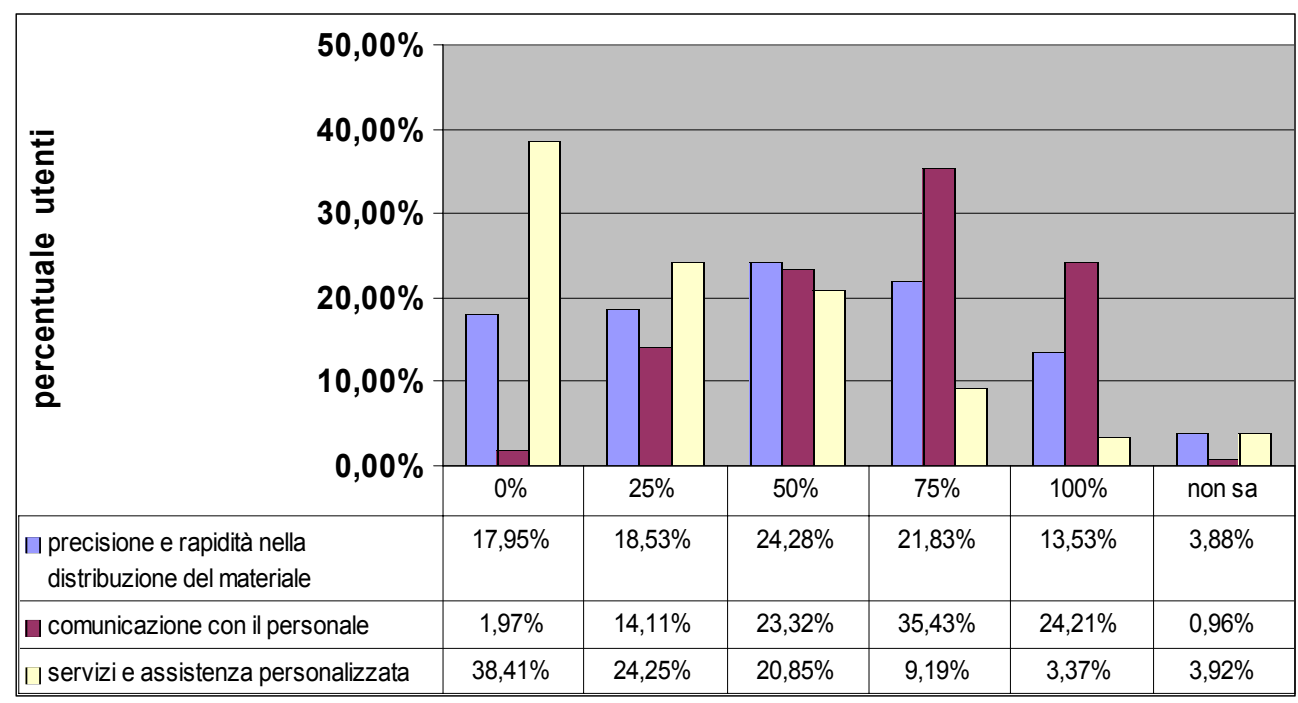

Fig. 18 - Soddisfazione percepita per il Personale

\begin{tabular}{|c|c|c|c|c|}
\hline \multirow{8}{*}{$\begin{array}{l}70 \\
60 \\
50 \\
40 \\
30 \\
20 \\
10\end{array}$} & \multicolumn{4}{|c|}{70,0} \\
\hline & \multicolumn{4}{|c|}{60,0} \\
\hline & \multicolumn{4}{|c|}{50,0} \\
\hline & \multicolumn{4}{|c|}{30,0} \\
\hline & \\
\hline & \multicolumn{4}{|c|}{10,0} \\
\hline & & & & $\square$ \\
\hline & $\begin{array}{c}\text { insoddisfatto o } \\
\text { poco insoddisfatto }\end{array}$ & indifferente & $\begin{array}{c}\text { abbastanza } \\
\text { soddisfatto o } \\
\text { soddisfatto }\end{array}$ & non sa \\
\hline \begin{tabular}{|l|} 
precisione e rapidità nella \\
distribuzione del materiale
\end{tabular} & 14,09 & 20,46 & 46,69 & 3,39 \\
\hline$\square$ comunicazione con il personale & 10,25 & 20,82 & 60,67 & 0,96 \\
\hline$\square$ servizi e assistenza personalizzata & 13,21 & 24,19 & 47,57 & 1,46 \\
\hline
\end{tabular}

Mettendo in relazione l'uso, l'importanza attribuita e il numero di utenti si nota che per le voci di questa dimensione vale quanto precedentemente rilevato e cioè sono coloro che più utilizzano un servizio che gli attribuiscono maggiore importanza (Fig. 19). Ugualmente la rappresentazione grafica della 
relazione uso, soddisfazione percepita e numero di utenti evidenzia la dipendenza fra giudizio positivo e maggior livello di frequenza di impiego; e anche in questo caso gli abbastanza soddisfatti superano i soddisfatti (Fig. 20).

Fig. 19 - Relazione fra uso, importanza attribuita al Personale e numero di utenti

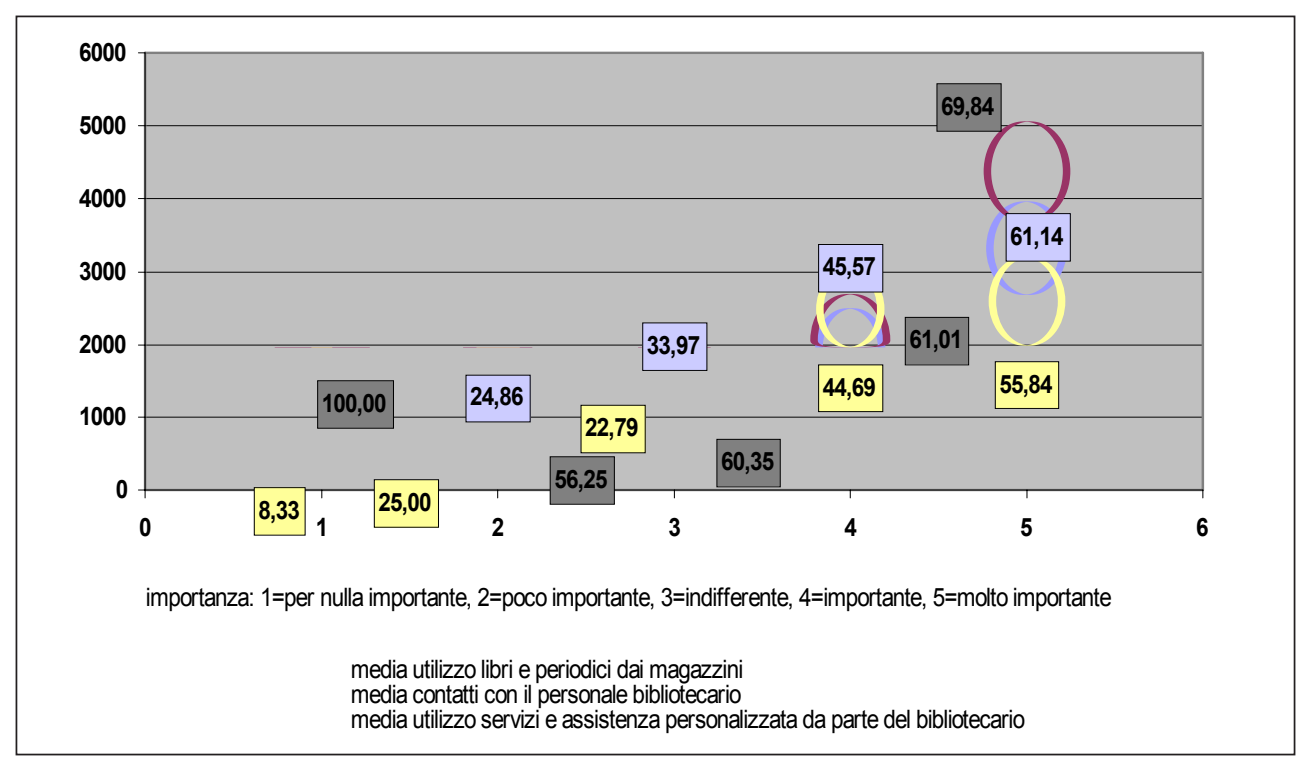

Fig. 20 - Relazione fra uso, soddisfazione percepita per il Personale e numero di utenti

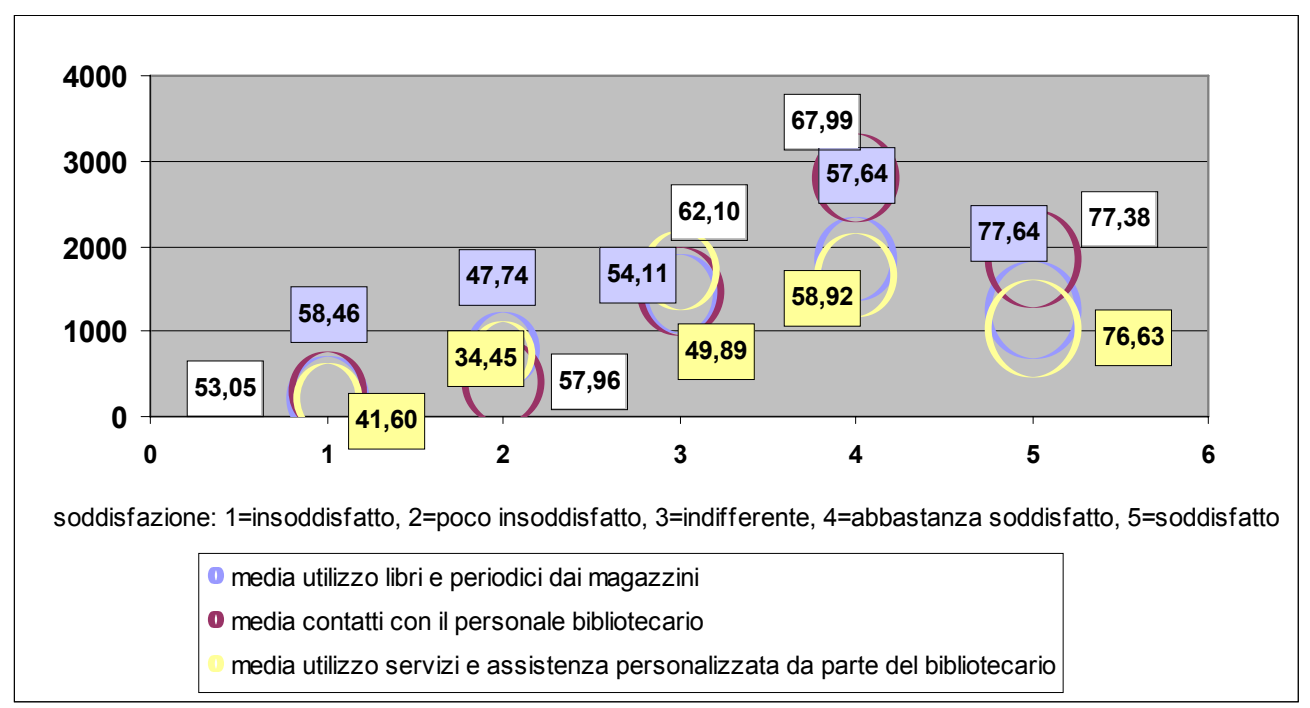




\section{Conclusioni}

Dopo la lettura delle tabelle, possiamo tentare di evidenziare alcune tendenze che sembrano emergere dal giudizio espresso dagli utenti/studenti della biblioteca, andando a confrontare le valutazioni raccolte mediamente per ciascuna dimensione sia per il livello di importanza che per la soddisfazione percepita. Ovviamente per un ulteriore approfondimento dell'analisi statistica delle tabelle e per uno specifico confronto fra le dimensioni si rimanda all'Appendice D "Stima degli errori di campionamento dell'Indagine sulla soddisfazione degli utenti", dove è riportata, per ciascuna variabile assunta da ogni voce del questionario, la stima dell'errore campionario che consente di calcolare l'intervallo di confidenza.

Dal confronto delle dimensioni, per quanto riguarda il livello di importanza attribuita, è la dimensione Personale quella alla quale mediamente il maggior numero di utenti/studenti attribuisce un giudizio positivo ed è anche quella che ottiene mediamente la percentuale più bassa di giudizi negativi (Fig. 21). Seguono le dimensioni Ambienti e l'Accessibilità delle risorse molto ravvicinate fra loro e, dato che può sorprendere, l'Offerta documentaria è quella che in media registra meno aspettative.

Fig. 21 - Importanza attribuita alle dimensioni

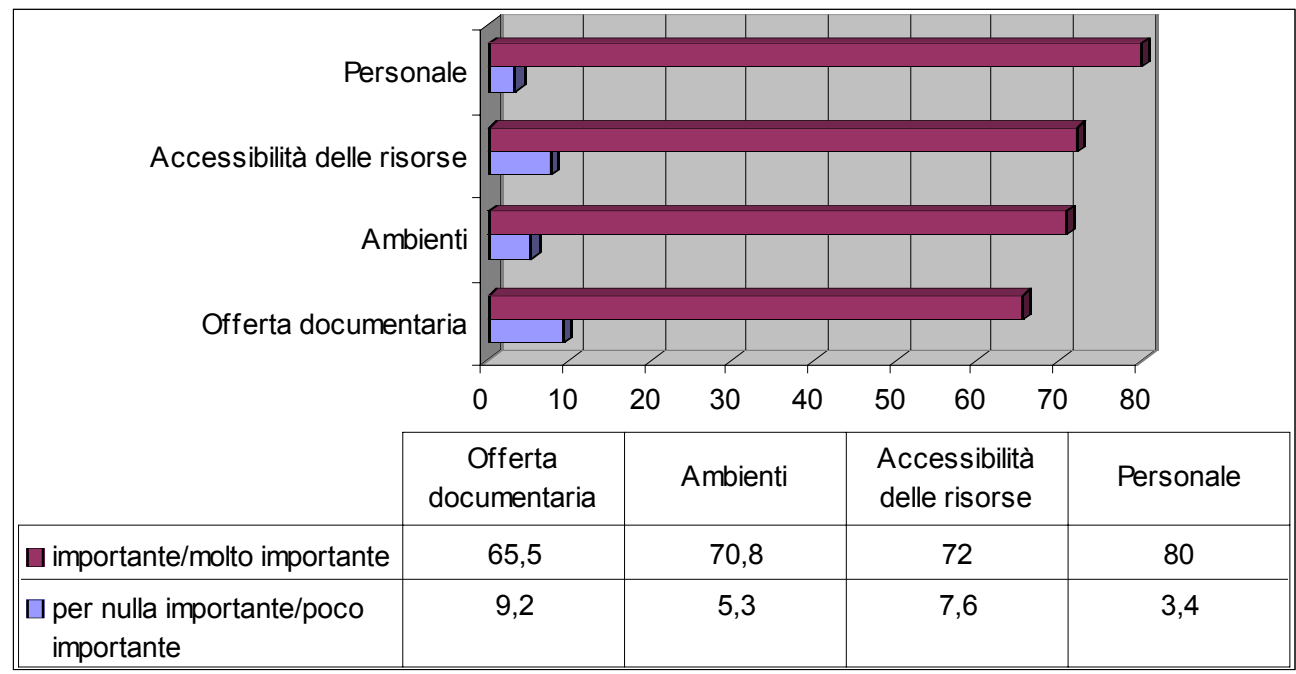

Se si considera invece l'aspetto relativo al grado di soddisfazione percepita, è sempre la dimensione Personale quella che in media ottiene la percentuale più alta di consensi; seguono ben distanziate l'Offerta documentaria 
(grazie soprattutto alla voce relativa ai libri, periodici e cd rom) e l'Accessibilità delle risorse (Fig. 22). Il risultato peggiore spetta alla dimensione Ambienti, che raccoglie mediamente la più bassa percentuale di giudizi positivi e anche la percentuale più alta di giudizi negativi.

Fig. 22 - Soddisfazione percepita per le dimensioni

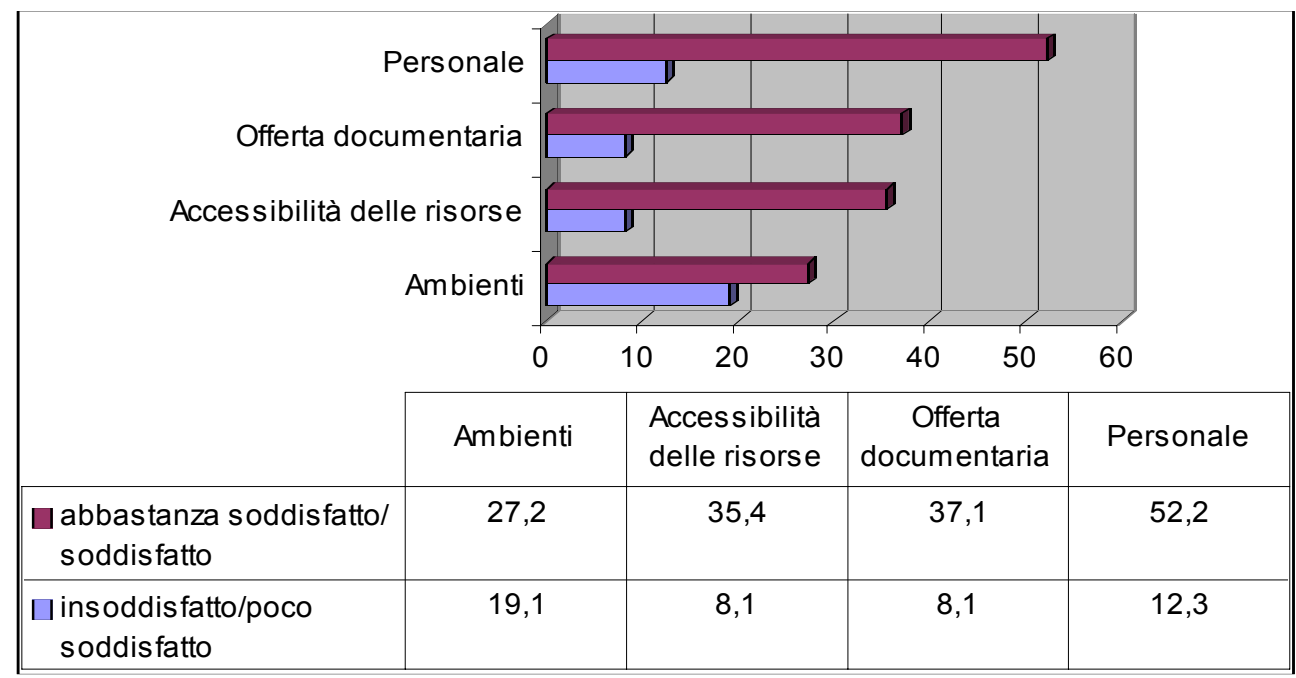

Ancora una riflessione merita la consistente fetta di utenti/studenti, che costantemente per tutta l'indagine si dichiara indifferente e non esprime una valutazione né per il grado di importanza attribuita né per il grado di soddisfazione percepita. Tale risultato, che ovviamente richiede caso per caso valutazioni più approfondite, sembra suggerire la necessità di una maggiore attenzione agli aspetti relativi alla comunicazione e promozione delle attività e dei servizi della biblioteca. Sorprende infatti che mediamente la percentuale degli indifferenti si attesta al 13,7\% per l'aspetto importanza, mentre sale al $17,6 \%$ per l'aspetto soddisfazione; ed ecco i valori che assume in media per ciascuna dimensione, nei due aspetti dell'importanza e della soddisfazione:

\begin{tabular}{|l|c|c|}
\hline Dimensione & \% Importanza & \% Soddisfazione \\
\hline Offerta documentaria & 16,7 & 19,5 \\
\hline Accessibilità delle risorse & 13,1 & 6,9 \\
\hline Ambienti & 11,7 & 22,2 \\
\hline Personale & 13,5 & 21,7 \\
\hline
\end{tabular}


Concludendo quindi possiamo ritenere con una certa approssimazione che la dimensione del personale rappresenta il punto di forza della biblioteca, in quanto raccoglie mediamente il più alto grado di importanza (qualità attesa) e il più alto grado di soddisfazione (qualità percepita) nel giudizio degli utenti/studenti. Tale risultato conferma quanto già rilevato dal Nucleo di valutazione interna nel Rapporto del $2000^{11}$, dove emergeva una netta soddisfazione degli studenti dell'area delle Scienze sociali per il personale delle loro biblioteche di riferimento.

I punti critici sembrano invece essere quelli relativi agli Ambienti (in particolare, come era prevedibile, per la disponibilità dei posti a sedere nelle sale di lettura) per i quali la biblioteca non soddisfa in media quasi il $20 \%$ dei propri utenti/studenti. Una forte criticità si ritrova anche nel dato relativo all'Accessibilità delle risorse, soprattutto per quanto riguarda la disponibilità di postazioni dedicate alla navigazione in rete e alla disponibilità di opere di consultazione.

Infine per un'analisi più particolareggiata sono state elaborate delle tabelle di raffronto dove vengono riportate per ciascuna voce del questionario, indipendentemente dalla dimensione di appartenenza, la percentuale di utenti/studenti che hanno espresso un giudizio, la percentuale dei relativi non sa e anche degli indifferenti ${ }^{12}$.

Dalla prima tabella, in cui si riassumono i giudizi positivi in termini di importanza, si ricava che la voce per la quale un maggior numero di utenti dichiara un giudizio positivo è la comunicazione con il personale a cui seguono: l'accesso a Internet e la presenza dello scaffale aperto. (Tab. 1)

Nella tabella seguente si ordinano invece le voci in base alla percentuale di utenti che hanno assegnato un grado negativo di importanza e anche in questo caso si affiancano la percentuale dei non sa e degli indifferenti. Da questa lettura dei dati si evidenzia che il prestito interbibliotecario e depliant e segnaletica sono le voci per le quali più utenti esprimono meno aspettativa; all'opposto della graduatoria troviamo la voce comfort e luminosità della sala di lettura. (Tab. 2)

11 Università degli studi di Firenze 2001b. In particolare si vedano le pagine 163-164.

12 La quantificazione di coloro che non sanno esprimere un giudizio e degli indifferenti consente di valutare più appropriatamente il peso delle percentuali dei giudizi positivi o negativi totalizzati da ciascuna voce del questionario. 
Tab. 1

\begin{tabular}{|l|c|c|c|}
\hline Voce & $\begin{array}{c}\text { \% Importante/ } \\
\text { molto importante }\end{array}$ & $\begin{array}{c}\% \\
\text { Non sa }\end{array}$ & $\begin{array}{c}\% \\
\text { Indifferente }\end{array}$ \\
\hline Comunicazione con il personale & 91,3 & 0,4 & 5,7 \\
\hline Accesso a Internet & 84,4 & 2,9 & 6,3 \\
\hline Scaffale aperto & 81,2 & 2,9 & 10,1 \\
\hline Posti a sedere & 79,2 & 2,4 & 11,1 \\
\hline Comfort e luminosità in sala di lettura & 74,8 & 1,4 & 7,3 \\
\hline $\begin{array}{l}\text { Precisione e rapidità nella distribuzione } \\
\text { del materiale }\end{array}$ & 74,7 & 5,3 & 13,5 \\
\hline Silenzio in sala di lettura & 73,4 & 1,4 & 8,2 \\
\hline Servizi e assistenza personalizzata & 72,3 & 1,4 & 21,3 \\
\hline Libri, periodici e cd rom & 71,8 & 1,9 & 20,9 \\
\hline Novità librarie & 65,0 & 8,3 & 16,5 \\
\hline Prestito interbibliotecario & 64,2 & 9,2 & 15,0 \\
\hline Banche dati & 62,7 & 13,1 & 16,4 \\
\hline Ultimi n. dei periodici & 61,4 & 14,2 & 14,5 \\
\hline Opere di consultazione & 59,8 & 9,7 & 9,7 \\
\hline Depliant segnaletica & 56,9 & 11,2 & 20,3 \\
\hline
\end{tabular}

Tab. 2

\begin{tabular}{|l|c|c|c|}
\hline Voce & $\begin{array}{c}\text { \% Importante/ } \\
\text { molto importante }\end{array}$ & $\begin{array}{c}\% \\
\text { Non sa }\end{array}$ & $\begin{array}{c}\% \\
\text { Indifferente }\end{array}$ \\
\hline Comfort e luminosità in sala di lettura & 1,0 & 1,4 & 7,3 \\
\hline Silenzio in sala di lettura & 1,5 & 1,4 & 8,2 \\
\hline Comunicazione con il personale & 2,4 & 0,4 & 5,7 \\
\hline Servizi e assistenza personalizzata & 4,9 & 1,4 & 21,3 \\
\hline Libri, periodici e cd rom & 5,4 & 1,9 & 20,9 \\
\hline Scaffale aperto & 5,8 & 2,9 & 10,1 \\
\hline $\begin{array}{l}\text { Precisione e rapidità nella distribuzione } \\
\text { del materiale }\end{array}$ & 6,3 & 5,3 & 13,5 \\
\hline Accesso a Internet & 6,4 & 2,9 & 6,3 \\
\hline Posti a sedere & 7,3 & 2,4 & 11,1 \\
\hline Banche dati & 7,8 & 13,1 & 16,4 \\
\hline Ultimi n. dei periodici & 9,8 & 14,2 & 14,6 \\
\hline Novità librarie & 10,2 & 8,3 & 16,5 \\
\hline Opere di consultazione & 10,7 & 9,7 & 19,7 \\
\hline Depliant e segnaletica & 11,6 & 11,2 & 20,3 \\
\hline Prestito interbibliotecario & 11,6 & 9,2 & 15,0 \\
\hline
\end{tabular}


Per quanto riguarda l'aspetto della soddisfazione percepita, indipendentemente dalla dimensione di appartenenza, troviamo al primo posto le risorse documentarie (libri, periodici e cd rom), seguono le capacità comunicative e le competenze professionali del personale (servizi di assistenza personalizzata, precisione e rapidità nella distribuzione) e la disponibilità di volumi a scaffale aperto. I punti critici invece riguardano la disponibilità delle opere di reference, le postazioni messe a disposizione per la ricerca in rete, i depliant e la segnaletica (Tab. 3).

Tab. 3

\begin{tabular}{|l|c|c|c|}
\hline Voce & $\begin{array}{c}\text { \% Abbastanza } \\
\text { soddisfatto } \\
\text { soddisfatto }\end{array}$ & $\begin{array}{c}\% \\
\text { Non sa }\end{array}$ & $\begin{array}{c}\% \\
\text { Indifferente }\end{array}$ \\
\hline Libri, periodici e cd rom & 63,6 & 0,9 & 22,3 \\
\hline Comunicazione con il personale & 60,6 & 0,9 & 20,8 \\
\hline Scaffale aperto & 54,0 & 1,9 & 7,3 \\
\hline Servizi e assistenza personalizzata & 47,5 & 1,4 & 24,1 \\
\hline $\begin{array}{l}\text { Precisione e rapidità nella distribuzione } \\
\text { del materiale }\end{array}$ & 46,0 & 3,3 & 20,4 \\
\hline Comfort e luminosità in sala di lettura & 6,0 & 1,9 & 22,7 \\
\hline Silenzio in sala di lettura & 45,3 & 1,4 & 24,2 \\
\hline Banche dati & 39,4 & 28,1 & 5,3 \\
\hline Novità librarie & 34,5 & 4,4 & 21,8 \\
\hline Posti a sedere & 30,1 & 1,9 & 21,3 \\
\hline Prestito interbibliotecario & 28,9 & 4,8 & 18,0 \\
\hline Ultimi n. dei periodici & 27,0 & 4,8 & 16,0 \\
\hline Opere di consultazione & 24,2 & 4,8 & 4,8 \\
\hline Accesso a Internet & 24,2 & 2,8 & 18,9 \\
\hline Depliant segnaletica & 21,9 & 3,9 & 20,8 \\
\hline
\end{tabular}

Ed infine ecco, in ordine crescente, le voci in base alla percentuale di utenti che hanno espresso un giudizio negativo, sempre in relazione all'aspetto della soddisfazione (Tab. 4). Al primo posto troviamo la voce libri, periodici e cd rom, mentre all'ultimo l'accesso a Internet e i posti a sedere, che raccolgono la percentuale più alta di insoddisfazione. 
Tab. 4

\begin{tabular}{|l|c|c|c|}
\hline Voce & $\begin{array}{c}\text { \% Abbastanza } \\
\text { soddisfatto } \\
\text { soddisfatto }\end{array}$ & $\begin{array}{c}\% \\
\text { Non sa }\end{array}$ & $\begin{array}{c}\% \\
\text { Indifferente }\end{array}$ \\
\hline Libri, periodici e cd rom & 3,9 & 0,9 & 22,3 \\
\hline Prestito interbibliotecario & 5,8 & 4,8 & 18,0 \\
\hline Opere di consultazione & 6,2 & 4,8 & 4,8 \\
\hline Scaffale aperto & 9,2 & 1,9 & 7,3 \\
\hline Ultimi n. dei periodici & 9,2 & 4,8 & 16,0 \\
\hline Banche dati & 9,6 & 28,1 & 5,3 \\
\hline Comunicazione con il personale & 10,2 & 0,9 & 20,8 \\
\hline Comfort e luminosità in sala di lettura & 12,6 & 1,9 & 22,7 \\
\hline Servizi e assistenza personalizzata & 13,2 & 1,4 & 24,1 \\
\hline Novità librarie & 13,6 & 4,4 & 21,8 \\
\hline $\begin{array}{l}\text { Precisione e rapidità nella distribuzione } \\
\text { del materiale }\end{array}$ & 14,0 & 3,3 & 20,4 \\
\hline Depliant segnaletica & 14,9 & 3,9 & 20,8 \\
\hline Silenzio in sala di lettura & 16,5 & 1,4 & 24,2 \\
\hline Accesso a Internet & 27,6 & 2,8 & 18,9 \\
\hline Posti a sedere & 32,4 & 1,9 & 21,3 \\
\hline
\end{tabular}




\section{Appendice A}

\section{Dati e indicatori in uso presso il Sistema Bibliotecario di Ateneo (aggiornato al 2002)}

\section{Dati}

\begin{tabular}{|c|c|l|c|}
\hline \multicolumn{2}{|c|}{ UTENZA } & Periodicità \\
\hline D1.01 & Docenti e ricercatori & $\begin{array}{l}\text { Descrizione } \\
\text { Numero dei docenti delle tre fasce } \\
\text { che lavorano nell'Ateneo. }\end{array}$ & Annuale \\
\hline D1.02 & Studenti & $\begin{array}{l}\text { Numero degli iscritti ai corsi di } \\
\text { laurea, di diploma universitario ed } \\
\text { alle scuole di specializzazione. }\end{array}$ & Annuale \\
\hline D1.03 & Utenti attivi & $\begin{array}{l}\text { Numero totale di utenti che hanno } \\
\text { effettuato almeno un prestito nel } \\
\text { periodo considerato. }\end{array}$ & Quadrimestrale \\
\hline
\end{tabular}

\begin{tabular}{|c|c|c|c|}
\hline \multicolumn{4}{|c|}{ RISORSE } \\
\hline \multicolumn{2}{|c|}{ Denominazione } & Descrizione & Periodicità \\
\hline $\mathrm{D} 2.01$ & $\begin{array}{c}\text { Patrimonio } \\
\text { bibliografico }\end{array}$ & $\begin{array}{l}\text { Il dato risulta dalla somma di: } \\
\text { a) numero di inventari corrispon- } \\
\text { denti all'inventariazione manua- } \\
\text { le precedente alla gestione auto- } \\
\text { matizzata con stima degli in- } \\
\text { ventari doppi relativi ad opere in } \\
\text { più volumi; } \\
\text { b) numero di inventari inseriti in } \\
\text { SBN dall'inizio dell'automazione. }\end{array}$ & Annuale \\
\hline $\mathrm{D} 2.02$ & Periodici correnti & Numero degli abbonamenti correnti. & Annuale \\
\hline $\mathrm{D} 2.03$ & Periodici disdetti & $\begin{array}{l}\text { Numero degli abbonamenti non rin- } \\
\text { novati nell'anno precedente. }\end{array}$ & Annuale \\
\hline D2.04 & $\begin{array}{l}\text { Volumi e periodici } \\
\text { ad accesso libero }\end{array}$ & $\begin{array}{l}\text { Numero di volumi collocati a scaffa- } \\
\text { le aperto. Il dato è conteggiato o } \\
\text { stimato in base alla lunghezza degli } \\
\text { scaffali occupati. }\end{array}$ & Annuale \\
\hline $\mathrm{D} 2.05$ & Posti di lettura & $\begin{array}{l}\text { Numero dei posti a sedere disponibili } \\
\text { nelle sale di lettura di ciascun punto } \\
\text { di servizio. }\end{array}$ & Annuale \\
\hline
\end{tabular}




\begin{tabular}{|c|c|c|c|}
\hline D2.06 & $\begin{array}{l}\text { PC destinati al } \\
\text { pubblico }\end{array}$ & $\begin{array}{l}\text { Numero dei personal e dei network } \\
\text { computer destinati al pubblico. }\end{array}$ & Annuale \\
\hline D2.07 & $\begin{array}{l}\text { Metri quadrati al } \\
\text { pubblico }\end{array}$ & $\begin{array}{l}\text { Estensione degli spazi delle biblio- } \\
\text { teche destinati al pubblico, espressa } \\
\text { in metri quadrati: sale di lettura, sale } \\
\text { cataloghi, distribuzione. Sono esclusi } \\
\text { i locali destinati al magazzino, al } \\
\text { personale e ad attività interne. }\end{array}$ & Annuale \\
\hline D2.08 & Metri quadrati totali & $\begin{array}{l}\text { Estensione di tutti i locali delle bi- } \\
\text { blioteche, espressa in metri quadrati. }\end{array}$ & Annuale \\
\hline D2.09 & Punti di servizio & $\begin{array}{l}\text { Numero dei punti di servizio del } \\
\text { SBA (ex biblioteche di facoltà o di } \\
\text { dipartimento) aperti al pubblico, co- } \\
\text { me stabiliti dall'Ordinanza n. } 102 \text { del } \\
29 / 03 / 1996 \text {. }\end{array}$ & Annuale \\
\hline D2.10 & $\begin{array}{l}\text { Ore settimanali di } \\
\text { apertura }\end{array}$ & $\begin{array}{l}\text { Totale delle ore di apertura dei punti } \\
\text { di servizio di ciascuna biblioteca, } \\
\text { aperti al pubblico ad opera dei bi- } \\
\text { bliotecari. }\end{array}$ & Quadrimestrale \\
\hline D2.11 & Personale per livelli & $\begin{array}{l}\text { Personale attribuito alle biblioteche } \\
\text { di area, suddiviso per qualifiche fun- } \\
\text { zionali. }\end{array}$ & Annuale \\
\hline D2.12 & $\begin{array}{l}\text { Personale per } \\
\text { attività (carichi di } \\
\text { lavoro) }\end{array}$ & $\begin{array}{l}\text { Stima del tempo di lavoro dedicato } \\
\text { alle diverse attività (a: catalogazione, } \\
\text { b: inventariazioni, c: prestiti) da parte } \\
\text { dei bibliotecari. }\end{array}$ & Annuale \\
\hline D2.13 & $\begin{array}{l}\text { Formule di } \\
\text { ripartizione delle } \\
\text { risorse umane }\end{array}$ & $\begin{array}{l}\text { Unità di personale necessarie per lo } \\
\text { svolgimento del complesso delle } \\
\text { attività di ciascuna biblioteca, secon- } \\
\text { do lo standard della Association of } \\
\text { College \& Research Libraries. }\end{array}$ & Annuale \\
\hline
\end{tabular}




\begin{tabular}{|c|c|c|c|}
\hline \multicolumn{4}{|c|}{ INPUTS } \\
\hline \multicolumn{2}{|c|}{ Denominazione } & Descrizione & Periodicità \\
\hline D3.01 & Budget Università & Dotazione finanziaria dell'Ateneo. & Annuale \\
\hline D3.02 & Spese SBA & $\begin{array}{l}\text { Spese sostenute dalle biblioteche di } \\
\text { area e dallo SBA, come risultano in } \\
\text { base ai mandati emessi. }\end{array}$ & Annuale \\
\hline D3.03 & $\begin{array}{l}\text { Spese libri: serie } \\
\text { storica }\end{array}$ & Spesa per l'acquisto di monografie. & Annuale \\
\hline D3.04 & $\begin{array}{l}\text { Spese periodici: } \\
\text { serie storica }\end{array}$ & Spesa per l'acquisto di periodici. & Annuale \\
\hline D3.05 & $\begin{array}{l}\text { Spese rilegature: } \\
\text { serie storica }\end{array}$ & Spesa per le rilegature. & Annuale \\
\hline D3.06 & $\begin{array}{l}\text { Spese risorse } \\
\text { elettroniche: serie } \\
\text { storica }\end{array}$ & $\begin{array}{l}\text { Spesa per l'acquisto di risorse elet- } \\
\text { troniche. }\end{array}$ & Annuale \\
\hline D3.07 & $\begin{array}{l}\text { Spese periodici } \\
\text { online }\end{array}$ & $\begin{array}{l}\text { Spesa sostenuta dal Coordinamento } \\
\text { Centrale Biblioteche e dalle singole } \\
\text { biblioteche per l'acquisto di periodici } \\
\text { elettronici. }\end{array}$ & Annuale \\
\hline
\end{tabular}

\begin{tabular}{|c|c|l|c|}
\hline \multicolumn{2}{|c|}{ Denominazione } & \multicolumn{1}{c|}{ Descrizione } & Periodicità \\
\hline D4.01 & Inventari & $\begin{array}{l}\text { Numero dei nuovi inventari, distinti } \\
\text { tra monografie, periodici e altri sup- } \\
\text { porti. }\end{array}$ & Quadrimestrale \\
\hline D4.02 & Catalogazioni & $\begin{array}{l}\text { Numero totale dei nuovi inventari } \\
\text { catalogati e collocati. }\end{array}$ & Quadrimestrale \\
\hline D4.03 & $\begin{array}{l}\text { Catture (a) e } \\
\text { creazioni (b) }\end{array}$ & $\begin{array}{l}\text { Numero delle nuove notizie biblio- } \\
\text { grafiche, distinte tra catture dall'In- } \\
\text { dice nazionale SBN e creazioni ex } \\
\text { novo. }\end{array}$ & Quadrimestrale \\
\hline D4.04 & $\begin{array}{c}\text { Recupero del } \\
\text { pregresso }\end{array}$ & $\begin{array}{l}\text { Numero degli inventari recuperati nel } \\
\text { catalogo automatizzato. }\end{array}$ & Quadrimestrale \\
\hline D4.05 & $\begin{array}{c}\text { Tempi dell'iter del } \\
\text { libro }\end{array}$ & $\begin{array}{l}\text { Totale dei giorni necessari per le } \\
\text { diverse fasi della catalogazione, dalla } \\
\text { ricezione alla collocazione. }\end{array}$ & Quadrimestrale \\
\hline D4.06 & Prestiti & Numero totale dei prestiti. & Quadrimestrale \\
\hline
\end{tabular}




\begin{tabular}{|c|c|l|c|}
\hline $\begin{array}{c}\text { D4.06 } \\
\text { bis }\end{array}$ & Prestiti BID & $\begin{array}{l}\text { Numero totale di edizioni di un'opera } \\
\text { prestate indipendentemente dal nu- } \\
\text { mero di copie possedute. }\end{array}$ & Quadrimestrale \\
\hline D4.07 & $\begin{array}{c}\text { Prestiti } \\
\text { interbibliotecari }\end{array}$ & $\begin{array}{l}\text { Numero totale delle transazioni di } \\
\text { Lending e Borrowing. }\end{array}$ & Quadrimestrale \\
\hline D4.08 & $\begin{array}{c}\text { Documenti } \\
\text { elettronici trasmessi } \\
\text { ad utenti interni }\end{array}$ & $\begin{array}{l}\text { Numero di documenti elettronici } \\
\text { trasmessi ad utenti interni tramite il } \\
\text { software Ariel. }\end{array}$ & Quadrimestrale \\
\hline D4.09 & Ricerche in OPAC & Numero degli accessi all'OPAC. & Quadrimestrale \\
\hline D4.10 & $\begin{array}{c}\text { Ricerche in banche } \\
\text { dati }\end{array}$ & $\begin{array}{l}\text { Numero totale delle sessioni di ricerca } \\
\text { effettuate per le singole banche dati. }\end{array}$ & Quadrimestrale \\
\hline D4.12 & $\begin{array}{c}\text { Corsi per studenti } \\
\text { D4.13 }\end{array}$ & $\begin{array}{l}\text { Numero dei corsi organizzati dalle } \\
\text { singole biblioteche. }\end{array}$ & Quadrimestrale \\
\hline D4.14 & $\begin{array}{c}\text { Periodici elettronici } \\
\text { attivi }\end{array}$ & $\begin{array}{l}\text { Numero dei titoli di periodici in } \\
\text { versione elettronica. }\end{array}$ & Semestrale \\
\hline D4.16 & $\begin{array}{c}\text { Accessi periodici } \\
\text { elettronici }\end{array}$ & $\begin{array}{l}\text { Numero totale degli accessi ai perio- } \\
\text { dici, distinto in TOC, Abstract, Full- } \\
\text { text (PDF o HTML). }\end{array}$ & Semestrale \\
\hline
\end{tabular}

\section{Indicatori}

\begin{tabular}{|c|c|l|c|}
\hline \multicolumn{4}{|c|}{ GENERALI } \\
\hline I1.01 & $\begin{array}{c}\text { Posti di lettura / } \\
\text { Iscritti }\end{array}$ & $\begin{array}{l}\text { Dumero di posti di lettura } \\
\text { disponibili per iscritto }\end{array}$ & D2.05 / D1.02 \\
\hline I1.02 & $\begin{array}{c}\text { Metri quadrati } \\
\text { biblioteche / Iscritti }\end{array}$ & $\begin{array}{l}\text { Spazi disponibili per iscritto, } \\
\text { espressi in metri quadrati }\end{array}$ & D2.07 / D1.02 \\
\hline I1.03 & Volumi / Iscritti & $\begin{array}{l}\text { Numero di volumi disponibili } \\
\text { per ogni iscritto }\end{array}$ & D2.01 / D1.02 \\
\hline I1.04 & $\begin{array}{l}\text { Spesa del SBA / } \\
\text { Budget d'Ateneo }\end{array}$ & $\begin{array}{l}\text { Percentuale del budget di Ate- } \\
\text { neo destinata allo SBA }\end{array}$ & (D3.02 / D3.01) $\times 100$ \\
\hline I1.05 & $\begin{array}{c}\text { Corsi per gli } \\
\text { studenti / Iscritti }\end{array}$ & $\begin{array}{l}\text { Numero di corsi organizzati per } \\
\text { iscritto }\end{array}$ & D4.12/ D1.02 \\
\hline I1.06 & $\begin{array}{c}\text { Volumi ad accesso } \\
\text { libero / Volumi } \\
\text { totali }\end{array}$ & $\begin{array}{l}\text { Percentuale di patrimonio bi- } \\
\text { bliografico collocata a scaffale } \\
\text { aperto rispetto al posseduto } \\
\text { delle biblioteche. }\end{array}$ & (D2.04 / D2.01) x 100 \\
\hline
\end{tabular}

1 Numero identificativo della notizia bibliografica SBN. 


\begin{tabular}{|c|c|c|c|}
\hline \multicolumn{4}{|c|}{ DI EFFICACIA } \\
\hline \multicolumn{2}{|c|}{ Denominazione } & Descrizione & Formula di calcolo \\
\hline $\mathrm{I} 2.01$ & $\begin{array}{c}\text { Indice di } \\
\text { circolazione } \\
\text { Prestiti / Inventari }\end{array}$ & $\begin{array}{l}\text { Percentuale di patrimonio do- } \\
\text { cumentario data in prestito } \\
\text { (prestito esterno, interno o } \\
\text { notturno). }\end{array}$ & $(\mathrm{D} 4.06 / \mathrm{D} 2.01) \times 100$ \\
\hline $\mathrm{I} 2.02$ & Prestiti / BID & $\begin{array}{l}\text { Frequenza con la quale una } \\
\text { determinata edizione è andata } \\
\text { in prestito, indipendentemente } \\
\text { dal numero di esemplari pos- } \\
\text { seduti. }\end{array}$ & D4.06 / D4.06 bis \\
\hline $\mathrm{I} 2.03$ & $\begin{array}{l}\text { Indice di } \\
\text { penetrazione } \\
\text { Percentuale di } \\
\text { utenti attivi }\end{array}$ & $\begin{array}{l}\text { Percentuale di studenti che usu- } \\
\text { fruiscono del servizio di presti- } \\
\text { to rispetto al totale degli } \\
\text { iscritti. }\end{array}$ & $\begin{array}{l}(\mathrm{D} 1.03 /(\mathrm{D} 1.01+ \\
\mathrm{D} 1.02) \times 100\end{array}$ \\
\hline $\mathrm{I} 2.04$ & Prestiti / Iscritti & $\begin{array}{l}\text { Numero medio annuale di vo- } \\
\text { lumi presi in prestito da ogni } \\
\text { studente. }\end{array}$ & D4.06 / D1.02 \\
\hline $\mathrm{I} 2.05$ & $\begin{array}{l}\text { Prestiti } \\
\text { interbibliotecari / } \\
\text { Iscritti }\end{array}$ & $\begin{array}{l}\text { Numero medio annuale di } \\
\text { prestiti interbibliotecari (borro- } \\
\text { wing + lending) fruiti da ogni } \\
\text { studente. }\end{array}$ & D4.07 / D1.02 \\
\hline $\mathrm{I} 2.06$ & $\begin{array}{l}\text { Ricerche in OPAC / } \\
\text { Iscritti }\end{array}$ & $\begin{array}{l}\text { Numero medio delle ricerche } \\
\text { condotte in OPAC da ogni } \\
\text { studente. }\end{array}$ & D4.09 / D1.02 \\
\hline $\mathrm{I} 2.07$ & $\begin{array}{l}\text { Uso delle banche } \\
\text { dati }\end{array}$ & $\begin{array}{l}\text { Numero medio delle sessioni di } \\
\text { ricerca complessivamente ef- } \\
\text { fettuate nelle banche dati da } \\
\text { ogni studente. }\end{array}$ & D4.10 / D1.02 \\
\hline $\mathrm{I} 2.8$ & $\begin{array}{l}\text { Partecipanti ai corsi } \\
\text { / Iscritti }\end{array}$ & $\begin{array}{l}\text { Percentuale di studenti che ha } \\
\text { partecipato ai corsi di forma- } \\
\text { zione per gli studenti. }\end{array}$ & D4.13/ D1.02 \\
\hline $\mathrm{I} 2.9$ & $\begin{array}{l}\text { Percentuale di } \\
\text { recupero }\end{array}$ & $\begin{array}{l}\text { Percentuale dei recuperi sul to- } \\
\text { tale delle catalogazioni. }\end{array}$ & $\begin{array}{c}{[\mathrm{D} 4.04 /(\mathrm{D} 4.02+\mathrm{D} 4.04)]} \\
\text { x } 100\end{array}$ \\
\hline $\mathrm{I} 2.10$ & $\begin{array}{l}\text { Percentuale di } \\
\text { catalogazione } \\
\text { derivata }\end{array}$ & $\begin{array}{l}\text { Percentuale di notizie biblio- } \\
\text { grafiche catturate da SBN sul } \\
\text { totale delle catalogazioni. }\end{array}$ & $(\mathrm{D} 4.03 \mathrm{a} / \mathrm{D} 4.02) \times 100$ \\
\hline $\mathrm{I} 2.11$ & $\begin{array}{l}\text { Uso periodici } \\
\text { elettronici }\end{array}$ & $\begin{array}{l}\text { Numero degli usi remoti in } \\
\text { rapporto al numero di utenti }\end{array}$ & D4.16 / (D1.01+D1.02) \\
\hline $\mathrm{I} 2.12$ & Costo per accesso & $\begin{array}{l}\text { Spesa annuale per periodici } \\
\text { elettronici in rapporto al nu- } \\
\text { mero degli accessi. }\end{array}$ & D3.07 / D4.16 \\
\hline
\end{tabular}




\begin{tabular}{|c|c|l|c|}
\hline \multicolumn{3}{|c|}{ DI PRODUTTIVITA } \\
\hline I3.01 & $\begin{array}{c}\text { Numero delle } \\
\text { catalogazioni in } \\
\text { un'ora }\end{array}$ & $\begin{array}{l}\text { Descrizione } \\
\text { Numero di volumi catalogati in } \\
\text { un'ora di lavoro dedicata alla } \\
\text { catalogazione. }\end{array}$ & Formula di calcolo \\
\hline I3.02 & $\begin{array}{c}\text { Numero delle } \\
\text { acquisizioni in } \\
\text { un'ora }\end{array}$ & $\begin{array}{l}\text { Numero di acquisizioni effet- } \\
\text { tuate in un'ora di lavoro de- } \\
\text { dicata alle acquisizioni. }\end{array}$ & D4.01/D2.12b \\
\hline I3.03 & $\begin{array}{l}\text { Numero di } \\
\text { operazioni } \\
\text { ILL+Prestito in } \\
\text { un'ora }\end{array}$ & $\begin{array}{l}\text { Numero di operazioni di pre- } \\
\text { stito interbibliotecario / docu- } \\
\text { ment delivery e prestito locale } \\
\text { effettuate in un'ora di lavoro } \\
\text { dedicata a queste attività. }\end{array}$ & $\begin{array}{c}\text { (D4.06+D4.07+D4.08)/ } \\
\text { D2.12c }\end{array}$ \\
\hline
\end{tabular}




\section{Appendice B \\ Questionari dell'indagine sulla soddisfazione degli utenti}

\section{Questionario sulla valutazione dei servizi bibliotecari d'Ateneo sommi- nistrato agli studenti}

QU01 Buona sera, sono [ ], chiamo dal Dipartimento di Statistica dell'Università di Firenze.

Stiamo svolgendo un'indagine per conto del Sistema Bibliotecario d'Ateneo.

Posso parlare 5 minuti per rivolgere alcune semplici domande al Sig. ... ?

Dal servizio bibliotecario di Ateneo ci risulta che l'anno scorso sei stato utente della biblioteca di [ ].

L'Università degli Studi di Firenze sta conducendo un sondaggio per conoscere qual è il grado di soddisfazione degli utenti dei servizi bibliotecari. Siamo interessati a sapere che cosa, secondo il tuo parere, funziona e che cosa, invece, deve migliorare.

I dati che fornirai saranno registrati ed utilizzati in forma anonima, in conformità alla normativa sulla tutela della privacy.

Facendo riferimento alla biblioteca [ ] di cui sei stato utente, per ciascun servizio o caratteristica che ti leggerò mi dovresti dire:

\begin{tabular}{|l|l|}
\hline \multirow{4}{*}{$\begin{array}{l}\text { L'importanza che gli attribuisci indipendentemente } \\
\text { dal modo in cui è gestito in biblioteca }\end{array}$} & $1=$ Per nulla importante \\
\cline { 2 - 2 } & 2 = Poco importante \\
\cline { 2 - 2 } & $3=$ Indifferente \\
\cline { 2 - 2 } & $4=$ Importante \\
\cline { 2 - 2 } & $5=$ Molto importante \\
\hline
\end{tabular}

\begin{tabular}{|l|l|}
\hline \multirow{4}{*}{$\begin{array}{l}\text { Quante volte (in percentuale) lo utilizzi sul totale } \\
\text { delle volte che vai in biblioteca }\end{array}$} & $0 \%$ \\
& $50 \%$ \\
& $50 \%$ \\
& $75 \%$ \\
\hline
\end{tabular}

\begin{tabular}{|l|l|}
\hline \multirow{4}{*}{$\begin{array}{l}\text { Il tuo grado di soddisfazione per quel servizio o o } \\
\text { quella caratteristica }\end{array}$} & $1=$ insoddisfatto \\
\cline { 2 - 2 } & $2=$ poco insoddisfatto \\
\cline { 2 - 2 } & $3=$ Indifferente \\
\cline { 2 - 2 } & $4=$ abbastanza soddisfatto \\
\cline { 2 - 2 } & $5=$ soddisfatto \\
\hline
\end{tabular}


Per i rilevatori:

$96=$ Non sa

$97=$ Non vuole rispondere

$98=$ Interrompe l'intervista

$99=$ Cade la linea

Libri, periodici attualmente e cd-rom posseduti dalla biblioteca di [ ]

QU02A1. Importanza

QU02B1. Percentuale d'uso [se 0\% vai alla domanda QU02A2]

QU02C1. Grado di soddisfazione

Disponibilità in biblioteca delle novità librarie nelle discipline di suo interesse

QU02A2. Importanza

QU02B2. Percentuale d'uso [se 0\% vai alla domanda QU02A3]

QU02C2. Grado di soddisfazione

Disponibilità in biblioteca degli ultimi numeri dei periodici nelle discipline di suo interesse

QU02A3. Importanza

QU02B3. Percentuale d'uso [se 0\% vai alla domanda QU02A4]

QU02C3. Grado di soddisfazione

Precisione e rapidità nella distribuzione di libri e periodici dai magazzini QU02A4. Importanza

QU02B4. Percentuale d'uso [se 0\% vai alla domanda QU02A5]

QU02C4. Grado di soddisfazione

Facilità del recupero di libri e periodici dagli scaffali liberamente accessibili ai lettori

QU02A5. Importanza

QU02B5. Percentuale d'uso [se 0\% vai alla domanda QU03A6]

QU02C5. Grado di soddisfazione

Disponibilità in sala di lettura di posti a sedere per l'uso di materiale della biblioteca

QU03A6. Importanza

QU03B6. Percentuale d'uso [se 0\% vai alla domanda QU03A9]

QU03C6. Grado di soddisfazione 
Silenzio in sala di lettura

QU03A7. Importanza

QU03B7. Percentuale d'uso [se 0\% vai alla domanda QU03A8]

QU03C7. Grado di soddisfazione

Comfort e luminosità della sala di lettura

QU03A8. Importanza

QU03B8. Percentuale d'uso [se 0\% vai alla domanda QU03A9]

QU03C8. Grado di soddisfazione

Accesso a Internet in Biblioteca

QU03A9. Importanza

QU03B9. Percentuale d'uso [se 0\% vai alla domanda QU03A10]

QU03C9. Grado di soddisfazione

Prestito interbibliotecario: recupero di libri e articoli da altre biblioteche universitarie

QU03A10. Importanza

QU03B10. Percentuale d'uso [se 0\% vai alla domanda QU03A11]

QU03C10. Grado di soddisfazione

Facilità del recupero e consultazione di bibliografie, repertori, enciclopedie

QU03A11. Importanza

QU03B11. Percentuale d'uso [se 0\% vai alla domanda QU03A12]

QU03C11. Grado di soddisfazione

Banche dati: modo in cui sono messe a disposizione del pubblico

QU03A12. Importanza

QU03C12. Grado di soddisfazione

Comunicazione con il personale delle biblioteche (disponibilità e cortesia dei bibliotecari nei confronti del pubblico)

QU03A13. Importanza

QU03B13. Percentuale d'uso [se 0\% vai alla domanda QU04A14]

QU03C13. Grado di soddisfazione 
Servizi e assistenza personalizzata al lettore da parte del bibliotecario QU04A14. Importanza

QU04B14. Percentuale d'uso [se 0\% vai alla domanda QU03A15] QU04C14. Grado di soddisfazione

Depliant sulle biblioteche e segnaletica in biblioteca per facilitare l'uso dei servizi

\section{QU04A15. Importanza}

QU04B15. Percentuale d'uso [se 0\% vai alla domanda QU05]

QU04C15. Grado di soddisfazione

QU05 Quali altre biblioteche frequenti

\begin{tabular}{|l|l|}
\hline Antropologia & \\
\hline Agraria & \\
\hline Architettura & \\
\hline Biologia Animale & \\
\hline Botanica & \\
\hline Chimica & \\
\hline Dip. Costruzioni & \\
\hline Dip. di Diritto Comparato e Penale e Dip. di Diritto Privato e Processuale & \\
\hline Dip. di Diritto dell'Economia & \\
\hline Dip. di Diritto Pubblico & \\
\hline Dip. Economico Estimativo & \\
\hline Dip. Farmacologia & \\
\hline Dip. Filosofia & \\
\hline Dip. Ing. Civile & \\
\hline Dip. Italianistica & \\
\hline Dip. Linguistica & \\
\hline Dip. Matematica Applicata "G. Sansone" & \\
\hline Dip. Meccanica & \\
\hline Dip. Pediatria & \\
\hline Dip. Processi e Metodi & \\
\hline Dip. Progettazione & \\
\hline Dip. Psicologia & \\
\hline Dip. Sanità Pubblica & \\
\hline & \\
\hline & \\
\hline & \\
\hline & \\
\hline & \\
\hline
\end{tabular}




\begin{tabular}{|l|l|}
\hline Dip. Scienze Neurologiche e Psichiatriche & \\
\hline Dip. Sistemi & \\
\hline Dip. Storia dell'Architettura & \\
\hline Dip. Scienze dell'Antichità & \\
\hline Dip. Studi sul Medioevo & \\
\hline Dip. Storia delle Arti e dello Spettacolo & \\
\hline Dip. Teoria e Storia del Diritto & \\
\hline Dip. Urbanistica & \\
\hline Economia & \\
\hline Emeroteca & \\
\hline Fisica & \\
\hline Farmacia & \\
\hline Geografia & \\
\hline Giurisprudenza & \\
\hline Geomineralogia & \\
\hline Ingegneria & \\
\hline Lettere & \\
\hline Medicina & \\
\hline Matematica & \\
\hline Scienze Politiche & \\
\hline Statistica & \\
\hline Scienze della Formazione & \\
\hline Storia e Lett. Nordamericana & \\
\hline Nessuna & \\
\hline
\end{tabular}

Facendo riferimento ai servizi bibliotecari di ateneo nel loro complesso, ci valuteresti come hai fatto precedentemente i seguenti servizi o caratteristiche:

Sito web del Sistema Bibliotecario di Ateneo

QU06aA17. Importanza

QU06aB17. Percentuale d'uso [se 0\% vai alla domanda QU08sex] QU06aC17. Grado di soddisfazione 
Uso del catalogo della biblioteca su internet: ricerca di libri o periodici per titolo e ricerca di libri per autore

QU06aA18. Importanza

QU06aB18. Percentuale d'uso

QU06aC18. Grado di soddisfazione

Uso del catalogo su internet: ricerca di libri per argomento (nel campo: soggetto) o materia (nel campo: classificazione)

QU06aA19. Importanza

QU06aB19. Percentuale d'uso

QU06aC19. Grado di soddisfazione

Uso del catalogo della biblioteca su internet: ricerca di libri o periodici nel campo parole chiave

QU06aA20. Importanza

QU06aB20. Percentuale d'uso

QU06aC20. Grado di soddisfazione

Uso del catalogo della biblioteca su internet: prenotazione o proroga del prestito di un libro

QU07A21. Importanza

QU07B21. Percentuale d'uso

QU07C21. Grado di soddisfazione

Banche dati attualmente disponibili nelle biblioteche (via web, su cd-rom)

QU07A22. Importanza

QU07B22. Percentuale d'uso

QU07C22. Grado di soddisfazione

Risultati ottenuti dall'interrogazione delle banche dati

QU07A23. Importanza

QU07C23. Grado di soddisfazione

Uso dei periodici elettronici nelle discipline di suo interesse

QU07A24. Importanza

QU07B24. Percentuale d'uso

QU07C24. Grado di soddisfazione 
Adesso le faremo alcune domande di carattere personale. Queste domande possono essere utili a capire meglio il profilo degli utenti del Sistema Bibliotecario di Ateneo. La preghiamo di rispondere ricordandole il completo anonimato.

QU08sex Sesso del rispondente

1 Maschio

2 Femmina

QU08resfi Risiede nel comune di Firenze?

1 Sì [vai alla domanda QU08anno]

2 No

Qu08respr In quale provincia risiedi?

QU08dom Sei domiciliato a Firenze

QU08anno In quale anno è nato/a? (indicare l'anno in 4 cifre)

QU08lav Hai un lavoro?

1 Sì [vai alla domanda QU08tesi]

2 No

QU08tlav Lavori

1 Full-time

2 Part-time

QU08tesi Hai già chiesto la tesi?

1 Già laureato

2 Non ha ancora chiesto la tesi

3 Ha già chiesto la tesi 


\section{Questionario sulla valutazione dei servizi bibliotecari d'Ateneo sommi- nistrato ai docenti}

Gentile Professore, gentile Professoressa,

L'Università degli Studi di Firenze sta conducendo un sondaggio per conoscere quale è il grado di soddisfazione degli utenti dei servizi bibliotecari. I questionari sono rigorosamente anonimi. Siamo interessati a sapere che cosa, secondo il suo parere, funziona e che cosa, invece, dobbiamo migliorare.

Solo conoscendo le opinioni degli utenti l'Università potrà erogare servizi sempre più efficaci e soddisfacenti. Le chiediamo di rispondere con sincerità quand'anche il suo giudizio dovesse essere negativo. Le valutazioni che le chiediamo di effettuare riguardano sia la biblioteca che utilizza più frequentemente sia i servizi bibliotecari di Ateneo nel loro complesso. Ai fini dell'indagine lei risulta utente della biblioteca che utilizza più frequentemente.

Istruzioni per la compilazione

Per ogni domanda barrare la casella che meglio risponde alle sue opinioni o abitudini, in relazione a:

\begin{tabular}{|c|c|}
\hline \multirow{7}{*}{$\begin{array}{l}\text { L'importanza che attribuisce al servizio, } \\
\text { indipendentemente da come è gestito in } \\
\text { biblioteca }\end{array}$} & $97=$ Non $\mathrm{sa}$ \\
\hline & $98=$ Non risponde \\
\hline & $1=$ Per nulla importante \\
\hline & $2=$ Poco importante \\
\hline & $3=$ Indifferente \\
\hline & $4=$ Importante \\
\hline & $5=$ Molto importante \\
\hline \multirow{7}{*}{$\begin{array}{l}\text { Percentuale d'uso del servizio nelle } \\
\text { biblioteche dell'Università di Firenze }\end{array}$} & $97=$ Non sa \\
\hline & $98=$ Non risponde \\
\hline & $0 \%$ \\
\hline & $25 \%$ \\
\hline & $50 \%$ \\
\hline & $75 \%$ \\
\hline & $100 \%$ \\
\hline
\end{tabular}




\begin{tabular}{|l|l|}
\hline \multirow{4}{*}{ il suo grado di soddisfazione } & $97=$ Non sa \\
\cline { 2 - 2 } & $98=$ Non risponde \\
\hline & $1=$ Insoddisfatta/o \\
\cline { 2 - 2 } $2=$ Poco insoddisfatta/o \\
\cline { 2 - 2 } $3=$ Indifferente \\
\hline $4=$ Abbastanza soddisfatta/o \\
\hline $5=$ Soddisfatta/o \\
\hline
\end{tabular}

\begin{tabular}{|l|l|l|}
\hline & $\begin{array}{l}\text { 1.0a } \\
\text { Quali biblioteche } \\
\text { del Sistema } \\
\text { Bibliotecario d'Ateneo } \\
\text { frequenta abitualmente? }\end{array}$ & $\begin{array}{l}\text { Fra le biblioteche che } \\
\text { frequenta abitualmente, } \\
\text { quale frequenta di più? } \\
\text { (indicare una sola } \\
\text { scelta) }\end{array}$ \\
\hline Antropologia & & \\
\hline Agraria & & \\
\hline Architettura & & \\
\hline Biologia Animale & & \\
\hline Botanica & & \\
\hline Chimica & & \\
\hline Dip. Costruzioni & & \\
\hline Dip. di Diritto Comparato e Penale e Dip. di & & \\
\hline Diritto Privato e Processuale & & \\
\hline Dip. di Diritto dell'Economia & & \\
\hline Dip. di Diritto Pubblico & & \\
\hline Dip. Economico Estimativo & & \\
\hline Dip. Farmacologia & & \\
\hline Dip. Filosofia & & \\
\hline Dip. Ing. Civile & & \\
\hline Dip. Italianistica & & \\
\hline Dip. Linguistica & & \\
\hline Dip. Matematica Applicata "G. Sansone" & & \\
\hline Dip. Meccanica & & \\
\hline Dip. Pediatria & & \\
\hline Dip. Processi e Metodi & & \\
\hline Dip. Progettazione & & \\
\hline Dip. Psicologia & & \\
\hline
\end{tabular}




\begin{tabular}{|c|c|c|}
\hline \multicolumn{3}{|l|}{ Dip. Sanità Pubblica } \\
\hline Dip. Scienze Neurologiche e Psichiatriche & & \\
\hline Dip. Sistemi & & \\
\hline Dip. Storia dell'Architettura & & \\
\hline Dip. Scienze dell'Antichità & & \\
\hline Dip. Studi sul Medioevo & & \\
\hline Dip. Storia delle Arti e dello Spettacolo & & \\
\hline Dip. Teoria e Storia del Diritto & & \\
\hline Dip. Urbanistica & & \\
\hline Economia & & \\
\hline Emeroteca & & \\
\hline Fisica & & \\
\hline Farmacia & & \\
\hline Geografia & & \\
\hline Giurisprudenza & & \\
\hline Geomineralogia & & \\
\hline Ingegneria & & \\
\hline Lettere & & \\
\hline Medicina & & \\
\hline Matematica & & \\
\hline Scienze Politiche & & \\
\hline Statistica & & \\
\hline Scienze della Formazione & & \\
\hline Storia e Lett. Nordamericana & & \\
\hline \begin{tabular}{|l|} 
Nessuna \\
FILTRO: Chi risponde Nessuna deve andare \\
alla domanda 1.1 e poi alla1.17, chi ha in- \\
dicato almeno una biblioteca deve andare alla \\
domanda 1.2.
\end{tabular} & & \\
\hline
\end{tabular}

1.1 Perché non utilizza nessuna biblioteca del Servizio Bibliotecario di Ateneo?

Orari di apertura non adeguati alle sue esigenze

Indisponibilità degli strumenti/servizi di suo interesse

Preferenza di biblioteche non appartenenti al Sistema Bibliotecario di Ateneo (Specificare quali) ...

Altro (specificare) ... 
FILTRO: Chi ha risposto alla domanda 1.1 deve saltare alla domanda 1.17

Le ricordiamo che le successive valutazioni dalla domanda 1.2 alla domanda 1.16 si riferiscono alla biblioteca che lei frequenta di più.

1.2 - Libri, periodici e cd-rom attualmente posseduti dalla biblioteca

\begin{tabular}{|l|c|c|c|c|c|c|c|}
\hline $\begin{array}{l}\text { Quanto sono importanti in relazione alle } \\
\text { sue esigenze di studio e ricerca? }\end{array}$ & 97 & 98 & 1 & 2 & 3 & 4 & 5 \\
\hline $\begin{array}{l}\text { In quale percentuale le sue visite in } \\
\text { biblioteca sono dedicate principalmente } \\
\text { alla consultazione o al prestito di tale } \\
\text { materiale? }\end{array}$ & 97 & 98 & $0 \%$ & $25 \%$ & $50 \%$ & $75 \%$ & $100 \%$ \\
\hline $\begin{array}{l}\text { Qual è il suo grado di soddisfazione per } \\
\text { l'offerta documentaria delle } \\
\text { biblioteche? }\end{array}$ & 97 & 98 & 1 & 2 & 3 & 4 & 5 \\
\hline
\end{tabular}

1.3 - Disponibilità in biblioteca delle novità librarie nelle discipline di suo interesse

\begin{tabular}{|l|c|c|c|c|c|c|c|}
\hline $\begin{array}{l}\text { Quanto è importante in relazione alle } \\
\text { sue esigenze di studio e ricerca? }\end{array}$ & 97 & 98 & 1 & 2 & 3 & 4 & 5 \\
\hline $\begin{array}{l}\text { In quale percentuale le sue visite in } \\
\text { biblioteca sono dedicate principalmente } \\
\text { alla consultazione delle novità librarie? }\end{array}$ & 97 & 98 & $0 \%$ & $25 \%$ & $50 \%$ & $75 \%$ & $100 \%$ \\
\hline $\begin{array}{l}\text { Qual è il suo grado di soddisfazione al } \\
\text { proposito? }\end{array}$ & 97 & 98 & 1 & 2 & 3 & 4 & 5 \\
\hline
\end{tabular}

1.4 - Disponibilità in biblioteca degli ultimi numeri dei periodici nelle discipline di suo interesse

\begin{tabular}{|l|c|c|c|c|c|c|c|}
\hline $\begin{array}{l}\text { Quanto è importante in relazione alle } \\
\text { sue esigenze di studio e ricerca? }\end{array}$ & 97 & 98 & 1 & 2 & 3 & 4 & 5 \\
\hline $\begin{array}{l}\text { In quale percentuale le sue visite in } \\
\text { biblioteca sono dedicate principalmente } \\
\text { alla consultazione degli ultimi numeri } \\
\text { dei periodici? }\end{array}$ & 97 & 98 & $0 \%$ & $25 \%$ & $50 \%$ & $75 \%$ & $100 \%$ \\
\hline $\begin{array}{l}\text { Qual è il suo grado di soddisfazione al } \\
\text { proposito? }\end{array}$ & 97 & 98 & 1 & 2 & 3 & 4 & 5 \\
\hline
\end{tabular}


APPENDICE B

1.5 - Precisione e rapidità nella distribuzione di libri e periodici dai magazzini

\begin{tabular}{|l|c|c|c|c|c|c|c|}
\hline $\begin{array}{l}\text { Quanto è importante in relazione alle } \\
\text { sue esigenze di studio e ricerca? }\end{array}$ & 97 & 98 & 1 & 2 & 3 & 4 & 5 \\
\hline $\begin{array}{l}\text { In quale percentuale le sue visite in } \\
\text { biblioteca prevedono l'uso di materiali } \\
\text { collocati in un magazzino? }\end{array}$ & 97 & 98 & $0 \%$ & $25 \%$ & $50 \%$ & $75 \%$ & $100 \%$ \\
\hline $\begin{array}{l}\text { Qual è il suo grado di soddisfazione al } \\
\text { proposito? }\end{array}$ & 97 & 98 & 1 & 2 & 3 & 4 & 5 \\
\hline
\end{tabular}

1.6 - Facilità del recupero di libri e periodici dagli scaffali liberamente accessibili ai lettori

\begin{tabular}{|l|c|c|c|c|c|c|c|}
\hline $\begin{array}{l}\text { Quanto è importante in relazione alle } \\
\text { sue esigenze di studio e ricerca? }\end{array}$ & 97 & 98 & 1 & 2 & 3 & 4 & 5 \\
\hline $\begin{array}{l}\text { In quale percentuale le sue visite in } \\
\text { biblioteca prevedono l'uso di materiali } \\
\text { collocati a scaffale aperto? }\end{array}$ & 97 & 98 & $0 \%$ & $25 \%$ & $50 \%$ & $75 \%$ & $100 \%$ \\
\hline $\begin{array}{l}\text { Qual è il suo grado di soddisfazione al } \\
\text { proposito? }\end{array}$ & 97 & 98 & 1 & 2 & 3 & 4 & 5 \\
\hline
\end{tabular}

1.7 - Disponibilità in sala di lettura di posti a sedere per l'uso di materiale della biblioteca

\begin{tabular}{|l|c|c|c|c|c|c|c|}
\hline $\begin{array}{l}\text { Quanto è importante in relazione alle } \\
\text { sue esigenze di studio e ricerca? }\end{array}$ & 97 & 98 & 1 & 2 & 3 & 4 & 5 \\
\hline $\begin{array}{l}\text { In quale percentuale le sue visite in } \\
\text { biblioteca prevedono l'uso della sala di } \\
\text { lettura per la consultazione di materiale } \\
\text { della biblioteca? }\end{array}$ & 97 & 98 & $0 \%$ & $25 \%$ & $50 \%$ & $75 \%$ & $100 \%$ \\
\hline $\begin{array}{l}\text { Qual è il suo grado di soddisfazione al } \\
\text { proposito? }\end{array}$ & 97 & 98 & 1 & 2 & 3 & 4 & 5 \\
\hline
\end{tabular}

FILTRO: Chi risponde $0 \%$ alla domanda 1.7 deve saltare alla domanda 1.10

1.8 - Silenzio in sala di lettura

\begin{tabular}{|l|c|c|c|c|c|c|c|}
\hline $\begin{array}{l}\text { Quanto è importante in relazione alle } \\
\text { sue } \\
\text { esigenze di studio e ricerca? }\end{array}$ & 97 & 98 & 1 & 2 & 3 & 4 & 5 \\
\hline $\begin{array}{l}\text { In che percentuale delle sue visite in } \\
\text { biblioteca ha trovato silenziose le sale di } \\
\text { lettura? }\end{array}$ & 97 & 98 & $0 \%$ & $25 \%$ & $50 \%$ & $75 \%$ & $100 \%$ \\
\hline $\begin{array}{l}\text { Qual è il suo grado di soddisfazione al } \\
\text { proposito? }\end{array}$ & 97 & 98 & 1 & 2 & 3 & 4 & 5 \\
\hline
\end{tabular}


1.9 - Comfort e luminosità della sala di lettura

\begin{tabular}{|l|c|c|c|c|c|c|c|}
\hline $\begin{array}{l}\text { Quanto è importante in relazione alle } \\
\text { sue esigenze di studio e ricerca? }\end{array}$ & 97 & 98 & 1 & 2 & 3 & 4 & 5 \\
\hline $\begin{array}{l}\text { In che percentuale delle sue visite in } \\
\text { biblioteca ha trovato confortevoli e bene } \\
\text { illuminate le sale di lettura? }\end{array}$ & 97 & 98 & $0 \%$ & $25 \%$ & $50 \%$ & $75 \%$ & $100 \%$ \\
\hline $\begin{array}{l}\text { Qual è il suo grado di soddisfazione al } \\
\text { proposito? }\end{array}$ & 97 & 98 & 1 & 2 & 3 & 4 & 5 \\
\hline
\end{tabular}

1.10 - Accesso a Internet in Biblioteca

\begin{tabular}{|l|c|c|c|c|c|c|c|}
\hline $\begin{array}{l}\text { Quanto è importante in relazione alle } \\
\text { sue esigenze di studio e ricerca? }\end{array}$ & 97 & 98 & 1 & 2 & 3 & 4 & 5 \\
\hline $\begin{array}{l}\text { In quale percentuale le sue visite in } \\
\text { biblioteca prevedono l'accesso a } \\
\text { internet per ragioni di studio o ricerca? }\end{array}$ & 97 & 98 & $0 \%$ & $25 \%$ & $50 \%$ & $75 \%$ & $100 \%$ \\
\hline $\begin{array}{l}\text { Qual è il suo grado di soddisfazione al } \\
\text { proposito? }\end{array}$ & 97 & 98 & 1 & 2 & 3 & 4 & 5 \\
\hline
\end{tabular}

1.11 - Prestito interbibliotecario: recupero di libri e articoli da altre biblioteche universitarie

\begin{tabular}{|l|c|c|c|c|c|c|c|}
\hline $\begin{array}{l}\text { Quanto è importante in relazione alle } \\
\text { sue esigenze di studio e ricerca? }\end{array}$ & 97 & 98 & 1 & 2 & 3 & 4 & 5 \\
\hline $\begin{array}{l}\text { In quale percentuale i documenti } \\
\text { utilizzati nelle sue ricerche ti sono stati } \\
\text { forniti tramite il servizio di prestito } \\
\text { interbibliotecario? }\end{array}$ & 97 & 98 & $0 \%$ & $25 \%$ & $50 \%$ & $75 \%$ & $100 \%$ \\
\hline $\begin{array}{l}\text { Qual è il suo grado di soddisfazione } \\
\text { al proposito? }\end{array}$ & 97 & 98 & 1 & 2 & 3 & 4 & 5 \\
\hline
\end{tabular}

1.12 - Facilità del recupero e consultazione di bibliografie, repertori, enciclopedie

\begin{tabular}{|l|c|c|c|c|c|c|c|}
\hline $\begin{array}{l}\text { Quanto è importante in relazione alle } \\
\text { sue esigenze di studio e ricerca? }\end{array}$ & 97 & 98 & 1 & 2 & 3 & 4 & 5 \\
\hline $\begin{array}{l}\text { In quale percentuale le sue visite in } \\
\text { biblioteca prevedono l'uso di tali } \\
\text { pubblicazioni? }\end{array}$ & 97 & 98 & $0 \%$ & $25 \%$ & $50 \%$ & $75 \%$ & $100 \%$ \\
\hline $\begin{array}{l}\text { Qual è il suo grado di soddisfazione per } \\
\text { il modo in cui la biblioteca mette a } \\
\text { disposizione questo materiale? }\end{array}$ & 97 & 98 & 1 & 2 & 3 & 4 & 5 \\
\hline
\end{tabular}


APPENDICE B

1.13 - Banche dati: modo in cui sono messe a disposizione del pubblico

\begin{tabular}{|l|c|c|c|c|c|c|c|}
\hline $\begin{array}{l}\text { Quanto è importante in relazione alle } \\
\text { sue esigenze di studio e ricerca? }\end{array}$ & 97 & 98 & 1 & 2 & 3 & 4 & 5 \\
\hline $\begin{array}{l}\text { Qual è il suo grado di soddisfazione per } \\
\text { il modo in cui la biblioteca mette le } \\
\text { Banche Dati a disposizione del } \\
\text { pubblico? }\end{array}$ & 97 & 98 & 1 & 2 & 3 & 4 & 5 \\
\hline
\end{tabular}

1.14 - Comunicazione con il personale delle biblioteche (disponibilità e cortesia dei bibliotecari nei confronti del pubblico)

\begin{tabular}{|l|c|c|c|c|c|c|c|}
\hline $\begin{array}{l}\text { Quanto è importante in relazione alle } \\
\text { sue esigenze di studio e ricerca? }\end{array}$ & 97 & 98 & 1 & 2 & 3 & 4 & 5 \\
\hline $\begin{array}{l}\text { In quale percentuale delle sue visite in } \\
\text { biblioteca ha contatti diretti con } i \\
\text { bibliotecari? }\end{array}$ & 97 & 98 & $0 \%$ & $25 \%$ & $50 \%$ & $75 \%$ & $100 \%$ \\
\hline $\begin{array}{l}\text { Qual è il suo grado di soddisfazione } \\
\text { al proposito? }\end{array}$ & 97 & 98 & 1 & 2 & 3 & 4 & 5 \\
\hline
\end{tabular}

1.15 - Servizi e assistenza personalizzata al lettore da parte del bibliotecario

\begin{tabular}{|l|c|c|c|c|c|c|c|}
\hline $\begin{array}{l}\text { Quanto è importante in relazione alle } \\
\text { sue esigenze di studio e ricerca? }\end{array}$ & 97 & 98 & 1 & 2 & 3 & 4 & 5 \\
\hline $\begin{array}{l}\text { Quando deve effettuare ricerche } \\
\text { complesse, in che percentuale si rivolge } \\
\text { al bibliotecario per un servizio } \\
\text { personalizzato? }\end{array}$ & 97 & 98 & $0 \%$ & $25 \%$ & $50 \%$ & $75 \%$ & $100 \%$ \\
\hline $\begin{array}{l}\text { Qual è il suo grado di soddisfazione per } \\
\text { la consulenza? }\end{array}$ & 97 & 98 & 1 & 2 & 3 & 4 & 5 \\
\hline
\end{tabular}

1.16 - Depliant sulle biblioteche e segnaletica in biblioteca per facilitare l'uso dei servizi

\begin{tabular}{|l|c|c|c|c|c|c|c|}
\hline $\begin{array}{l}\text { Quanto è importante in relazione alle } \\
\text { sue esigenze di studio e ricerca? }\end{array}$ & 97 & 98 & 1 & 2 & 3 & 4 & 5 \\
\hline $\begin{array}{l}\text { In quale percentuale delle sue visite in } \\
\text { biblioteca usa, anche in modo rapido, } \\
\text { questi strumenti? }\end{array}$ & 97 & 98 & $0 \%$ & $25 \%$ & $50 \%$ & $75 \%$ & $100 \%$ \\
\hline $\begin{array}{l}\text { Qual è il suo grado di soddisfazione al } \\
\text { proposito? }\end{array}$ & 97 & 98 & 1 & 2 & 3 & 4 & 5 \\
\hline
\end{tabular}


Le successive valutazioni dalla domanda 1.17 alla domanda 1.25 riguardano il Sistema Bibliotecario nel suo complesso

1.17 Lei utilizza il sito web del Sistema Bibliotecario di Ateneo?

$\mathrm{Si}$

No $\square$ FILTRO: Passare a domanda 1.26

1.18 - Uso del catalogo della biblioteca su internet: ricerca di libri o periodici per titolo e ricerca di libri per autore

\begin{tabular}{|l|c|c|c|c|c|c|c|}
\hline $\begin{array}{l}\text { Quanto è importante in relazione alle } \\
\text { sue esigenze di studio e ricerca? }\end{array}$ & 97 & 98 & 1 & 2 & 3 & 4 & 5 \\
\hline $\begin{array}{l}\text { Su 10 consultazioni del catalogo, quante } \\
\text { volte usa questa opzione di ricerca? }\end{array}$ & 97 & 98 & $0 \%$ & $25 \%$ & $50 \%$ & $75 \%$ & $100 \%$ \\
\hline $\begin{array}{l}\text { Qual è il suo grado di soddisfazione } \\
\text { al proposito? }\end{array}$ & 97 & 98 & 1 & 2 & 3 & 4 & 5 \\
\hline
\end{tabular}

1.19 - Uso del catalogo su internet: ricerca di libri per argomento (nel campo: soggetto) o materia (nel campo: classificazione)

\begin{tabular}{|l|c|c|c|c|c|c|c|}
\hline $\begin{array}{l}\text { Quanto è importante in relazione alle } \\
\text { sue esigenze di studio e ricerca? }\end{array}$ & 97 & 98 & 1 & 2 & 3 & 4 & 5 \\
\hline $\begin{array}{l}\text { Su 10 consultazioni del catalogo, quante } \\
\text { volte usa questa opzione di ricerca? }\end{array}$ & 97 & 98 & $0 \%$ & $25 \%$ & $50 \%$ & $75 \%$ & $100 \%$ \\
\hline $\begin{array}{l}\text { Qual è il suo grado di soddisfazione } \\
\text { al proposito? }\end{array}$ & 97 & 98 & 1 & 2 & 3 & 4 & 5 \\
\hline
\end{tabular}

1.20 - Uso del catalogo della biblioteca su internet: ricerca di libri o periodici nel campo parole chiave

\begin{tabular}{|l|c|c|c|c|c|c|c|}
\hline $\begin{array}{l}\text { Quanto è importante in relazione alle } \\
\text { sue esigenze di studio e ricerca? }\end{array}$ & 97 & 98 & 1 & 2 & 3 & 4 & 5 \\
\hline $\begin{array}{l}\text { Su 10 consultazioni del catalogo, quante } \\
\text { volte usa questa opzione di ricerca? }\end{array}$ & 97 & 98 & $0 \%$ & $25 \%$ & $50 \%$ & $75 \%$ & $100 \%$ \\
\hline $\begin{array}{l}\text { Qual è il suo grado di soddisfazione } \\
\text { al proposito? }\end{array}$ & 97 & 98 & 1 & 2 & 3 & 4 & 5 \\
\hline
\end{tabular}




\section{Appendice B}

1.21 - Uso del catalogo della biblioteca su internet: prenotazione o proroga del prestito di un libro

\begin{tabular}{|l|c|c|c|c|c|c|c|}
\hline $\begin{array}{l}\text { Quanto è importante in relazione alle } \\
\text { sue esigenze di studio e ricerca? }\end{array}$ & 97 & 98 & 1 & 2 & 3 & 4 & 5 \\
\hline $\begin{array}{l}\text { Quando deve rinnovare un prestito o } \\
\text { prenotare un libro, quante volte usa la } \\
\text { procedura su web? }\end{array}$ & 97 & 98 & $0 \%$ & $25 \%$ & $50 \%$ & $75 \%$ & $100 \%$ \\
\hline $\begin{array}{l}\text { Qual è il suo grado di soddisfazione } \\
\text { al proposito? }\end{array}$ & 97 & 98 & 1 & 2 & 3 & 4 & 5 \\
\hline
\end{tabular}

1.22 - Banche dati attualmente disponibili nelle biblioteche (via web, su cd-rom)

\begin{tabular}{|l|c|c|c|c|c|c|c|}
\hline $\begin{array}{l}\text { Quanto sono importanti le banche dati } \\
\text { in relazione alle sue esigenze di studio } \\
\text { e ricerca? }\end{array}$ & 97 & 98 & 1 & 2 & 3 & 4 & 5 \\
\hline $\begin{array}{l}\text { In quale percentuale le sue visite in } \\
\text { biblioteca (o i contatti col sito web della } \\
\text { biblioteca) prevedono l'uso di tali } \\
\text { risorse? }\end{array}$ & 97 & 98 & $0 \%$ & $25 \%$ & $50 \%$ & $75 \%$ & $100 \%$ \\
\hline $\begin{array}{l}\text { Qual è il suo grado di soddisfazione per } \\
\text { le materie che le banche dati trattano? }\end{array}$ & 97 & 98 & 1 & 2 & 3 & 4 & 5 \\
\hline
\end{tabular}

1.23 - Risultati ottenuti dall'interrogazione delle banche dati

\begin{tabular}{|l|c|c|c|c|c|c|c|}
\hline $\begin{array}{l}\text { Quanto sono importanti in relazione alle } \\
\text { sue esigenze di studio e ricerca? }\end{array}$ & 97 & 98 & 1 & 2 & 3 & 4 & 5 \\
\hline $\begin{array}{l}\text { Qual è il suo grado di soddisfazione per } \\
\text { le indicazioni bibliografiche tratte della } \\
\text { consultazione delle banche dati? }\end{array}$ & 97 & 98 & 1 & 2 & 3 & 4 & 5 \\
\hline
\end{tabular}

1.24 - Uso dei periodici elettronici nelle discipline di suo interesse

\begin{tabular}{|l|c|c|c|c|c|c|c|}
\hline $\begin{array}{l}\text { Quanto è importante in relazione alle } \\
\text { sueesigenze di studio e ricerca? }\end{array}$ & 97 & 98 & 1 & 2 & 3 & 4 & 5 \\
\hline $\begin{array}{l}\text { Quando deve consultare un periodico, } \\
\text { in quale percentuale usa la versione } \\
\text { elettronica che l'università rende } \\
\text { disponibile dai suoi computer? }\end{array}$ & 97 & 98 & $0 \%$ & $25 \%$ & $50 \%$ & $75 \%$ & $100 \%$ \\
\hline $\begin{array}{l}\text { Qual è il suo grado di soddisfazione } \\
\text { al proposito? }\end{array}$ & 97 & 98 & 1 & 2 & 3 & 4 & 5 \\
\hline
\end{tabular}


1.25 - Uso sito web del Sistema Bibliotecario di Ateneo

\begin{tabular}{|l|c|c|c|c|c|c|c|}
\hline $\begin{array}{l}\text { Quanto è importante in relazione alle } \\
\text { sue esigenze di studio e ricerca? }\end{array}$ & 97 & 98 & 1 & 2 & 3 & 4 & 5 \\
\hline $\begin{array}{l}\text { Con quale percentuale, rispetto ai } \\
\text { contatti che ha con l'università per } \\
\text { acquisire informazioni sulle biblioteche, } \\
\text { usa il sito internet del Sistema } \\
\text { Bibliotecario di Ateneo? }\end{array}$ & 97 & 98 & $0 \%$ & $25 \%$ & $50 \%$ & $75 \%$ & $100 \%$ \\
\hline $\begin{array}{l}\text { Qual è il suo grado di soddisfazione al } \\
\text { proposito? }\end{array}$ & 97 & 98 & 1 & 2 & 3 & 4 & 5 \\
\hline
\end{tabular}

Adesso le faremo alcune domande di carattere personale. Queste domande possono essere utili a capire meglio il profilo degli utenti del Sistema Bibliotecario di Ateneo. La preghiamo di rispondere ricordandole il completo anonimato.

1.26 - Sesso del rispondente

Maschio

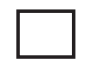

Femmina

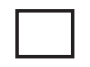

1.27 - Lei risiede nel comune di Firenze?

Sì $\square$ FILTRO: Passare a domanda 1.30

No $\square$ FILTRO: Passare a domanda 1.28

1.28 - Qual è la sua provincia di residenza?

1.29 - Lei è domiciliato a Firenze?

Sì

No 
APPENDICE B

1.30 - Qual è il suo anno di nascita? $\square$

Fine del questionario

Il Sistema Bibliotecario di Ateneo la ringrazia per la cortese collaborazione 


\section{Appendice C}

\section{Spoglio statistico dei dati del Sistema Bibliotecario di Ateneo}

Gli spogli dei quesiti sono riferiti alla biblioteca di area di cui l'intervista-

to risulta utente. I dati campionari sono stati riportati all'universo costituito dagli utenti unici delle biblioteche di area 
Libri, periodici e cd-rom attualmente posseduti dalla biblioteca (di cui risulti utente).

Quanto sono importanti in relazione alle tue esigenze di studio e ricerca?

\begin{tabular}{|c|c|c|c|c|}
\hline QU02A1 & Frequenze & Percentuali & $\begin{array}{l}\text { Frequenze } \\
\text { cumulate }\end{array}$ & $\begin{array}{l}\text { Percentual } \\
\text { cumulate }\end{array}$ \\
\hline Per nulla importante & $\ldots 605$ & 2.18 & .605 & 2.18 \\
\hline Poco importante . . & $\ldots 1108$ & 3.99 & 1713 & .6 .17 \\
\hline Indifferente $\ldots \ldots$ & . . .5548 & .19 .99 & .7261 & 26.17 \\
\hline Importante $\ldots . .$. & .10721 & .38 .64 & 17982 & 64.81 \\
\hline Molto importante & . . 9462 & .34 .10 & 27444 & .98 .91 \\
\hline Non sa $\ldots . . \ldots$. & $\ldots 303$ & . . 1.09 & 27747 & .100 .00 \\
\hline
\end{tabular}

Libri, periodici e cd-rom attualmente posseduti dalla biblioteca (di cui risulti utente).

In quale percentuale le tue visite in biblioteca sono dedicate principalmente alla consultazione o al prestito di tale materiale?

\begin{tabular}{|c|c|c|c|c|}
\hline QU02B1 & Frequenze & Percentuali & $\begin{array}{l}\text { Frequenze } \\
\text { cumulate }\end{array}$ & $\begin{array}{l}\text { Percentuali } \\
\text { cumulate }\end{array}$ \\
\hline $0 \%$ & 2345 & .8 .45 & 2345 & 8.45 \\
\hline $25 \%$ & .7290 & .26 .27 & 9635 & 34.73 \\
\hline $50 \%$ &. .7131 & .25 .70 & 16766 & . 60.43 \\
\hline $75 \%$ & .6265 & .22 .58 & .23031 & . 83.01 \\
\hline $100 \%$ & .4614 & .16 .63 & .27645 & 99.64 \\
\hline Non sa .. & $\ldots 101$ & $\ldots 0.36$ & .27746 & .100 .00 \\
\hline
\end{tabular}

Libri, periodici e cd-rom attualmente posseduti dalla biblioteca (di cui risulti utente).

Qual è il tuo grado di soddisfazione per l'offerta documentaria della biblioteca?

\begin{tabular}{|c|c|c|c|c|}
\hline QU02C1 & Frequenze & Percentuali & $\begin{array}{c}\text { Frequenze } \\
\text { cumulate }\end{array}$ & $\begin{array}{l}\text { Percentuali } \\
\text { cumulate }\end{array}$ \\
\hline Insoddisfatto & 315 & 1.24 & .315 & 1.24 \\
\hline Poco insoddisfatto & .2574 & .10 .13 & 2889 & .11 .37 \\
\hline Indifferente . & . 6895 & .27 .14 & .9784 & 38.52 \\
\hline Abbastanza soddisfatto & .11421 & . . 44.96 & 21205 & .83 .48 \\
\hline Soddisfatto ...... & . . .4040 & .15 .90 & 25245 & . . 99.39 \\
\hline Non sa & .156 & $\ldots 0.61$ & 25401 & .100 .00 \\
\hline
\end{tabular}

Dati Mancanti $=2345$ 
Disponibilità in biblioteca delle novità librarie nelle discipline di tuo interesse.

Quanto è importante in relazione alle tue esigenze di studio e ricerca?

\begin{tabular}{|c|c|c|c|c|}
\hline QU02A2 & Frequenze & Percentuali & $\begin{array}{c}\text { Frequenze } \\
\text { cumulate }\end{array}$ & $\begin{array}{l}\text { Percentuali } \\
\text { cumulate }\end{array}$ \\
\hline Per nulla importante & 1497 & 5.40 & 1497 & 5.40 \\
\hline Poco importante & .2144 & 7.73 & 3641 & .13 .12 \\
\hline Indifferente $\ldots$ & .5449 & .19 .64 & 9090 & 32.76 \\
\hline Importante $\ldots$ & $\ldots 6567$ & .23 .67 & .15657 & $\ldots 56.43$ \\
\hline Molto importante & .10062 & . . 36.26 & . . 25719 & . 92.69 \\
\hline Non sa $\ldots . . .$. & .2028 & $\ldots 7.31$ & . . 27747 & .100 .00 \\
\hline
\end{tabular}

Disponibilità in biblioteca delle novità librarie nelle discipline di tuo interesse.

In quale percentuale le tue visite in biblioteca sono dedicate principalmente alla consultazione delle novità librarie?

\begin{tabular}{|c|c|c|c|c|}
\hline QU02B2 & Frequenze & Percentuali & $\begin{array}{c}\text { Frequenze } \\
\text { cumulate }\end{array}$ & $\begin{array}{l}\text { Percentuali } \\
\text { cumulate }\end{array}$ \\
\hline $0 \%$ & .8243 & 29.71 & .8243 & .29 .71 \\
\hline $25 \%$ & .6419 & .23 .13 . & .14662 & . . 52.84 \\
\hline $50 \%$ & . 6403 & .23 .08 & .21065 & . 75.92 \\
\hline $75 \%$ & $\ldots 4337$ & .15 .63$. & .25402 . & $\ldots 91.55$ \\
\hline $100 \%$ & . . 1423 & $\ldots 5.13$ & . . 26825. & . . 969.68 \\
\hline Non sa & $\ldots 921$ & $\ldots 3.32$. & .27746 & . . 100.00 \\
\hline
\end{tabular}

Disponibilità in biblioteca delle novità librarie nelle discipline di tuo interesse.

Qual è il tuo grado di soddisfazione al proposito?

\begin{tabular}{|c|c|c|c|c|}
\hline QU02C2 & Frequenze & Percentuali & $\begin{array}{c}\text { Frequenze } \\
\text { cumulate }\end{array}$ & $\begin{array}{c}\text { Percentuali } \\
\text { cumulate }\end{array}$ \\
\hline Insoddisfatto & .1014 & .5 .20 & 1014 & .5 .20 \\
\hline Poco insoddisfatto & .3593 & .18 .42 & 4607 & 23.62 \\
\hline Indifferente $\ldots$. & . 6207 & 31.83 & .10814 & 55.45 \\
\hline Abbastanza soddisfatto & .5508 & .28 .24 & .16322 & .83 .69 \\
\hline Soddisfatto & .2244 & .11 .51 & . 18566 & .95 .20 \\
\hline Non sa ... & $\ldots 937$ & $\ldots 4.80$ & .19503 & .100 .00 \\
\hline
\end{tabular}

Dati Mancanti $=8243$ 
Disponibilità in biblioteca degli ultimi numeri dei periodici nelle discipline di tuo interesse. Quanto è importante in relazione alle tue esigenze di studio e ricerca?

\begin{tabular}{|c|c|c|c|c|}
\hline QU02A3 & Frequenze & Percentuali & $\begin{array}{l}\text { Frequenze } \\
\text { cumulate }\end{array}$ & $\begin{array}{l}\text { Percentuali } \\
\text { cumulate }\end{array}$ \\
\hline Per nulla importante & 2604 & .9 .39 & 2604 & .9 .39 \\
\hline Poco importante & .1894 & .6 .83 & 4498 & .16 .21 \\
\hline Indifferente $\ldots$ & .4359 & .15 .71 & $\ldots 8857$ & .31 .92 \\
\hline Importante $\ldots$. & .6027 & .21 .72 & .14884 & 53.65 \\
\hline Molto importante & .9263 & 33.39 & .24147 & .87 .03 \\
\hline Non sa $\ldots \ldots \ldots$ & .3598 & 12.97 & .27745 & 100.00 \\
\hline
\end{tabular}

Disponibilità in biblioteca degli ultimi numeri dei periodici nelle discipline di tuo interesse. In quale percentuale le tue visite in biblioteca sono dedicate principalmente alla consultazione degli ultimi numeri dei periodici?

\begin{tabular}{|c|c|c|c|c|}
\hline QU02B3 & Frequenze & Percentuali & $\begin{array}{l}\text { Frequenze } \\
\text { cumulate }\end{array}$ & $\begin{array}{l}\text { Percentuali } \\
\text { cumulate }\end{array}$ \\
\hline $0 \%$ & 12305 & 44.35 & .12305 & 44.35 \\
\hline $25 \%$ & 4827 & 17.40 & .17132 & 61.75 \\
\hline $50 \%$ & . 4962 . & 17.88 & . . 22094 & 79.63 \\
\hline $75 \%$ & 2913 . & .10 .50 & . . 25007 & 90.13 \\
\hline $100 \%$ & .1455 . & $\ldots 5.24$ & . . 26462 & . .95.38 \\
\hline Non sa $\ldots$ & 1283 & .. 4.62 & $\ldots .27745$ & . . 100.00 \\
\hline
\end{tabular}

Disponibilità in biblioteca degli ultimi numeri dei periodici nelle discipline di tuo interesse. Qual è il tuo grado di soddisfazione al proposito?

\begin{tabular}{|c|c|c|c|c|}
\hline QU02C3 & Frequenze & Percentuali & $\begin{array}{c}\text { Frequenze } \\
\text { cumulate }\end{array}$ & $\begin{array}{l}\text { Percentuali } \\
\text { cumulate }\end{array}$ \\
\hline Insoddisfatto & .784 & .5 .08 & .784 & 5.08 \\
\hline Poco insoddisfatto & $\ldots 1781$ & $\ldots 11.53$ & .2565 & 16.61 \\
\hline Indifferente $\ldots .$. & .5020 & .32 .51 & .7585 & 49.12 \\
\hline Abbastanza soddisfatto & .4991 & .32 .32 & .12576 & .81 .44 \\
\hline Soddisfatto . . & 1685 & . . 10.91 & .14261 & .92 .35 \\
\hline Non sa ... & .1181 & $\ldots 7.65$ & 15442 & .100 .00 \\
\hline
\end{tabular}

Dati Mancanti $=12305$ 
Precisione e rapidità nella distribuzione di libri e periodici dai magazzini.

Quanto è importante in relazione alle tue esigenze di studio e ricerca?

\begin{tabular}{|c|c|c|c|c|}
\hline QU02A4 & Frequenze & Percentuali & $\begin{array}{l}\text { Frequenze } \\
\text { cumulate }\end{array}$ & $\begin{array}{l}\text { Percentuali } \\
\text { cumulate }\end{array}$ \\
\hline Per nulla importante & .1259 & 4.54 & .1259 & 4.54 \\
\hline Poco importante & .1167 & 4.21 & 2426 & 8.74 \\
\hline Indifferente $\ldots$ & .4384 & $\ldots 15.80$ & . 6810 & .24 .54 \\
\hline Importante $\ldots \ldots$ & $\ldots 7411$ & $\ldots .26 .71$ & $\ldots 14221$ & $\ldots .51 .25$ \\
\hline Molto importante .. & $\ldots 11601$ & .41 .81 & $\ldots 25822$ & $\ldots 93.07$ \\
\hline Non sa $\ldots . .$. & .1924 & $\ldots 6.93$ & . . 27746 & . . 100.00 \\
\hline
\end{tabular}

Precisione e rapidità nella distribuzione di libri e periodici dai magazzini.

In quale percentuale le tue visite in biblioteca prevedono l'uso di materiali collocati in magazzino?

\begin{tabular}{|c|c|c|c|c|}
\hline QU02B4 & Frequenze & Percentuali & $\begin{array}{c}\text { Frequenze } \\
\text { cumulate }\end{array}$ & $\begin{array}{l}\text { Percentuali } \\
\text { cumulate }\end{array}$ \\
\hline $0 \%$ & .6823 & .24 .59 & . 6823 & . . 24.59 \\
\hline $25 \%$ & .5051 & .18 .20 & .11874 & . . 42.80 \\
\hline $50 \%$ & . 6259 & . . 22.56 & 18133 & $\ldots 65.35$ \\
\hline $75 \%$ & .5344 & .19 .26 & 23477 & $\ldots 84.61$ \\
\hline $100 \%$ & . .3239 & .11 .67 & . . 26716 & . . 96.29 \\
\hline Non sa & . 1030 & $\ldots 3.71$ & .27746 & . . 100.00 \\
\hline
\end{tabular}

Precisione e rapidità nella distribuzione di libri e periodici dai magazzini. Qual è il tuo grado di soddisfazione al proposito?

\begin{tabular}{|c|c|c|c|c|}
\hline QU02C4 & Frequenze & Percentuali & $\begin{array}{c}\text { Frequenze } \\
\text { cumulate }\end{array}$ & $\begin{array}{l}\text { Percentuali } \\
\text { cumulate }\end{array}$ \\
\hline Insoddisfatto & .852 & 4.07 & .852 & .4 .07 \\
\hline Poco insoddisfatto & .2535 & .12 .12 & 3387 & .16 .19 \\
\hline Indifferente $\ldots$ & .5806 & .27 .75 & 9193 & .43 .94 \\
\hline Abbastanza soddisfatto & . .6597 & .31 .53 & 15790 & .75 .47 \\
\hline Soddisfatto & 4387 & 20.97 & 20177 & .96 .44 \\
\hline Non sa $\ldots .$. & .745 & . . 3.56 & 20922 & 100.00 \\
\hline
\end{tabular}

Dati Mancanti $=6823$ 
Facilità del recupero di libri e periodici dagli scaffali liberamente accessibili ai lettori.

Quanto è importante in relazione alle tue esigenze di studio e ricerca?

\begin{tabular}{|c|c|c|c|c|}
\hline QU02A5 & Frequenze & Percentuali & $\begin{array}{c}\text { Frequenze } \\
\text { cumulate }\end{array}$ & $\begin{array}{l}\text { Percentuali } \\
\text { cumulate }\end{array}$ \\
\hline Per nulla importante & .784 & 2.83 & $\ldots .784$ & 2.83 \\
\hline Poco importante . . & . . 1191 & $\ldots 4.29$ & . 1975 & 7.12 \\
\hline Indifferente $\ldots$ & $\ldots 2931$ & $\ldots 10.56$ & . . 4906 & .17 .68 \\
\hline Importante ... & $\ldots 7848$ & .28 .28 & $\ldots 12754$ & .45 .97 \\
\hline Molto importante & .14064 & $\ldots 50.69$ & . . 26818 & .96 .65 \\
\hline Non sa & . . .929 & $\ldots 3.35$ & .27747 & 100.00 \\
\hline
\end{tabular}

Facilità del recupero di libri e periodici dagli scaffali liberamente accessibili ai lettori. In quale percentuale le tue visite in biblioteca prevedono l'uso di materiali collocati a scaffale aperto?

\begin{tabular}{|c|c|c|c|c|}
\hline QU02B5 & Frequenze & Percentuali & $\begin{array}{c}\text { Frequenze } \\
\text { cumulate }\end{array}$ & $\begin{array}{l}\text { Percentuali } \\
\text { cumulate }\end{array}$ \\
\hline $0 \%$ & .3365 & . .12.13 & . . 3365 & .12 .13 \\
\hline $25 \%$ & . . 4169 & .15 .03 & $\ldots 7534$ & .27 .15 \\
\hline $50 \%$ & . . 6486 & . .23.38 & 14020 & .50 .53 \\
\hline $75 \%$ & $\ldots 7851$ & . . 28.30 & . . 21871 & $\ldots 78.83$ \\
\hline $100 \%$ &. .5244 & . 18.90 & .27115 & . .97.73 \\
\hline Non sa & $\ldots 631$ & . . 2.27 & 27746 & 100.00 \\
\hline
\end{tabular}

Facilità del recupero di libri e periodici dagli scaffali liberamente accessibili ai lettori. Qual è il tuo grado di soddisfazione al proposito?

\begin{tabular}{|c|c|c|c|c|}
\hline QU02C5 & Frequenze & Percentuali & $\begin{array}{c}\text { Frequenze } \\
\text { cumulate }\end{array}$ & $\begin{array}{l}\text { Percentuali } \\
\text { cumulate }\end{array}$ \\
\hline Insoddisfatto & .1615 & .6 .62 &. .1615 & .6 .62 \\
\hline Poco insoddisfatto & 4025 & $\ldots 16.51$ & . . 5640 & 23.13 \\
\hline Indifferente $\ldots$. & $\ldots 5717$ & $\ldots 23.45$ & . 11357 & 46.58 \\
\hline Abbastanza soddisfatto & .8123 & . . 33.32 & . .19480 & 79.90 \\
\hline Soddisfatto $\ldots . \ldots \ldots$ & 4492 & $\ldots 18.42$ & . . 23972 & 98.33 \\
\hline Non sa $\ldots$ & . . 408 & $\ldots 1.67$ & .24380 & 100.00 \\
\hline
\end{tabular}

Dati Mancanti $=3365$ 
Disponibilità in sala di lettura di posti a sedere per l'uso di materiale della biblioteca.

Quanto è importante in relazione alle tue esigenze di studio e ricerca?

\begin{tabular}{cccc} 
QU03A6 Frequenze & Percentuali & $\begin{array}{c}\text { Frequenze } \\
\text { cumulate }\end{array}$ & $\begin{array}{c}\text { Percentuali } \\
\text { cumulate }\end{array}$ \\
\hline
\end{tabular}

\begin{tabular}{|c|c|c|c|}
\hline Per nulla importante $\ldots . .$. & 2.63 & 731 & 2.63 \\
\hline Poco importante & 4.34 & 1934 & 5.97 \\
\hline 3621 & 13.05 & 5555 & .02 \\
\hline .6328 & 22.81 & .11883 & .42 .83 \\
\hline 15300 & 55.14 & .27183 & .97 .97 \\
\hline Jon sa & .2 .03 & .27747 & 100.00 \\
\hline
\end{tabular}

Disponibilità in sala di lettura di posti a sedere per l'uso di materiale della biblioteca. In quale percentuale le tue visite in biblioteca prevedono l'uso della sala per la consultazione di materiale della biblioteca?

\begin{tabular}{|c|c|c|c|c|}
\hline QU03B6 & Frequenze & Percentuali & $\begin{array}{c}\text { Frequenze } \\
\text { cumulate }\end{array}$ & $\begin{array}{l}\text { Percentuali } \\
\text { cumulate }\end{array}$ \\
\hline $0 \%$ & 4224 & .15 .22 & . . 4224 & .15 .22 \\
\hline $25 \%$ & .5076 & .18 .29 & 9300 & .33 .52 \\
\hline $50 \%$ & . 6355 & . . 22.90 & .15655 & .56 .42 \\
\hline $75 \%$ & .6301 & .22 .71 & . . 21956 & $\ldots 79.13$ \\
\hline $100 \%$ &. .5412 & .19 .51 & . . 27368 & . .98.64 \\
\hline Non sa & . 378 & $\ldots 1.36$ & .27746 & 100.00 \\
\hline
\end{tabular}

Disponibilità in sala di lettura di posti a sedere per l'uso di materiale della biblioteca Qual è il tuo grado di soddisfazione al proposito?

\begin{tabular}{|c|c|c|c|c|}
\hline QU03C6 & Frequenze & Percentuali & $\begin{array}{c}\text { Frequenze } \\
\text { cumulate }\end{array}$ & $\begin{array}{l}\text { Percentuali } \\
\text { cumulate }\end{array}$ \\
\hline Insoddisfatto & .2513 & 10.68 & 2513 & .10 .68 \\
\hline Poco insoddisfatto & .5523 & 23.48 & .8036 & $\ldots 34.16$ \\
\hline Indifferente $\ldots$ & . . 5895 & 25.06 & 13931 & $\ldots 59.23$ \\
\hline Abbastanza soddisfatto & . .5967 & 25.37 & 19898 & $\ldots 84.59$ \\
\hline Soddisfatto $\ldots . . \ldots$ & . . 3211 & .13 .65 & 23109 & . . 98.24 \\
\hline Non sa $\ldots .$. & $\ldots 413$ & .1 .76 & 23522 & .100 .00 \\
\hline
\end{tabular}

Dati Mancanti $=4224$ 
Silenzio in sala di lettura.

Quanto è importante in relazione alle tue esigenze di studio e ricerca?

\begin{tabular}{|c|c|c|c|c|}
\hline QU03A7 & Frequenze & Percentuali & $\begin{array}{c}\text { Frequenze } \\
\text { cumulate }\end{array}$ & $\begin{array}{l}\text { Percentuali } \\
\text { cumulate }\end{array}$ \\
\hline Per nulla importante & .202 & 0.86 & .202 & 0.86 \\
\hline Poco importante .. & $\ldots 554$ & 2.36 & .756 & 3.21 \\
\hline Indifferente $\ldots$ & .2257 & .9 .60 & . . 3013 & .12 .81 \\
\hline Importante $\ldots . .$. & $\ldots .5444$ & .23 .14 & $\ldots 8457$ & .35 .95 \\
\hline Molto importante & .14823 & . .63.02 & . . 23280 & .98 .97 \\
\hline Non sa $\ldots \ldots \ldots$ & $\ldots 242$ & $\ldots 1.03$ & .23522 & 100.00 \\
\hline
\end{tabular}

Dati Mancanti $=4224$

Silenzio in sala di lettura.

In che percentuale delle tue visite in biblioteca hai trovato silenziose le sale di lettura?

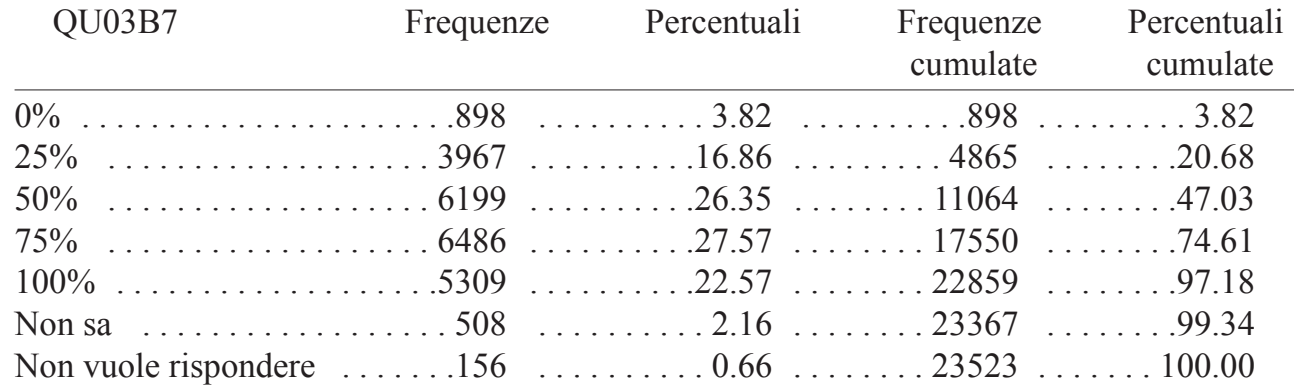

Dati Mancanti $=4224$

Silenzio in sala di lettura.

Qual è il tuo grado di soddisfazione al proposito?

\begin{tabular}{|c|c|c|c|c|}
\hline QU03C7 & Frequenze & Percentuali & $\begin{array}{c}\text { Frequenze } \\
\text { cumulate }\end{array}$ & $\begin{array}{c}\text { Percentuali } \\
\text { cumulate }\end{array}$ \\
\hline Insoddisfatto & .1435 & 6.34 & 1435 & 6.34 \\
\hline Poco insoddisfatto & .2727 & .12 .05 & 4162 & .18 .39 \\
\hline Indifferente & . 6030 & .26 .65 & 10192 & .45 .05 \\
\hline Abbastanza soddisfatto & . .6889 & .30 .45 & .17081 & .75 .49 \\
\hline Soddisfatto $\ldots \ldots$ & .5099 & .22 .54 & 22180 & .98 .03 \\
\hline Non sa . . & . . 446 & . . 1.97 & .22626 & .100 .00 \\
\hline
\end{tabular}

Dati Mancanti $=5121$ 
Comfort e luminosità della sala di lettura.

Quanto è importante in relazione alle tue esigenze di studio e ricerca?

\begin{tabular}{|c|c|c|c|c|}
\hline QU03A8 & Frequenze & Percentuali & $\begin{array}{c}\text { Frequenze } \\
\text { cumulate }\end{array}$ & $\begin{array}{l}\text { Percentuali } \\
\text { cumulate }\end{array}$ \\
\hline Per nulla importante & .176 & 0.75 & .176 & 0.75 \\
\hline Poco importante . . & $\ldots 386$ & 1.64 & .562 & 2.39 \\
\hline Indifferente $\ldots$ & $\ldots 2127$ & . 9.04 & 2689 & .11 .43 \\
\hline Importante .... & .6074 & .25 .82 & .8763 & .37 .25 \\
\hline Molto importante . & .14506 & . .61.67 & 23269 & .98 .92 \\
\hline Non sa $\ldots . .$. & .254 & .1 .08 & 23523 & 100.00 \\
\hline
\end{tabular}

Dati Mancanti $=4224$

Comfort e luminosità della sala di lettura.

In che percentuale delle tue visite in biblioteca hai trovato confortevoli e bene illuminate le sale di lettura?

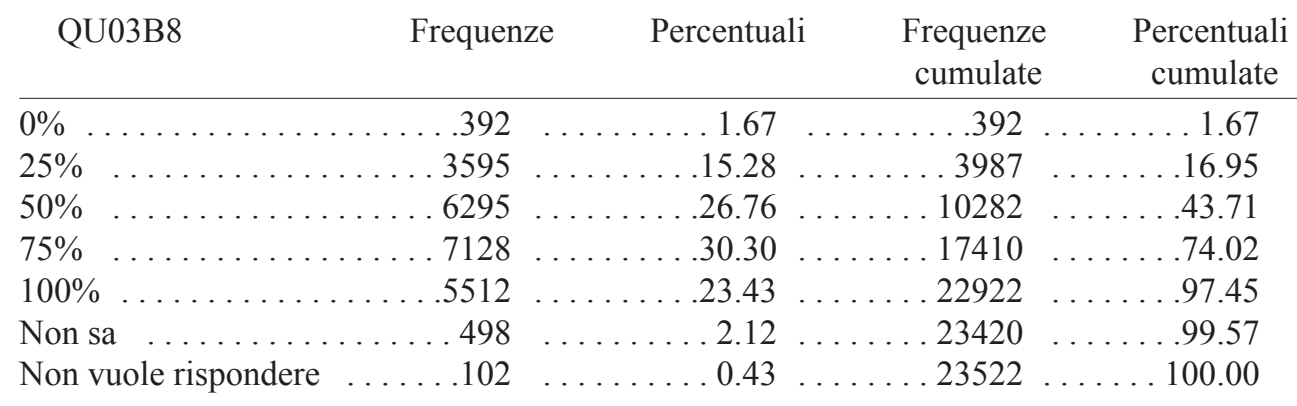

Dati Mancanti $=4224$

Comfort e luminosità della sala di lettura.

Qual è il tuo grado di soddisfazione al proposito?

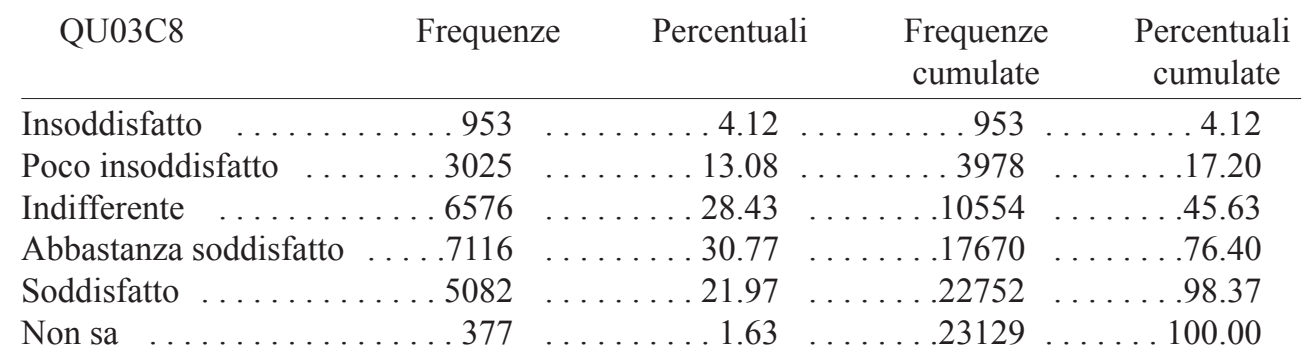

Dati Mancanti $=4616$ 
Accesso a internet in biblioteca.

Quanto è importante in relazione alle tue esigenze di studio e ricerca?

\begin{tabular}{|c|c|c|c|c|}
\hline QU03A9 & Frequenz & Percentuali & $\begin{array}{c}\text { Frequenze } \\
\text { cumulate }\end{array}$ & $\begin{array}{l}\text { Percentuali } \\
\text { cumulate }\end{array}$ \\
\hline Per nulla importante & 1307 & 4.71 & .1307 & 4.71 \\
\hline Poco importante .. & .1276 & .4 .60 & .2583 & 9.31 \\
\hline Indifferente.. & .2333 & $\ldots 8.41$ & $\ldots 4916$ & .17 .72 \\
\hline Importante $\ldots . .$. & $\ldots .5603$. & . . . 20.19 & . 10519 & .37 .91 \\
\hline Molto importante & 16486 & . . . . 59.42 & . . 27005 & .97 .33 \\
\hline Non sa $\ldots . . .$. & $\ldots 742$. & $\ldots \ldots \ldots 2.67$ & . . 27747 & 100.00 \\
\hline
\end{tabular}

Accesso a internet in biblioteca.

In quale percentuale le tue visite in biblioteca prevedono l'accesso a internet per ragioni di studio o ricerca?

\begin{tabular}{|c|c|c|c|c|}
\hline QU03B9 & Frequenze & Percentuali & $\begin{array}{c}\text { Frequenze } \\
\text { cumulate }\end{array}$ & $\begin{array}{l}\text { Percentuali } \\
\text { cumulate }\end{array}$ \\
\hline $0 \%$ & .6666 & .24 .03 & $\ldots 6666$ & .24 .03 \\
\hline $25 \%$ & . . 5352 & .19 .29 & 12018 & .43 .32 \\
\hline $50 \%$ & . . 5019 & . .18.09 & . 17037 & . .61.41 \\
\hline $75 \%$ & . . 5672 & .20 .44 & . . 22709 & .81 .85 \\
\hline $100 \%$ & . .4508 & .16 .25 & .27217 & .98 .10 \\
\hline Non sa. & . . 528 & .1 .90 & .27745 & 100.00 \\
\hline
\end{tabular}

Accesso a internet in biblioteca.

Qual è il tuo grado di soddisfazione al proposito?

\begin{tabular}{|c|c|c|c|c|}
\hline QU03C9 & Frequenze & Percentuali & $\begin{array}{c}\text { Frequenze } \\
\text { cumulate }\end{array}$ & $\begin{array}{l}\text { Percentuali } \\
\text { cumulate }\end{array}$ \\
\hline Insoddisfatto & .3309 & .15 .70 & 3309 & .15 .70 \\
\hline Poco insoddisfatto & $\ldots 5350$ & .25 .38 & 8659 & .41 .08 \\
\hline Indifferente & $\ldots 5182$ & $\ldots .24 .58$ & . . 13841 & $\ldots \ldots 65.66$ \\
\hline Abbastanza soddisfatto & $\ldots 4007$ & $\ldots 19.01$ & . 17848 & $\ldots 84.67$ \\
\hline Soddisfatto $\ldots \ldots \ldots$ & $\ldots 2659$ & .12 .61 & . . 20507 & .97 .28 \\
\hline Non sa $\ldots \ldots \ldots$ & $\ldots 573$ & $\ldots 2.72$ & 21080 & 100.00 \\
\hline
\end{tabular}


Prestito interbibliotecario: recupero di libri e articoli da altre biblioteche universitarie.

Quanto è importante in relazione alle tue esigenze di studio e ricerca?

\begin{tabular}{|c|c|c|c|c|}
\hline QU03A10 & Frequenze & Percentuali & $\begin{array}{c}\text { Frequenze } \\
\text { cumulate }\end{array}$ & $\begin{array}{c}\text { Percentuali } \\
\text { cumulate }\end{array}$ \\
\hline Per nulla importante & 1783 & 6.43 & 1783 & 6.43 \\
\hline Poco importante & . . 1776 & . . 6.40 & . . 3559 & .12 .83 \\
\hline Indifferente $\ldots$ & 4181 & .15 .07 & .7740 & .27 .90 \\
\hline Importante &. .5442 & .19 .61 & . . 13182 & .47 .51 \\
\hline Molto importante & .11679 & .42 .09 & .24861 & .89 .60 \\
\hline Non sa $\ldots . .$. & $\ldots 2885$ & .10 .40 & .27746 & 100.00 \\
\hline
\end{tabular}

Prestito interbibliotecario: recupero di libri e articoli da altre biblioteche universitarie. In quale percentuale i documenti utilizzati nelle tue ricerche ti sono stati forniti tramite il servizio di prestito interbibliotecario?

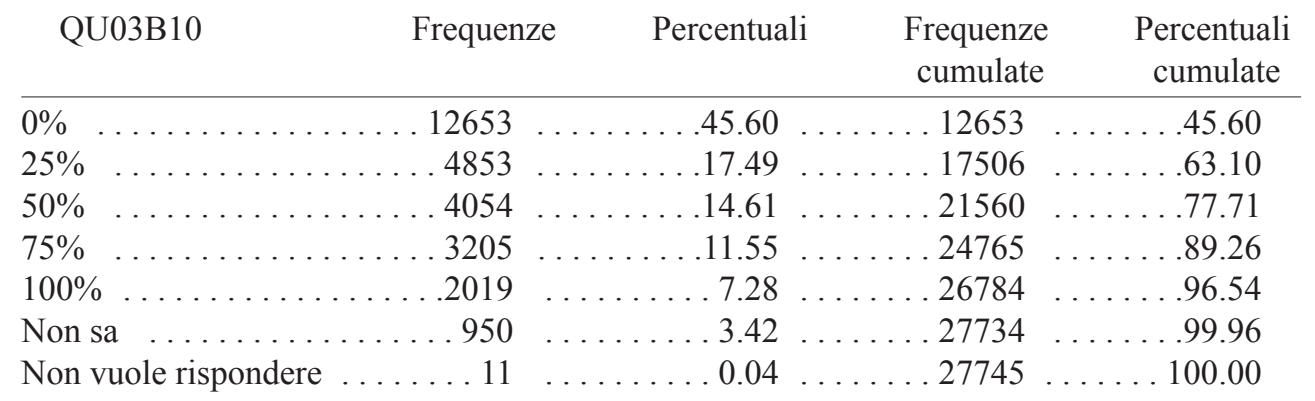

Prestito interbibliotecario: recupero di libri e articoli da altre biblioteche universitarie. Qual è il tuo grado di soddisfazione al proposito?

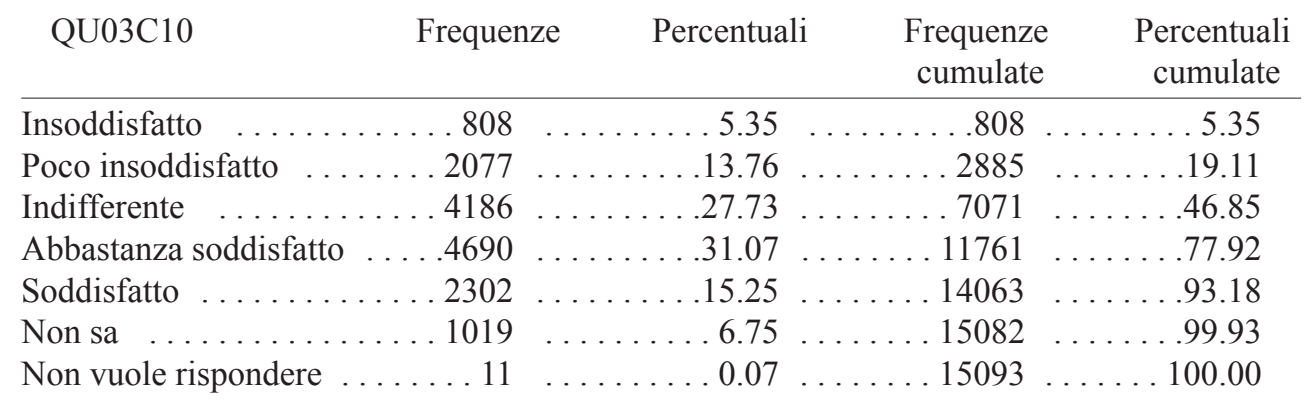

Dati Mancanti $=12653$ 
Facilità del recupero e consultazione di bibliografie, repertori, enciclopedie.

Quanto è importante in relazione alle tue esigenze di studio e ricerca?

\begin{tabular}{|c|c|c|c|}
\hline QU03A11 & Percentuali & $\begin{array}{c}\text { Frequenze } \\
\text { cumulate }\end{array}$ & $\begin{array}{c}\text { Percentuali } \\
\text { cumulate }\end{array}$ \\
\hline Per nulla importante . . . . . 1882 & 6.78 & 1882 & 6.78 \\
\hline Poco importante . . . . . . . . 1912 & 6.89 & 3794 & .13 .67 \\
\hline Indifferente & $\ldots 20.63$ & . 9519 & .34 .31 \\
\hline Importante . . . . . . . . 6683 . & . . 24.09 & . 16202 & .58 .39 \\
\hline Molto importante . . . . . .99109 & . . 32.83 & .25311 & .91 .22 \\
\hline Non sa $\ldots .$. & .8 .74 & .27736 & .99 .96 \\
\hline Non vuole rispondere $\ldots . . .11$ & 0.04 & .27747 & 100.00 \\
\hline
\end{tabular}

Facilità del recupero e consultazione di bibliografie, repertori, enciclopedie.

In quale percentuale le tue visite in biblioteca prevedono l'uso di tali materiali?

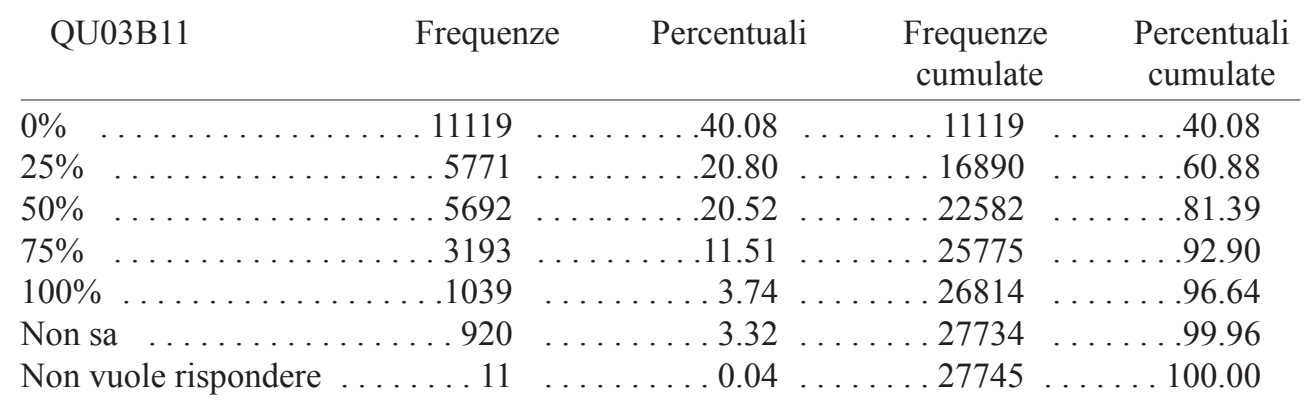

Facilità del recupero e consultazione di bibliografie, repertori, enciclopedie.

Qual è il tuo grado di soddisfazione per il modo in cui la biblioteca mette a disposizione questo materiale?

\begin{tabular}{|c|c|c|c|c|}
\hline QU03C11 & Frequenze & Percentuali & $\begin{array}{c}\text { Frequenze } \\
\text { cumulate }\end{array}$ & $\begin{array}{c}\text { Percentuali } \\
\text { cumulate }\end{array}$ \\
\hline Insoddisfatto & .546 & .3 .28 & $\ldots .546$ & 3.28 \\
\hline Poco insoddisfatto & 2162 & .13 .00 & 2708 & .16 .29 \\
\hline Indifferente $\ldots$ & 6069 & .36 .50 & .8777 & .52 .79 \\
\hline Abbastanza soddisfatto & .5292 & . .31.83 & .14069 & .84 .62 \\
\hline Soddisfatto $\ldots . . \ldots$ & . . 1489 & $\ldots 8.96$ & .15558 & .93 .57 \\
\hline Non sa & .1058 & $\ldots 6.36$ & . . 16616 & . .99.93 \\
\hline Non vuole rispondere & $\ldots 11$ & $\ldots 0.07$ & $\ldots 16627$ & 100.00 \\
\hline
\end{tabular}

Dati Mancanti $=11119$ 
Banche dati: modo in cui sono messe a disposizione del pubblico.

Quanto è importante in relazione alle tue esigenze di studio e ricerca?

\begin{tabular}{|c|c|c|c|}
\hline QU03A12 & Percentuali & $\begin{array}{c}\text { Frequenze } \\
\text { cumulate }\end{array}$ & $\begin{array}{l}\text { Percentuali } \\
\text { cumulate }\end{array}$ \\
\hline Per nulla importante .... & 5.01 & 1389 & 5.01 \\
\hline Poco importante . . & .3 .36 & 2321 & .8 .37 \\
\hline Indifferente $\ldots \ldots \ldots$ & .18 .19 & 7367 & .26 .55 \\
\hline Importante & .25 .96 & .14569 & .52 .51 \\
\hline Molto importante ... & $\ldots 35.51$ & .24422 & .88 .02 \\
\hline Non sa & $\ldots 11.85$ & .27711 & .99 .88 \\
\hline Non vuole rispondere . & 0.12 & .27745 & 100.00 \\
\hline
\end{tabular}

Banche dati: modo in cui sono messe a disposizione del pubblico.

Qual è il tuo grado di soddisfazione per il modo in cui la biblioteca mette a disposizione questo materiale?

\begin{tabular}{|c|c|c|c|c|}
\hline QU03C12 & Frequenze & Percentuali & $\begin{array}{c}\text { Frequenze } \\
\text { cumulate }\end{array}$ & $\begin{array}{c}\text { Percentuali } \\
\text { cumulate }\end{array}$ \\
\hline Insoddisfatto & .1038 & 3.74 & .1038 & .3 .74 \\
\hline Poco insoddisfatto & 2075 & 7.48 & .3113 & .11 .22 \\
\hline Indifferente $\ldots$. & .7139 & .25 .73 & 10252 & .36 .95 \\
\hline Abbastanza soddisfatto & . . 6666 & .24 .03 & . . 16918 & .60 .97 \\
\hline Soddisfatto ..... & .2652 & 9.56 & . . 19570 & .70 .53 \\
\hline Non sa $\ldots$. & .8176 & .29 .47 & 27746 & 100.00 \\
\hline
\end{tabular}


Comunicazione con il personale delle biblioteche (disponibilità e cortesia dei bibliotecari nei confronti del pubblico).

Quanto è importante in relazione alle tue esigenze di studio e ricerca?

\begin{tabular}{|c|c|c|c|c|}
\hline QU03A13 & Frequenze & Percentuali & $\begin{array}{l}\text { Frequenze } \\
\text { cumulate }\end{array}$ & $\begin{array}{c}\text { Percentuali } \\
\text { cumulate }\end{array}$ \\
\hline Per nulla importante & .157 & .0 .57 & .157 & 0.57 \\
\hline Poco importante ... & .575 & .2 .07 & .732 & 2.64 \\
\hline Indifferente & .3304 & $\ldots 11.91$ & 4036 & .14 .55 \\
\hline Importante $\ldots . .$. & $\ldots 7915$ & .28 .53 & .11951 & .43 .07 \\
\hline Molto importante & .15406 & .55 .53 & $\ldots 27357$ & . .98.60 \\
\hline Non sa $\ldots \ldots \ldots$. & . . 389 & $\ldots 1.40$ & . . 27746 & $\ldots 100.00$ \\
\hline
\end{tabular}

Comunicazione con il personale delle biblioteche (disponibilità e cortesia dei bibliotecari nei confronti del pubblico).

In quale percentuale delle tue visite in biblioteca hai contatti diretti con i bibliotecari?

\begin{tabular}{|c|c|c|c|c|}
\hline QU03B13 & Frequenze & Percentuali & $\begin{array}{c}\text { Frequenze } \\
\text { cumulate }\end{array}$ & $\begin{array}{l}\text { Percentuali } \\
\text { cumulate }\end{array}$ \\
\hline $0 \%$ & .443 & 1.60 & .443 & 1.60 \\
\hline $25 \%$ & . . 3417 & .12 .31 & .3860 & .13 .91 \\
\hline $50 \%$ & $\ldots 8417$ & .30 .33 & .12277 & .44 .25 \\
\hline $75 \%$ & .8766 & . .31.59 & . . 21043 & .75 .84 \\
\hline $100 \%$ & .6376 & . . 22.98 & .27419 & .98 .82 \\
\hline Non sa & . . 328 & . . 1.18 & .27747 & 100.00 \\
\hline
\end{tabular}

Comunicazione con il personale delle biblioteche (disponibilità e cortesia dei bibliotecari nei confronti del pubblico).

Qual è il tuo grado di soddisfazione al proposito?

\begin{tabular}{|c|c|c|c|c|}
\hline QU03C13 & Frequenze & Percentuali & $\begin{array}{c}\text { Frequenze } \\
\text { cumulate }\end{array}$ & $\begin{array}{c}\text { Percentuali } \\
\text { cumulate }\end{array}$ \\
\hline Insoddisfatto & .1216 & 4.45 & 1216 & 4.45 \\
\hline Poco insoddisfatto & $\ldots 2575$ & 9.43 & .3791 & .13 .88 \\
\hline Indifferente & . . 6264 & .22 .94 & 10055 & .36 .83 \\
\hline Abbastanza soddisfatto & .10575 & .38 .73 & .20630 & .75 .56 \\
\hline Soddisfatto . . & $\ldots 6464$ & .23 .68 & .27094 & .99 .23 \\
\hline Non sa .. & . . 209 & $\ldots 0.77$ & .27303 & .100 .00 \\
\hline
\end{tabular}

Dati Mancanti $=443$ 
Servizi e assistenza personalizzata al lettore da parte del bibliotecario.

Quanto è importante in relazione alle tue esigenze di studio e ricerca?

\begin{tabular}{|c|c|c|c|c|}
\hline QU04A14 & Frequenze & Percentuali & $\begin{array}{l}\text { Frequenze } \\
\text { cumulate }\end{array}$ & $\begin{array}{l}\text { Percentuali } \\
\text { cumulate }\end{array}$ \\
\hline Per nulla importante & . . .692 & 2.49 & .692 & 2.49 \\
\hline Poco importante & .1121 & .4 .04 & 1813 & .6 .53 \\
\hline Indifferente $\ldots$ & $\ldots 5403$ & .19 .47 & .7216 & .26 .01 \\
\hline Importante .... & $\ldots 9569$ & . .34.49 & . . 16785 & $\ldots 60.50$ \\
\hline Molto importante & .10275 & .37 .03 & . . 27060 & $\ldots 97.53$ \\
\hline Non sa $\ldots . .$. & $\ldots 685$ & . . 2.47 & .27745 & .100 .00 \\
\hline
\end{tabular}

Servizi e assistenza personalizzata al lettore da parte del bibliotecario.

Quando devi effettuare ricerche complesse, in che percentuale ti rivolgi al bibliotecario per un servizio personalizzato?

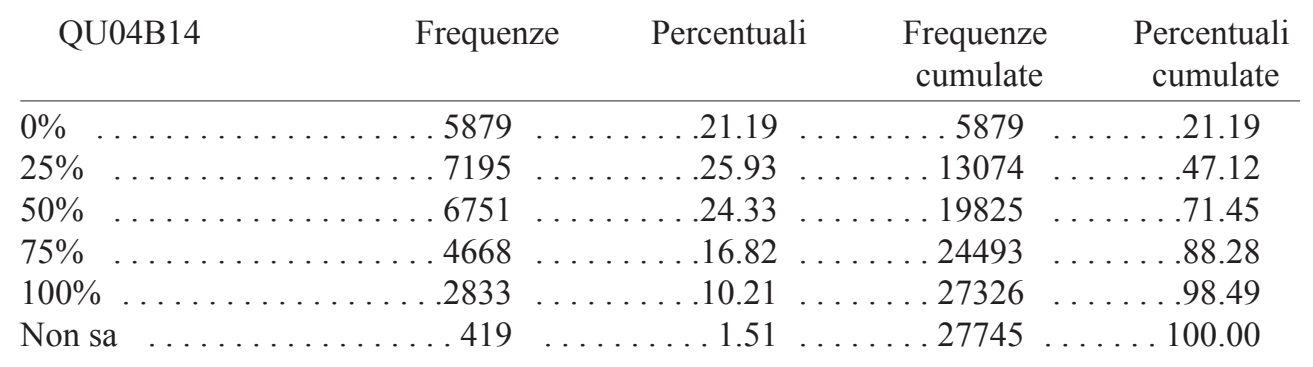

Servizi e assistenza personalizzata al lettore da parte del bibliotecario.

Qual è il tuo grado di soddisfazione per la consulenza?

\begin{tabular}{|c|c|c|c|c|}
\hline QU04C14 & Frequenze & Percentuali & $\begin{array}{c}\text { Frequenze } \\
\text { cumulate }\end{array}$ & $\begin{array}{c}\text { Percentuali } \\
\text { cumulate }\end{array}$ \\
\hline Insoddisfatto & .1014 & 4.64 & .1014 & .4 .64 \\
\hline Poco insoddisfatto & .2879 & .13 .17 & 3893 & .17 .80 \\
\hline Indifferente $\ldots$ & 6005 & .27 .46 & 9898 & .45 .26 \\
\hline Abbastanza soddisfatto & .7019 & .32 .10 & 16917 & .77 .36 \\
\hline Soddisfatto & 4418 & .20 .20 & 21335 & .97 .57 \\
\hline Non sa $\ldots$ & . . 532 & 2.43 & 21867 & 100.00 \\
\hline
\end{tabular}

Dati Mancanti $=5879$ 
Depliant sulle biblioteche e segnaletica in biblioteca per facilitare l'uso dei servizi.

Quanto è importante in relazione alle tue esigenze di studio e ricerca?

\begin{tabular}{|c|c|c|c|c|}
\hline QU04A15 & Frequenze & Percentuali & $\begin{array}{c}\text { Frequenze } \\
\text { cumulate }\end{array}$ & $\begin{array}{c}\text { Percentuali } \\
\text { cumulate }\end{array}$ \\
\hline Per nulla importante & .2693 & 9.71 & . . 2693 & 9.71 \\
\hline Poco importante & .2065 & .7 .44 & . . 4 4758 & .17 .15 \\
\hline Indifferente $\ldots$ & $\ldots 6424$ & .23 .15 & . . 11182 & .40 .30 \\
\hline Importante .... & $\ldots 6901$ & .24 .87 & . . 18083 & . .65.17 \\
\hline Molto importante . & . . 6892 & .24 .84 & . . 24975 & . .90.01 \\
\hline Non sa & .2771 & 9.99 & .27746 & 100.00 \\
\hline
\end{tabular}

Depliant sulle biblioteche e segnaletica in biblioteca per facilitare l'uso dei servizi.

In quale percentuale delle tue visite in biblioteca usi, anche in modo rapido, questi strumenti?

\begin{tabular}{|c|c|c|c|c|}
\hline QU04B15 & Frequenze & Percentuali & $\begin{array}{c}\text { Frequenze } \\
\text { cumulate }\end{array}$ & $\begin{array}{l}\text { Percentuali } \\
\text { cumulate }\end{array}$ \\
\hline $0 \%$ & .10670 & .38 .46 & .10670 & .38 .46 \\
\hline $25 \%$ & . . 6473 & .23 .33 & . . 17143 & . .61.79 \\
\hline $50 \%$. & . . 5621 & . . . 20.26 & . . 22764 & .82 .04 \\
\hline $75 \%$ & $\ldots 2795$ & .10 .07 & . . 25559 & . . 92.12 \\
\hline $100 \% \ldots$ & . . 1366 & . . 4.92 & . . 26925 & . . .97.04 \\
\hline Non sa & $\ldots 821$ & . . 2.96 & .27746 & 100.00 \\
\hline
\end{tabular}

Depliant sulle biblioteche e segnaletica in biblioteca per facilitare l'uso dei servizi. Qual è il tuo grado di soddisfazione al proposito?

\begin{tabular}{|c|c|c|c|c|}
\hline QU04C15 & Frequenze & Percentuali & $\begin{array}{c}\text { Frequenze } \\
\text { cumulate }\end{array}$ & $\begin{array}{c}\text { Percentuali } \\
\text { cumulate }\end{array}$ \\
\hline Insoddisfatto & $\ldots 712$ & $\ldots 4.17$ & $\ldots .712$ & $\ldots 4.17$ \\
\hline Poco insoddisfatto & 3443 & $\ldots .20 .16$ & . . 4155 & .24 .33 \\
\hline Indifferente . & . . 5945 & $\ldots .34 .81$ & $\ldots 10100$ & $\ldots .59 .15$ \\
\hline Abbastanza soddisfatto & . . .4429 & . . 25.94 & . . 14529 & $\ldots 85.08$ \\
\hline Soddisfatto ....... & $\ldots 1847$ & .10 .82 & . 16376 & .95 .90 \\
\hline Non sa $\ldots$. & $\ldots 700$ & . . 4.10 & . 17076 & 100.00 \\
\hline
\end{tabular}


Quali altre biblioteche frequenti? Almeno un'altra (prima indicata)

QU05A Frequenze Percentuali Frequenze Percentuali

\begin{tabular}{|c|c|c|c|}
\hline & & cumulate & cumula \\
\hline$\ldots \ldots \ldots 654$ & 2.36 & .654 & 2.36 \\
\hline Farmacia .............4419 & 1.51 & 1073 & 3.87 \\
\hline Dip. Farmacologia . . . . . 69 & 0.25 & 1142 & 4.12 \\
\hline Dip. Sanità Pubblica . . . . . . 12 & 0.04 & 1154 & 4.16 \\
\hline Antropologia .........193 & $\ldots \ldots$ & .1347 & 4.86 \\
\hline Biologia Animale . . . . . 223 & 0.80 & .1570 & .5 .66 \\
\hline Botanica . . . . . . . . . 248 & 0.89 & $\ldots 1818$ & 6.55 \\
\hline Chimica ...........76 & $\ldots \ldots$ & .1894 & .6 .83 \\
\hline 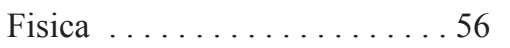 & $\ldots \ldots$ & . 1950 & 7.03 \\
\hline Geomineralogia $\ldots \ldots \ldots 68$ & $\ldots \ldots$ & 2018 & 7.27 \\
\hline Matematica & $\ldots \ldots \ldots 1.55$ & . . 2449 & .8 .83 \\
\hline Dip. Matematica Applicata . . .23 & .0 .08 & 2472 & .8 .91 \\
\hline Economia & .2 .73 & . 3230 & .11 .64 \\
\hline Giurisprudenza . . . . . . 1706 & 6.15 & .4936 & .17 .79 \\
\hline Scienze Politiche . . . . . . . 427 & .1 .54 & . . 5363 & .19 .33 \\
\hline Statistica . . . . . . . . . 629 & .2 .27 & .5992 & .21 .60 \\
\hline Dip. Diritto Comparato & 0.25 & . . 6060 & .21 .84 \\
\hline Dip. Diritto dell'Economia . . . 39 & 0.14 & 6099 & .21 .98 \\
\hline Dip. Diritto Pubblico & 0.37 & . . 6203 & .22 .36 \\
\hline Dip. Teoria e St. del Diritto $\ldots 35$ & 0.13 & .6238 & .22 .49 \\
\hline Agraria . . . . . . . . . 195 & 0.70 & . 6433 & .23 .19 \\
\hline Architettura . . . . . . . .988 & $\ldots 3.56$ & $\ldots 7421$ & $\ldots 26.75$ \\
\hline Ingegneria $\ldots \ldots \ldots \ldots .948$ & . . 3.42 & $\ldots 8369$ & .30 .17 \\
\hline Dip. Costruzioni $\ldots \ldots \ldots 112$ & $\ldots 0.40$ & $\ldots 8481$ & $\ldots 30.57$ \\
\hline Dip. Meccanica .......... 39 & $\ldots 0.14$ & . 8520 & .30 .71 \\
\hline Dip. Progettazione . . . . . .157 & $\ldots 0.57$ & $\ldots 8677$ & .31 .28 \\
\hline Dip. Urbanistica . . . . . . . 39 & $\ldots \ldots \ldots 0.14$ & . 8716 & . . .31.42 \\
\hline Dip. Storia Architettura . . . .108 & $\ldots 0.39$ & .8824 & .31 .81 \\
\hline Geografia ...........181 & $\ldots 0.65$ & $\ldots 9005$ & $\ldots .32 .46$ \\
\hline$\ldots \ldots \ldots \ldots 2563$ & . 9.24 & 11568 & .41 .70 \\
\hline Scienze della Formazione $\ldots 756$ & . . 2.73 & .12324 & $\ldots 44.42$ \\
\hline St. e Letteratura Nordamericana 34 & $\ldots 0.12$ & .12358 & .44 .55 \\
\hline Dip. Filosofia . . . . . . . 309 & $\ldots 1.11$ & .12667 & $\ldots 45.66$ \\
\hline Dip. Italianistica $\ldots \ldots \ldots \ldots 34$ & $\ldots 0.12$ & . . 12701 & $\ldots 45.78$ \\
\hline Dip. Psicologia . . . . . . . . 125 & $\ldots \ldots \ldots 0.45$ & . 12826 & $\ldots .46 .23$ \\
\hline Dip. St. Arti e Spettacolo _ . 221 & $\ldots 0.80$ & 13047 & .47 .03 \\
\hline$\ldots \ldots \ldots \ldots 14695$ & $\ldots \ldots \ldots 52.97$ & 27742 & . . 100.00 \\
\hline
\end{tabular}


Quali altre biblioteche frequenti? Almeno altre due (seconda indicata)

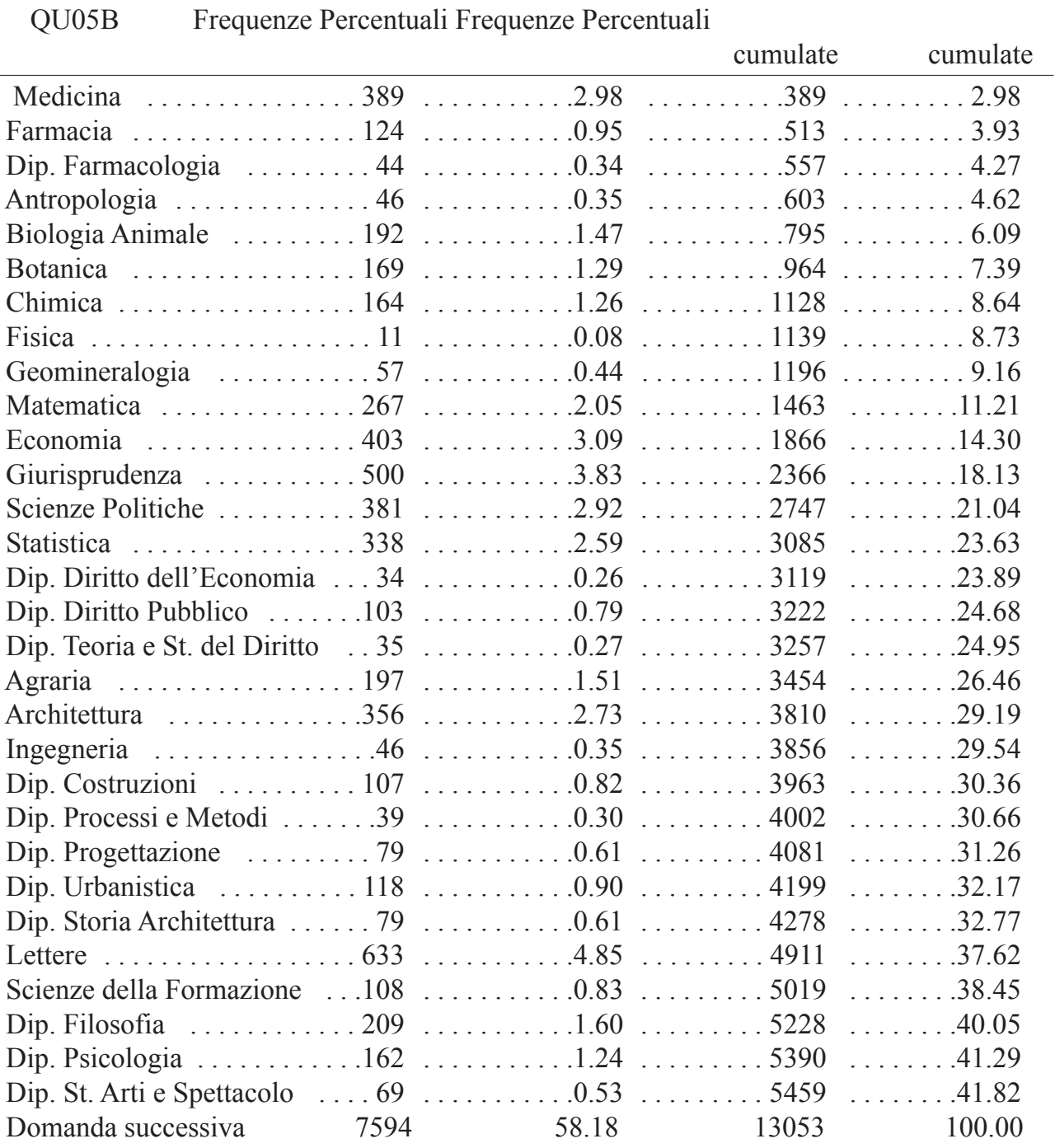

Dati Mancanti $=14695$ 
Quali altre biblioteche frequenti? Almeno altre tre (terza indicata)

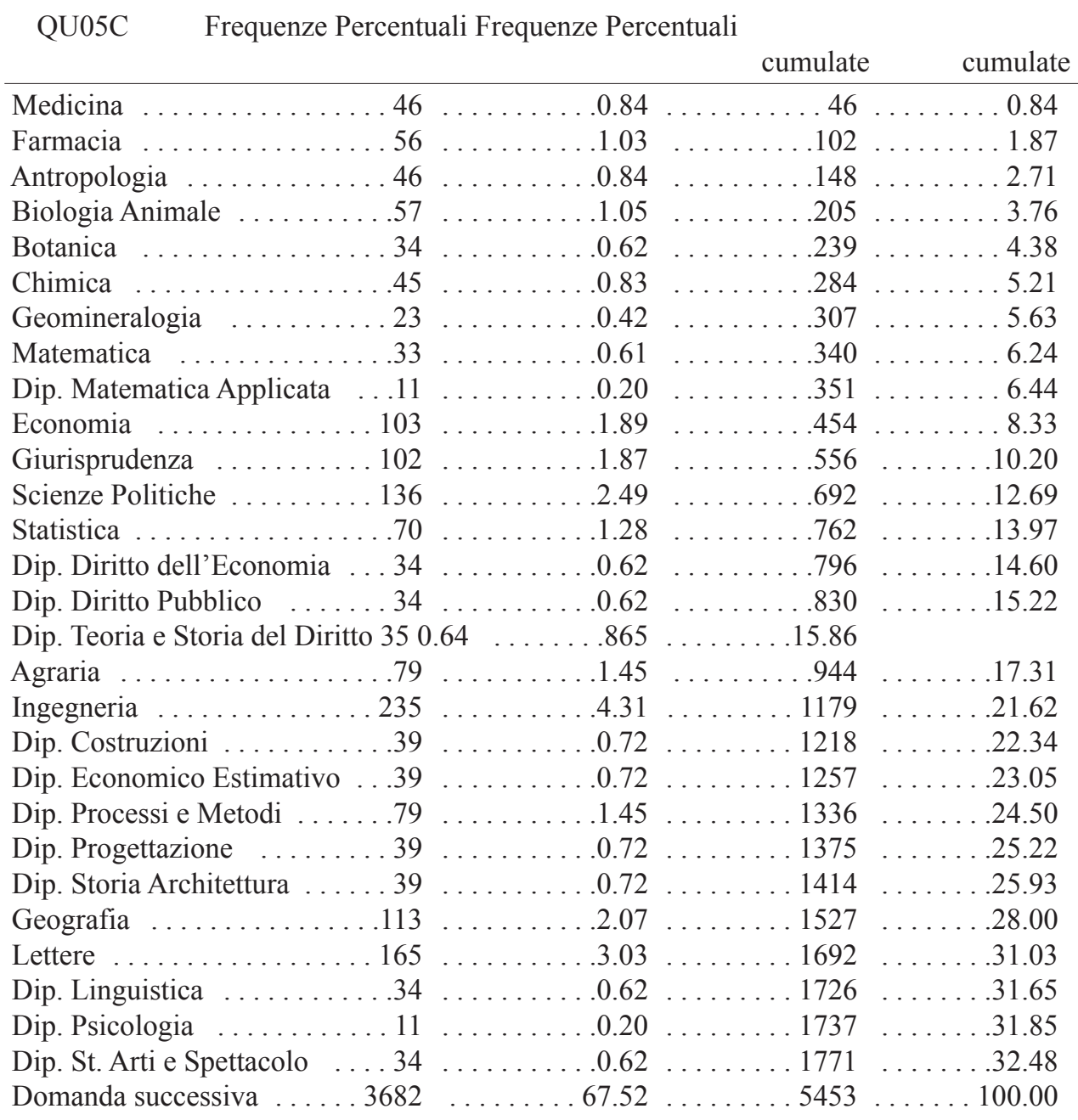

Dati Mancanti $=22290$ 
Quali altre biblioteche frequenti? Almeno altre quattro (quarta indicata)

QU05D Frequenze Percentuali Frequenze Percentuali

\begin{tabular}{|c|c|c|c|}
\hline & & $\mathrm{c}$ & cumulate \\
\hline Medicina & .4 .74 & .84 & 4.74 \\
\hline Farmacia & .0 .62 & 95 & .5 .36 \\
\hline Biologia Animale $\ldots . \ldots \ldots$ & .0 .62 & .106 & .5 .98 \\
\hline Botanica $\ldots . \ldots$. & .1 .30 & .129 & 7.28 \\
\hline Chimica .............79 & .4 .46 & .208 & .11 .73 \\
\hline Geomineralogia . . & .0 .62 & .219 & .12 .35 \\
\hline Matematica $\ldots \ldots \ldots \ldots 35$ & .1 .97 & .254 & .14 .33 \\
\hline Economia & .1 .97 & .289 & .16 .30 \\
\hline Agraria $\ldots \ldots \ldots \ldots$ & .0 .62 & .300 & .16 .92 \\
\hline Architettura & .1 .97 & .335 & . 18.89 \\
\hline Dip. Ingegneria Civile . . . . .39 & .2 .20 & .374 & .21 .09 \\
\hline Dip. Progettazione ....... 39 & .2 .20 & .413 & .23 .29 \\
\hline Lettere ...............34 & $\ldots 1.92$ & .447 & $\ldots .25 .21$ \\
\hline Scienze della Formazione . . . 69 & . .3.89 & $\ldots .516$ & .29 .10 \\
\hline Dip. Filosofia $\ldots \ldots \ldots \ldots 68$ & .3 .84 & $\ldots .584$ & $\ldots .32 .94$ \\
\hline Scienze Antichità & . 1.92 & $\ldots 618$ & $\ldots 34.86$ \\
\hline Domanda successiva . . . . 1155 & $\ldots 65.14$ & $\ldots 1773$ & . . 100.00 \\
\hline
\end{tabular}

Dati Mancanti $=25972$

Quali altre biblioteche frequenti? Almeno altre cinque (quinta indicata)

QU05E Frequenze Percentuali Frequenze Percentuali

\begin{tabular}{|c|c|c|c|}
\hline & & cumulate & cumulate \\
\hline Medicina & $\ldots 1.78$ & 11 & 1.78 \\
\hline$\ldots \ldots 11$ & .1 .78 & 22 & .3 .57 \\
\hline Dip. Processi e Metodi . . . . . .39 & .6 .32 & $\ldots 61$ & 9.89 \\
\hline Dip. Urbanistica . . . . . . . . 39 & $\ldots 6.32$ & .100 & .16 .21 \\
\hline Lettere & .6 .32 & .139 & .22 .53 \\
\hline Dip. Psicologia & .5 .51 & .173 & .28 .04 \\
\hline Domanda successiva $\ldots$ & $\ldots 71.96$ & .617 & 100.00 \\
\hline
\end{tabular}

Dati Mancanti $=27127$ 
Quali altre biblioteche frequenti? Almeno altre sei (sesta indicata)

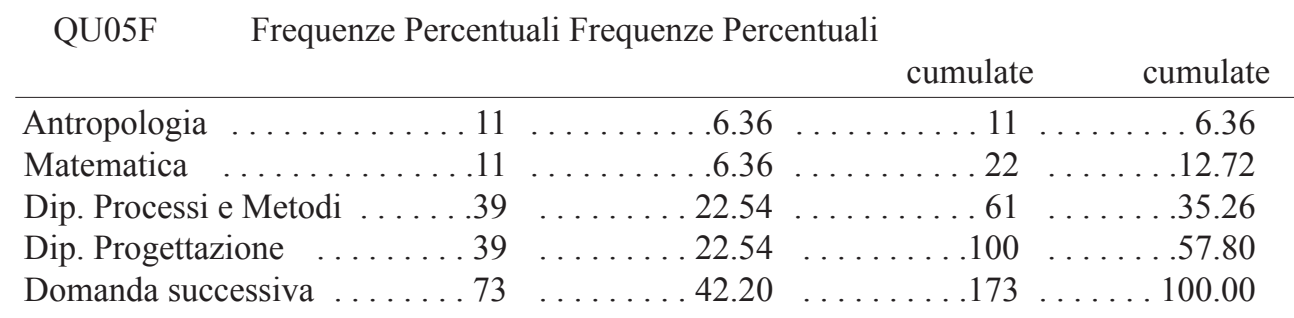

Dati Mancanti $=27572$

Quali altre biblioteche frequenti? Almeno altre sette (settima indicata)

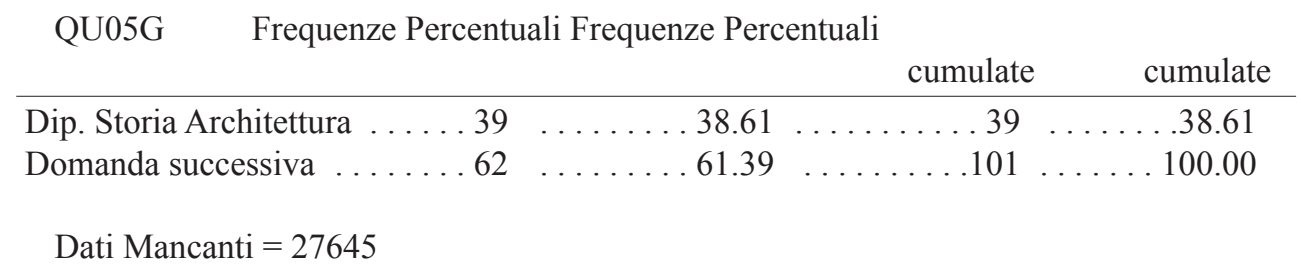

Quali altre biblioteche frequenti? Almeno altre otto (ottava indicata)

QU05H Frequenze Percentuali Frequenze Percentuali $\quad$ cumulate cumulate

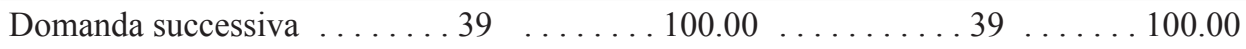

Dati Mancanti $=27707$ 
(Uso) Sito web del Sistema Bibliotecario di Ateneo.

Quanto è importante in relazione alle tue esigenze di studio e ricerca?

\begin{tabular}{|c|c|c|c|c|}
\hline QU06aA17 & Frequenze & Percentuali & $\begin{array}{c}\text { Frequenze } \\
\text { cumulate }\end{array}$ & $\begin{array}{l}\text { Percentuali } \\
\text { cumulate }\end{array}$ \\
\hline Per nulla importante & .1227 & 4.42 & .1227 & .4 .42 \\
\hline Poco importante ... & $\ldots 891$ & .3 .21 & .2118 &. .7 .63 \\
\hline Indifferente $\ldots$ & $\ldots 2570$ & .9 .26 & .4688 & 16.90 \\
\hline Importante ..... & . . .5908 & 21.29 & .10596 & .38 .19 \\
\hline Molto importante & .15692 & .56 .55 & .26288 & .94 .74 \\
\hline Non sa $\ldots \ldots$. & . . 1459 & . . 5.26 & .27747 & 100.00 \\
\hline
\end{tabular}

(Uso) Sito web del Sistema Bibliotecario di Ateneo.

Con quale percentuale, rispetto ai contatti che hai con l'Università per acquisire informazioni sulle biblioteche, usi il sito internet del Sistema Bibliotecario di Ateneo?

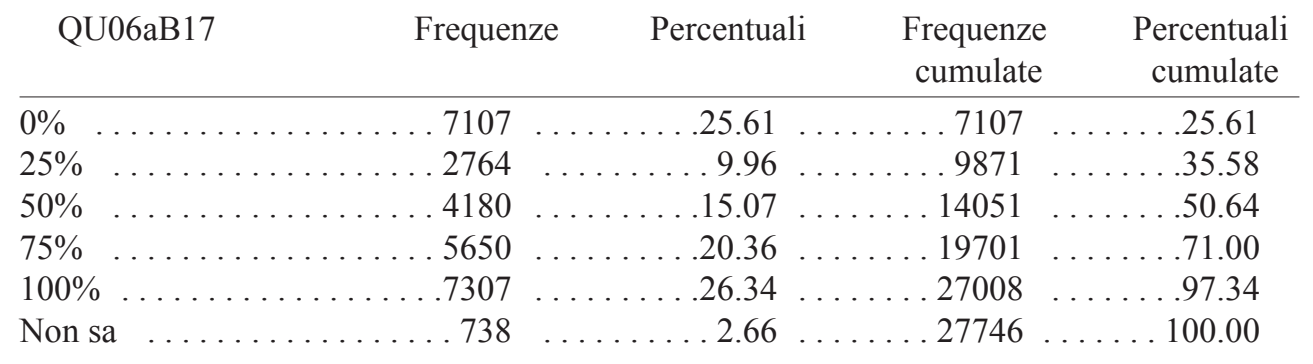

(Uso) Sito web del Sistema Bibliotecario di Ateneo.

Qual è il tuo grado di soddisfazione al proposito?

\begin{tabular}{|c|c|c|c|c|}
\hline QU06aC17 & Frequenze & Percentuali & $\begin{array}{c}\text { Frequenze } \\
\text { cumulate }\end{array}$ & $\begin{array}{c}\text { Percentuali } \\
\text { cumulate }\end{array}$ \\
\hline Insoddisfatto & $\ldots 292$ & $\ldots 1.41$ & $\ldots .292$ & $\ldots 1.41$ \\
\hline Poco insoddisfatto & .1245 & $\ldots 6.03$ & $\ldots 1537$ & $\ldots 7.45$ \\
\hline Indifferente . & $\ldots 4053$ & $\ldots 19.64$ & . . 5590 & . . 27.09 \\
\hline Abbastanza soddisfatto & $\ldots 8830$ & $\ldots .42 .79$ & . . 14420 & . . . 69.87 \\
\hline Soddisfatto ........ & $\ldots 5372$ & . .26.03 & . . 19792 & .95 .90 \\
\hline Non sa ... & $\ldots 846$ & 4.10 & 20638 & 100.00 \\
\hline
\end{tabular}


Uso del catalogo della biblioteca su internet: ricerca libri o periodici per titolo e ricerca di libri per autore.

Quanto è importante in relazione alle tue esigenze di studio e ricerca?

\begin{tabular}{|c|c|c|c|c|}
\hline QU06aA18 & Frequenze & Percentuali & $\begin{array}{c}\text { Frequenze } \\
\text { cumulate }\end{array}$ & $\begin{array}{l}\text { Percentuali } \\
\text { cumulate }\end{array}$ \\
\hline Per nulla importante & .77 & 0.37 & 77 & 0.37 \\
\hline Poco importante & .192 & 0.93 & .269 & 1.30 \\
\hline Indifferente $\ldots$ & $\ldots 587$ & .2 .84 & .856 & 4.15 \\
\hline Importante ... & $\ldots 4767$ & .23 .10 & .5623 & .27 .24 \\
\hline Molto importante & .14535 & .70 .42 & .20158 & $\ldots 97.67$ \\
\hline Non sa $\ldots \ldots \ldots$ & $\ldots 481$ & .2 .33 & 20639 & .100 .00 \\
\hline
\end{tabular}

Dati Mancanti $=7107$

Uso del catalogo della biblioteca su internet: ricerca libri o periodici per titolo e ricerca di libri per autore.

$\mathrm{Su} 10$ consultazioni del catalogo, quante volte usi questa opzione di ricerca?

\begin{tabular}{|c|c|c|c|c|}
\hline QU06aB18 & Frequenze & Percentuali & $\begin{array}{c}\text { Frequenze } \\
\text { cumulate }\end{array}$ & $\begin{array}{c}\text { Percentuali } \\
\text { cumulate }\end{array}$ \\
\hline $0 \%$ & $\ldots .627$ & .3 .04 & .627 & 3.04 \\
\hline $25 \%$ & . 1527 & .7 .40 & 2154 & .10 .44 \\
\hline $50 \% \quad \ldots$ & . 2360 & . .11.43 & 4514 & .21 .87 \\
\hline $75 \% \quad \ldots$ & . 6129 & . .29.69 & 10643 & .51 .56 \\
\hline $100 \% \ldots$ & . .9672 & .46 .86 & 20315 & .98 .43 \\
\hline Non sa . . & . . 325 & $\ldots 1.57$ & 20640 & 100.00 \\
\hline
\end{tabular}

Dati Mancanti $=7107$

Uso del catalogo della biblioteca su internet: ricerca libri o periodici per titolo e ricerca di libri per autore.

Qual è il tuo grado di soddisfazione al proposito?

\begin{tabular}{|c|c|c|c|}
\hline QU06aC18 & Percentuali & $\begin{array}{c}\text { Frequenze } \\
\text { cumulate }\end{array}$ & $\begin{array}{l}\text { Percentuali } \\
\text { cumulate }\end{array}$ \\
\hline Insoddisfatto $\ldots \ldots \ldots \ldots 161$ & $\ldots \ldots \ldots 0.80$. & $\ldots \ldots \ldots 161$ & $\ldots 0.80$ \\
\hline Poco insoddisfatto $\ldots \ldots \ldots 726$ & $\ldots \ldots \ldots 3.63$. & $\ldots \ldots \ldots 887$ & $\ldots 4.43$ \\
\hline Indifferente $\ldots \ldots \ldots \ldots 3524$. & $\ldots \ldots \ldots 17.61$. & $\ldots 4411$ & $\ldots \ldots .22 .04$ \\
\hline Abbastanza soddisfatto . . . 9034 . & $\ldots .45 .14$. & . . 13445 & $\ldots \ldots 67.18$ \\
\hline Soddisfatto . . . . . . . . 6209 & $\ldots 31.02$. & . . 19654 & $\ldots 98.21$ \\
\hline Non sa ..............359 & $\ldots \ldots \ldots 1.79$. & . . 20013. & $\ldots \ldots 100.00$ \\
\hline
\end{tabular}


Uso del catalogo su internet: ricerca libri per argomento (nel campo: soggetto) o materia (nel campo classificazione).

Quanto è importante in relazione alle tue esigenze di studio e ricerca?

\begin{tabular}{|c|c|c|c|c|}
\hline QU06aA19 & Frequenze & Percentuali & $\begin{array}{c}\text { Frequenze } \\
\text { cumulate }\end{array}$ & $\begin{array}{l}\text { Percentuali } \\
\text { cumulate }\end{array}$ \\
\hline
\end{tabular}

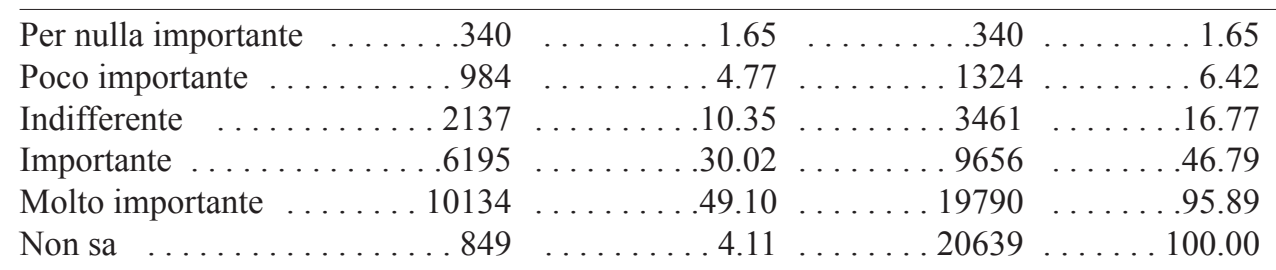

Dati Mancanti $=7107$

Uso del catalogo su internet: ricerca libri per argomento (nel campo: soggetto) o materia (nel campo classificazione).

$\mathrm{Su} 10$ consultazioni del catalogo, quante volte usi questa opzione di ricerca?

\begin{tabular}{|c|c|c|c|c|}
\hline QU06aB19 & Frequenze & Percentuali & $\begin{array}{c}\text { Frequenze } \\
\text { cumulate }\end{array}$ & $\begin{array}{c}\text { Percentuali } \\
\text { cumulate }\end{array}$ \\
\hline $0 \%$ & .2708 & .13 .12 & .2708 & .13 .12 \\
\hline $25 \%$ & .3928 & .19 .03 & .6636 & .32 .15 \\
\hline $50 \% \ldots$ & $\ldots 4057$ & . 19.66 & 10693 & .51 .81 \\
\hline $75 \% \ldots$ & . . 5591 & . .27.09 & .16284 & .78 .90 \\
\hline $100 \%$. & . .3985 & .19 .31 & 20269 & .98 .21 \\
\hline Non sa & . . 369 & . . 1.79 & .20638 & 100.00 \\
\hline
\end{tabular}

Dati Mancanti $=7107$

Uso del catalogo su internet: ricerca libri per argomento (nel campo: soggetto) o materia (nel campo classificazione).

Qual è il tuo grado di soddisfazione al proposito?

\begin{tabular}{|c|c|c|c|c|}
\hline QU06aC19 & Frequenze & Percentuali & $\begin{array}{c}\text { Frequenze } \\
\text { cumulate }\end{array}$ & $\begin{array}{l}\text { Percentuali } \\
\text { cumulate }\end{array}$ \\
\hline Insoddisfatto & 442 & 2.47 & .442 & 2.47 \\
\hline Poco insoddisfatto & .1878 & .10 .47 & 2320 & .12 .94 \\
\hline Indifferente $\ldots$. & 4733 & .26 .40 & 7053 & .39 .33 \\
\hline Abbastanza soddisfatto & .6653 & .37 .10 & . . 13706 & .76 .44 \\
\hline Soddisfatto ... & .3878 & .21 .63 & $\ldots 17584$ & .98 .06 \\
\hline Non sa ... & . . 347 & . . 1.94 & .17931 & 100.00 \\
\hline
\end{tabular}

Dati Mancanti $=9815$ 
Uso del catalogo su internet: ricerca libri o periodici nel campo parola chiave.

Quanto è importante in relazione alle tue esigenze di studio e ricerca?

\begin{tabular}{|c|c|c|c|c|}
\hline QU06aA20 & Frequenze & Percentuali & $\begin{array}{c}\text { Frequenze } \\
\text { cumulate }\end{array}$ & $\begin{array}{c}\text { Percentuali } \\
\text { cumulate }\end{array}$ \\
\hline Per nulla i & 1440 & 6.98 & 1440 & 6.98 \\
\hline Poco importante & .1513 & 7.33 & . . 2953 & .14 .31 \\
\hline Indifferente $\ldots$ & 3807 & .18 .45 & . 6760 & .32 .75 \\
\hline Importante & $\ldots .5484$ & .26 .57 & . . 12244 & .59 .32 \\
\hline Molto importante & . .6886 & .33 .36 & . 19130 & . .92.69 \\
\hline Non sa $\ldots \ldots$. & .1509 & .7 .31 & 20639 & 100.00 \\
\hline
\end{tabular}

Dati Mancanti $=7107$

Uso del catalogo su internet: ricerca libri o periodici nel campo parola chiave.

$\mathrm{Su} 10$ consultazioni del catalogo, quante volte usi questa opzione di ricerca?

\begin{tabular}{|c|c|c|c|c|}
\hline QU06aB20 & Frequenze & Percentuali & $\begin{array}{c}\text { Frequenze } \\
\text { cumulate }\end{array}$ & $\begin{array}{l}\text { Percentuali } \\
\text { cumulate }\end{array}$ \\
\hline $0 \%$ & .7118 & .34 .49 & $\ldots 7118$ & .34 .49 \\
\hline $25 \%$ & .3656 & .17 .71 & 10774 & .52 .20 \\
\hline $50 \%$ & . 3093 & .14 .99 & .13867 & . .67.19 \\
\hline $75 \%$ & 3903 & . .18.91 & . . 17770 & .86 .10 \\
\hline $100 \%$ & .2362 & .11 .44 & . . 20132 & .97 .54 \\
\hline Non sa & $\ldots 507$ & $\ldots 2.46$ & 20639 & .100 .00 \\
\hline
\end{tabular}

Dati Mancanti $=7107$

Uso del catalogo su internet: ricerca libri o periodici nel campo parola chiave.

Qual è il tuo grado di soddisfazione al proposito?

\begin{tabular}{|c|c|c|c|c|}
\hline QU06aC20 & Frequenze & Percentuali & $\begin{array}{c}\text { Frequenze } \\
\text { cumulate }\end{array}$ & $\begin{array}{c}\text { Percentuali } \\
\text { cumulate }\end{array}$ \\
\hline Insoddisfatto & 618 & 4.57 & .618 & 4.57 \\
\hline Poco insoddisfatto & 1848 & .13 .67 & 2466 & .18 .24 \\
\hline Indifferente . & 4007 & .29 .64 & 6473 & .47 .88 \\
\hline Abbastanza soddisfatto & .4430 & .32 .77 & 10903 & .80 .64 \\
\hline Soddisfatto & .2121 & .15 .69 & .13024 & .96 .33 \\
\hline Non sa ... & $\ldots 496$ & .3 .67 & .13520 & 100.00 \\
\hline
\end{tabular}

Dati Mancanti $=14225$ 
Uso del catalogo della biblioteca su internet: prenotazione o proroga del prestito di un libro. Quanto è importante in relazione alle tue esigenze di studio e ricerca?

\begin{tabular}{|c|c|c|c|c|}
\hline QU07A21 & Frequenz & Percentuali & $\begin{array}{c}\text { Frequenze } \\
\text { cumulate }\end{array}$ & $\begin{array}{l}\text { Percentuali } \\
\text { cumulate }\end{array}$ \\
\hline Per nulla importante & .977 & 4.73 & .977 & 4.73 \\
\hline Poco importante .. & $\ldots 457$ & .2 .21 & $\ldots 1434$ & 6.95 \\
\hline Indifferente $\ldots$ & $\ldots 1954$ & 9.47 & . . 3388 & .16 .42 \\
\hline Importante . & . . .4804 & .23 .28 & 8192 & .39 .69 \\
\hline Molto importante & .11051. & .53 .54 & . . 19243 & $\ldots 93.24$ \\
\hline Non sa $\ldots .$. & . . 1396 & $\ldots 6.76$ & . . 20639 & .100 .00 \\
\hline
\end{tabular}

Dati Mancanti $=7107$

Uso del catalogo della biblioteca su internet: prenotazione o proroga del prestito di un libro. Quando devi rinnovare un prestito o prenotare un libro, quante volte usi la procedura su web?

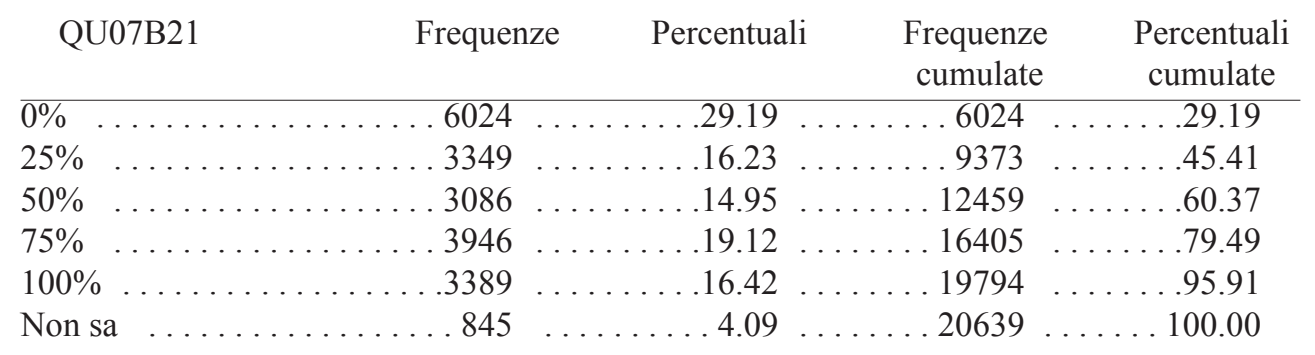

Dati Mancanti $=7107$

Uso del catalogo della biblioteca su internet:prenotazione o proroga del prestito di un libro. Qual è il tuo grado di soddisfazione al proposito?

\begin{tabular}{|c|c|c|c|c|}
\hline QU07C21 & Frequenze & Percentuali & $\begin{array}{c}\text { Frequenze } \\
\text { cumulate }\end{array}$ & $\begin{array}{c}\text { Percentuali } \\
\text { cumulate }\end{array}$ \\
\hline Insoddisfatto & 496 & .3 .39 & .496 & .3 .39 \\
\hline Poco insoddisfatto & 1131 & $\ldots 7.74$ & .1627 & .11 .13 \\
\hline Indifferente $\ldots$. & 2662 & $\ldots 18.21$ & 4289 & .29 .35 \\
\hline Abbastanza soddisfatto & .4807 & .32 .89 & 9096 & .62 .24 \\
\hline Soddisfatto & 4669 & .31 .95 & . 13765 & .94 .18 \\
\hline Non sa & .850 & . . 5.82 & .14615 & .100 .00 \\
\hline
\end{tabular}

Dati Mancanti $=13132$ 
Banche dati attualmente disponibili nelle biblioteche (via web, su cd-rom).

Quanto sono importanti in relazione alle tue esigenze di studio e ricerca?

\begin{tabular}{|c|c|c|c|c|}
\hline QU07A22 & Frequenze & Percentuali & $\begin{array}{c}\text { Frequenze } \\
\text { cumulate }\end{array}$ & $\begin{array}{l}\text { Percentuali } \\
\text { cumulate }\end{array}$ \\
\hline Per nulla importante & .1024 & 4.96 &. .1024 & 4.96 \\
\hline Poco importante & $\ldots 614$ & $\ldots 2.97$ & . . 1638 & .7 .94 \\
\hline Indifferente $\ldots$ & . 4050 & .19 .62 & . . 5688 & 27.56 \\
\hline Importante $\ldots .$. & . . 4700 & .22 .77 & . 10388 & .50 .33 \\
\hline Molto importante & . .6219 & .30 .13 & .16607 & .80 .46 \\
\hline Non sa $\ldots . .$. & .4032 & .19 .54 & .20639 & .100 .00 \\
\hline
\end{tabular}

Dati Mancanti $=7107$

Banche dati attualmente disponibili nelle biblioteche (via web, su cd-rom).

In quale percentuale le tue visite in biblioteca (o i contatti con il sito web della biblioteca) prevedono l'uso di tali risorse?

\begin{tabular}{|c|c|c|c|c|}
\hline QU07B22 & Frequenze & Percentuali & $\begin{array}{c}\text { Frequenze } \\
\text { cumulate }\end{array}$ & $\begin{array}{c}\text { Percentuali } \\
\text { cumulate }\end{array}$ \\
\hline $0 \%$ & 8938 & .43 .31 & 8938 & .43 .31 \\
\hline $25 \%$ & 3627 & .17 .57 & 12565 & .60 .88 \\
\hline $50 \%$ & .3624 & .17 .56 & 16189 & .78 .44 \\
\hline $75 \%$ & 2372 & .11 .49 & .18561 & . .89.93 \\
\hline $100 \%$ & $\ldots 917$ & . . 4.44 & .19478 & . . 94.37 \\
\hline Non sa & . . 1161 & .5 .63 & 20639 & .100 .00 \\
\hline
\end{tabular}

Dati Mancanti $=7107$

Banche dati attualmente disponibili nelle biblioteche (via web, su cd-rom).

Qual è il tuo grado di soddisfazione per le materie che le banche dati trattano?

\begin{tabular}{|c|c|c|c|}
\hline QU07C22 & Percentuali & $\begin{array}{c}\text { Frequenze } \\
\text { cumulate }\end{array}$ & $\begin{array}{c}\text { Percentuali } \\
\text { cumulate }\end{array}$ \\
\hline Insoddisfatto & 2.43 & .284 & 2.43 \\
\hline Poco insoddisfatto & .10 .28 . & .1487 & .12 .71 \\
\hline Indifferente $\ldots \ldots$ & .33 .31 . & $\ldots 5384$ & .46 .02 \\
\hline Abbastanza soddisfatto . . . .3710 & .31 .71 . & 9094 & .77 .73 \\
\hline Soddisfatto . & .11 .88 & 10484 & .89 .61 \\
\hline Non sa $\ldots \ldots \ldots \ldots 1216$ & .10 .39 . & .11700 & 100.00 \\
\hline
\end{tabular}

Dati Mancanti $=16046$ 
Risultati ottenuti dall'interrogazione delle banche dati.

Quanto sono importanti in relazione alle tue esigenze di studio e ricerca?

\begin{tabular}{|c|c|c|c|c|}
\hline QU07A23 & Frequenze & Percentuali & $\begin{array}{c}\text { Frequenze } \\
\text { cumulate }\end{array}$ & $\begin{array}{l}\text { Percentual } \\
\text { cumulate }\end{array}$ \\
\hline Per nulla importante & .419 & .2 .03 & . . .419 & 2.03 \\
\hline Poco importante .. & $\ldots 446$ & .2 .16 & $\ldots 865$ & 4.19 \\
\hline Indifferente.. & .3810 & $\ldots 18.46$ & .4675 & .22 .65 \\
\hline Importante ... & $\ldots 4286$ & . . 20.77 & .8961 & .43 .42 \\
\hline Molto importante & . .5591 & . .27.09 & 14552 & .70 .51 \\
\hline Non sa $\ldots . .$. & . 6085 & .29 .49 & 20637 & 100.00 \\
\hline
\end{tabular}

Dati Mancanti $=7107$

Risultati ottenuti dall'interrogazione delle banche dati.

Qual è il tuo grado di soddisfazione per le indicazioni bibliografiche tratte dalla consultazione delle banche dati?

\begin{tabular}{|c|c|c|c|}
\hline QU07C23 & Percentuali & $\begin{array}{c}\text { Frequenze } \\
\text { cumulate }\end{array}$ & $\begin{array}{l}\text { Percentuali } \\
\text { cumulate }\end{array}$ \\
\hline Insoddisfatto & 2.91 & .601 & 2.91 \\
\hline Poco insoddisfatto & 4.92 & 1617 & 7.83 \\
\hline Indifferente & .22 .74 & .6311 & .30 .58 \\
\hline Abbastanza soddisfatto . . . .4497 & .21 .79 & 10808 & .52 .37 \\
\hline Soddisfatto & .8 .77 & .12618 & .61 .14 \\
\hline$\ldots \ldots \ldots 8021$. & $\ldots 38.86$ & 20639 & 100.00 \\
\hline
\end{tabular}

Dati Mancanti $=7107$ 
Uso dei periodici elettronici nelle discipline di tuo interesse.

Quanto è importante in relazione alle tue esigenze di studio e ricerca?

\begin{tabular}{|c|c|c|c|c|}
\hline QU07A24 & Frequenze & Percentuali & $\begin{array}{l}\text { Frequenze } \\
\text { cumulate }\end{array}$ & $\begin{array}{l}\text { Percentuali } \\
\text { cumulate }\end{array}$ \\
\hline Per nulla importante & .1899 & 9.20 & 1899 & 9.20 \\
\hline Poco importante ... & .1804 & .8 .74 & 3703 & .17 .94 \\
\hline Indifferente $\ldots$ & $\ldots 3213$ & .15 .57 & .6916 & .33 .51 \\
\hline Importante $\ldots$. & . . 3869 & .18 .75 & .10785 & .52 .26 \\
\hline Molto importante & .5122 & .24 .82 & .15907 & .77 .08 \\
\hline Non sa $\ldots . .$. & .4731 & . .22.92 & 20638 & 100.00 \\
\hline
\end{tabular}

Dati Mancanti $=7107$

Uso dei periodici elettronici nelle discipline di tuo interesse.

Quando devi consultare un periodico, in quale percentuale usi la versione elettronica che l'Università rende disponibile dai suoi computer?

\begin{tabular}{|c|c|c|c|c|}
\hline QU07B24 & Frequenze & Percentuali & $\begin{array}{c}\text { Frequenze } \\
\text { cumulate }\end{array}$ & $\begin{array}{c}\text { Percentuali } \\
\text { cumulate }\end{array}$ \\
\hline $0 \%$ & 11514 & .55 .79 & 11514 & .55 .79 \\
\hline $25 \%$ & $\ldots 2885$ & .13 .98 & 14399 & .69 .77 \\
\hline $50 \%$ & . . 2266 & .10 .98 & 16665 & .80 .75 \\
\hline $75 \%$ & . 1620 & 7.85 & . 18285 & .88 .60 \\
\hline $100 \%$ & $\ldots 873$ & .4 .23 & 19158 & .92 .83 \\
\hline Non sa & . 1480 & .7 .17 & .20638 & 100.00 \\
\hline
\end{tabular}

Dati Mancanti $=7107$

Uso dei periodici elettronici nelle discipline di tuo interesse.

Qual è il tuo grado di soddisfazione al proposito?

\begin{tabular}{|c|c|c|c|}
\hline QU07C24 & Percentuali & $\begin{array}{c}\text { Frequenze } \\
\text { cumulate }\end{array}$ & $\begin{array}{c}\text { Percentuali } \\
\text { cumulate }\end{array}$ \\
\hline Insoddisfatto & 1.74 & .159 & 1.74 \\
\hline Poco insoddisfatto & .8 .97 & .977 & .10 .71 \\
\hline Indifferente $\ldots$. & .32 .95 & 3983 & .43 .65 \\
\hline Abbastanza soddisfatto . . . 2469 & .27 .06 & 6452 & .70 .71 \\
\hline Soddisfatto & .13 .70 & 7702 & .84 .41 \\
\hline$\ldots \ldots 1422$ & .15 .59 & 9124 & 100.00 \\
\hline
\end{tabular}

Dati Mancanti $=18622$ 
Sesso

\begin{tabular}{|c|c|c|c|c|}
\hline QU08sex & Frequenze & Percentuali & $\begin{array}{c}\text { Frequenze } \\
\text { cumulate }\end{array}$ & $\begin{array}{c}\text { Percentuali } \\
\text { cumulate }\end{array}$ \\
\hline & 16590 & .59 .79 & . . 16590 & .59 .79 \\
\hline Maschio & .11156 & .40 .21 & . . 27746 & . . 100.00 \\
\hline
\end{tabular}

Risiedi nel comune di Firenze?

\begin{tabular}{|c|c|c|c|c|}
\hline QU08resfi & Frequenze & Percentuali & $\begin{array}{c}\text { Frequenze } \\
\text { cumulate }\end{array}$ & $\begin{array}{l}\text { Percentuali } \\
\text { cumulate }\end{array}$ \\
\hline $\mathrm{NO}$ & 16703 & .60 .20 & 16703 & .60 .20 \\
\hline Sİ & 11043 & .39 .80 & 27746 & 100.00 \\
\hline
\end{tabular}

Provincia di residenza

\begin{tabular}{|c|c|c|c|c|}
\hline \multicolumn{2}{|c|}{ QU08respr } & Percentuali & $\begin{array}{c}\text { Frequenze } \\
\text { cumulate }\end{array}$ & $\begin{array}{c}\text { Percentuali } \\
\text { cumulate }\end{array}$ \\
\hline$\overline{\mathrm{AN}}$ & $\ldots \ldots \ldots$ & 0.88 & $\ldots 147$ & $\ldots 0.88$ \\
\hline AP & $\ldots \ldots \ldots \ldots \ldots$ & 0.46 . & 224 & .1 .34 \\
\hline AR & . . . . . . . . . . . . 1747 & 10.46 & 1971 & 11.80 \\
\hline BA & $\ldots \ldots \ldots \ldots \ldots . \ldots . \ldots 39$ & 0.23 & 2010 & 12.03 \\
\hline BO & .124 & 0.74 & 2134 & 12.78 \\
\hline $\mathrm{BS}$ & $\ldots \ldots \ldots \ldots \ldots$ & 0.47. & 2213 & 13.25 \\
\hline $\mathrm{BZ}$ & .51 & 0.31 . & 2264 & 13.56 \\
\hline $\mathrm{CA}$ & $\ldots \ldots \ldots$ & . 0.69 . & 2379 & 14.24 \\
\hline $\mathrm{CH}$ & $\ldots \ldots \ldots \ldots \ldots$ & $\ldots 0.44$. & 2452 & 14.68 \\
\hline $\mathrm{CN}$ & $\ldots \ldots \ldots \ldots \ldots \ldots \ldots$ & $\ldots \ldots \ldots 0.07$. & 2464 & 14.75 \\
\hline $\mathrm{CO}$ & $\ldots \ldots \ldots \ldots \ldots \ldots \ldots \ldots$ & $\ldots \ldots 0.07$. & 2476 & .14 .82 \\
\hline $\mathrm{CS}$ & $\ldots \ldots \ldots \ldots \ldots \ldots 161$ & $\ldots \ldots \ldots 0.96$. & 2637 & .15 .79 \\
\hline CT & $\ldots \ldots \ldots \ldots \ldots \ldots \ldots \ldots$ & $\ldots 0.21$. & $\ldots 2672$ & .16 .00 \\
\hline $\mathrm{CZ}$ & $\ldots \ldots \ldots \ldots \ldots \ldots 220$ & $\ldots .1 .32$ & . . 2892 & .17 .32 \\
\hline FG & $\ldots \ldots \ldots \ldots \ldots \ldots \ldots 12$ & $\ldots 0.07$. & 2904 & .17 .39 \\
\hline FI & $\ldots \ldots \ldots \ldots \ldots . \ldots 5787$ & $\ldots 34.65$. & 8691 & . . 52.04 \\
\hline FO & $\ldots \ldots \ldots \ldots \ldots \ldots \ldots 79$ & $\ldots 0.47$. & $\ldots 8770$ & .52 .51 \\
\hline GE & $\ldots \ldots \ldots \ldots \ldots \ldots 43$ & $\ldots \ldots \ldots 0.26$. & $\ldots 8813$ & .52 .77 \\
\hline GR & $\ldots \ldots \ldots \ldots \ldots \ldots \ldots 409$ & $\ldots 2.45$. & . . 9222 & .55 .21 \\
\hline IS & $\ldots \ldots \ldots \ldots \ldots \ldots \ldots \ldots \ldots$ & $\ldots 0.20$ & .9256 & .55 .42 \\
\hline KR & $\ldots \ldots \ldots \ldots \ldots$ & $\ldots \ldots \ldots 0.68$. & . . .9369 & .56 .10 \\
\hline LE & 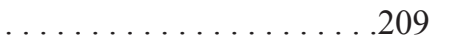 & $\ldots \ldots \ldots 1.25$. & $\ldots 9578$ & .57 .35 \\
\hline LI & $\ldots \ldots \ldots \ldots \ldots \ldots \ldots .243$ & $\ldots \ldots \ldots 1.45$. & $\ldots \ldots \ldots 9821$. & $\ldots \ldots 58.80$ \\
\hline
\end{tabular}




\begin{tabular}{|c|c|c|c|c|}
\hline $\mathrm{U}$ & 594 & 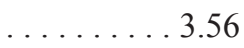 & & \\
\hline $\mathrm{MC}$ & .107 & 0.64 & .10522 & 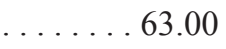 \\
\hline IE & $\ldots \ldots \ldots \ldots \ldots$ & 0.23 & 561 & \\
\hline$S$ & $\ldots \ldots \ldots \ldots \ldots$ & 0.60 & 62 & \\
\hline $\mathrm{O}$ & $\ldots \ldots \ldots \ldots \ldots \ldots$ & 0.26 & .10705 & \\
\hline U & $\ldots \ldots \ldots \ldots$ & $\ldots \ldots$ & 78 & \\
\hline $\mathrm{R}$ & $\ldots \ldots \ldots \ldots \ldots$ & $\ldots$ & .10812 & 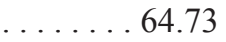 \\
\hline A & $\ldots \ldots \ldots \ldots \ldots$ & $\ldots \ldots$ & $\ldots$ & \\
\hline$E$ & $\ldots \ldots \ldots \ldots \ldots \ldots$ & $\ldots \ldots$ & .10945 & . . \\
\hline $\mathrm{G}$ & $\ldots \ldots \ldots$ & $\ldots \ldots$ & $\ldots 1$ & \\
\hline & $\ldots \ldots \ldots \ldots \ldots$ & .2 .58 & 72 & . \\
\hline 0 & 1856 & $\ldots \ldots \ldots 11.11$ & .13 & 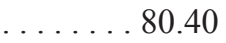 \\
\hline & $\ldots \ldots \ldots$ & $\ldots \ldots$ & 95 & \\
\hline T & 1676 & 10.03 & 71 & \\
\hline & $\ldots \ldots \ldots$ & . . & .1 & \\
\hline V & .34 & 0.2 & .15 & \\
\hline Z & .70 & . & 54 & $\cdots$ \\
\hline A & .76 & 0.46 & .15 & 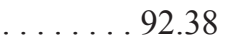 \\
\hline & . 39 & . & $\ldots$ & 3 \\
\hline $\mathrm{O}$ & $\ldots \ldots \ldots \ldots$ & 0 & $\ldots$ & 9 \\
\hline A & $\ldots \ldots \ldots \ldots \ldots$ & $\ldots$ & $\ldots$ & .. \\
\hline & . . 397 & $\ldots 2$ & .1 & 9 \\
\hline & .45 & 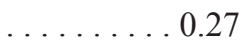 & 08 & 9 \\
\hline & $\ldots 74$ & $\ldots 0$ & .16 & . . \\
\hline & .182 & $\ldots$ & $\ldots$ & 97 \\
\hline A & .157 & 0 & .16 & 9 \\
\hline TE & .82 & 0 & $\ldots$ & 98 \\
\hline & .33 & 0 & 36 & 9 \\
\hline D & .43 & 0 & $\ldots$ & \\
\hline 工 & .11 & 0 & .16 & 9 \\
\hline & .39 & 0.23 & $\ldots$ & 95 \\
\hline & . . 39 & $\ldots \ldots$ & $\ldots$ & .99 \\
\hline $\mathrm{V}$ & $\ldots \ldots \ldots \ldots \ldots$ & $\ldots 0.20$ & . .16702 & 100 \\
\hline
\end{tabular}

Dati Mancanti $=11043$ 
Domicilio a Firenze

\begin{tabular}{|c|c|c|c|c|}
\hline QU08dom & Frequenze & Percentuali & $\begin{array}{c}\text { Frequenze } \\
\text { cumulate }\end{array}$ & $\begin{array}{c}\text { Percentuali } \\
\text { cumulate }\end{array}$ \\
\hline $\mathrm{NO}$ & .13163 & 78.81 & .13163 & 78.81 \\
\hline Sİ & . . 3540 & 21.19 & .16703 & 100.00 \\
\hline
\end{tabular}

Dati Mancanti $=11043$

Anno di nascita

\begin{tabular}{|c|c|c|c|c|}
\hline \multicolumn{2}{|c|}{ QU08anno } & Percentuali & $\begin{array}{c}\text { Frequenze } \\
\text { cumulate }\end{array}$ & $\begin{array}{c}\text { Percentuali } \\
\text { cumulate }\end{array}$ \\
\hline Non ris & sp. $\ldots \ldots \ldots \ldots$ & .0 .14 & .39 & .0 .14 \\
\hline 1944 & $\ldots \ldots \ldots \ldots \ldots$ & $\ldots 0.04$ & $\ldots .50$ & .0 .18 \\
\hline 1946 & $\ldots \ldots \ldots \ldots \ldots \ldots \ldots$ & $\ldots \ldots \ldots 0.12$ & $\ldots \ldots \ldots 84$ & .0 .30 \\
\hline 1952 & $\ldots \ldots \ldots \ldots \ldots \ldots 35$ & $\ldots \ldots \ldots 0.13$ & $\ldots \ldots \ldots 119$ & .0 .43 \\
\hline 1955 & $\ldots \ldots \ldots \ldots \ldots 69$ & $\ldots \ldots \ldots 0.25$ & $\ldots 188$ & .0 .68 \\
\hline 1958 & $\ldots \ldots \ldots \ldots \ldots \ldots 34$ & $\ldots \ldots \ldots 0.12$ & $\ldots 222$ & .0 .80 \\
\hline 1961 & $\ldots \ldots \ldots \ldots \ldots \ldots \ldots 39$ & $\ldots \ldots \ldots 0.14$ & $\ldots 261$ & .0 .94 \\
\hline 1962 & $\ldots \ldots \ldots \ldots \ldots \ldots 33$ & $\ldots \ldots \ldots 0.12$ & . . 294 & .1 .06 \\
\hline 1963 & $\ldots 176$ & $\ldots 0.63$ & $\ldots 470$ & .1 .69 \\
\hline 1964 & $\ldots \ldots \ldots \ldots \ldots \ldots 108$ & $\ldots \ldots \ldots 0.39$ & . . 578 & .2 .08 \\
\hline 1965 & $\ldots 127$ & $\ldots 0.46$ & $\ldots 705$ & .2 .54 \\
\hline 1966 & $\ldots \ldots \ldots \ldots \ldots \ldots 58$ & $\ldots \ldots \ldots 0.21$ & $\ldots 763$ & $\ldots 2.75$ \\
\hline 1967 & .244 & $\ldots 0.88$ & .1007 & .3 .63 \\
\hline 1968 & $\ldots 306$ & $\ldots 1.10$ & .1313 & .4 .73 \\
\hline 1969 & . . 365 & . . 1.32 & .1678 & .6 .05 \\
\hline 1970 & $\ldots 615$ & $\ldots 2.22$ & .2293 & $\ldots 8.26$ \\
\hline 1971 & . .1040 & $\ldots 3.75$ & . . 3333 & .12 .01 \\
\hline 1972 & . .1290 & $\ldots 4.65$ & . . 4623 & .16 .66 \\
\hline 1973 & . . 2103 & $\ldots 7.58$ & $\ldots 6726$ & .24 .24 \\
\hline 1974 & $\ldots .2663$ & $\ldots .9 .60$ & $\ldots 9389$ & .33 .84 \\
\hline 1975 & . . .2660 & . . . . 99.59 & . . 12049 & .43 .43 \\
\hline 1976 & . . .3824 & $\ldots \ldots \ldots 13.78$ & $\ldots 15873$ & .57 .21 \\
\hline 1977 & . . .3154 & $\ldots 11.37$ & . . 19027 & 68.58 \\
\hline 1978 & . . 2763 & $\ldots \ldots \ldots 9.96$ & . . 21790 & . . 78.54 \\
\hline 1979 & . . 3368 & $\ldots \ldots 12.14$ & $\ldots .25158$ & . .90.68 \\
\hline 1980 & $\ldots .2155$ & $\ldots \ldots \ldots .7 .77$ & . . . . 27313 & . .98.45 \\
\hline 1981 & $\ldots 386$ & . . . . . 1.39 & . . .27699 & . . 99.84 \\
\hline 1984 & $\ldots \ldots \ldots \ldots \ldots \ldots 45$ & $\ldots \ldots \ldots 0.16$ & . . . . 27744 & $\ldots 100.00$ \\
\hline
\end{tabular}


Lavori?

\begin{tabular}{|c|c|c|c|c|}
\hline QU08lav & Frequenze & Percentuali & $\begin{array}{c}\text { Frequenze } \\
\text { cumulate }\end{array}$ & $\begin{array}{l}\text { Percentuali } \\
\text { cumulate }\end{array}$ \\
\hline $\begin{array}{l}\text { NO } \\
\text { sì }\end{array}$ & $\begin{array}{l}.17600 \\
10106\end{array}$ & $\begin{array}{l}.63 .52 \\
3648\end{array}$ & $\begin{array}{l}17600 \\
27706\end{array}$ & $\begin{array}{r}.63 .52 \\
10000\end{array}$ \\
\hline
\end{tabular}

Dati Mancanti $=39$

Tempo pieno o part-time

QU08tlav Frequenze Percentuali $\begin{gathered}\text { Frequenze } \\ \text { cumulate }\end{gathered} \begin{gathered}\begin{array}{c}\text { Percentuali } \\ \text { cumulate }\end{array} \\ \text { cumbing }\end{gathered}$

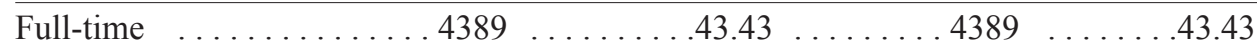

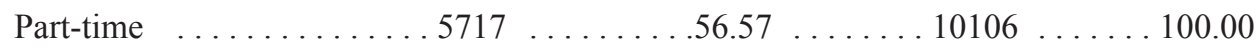

Dati Mancanti $=17640$

Già laureato oppure ha chiesto o non ha chiesto la tesi?

\begin{tabular}{|c|c|c|c|c|}
\hline QU08tesi & Frequenze & Percentuali & $\begin{array}{c}\text { Frequenze } \\
\text { cumulate }\end{array}$ & $\begin{array}{c}\text { Percentuali } \\
\text { cumulate }\end{array}$ \\
\hline Laure & 3541 & 12.78 & .3541 & 12.78 \\
\hline Non ha ancor & si 14827 & 53.51 & 18368 & 66.29 \\
\hline Ha gia chiesto & .9339 & 33.71 & .27707 & 100.00 \\
\hline
\end{tabular}

Dati Mancanti $=39$ 



\section{Appendice D}

\section{Stima degli errori di campionamento dell'indagine sulla soddisfazione degli utenti}

\section{Nota tecnica: Stima degli errori di campionamento}

Per l'estrazione del campione è stato adottato uno schema di campionamento stratificato, utilizzando come variabili di stratificazione la ex-biblioteca di facoltà e l'area. Per raggiungere lo stesso livello di precisione per le stime relative alle diverse aree, la dimensione complessiva del campione (1000 studenti) è stata suddivisa tra le 5 aree. Successivamente all'interno di ciascun area la dimensione del campione d'area è stata ripartita proporzionalmente tra i corrispondenti strati. In ciascuno strato gli studenti da intervistare sono stati selezionati con un'estrazione casuale semplice senza ripetizione.

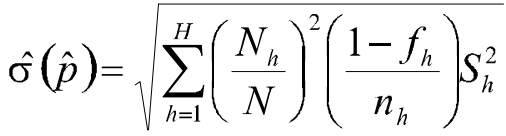

dove $N_{h}$ è la dimensione dello strato $h$ da cui sono stati selezionati $n_{h}$ studenti, $f_{h}=\frac{n_{h}}{N_{h}} \quad, N$ è la dimensione della popolazione ed infine $S_{h}^{2}$ è la varianza elementare campionaria nello strato.

Nelle tabella seguente sono riportati gli errori di campionamento per le principali stime $\hat{p}$ per le 5 aree del Sistema Bibliotecario e per l'intera popolazione.

Il calcolo dell'intervallo di confidenza per una stima $\hat{p}$ può essere effettuato come per il seguente esempio, che riguarda la variabile QU02A1, importanza dei libri e periodici posseduti dalla biblioteca per proprie esigenze di studio e di ricerca.

1. Scelta dell'ambito di riferimento della stima di interesse: proporzione di coloro che tra gli utenti di tutte le biblioteche, hanno definito molto importanti per le proprie esigenze di studio e di ricerca i libri e periodici posse- 
duti dalla biblioteca di cui sono utenti (QU02A1 = molto importante). Secondo i dati d'indagine tale stima è uguale a $0,3410(34,10 \%)$.

2. Scelta del livello di confidenza dell'intervallo di confidenza voluto: generalmente tale livello è posto pari al $95 \%$, di conseguenza i percentili in base ai quali calcolare l'intervallo sono pari a 2 (approssimazione di 1,96). Possono essere scelti altri valori del livello di confidenza: per esempio per il $90 \%$ abbiamo percentili uguali a 1,645 (cfr. Tavole della distribuzione normale) ${ }^{1}$.

3. Lettura del valore dell'errore di campionamento corrispondente alla variabile di interesse e all'ambito di riferimento scelto nella Tabella 1 (Stima dell'errore campionario per il totale biblioteche e le singole aree).

4. Calcolo dell'intervallo di confidenza: nel caso d'interesse nell'esempio, al livello del 95\% abbiamo per una stima uguale a 0,3410 (34,10\%), il corrispondente intervallo stimato pari a [30,99\%-37,21\%], ottenuto con i seguenti calcoli

estremo inferiore $=0,3410-2 \times 0,01553=0,309934(30,99 \%)$

estremo superiore $=0,3410+2 \times 0,01553=0,372066(37,21 \%)$

1 Cicchitelli 2000. 
Tabella 1: Stima dell'errore campionario per il totale biblioteche e le singole aree

\begin{tabular}{|c|c|c|c|c|c|c|}
\hline \multirow[b]{2}{*}{ VARIABILI } & \multicolumn{6}{|c|}{ Ambiti di riferimento } \\
\hline & $\begin{array}{l}\text { Totale } \\
\text { biblio. } \\
\sigma(\hat{p})\end{array}$ & $\begin{array}{l}\text { Biblio. } \\
\text { Bio- } \\
\text { medica } \\
\sigma(\hat{p})\end{array}$ & $\begin{array}{c}\text { Biblio. } \\
\text { Scienze } \\
\text { Sociali } \\
\sigma(\hat{p})\end{array}$ & $\begin{array}{l}\text { Biblio. } \\
\text { Scienze } \\
\sigma(\hat{p})\end{array}$ & $\begin{array}{c}\text { Biblio. } \\
\text { Scienze } \\
\text { Tecnolog. } \\
\sigma(\hat{p})\end{array}$ & $\begin{array}{c}\text { Biblio } \\
\text { Umanista } \\
\sigma(\hat{p})\end{array}$ \\
\hline QU02A1 = per nulla importante & 0,00487 & 0,010257 & 0,008276 & 0,006541 & 0,008777 & 0,012826 \\
\hline QU02A1 = poco importante & 0,00765 & 0,013439 & 0,014161 & 0,012086 & 0,012508 & 0,013737 \\
\hline QU02A1 = indifferente & 0,0134 & 0,025363 & 0,028052 & 0,026132 & 0,025548 & 0,028252 \\
\hline QU02A1 = importante & 0,01624 & 0,032183 & 0,033773 & 0,031906 & 0,031416 & 0,033533 \\
\hline QU02A1 = molto importante & 0,01553 & 0,031859 & 0,032099 & 0,03278 & 0,028944 & 0,033648 \\
\hline QU02A1 = non sa & 0,00481 & 0,012811 & 0,009306 & 0,004636 & 0,008039 & 0,007025 \\
\hline QU02B1 = 0\% & 0,00918 & 0,01825 & 0,019992 & 0,019301 & 0,01636 & 0,020036 \\
\hline QU02B1 $=25 \%$ & 0,01448 & 0,030502 & 0,031762 & 0,027992 & 0,026729 & 0,030001 \\
\hline QU02B1 $=50 \%$ & 0,01458 & 0,02861 & 0,027801 & 0,027562 & 0,029092 & 0,031325 \\
\hline QU02B1 $=75 \%$ & 0,01383 & 0,027271 & 0,02833 & 0,030156 & 0,026515 & 0,029049 \\
\hline QU02B1 = 100\% & 0,01239 & 0,022374 & 0,025548 & 0,025332 & 0,023857 & 0,026153 \\
\hline QU02B1 = non sa & 0,00791 & & 0,008136 & & & \\
\hline QU02C1 = insoddisfatto & 0,00966 & 0,008242 & & 0,007235 & 0,010603 & 0,020161 \\
\hline QU02C1 = poco insoddisfatto & 0,01053 & 0,020761 & 0,01458 & 0,021251 & 0,022962 & 0,023238 \\
\hline QU02C1 = indifferente & 0,01557 & 0,029906 & 0,031256 & 0,030931 & 0,030857 & 0,031891 \\
\hline QU02C1 = abbastanza soddisfatto & 0,01729 & 0,034533 & 0,036156 & 0,034744 & 0,032463 & 0,036911 \\
\hline QU02C1 = soddisfatto & 0,01316 & 0,026243 & 0,029646 & 0,028304 & 0,021283 & 0,026509 \\
\hline QU02C1 = non sa & 0,00909 & 0,018334 & 0,012804 & & 0,013171 & \\
\hline QU02A2 = per nulla importante & 0,00798 & 0,011972 & 0,014049 & 0,009204 & 0,015486 & 0,019673 \\
\hline QU02A2 = poco importante & 0,00885 & 0,01764 & 0,018308 & 0,017332 & 0,015624 & 0,020699 \\
\hline QU02A2 = indifferente & 0,01328 & 0,025939 & 0,025594 & 0,026132 & 0,026525 & 0,028123 \\
\hline QU02A2 = importante & 0,01429 & 0,025577 & 0,029786 & 0,027338 & 0,027614 & 0,02987 \\
\hline QU02A2 = molto importante & 0,01588 & 0,031973 & 0,033772 & 0,033043 & 0,02991 & 0,033029 \\
\hline QU02A2 = non sa & 0,00833 & 0,023638 & 0,01902 & 0,017332 & 0,015354 & 0,015278 \\
\hline QU02B2 = 0\% & 0,01517 & 0,029642 & 0,031652 & 0,029509 & 0,028285 & 0,03303 \\
\hline QU02B2 = 25\% & 0,01389 & 0,029142 & 0,030524 & 0,029678 & 0,025738 & 0,028256 \\
\hline QU02B2 $=50 \%$ & 0,01405 & 0,026538 & 0,026899 & 0,02778 & 0,027473 & 0,030969 \\
\hline QU02B2 $=75 \%$ & 0,01222 & 0,023039 & 0,025323 & 0,022152 & 0,025079 & 0,022966 \\
\hline QU02B2 $=100 \%$ & 0,00722 & 0,018669 & 0,013378 & 0,017332 & 0,013628 & 0,01612 \\
\hline QU02B2 = non sa & 0,00708 & 0,013831 & 0,014049 & 0,012887 & 0,014511 & 0,011954 \\
\hline QU02C2 = insoddisfatto & 0,00891 & 0,015805 & 0,016486 & 0,01769 & 0,01876 & 0,017907 \\
\hline QU02C2 = poco insoddisfatto & 0,01554 & 0,031882 & 0,029909 & 0,029355 & 0,031549 & 0,032143 \\
\hline QU02C2 = indifferente & 0,01872 & 0,034039 & 0,038493 & 0,036123 & 0,035691 & 0,040923 \\
\hline QU02C2 = abbastanza soddisfatto & 0,01775 & 0,036767 & 0,036224 & 0,036567 & 0,032172 & 0,041146 \\
\hline QU02C2 = soddisfatto & 0,01253 & 0,02769 & 0,031448 & 0,026193 & 0,023369 & 0,019196 \\
\hline QU02C2 = non sa & 0,0084 & 0,019885 & 0,020032 & 0,016606 & 0,014065 & 0,017755 \\
\hline
\end{tabular}




\begin{tabular}{|c|c|c|c|c|c|c|}
\hline QU02A3 = per nulla importante & 0,0096 & 0,017278 & 0,017386 & 0,018355 & 0,017588 & 0,023925 \\
\hline QU02A3 = poco importante & 0,00871 & 0,014563 & 0,018052 & 0,012086 & 0,017022 & 0,022441 \\
\hline QU02A3 = indifferente & 0,01181 & 0,027168 & 0,024356 & 0,028199 & 0,022674 & 0,02377 \\
\hline QU02A3 = importante & 0,01371 & 0,026146 & 0,029302 & 0,028598 & 0,026482 & 0,027354 \\
\hline QU02A3 = molto importante & 0,01563 & 0,0285 & 0,033001 & 0,030454 & 0,030529 & 0,03148 \\
\hline QU02A3 = non sa & 0,01083 & 0,027324 & 0,023976 & 0,021008 & 0,017636 & 0,025024 \\
\hline QU02B3 $=0 \%$ & 0,01608 & 0,033053 & 0,033903 & 0,033225 & 0,02956 & 0,034913 \\
\hline QU02B3 $=25 \%$ & 0,0126 & 0,023821 & 0,025204 & 0,025332 & 0,026421 & 0,023317 \\
\hline QU02B3 $=50 \%$ & 0,01283 & 0,021243 & 0,024574 & 0,025606 & 0,026489 & 0,026153 \\
\hline QU02B3 $=75 \%$ & 0,01026 & 0,018167 & 0,023083 & 0,020602 & 0,020787 & 0,01739 \\
\hline QU02B3 $=100 \%$ & 0,00763 & 0,016361 & 0,017723 & 0,012887 & 0,015411 & 0,012067 \\
\hline QU02B3 = non sa & 0,00759 & 0,017015 & 0,015513 & 0,013635 & 0,016802 & 0,015359 \\
\hline QU02C3 = insoddisfatto & 0,01028 & 0,027991 & 0,021821 & 0,023854 & 0,016522 & 0,025076 \\
\hline QU02C3 = poco insoddis & 0,01486 & 0,02751 & 0,027828 & 0,025179 & 0,026573 & 0,037134 \\
\hline QU02C3 = indifferente & 0,02132 & 0,045582 & 0,0412 & 0,042931 & 0,038705 & 0,047885 \\
\hline QU02C3 = abbastanza so & 0,02115 & 0,045875 & 0,041496 & 0,044618 & 0,036571 & 0,051965 \\
\hline QU02C3 = soddisfatto & 0,01403 & 0,034456 & 0,034564 & 0,026412 & 0,022972 & 0,025076 \\
\hline QU02C3 = non sa & 0,01246 & 0,036086 & 0,025658 & 0,025179 & 0,015877 & 0,032532 \\
\hline QU02A4 = per nul & 0,00726 & 0,011054 & 0,017251 & 0,006541 & 0,015093 & 0,017259 \\
\hline QU02A4 = poco importa & 0,00681 & 0,01213 & 0,014157 & 0,012086 & 0,013915 & 0,013 \\
\hline QU02A4 = indifferente & 0,01222 & 0,024393 & 0,023615 & 152 & 0,025956 & 0,022966 \\
\hline QU02A4 = importante & 0,01468 & 0,028242 & 0,03074 & 0,030735 & 0,02822 & 0,030255 \\
\hline QU02A4 = molto impo & 0,01623 & 0,032266 & 0,034431 & 0,03278 & 0,03004 & 0,034872 \\
\hline QU02A4 = non sa & 0,00794 & 0,023383 & 0,015513 & 0,021401 & 0,016582 & 0,012926 \\
\hline QU02B4 = 0\% & 0,01407 & 0,030338 & 0,026471 & 0,029678 & 0,029763 & 0,026648 \\
\hline QU02B4 = 25\% & 0,0128 & 0,024936 & 0,02672 & 0,027992 & 0,025216 & 0,025213 \\
\hline QU02B4 $=50 \%$ & 0,01401 & 0,026735 & 0,029584 & 0,026132 & 0,026865 & 0,02909 \\
\hline QU02B4 $=75 \%$ & 0,01303 & 0,026304 & & 0,025606 & & \\
\hline QU02B4 = 100\% & 0,0107 & 0,017278 & 0,023482 & 0,018837 & 0,017003 & 0,026718 \\
\hline QU02B4 = non & & & & 0,013635 & & \\
\hline QU02C4 = insoddisfatto & 0,00908 & 0,011656 & 0,014164 & 0,014228 & 0,021181 & 0,022659 \\
\hline QU02C4 = pocc & & & & 662 & 0,024648 & 0,027588 \\
\hline QU02C4 = indifferente & 0,01732 & 0,037489 & 0,032982 & 0,034766 & 0,036728 & 0,03378 \\
\hline QU02C4 = abbastanza soddisfatto & 0,01793 & 0,038785 & 659 & 546 & 0,035889 & 0,03599 \\
\hline QU02C4 = soddisfatto & 0,01535 & 0,033898 & 0,031343 & 0,034167 & 0,027114 & 0,033735 \\
\hline QU02C4 = non sa & 0,00703 & 0,018601 & 0,015189 & 0,016718 & 0,015038 & 0,01047 \\
\hline QU02A5 = per nulla importante & 0,00563 & 0,010038 & 0,011389 & 0,006541 & 0,009309 & 0,014662 \\
\hline QU02A5 = poco importante & 0,00649 & 0,015896 & 0,011524 & 0,015624 & 0,011492 & 0,01612 \\
\hline QU02A5 = indifferente & 0,00995 & 0,021581 & 0,020622 & 0,021782 & 0,015338 & 0,025531 \\
\hline QU02A5 = importante & 0,01493 & 0,029915 & 0,031406 & 0,030156 & 0,028011 & 0,031732 \\
\hline QU02A5 = molto impo1 & 0,01626 & 0,033033 & 0,033839 & 0,033351 & 0,030873 & 0,034208 \\
\hline QU02A5 = non sa & 0,00671 & 0,014308 & 0,011524 & 0,009204 & 0,016028 & 0,017222 \\
\hline QU02B5 = $0 \%$ & 0,01058 & 0,023023 & 0,023027 & 0,01975 & 0,012355 & 0,028469 \\
\hline QU02B5 = 25\% & 0,01157 & 0,02506 & 0,027306 & 0,025051 & 0,017036 & 0,027387 \\
\hline QU02B5 $=50 \%$ & 0,01405 & 0,029217 & 0,027538 & 0,028199 & 0,026894 & 0,030945 \\
\hline QU02B5 $=75 \%$ & 0,01498 & 0,029346 & 0,030403 & 0,030735 & 0,030023 & 0,029719 \\
\hline
\end{tabular}


STIMA DEGLI ERRORI DI CAMPIONAMENTO DELL'INDAGINE

\begin{tabular}{|c|c|c|c|c|c|c|}
\hline QU02B5 = 100\% & 0,01282 & 0,022919 & 0,025631 & 0,025606 & 0,029124 & 0,018487 \\
\hline QU02B5 = non sa & 0,00706 & 0,006317 & 0,011524 & 0,007991 & 0,01744 & 0,010982 \\
\hline QU02C5 = insoddisfatto & 0,00913 & 0,010699 & 0,011177 & 0,012464 & 0,02113 & 0,017674 \\
\hline QU02C5 = poco insoddisfatto & 0,01331 & 0,021449 & 0,020925 & 0,019209 & 0,025795 & 0,034487 \\
\hline QU02C5 = indifferente & 0,01501 & 0,030984 & 0,031809 & 0,031276 & 0,02641 & 0,034646 \\
\hline QU02C5 = abbastanza soddisfatto & 0,01656 & 0,034676 & 0,036637 & 0,033514 & 0,029671 & 0,035182 \\
\hline QU02C5 = soddisfatto & 0,01335 & 0,029631 & 0,03165 & 0,030656 & 0,022284 & 0,028251 \\
\hline QU02C5 = non sa & 0,00568 & 0,007389 & 0,011056 & 0,010233 & 0,011083 & 0,012496 \\
\hline QU03A6 = per nulla importante & 0,00601 & 0,010257 & 0,010602 & 0,011217 & 0,013808 & 0,012984 \\
\hline QU03A6 = poco importante & 0,00704 & 0,011054 & 0,014883 & 0,012887 & 0,023095 & 0,017405 \\
\hline QU03A6 = indifferente & 0,01146 & 0,018961 & 0,021458 & 0,014997 & 0,024025 & 0,023922 \\
\hline QU03A6 = importante & 0,014 & 0,026483 & 0,027731 & 0,027992 & 0,026741 & 0,030966 \\
\hline QU03A6 = molto impo & 0,01654 & 0,031535 & 0,033966 & 0,032003 & 0,03176 & 0,035165 \\
\hline QU03A6 = non sa & 0,00617 & 0,022304 & 0,010483 & 0,006541 & 0,010091 & 0,012033 \\
\hline QU03B6 = 0\% & 0,01178 & 0,023012 & 0,024982 & 0,024162 & 0,02182 & 0,025304 \\
\hline QU03B6 = 25\% & 0,01276 & 0,023759 & 0,028833 & 0,024466 & 0,02252 & 0,02752 \\
\hline $6=50 \%$ & 0,01416 & 0,024393 & 0,028435 & 0,02385 & 0,027767 & 0,030493 \\
\hline QU03B6 = 75\% & 0,01377 & 0,029527 & 0,027803 & 0,029841 & 0,026877 & 0,028303 \\
\hline QU03B6 = 100\% & & 0,028429 & 0,025905 & 0,029159 & 0,02577 & 0,025337 \\
\hline QU03B6 = non sa & 0,00913 & & 0,010483 & & 0,017386 & 0,02576 \\
\hline QU03C6 = insoddi & 0,01109 & 571 & 0,022373 & 0,0 & 0,022228 & 0,022133 \\
\hline QU03C6 $=$ poco in & 0,01535 & 0,029282 & 0,033777 & 0,029633 & 0,030019 & 0,029089 \\
\hline QU03C6 = indifferente & 0,01584 & 0,027634 & 0,03256 & 0,029633 & 0,031807 & 0,031602 \\
\hline QU03C6 = abbastanza s & 0,01553 & 0,032515 & 0,031372 & 0,032482 & 0,028554 & 0,03503 \\
\hline QU03C6 = soddisfatto & 0,01255 & 0,030121 & 0,022946 & 0,029633 & 0,021767 & 0,028784 \\
\hline QU03C6 = non sa & 0,00878 & & 0,011213 & & 0,013176 & 0,014937 \\
\hline QU03A7 = per nulla importante & 0,0081 & 0,007447 & 0,009647 & 0,009522 & 0,026555 & 0,011611 \\
\hline QU0 & & & & & & \\
\hline QU03A7 = indifferente & 0,01087 & 0,019591 & 0,022078 & 0,017802 & 0,022975 & 0,01984 \\
\hline QU0 & & & & 0,032696 & & \\
\hline QU03A7 = molto impo & 0,01749 & 0,034369 & 0,036506 & 0,035388 & 0,033602 & 0,036273 \\
\hline QU03A7 $=\mathrm{n}$ & 0,00566 & & 0,009647 & & 0,015696 & 0,015384 \\
\hline QU03B7 = 0\% & 0,00679 & 0,019485 & 0,013646 & 0,012221 & 0,012804 & 0,01429 \\
\hline QU03B7 = 25\% & 0,01372 & 0,023419 & 0,031419 & 0,02608 & 0,026983 & 0,024399 \\
\hline QU03B7 $=50 \%$ & 0,01605 & 0,030225 & 0,033358 & 0,03226 & 0,030282 & 0,034536 \\
\hline QU03B7 = 75\% & 0,01614 & 0,033541 & 0,032985 & 0,033486 & 0,031147 & 0,033614 \\
\hline QU03B7 $=100 \%$ & 0,01511 & 0,030993 & 0,029531 & 0,031046 & 0,028324 & 0,034322 \\
\hline QU03B7 = non sa & 0,00731 & 0,022489 & 0,011022 & 0,007798 & 0,008475 & 0,018911 \\
\hline QU03B7 = non vuole risp & 0,00729 & 0,010085 & & 0,007798 & 0,017217 & \\
\hline QU03C7 = insoddisfatto & 0,00884 & 0,02099 & 0,019415 & 0,019804 & 0,016016 & 0,018001 \\
\hline QU03C7 = poco insoddis & 0,01214 & 0,023198 & 0,025852 & 0,020485 & 0,024087 & 0,023441 \\
\hline QU03C7 = indifferente & 0,01634 & 0,035081 & 0,035032 & 0,029794 & 0,031937 & 0,031469 \\
\hline QU03C7 = abbastanza sc & 0,01707 & 0,034181 & 0,034202 & 0,035694 & 0,033001 & 0,036014 \\
\hline QU03C7 = soddisfatto & 0,01534 & 0,029083 & 0,031158 & 0,0333 & 0,02757 & 0,035311 \\
\hline QU03C7 = non sa & 0,00725 & & 0,009992 & & 0,012409 & 0,016646 \\
\hline QU03A8 = per nulla importante & 0,00788 & 0,019051 & 0,007979 & 0,007798 & 0,016081 & 0,008347 \\
\hline
\end{tabular}




\begin{tabular}{|c|c|c|c|c|c|c|}
\hline QU03A8 = poco importante & 0,00494 & 0,009271 & 0,007979 & 0,009522 & 0,009926 & 0,011724 \\
\hline QU03A8 = indifferente & 0,01118 & 0,02076 & 0,021103 & 0,019898 & 0,02098 & 0,021982 \\
\hline QU03A8 = importante & 0,01588 & 0,032081 & 0,033103 & 0,031797 & 0,030089 & 0,033592 \\
\hline QU03A8 = molto importante & 0,01759 & 0,035166 & 0,036325 & 0,035157 & 0,033314 & 0,03765 \\
\hline QU03A8 = non sa & 0,00528 & 0,007447 & 0,009647 & & 0,016081 & 0,013015 \\
\hline QU03B8 $=0 \%$ & 0,00787 & 0,01241 & 0,011022 & 0,00553 & 0,009797 & 0,027138 \\
\hline QU03B8 $=25 \%$ & 0,01331 & 0,018794 & 0,028836 & 0,022815 & 0,024246 & 0,029813 \\
\hline QU03B8 $=50 \%$ & 0,01608 & 0,029674 & 0,031328 & 0,03226 & 0,031795 & 0,034339 \\
\hline QU03B8 $=75 \%$ & 0,01654 & 0,03518 & 0,034088 & 0,034333 & 0,031273 & 0,035205 \\
\hline QU03B8 $=100 \%$ & 0,01514 & 0,032896 & 0,032431 & 0,032903 & 0,028873 & 0,030154 \\
\hline QU03B8 = non sa & 0,00894 & 0,018794 & 0,012203 & 0,00553 & 0,008475 & 0,029269 \\
\hline QU03B8 = non vuole risp & 0,00899 & 0,011113 & & 0,00553 & 0,009797 & \\
\hline QU03C8 = insoddisfatto & 0,00741 & 0,017599 & 0,016013 & 0,012294 & 0,01087 & 0,019289 \\
\hline QU03C8 $=$ poco insoddisf & 0,01254 & 0,019139 & 0,023469 & 0,022405 & 0,025281 & 0,027664 \\
\hline QU03C8 = indifferente & 0,01655 & 0,02908 & 0,033622 & 0,031205 & 0,031911 & 0,03588 \\
\hline QU03C8 = abbastanza sod & 0,01668 & 0,036012 & 0,035198 & 0,034927 & 0,031633 & 0,034447 \\
\hline QU03C8 = soddisfatto & 0,01491 & 0,031637 & 0,032203 & 0,033064 & 0,029295 & 0,027986 \\
\hline QU03C8 = non sa & 0,00688 & & 0,011275 & & 0,018092 & 0,020753 \\
\hline QU03A9 = per nul & 0,00738 & 439 & 0,010675 & 217 & 0,014546 & 0,020215 \\
\hline QU03A9 = poco importa & 0,00694 & 0,016141 & 0,013395 & 0,012887 & 0,012358 & 0,016727 \\
\hline QU03A9 = indifferente & 0,00927 & 0,018316 & 0,016762 & 0,01622 & 0,018887 & 0,020215 \\
\hline QU03A9 = importante & 0,0132 & 0,028063 & 0,026413 & 0,029509 & 0,026011 & 0,026822 \\
\hline QU03A9 = molto impo & 0,01621 & 0,032594 & 0,032353 & 0,032895 & 0,031328 & 0,034771 \\
\hline QU03A9 = non sa & 0,00539 & 0,01024 & 0,011524 & 0,009204 & 0,009064 & 0,012984 \\
\hline QU03B9 = 0\% & 0,01409 & 0,028732 & 0,028761 & 0,026874 & 0,026831 & 0,030357 \\
\hline QU03B9 $=25 \%$ & 0,01298 & 0,027636 & 0,027561 & 0,027562 & 0,025181 & 0,025476 \\
\hline QU03B9 $=50 \%$ & 0,01269 & 0,027578 & 0,027561 & 0,026386 & 0,023262 & 0,02653 \\
\hline QU03B9 $=75 \%$ & & & & & & \\
\hline QU03B9 = 100\% & 0,01224 & 0,022002 & 0,022967 & 0,024762 & 0,023782 & 0,027712 \\
\hline $9=$ non sa & & 0,020521 & 0,011524 & & & 0,009798 \\
\hline QU03C9 = insoddisfatto & 0,01393 & 0,026251 & 0,024951 & 0,025744 & 0,02972 & 0,028535 \\
\hline QU03C9 = poco & & & & 423 & 0,034217 & 0,033999 \\
\hline QU03C9 = indifferente & 0,01649 & 0,033909 & 0,035518 & 0,032709 & 0,030856 & 0,034428 \\
\hline QU03C9 = abbastanza soddisfatto & & & 0,030829 & 709 & 0,026218 & 0,033689 \\
\hline QU03C9 = soddisfatto & 0,01247 & 0,026987 & 0,026686 & 0,028013 & 0,020716 & 0,029557 \\
\hline QU03C9 = non sa & 0,00787 & 0,031847 & 0,014963 & 0,012987 & 0,01288 & 0,014546 \\
\hline QU03A10 = per nulla importante & 0,00809 & 0,016629 & 0,01412 & 0,014336 & 0,014752 & 0,020565 \\
\hline QU03A10 = poco importante & 0,00816 & 0,016032 & 0,017723 & 0,012887 & 0,013127 & 0,020233 \\
\hline QU03A10 = indifferente & 0,01195 & 0,023934 & 0,024645 & 0,02385 & 0,024772 & 0,02167 \\
\hline QU03A10 = importante & 0,01316 & 0,02633 & 0,028262 & 0,028199 & 0,025781 & 0,025213 \\
\hline QU03A10 = molto impo & 0,01637 & 0,031795 & 0,034128 & 0,033195 & 0,030913 & 0,034937 \\
\hline QU03A10 = non sa & 0,01012 & 0,024149 & 0,019907 & 0,017854 & 0,020421 & 0,020233 \\
\hline QU03B10 $=0 \%$ & 0,01635 & 0,03313 & 0,033928 & 0,032434 & 0,031353 & 0,034193 \\
\hline QU03B10 = 25\% & 0,01251 & 0,024274 & 0,026413 & 0,028401 & 0,023571 & 0,026036 \\
\hline QU03B10 $=50 \%$ & 0,01182 & 0,022865 & 0,025928 & 0,021008 & 0,021928 & 0,024788 \\
\hline QU03B10 $=75 \%$ & 0,01067 & 0,019443 & 0,022149 & 0,023199 & 0,020295 & 0,022539 \\
\hline
\end{tabular}


STIMA DEGLI ERRORI DI CAMPIONAMENTO DELL'INDAGINE

\begin{tabular}{|c|c|c|c|c|c|c|}
\hline QU03B10 = 100\% & 0,00861 & 0,016032 & 0,012535 & 0,019301 & 0,018528 & 0,019148 \\
\hline QU03B10 = non sa & 0,00676 & 0,013045 & 0,013943 & 0,011217 & 0,014117 & 56 \\
\hline QU03B10 = non vuole rispo & 0,0067 & & & 0,004636 & & \\
\hline QU03C10 = insoddisfatto & 0,0117 & 0,018763 & 0,012418 & 0,025343 & 0,024159 & 0,021543 \\
\hline QU03C10 = poco insoddisfatto & 0,01602 & 0,030924 & 0,028055 & 0,023335 & 0,033177 & 0,036053 \\
\hline QU03C10 = indifferente & 0,02032 & 0,042549 & 0,044276 & 0,03445 & 036 & 795 \\
\hline QU03C10 = abbastanza sc & 0,02086 & 0,044783 & 0,045617 & 0,041833 & 0,040608 & 0,040323 \\
\hline QU03C10 = soddisfatto & 0,016 & 0,038254 & 0,028021 & 0,03496 & 0,03132 & 0,03661 \\
\hline QU03C10 = non sa & 0,01142 & 0,026305 & 0,026538 & 0,018382 & 0,019437 & 0,024779 \\
\hline QU03C10 = non vuole rispondere & 0,0113 & & & 0,007658 & & \\
\hline QU03A11 = per nulla importante & 0,00816 & 724 & 378 & 0,016788 & 862 & 198 \\
\hline QU03A11 = poco in & 0,00841 & 0,01825 & 0,01737 & 0,014997 & 0,015173 & 0,019153 \\
\hline QU03A11 = indifferente & 0,01348 & 0,024543 & 0,026806 & 0,027109 & 0,027285 & 0,027138 \\
\hline QU03 & 0,01419 & 0,028285 & 0,030128 & 0,029841 & 0,027937 & 427 \\
\hline QU03A11 = molto impor & 0,01558 & 0,029278 & 0,032659 & 0,030156 & 0,028955 & 0,034047 \\
\hline QU03 & 0,00903 & 0,02485 & 0,020459 & 183 & 654 & 397 \\
\hline QU03A11 = non vuc & 0,00832 & & & 0,004636 & & \\
\hline QU03 & 588 & 077 & 887 & 252 & 943 & 298 \\
\hline QU03B & 358 & 0,025363 & 0,027306 & 6633 & 749 & 0,027066 \\
\hline QU03 & & 0,025094 & 303 & 0,025332 & 075 & 416 \\
\hline QU03 & 077 & 361 & 908 & 2152 & 553 & 968 \\
\hline QU03 & 0,00677 & 0,007874 & 0,01486 & 0,004636 & 867 & 649 \\
\hline QU03 & 0,00651 & 831 & 717 & 217 & & 926 \\
\hline QU03 & 0,00677 & & & 636 & & \\
\hline QU03C11 = insoddis & 0,01157 & 0,021282 & 0,013875 & & 388 & 0,01806 \\
\hline QU03 & 0,01484 & 855 & 0,024955 & 0,026645 & 245 & 4212 \\
\hline QU03C11 = indifferente & & 0,047739 & & 0,04468 & 3372 & 715 \\
\hline QU03C11 = abbas & 0,02044 & 0,044603 & 556 & 0,045844 & 636 & 831 \\
\hline QU03C11 = soddisfatto & & 0,029306 & & 0,024067 & 1639 & \\
\hline QU03C11 = non sa & 0,01168 & 0,031684 & 0,024955 & 044 & 529 & 479 \\
\hline QU & & & & & & \\
\hline QU03A12 = per nulla importar & 737 & 0,012642 & 0,014883 & 4336 & 958 & 662 \\
\hline QU03 & & & & & & \\
\hline QU03A12 = indifferente & 0,01278 & 0,025736 & 0,025399 & 0,026386 & 0,025423 & 0,026227 \\
\hline QU03A12 = importante & 0,01456 & 0,02882 & 0,030297 & 0,031126 & 0,02841 & 0,029258 \\
\hline QU03A12 = molto impo1 & 0,01588 & 0,031825 & 0,033166 & 0,030735 & 0,029693 & 0,034308 \\
\hline QU03A12 = non sa & 0,01083 & 0,021611 & 0,023235 & 0,018355 & 0,019539 & 0,024127 \\
\hline QU03A12 = non vuc & 0,00551 & & & & 0,008332 & \\
\hline QU03C12 = insoddisfatto & 0,00639 & 0,010038 & 0,01412 & 0,011217 & 0,012677 & 0,012033 \\
\hline QU03C12 = poco insoddis & 0,00878 & 0,017088 & 0,015441 & 0,015624 & 0,018097 & 0,01918 \\
\hline QU03C12 = indifferente & 0,01459 & 0,028868 & 0,030874 & 0,02879 & 0,027479 & 0,03076 \\
\hline QU03C12 = abbastanza sc & 0,01431 & 0,027455 & 0,030128 & 0,027562 & 0,027467 & 0,029663 \\
\hline QU03C12 = soddisfatto & 0,00971 & 0,021514 & 0,019398 & 0,020602 & 0,019462 & 0,019178 \\
\hline QU03C12 = non sa & 0,01512 & 0,030588 & 0,030979 & 0,031594 & 0,028428 & 0,032774 \\
\hline QU03A13 = per nulla importante & 0,00405 & & 0,006724 & 0,004636 & 0,022684 & 0,007025 \\
\hline QU03A13 = poco importante & 0,00629 & 0,009153 & 0,010675 & 0,009204 & 0,009441 & 0,010982 \\
\hline
\end{tabular}




\begin{tabular}{|c|c|c|c|c|c|c|}
\hline QU03A13 = indifferente & 0,01079 & 0,021514 & 0,015768 & 0,019301 & 0,02419 & 0,022502 \\
\hline QU03A13 = importante & 0,01502 & 0,029672 & 0,031325 & 0,030307 & 0,028056 & 0,032478 \\
\hline QU03A13 = molto importante & 0,01645 & 0,032979 & 0,033469 & 0,03278 & 0,031715 & 0,034849 \\
\hline QU03A13 = non sa & 0,01002 & 0,011777 & 0,026318 & & 0,010046 & 0,013617 \\
\hline QU03B13 $=0 \%$ & 0,00542 & 0,011543 & 0,010675 & 0,009204 & 0,013744 & 0,007025 \\
\hline QU03B13 $=25 \%$ & 0,01081 & 0,024423 & 0,024019 & 0,01975 & 0,022992 & 0,021497 \\
\hline QU03B13 $=50 \%$ & 0,01527 & 0,029461 & 0,029175 & 0,030307 & 0,029749 & 0,03382 \\
\hline QU03B13 = 75\% & 0,01546 & 0,030487 & 0,032998 & 0,031594 & 0,029424 & 0,03169 \\
\hline QU03B13 = $100 \%$ & 0,01403 & 0,026983 & 0,029471 & 0,02879 & 0,026483 & 0,029701 \\
\hline QU03B13 = non sa & 0,00709 & 0,007874 & 0,006724 & 0,004636 & 0,01463 & 0,007025 \\
\hline QU03C13 = insoddisfatto & 0,00694 & 0,010926 & 0,014438 & 0,013912 & 0,012735 & 0,015559 \\
\hline QU03C13 = poco insoddisfatto & 0,00982 & 0,021467 & 0,016525 & 0,016546 & 0,018809 & 0,023956 \\
\hline QU03C13 = indifferente & 0,0139 & 0,031073 & 0,028454 & 0,029481 & 0,027089 & 0,027947 \\
\hline QU03C13 = abbastanza sod & 0,01638 & 0,031506 & & 0,032578 & 0,031175 & 0,034423 \\
\hline QU03C13 = soddisfatto & 0,01426 & 0,027558 & 0,030732 & 0,029663 & 0,026385 & 0,029982 \\
\hline QU03C13 = non sa & 0,00838 & & 0,006861 & & 0,008139 & \\
\hline QU04A14 = per null & 0,00531 & 0,01213 & 0,009561 & 086 & 0,010883 & 988 \\
\hline QU04A14 = poco ir & 0,00634 & & 0,012569 & 0,016788 & 0,01 & 0,013816 \\
\hline QU04A14 = indifferente & 0,01322 & 0,024423 & 0,028206 & 0,024162 & 0,024592 & 0,028492 \\
\hline QU04 & & & & & & \\
\hline QU04A14 = molto impor & 0,01599 & 0,032183 & 0,033286 & 0,032652 & 0,030399 & 0,033663 \\
\hline QU04 & & & & & & \\
\hline QU04B14 = $0 \%$ & 0,01337 & 0,027223 & 0,029122 & 0,02879 & 0,024958 & 0,027261 \\
\hline QU04 & & & & 0,030307 & 0,027996 & \\
\hline QU04B14 $=50 \%$ & 0,01433 & 0,027916 & 0,028759 & 0,027109 & 0,027887 & 0,030539 \\
\hline QU04B14 = 75\% & 0,01235 & 0,027916 & 0,025594 & 0,024162 & 0,023113 & 0,026446 \\
\hline QU04B14 = 100\% & 0,01013 & 0,017209 & 0,022241 & 0,019301 & 0,018759 & 0,02139 \\
\hline QU04B14 = non sa & 0,00508 & 0,006317 & 0,008276 & 0,004636 & 0,009064 & 0,009906 \\
\hline QU04C14 = insoddisfatto & 0,00787 & 0,015926 & 0,015199 & 0,019996 & 0,014955 & 0,017029 \\
\hline QU04C14 = poco insoddisf & 0,0128 & 0,02685 & 0,026794 & 0,019996 & 0,024037 & 0,027323 \\
\hline QU04C14 = indifferente & 0,01672 & 0,034819 & 0,036487 & 0,033792 & 0,031516 & 0,033401 \\
\hline QU04C14 = abbastanza soc & 0,01759 & 0,034091 & 0,036331 & 0,037684 & 0,034575 & 0,034922 \\
\hline QU04C14 = soddisfatto & 0,01511 & 0,029263 & 0,030747 & 0,03256 & 0,027525 & 0,033855 \\
\hline QU04C14 = non sa & 0,00673 & 0,012883 & 0,010815 & 0,006235 & 0,018355 & 0,012202 \\
\hline QU04A15 = per null & 0,00971 & 0,019799 & 0,017222 & 0,020183 & 0,018428 & 0,023212 \\
\hline QU04A15 = poco importante & 0,00855 & 0,020254 & 0,01486 & 0,018355 & 0,016293 & 0,020233 \\
\hline QU04A15 = indifferente & 0,01412 & 0,026845 & 0,027509 & 0,026132 & 0,028387 & 0,029493 \\
\hline QU04A15 = importante & 0,01441 & 0,026667 & 0,031933 & 0,028598 & 0,026895 & 0,029354 \\
\hline QU04A15 = molto impor & 0,01432 & 0,028448 & 0,030128 & 0,029841 & 0,026861 & 0,030368 \\
\hline QU04A15 = non sa & 0,00985 & 0,023037 & 0,021752 & 0,020602 & 0,018565 & 0,019089 \\
\hline QU04B15 = 0\% & 0,01602 & 0,03281 & 0,033514 & 0,032652 & 0,029848 & 0,034489 \\
\hline QU04B15 = 25\% & 0,01406 & 0,028063 & 0,029562 & 0,026874 & 0,026057 & 0,030566 \\
\hline QU04B15 $=50 \%$ & 0,01344 & 0,026854 & 0,027998 & 0,025332 & 0,026591 & 0,026718 \\
\hline QU04B15 $=75 \%$ & 0,01003 & 0,017337 & 0,019907 & 0,023199 & 0,019872 & 0,020702 \\
\hline QU04B15 = 100\% & 0,0074 & 0,010257 & 0,012354 & 0,014336 & 0,017598 & 0,012055 \\
\hline QU04B15 = non sa & 0,0058 & 0,010038 & 0,013395 & 0,012086 & 0,014117 & 0,012033 \\
\hline
\end{tabular}


STIMA DEGLI ERRORI DI CAMPIONAMENTO DELL'INDAGINE

\begin{tabular}{|c|c|c|c|c|c|c|}
\hline QU04C15 = insoddisfatto & 0,00883 & 0,011361 & 0,01844 & 0,015504 & 0,016708 & 0,018823 \\
\hline QU04C15 = poco insoddisfatto & 0,017 & 0,041987 & 0,034983 & 0,030182 & 0,029518 & 0,039709 \\
\hline QU04C15 = indifferente & 0,02064 & 0,039962 & 0,041938 & 0,040455 & 0,038674 & 0,044378 \\
\hline QU04C15 = abbastanza soddisfatto & 0,01888 & 0,035666 & 0,039915 & 0,041282 & 0,034974 & 0,03913 \\
\hline QU04C15 = soddisfatto & 0,01317 & 0,032875 & 0,022784 & 0,030911 & 0,026519 & 0,027174 \\
\hline QU04C15 = non sa & 0,00999 & 0,017435 & 0,021554 & 0,01883 & 0,013355 & 0,01673 \\
\hline QU06aA17 = per nulla & 0,00677 & 0,017209 & 0,015564 & 0,010265 & 0,011983 & 0,013856 \\
\hline QU06aA17 = poco importante & 0,0061 & 0,007543 & 0,011524 & 0,007991 & 0,013179 & 0,012067 \\
\hline QU06aA17 = indifferente & 0,01054 & 0,018961 & 0,019992 & 0,017854 & 0,018633 & 0,021665 \\
\hline QU06aA17 = importante & 0,01358 & 0,029337 & 0,027038 & 0,026386 & 0,02783 & 0,026199 \\
\hline QU06aA17 = molto & 0,01647 & 0,032517 & 0,033976 & 0,032003 & 0,031654 & 684 \\
\hline QU06aA17 = non sa & 0,00729 & 0,01413 & 0,016807 & 0,014336 & 0,010179 & 0,018562 \\
\hline QU06aB17 $=0 \%$ & 0,01444 & 632 & 0,032167 & 606 & 0,026415 & 009 \\
\hline QU06 & & 0,021243 & 0,018996 & 0,021401 & 0,021201 & 998 \\
\hline QU06 & 201 & 919 & 019 & 401 & 571 & 575 \\
\hline QU06 & & & & & & \\
\hline QU06 & 456 & 987 & 802 & 596 & 241 & 466 \\
\hline & & & & & & \\
\hline QU06 & 22 & 495 & 721 & 593 & 054 & \\
\hline & & & & & & \\
\hline QU06: & 0,01543 & 0,03 & 132 & 398 & 173 & 075 \\
\hline QU0C & & & & & & \\
\hline QU06aC17 $=\mathrm{s}$ & 0,01703 & 503 & 753 & 237 & 617 & 947 \\
\hline QU06 & & 529 & & 2593 & & \\
\hline QU06aA18 = p & 0,00567 & & 0,010029 & & 652 & \\
\hline QU06aA18 = poco importante & 0,00653 & & 0,017275 & & 0,011652 & 138 \\
\hline QU06aA18 = i & 0,00664 & 0,018426 & 0,019724 & 57 & 338 & 0,01138 \\
\hline QU06aA18 = importante & 0,01605 & 0,035828 & 0,036729 & 0,031858 & 0,030033 & 0,031215 \\
\hline QU06aA18 = n & 738 & 0,038079 & 215 & 285 & 157 & 436 \\
\hline QU06 & 585 & 629 & 328 & 037 & 0,011652 & 0,0113 \\
\hline QU06aB18 = 0\% & 0,00708 & 0,016119 & 0,019399 & & & 0,01138 \\
\hline QU & 03 & 0,0 & 035 & 688 & 605 & 582 \\
\hline QU06aB18 = 50\% & 0,01213 & 0,029561 & 0,028403 & & 0,02105 & 0,025634 \\
\hline QU06 & 0,01767 & 0,035425 & 814 & 0,035937 & 3214 & 037 \\
\hline QU06aB18 = 100\% & & & 0,040662 & 0,036971 & 0,035729 & 0,040178 \\
\hline QU06aB18 = non sa & 0,00659 & 0,013578 & 0,010029 & 0,008037 & 0,013708 & 0,0113 \\
\hline QU06aC18 = insoddisfatto & 0,00648 & 0,01146 & 0,015926 & 0,011298 & 0,013902 & \\
\hline QU06aC18 = poco insoddisfatto & 0,00735 & 0,013478 & 0,018891 & 0,014809 & 0,013239 & 329 \\
\hline QU06aC18 = indifferente & 0,01492 & 0,032856 & 0,036155 & 0,025483 & 0,024886 & 0,032662 \\
\hline QU06aC18 = abbastanza soddisfatto & 0,0194 & 0,040786 & 0,042122 & 0,036444 & 0,036952 & 0,039676 \\
\hline QU06aC18 = soddisfatto & 0,01793 & 0,038776 & 0,037379 & 0,036157 & 0,032718 & \\
\hline QU06aC18 = non sa & 0,00851 & 0,0142 & 0,012388 & 0,008037 & 0,021534 & 0,011532 \\
\hline QU06aA19= per nulla importante & 0,00708 & 0,009164 & 0,012328 & & 0,012808 & 0,017122 \\
\hline QU06aA19 = poco importante & 0,00849 & 0,011393 & 0,017236 & 0,012593 & 0,014239 & 0,021941 \\
\hline QU06aA19 = indifferente & 0,01275 & 0,024545 & 0,026288 & 0,022927 & 0,02172 & 0,026187 \\
\hline QU06aA19 = importante & 0,01766 & 0,038516 & 0,03911 & 0,033079 & 0,033279 & 0,035618 \\
\hline
\end{tabular}




\begin{tabular}{|c|c|c|c|c|c|c|}
\hline QU06aA19 = molto importante & 0,01926 & 0,040093 & 0,041977 & 0,036924 & 0,035792 & 0,04044 \\
\hline QU06aA19 = non sa & 0,00778 & 0,015548 & 0,017275 & 0,012593 & 0,013999 & 0,017122 \\
\hline QU06aB19 = 0\% & 0,01293 & 0,027542 & 0,028418 & 0,025008 & 0,022548 & 0,02922 \\
\hline QU06aB19 $=25 \%$ & 0,01525 & 0,032228 & 0,032485 & 0,028763 & 0,029164 & 0,031198 \\
\hline QU06aB19 $=50 \%$ & 0,01523 & 0,03603 & 0,032956 & 0,028021 & 0,027889 & 0,032512 \\
\hline QU06aB19 = 75\% & 0,01722 & 0,033469 & 0,038 & 0,035137 & 0,03277 & 0,034303 \\
\hline QU06aB19 $=100 \%$ & 0,01545 & 0,027912 & 0,033795 & 0,02723 & 0,029148 & 0,032232 \\
\hline QU06aB19= non sa & 0,00585 & 0,014629 & 0,010029 & 0,009814 & 0,017496 & 0,012927 \\
\hline QU06aC19 = insoddisfatto & 0,00945 & 0,014313 & 0,025602 & 0,009282 & 0,014698 & 0,020849 \\
\hline QU06aC19 = poco insoddisfatto & 0,01244 & 0,028426 & 0,025602 & 0,026265 & 0,019953 & 0,031835 \\
\hline QU06aC19= indifferente & 0,01826 & 0,039519 & 0,041878 & 0,033785 & 0,033775 & 0,036348 \\
\hline QU06aC19 = abbastanza so & 0,02017 & 0,041463 & 0,043566 & 0,038773 & 0,037847 & 0,041853 \\
\hline QU06aC19 = soddisfatto & 0,01696 & 0,035453 & 0,035736 & 0,033785 & 0,030558 & 0,038121 \\
\hline QU06aC19= non sa & 0,00539 & 0,014852 & 0,021405 & 0,013036 & 0,012059 & 0,01704 \\
\hline $0=$ per null & 0,00987 & 0,020629 & 0,01974 & 0,014809 & 0,016204 & 0,025585 \\
\hline QU06aA20 = poco im & 0,01016 & 0,02147 & 0,020817 & 0,016688 & 0,017805 & 0,024359 \\
\hline QU06aA20 = indiffe & 0,01491 & 0,028471 & 0,030158 & 0,030733 & 0,027418 & 0,033662 \\
\hline QU06aA20 = imp & 0,01699 & 0,038911 & 0,037713 & 0,032614 & 0,032512 & 0,032909 \\
\hline QU06aA20 = molto impo & 0,01822 & 0,034523 & 0,040662 & 0,035694 & 0,034495 & 0,036453 \\
\hline QU06a & 0,01146 & 0,023318 & 0,01974 & 0,018333 & 0,019831 & 0,02 \\
\hline QU06 & & 0,039157 & 0,036334 & 427 & 0,034274 & \\
\hline QU06aB20 = 25\% & 0,01481 & 0,031304 & 0,031389 & 0,029461 & 0,028587 & 0,029799 \\
\hline $0=50 \%$ & 0,0136 & 0,032292 & 0,030246 & 0,025483 & 0,023738 & 0,030159 \\
\hline QU06aB20 = 75\% & 0,01512 & 0,025865 & 0,035611 & 0,031589 & 0,030071 & 0,025299 \\
\hline QU06aB20 $=100 \%$ & 0,01248 & 0,021547 & 0,030918 & 0,018333 & 0,022459 & 0,02491 \\
\hline QU06aB20 = non sa & 0,00787 & 0,016119 & 0,024312 & 0,009814 & 0,01 & 0,014493 \\
\hline QU06aC20 = insoddisfatto & 0,01189 & 0,030052 & 0,021495 & 0,01523 & 0,020301 & 0,025081 \\
\hline QU06aC20 $=$ poco insoddis & & & & & & \\
\hline QU06aC20 = indifferente & 0,02192 & 0,050273 & 0,046435 & 0,043212 & 0,039013 & 0,04 \\
\hline QU06aC20 = abbast & & & & & & \\
\hline QU06aC20 = soddisfatto & 0,01727 & 0,037676 & 0,037639 & 0,039187 & 0,031219 & 0,03 \\
\hline QU0C & & & & 504 & & \\
\hline QU07A21 = per nulla importante & 0,01007 & 0,013218 & 0,024558 & 0,016688 & 0,014635 & 0,018255 \\
\hline ortante & 0,00727 & & 0,019116 & 0,008037 & 0,012808 & 0,013097 \\
\hline QU07A21 = indifferente & 0,01155 & 0,019647 & 0,022625 & 0,020485 & 0,023354 & 0,023635 \\
\hline QU07A21 = importante & 0,0163 & 0,03409 & 0,038113 & 0,030429 & 0,028602 & 0,034712 \\
\hline QU07A21 = molto impo1 & 0,01913 & 0,039027 & 0,040603 & 0,036609 & 0,035873 & 0,040641 \\
\hline QU07A21 = non sa & 0,00982 & 0,017457 & 0,023798 & 0,016688 & 0,015837 & 0,022662 \\
\hline QU07B21 $=0 \%$ & 0,01772 & 0,03098 & 0,038422 & 0,033079 & 0,032551 & 0,038672 \\
\hline QU07B21 $=25 \%$ & 0,01435 & 0,029527 & 0,03432 & 0,021758 & 0,026495 & 0,028186 \\
\hline QU07B21 $=50 \%$ & 0,01385 & 0,02902 & 0,027739 & 0,026815 & 0,026213 & 0,030287 \\
\hline QU07B21 $=75 \%$ & 0,01508 & 0,032874 & 0,030918 & 0,031858 & 0,029926 & 0,029286 \\
\hline QU07B21 = 100\% & 0,01388 & 0,035141 & 0,028788 & 0,029794 & 0,026413 & 0,028122 \\
\hline QU07B21 = non sa & 0,00802 & 0,015172 & 0,020864 & 0,013753 & 0,012949 & 0,017023 \\
\hline QU07C21 = insoddisfatto & 0,01328 & 0,016247 & 0,019615 & 0,013595 & 0,018248 & 0,022518 \\
\hline QU07C21 = poco insoddisfatto & 0,01255 & 0,022573 & 0,02607 & 0,013595 & 0,021715 & 0,033123 \\
\hline
\end{tabular}


STIMA DEGLI ERRORI DI CAMPIONAMENTO DELL'INDAGINE

\begin{tabular}{|c|c|c|c|c|c|c|}
\hline QU07C21 = indifferente & 0,01797 & 0,030012 & 0,037193 & 0,03185 & 0,03527 & 0,038614 \\
\hline QU07C21 = abbastanza soddisfatto & 0,02145 & 0,043468 & 0,048399 & 0,042243 & 0,039201 & 0,046434 \\
\hline QU07C21 = soddisfatto & 0,02137 & 0,041808 & 0,045319 & 0,04299 & 0,040426 & 0,046424 \\
\hline QU07C21 = non sa & 0,01089 & 0,018518 & 0,027803 & 0,018984 & 0,017031 & 0,026163 \\
\hline QU07A22 = per nulla importante & 0,00884 & 0,013218 & 0,018566 & 0,019804 & 0,01516 & 0,025493 \\
\hline QU07A22 = poco importante & 0,0106 & 0,013218 & 0,017275 & 0,011298 & 0,011652 & 0,015876 \\
\hline QU07A22 = indifferente & 0,01539 & 0,025452 & 0,028345 & 0,030733 & 0,031567 & 0,031665 \\
\hline QU07A22 = importante & 0,01608 & 0,036581 & 0,037678 & 0,03285 & 0,029742 & 0,030768 \\
\hline QU07A22 = molto impor & 0,01777 & 0,037089 & 0,039864 & 0,031313 & 0,032649 & 0,037244 \\
\hline QU07A22 = non sa & 0,01521 & 0,034418 & 0,030179 & 0,028763 & 0,02784 & 0,034837 \\
\hline QU07B22 $=0 \%$ & 0,01905 & 0,039742 & 0,04039 & 0,037081 & 0,035844 & 0,040126 \\
\hline QU07B22 = 25\% & 0,01478 & & 0,031474 & 0,025483 & 0,028456 & 0,0 \\
\hline QU07B22 = 50\% & 0,01473 & 0,032292 & 0,033795 & 0,026815 & 0,026836 & 0,030245 \\
\hline QU07 & 0,01246 & 0,026057 & 0,027024 & 0,022354 & 0,024506 & 0,023958 \\
\hline QU07B22 = 100\% & 0,00827 & 0,017241 & 0,022468 & 0,013753 & 0,018126 & 0,012927 \\
\hline $2=$ non sa & 0,00899 & 0,016357 & 0,020876 & 0,014809 & 0,014239 & 0,021929 \\
\hline QU07C22 = insoddis & 0,01404 & & 0,023466 & 0,024403 & 0,032637 & 0,020958 \\
\hline $2=$ poco & 0,0161 & 0,026575 & 0,028093 & 578 & 0,034581 & 362 \\
\hline QU07C22 = indiff & 0,0244 & 0,049475 & 0,052008 & 0,051767 & 0,045421 & 0,049571 \\
\hline $2=$ abbastanza $\mathrm{s}$ & 0,02416 & 0,049716 & 0,048002 & 0,0 & 4952 & 983 \\
\hline QU07C22 = sodc & 0,01671 & 0,0401 & 0,033368 & 0,03163 & 0,032637 & 0,033273 \\
\hline QU07C22 = non sa & & 0,029187 & 0,030171 & 0,03163 & 0,028502 & 0,03 \\
\hline QU07A23 = per null & 0,00685 & 0,018262 & 0,012328 & 0,012593 & 7197 & 0,02 \\
\hline $23=$ poco importante & 0,00588 & 0,020597 & 328 & 298 & 963 & 306 \\
\hline QU07A23 = indifferente & 0,01501 & 0,030451 & 0,029696 & 0,030429 & 0,029434 & 0,031198 \\
\hline QU07A23 = impor & 0,01575 & 0,03266 & 0,036724 & 9461 & 0,028377 & 0,032482 \\
\hline QU07A23 = molto impo & 0,01701 & 0,034546 & 0,03862 & 0,03285 & 0,031433 & 0,034664 \\
\hline QU07A23 = non sa & 0,01749 & 0,038516 & 0,034833 & 0,033079 & 0,033013 & 0,038407 \\
\hline QU07C23 = insoddi & & & 0,017236 & & 0,021398 & \\
\hline QU07C23 = poco insoddisfatto & 0,00856 & 0,019313 & 0,020384 & 0,013753 & 0,017436 & 0,0 \\
\hline & & & & & & \\
\hline QU07C23 = abbastanza sc & 0,016 & 0,03229 & 0,038265 & 0,029461 & 0,027921 & 0,033669 \\
\hline QU0 & & & & & & \\
\hline QU07C23 = non sa & 0,01882 & 0,03953 & 0,038265 & 0,036682 & 0,035895 & 0,040127 \\
\hline QU07A24 = per nulla importante & 0,01123 & 0,023216 & 0,023728 & 0,019804 & 0,020134 & 0,025585 \\
\hline QU07A24 = poco importante & 0,01114 & 0,016183 & 0,023625 & 0,015782 & 0,019015 & 0,027618 \\
\hline QU07A24 = indifferente & 0,01391 & 0,02494 & 0,023746 & 0,030116 & 0,026588 & 0,032863 \\
\hline QU07A24 = importante & 0,0151 & 0,033703 & 0,035222 & 0,027632 & 0,028962 & 0,027595 \\
\hline QU07A24 = molto importante & 0,01652 & 0,033844 & 0,036988 & 0,032614 & 0,032022 & 0,031161 \\
\hline QU07A24 = non sa & 0,01602 & 0,037176 & 0,034219 & 0,031858 & 0,029448 & 0,034209 \\
\hline QU07B24 = 0\% & 0,01899 & 0,040307 & 0,041994 & 0,036529 & 0,036191 & 0,037586 \\
\hline QU07B24 = 25\% & 0,01339 & 0,0305 & 0,03146 & 0,025008 & 0,024834 & 0,025725 \\
\hline QU07B24 = 50\% & 0,01209 & 0,024706 & 0,028345 & 0,024006 & 0,023569 & 0,021185 \\
\hline QU07B24 = 75\% & 0,01038 & 0,022934 & 0,025126 & 0,018333 & 0,020011 & 0,017839 \\
\hline QU07B24 = 100\% & 0,00788 & 0,014495 & 0,017721 & 0,013753 & 0,015618 & 0,014493 \\
\hline QU07B24 = non sa & 0,01005 & 0,020485 & 0,021915 & 0,017535 & 0,018844 & 0,021014 \\
\hline
\end{tabular}


Appendice D

\begin{tabular}{|l|c|c|c|c|c|c|}
\hline QU07C24 = insoddisfatto & 0,01943 & 0,028248 & 0,019504 & 0,014278 & 0,030372 & 0,045893 \\
\hline QU07C24 = poco insoddisfatto & 0,01728 & 0,033802 & 0,03934 & 0,036071 & 0,0332 & 0,032803 \\
\hline QU07C24 = indifferente & 0,02757 & 0,053784 & 0,056011 & 0,058971 & 0,04993 & 0,064161 \\
\hline QU07C24 = abbastanza soddisfatto & 0,02632 & 0,055771 & 0,051014 & 0,052461 & 0,049197 & 0,0609 \\
\hline QU07C24 = soddisfatto & 0,02062 & 0,039722 & 0,040635 & 0,027919 & 0,037229 & 0,05255 \\
\hline QU07C24 = non sa & 0,02113 & 0,043949 & 0,03934 & 0,042057 & 0,038044 & 0,055176 \\
\hline
\end{tabular}




\section{Bibliografia $^{1}$}

ACRL \& ALA, 1995, Standards for college libraries 1995 edition. College and research libraries news, 56 (4), 245-257.

Agostini N, 2001, Se la biblioteca è orientata all'utente: la ricerca della qualità dei servizi attraverso l'uso strategico dei nuovi strumenti di programmazione e gestione dell'ente locale. Biblioteche oggi, 19 (2), 24-32.

Bellini P \& Rizzi I, 2001, ISO 11620. Stima della Target population. Indicatore B.1.1.1 - User satisfaction. Biblioteche oggi, 19 (1), 52-68.

Catinella B, Corbolante M \& Romeo MA, 2001, Indicatori di performance per biblioteche di ateneo. Un esperimento di descrizione statistica e un approccio alla valutazione presso l'Università di Padova. In appendice un saggio di Marina Duzzin. Roma, AIB.

Cicchitelli G, 20002, Probabilità e statistica. Rimini, Maggioli.

Chiaro M, 1996, I sondaggi telefonici. Roma, CISU.

Corbetta P, 2000, Metodologia e tecniche della ricerca sociale. Bologna, il Mulino.

Corbolante M, 2000, Monitorare la qualità. Modelli applicativi di misurazione dei servizi nelle biblioteche di università. Biblioteche oggi, 18 (2), 58-65.

Dautriat H, 199910, Il questionario. Guida per la preparazione e l'impiego nelle ricerche sociali di psicologia sociale e di mercato. Milano, F. Angeli.

Di Domenico G \& Rosco M, 1998, Comunicazione e marketing della biblioteca. La prospettiva del cambiamento per la gestione efficace dei servizi. Milano, Editrice Bibliografica.

Di Domenico G, 1996, Progettare la user satisfaction. Come la biblioteca efficace gestisce gli aspetti immateriali del servizio. Biblioteche oggi, 14 (9), 52-65.

Fabris G \& Rolando S (a cura di), 2000, La customer satisfaction nel settore pubblico. Milano, F. Angeli. Supplemento a: Rivista italiana di comunicazione pubblica, 1 (3).

Fink A \& Kosecoff J, 1985, How to conduct surveys. A step-by-step guide. Beverly Hills, Sage Publications.

1 Comprende le opere citate nei precedenti contributi e le opere consultate dagli autori ai fini delle attività di misurazione e valutazione dei servizi bibliotecari e dell'indagine sulla soddisfazione dell'utenza. L'ultima consultazione dei siti internet è stata effettuata il 24 febbraio 2004. 
Foglieni O (a cura di), 2002, Comunicare la biblioteca. Nuove strategie di marketing e modelli di interazione. Milano, Editrice Bibliografica.

Galluzzi A, 2001, La valutazione del reference nell'era digitale. Bibliotime, 4 (1), http://www.spbo.unibo.it/bibliotime/num-iv-1/galluzzi.htm

Galluzzi A, 2001, Strumenti di valutazione per i servizi digitali. Quali strategie in un contesto "ibrido"? Biblioteche oggi, 19 (10), 6-14.

Hayes BE, 2000, Misurare la soddisfazione dei clienti. Sviluppo, controllo, utilizzazione dei questionari, tecniche per l'analisi dei risultati, edizione italiana a cura di G Ughetto, prefazione di L Barda. Milano, F. Angeli.

IFLA \& AIB, 1999, Linee guida per la valutazione delle biblioteche universitarie: edizione italiana di Measuring quality. Roma, AIB.

IFLA, Poll R \& te Boekhorst P, 1996, Measuring Quality. International Guidelines for Performance Measurement in Academic Libraries. Munchen, New Providence, Paris, K. J. Saur.

ISO, 1998, International standard ISO 11620. Information and documentation - Library performance indicators. Information et documentation Indicateurs de performance des bibliothèques. Genève, ISO.

ISTAT, 1989, Manuale di tecniche di indagine. Roma, ISTAT (vol. 2, Il questionario: progettazione, redazione e verifica; vol. 3, Tecniche di somministrazione del questionario).

Maggino F, 1995, Il questionario. Aspetti metodologici, informatici e statistici. Firenze, Centro Editoriale Toscano.

Mamoli F, 2001, La gestione consapevole della biblioteca. Progetto riorganizzativo del sistema bibliotecario dell'Università di Parma. In: AIB 99. Atti del XLV Congresso nazionale dell'Associazione italiana biblioteche, Roma, 16-19 maggio 1999 (a cura di E Frustaci \& M Guerrini), pp. 73-81. Roma, AIB. Anche in: http://www.aib.it/aib/congr/co99 mamoli.htm.

Maraviglia MG (a cura di), 2001, Rapporto 1996-2000. http://www.unifi.it/ universita/biblioteche/rapporti/ rapporto96_00.pdf.

Ministero dell'Università e della Ricerca Scientifica e Tecnologica, 1999, Programma di ricerca Misurazione e valutazione delle biblioteche universitarie. Rapporto preliminare del gruppo di ricerca. http:/www.miur.it/ osservatorio/ricbibl.htm.

Negro G \& Susio B, 1998, La qualità totale nella pubblica amministrazione. Modelli ed esperienze. Milano, Il sole-24 ore.

Nelli R \& Sada E (a cura di), 1998, La ricerca sul profilo di immagine e customer satisfaction della Biblioteca dell'Università Cattolica di Milano. http://www3.unicatt.it/unicattolica/sistema_bibliotecario/Milano/Allega ti/1998prog2.pdf. 
Papa J \& Scimone T (a cura di), 2000, Sessione poster. Catalogo degli abstracts. Per il convegno CRUI "Le biblioteche accademiche del futuro. Idee, progetti, risorse". Roma, 22-23 maggio 2000. [Campobasso], Università degli Studi del Molise.

Pilia E, 1997, La misurazione dei servizi delle biblioteche dell'Università. Bollettino AIB, 37 (3), 281-326.

Redazione (a cura della), 1995, Dalla quantità alla qualità. Un'indagine condotta dall'AIB su un campione di 200 biblioteche pubbliche di base propone nuovi criteri valutativi. Biblioteche oggi, 13 (5), 8-15.

Revelli C, 1998, La valutazione del servizio. Una funzione cruciale del management che acquista sempre più rilevanza nella cultura della biblioteca. Biblioteche oggi, 16 (7), 32-39.

Sacchetti L \& Gava S, [2003], L'attenzione per l'utente: esperienze di focus group. Bologna, Università degli studi, Dipartimento di Scienze Statistiche, Biblioteca. http://consiglio.regione.emilia-romagna.it/biblio/incon tro17022003/attenzione_utente.pdf.

Sardelli A, 2001, Dalla certificazione alla Qualità Totale. Milano, Editrice Bibliografica.

Tammaro AM \& Landi F, 1997, La biblioteca universitaria quantificabile. Un modello operativo per la gestione consapevole del sistema bibliotecario d'ateneo. Biblioteche oggi, 15 (2), 66-77.

Tammaro AM (a cura di), 1995, Progetto di riorganizzazione del Sistema Bibliotecario d'Ateneo e sintesi delle relazioni dei coordinatori di polo (1995). http://www.unifi.it/universita/biblioteche/progetto/progetto.htm.

Tammaro AM (a cura di), 1997, Relazione sull'attività di coordinamento del Sistema Bibliotecario d'Ateneo: 1997. http://www.unifi.it/universita/bi blioteche/relazioni/relazione97.htm.

Tammaro AM (a cura di), 1997, Sistema Bibliotecario d'Ateneo: rapporto 1997 [Parte prima]. http://www.unifi.it/universita/biblioteche/rappor ti/rapporto97a.htm.

Tammaro AM (a cura di), 1998, Progetto di riorganizzazione del Sistema Bibliotecario d'Ateneo (1998). http://www.unifi.it/universita/bibliote che/progetto/progetto98.htm.

Tammaro AM, 1999, Document delivery come alternativa all'abbonamento. The digital library: challenges and solutions for the new millenium, Bologna, 17-18 giugno 1999. http://www.aib.it/aib/commiss/cnur/dlttam ma.htm.

Tammaro AM, 2000, Misurazione e valutazione della biblioteca digitale. Come definire i nuovi indicatori di rendimento. Biblioteche oggi, 18 (1), 66-70. 
UNI, 1996, Informazione e documentazione. Statistiche internazionali per le biblioteche. UNI EN ISO 2789. Milano, UNI.

Università degli Studi di Firenze, 1998, Rapporto 1998 del Nucleo di valutazione interna. Firenze, Università degli Studi. Anche in: http://www.uni fi.it/organizzazione/org ateneo/val int/cover.html.

Università degli Studi di Firenze, 2000, Progetto di Riorganizzazione del Sistema Bibliotecario di Ateneo (Aggiornamento 2000). http://www.uni fi.it/universita/biblioteche/progetto/progetto2000.htm.

Università degli Studi di Firenze, 2000, Rapporto 1999 del Nucleo di valutazione interna. Firenze, Università degli Studi. Anche in: http://www.uni fi.it/organizzazione/org_ateneo/val_int/1999/rap1999_index.html.

Università degli Studi di Firenze, 2000, Regolamento del Sistema Bibliotecario di Ateneo. http://www.unifi.it/statuto/biblio.html.

Università degli Studi di Firenze, 2001, Ordinanze e decreti. http://www.uni fi.it/universita/biblioteche/ordinanze/ordinanze.htm.

Università degli Studi di Firenze, 2001, Rapporto 2000. Nucleo di valutazione interna. Firenze, Università degli Studi. Anche in: http://www.unifi.it/ organizzazione/org_ateneo/val_int/2000/rap2000_index.html.

Università degli Studi di Firenze, 2002, Carta dei servizi all'utenza (approvata dalla Commissione Biblioteche il 26 settembre 2002). http://www.uni fi.it/biblioteche/servizi/carta.htm.

Università degli Studi di Firenze, 2002, Statuto dell'Università degli Studi di Firenze (Emanato con Decreto Rettorale n. 577 del 20.6.95 e pubblicato sulla G.U. n. 156 del 6.7.95 e modificato con Decreti Rettorali nn. 1012 del 25. 11. 96; n. 712 del 18. 05. 99; n. 916 del 26. 07. 2001; n. 300 del 15. 2. 2002 e n. 555 del 17. 06. 2002). http://www.unifi.it/statuto/in dex.html.

Ventura R, 1998, La customer satisfaction in biblioteca. Introduzione alla qualità totale nei servizi bibliotecari, per una biblioteconomia della qualità totale. Con un sondaggio svolto presso la Biblioteca Chelliana di Grosseto. Tesi di diploma in Biblioteconomia. Università degli Studi "La Sapienza" di Roma (Scuola Speciale per Archivisti e Bibliotecari).

Zammuner VL, [1998], Tecniche dell'intervista e del questionario. Bologna, il Mulino.

Zeithaml VA, Parasuraman A \& Berry LL, 1991, Servire qualità, edizione italiana a cura di Gramma. Milano, McGraw-Hill Libri Italia. 

Finito di stampare nel 2004 da

Global Print

Gorgonzola (MI) 